\title{
Die Grammatik prädikativer Ausdrücke im Polnischen und Russischen
}

\author{
Dissertation \\ zur Erlangung des philosophischen Doktorgrades
}

an der Philosophischen Fakultät

der Georg-August-Universität Göttingen

vorgelegt von

Hagen Pitsch

aus Magdeburg

Göttingen 2014 
1. Gutachter: Prof. Dr. Uwe Junghanns

2. Gutachterin: Prof. Dr. Gerhild Zybatow

3. Gutachter: Prof. Dr. Sascha Gaglia

Tag der mündlichen Prüfung: 28. Februar 2014 


\section{Inhaltsverzeichnis}

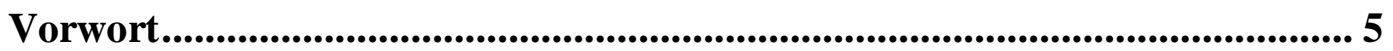

1. Einleitung...............................................................................................................8 8

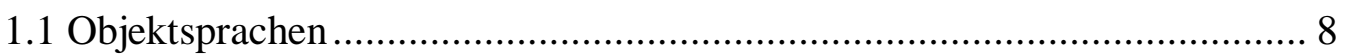

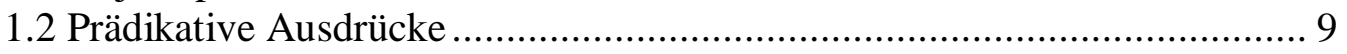

1.3 Variationen von Prädikativformen ........................................................ 11

1.4 Interpretative Effekte (kursorischer Forschungsstand)........................ 14

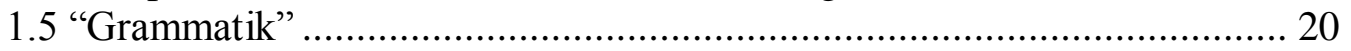

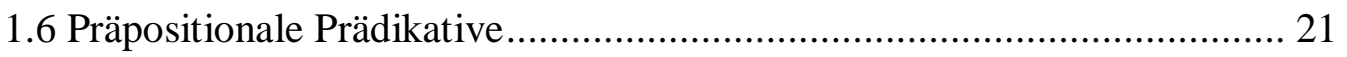

2. Bestandsaufnahme: Kopulasätze im Polnischen und Russischen ............... 24

2.1 Substantivische Prädikative ..................................................................... 24

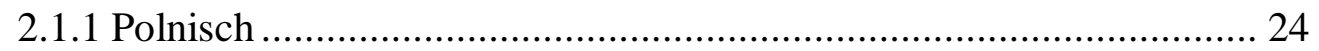

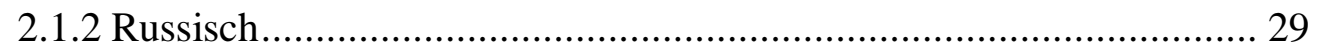

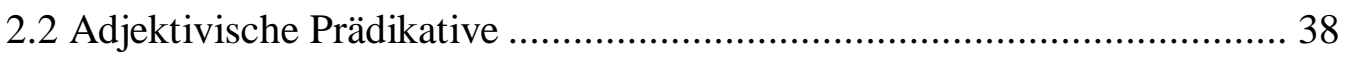

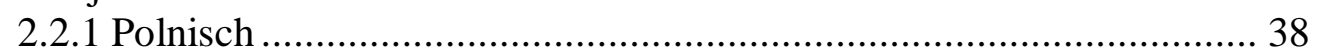

2.2.2 Russisch............................................................................ 44

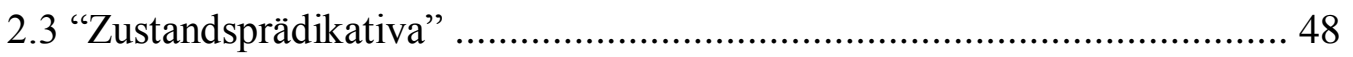

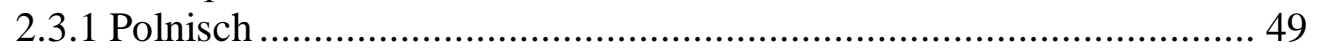

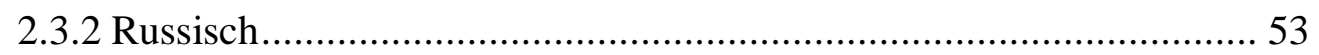

3. Besprechung relevanter Analysen zu den Variationen ............................... 57

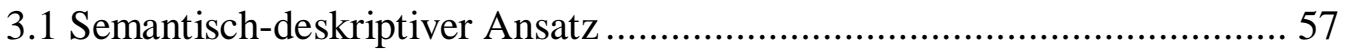

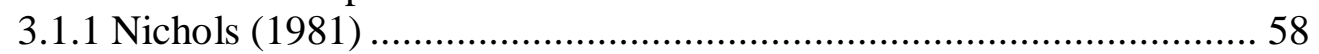

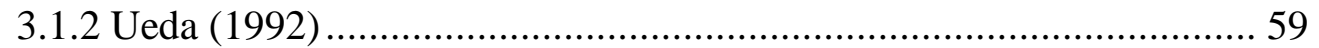

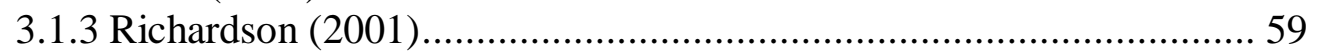

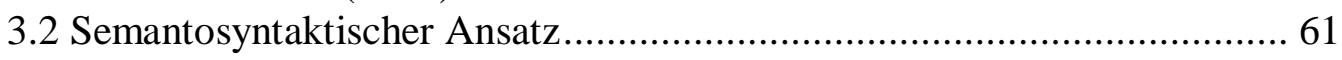

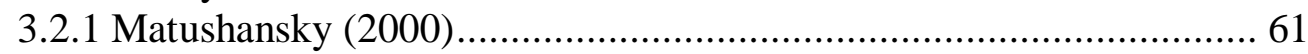

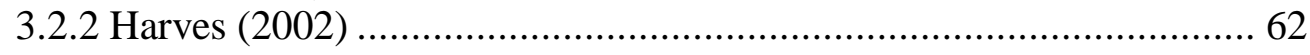

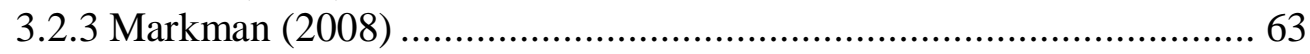

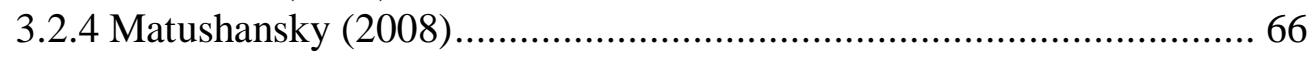

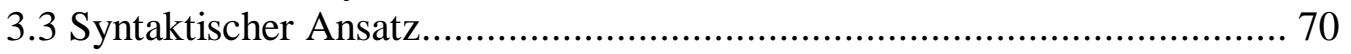

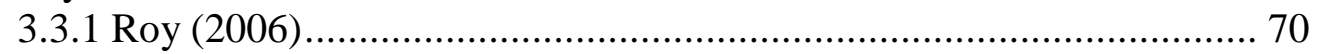

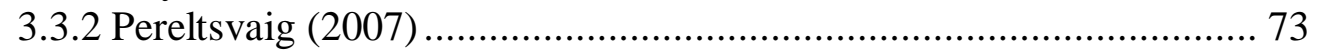

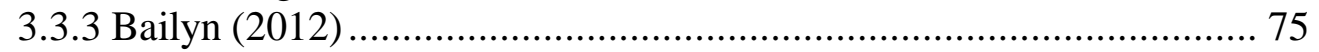

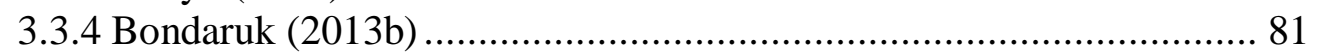

3.4 Semantopragmatischer Ansatz .................................................... 84

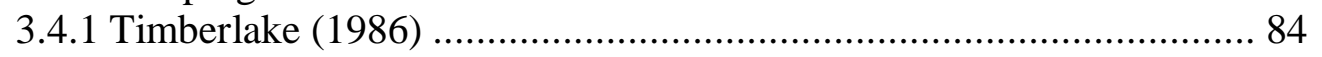

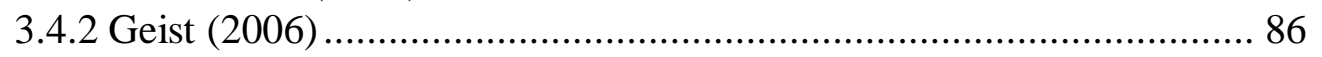

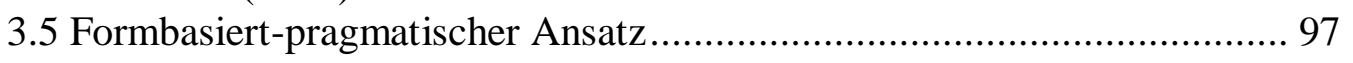

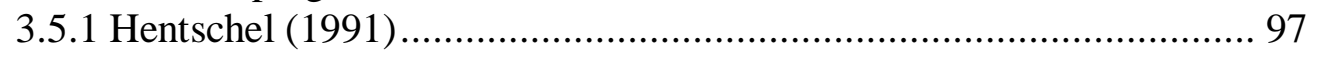

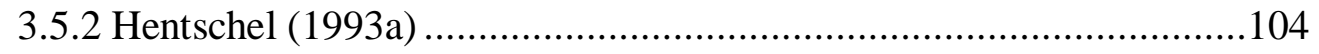

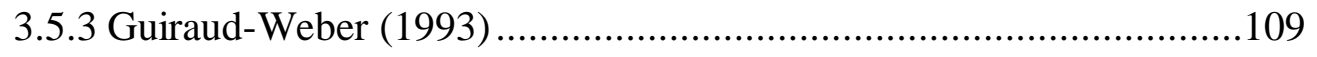

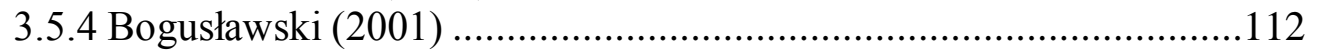

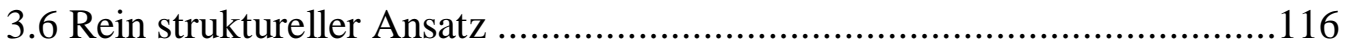

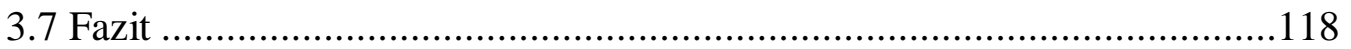


4. Theoretischer Rahmen ......................................................................120

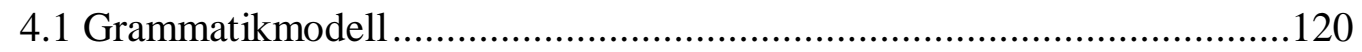

4.2 Mentales Lexikon und Lexikoneinträge ............................................123

4.3 Morphosyntaktische Merkmale .............................................................126

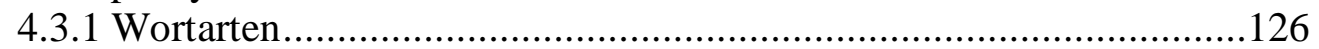

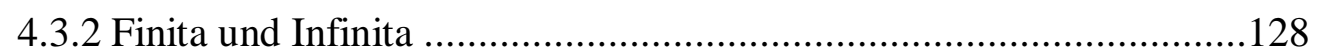

4.3.3 Aktiv und Passiv ........................................................................130

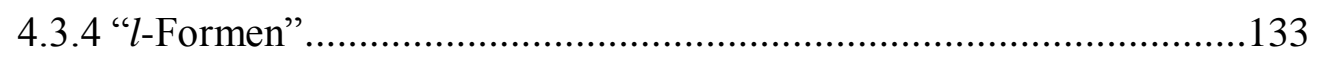

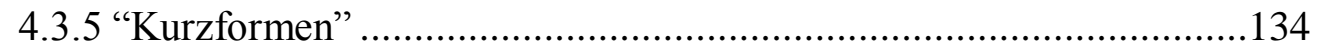

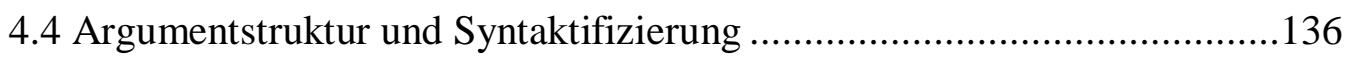

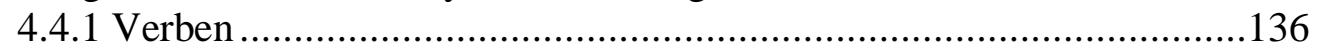

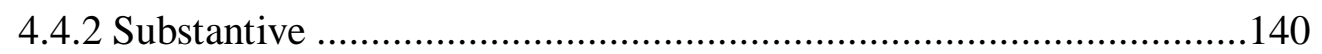

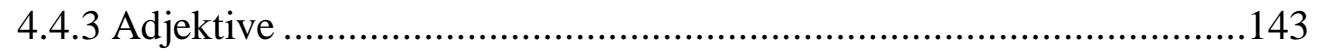

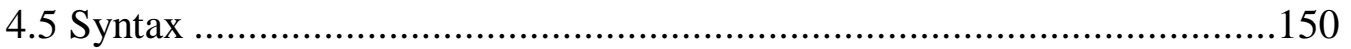

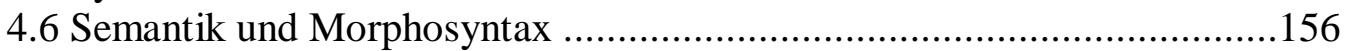

4.6.1 Flexionsmorphologie als Reflex semantischer Operatoren.................156

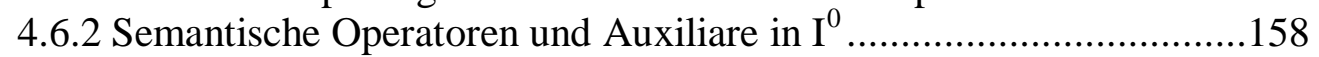

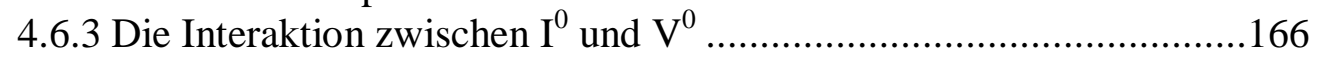

4.7 Zwei-Ebenen-Semantik............................................................... 175

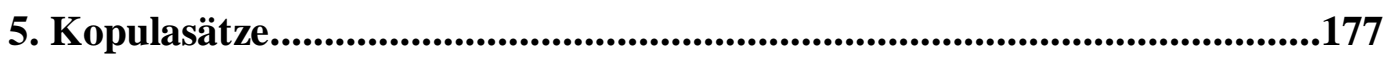

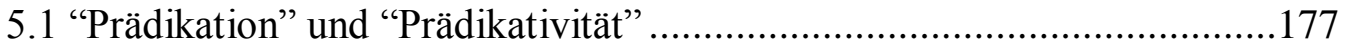

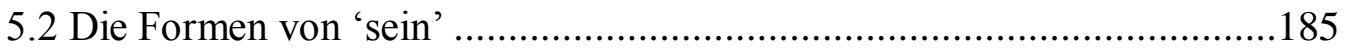

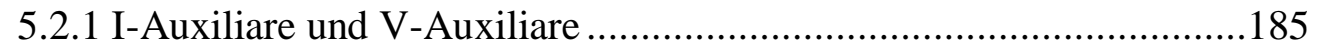

5.2.2 Bestand und Distribution der V-Auxiliare .....................................190

5.2.3 Exkurs: Vorgangspassiv und "Zustandspassiv" im Russischen .........196

5.2.4 Bestand und Distribution der I-Auxiliare........................................201

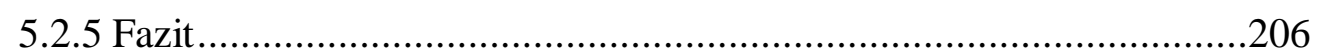

5.3 Primäre und sekundäre Prädikation .................................................2207

5.3.1 Primäre Prädikation ..................................................................207

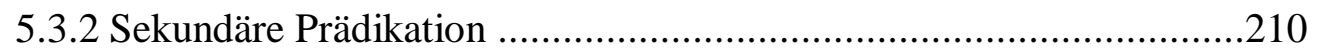

5.3.2.1 Generelle Annahmen ............................................................210

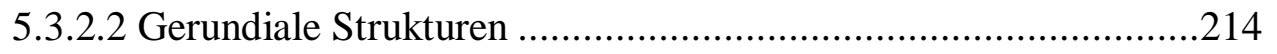

5.3.2.3 Strukturen ohne Gerundien..............................................218

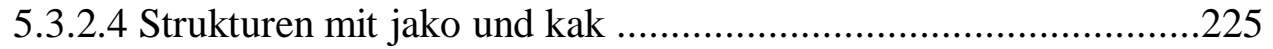

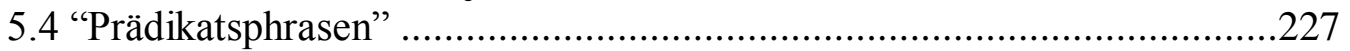

5.5 Kasuskongruenz und Kasuszuweisung ..................................................230

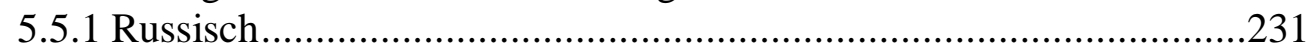

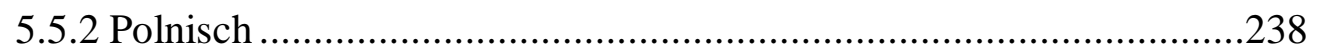

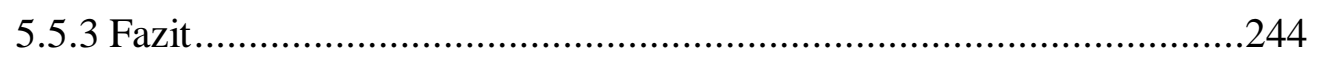

5.6 Die Gebrauchsweisen von (prädikativen) Adjektiven ............................245

5.6.1 Lang- und Kurzformen............................................................246

5.6.2 Mögliche syntaktische Strukturen ..............................................250

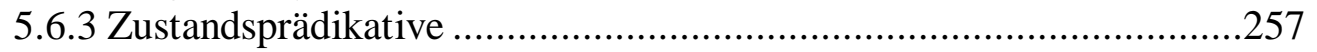


6. Der "formbasierte" Vorschlag .................................................................269

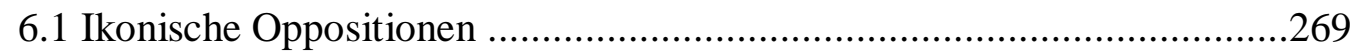

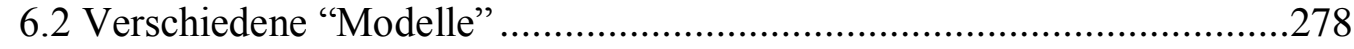

6.2.1 Prädikative Adjektive im Russischen ........................................278

6.2.2 Prädikative Substantive im Russischen.........................................286

6.2.3 Prädikative Adjektive im Polnischen .............................................2.289

6.2.4 Prädikative Substantive im Polnischen ...........................................290

7. Zusammenfassung und Ausblick .........................................................292

Abkürzungsverzeichnis .................................................................................295

Literaturverzeichnis ...................................................................................296

Quellenverzeichnis...........................................................................................317

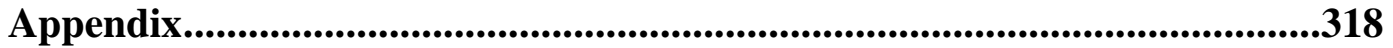




\section{Vorwort}

Die vorliegende Dissertationsschrift kann als Erweiterung des Themas der Magisterarbeit des Verfassers (Pitsch 2008) betrachtet werden, d. h. der Kasusvariation an Prädikatsnomina in russischen Kopulasätzen. Obwohl (oder weil) es sich um einen zentralen Bereich der russischen Grammatik handelt, mit dem sich im Laufe mehr als eines Jahrhunderts eine Vielzahl sprachwissenschaftlicher Arbeiten auseinandergesetzt hat, konnte die slavistische Linguistik in Bezug auf die Motivation, Funktion und (semantische/pragmatische/grammatische) 'Bedeutung' dieser Erscheinung bis heute keine allgemein akzeptierte Erklärung finden. Die vorgelegte Arbeit geht über das genannte Untersuchtungsobjekt jedoch in mehreren Punkten hinaus:

Zum ersten wird nicht nur das moderne Russische, sondern auch die moderne polnische Sprache behandelt, die ähnliche, jedoch keineswegs identische Variationen in Kopulasätzen kennt.

Zum zweiten geht es nicht lediglich um die erwähnte Kasusvariation (Nominativ vs. Instrumental), sondern auch um die Alternation zwischen sog. Kurz- und Langformadjektiven, die primär in russischen Kopulasätzen regelmäßig auftritt. Zum dritten werden nicht ausschließlich Kopulasätze thematisiert, die dem Ausdruck 'primärer' Prädikation dienen, sondern auch Strukturen, die als 'sekundäre' Prädikation bekannt sind oder mindestens dafür gehalten werden.

Zum vierten wird v. a. im Rahmen der Erörtung der theoretischen Grundannahmen dieser Arbeit der engere thematische Bereich der Kopulasätze verlassen und die "Grammatik" aller potenziell als (Satz-)Prädikat fungierender Ausdrücke betrachtet. Es wird also, mit anderen Worten, nicht nur um nominale, sondern auch um verbale Prädikatausdrücke gehen. Insbesondere wird hierbei das Verhältnis zwischen (Flexions-)Morphologie und Semantik (im Sinne von 'grammatischer Bedeutung') im Mittelpunkt stehen. So ist diese Arbeit auch als Beitrag zur Forschung über die nordslavische Satzstruktur, über deren internes Funktionieren und über die Schnittstelle zwischen Morphologie und Syntax bzw. zwischen Semantik und (Morpho-)Syntax zu verstehen.

Die Arbeit gliedert sich wie folgt:

In Abschnitt 1 werden die Themen der Arbeit definiert und besprochen, d. h. die Objektsprachen, der Begriff “prädikativer Ausdrucks”, das relevante Phänomen in 
polnischen und russischen Kopulasätzen sowie mein Begriff von "Grammatik". Im Zusammenhang mit den Variationen werden auch die meisten der in der Fachliteratur präsentierten Analysen eingeführt (quasi als kursorischer Forschungsstand). Schließlich werden, soweit als nötig, auch die hier ansonsten ausgeblendeten präpositionalen Prädikative diskutiert.

Abschnitt 2 führt die sprachlichen Daten ein, mit denen in der weiteren Analyse gearbeitet wird. Hier werden jeweils nacheinander polnische und russische prädikative Substantive sowie Adjektive und auch die in der slavistischen Forschung umstrittenen "Zustandsprädikativa" in ihren Kontexten präsentiert. Naturgemäß stehen hier die möglichen Variationen im Mittelpunkt.

Abschnitt 3 könnte als 'detaillierter Forschungsstand' bezeichnet werden, da er die wichtigsten linguistischen Analysen zu den Variationen in russischen und polnischen Kopulasätzen in jeweils geboten detaillierter Form bespricht. Sämtliche Analysen werden bereits in Abschnitt 1 erwähnt, wo auch deren Einteilung in sechs theoretische Hauptrichtungen ("Lager") erfolgt.

In Abschnitt 4 werden die zugrunde gelegten theoretischen Grundannahmen dargelegt. Besprochen werden das Grammatikmodell, der Aufbau von Lexikoneinträgen, die morphosyntaktische Merkmalsarchitektur, Spezifika prädikativ verwendbarer Kategorien, Annahmen zur Satzsyntax sowie zur Interaktion zwischen Semantik und Morphosyntax und schließlich die "Zwei-Ebenen-Semantik".

Abschnitt 5 widmet sich den Kopulasätzen als dem Kernthema dieser Arbeit. Es geht folglich um die Grundbegriffe "Prädikation" und "Prädikativität", die Rolle der Flexionsformen von poln. być bzw. russ. byt' 'sein' sowie die Unterscheidung von primärer und sekundärer Prädikation sowie "Prädikatsphrasen". Ebenso wird ein Vorschlag zur Beantwortung der zentralen theoretischen Frage nach den Prinzipien und Mechanismen formuliert, durch die russische und polnische Prädikatsnomina Kasusmarkierungen erhalten. Schließlich werden in diesem wichtigen Abschnitt auch die diversen Gebrauchsweisen von (prädikativen) Adjektiven besprochen, darunter auch die der sog. "Zustandsprädikativa".

In Abschnitt 6 stelle ich eine "formbasierte" Analyse der Variationen und, daraus folgend, deren theoretische Bewertung und Verortung vor. Eingeführt, begründet und diskutiert wird in diesem Zusammenhang zum einen das Konzept ikonischer Oppositionen und zum anderen die Annahme verschiedener, nebeneinander bestehender "Modelle" innerhalb ein und derselbe Sprache (vgl. Guiraud-Weber 
1993), derer sich die Sprecher bedienen (können) und die sich voneinander v. a. in der Zahl der zur Auswahl stehenden (Prädikativ-)Formen unterscheiden.

Abschnitt 7 schließlich fasst die Arbeit zusammen. Ferner werden offene Fragen benannt, und es wird ein Ausblick auf zukünftige Forschungsaufgaben gegeben.

Der Appendix ermöglicht, die in der Arbeit gemachten Annahmen zu Aspekt, Tempus und Modus zusammenhängend und an Beispielen nachzuvollziehen.

\section{Danksagung}

Diese Arbeit hätte nie entstehen können ohne das grundlegende Vertrauen und die andauernde Unterstützung durch meinen "Doktorvater" Uwe Junghanns. Umso mehr gilt dies, als ich die slavistische Linguistik ohne ihn wohl niemals als meine Berufung erkannt und verstanden hätte. Ebenso tiefer Dank gilt meiner Betreuerin Gerhild Zybatow, deren großartige Menschlichkeit und Offenheit mich - nicht nur fachlich - immer vorwärts gebracht haben.

Ich möchte ferner allen JungslavistInnen danken, die mir im Rahmen der JuSlaTreffen immer wieder wertvolle Hinweise und Ideen geschenkt haben. Dasselbe gilt für die BesucherInnen der folgenden wissenschaftlichen Veranstaltungen: des Kolloquiums zur slavistischen Linguistik am Seminar für Slavische Philologie der Universität Göttingen, des Slavistischen Kolloquiums am Institut für Slavistik der Universität Leipzig, des Sprachwissenschaftlichen Kolloquiums am Seminar für Deutsche Philologie der Universität Göttingen, der Linguistischen Vortragsreihe am Sprachwissenschaftlichen Seminar der Universität Göttingen sowie der 9. Internationalen Konferenz Formal Description of Slavic Languages (FDSL 9), die im Dezember 2011 in Göttingen stattfand.

Mein ganz persönlicher Dank gilt Denisa, Doro, Eugen, Joost, Ljudmila, Marcel, Markus, Sascha und Thomas - ohne die Gespräche mit euch wäre ich auf halber Strecke stecken geblieben.

Schließlich danke ich von Herzen meiner Familie, meinen Eltern und Großeltern, für die verständnisvolle Unterstützung, die sie mir gewährt haben.

\section{Für Steffi.}




\section{Einleitung}

Die vorliegende Arbeit befasst sich mit der Grammatik prädikativer Ausdrücke bzw. mit den grammatischen Eigenschaften von Prädikatsnomina im Russischen und Polnischen. Diese Themenstellung bedarf der mehrfachen Konkretisierung, die im Mittelpunkt der folgenden Abschnitte stehen wird.

\subsection{Objektsprachen}

Die Grundlage meiner Betrachtungen sind das moderne Russische und Polnische. Ich nehme eine synchrone Perspektive ein, was nicht ausschließt, dass an geeigneten Stellen - stets aber im Sinne von Hintergrundinformationen und in gebotener Kürze - auf sprachgeschichtliche Aspekte rekurriert werden kann. Was die Objektsprachen betrifft, so stellen Daten aus deren Standardvarietäten den Großteil des sprachlichen Materials. Jedoch erweist sich dieser Rahmen als mitunter zu eng, so dass ggf. auch andere Varietäten des Polnischen und Russischen (etwa die Umgangssprache in ihren diversen Abtönungen) Erwähnung finden.

Polnisch und Russisch als moderne Einzelsprachen werden genetisch-typologisch der indoeuropäischen Sprachfamilie, und innerhalb dieser dem slavischen Sprachzweig zugeordnet. Innerhalb des letzteren wird Russisch der ostslavischen, Polnisch dagegen der westslavischen Gruppe zugerechnet, sofern man der innerhalb der Slavistik weithin akzeptierten Dreiteilung folgt. ${ }^{1}$

Die Wahl der Objektsprachen Polnisch und Russisch ist mehrfach begründbar. Zum einen sind beide Sprachen die jeweils 'sprecherstärksten' Vertreter ihrer

1 Es gibt andere Gruppierungsvorschläge (siehe z. B. Hock ${ }^{3} 1998,31$ ff.). Ost- und Westslavisch werden mitunter auch dem Südslavischen gegenübergestellt und zusammen als "Nordslavisch" bezeichnet (vgl. u. a. Bartula 1969; Moser 1994). Diese Gegenüberstellung ist insofern legitim, als Ost- und Westslavisch geographisch benachbart sind und - im Sinne eines sprachlichen Kontinuums - fließend ineinander übergehen. Das Südslavische ist geographisch von dieser "Nordslavia" deutlich getrennt. Die südslavischen Sprachen haben sich über Jahrhunderte unabhängig entwickelt, in denen die nordslavischen Sprachen kontinuierlichen Kontakt hatten. So hat das Südslavische Eigenheiten bewahrt bzw. erst ausgebildet, die im Norden oft fehlen. Umgekehrt gilt dies nur bedingt; vielmehr übten die südslavischen Sprachen in Form des Kirchenslavischen mehrfach Einfluss auf das Nord(ost)slavische, besonders auf das Russische, aus. 
Gruppe und können so gewissermaßen als 'repräsentativ' gelten. ${ }^{2}$ Relevanter ist, dass sie als nordslavische Sprachen viele prinzipielle grammatische Parallelen aufweisen, innerhalb derer jedoch häufig (mikro)parametrische Differenzen vorliegen. Demnach besteht gewissermaßen Variation bei grundsätzlicher Ähnlichkeit, was die geeignete Grundlage einer vergleichenden Analyse darstellt. Schließlich kommen sowohl im Polnischen als auch im Russischen in vergleichbarer Qualität und Quantität nominale Prädikative (Prädikatsnomina) zum Einsatz, die das zentrale Thema dieser Arbeit bilden, wobei auch bei dieser prinzipiellen Parallele im Detail wichtige Unterschiede herrschen. Die derart festzustellenden Gemeinsamkeiten und Differenzen im Hinblick auf die Verwendung prädikatsnominaler Formen im Polnischen und Russischen können so m. E. zu Antworten auf bis dato oft nur unzureichend beantwortete Fragen nach deren sprachspezifischen sowie sprachübergreifenden Eigenschaften führen.

\subsection{Prädikative Ausdrücke}

Der Begriff "prädikativer Ausdruck" meint hier zunächst ganz generell eine (flektierte) Wortform, die in Sätzen als Prädikat fungiert. Hierunter fallen also alle Wortformen, die die inhaltliche Basis eines Satzes bilden, darunter finite und infinite Verbformen, prädikative Substantive, prädikative Adjektive (u. U. auch prädikative Adverbien) sowie prädikative Präpositionalphrasen. Da in dieser Arbeit aber im Besonderen Kopula-Prädikativ-Strukturen (Kopulasätze) im Mittelpunkt stehen, wird es besonders um nominale oder mindestens 'verbalnominale' Ausdrücke gehen, die als Prädikat fungieren. Das engere Untersuchungsobjekt sind somit prädikative Ausdrücke, die nicht der Wortart "Verb" angehören.

Meist und auch hier wird davon ausgegangen, dass jede satzwertige Struktur auf einer flektierten Verbform basiert. Die invariante (deskriptive, grammatische) Bedeutung eines Verblexems wird von einer Proposition gestellt, die im Kontext des Satzes in eine Äußerung (bzw. Assertion als kommunikativer Grundeinheit) überführt wird. Ein Verb referiert jeweils auf einen Sachverhalt (Zustand, Prozess oder Ereignis), der in der außersprachlichen Welt besteht und durch die Assertion als konkrete Instanz der fraglichen verbalen Proposition ausgewiesen wird. Ferner

2 Das heißt nicht, dass die übrigen Sprachen der Gruppen durch bloße Übertragung der Befunde erfassbar wären. Jedoch stehen sie dem Polnischen bzw. Russischen in vielen Charakteristika näher als der jeweils anderen 'repräsentativen' Sprache. 
bringt jedes Verb die ggf. an 'seinem' Sachverhalt beteiligten Partizipanten mit sich. Diese Argumentausdrücke erfahren in der Folge eine bestimmte syntaktische Realisierung.

Es ist jedoch offensichtlich, dass die "rein verbische" Form der Prädikation nicht die einzig mögliche in natürlichen Sprachen ist. Nicht für alle Sachverhalte bzw. Sachverhaltstypen, die in der außersprachlichen Welt bestehen, gibt es verbale Lexeme. Dies gilt insbesondere für Zustandscharakterisierungen bzw. Eigenschaftszuschreibungen, die primär durch Kopulasätze versprachlicht werden. ${ }^{3}$ Hierher gehören auch russische und polnische Sätze mit sog. "Prädikativa". 4

Als konkrete Untersuchungsobjekte der vorliegenden Arbeit ergeben sich demnach Prädikative sowie "Prädikativa". Ich werde jedoch versuchen zu zeigen, dass letztere ebenfalls als Prädikative gelten können, was die Annahme einer eigenständigen Wortart "Prädikativum” überflüssig macht. Im Fokus stehen somit Prädikative in ihren diversen Erscheinungsformen, v. a. aber Prädikatsnomina, d. h. Substantive sowie Adjektive. Diese Beschränkung begründet sich damit, dass nur letztere flektierbar und daher imstande sind, morphologische bzw. morphosyntaktische Markierungen zu erhalten. Die Tatsache, dass ein und dasselbe prädikative Lexem in russischen sowie polnischen Kopulasätzen häufig in zwei oder mehr Formen bzw. mit zwei oder mehr Markierungen erscheinen kann, ist in der Forschung seit jeher Ursprung diverser, zum Teil deutlich divergierender Beschrei-

3 "In Termen von G[overnment\&]B[inding] ist die[s] eine Rekonstruktion des bekannten Faktums: Prädikativ + Kopula erfüllen zusammen die Funktion eines Vollverbs und können deshalb zufällige Lücken im Verbsystem auffüllen, vgl. arm werden - verarmen[,] reich werden *verreichen o.ä.[,] Lehrer sein - lehren." (Steinitz 1990, 122). Ein Unterschied zwischen "verbischer" und "nominaler" Prädikation bleibt hier unerwähnt, dass nämlich durch erstere meist (dynamische) Aktivitäten/Ereignisse, durch letztere hingegen (statische) Zustände versprachlicht werden. So bezeichnet das "nominale" Lehrer sein einen Zustand, das "verbische" lehren eine Aktivität. Demnach füllt der "nominale" Prädikationstyp nicht einfach Lücken, die der "verbische" offen lässt; er hat vielmehr die Allgemeinbedeutung der Zustandscharakterisierung und so eine spezifische Rolle im grammatischen Gefüge. Das schließt nicht aus, dass zwischen "verbischer" und "nominaler" Prädikation Synonymie bestehen kann, vgl. verarmen und arm werden (beachte aber, dass werden einen Zustandswechsel denotiert). Meist wird aber ein Prädikationstyp bevorzugt, worauf Faktoren wie Usualität und Kontext Einfluss nehmen. So zeigt sich, was auch für die Betrachtung russischer und polnischer Kopulasätze relevant sein wird, dass nämlich jenseits strikter Grammatikalität die Akzeptabilität für die Wahl der Ausdrucksform relevant ist. Oft ist nicht zu sagen, ein Satz sei strikt ungrammatisch, wohl aber liegen häufig deutliche Unterschiede in seiner Akzeptabilität vor.

4 Siehe hierzu insbesondere die Abschnitte 2.3 und 5.6.3. 
bungen und Erklärungen. Auf die erwähnten Variationen und auf ihre wichtigsten bis dato vorliegenden Analysen wird im folgenden Abschnitt eingegangen.

\subsection{Variationen von Prädikativformen}

Zu den erwähnten Erscheinungsformen von Prädikativen in Kopulasätzen gehören (i) der "doppelte Nominativ" im Gegensatz zum "prädikativen Instrumental" sowie (ii) die adjektivische Kurzform im Gegensatz zu ihrer Langform. ${ }^{5}$ Die theoretische Diskussion in diesem Zusammenhang dreht sich naturgemäß um die Frage, ob diese Variationen in der Form der Prädikatsnomina mit einem Unterschied in ihrer 'Bedeutung' bzw. in der Interpretation der aus ihnen resultierenden Kopulasätze einhergehen. Sofern dies bejaht wird, was mindestens für das Russische auf der Basis von empirischen Beobachtungen und Aussagen kompetenter Sprecher meist der Fall ist, schließt sich folgerichtig die Frage an, worauf dieser 'Bedeutungsunterschied' beruht bzw. wie er zustande kommt.

In diesem Kontext hält sich besonders in der russistischen Grammatikschreibung hartnäckig die Ansicht, der angedeutete Bedeutungsunterschied sei im Sprachsystem angelegt. Dabei variieren die Analysen zum einen in der Beschreibung der Bedeutungsunterschiede an sich und zum anderen in Bezug auf die Verortung ihrer Quelle/n im Sprachsystem. Entweder werden die feststellbaren interpretativen Effekte ${ }^{6}$ direkt auf die Prädikativformen bzw. auf deren jeweilige morphologische Markierung zurückgeführt (vgl. u. a. Geist 2006, 2010), oder es wird von verschiedenen "Konstruktionen" ausgegangen, denen die jeweilige 'Bedeutung' zueigen sei (vgl. u. a. Kuznetsova \& Rakhilina 2010); schließlich wird vorgeschlagen, die alternierenden Prädikativformen bzw. Interpretationen der Kopulasätze folgten aus distinkten syntaktischen Strukturen (siehe u. a. Matushansky 2000; Markman 2008; Bailyn 2012).

5 Der Vergleich mit dem Polnischen, für das - trotz ähnlicher, wenn nicht identischer morphosyntaktischer Markierungen - i. d. R. keine semantischen Distinktionen behauptet werden, erweist sich bei der Untersuchung dieser Phänomene als äußerst fruchtbar. Die Distinktion von Kurz- und Langform existiert zwar auch im Polnischen, jedoch weitaus eingeschränkter als im Russischen, da Kurzformen nur noch rudimentär auftreten.

6 Mehr als 'Effekte' sind es offenbar nicht (vgl. u. a. Hentschel 1991, 1993a). Obwohl die Analyse von Geist (2006) zu jenen gehört, die die Quelle der Bedeutungsunterschiede in der Grammatik verorten, spricht sie durchgängig von “interpretativen Effekten”, die abhängig vom Kontext aus einer Präsupposition folgen, die Teil der Bedeutung des Instrumentalsuffixes sein soll. 
Was in der vorliegenden Arbeit insbesondere bezweifelt wird, ist die oftmals nicht hinterfragte bzw. stillschweigend vorausgesetzte Grundannahme, die Form- bzw. Markierungsvarianten der Prädikative reflektierten etwas im grammatischen System per se Vorhandenes, d. h. etwas dort Angelegtes. Dies impliziert, dass (a) im mentalen Lexikon eine invariante semantische Komponente vorhanden sei, die direkt oder indirekt durch die variierenden Prädikativformen sprachlich realisiert bzw. reflektiert wird, oder dass (b) distinkte syntaktische Strukturen (also "Konstruktionen") vorgegeben seien, die ihrerseits die alternierenden Prädikativformen und somit auch die interpretativen Effekte determinieren und erklären.

Es gibt jedoch gute Gründe, an diesem Annahme zu zweifeln. Dabei geht es durchaus nicht darum, die interpretativen Effekte an sich in Frage zu stellen. Allein ihre ständige Erwähnung in Grammatiken und Analysen belegt, dass sie real sind. ${ }^{7}$ Vorsicht ist jedoch geboten, wenn diese Effekte auf eine fixe Quelle im Sprachsystem zurückgeführt werden, unabhängig davon, ob diese ihrer Natur nach semantisch oder syntaktisch sei.

Dagegen spricht allein schon, dass die Effekte selbst überaus heterogen beschrieben werden. Offenbar fällt es nicht nur kompetenten Sprechern ${ }^{8}$, sondern auch LinguistInnen schwer, für sie eine stabile Beschreibung zu finden. Ferner treten die Effekte in russischen und (sofern für sie überhaupt angenommen) auch in polnischen Kopulasätzen - anders als z. B. im Spanischen - keineswegs konsequent auf. ${ }^{9} \mathrm{Zu}$ ihnen kommt es überhaupt nur dann, wenn mindestens zwei Prädi-

7 Auf Grund dieses Befundes sind m. E. Ansätze mit Vorsicht zu behandeln, die in bestimmten Prädikativmarkierungen eine lediglich strukturelle Markierung des Prädikats sehen (so z. B. Krasovitsky et al. 2008). Hentschel (1991) ist moderater, wenn er die Effekte nicht absolut verneint, in ihnen aber nicht den zentralen Faktor für die Variationen sieht. Ihm zufolge ist der Instrumental an russischen Prädikatsnomina das markierte Signal für den prädikativen Status. Er folgert, dass der Hauptfaktor für die Kasuswahl Un-/belebtheit bzw. die ikonische Absetzung vom Subjektausdruck sei und zieht eine Parallele zur sog. 'Belebtheitskategorie'.

Analysen wie Krasovitsky et al. (2008) bilden einen Extrempol, dem semantisch und/oder syntaktisch orientierte Arbeiten wie Matushansky (2000), Geist (2006), Markman (2008) etc. gegenüberstehen. Die vorliegende Arbeit steht zwischen beiden Polen und Hentschel (1991, 1993a) am nächsten, da die Realität der Effekte nicht bestritten, aber dafür plädiert wird, sie nicht als tiefere Motivation für die Markierungen (sondern als deren Folge) zu betrachten.

8 Ich werde in dieser Arbeit durchgängig die generisch-maskuline Form "Sprecher" verwenden.

9 Maienborn (2003a, 2005a) zeigt, dass in spanischen Kopulasätzen mit adjektivischen Prädikativen eine semantisch basierte und pragmatisch gesteuerte Distinktion vorliegt, die hinsichtlich der interpretativen Effekte dem Russischen vergleichbar ist. Sie fußt auf den distinkten Kopulalexemen ser und estar und findet völlig konsequent statt. 
kativformen bzw. -markierungen in potenzieller Opposition zueinander stehen. Wichtig ist zudem, dass die meisten Arbeiten zum Russischen von der Situation ausgehen, wie sie für die normierte Standardsprache (russkij literaturnyj jazyk) besteht bzw. per definitionem bestehen soll. In anderen Varietäten des Russischen, z. B. in der "gehobenen Umgangssprache" (russkaja razgovornaja reč'), in der “einfachen Umgangssprache” (prostorečie) oder in der journalistischen Sprache etc., gelten offenbar mitunter eigene, abweichende "Regeln". ${ }^{10}$ So ergibt sich eine recht diffuse Gesamtlage. Zwar lässt sich die Existenz interpretativer Effekte nicht bestreiten, ihr Charakter jedoch ist offenbar nur schwerlich einheitlich erfassbar, da sich je nach Varietät allein schon das Spektrum möglicher Prädikativformen, auf denen die Effekte offensichtlich beruhen, unterscheidet.

Dieses komplexe Gesamtbild spricht gegen die (deterministische) Annahme einer semantisch-lexikalisch, konstruktionsbasiert und/oder syntaktisch-strukturell vorbestimmten, also im Sprachsystem manifesten und somit invarianten Distinktion, die sich mittelbar oder unmittelbar in den alternierenden Prädikativformen realisiere. Von einer solchen Distinktion wäre ja gerade zu erwarten, dass sie konsequent stattfindet. Jedoch trifft dies v. a. für das Russische gerade in dem Maße nicht $\mathrm{zu}$, wie es umgekehrt etwa für das Spanische bei adjektivischen Prädikativen zutrifft (vgl. Maienborn 2003a, 2005a). Dieser Unterschied muss im Rahmen einer theoretischen Rekonstruktion Beachtung finden, für die also schon an dieser Stelle prognostiziert werden kann, dass sie für das Russische deutlich anders ausfallen dürfte als für das Spanische, wenn auch z. B. Geist (2006) entgegengesetzt argumentiert. Freilich bestehen auch Unterschiede hinsichtlich der Prädikativmarkierungen und ihrer Effekte zwischen dem Russischen und Polnischen - sie sind aber anderer Natur und gewissermaßen 'weniger gewichtig' als jene Unterschiede, die beide slavischen Sprachen im Vergleich zum Spanischen zeigen. Was sowohl

10 So bemerkt Isačenko $(1962,151)$, dass russische Kurzformen in der "gepflegten Hochsprache [...] eine durchaus lebendige und entwicklungsfähige Kategorie" bilden. Damit spricht er sich dagegen aus, dass die Kurzformen (generell) im Rückgang begriffen seien. Seine detaillierten Ausführungen zum Kurzformgebrauch beziehen sich v. a. auf die "gepflegte Hochsprache" und scheinen demnach nicht in gleichem Maße für andere Varietäten zu gelten. So sind Kurzformen laut Corbett $(2004,208)$ in der gesprochenen Sprache stark eingeschränkt. Er bemerkt, dass sie im Laufe des 19. und 20. Jh. zurückgegangen und von den prädikativen Langformen "verdrängt" worden seien (vgl. Abschnitte 4.4.3, 5.6). Es zeigt sich, dass je nach Varietät in unterschiedlichem Maße von bestimmten Prädikativformen Gebrauch gemacht wird, woraus zu folgern ist, dass auch die interpretativen Effekte in verschiedenen Varietäten unterschiedlich ausfallen dürften. Dies zeigt auch Guiraud-Weber (1993) bzgl. der russischen Kurzform. 
Polnisch als auch Russisch vom Spanischen absetzt - so die hier vertretene These -, ist das Fehlen einer im engeren Sinne grammatisch determinierten Distinktion: ${ }^{11}$

\begin{abstract}
Arbeitshypothese:
Polnisch und Russisch verfügen über variierende morphosyntaktische Formen von bzw. Markierungen an Prädikatsnomina. Die resultierenden Variationen werden in beiden Sprachen verschiedentlich ausgenutzt, um bestimmte interpretative Effekte zu erzielen. Den Variationen liegen jedoch keine semantischen und/oder syntaktischen Invarianten zugrunde.
\end{abstract}

Anders formuliert: "Sprachlich real" sind im Polnischen und Russischen allein die ggf. verfügbaren Formen von Prädikatsnomina. Sie werden in beiden Sprachen zwar unterschiedlich eingesetzt, haben jedoch gemeinsam, dass es sich bei ihnen in der Tat lediglich um Varianten in der Form handelt, denen von sich aus keine (grammatische) Bedeutung zukommt. Mit Hentschel (1993a) lässt sich auch von “diakritischen" Markierungen oder Formen sprechen. Es ist zu betonen, dass eine bestimmte Form bzw. Markierung überhaupt nur dann eine 'Bedeutung' oder Lesart erhalten kann, wenn sie in Opposition zu einer alternativen Form bzw. Markierung steht, d. h. wenn eine "Variation im eigentlichen Sinne" (Bogusławski 2001, 127) gegeben ist.

\title{
1.4 Interpretative Effekte (kursorischer Forschungsstand)
}

Die hauptsächlich für das Russische (und hier wiederum v. a. für Prädikatssubstantive) beschriebenen interpretativen Effekte treten, wie bereits erwähnt, nur dann auf, wenn mindestens zwei variierende Prädikativformen bzw. -markierungen potenziell konkurrieren. Nur in diesem Fall besteht eine Opposition zwischen ihnen, und nur dann kann Isačenkos (1962, 146-147) Formulierung

11 Mit den Begriffen der Zwei-Ebenen-Theorie der Bedeutung (siehe Abschnitt 4.7) lässt sich dies derart paraphrasieren, dass im Spanischen eine intragrammatisch determinierte Distinktion vorliegt, während im Polnischen und Russischen eine lediglich extragrammatisch determinierte Unterscheidung vorliegt/vorliegen kann. In spanischen Kopulasätzen ist in Form der beiden Lexeme ser und estar eine lexikalisch angelegte Distinktion vorgegeben. Im Russischen und Polnischen stehen dagegen lediglich alternative morphosyntaktische Formen/Marker von Prädikatsnomina zur Verfügung, die 'am Ende' ggf. - und stets im Zusammenspiel mit weiteren (extragrammatischen) Faktoren - eine bestimmte Lesart verursachen/verursachen können. 
wörtlich gelten, dass nämlich jede grammatische Form auch etwas "meine". ${ }^{12}$ Freilich bedeutet das nicht, dass in Fällen, in denen nur eine Prädikativform verwendet werden kann, diese nicht dennoch einen Bedeutung trägt. An sich hat sie selbstverständlich ihre deskriptive Bedeutung und Funktion im Satz. Jedoch steht sie in einem solchen Fall nicht in Opposition zu einer alternativen Form (oder alternativen Formen) und kann insofern nichts Zusätzliches "meinen", das sich erst aus dem möglichen Gegensatz verschiedener sprachlicher Zeichen in ein und derselben Funktion/Position ergibt, d. h. ein 'interpretativer Effekt'.

Die interpretativen Effekte im Zusammenhang mit russischen Kopulasätzen werden in der Fachliteratur sehr heterogen beschrieben und - wenn überhaupt auch sehr unterschiedlich hergeleitet. In den Tabellen 1 bis 5 sind einige Arbeiten zusammengestellt, die Bezug auf die in Konkurrenz zueinander stehenden Kasusformen an russischen Prädikatsnomina (Nominativ vs. Instrumental, mehrheitlich Substantive) nehmen (vgl. auch Geist 2006; Pitsch 2008). Ich ordne die Ansätze in folgende "Lager", die durch den Faktor bestimmt werden, der für die Wahl der Prädikativform bzw. des Kasus als ausschlaggebend betrachtet wird: ${ }^{13}$

1. semantisch-deskriptive Ansätze

2. semantosyntaktische Ansätze

3. syntaktische Ansätze

4. semantopragmatische Ansätze

5. formbasiert-pragmatische Ansätze

6. rein strukturelle Ansätze

Die Mehrzahl der traditionellen - und dabei so gut wie immer deskriptiven - Beschreibungen, aber auch eine Reihe jüngerer, explikativ orientierter Arbeiten können als rein semantisch bezeichnet werden (Tab. 1). Hier wird davon ausgegangen, eine bestimmte Semantik bzw. ein bestimmter Bedeutungsanteil sei für die Wahl der Prädikativform ursächlich. Dieser Bedeutungsanteil muss - was oft

12 Hier kann man auch auf eine Grundannahme des sprachwissenschaftlichen Strukturalismus (de Saussure) verweisen, der zufolge sprachliche Zeichen ein System von Werten bilden, die zueinander in Opposition stehen. Ein Zeichen definiert sich (erst) durch seine Beziehung zu den übrigen Zeichen desselben Systems.

13 Vgl. die Trennung von semantischen und syntaktischen Ansätzen bei Bailyn (2001, 1-2; 2012, 198-199). Freilich sind auch andere Einteilungen denkbar. 
nicht thematisiert oder offengelassen wird - in irgendeiner Form im Sprachsystem angelegt sein, höchstwahrscheinlich als Teil der grammatischen Bedeutung einer bestimmten lexikalischen Einheit im mentalen Lexikon (d. h. als Komponente in ihrem Lexikoneintrag).

Als semantosyntaktisch bezeichne ich Analysen, die eine semantische Distinktion als Grundlage für differente syntaktische Strukturen betrachten, die ihrerseits die variierenden Prädikativformen determinieren (Tab. 2).

Die zuletzt genannten Analysen stehen zwischen den anfangs erwähnten (rein) semantischen Arbeiten und einem weiteren "Lager", das ich syntaktisch nenne (Tab. 3), da die interpretativen Effekte hier als "Produkt" distinkter syntaktischer Strukturen betrachtet werden. Folglich wird die Syntax als zentraler (meist einziger) Faktor benannt, der sowohl die Prädikativform(en) als auch die schließliche Satzinterpretation determiniere. Der Übergang zu den semantosyntaktischen Analysen ist mitunter fließend.

Ferner liegt eine Sichtweise vor, die wohl am geeignetsten semantopragmatisch zu nennen ist (Tab. 4). Hier wird einerseits zwar von einer semantischen Grundlage für die variierenden Prädikativformen ausgegangen, jedoch wird die Pragmatik (der Kontext) als bestimmender Faktor für das Zustandekommen eines konkreten interpretativen Effekts betrachtet.

Als formbasiert-pragmatisch (Tab. 5) bezeichne ich Arbeiten, die von keiner semantischen und/oder syntaktischen Basierung der Variationen ausgehen. Die möglichen Prädikativvarianten werden vielmehr als bloße Formen bzw. Marker betrachtet, die per se keine Eigenbedeutung haben, jedoch eine bestimmte Deutung des Satzes bewirken (können).

Die Arbeit von Krasovitsky et al. (2008) sticht insofern hervor, als sie die Kasuswahl am russischen Prädikatsnomen als rein strukturell determiniert betrachtet (Tab. 6). Sie sei demnach weder semantisch noch syntaktisch bestimmt; vielmehr wird hier behauptet, der Instrumental sei im heutigen Russischen der Default für substantivische Prädikative.

Bewertungsgrundlage für jede Analyse sollte sein, inwieweit sie imstande ist, die Verbindung zwischen einer bestimmten Prädikativmarkierung, ihrer Gebrauchsweise sowie den resultierenden interpretativen Effekten herzustellen. Sie sollte ferner nicht nur auf die Kasusvariation am Prädikativ, sondern auch auf die Alternation zwischen Kurz- und Langformen (v. a. im Russischen) eingehen und beide Erscheinungen gleichermaßen und einheitlich erfassen. 
Die in den folgenden Tabellen zusammengestellten Arbeiten stellen lediglich eine Auswahl dar. Aber schon sie dokumentiert hinreichend, wie heterogen dieses Phänomen in der Fachliteratur beschrieben und erklärt wird. In Abschnitt 3 werde ich auf einige besonders relevante Arbeiten näher eingehen.

Tab. 1: Semantisch-deskriptiv.

\begin{tabular}{|c|c|c|}
\hline NOMINATIV & INSTRUMENTAL & QUELLE \\
\hline Ganzes-Lesart & Teil-Lesart & Potebnja (1958) \\
\hline Feststellung / Identifizierung & Werturteil / Charakterisierung & $\begin{array}{l}\text { Švedova (1952) } \\
\text { Isačenko (1962) }\end{array}$ \\
\hline $\begin{array}{l}\text { neutral bzgl. resultativer } \\
\text { Aktualisierung }\end{array}$ & $\begin{array}{l}\text { Marker für resultative } \\
\text { Akualisierung }\end{array}$ & Mrázek (1964) \\
\hline $\begin{array}{l}\text { kein Zeitbezug (non- } \\
\text { episodisch) }\end{array}$ & Zeitbezug (episodisch) & Jakobson (1971a) \\
\hline $\begin{array}{l}\text { a. "Essiv" (vgl. Finnisch); } \\
\text { b. bei NPn mit den Merkmalen } \\
\text { [+Klasse], [-Klasse,-Zeit]; } \\
\text { c. mit merkmallosen Verben }\end{array}$ & $\begin{array}{l}\text { a. "Translativ" (vgl. Finnisch); } \\
\text { b. bei NPn mit den Merkmalen } \\
\text { [-Klasse], [-Klasse,+Zeit]; } \\
\text { c. mit merkmalhaften Verben }\end{array}$ & Chvany (1975) \\
\hline unmarkiert in Umgangsspr. & unmarkiert im Standard & Křížková (1969) \\
\hline stilistisch markiert & stilistisch neutral & AG (1980) \\
\hline permanente Geltung & temporäre Geltung & $\begin{array}{l}\text { Vinogradov \& Istrina (1960) } \\
\text { Galkina-Fedoruk et al. } \\
\text { (1964) } \\
\text { Berneker \& Vasmer }\left({ }^{7} 1971\right) \\
\text { Schaller (1975) } \\
\text { Wierzbicka (1980) } \\
\text { AG (1980) } \\
\text { Gabka et al. (1989) } \\
\text { Cubberley (2002) }\end{array}$ \\
\hline essenzielle Eigenschaft & akzidentelle Eigenschaft & \\
\hline idiomatische Lesart & agentive Interpretation & \\
\hline \multicolumn{2}{|c|}{ abhängig von der lexikosemantischen Klasse des Prädikativs } & $\begin{array}{l}\text { Røed (1966) } \\
\text { Nichols (1981, 1985) }\end{array}$ \\
\hline unique Interpretation & non-unique Interpretation & Ueda (1992) \\
\hline $\begin{array}{l}\text { non-eventive Interpretation } \\
\text { (ein Zustand denotiert) }\end{array}$ & $\begin{array}{l}\text { eventive Interpretation } \\
\text { (zwei Zustände denotiert) }\end{array}$ & Richardson (2001) \\
\hline non-kontrastive Kontexte & kontrastive Kontexte & $\begin{array}{l}\text { Kuznetsova \& Rakhilina (2010) } \\
\text { Kuznetsova (2013) }\end{array}$ \\
\hline
\end{tabular}


Tab. 2: Syntaktosemantisch.

\begin{tabular}{|c|c|c|}
\hline NOMINATIV & INSTRUMENTAL & QUELLE \\
\hline funktionale Kategorie $\left(\mathrm{T}^{0}\right)$ & lexikalische Kategorie $\left(\mathrm{V}^{0}\right)$ & Geist (1999) \\
\hline keine aspektuelle Spezifikation & aspektuelle Spezifikation & $\begin{array}{l}\text { Matushansky } \\
(2000)\end{array}$ \\
\hline non-eventiv & eventiv & Roy (2004) \\
\hline non-eventiv (nur Default-Aspekt) & eventiv & Markman (2008) \\
\hline non-eventiv / äquativ & eventiv / prädikativ & $\begin{array}{l}\text { Matushansky } \\
\text { (2008) }\end{array}$ \\
\hline \multicolumn{2}{|c|}{$\begin{array}{l}\text { Dreiteilung von Kopulasätzen: } \\
\text { a. } \quad \text { situationsbeschreibend [nur Adjektive }=\mathrm{KF} \text { ] } \\
\text { b. } \\
\text { charakterisierend [nur Substantive, darunter auch } \mathrm{LF}_{\mathrm{INS}}+\varnothing_{\mathrm{N}} \text { ] } \\
\text { c. } \quad \text { definierend [nur Substantive }=\mathrm{LF}_{\mathrm{NOM}}+\varnothing_{\mathrm{N}} \text { ] }\end{array}$} & Roy (2013) \\
\hline
\end{tabular}

Tab. 3: Syntaktisch.

\begin{tabular}{|c|c|c|}
\hline NOMINATIV & INSTRUMENTAL & QUELLE \\
\hline ohne overte Verbform & mit overter Verbform & Chvany (1975) \\
\hline $\begin{array}{l}\mathrm{SC}=\text { PredP }=\text { 'schwache' Phase } \\
\mathrm{Asp}^{0}=[-\mathrm{INST},-\mathrm{EPP},- \text { Asp }]\end{array}$ & $\begin{array}{l}\mathrm{SC}=\text { PredP }=\text { 'starke' Phase } \\
\mathrm{Asp}^{0}=[+\mathrm{INST},+\mathrm{EPP},+\mathrm{Asp}]\end{array}$ & Harves (2002) \\
\hline $\begin{array}{l}\text { keine AspP; } \\
\text { Kompl. von } \text { Pred }^{0}: \text { Num[eral]P }\end{array}$ & $\begin{array}{l}\text { AspP vorhanden; } \\
\text { Kompl. von Pred }^{0} \text { : Cl[assifier]P }\end{array}$ & Roy (2006) \\
\hline symmetrische Struktur & asymmetrische Struktur & Pereltsvaig (2007) \\
\hline primäre Prädikation $\left(1 \mathrm{x} \operatorname{Pred}^{0}\right)$ & sekundäre Prädikation $\left(2 \times \operatorname{Pred}^{0}\right)$ & Bailyn (2012) \\
\hline
\end{tabular}

Tab. 4: Semantopragmatischer Ansatz

\begin{tabular}{|l|l|l|}
\hline \multicolumn{1}{|c|}{ NOMINATIV } & \multicolumn{1}{|c|}{ INSTRUMENTAL } & \multicolumn{1}{c|}{ QUELLE } \\
\hline $\begin{array}{l}\text { keine Abweichung von } \\
\text { Vorzustand/Sprechererwartung }\end{array}$ & $\begin{array}{l}\text { Abweichung von } \\
\text { Vorzustand/Sprechererwartung }\end{array}$ & $\begin{array}{l}\text { Timberlake } \\
(1986)\end{array}$ \\
\hline kein Bezug auf spezifische Topiksit. & Bezug auf spezifische Topiksituation & Geist (2006) \\
\hline
\end{tabular}

Tab. 5: Formbasiert-pragmatisch.

\begin{tabular}{|l|l|l|}
\hline \multicolumn{1}{|c|}{ NOMINATIV } & \multicolumn{1}{|c|}{ INSTRUMENTAL } & QUELLE \\
\hline unmarkiertes Signal & markiertes Signal & Hentschel (1991) \\
\hline
\end{tabular}


Tab. 6: Rein strukturell.

\begin{tabular}{|l|l|l|}
\hline \multicolumn{1}{|c|}{ NOMINATIV } & \multicolumn{1}{|c|}{ INSTRUMENTAL } & \multicolumn{1}{c|}{ QUELLE } \\
\hline bei Präsensformen der Kopula & $\begin{array}{l}\text { bei Präterital-, Imperativ-, .. } \\
\text { Futurformen der Kopula }\end{array}$ & $\begin{array}{l}\left.\text { Comrie et al. } \text { ( }^{2} 1996\right) \\
\text { Bendixen \& Voigt } \\
(1999)\end{array}$ \\
\hline heute nur noch rudimentär & heute Default & $\begin{array}{l}\text { Krasovitsky et al. } \\
(2008)\end{array}$ \\
\hline
\end{tabular}

Mit Bezug auf polnische Kopulasätze liegen deutlich weniger theoretische Arbeiten vor. Dies ist sicherlich der Tatsache geschuldet, dass für das Polnische meist von keiner semantischen Distinktion ausgegangen wird. Jedoch muss auch für polnische Prädikative das Zustandekommen der Kasusmarkierung geklärt werden, da auch hier Nominativ (bzw. der Kongruenzkasus) und Instrumental variieren (können). Einschränkend ist zu bemerken, dass im Falle prädikativer Substantive der Instrumental gegenüber dem Nominativ absolut dominiert (siehe u. a. Rothstein 1993, 729; Bąk ${ }^{11}$ 1999, 413; Bogusławski 2001, 127; Bondaruk 2013b, 148), während bei prädikativen Adjektiven ganz überwiegend der Kongruenzkasus auftritt (siehe Abschnitt 2).

Eine Auswahl von theoretischen Arbeiten zur Kasusvariation im Polnischen zeigt Tab. 7. Diese Arbeiten lassen sich nach dem oben angewandten Gruppierungsschema wie folgt ordnen: Klemensiewicz (1926), Rothstein (1986, 1993) sowie Bąk ( $\left.{ }^{11} 1999\right)$ (und sicherlich die Mehrzahl der hier unerwähnten deskriptiven Arbeiten und Grammatiken) sind semantisch-deskriptiv, indem sie für die variierenden Kasusformen ‘lediglich’ Gebrauchsregeln auf Grundlage der von ihnen jeweils behaupteten Deutungen formulieren. Als syntaktisch kann man Bailyn \& Citko (1999), Przepiórkowski (2001), Witkoś (2010) und Bondaruk (2013b) bezeichnen. Es ist anzumerken, dass sich Bailyn \& Citko (1999) und Witkoś (2010) ebenso dem rein strukturellen Lager zuordnen ließen, behandeln sie den Instrumental doch als Default. Schließlich ist Bogusławski (2001) Vertreter des formbasiert-pragmatischen Lagers, der die "Ausdeutung" der Kasusvariation als Folgeerscheinung rein formaler Oppositionen zwischen zwei morphologischen Markierungen erklärt und eine semantische Distinktion verneint.

Eine semantische Distinktion im engeren Sinne, d. h. einen im mentalen Lexikon (in welcher lexikalischen Einheit auch immer) angelegten bzw. in der Satzsyntax in der einen oder anderen Form manifesten Bedeutungsanteil, nimmt keine der 
aufgeführten Arbeiten an. In dieser Tatsache spiegelt sich die bereits gemachte Aussage, dass für das Polnische üblicherweise von keiner ('echten') semantischen Distinktion ausgegangen wird. Woraus aber die Interpretationen der Sätze folgen (die folglich wiederum bloße 'interpretative Effekte' sind), bleibt meist offen.

Tab. 7: Ansätze zur Kasusvariation in polnischen Kopulasätzen

\begin{tabular}{|l|l|l|}
\hline \multicolumn{1}{|c|}{ NOMINATIV } & \multicolumn{1}{|c|}{ INSTRUMENTAL } & \multicolumn{1}{c|}{ QUELLE } \\
\hline das Subjekt definierend & das Subjekt beschreibend & Klemensiewicz (1926) \\
\hline ohne "andere Assoziationen" & impliziert Alternativen & Rothstein (1986) \\
\hline $\begin{array}{l}\text { u. U. expressive Lesart } \\
\text { [angeblich ungrammatisch; } \\
\text { vgl. Bondaruk (2013b, 157)] }\end{array}$ & durch Pred ${ }^{0}$ zugewiesen & Rothstein (1993) \\
\hline $\begin{array}{l}\text { Regelfall bei Adjektiven; } \\
\text { markierter Fall bei Substantiven }\end{array}$ & Regelfall bei Substantiven & Bailyn \& Citko (1999) \\
\hline setzt Zusatzbedingungen voraus & (negativ bestimmter) Default & Bąk (11 1999) \\
\hline ohne Subjekt-Raising & Bei Subjekt-Raising & Przepiórkowski (2001) \\
\hline [wird lediglich erwähnt] & durch $\pi^{0}$ valuiert & Citko (2008) \\
\hline ['markierte' Kasusmarkierung] & Default für Prädikative \\
\hline $\begin{array}{l}\text { defektives Pred } \text { (keine Merkmale) } \\
\text { feature sharing mit Subjekt-DP }\end{array}$ & $\begin{array}{l}\text { non-defektives Pred }{ }^{0}(\varphi, \text { INS) } \\
\approx \text { Kasuszuweisung }\end{array}$ & Witkoś (2010) \\
\hline
\end{tabular}

\section{5 "Grammatik"}

Schließlich ist der im Titel verwendete Begriff "Grammatik" zu erläutern. Hierunter verstehe ich die Menge der phonetischen, argumentstrukturellen, morphosyntaktischen und semantischen Eigenschaften lexikalischer Einheiten bzw. der mittels solcher Einheiten gebildeten Wortformen. ${ }^{14}$ Eine Grundannahme dieser Arbeit lautet, dass die genannten Eigenschaften parallele Bestandteile von Lexikoneinträgen im mentalen Lexikon sind (vgl. u. a. Bierwisch 1997, siehe Abschnitt 4.2). Dieser lexikalistischen Annahme zufolge wird das syntaktische "Verhalten" einer Wortform durch den ihr zugrunde liegenden Lexikoneintrag deter-

14 Da die phonetische Form lexikalischer Einheiten als gegeben behandelt wird, werden lautliche Eigenschaften nicht näher betrachtet. Freilich ist die Lautform stets Teil der "Grammatik" von lexikalischen Einheiten. 
miniert. Die phonetischen, argumentstrukturellen, morphosyntaktischen und semantischen Eigenschaften von Wortformen - wobei natürlich Prädikatsnomina und die Formen der Lexeme byćlbyt' 'sein' im Mittelpunkt stehen - bilden in der hier gebrauchten Terminologie folglich deren "Grammatik". Diese zu erfassen, ist ein erklärtes Ziel der vorliegenden Arbeit.

\subsection{Präpositionale Prädikative}

Es wurde erwähnt, dass vornehmlich substantivische und adjektivische Prädikatsnomina betrachtet werden. Diese Einengung des Untersuchungsgegenstands erfordert einige Bemerkungen zu den ausgeblendeten präpositionalen Prädikativen.

Zunächst ist zu klären, ob Sätze mit einer Form des Lexems 'sein' und einer Präpositionalphrase (PP) überhaupt Kopulasätze sind. Manche Analysen verneinen dies (siehe u. a. Heidolph, Flämig \& Motsch 1981; Bickes 1984; Eisenberg 1998, Geist 2006). Ihnen zufolge sind PPn nicht Komplemente der Kopula, sondern treten mit dem Vollverb 'sein' auf und sind somit Adverbiale. Die Komplementposition der Kopula wird demnach nur von Prädikatsnomina besetzt. Andere AutorInnen (z. B. Helbig \& Buscha 2001; Erben 1980; Zifonun, Hoffmann \& Strecker 1997; Steinitz 1990, 1992, 1997; Bierwisch 1998) betrachten PPn hingegen (auch) als Komplemente der Kopula.

Daten aus dem Russischen und Polnischen sprechen gegen die pauschale Annahme, PPn träten ausschließlich mit dem Voll- oder Existenzverb 'sein' auf. Zwar ist dies möglich, jedoch können PPn ebenso mit der "Kopula" auftreten:
(1) Kniga byla v biblioteke.
Buch-NOM.SG.F war-SG.F in Bibliothek-LOC
'Das Buch \{war, gab es\} in der Bibliothek.'

(Rus)

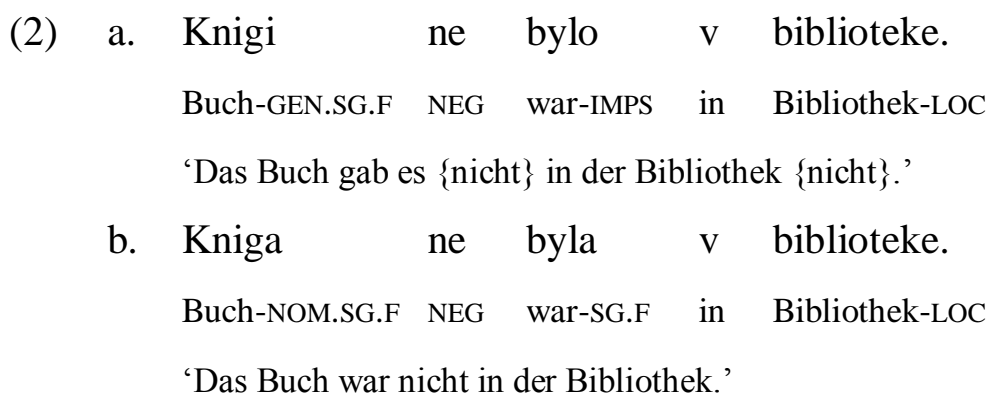


Der affirmative Satz in (1) kann entweder existenzielle oder prädikative Lesart haben. Seine Oberfläche lässt ohne Äußerungskontext nicht erkennen, welche Lesart vorliegt. Bei der Negation unterscheiden sich beide Lesarten hingegen strukturell, da die negierte Existenzaussage in (2a) den Genitiv der Negation am 'Subjekt'15 sowie die 'unpersönliche' Form bylo zeigt, wogegen der negierte Kopulasatz in (2b) sich von der affirmativen Variante in (1) lediglich durch die Anwesenheit von ne 'nicht' absetzt. Die PP kann in (2a) daher als adverbiales Adjunkt, in (2b) aber als Komplement der Kopula analysiert werden: ${ }^{16}$

(3) a. [VP [vP knigi $i_{\mathrm{i}}$ ne bylo $\left.\mathrm{t}_{\mathrm{i}}\right]$ [PP $\boldsymbol{v}$ biblioteke $\left.]\right]$

(Adjunkt-PP)

b. [vp kniga ne byla [pp v biblioteke ]]

(Komplement-PP)

Auch entsprechende Daten aus dem Polnischen untermauern diese Position. Ich wähle präsentische Sätze, da sich bei der Negation der Unterschied zwischen Existenz- und Kopulasätzen im Polnischen hier dadurch noch deutlicher als im Russischen zeigt, dass die negierte Existenzform idiosynkratisch nie ma 'es gibt nicht' [wörtlich: 'es hat nicht'] lautet:

(4) Książka jest w bibliotece.

Buch-NOM.SG.F ist-3SG in Bibliothek-LOC

'Das Buch \{ist, gibt es\} in der Bibliothek.'
a. Książki
nie ma
W bibliotece.
Buch-GEN.SG.F NEG hat-IMPS in Bibliothek-LOC
'Das Buch gibt es \{nicht\} in der Bibliothek \{nicht\}.'

b. Książka nie jest w bibliotece.

Buch-NOM.SG.F NEG ist-3SG in Bibliothek-LOC

'Das Buch ist nicht in der Bibliothek.'

15 Zugrunde liegend handelt es sich bei knigi um das 'logische Objekt' des Existenzverbs. Dass diese Konstituente in (2a) satzinitial erscheint, ist nicht obligatorisch, sondern nur analog zu (1). Die Aussage (2a) kann auch durch die Abfolge Ne bylo knigi v biblioteke realisiert werden. Die Wortfolgen sind informationsstrukturell begründbar.

16 Die Repräsentationen sind vereinfacht (syntaktische Grundannahmen in Abschnitt 4.5). " $t$ " markiert als 'Spur' (trace) die Basisposition des 'bewegten' (indizierten) Ausdrucks. Die 'Bewegung' von knigi in (3a) in eine Position linear vor ne gibt lediglich die Oberflächenabfolge wieder und impliziert keine theoretischen Annahmen. 
Der syntaktische Unterschied ist identisch mit dem, der bereits für das Russische diagnostiziert wurde: In (5a) ist die PP Adjunkt, in (5b) dagegen nimmt sie die Komplementposition der präsentischen Kopulaform jest ein:

(6) a. [VP [VP książki nie $\left.m a \mathrm{t}_{\mathrm{i}}\right]$ [PP $\boldsymbol{w}$ bibliotece $\left.]\right]$

(Adjunkt-PP)

b. [VP ksiązka nie jest [PP $\boldsymbol{w}$ bibliotece ] ]

(Komplement-PP)

PPn treten also in russischen und polnischen Kopulasätzen als Prädikative auf. Sie werden in dieser Arbeit dennoch ausgeblendet, da Präpositionen nicht flektieren, also keine morphosyntaktischen Markierungen erhalten. Vor dem Hintergrund der Tatsache, dass die variierenden Markierungen an Prädikativen die formale Basis des Zustandekommens interpretativer Effekte darstellen, sind PPn auf Grund ihrer 'Markierungslosigkeit' - auch als Prädikative - gewissermaßen 'uninteressant' ${ }^{17}$

17 Prädikative Adverbien (z. B. russ. doma 'zu Hause'/poln. dzisiaj 'heute') können als PPn mit koverten P-Köpfen analysiert werden. Vgl. Reyle, Rossdeutscher \& Kamp (2007, 580): “We analyse the adverb gestern as a kind of prepositional phrase with silent preposition and an occurrence of gestern as NP which is governed by this preposition". Auch genitivische Prädikative kann man als PPn behandeln, z. B. russ. srednego rosta/poln. średniego wzrostu 'mittleren Wuchses'. Mit Bailyn (2004, 2012) ließe sich ein koverter Quantifizierer denken, der den Genitiv und die 'Umfangsbedeutung' einbringt. Eine PP-Analyse scheint auch für poln. Sätze wie Ale z ciebie osioł! 'Du bist aber ein Esel!' machbar (vgl. Bogusławski 2001, 104f.). 


\section{Bestandsaufnahme: Kopulasätze im Polnischen und Russischen}

In diesem Abschnitt werden polnische und russische Kopulasätze, die das 'natürliche Habitat' von Prädikatsnomina darstellen, deskriptiv präsentiert. Diese Darstellung bildet eine Einführung in den Gegenstand und die Materialbasis der folgenden Ausführungen. Nacheinander wird jeweils die Situation im Polnischen und Russischen beschrieben. Der Abschnitt gliedert sich in drei Teile, die nacheinander substantivische, adjektivische und schließlich die sog. Zustandsprädikative thematisieren. ${ }^{18}$

\subsection{Substantivische Prädikative}

\subsubsection{Polnisch}

Sofern eine Variation zwischen Nominativ und Instrumental überhaupt möglich ist, erscheinen Prädikatssubstantive in polnischen Kopulasätzen ganz überwiegend im Instrumental (vgl. u. a. Rothstein 1993, 729; Bailyn \& Citko 1999; Bąk ${ }^{11}$ 1999, 413; Bogusławski 2001, 127; Bondaruk 2013b, 148), vgl. (1)-(4): ${ }^{19}$
(1) Ten wysoki blondyn jest pilotem. (Rothstein 1993, 729) dieser große Blonde-NOM.SG.M ist-3SG Pilot-INS.SG.M
'Dieser große Blonde ist Pilot.'

18 Hier werden nur prädizierende Kopulasätze betrachtet, neben denen Higgins (1979) drei weitere Typen - Identitäts-, identifizierende und spezifizierende Sätze - ansetzt. Geist (2006, 16-63) greift seine Klassifikation auf und entwirft ein Szenario, das die einzelnen Typen voneinander abzuleiten erlaubt. Die Basis bildet ein non-overter IDENT-Operator, der eine 'normale' Kopula in eine 'Identitätskopula' überführt (Geist 2006, 34-35), was Identitätssätze hervorbringt. Der Operator kann aber auch referenzielle DPn in "IdentDPn" überführen, die “weder eine prädikative NP noch eine Argument-DP, sondern eine NP vom dritten Typ [...]: eine prädikative DP” (Geist 2006, 51) seien. Es handelt sich somit um einstellige Prädikate mit (verminderter) Referenzialität, die Komplement der 'normalen' Kopula sein können. Sätze mit satzfinalen IdentDPn nennt Geist prädizierend-identifizierend. Wird die IdentDP in eine satzinitiale (Topik-)Position bewegt, ergeben sich spezifizierende Sätze (Übersicht in Geist 2006, 61). Folglich involvieren einzig prädizierende Kopulasätze keinerlei Form von 'Identität'.

19 Da in polnischen Kopulasätzen mit dem Element to (z. B. Adam to lekarz, Adam to jest lekarz, Adam jest to lekarz, Lekarz to Adam etc.; vgl. eine Übersicht bei Bogusławski 2001, 103-104), das in der Literatur sehr unterschiedlich charakterisiert wird (vgl. u. a. Wiśniewski 1990; Bogusławski 2001; Citko 2008; Bondaruk 2010, 2013a, 2013b), nur der Nominativ als Markierung am Prädikatsnomen auftritt, werden diese Sätze hier nicht betrachtet. 
(2) Pani Krusiewicz jest szwaczką.

Frau Krusiewicz-NOM.SG.F ist-3SG Näherin-INS.SG.F

'Frau Krusiewicz ist Näherin.'

(3) $[\ldots]$ mój ociec jest chemikiem $[\ldots] \quad$ K06, 37 mein Vater-NOM.SG.M ist-3SG Chemiker-INS.SG.M '... mein Vater ist Chemiker ...'

(4) Chcę być prezydentem i będę nim. will-1SG sein-INF Präsident-INS.SG.M und werde sein-1SG er-INS 'Ich will Präsident sein und werde es sein.'

(Rothstein 1993, 743)

In allen Beispielen erscheint die prädikative NP postkopular bzw. satzfinal. Die folgenden Beispiele zeigen dagegen präkopulare bzw. satzinitiale substantivische Prädikative, die ebenfalls mit dem Instrumental markiert sind:

(5) Moim obowiązkiem jest prowadzić korespondencję. meine Pflicht-INS.SG.N ist-3SG führen-INF Korrespondenz-ACC.SG 'Meine Pflicht ist (es), die Korrespondenz zu führen.'

(Rothstein 1993, 738)

(6) Duszą towarzystwa był (Rothstein 1993, 743)

Seele-INS.SG.F Gesellschaft-GEN.SG war-SG.M

zięć Kowalskich [...].

Schwiegersohn-NOM.SG.M Kowalski-GEN.PL

'Die Seele der Gesellschaft war der Schwiegersohn der Kowalskis ...'

Erscheint die Kopula in partizipialer Form, ist am substantivischen Prädikativ ausschließlich der Instrumental zulässig (vgl. Bogusławski 2001, 112-113):

(7) monumentalny gmach, będący repliką NKJP monumentaler Bau-NOM.SG.M seiend-NOM.SG.M Replik-INS.SG.F wiedeńskiej katedry św. Stefana Wiener Stephansdom-GEN.SG 'dieser Monumentalbau, der eine Replik des Wiener Stephansdoms ist' 
Der Instrumental kann so zwar als dominierende, nicht aber als ausschließliche Markierungsoption für polnische Prädikatssubstantive gelten (vgl. Bogusławski 2001, 127-128). Neben ihm tritt auch der sog. "doppelte Nominativ" auf. Dies gilt nicht nur bei finiten Formen der Kopula, sondern auch bei ihrem Infinitiv (bei dem der Instrumental aber klar dominiert, siehe Bogusławski 2001, 113): ${ }^{20}$
(8) Zosia jest wojewodzianka.
(Bogusławski 2001, 107)
Zosia-NOM.SG.F ist-3SG Voivodentochter-NOM.SG.F
'Zosia ist Tochter eines Voivoden.'
(9) Stefan jest orzel.
Stefan-NOM.SG.M ist-3SG Adler-NOM.SG.M
'Stefan ist ein Adler.'
(Bogusławski 2001, 109)
$\begin{array}{llll}\text { (10) On chciał być } & \text { w swojej wsi } \\ \text { er-NOM wollte-SG.M } & \text { sein-INF } & \text { in seinem Dorf }\end{array}$
(Bogusławski 2001, 113)
(taki)
Walęsa.
solcher-NOM.SG.M Wałęsa-NOM.SG.M
'Er wollte in seinem Dorf(so) ein Wałęsa sein.'

Es ist nachzulesen, der Nominativ könne - als Alternative zum Instrumental eine expressive bzw. emotionale Lesart implizieren (siehe Rothstein 1993, 729; Bogusławski 2001, 117), vgl. (11)-(13):
(11) Jesteś idiota!
bist-2SG Idiot-NOM.SG.M
'Du bist ein Idiot!'

(Rothstein 1993, 729)

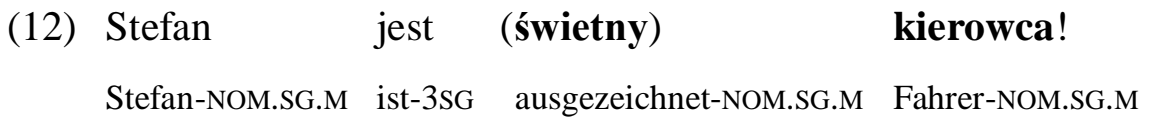

'Stefan ist (ein ausgezeichneter) Fahrer.'

(Bogusławski 2001, 104)

20 Bąk $\left({ }^{11} 1999,413-414\right)$ zufolge tritt der Nominativ in der gesprochenen Sprache und in der Literatur auf. Er werde primär im Falle des Fehlens einer (overten) Kopulaform, bei Eigennamen und bei Nationalitätenbezeichnungen verwendet (bei letzteren sei er heute archaisch). 
(13) Ale on jest wędkarz!

(Bogusławski 2001, 117)

aber er-NOM ist-3SG Angler-NOM.SG.M

'Der ist aber ein Angler!'

Diese Beobachtung bestätigt sich auch in der Tatsache, dass, sofern in diesen oder ähnlichen Beispielen der Instrumental anstelle des Nominativs verwendet wird, die entsprechenden 'neutralen' Äußerungsvarianten entstehen: ${ }^{21}$
Stefan
jest (świetnym)
kierowcą.
Stefan-NOM.SG.M ist-3SG ausgezeichnet-INS.SG.M Fahrer-INS.SG.M

'Stefan ist (ein ausgezeichneter) Fahrer.'

(Bogusławski 2001, 104)

(15) On jest zapalonym wędkarzem.

(Bogusławski 2001, 117)

er-NOM ist-3SG begeisterter Angler-INS.SG.M

'Er ist begeisterter Angler.'

(16)

$$
\begin{aligned}
& \ldots, \text { ale on jest wędkarzem. } \\
& \text { aber er-NOM ist-3SG Angler-INS.SG.M } \\
& \text { '..., aber er ist Angler.' }
\end{aligned}
$$

Kommt ferner der "Bewunderungs- oder Überraschungsausdruck" (Bogusławski 2001, 117) ale 'aber' zum Einsatz, wird der Instrumental völlig inakzeptabel, was die (mögliche) 'Expressivität' der Nominativmarkierung erneut bestätigt:

$\begin{array}{lllll}\text { (17) } & \text { Ale on jest wędkarz / *wędkarzem! } \\ \text { aber } & \text { er-NOM } & \text { ist-3SG } & \text { Angler-NOM.SG.M } & \text { Angler-INS.SG.M }\end{array}$

'Der ist aber ein Angler!'

(Bogusławski 2001, 117)

Ohne ale ist der Instrumental dagegen (auch in expressiven Äußerungen) zulässig:

21 Aber auch Sätze wie (14) bis (16) mit dem Instrumental können - mit einer entsprechenden Intonation - eine expressive bzw. emotionale Färbung erhalten (siehe auch (18) weiter unten). 
(18) Martens, jesteś idiotą!
Martens bist-2SG Idiot-INS.SG.M
'Martens, du bist ein Idiot!'

Zusammenfassend kann für polnische prädizierende Kopulasätze der Instrumental als "Standard" betrachtet werden. Bogusławski ist zuzustimmen, wenn er schreibt: "[O]ffensichtlich [ist] die instrumentalische Markierung negativ (by default) bestimmt." (Bogusławski 2001, 127). Ihr gegenüber ist "die nominativische Markierung nicht nur rein quantitativ (Frequenz), sondern auch in qualitativer Hinsicht $[\ldots]$ weitgehend in den Hintergrund gerückt worden $[\ldots]$. Nichtsdestoweniger ist die nominativische Markierung in einer Reihe von Fällen unentbehrlich, und zwar nicht nur in einigen lexikonbasierten Fällen, sondern auch in Fällen, die eine verallgemeinerte, klassenartige Charakterisierung zulassen oder erfordern.” (Bogusławski 2001, 127-128) Gleichzeitig ist der Instrumental - was durchaus keinen Widerspruch darstellt - ein explizites Signal für den prädikativen Status des betreffenden Ausdrucks (vgl. Hentschel 1991). Daraus folgt wiederum, dass auch die deskriptive Bedeutung der involvierten Nomina, der Kontext sowie das Weltwissen wichtige Faktoren für die Wahl der einen oder anderen Form bzw. Markierung am Prädikativ sind bzw. sein können. An das Ende dieses Abschnitts stelle ich ein 'griffiges' Fazit von Bogusławski, dem ich nur zustimmen kann:

Zwischen den Spracheinheiten "Kopula + Nom.", "Kopula + Instr." besteht tatsächlich das Verhältnis der Variation im eigentlichen Sinne; sie sind keine zwei verschiedenen semantischen Entitäten. Meine Voraussetzung dabei ist, daß wir den Terminus Variation eben auf das Fehlen der semantischen Unterschiede (im strengen Sinne) beziehen. (Bogusławski 2001, 127)

Eben hierin besteht m. E. eine grundsätzliche Parallele zum Russischen, wenngleich die Verwendungsbedingungen bzw. -kontexte für die Markierungen - und teilweise auch die Markierungen selbst - mitunter deutlich anders aussehen. Die Lage im Russischen wird im folgenden Unterabschnitt dargestellt. 


\subsubsection{Russisch}

In russischen präsentischen Kopulasätzen tritt in 'unmarkierten' Äußerungen die sog. "Nullkopula" auf. Die Annahme einer "stummen" Kopulaform folgt einer langen slavistischen Tradition (siehe u. a. Jakobson 1971b). ${ }^{22}$ Die Nullkopula als defiziente bzw. reduzierte Verbform ohne lautliche Matrix muss als finit gelten, ist aber hinsichtlich Person und Numerus unterspezifiziert bzw. 'variabel', so dass sie mit jeder möglichen Kombination dieser Merkmale, die ein potenzieller Subjektausdruck aufweist, kompatibel ist. Mit der Nullkopula erscheint am substantivischen Prädikativ konsequent der Nominativ; vgl. (19)-(26):

\footnotetext{
(19) $\mathrm{Ja} /$ ty/on $\quad-\quad$ prepodavatel'.

ich/du/er-NOM.M $\varnothing-1 / 2 / 3$ SG Dozent-NOM.SG.M

'Ich/du/er bin/bist/ist (ein) Dozent.'
}

(20) $\mathrm{Ja} /$ ty/ona $\quad-\quad$ prepodavatel'nica.

ich/du/sie-NOM.F $\varnothing-1 / 2 / 3$ SG Dozentin-NOM.SG.F

'Ich/du/sie bin/bist/ist (eine) Dozentin.'

(21) My/vy/oni - druz'ja.

wir/ihr/sie-NOM $\varnothing-1 / 2 / 3$ PL Freund-NOM.PL

'Wir/ihr/sie sind/seid/sind Freunde.'

(22) Otec - učitel'.

(Š\&L 1990, 428)

Vater-NOM.SG.M $\varnothing$-3SG Lehrer-NOM.SG.M

'(Der) Vater ist (ein) Lehrer.'

$\begin{array}{llll}\text { (23) Ona } & - & \text { ženščina } & \text { umnaja. } \\ \text { sie-NOM.SG.F } & \varnothing \text {-3SG } & \text { Frau-NOM.SG.F } & \text { klug LF-NOM.SG.F }^{2}\end{array}$

(Š\&L 1990, 430)

'Sie ist eine kluge Frau.'

22 Beobachtungen, die die Annahme einer "Nullkopula" im Russischen legitimieren, werden u. a. von Geist (2008) eingehend rekapituliert und besprochen. 


\section{(24) Satira - delo ser'ëznoe. \\ Satire-NOM.SG.F $\quad \varnothing$-3SG Sache-NOM.SG.N $\quad$ ernst $_{\text {LF-NOM.SG.N }}$}

( $\breve{S} \& L ~ 1990,428)$

'(Die) Satire ist eine ernste Angelegenheit.'

(25) Ėtot čelovek

$$
\text { - sama principial'nost'. }
$$

dieser Mensch-NOM.SG.M $\varnothing$-3SG selbst $_{\mathrm{KF}}$-NOM.SG.F Prinzipialität-NOM.SG.F

'Dieser Mensch ist die Prinzipientreue selbst.'

(Š\&L 1990, 428)

(26) A my - kollektiv.

NKRJ

aber wir-NOM $\varnothing$-1PL Kollektiv-NOM.SG.M

'Aber wir sind ein Kollektiv.'

Mitunter werden Beispiele angeführt, die zeigen sollen, dass mit der Nullkopula auch der prädikative Instrumental erscheinen könne, vgl. z. B. (27) und (28):
(27) Sergej u nas načal'nikom. (vgl. Geist 2006, 173)
Sergej-NOM.SG.M bei-PRP uns-GEN Chef-INS.SG.M
'Sergej ist bei uns der Chef.'
(28) Anna v bol'nice medsestroj. (vgl. Geist 2006, 173)
Anna-NOM.SG.F in-PRP Krankenhaus-LOC Krankenschwester-INS.SG.F
'Anna ist im Krankenhaus Krankenschwester.'

Diese Fälle werden hier zunächst lediglich erwähnt. Wie sich zeigt, taucht in ihnen stets weiteres Material auf, oft in Form einer lokalen PP. In Abschnitt 5.5 werde ich diese Fälle diskutieren. Vorerst lasse ich offen, ob auch die Nullkopula imstande ist, den Instrumental an ihrem Komplement zu lizenzieren.

Statt der Nullkopula kann - wenn auch heute nurmehr marginal bzw. auf markierte Kontexte beschränkt - deren overtes "Substitut" est' auftreten, das ebenso finit und 'variabel' wie die Nullkopula selbst ist (vgl. Geist 2006, 164-166). Als Voraussetzungen für den Gebrauch von est' nennt Geist Verum-Fokussierung oder 
temporalen Kontrast. Daneben tritt est' buchsprachlich auch in definitorischen Wendungen auf. Diese Fälle werden in (29) bis (31) illustriert: ${ }^{23}$

(29) [Vse dvigajutsja, a ètot ležit, kak trup.]

NKRJ

On i EST' trup!

er-NOM auch ist-3SG Leiche-NOM.SG.M

'[Alle bewegen sich, und der liegt da wie eine Leiche.] Er IST eine Leiche!'

(30) Sojuz Sovetskich Socialističeskich Respublik est'

UdSSR-NOM.SG.M

ist-3SG

\title{
socialističeskoe gosudarstvo rabočich i krest’jan.
}

Statt der Werktätigen-NOM.SG.N

'Die UdSSR ist ein sozialistischer Staat der Arbeiter und Bauern.'

\author{
(31) a. Ty byl, est' i budeš' (Geist 2006, 165) \\ du-NOM warst-SG.M bist-2SG und wirst sein-2SG \\ moj otec / moim otcom. \\ mein Vater-NOM.SG.M mein Vater-INS.SG.M \\ 'Du warst, bist und wirst mein Vater sein [bleiben].'
}

In präteritalen Kopulasätzen, die Geist $(2006,66)$ als “stabilen Kern” der Kasusvariation betrachtet, konkurrieren Nominativ und Instrumental regelmäßig (eine andere Sicht äußern z. B. Krasovitsky et al. 2008). Dies zeigen die folgenden Beispiele, wobei auf etwaige interpretative Unterschiede an dieser Stelle (noch) nicht eingegangen wird: ${ }^{24}$

23 Beispiel (31) zeigt, dass die Möglichkeit des Instrumentals am Komplement von est' (und damit wohl auch der "Nullkopula") nicht prinzipiell ausgeschlossen zu sein scheint. Dass er fast nie auftritt, scheint keine lexikalische Ursache zu haben. Siehe Abschnitt 5.5.

Nurmehr vereinzelt tritt die Form der 3. Ps. Pl. sut' 'sind' auf, die heute rein buchsprachlich und archaisch ist (vgl. Geist 2006, 166). Sie kann als "Relikt" gelten, zumal sie als markierte Form der 3. Ps. Pl. zunehmend von est' verdrängt wird, das durch seine 'variablen' Kongruenzmerkmale auch diese Kombination 'bedienen' kann.

24 Es sei bemerkt, dass die AG (1980, 239) erwähnt, der Nominativ könne als Alternative zum Instrumental archaischen Charakter tragen. Allerdings wird dies nur mit Blick auf "Verben des Bleibens, Zeigens und Nennens" (prebyvanie v sostojanii, vyjavlenie, nazyvanie) vermerkt, während die 'normale' Kopula in diesem Zusammenhang nicht erwähnt wird. 
(32) a. On byl moskvič.

( $\breve{S} \& L ~ 1990,429)$

er-nom war-SG.M Moskauer-NOM.SG.M

b. On byl moskvičom.

er-NOM war-SG.M Moskauer-INS.SG.M

'Er war (ein) Moskauer.'

(33) Boris byl muzykant.

(Bailyn 2012, 194)

Boris-NOM.SG.M war-3SG Musiker-NOM.SG.M

'Boris was a musician (in his very nature).'

(34) Boris byl muzykantom.

Boris-NOM.SG.M war-3SG Musiker-INS.SG.M

'Boris war Musiker [von Beruf].'

Folgt man Comrie, Stone \& Polinsky (1996, 170), so ist der prädikative Instrumental im heutigen Russischen mit einer Futur-, Konjunktiv- oder Imperativkopula nahezu obligatorisch. Ähnlich äußern sich auch Vinogradov \& Istrina (1960, 424, 432). Hentschel (1993, 288) erblickt hierin “irreale" Modus-/Tempusformen, die - verglichen mit den tendenziell "realen” Tempora Präsens und Präteritum verhältnismäßig 'undurchsichtige' modal-temporale Verhältnisse bezeichnen und so - in Hentschels Tendenzanalyse - die Neigung zum Instrumental verstärken. Festzuhalten ist, dass mit den genannten Modus- und Tempusformen der Instrumental tatsächlich deutlich häufiger als der Nominativ verwendet wird, dass letzterer jedoch nicht prinzipiell ausgeschlossen ist. Im Folgenden wird dies zunächst anhand eines futurischen Satzes illustriert:
(35) Ту
budeš'
artist
/ artistom.
du-NOM wirst sein-2SG Künstler-NOM.SG.M Künstler-INS.SG.M
'Du wirst (ein) Künstler sein.'

( $\breve{S} \& L ~ 1990,429)$

Das nächste Beispiel zeigt den Konjunktiv. Auch hier sind sowohl Nominativ als auch Instrumental möglich, obwohl auch hier der Nominativ als 'markiert' gilt: 
(36) Diskussija

byla by

( $\breve{S} \& L ~ 1990,429)$

Diskussion-NOM.SG.F war-SG.F KONJ

poleznoe delo / poleznym delom.

nützliche Sache-NOM.SG.N nützliche Sache-INS.SG.N

'Eine Diskussion wäre eine nützliche Sache.'

Schließlich bezeugen Beispiele mit dem Imperativ der Kopula, dass auch hier beide Kasusformen am Prädikativ prinzipiell verfügbar sind:

(37) $\mathrm{Nu}$, bud' drugom, Anatol'ič!

NKRJ

nun sei-IMP Freund-INS.SG.M Anatol'ič-NOM.SG

'Nun sei ein Freund, Anatoličc!'

(38)

Bud' drug $\quad-$ podelis' $\quad[\ldots]$
sei-IMP Freund-NOM.SG.M teile-IMP-REFL
'Sei ein Freund - teile ... [!]'

NKRJ

(39) Slušaj, perestan', bud' dobryj čelovek [...]

NKRJ höre-IMP höre auf-IMP sei-IMP guter Mensch-NOM.SG.M

'Höre zu, höre [damit] auf, sei ein guter Mensch ... [!]'

Bemerkenswert ist, dass Geist $(2006,105)$ bei russischen Kopulasätzen mit imperativischer Kopula eine syntaktische "Blockierung" des Nominativs behauptet:

\section{(40) Bud' porjadočnym čelovekom / *porjadočnyj čelovek!}

sei-IMP anständiger Mensch-INS.SG.M

'Sei ein anständiger Mensch!' anständiger Mensch-NOM.SG.M

(Geist 2006, 105)

Als Ausnahme davon gelten ihr nicht eingeleitete Nebensätze mit der Bedeutung einer unerfüllten Bedingung, in denen sie einen Subjektausdruck als syntaktisch vorhanden betrachtet. Dieser syntaktische Subjektausdruck (im Beispiel (41) kursiv) kann nach Geist als Kongruenzgeber für das Prädikativ dienen und so den Nominativ an ihm lizenzieren: 
(41) Bud' on porjadočnym čelovekom /

(Geist 2006, 106)

sei-IMP er-NOM anständiger Mensch-INS.SG.M

porjadočnyj čelovek, on by postupil inače.

anständiger Mensch-NOM.SG.M er würde handeln anders

'Wäre er ein anständiger Mensch, würde er anders handeln.'

Im Gegensatz dazu, so Geist, fehle in 'normalen' Imperativsätzen (wie den in (37)-(39) genannten!) ein "kongruenzgebendes, explizites Subjekt" (Geist 2006, 105), was den von ihr postulierten Ausschluss des Instrumentals erklären könne. In Anbetracht der oben angeführten Belege für den Nominativ auch in 'normalen' Imperativsätzen sind sowohl Geists Aussage als auch die strukturell-syntaktische Erklärung für ihre Beobachtungen mindestens zweifelhaft. Vielmehr scheint generell auch in imperativischen Sätzen die Wahl zwischen Nominativ und Instrumental am Prädikativ möglich zu sein, wenn auch der Instrumental signifikant überwiegt. Sofern keine strikt grammatischen Ursachen für den Ausschluss einer der beiden Kasusformen zu finden sind, weil ein solcher Ausschluss schlichtweg nicht vorliegt, muss dieser "Überhang” eine andere Ursache haben. Diese könnte in der Tat in dem tendenziell "modalen" bzw. "irrealen" Charakter des Imperativs (wie auch des Futurs und Konjunktivs) bestehen (vgl. Hentschel 1993, 288).

Der Nominativ sowie der Instrumental sind ferner in sog. Raising- bzw. Hebungsstrukturen wie (42a) als Alternativen möglich. Der Nominativ ist hingegen dann vollkommen ausgeschlossen, wenn eine sog. Kontrollstruktur wie in (42b) vorliegt (beide Beispiele nach Geist 2006, 105):

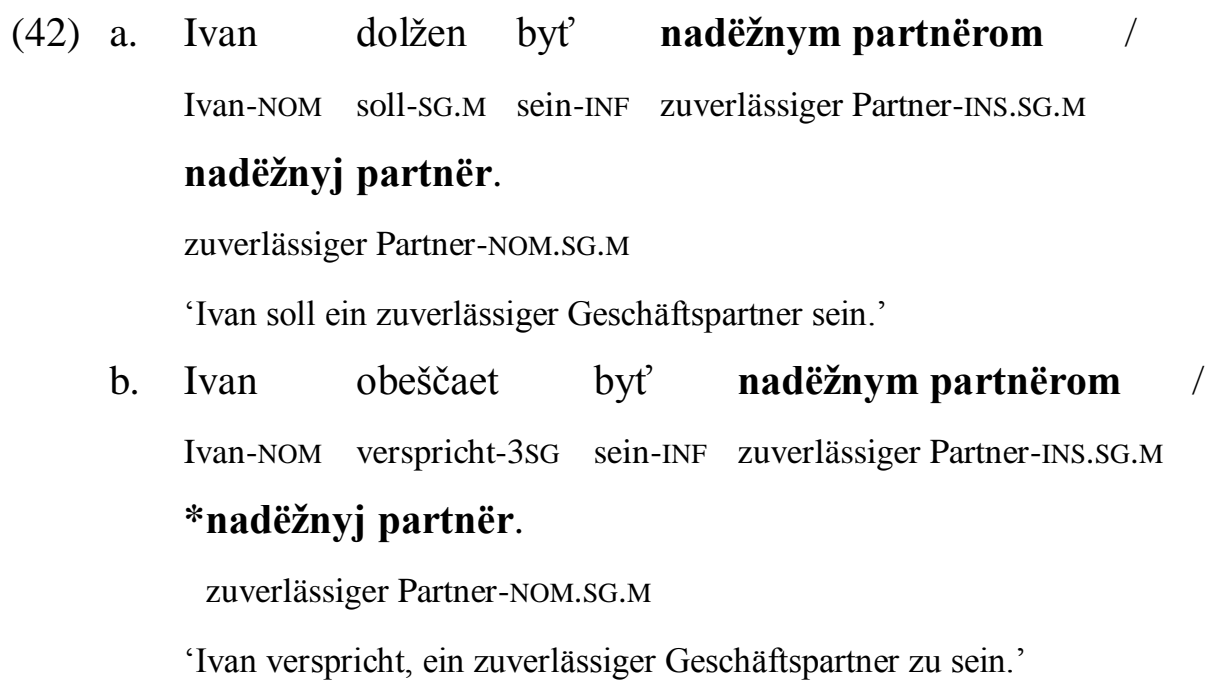


Wie schon in Bezug auf Imperativformen der Kopula, so begründet Geist (2006) den Ausschluss des Nominativs auch in Kontrollstrukturen wie (42b) damit, dass hier kein syntaktischer Kongruenzgeber im Nominativ verfügbar sei, über den der Kongruenzkasus lizenziert werden könne. Da die Datenlage hier eindeutiger als im Falle der Imperativsätze ist, kann diesem Schluss für Kontrollstrukturen m. E. zugestimmt werden. Während im Falle von Raising-Strukturen wie (42a) der Subjektausdruck des eingebetteten (Kopula-)Satzes in die Matrixstruktur angehoben wird und eine syntaktische Spur an seiner Basisposition zurücklässt, die als Kongruenzgeber für das Prädikativ fungieren kann, basieren Kontrollsatzgefüge wie (42b) auf eingebetteten satzwertigen Strukturen, in denen ein (aus der Matrix heraus oder arbiträr kontrolliertes) PRO-Subjekt vorliegt, das per definitionem "kasuslos" (Chomsky 1981, 56) oder lediglich mit einem "Nullkasus" (Chomsky 1995a, 118ff.) ausgestattet ist. In jedem Fall hat PRO kein Nominativmerkmal, weshalb dieser Kasus auch am Prädikativ nicht erscheinen kann. Einzige Option ist somit die Instrumentalmarkierung, da sie (in ihrer Lizenzierung unabhängig von PRO bzw. von der syntaktischen Relation der Kongruenz) unmittelbar durch die Kopula zugewiesen wird, also auf Rektion beruht.

Diese Analyse kann auch Fälle erklären, bei denen Prädikatssubstantive als sekundäre Prädikate verwendet werden, vgl. (43) (nach Geist 2010, 245):

(43) On vernulsja $\quad$ domoj oficerom $\quad /$ *oficer
er-NOM kehrte zurück-SG.M nach Hause
'Ivan kehrte als Offizier nach Hause zurück.'

Auch in diesem Fall ist der Nominativ ausgeschlossen. Für Adjektive, bei denen auch der Nominativ auftritt, sind weitere Erwägungen notwendig. Es zeigt sich aber, dass die vorgestellte Analyse Bestand hat. Die fraglichen nominativischen Formen sind nämlich keine Sekundärprädikate, sondern NP-Adjunkte (Wilder 1994) bzw. appositive Adjunkte (Bailyn 2012) (siehe hierzu Abschnitt 5.4). Es ist anzunehmen, dass die sekundäre Prädikation durch einen koverten verbalen (bzw. prädikativen) Kopf zustande kommt, der eine grammatische Bedeutung und Argumentstruktur hat, die einer infinit(ivisch)en Kopulaform entsprechen. ${ }^{25}$ Durch

25 Für diesen leeren Kopf verwende ich das Label V0 (Verb), da ich jede Form von "stummer" Kopula als Verbform betrachte. Alternativ sind auch in der Literatur anzutreffende Label wie 
seine faktische Infinitheit ist dieser Kopf unfähig, das externe Argument des Prädikativs als nominativischen Subjektausdruck zu syntaktifizieren. Demnach wird auch durch ihn lediglich ein PRO-Subjekt projiziert, das nicht als Kongruenzgeber fungieren kann. Vgl. die folgende vereinfachte syntaktische Struktur für (43), in der die (fett markierte) Projektion des koverten V-Kopfes Adjunkt am Matrixsatz ist. ${ }^{26}$ Der Instrumental wird direkt durch den V-Kopf vergeben:

(44) $\left[\mathrm{XP}\right.$ on $_{\mathrm{i}}$ vernulsja domoj [vP $\mathrm{PRO}_{\mathrm{i}} \varnothing_{\mathrm{V}}$ oficerom $\left.\left.\mathrm{INS}\right]\right]$

Kontrollstrukturen und sekundäre Prädikation stellen also diejenigen Bereiche der russischen Prädikativmarkierung dar, die keine Kasusvariation zulassen (was präsentische Kopulasätze mit der "Nullkopula" oder deren sog. Substitute angeht, so wurde schon oben darauf hingewiesen, dass erst noch zu klären ist, ob auch sie einen solchen Bereich darstellen oder nicht). In diesen Fällen hat bzw. erhält die Prädikativform keinerlei "Eigenbedeutung”. Anders formuliert, sie lässt keine interpretativen Effekte zu, die man mit ihr assoziieren könnte (vgl. u. a. Hentschel 1991; Kuznetsova \& Rakhilina 2010; Kuznetsova 2013). ${ }^{27}$

Solche Fälle stellen die primäre Evidenz für die in dieser Arbeit vertretene These dar, dass die möglichen Prädikativmarkierungen per se keine "Eigenbedeutung" haben, und zwar unabhängig davon, ob sie prinzipiell variieren können oder - wie in den zuvor genannten Beispielen - syntaktisch in ihrer Verfügbarkeit beschränkt sind und daher die jeweils einzige Wahlmöglichkeit darstellen. Die Festschreibung einer (ggf. auch unterspezifierten) invarianten Semantik für eine bestimmte Markierungsform läuft zwangsläufig darauf hinaus, dass diese Form die be-

$\operatorname{Pr}(\mathrm{ed})^{0}$ ('Prädikation'), $\operatorname{Rel}^{0}$ ('Relator') oder $v^{0}$ ('leichtes Verb') denkbar. Im Interesse möglichst weniger Zusatzannahmen verzichte ich, soweit möglich, auf solche "Spezialkategorien".

26 Ob es sich um ein VP- oder IP-Adjunkt handelt, lasse ich offen. Hinterhölzl $(2001,101)$ verbindet den Typ sekundärer Prädikation, d. h. (i) zirkumstanzial, (ii) rein depiktiv oder (iii) temporal restringiert, mit bestimmten Adjunktpositionen, namentlich (i) CP, (ii) IP oder (iii) VP. Somit ist möglich, dass Sekundärprädikate an verschiedenen Positionen 'andocken' und dadurch entsprechend verschiedenartige Beiträge zur Satzaussage leisten.

27 Unbesehen der Frage, ob die "Nullkopula" den Instrumental zuweisen kann oder nicht, gilt dies für präsentische Kopulasätze, in denen de facto nur der Nominativ auftritt, die aber trotzdem keine "permanente", "unspezifische" o. ä. Lesart haben können. Weiter unten werde ich zeigen, dass der Ausschluss des Instrumentals in Präsenssätzen nicht etwa auf eine 'Defizienz' der "Nullkopula", sondern auf die pragmatisch motivierte Vermeidung ambiger Strukturen zurückgeht. 
treffende 'Bedeutung' immer haben muss (auch dann, wenn sie im gegebenen Fall die einzig verwendbare Option ist). Wie später deutlich wird, scheitern Szenarien, die den relevanten Bedeutungsanteil (z. B. des Instrumentalsuffixes) dann 'neutralisieren' wollen, wenn keine Variation möglich ist, an prinzipiellen theoretischen Problemen, die teilweise sogar die Architektur des Sprachsystems bzw. die Interaktion der in ihm wirkenden Module betreffen und somit kaum zu ignorieren sind. Die semantische Determiniertheit der Prädikativformen und -markierungen, die solche Erklärungen erreichen, wird erkauft durch den Zwang nach der "Tilgung" ebendieser Determinierung in Fällen, in denen die Daten die vorhergesagten Bedeutungen/Interpretationen nicht bestätigen (siehe auch Pitsch 2014).

Gleiches gilt für Ansätze, die die interpretativen Effekte nicht semantisch, sondern syntaktisch zu erklären suchen. Hier wird einer bestimmten Markierungsform jeweils eine spezifische syntaktische Struktur zugeordnet, der sie gewissermaßen entspringt und die demnach auch dann gegeben sein muss, wenn die fragliche Form die einzige Option ist. Auch hier sind Zusatzannahmen notwendig, um das Fehlen einer bestimmten Interpretation in diesen Fällen zu erklären.

Eine wirksame Alternative zu solchen "deterministischen" Erklärungsmodellen bilden "diakritische" bzw. "formbasierte" Analysen wie die von Hentschel (1991, 1993a, 1995) oder Bogusławski (2001). Sie besagen, dass eine Kasusmarkierung bzw. Prädikativform per se keine lexikalische oder grammatische Eigenbedeutung hat. Vielmehr werde ihr eine bestimmte Lesart erst im Rahmen des Bestehens einer Opposition zu einer anderen, in derselben Position verwendbaren Form lediglich zugeschrieben. Somit handelt es sich um eine ihrer Natur nach semiotischpragmatische Erklärungsweise, deren Ausgangspunkt die bloße morphosyntaktische Form ist. Da mit diesen Formen keine fixe, d. h. im Lexikon oder in der Syntax invariant angelegte Bedeutung(skomponente) verbunden wird, kann es zu den o. g. Problemen im Zusammenhang mit Fällen, in denen keine Variationen möglich sind, nicht kommen. Dieser formbasierte Ansatz, der auch den Kern der vorliegenden Theorie bildet, wird detailliert in Abschnitt 6 besprochen. 


\subsection{Adjektivische Prädikative}

\subsubsection{Polnisch}

Adjektivische Prädikative erscheinen im modernen Polnischen in prädizierenden Kopulasätzen (primäre Prädikation; siehe auch Abschnitt 5.3.1) stets im Nominativ (vgl. u. a. Bąk $\left.{ }^{11} 1999,413\right)$ :

(45) Była pojętna.

K06, 16

war-SG.F gelehrig-NOM.SG.F

'Sie war gelehrig.'

(46) Plac

jest już pusty [...].

K06, 13

Platz-NOM.SG.M ist-3SG schon-ADV leer-NOM.SG.M

'Der Platz ist schon leer ...'

(47) Zadanie $[\ldots]$ jest bardzo trudne.

(Rothstein 1993, 740)

Aufgabe-NOM.SG.N ist-3SG sehr-ADV schwierig-NOM.SG.N

'Die Aufgabe ... ist sehr schwierig.'

(48) Są niezupełnie trzeźwi, chudzi, K06, 22 sind-3PL NEG-völlig-ADV nüchtern-NOM.PL.MPS hager-NOM.PL.MPS

żylaści, $\quad$ szczerbaci $\quad[\ldots]$.

sehnig-NOM.PL.MPS mit Zahnlücken(ADJ)-NOM.PL.MPS

'Sie sind nicht ganz nüchtern, hager, sehnig, haben Zahnlücken ...'

(49) Że język hiszpański wcale nie jest taki trudny. dass Spanisch-NOM.SG.M gänzlich NEG ist-3SG so schwierig-NOM.SG.M 'Dass die spanische Sprache überhaupt nicht so schwer ist.'

$\begin{array}{lllll}\text { (50) Jurek nie jest brzydki } & \text { nie }\end{array}$ Jurek-NOM.SG.M NEG ist-3SG hässlich-NOM.SG.M und NEG jest nudny.

ist-3SG langweilig-NOM.SG.M

'Jurek ist nicht hässlich und ist nicht langweilig.' 

(51) Pola Szwarcwald
była sympatyczna i
K06, 8
Pola Szwarcwald-NOM.SG.F war-SG.F sympathisch-NOM.SG.F und
mądra, a $[\ldots]$ naprawdę niebrzydka.
klug-NOM.SG.F und wirklich-ADV NEG-hässlich-NOM.SG.F
'Pola Szwarcwald war sympathisch und klug, und ... wirklich nicht hässlich.'

Der Nominativ erscheint auch dann, wenn eine infinitivische Kopula als Komplement eines Raising-Verbs (z. B. musieć 'müssen') auftritt. In einer solchen Konfiguration wird der Subjektausdruck durch das Raising-Verb "angehoben" und kongruiert daher mit letzterem, wenn er auch logisch der Einheit aus Kopula und Prädikatsnomen zuzuordnen ist; vgl. dazu das folgende Beispiel: ${ }^{28}$
Musieli
być
oślepieni
tym słońcem.
mussten-PL.MPS sein-INF geblendet-NOM.PL.MPS diese Sonne-INS.SG
'Sie mussten von dieser Sonne geblendet (gewesen/worden) sein.'

K06, 40

Als Fälle von Nominativmarkierungen betrachte ich auch Kurzformen, die im Polnischen jedoch nurmehr rudimentär von bestimmten Adjektivlexemen bildbar sind (vgl. Rothstein 1993, 705). Kurz- und Langformen liegen heute nebeneinander z. B. noch bei pewien/pewny 'gewiss' oder bei zdrów/zdrowy 'gesund' vor (im letzteren Fall ist die Kurzform allerdings deutlich archaisch; vgl. Rothstein 1993, 705):

\footnotetext{
Ojciec

nie był

pewien

jego pochodzenia

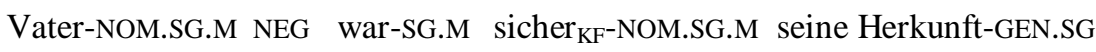

'Der Vater war seiner Herkunft nicht sicher[.]'
}

NKJP

28 U. a. Geist (2006, 104-106) zufolge wird bei Raising das Satzsubjekt “angehoben" und hinterlässt eine syntaktische Spur $(t)$, die als Kongruenzgeber für das Prädikativ fungiert. Mit dieser Spur kongruiert das adjektivische adjektivische Prädikativ auch im polnischen Beispiel in (i):

(i) [vP pro $_{\mathrm{NOM}}$ musieli [VP $\boldsymbol{t}_{(\mathrm{NOM})}$ być [AP Oślepieni $\left.\left.{ }_{\mathrm{NOM}}\right]\right]$ ].

Beachte, dass das non-overte pro im Polnischen, einer Nullsubjektsprache, die unmarkierte Realisierungsform pronominaler Subjekte ist. 
(54) Jednak Adaś

siebie.

NKJP

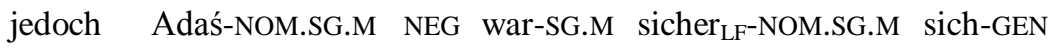

'Adaś jedoch war seiner nicht sicher.'

(55) “jestem zdrów,

przyślij coś do jedzenia” K06, 42

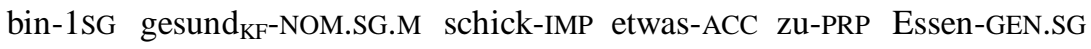

'>bin gesund, schick 'was zu essen`'

(56) Wiem, że nie jestem zdrowy.

NKJP

weiß-1SG dass NEG bin-1SG gesund $_{\text {LF }}$-NOM.SG.M

'Ich weiß, dass ich nicht gesund bin.'

(57) Brat

był wesól.

(Bąk $\left.{ }^{11} 1999,412\right)$

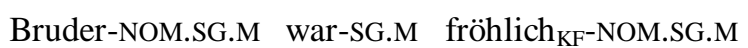

'Der Bruder war fröhlich.'

(58) Tym razem był wesoły.

NKJP

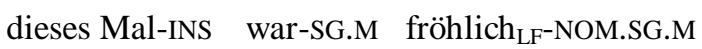

'Dieses Mal war er fröhlich.'

Einige Adjektive haben ausschließlich Kurzformen, so z. B. rad 'froh' oder wart 'wert' (vgl. Rothstein 1993, 705; Bąk ${ }^{11}$ 1999, 412):

(59) Tak

$$
\text { byłem rad! }
$$

solch-NOM.SG.M war-1SG froh-NOM.SG.M

'Ich war so froh!'

(60) Tyle wart jest ten pański świstek?

K06, 20

soviel-ADV wert-NOM.SG.M ist-3SG dieser Ihr Zettel-NOM.SG.M

'Soviel ist dieser Zettel von Ihnen wert?'

Alle bisherigen Belege sprechen dafür, den Nominativ bzw. Kongruenzkasus als

Default adjektivischer Prädikative im Polnischen zu betrachten. Es gibt jedoch einige Fälle, die eine Einschränkung bedeuten könnten: 


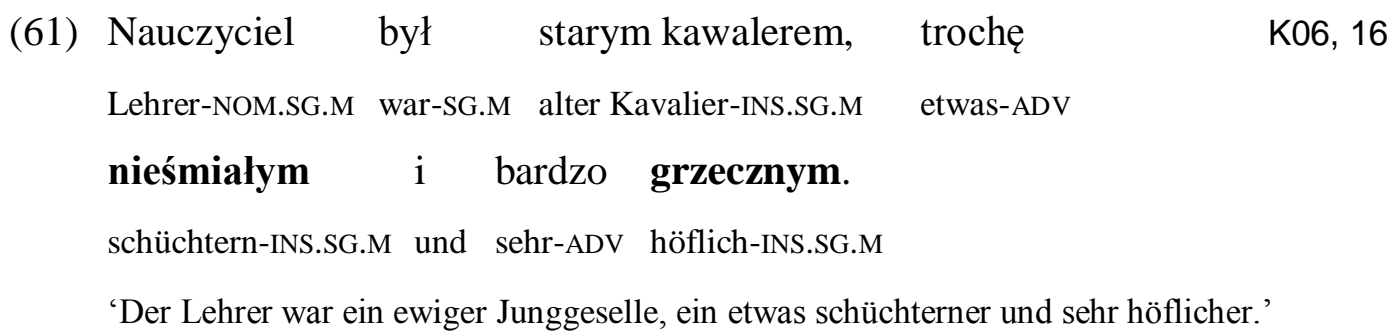

Hier kann die Instrumentalform der appositiv nachgestellten Adjektive nieśmiałym 'schüchtern' und grzecznym 'höflich' jedoch als Ellipse erklärt werden. Als Bezug für beide attributiven Adjektive dient, wie auch für starym 'alt', das Substantiv kawalerem 'Junggeselle', das das eigentliche Prädikatsnomen ist.

In den Bereich der Ellipse gehören m. E. auch Fälle mit possessiven Prädikativen:

\author{
(62) Ta książka jest moja / Jurka. (Rothstein 1993, 748) \\ dieses Buch-NOM.SG.F ist-3SG mein-NOM.SG.F Jurek-GEN.SG.M \\ 'Dieses Buch ist meines/Jureks.'
}

Jedoch betrifft die Ellipse in solchen Fällen nicht Prädikative im engeren Sinne, sondern vielmehr referenzielle Ausdrücke bzw. Determinansphrasen (DPn), da es sich, wie ich annehme, um Identitätssätze mit 'Identitätskopula' handelt. Dieser Kopulatyp, den Geist (2006, 34-35) durch einen IDENT-Operator aus der 'normalen' (prädizierenden) Kopula ableitet, setzt zwei referenzielle Ausdrücke einander gleich ("x = y"). Dies wird deutlich, wenn man die Ellipse in (62) gewissermaßen rückgängig macht; vgl. (63):
(63) Ta książka jest $\{$ moja $\}$ książka $\quad\{$ Jurka $\}$. dieses Buch-NOM.SG.F ist-3SG mein Buch-NOM.SG.F Jurek-GEN.SG.M 'Dieses Buch ist $\{$ mein Buch, das Buch Jureks\}.'

Auch die deutschen Übersetzungen zeigen die Referenzialität der postkopularen Ausdrücke, die sich zum einen im Possessivpronomen mein, zum anderen im Gebrauch des definiten Artikels äußert. Als weiteres Indiz für die Annahme, dass es sich um Identitätssätze handelt, kann gelten, dass anstelle von einfachem jest auch 
die Formen to oder to jest/jest to verwendet werden können, deren Gebrauch für diesen Satztyp charakteristisch ist (siehe u. a. Geist 2006, 44): ${ }^{29}$

\author{
(64) Ta książka to (jest) moja / Jurka. \\ dieses Buch-NOM.SG.F TO ist-3SG mein-NOM.SG.F Jurek-GEN.SG.M \\ 'Dieses Buch ist meines/Jureks.'
}

Vgl. ferner den ähnlichen Fall mit zwei substantivischen Vollausdrücken:

(65) A ta pani to moja żona.

NKJP

aber diese Dame-NOM.SG.F To meine Frau-NOM.SG.F

'Aber diese Dame ist meine Ehefrau.'

29 Für Bogusławski (2001) ist to jest (jest to) eine Einheit. Ganz ähnlich nimmt Bondaruk (2013b, 268-270) an, poln. to sei die overte klitische Belegung des sonst koverten Pred $^{0}$. Die Formen von być sitzen demnach in $v^{0}$. Die Einheit to jest folgt so aus der Klitisierung von to an jest bzw. von Pred ${ }^{0}$ and $v^{0}$.

Bondaruk (2013b) bezeichnet to być-Sätze als prädizierend-definierend (und deren Inversion als spezifizierend). Geists (2006) Klassifizierung entspricht dem, insofern ihre spezifizierenden Sätze aus der Inversion sog. prädizierend-identifizierender Sätze entstehen. Russ. èto wird von Geist als essenzieller Bestandteil von Identitätssätzen bzw. als overtes IDENT analysiert. Es könne sich nicht nur mit koverten, sondern auch overten Kopulaform verbinden, z. B. èto byl (byl èto). Bei overten Form von byt' kann IDENT laut Geist (2006) jedoch auch kovert sein, so dass z. B. auch byl allein die Identitätskopula bzw. -bedeutung vertreten kann.

Aus dem Vergleich von Geist (2006) und Bondaruk (2013b) geht hervor, dass poln. to als Teil von Kopulasätzen flexibler ist als russ. èto. Wie letzteres, kann auch poln. to zusammen mit einer być-Form als Identitätskopula fungieren (und wie im Russischen, kann auch im Polnischen eine bloße Form von być die Identitätskopula vertreten, sofern kovertes IDENT vorliegt). Dies scheint im o. g. Beispiel der Fall. Jedoch klassifiziert Bondaruk to być-Sätze als prädizierend-definierend (vgl. auch Roy 2013), was heißt, dass to dort nicht IDENT sein kann. Dennoch tritt to stets mit nominativischen Prädikatsnomina auf und triggert v. a. im zuletzt genannten Satztyp eine deutlich charakterisierende Lesart.

Es ist plausibel, dass partikelartige Einheiten wie to ähnlich 'expletiv' sein können wie z. B. der indefinite Artikel mit Prädikatsnomina in deutschen Kopulasätzen (vgl. u. a. Admoni 1973, 89; Geist 2006, 28-29, 120-121). Sie hätten dann keine deskriptive, wohl aber eine ikonische 'Bedeutung', indem sie die Lesart, die eine Konstituente oder der Satz selbst hat, syntaktisch verdeutlichen. So könnte man to in prädizierenden Sätzen als bloßen "Verstärker" der identifizierenden Lesart bzw. der Äquativmarkierung am Prädikativ (also des Nominativs) betrachten. In Identitätssätzen hingegen wäre es, ebenso wie russ. èto, ein overter IDENT-Operator. 
Solche Fälle sprechen also nicht gegen die bis dato gültige "Nominativregel" für adjektivische polnische Prädikative.

Rothstein (1993, 729) zufolge erscheinen prädikative Adjektive im Instrumental, wenn die Kopula in der Form des Adverbialpartizips (Gerundiums) erscheint:

Będąc jeszcze młodym, ...
seiend-GERUND noch-ADV jung-INS.SG.M
'Noch jung seiend, ...'

(Rothstein 1993, 729)

"Potenziell" sei dies auch dann der Fall, wenn der Infinitiv być die Bedeutung eines Zustandswechsels impliziere (Rothstein 1993, 730):

(67) Postanowił być oszczędnym. (Rothstein 1993, 730) beschloss-SG.M sein-INF sparsam-INS.SG.M 'Er beschloss, sparsam zu sein.'

Jedoch zeigen Korpusbelege, dass neben dem Instrumental in diesen Fällen auch der Nominativ durchaus zulässig ist:

(68)

Zaczynał nie będąc jeszcze pelnoletni. NKJP begann-SG.M NEG seiend-GERUND noch-ADV volljährig-NOM.SG.M 'Er begann, noch nicht volljährig seiend.'

(69) Martyna Noszczyk [...], będąc Martyna Noszczyk-NOM.SG.F seiend-GERUND schlechter-NOM.SG.F od dwukrotnej mistrzyni Polski von-PRP zweimalige Meisterin Polens-GEN.SG 'Martyna Noszczyk ..., schlechter als die zweimalige Meisterin Polens seiend'

(70) Prokurator chyba postanowił być bezpośredni. NKJP Staatsanwalt-NOM.SG.M wohl-ADV beschloss-SG.M sein-INF direkt-NOM.SG.M 'Der Staatsanwalt beschloss wohl, direkt zu sein [werden].'

Vollends ausgeschlossen ist der Nominativ dagegen, wenn der Infinitiv der Kopula nach einem "Modalprädikativum" wie trzeba 'notwendig' auftritt: 
(71)

$\begin{array}{lllll}\text { Przecież } & \text { trzeba } & \text { być } & \text { otwartym } & \text { [*otwarty }] \\ \text { doch-ADV } & \text { notwendig-IMPS } & \text { sein-INF } & \text { offen-INS.SG.M } & \text { offen-NOM.SG.M } \\ \text { na } & \text { świat. } & & & \end{array}$

auf-PRP Welt-ACC.SG

'Schließlich ist es notwendig, offen für die Welt zu sein.'

(72)

$\begin{array}{lllllll}\text { Czy jej } & \text { nie } & \text { wolno } & \text { być } & \text { głodną } & \text { [*głodna }] & \text { NKJP } \\ \text { ob } & \text { ihr-DAT.F } & \text { NEG } & \text { frei-IMPS } & \text { sein-INF } & \text { hungrig-INS.SG.F } & \text { hungrig-NOM.SG.F }\end{array}$

'Steht es (denn) ihr nicht frei, hungrig zu sein?'

Eine Erklärung für den Ausschluss des Nominativs in diesen Fällen wird in Abschnitt 5.5.2 skizziert. Damit beende ich die deskriptive Beschreibung polnischer prädikativer Adjektive und wende mich im Folgenden dem Russischen zu.

\subsubsection{Russisch}

Da die adjektivischen Kurzformen im heutigen Standardrussischen deulich verbreiteter sind als im Polnischen, ist - mindestens für den Fall der sog. Qualitätsadjektive $^{30}$ - mit drei Realisierungsformen adjektivischer Prädikative zu rechnen: (i) Langform im Nominativ, (ii) Kurzform (ebenfalls im Nominativ) ${ }^{31}$ und (iii) Langform im Instrumental:

${ }^{30}$ Qualitätsadjektive bezeichnen Eigenschaften/Merkmale ganz unmittelbar. Als Gegensatz gelten die sog. Beziehungsadjektive, die Eigenschaften über den Bezug auf eine bestimmte Entität bezeichnen. Beziehungsadjektive sind meist abgeleitet, Qualitätsadjektive dagegen primär (Geist 2006, 125). Dass nur Qualitätsadjektive Kurzformen bilden, ist insofern nicht verwunderlich, als es sich sowohl bei der morphosyntaktischen Klasse der Kurzformen als auch bei der lexikalisch-semantischen Klasse der Qualitätsadjektive um primär prädikative Ausdrucksformen handelt. In diesem Sinne kann von einer Korrelation zwischen Form und Bedeutung gesprochen werden. Beachte, dass Beziehungsadjektive nicht oder nur sehr eingeschränkt prädikativ auftreten; vielmehr sind sie auf den attributiven Gebrauch zugeschnitten, vgl. z. B. škol'naja $a_{\mathrm{LF}}$ kniga 'Schulbuch', aber 'kniga byla školnaja $\mathrm{LF}_{\mathrm{LF}}$ 'Das Buch war schulisch/*Das Buch war Schul-' bzw. *kniga byla školna $a_{\mathrm{KF}}$.

31 Mitunter wird den Kurzformen die Kategorie Kasus abgesprochen, vgl. u. a. Kempgen ( ${ }^{2} 2008$, $164,167)$ oder Geist $(2006,124,138)$. Auf diese Frage werde ich später zurückkommen. 
(73) a. Zagadka byla prostaja.

(Geist 2006, 124)

Rätsel-NOM.SG.F war-SG.F einfach LF -NOM.SG.F

b. Zagadka byla prosta.

Rätsel-NOM.SG.F war-SG.F einfach KF-NOM.SG.F

c. Zagadka byla prostoj.

Rätsel-NOM.SG.F war-SG.F einfach LF-INS.SG.F

alle: 'Das Rätsel war einfach.'

(74) a. Katja byla dobraja.

(Geist 2006, 156-157)

Katja-NOM.SG.F war-SG.F gut $_{\text {LF }}$-NOM.SG.F

'Katja war gut[artig].'

b. Katja byla dobra.

Katja-NOM.SG.F war-SG.F gut $_{\mathrm{KF}^{-N O M . S G . F}}$

'Katja war gut.'

(75) Katja byla dobroj.

Katja-NOM.SG.F war-SG.F gut $_{\text {LF-INS.SG.F }}$

'Katja war gut.'

(76) Ona byla sčastlivoj.

(Timberlake 1993, 862)

sie-NOM.SG.F war-SG.F glücklich LF-INS.SG.F

'Sie war glücklich.'

(77) Ona byla sčastliva.

sie-NOM.SG.F war-SG.F glücklich ${ }_{\text {KF-NOM.SG.F }}$

'Sie war glücklich.'

(78) Ona byla sčastlivaja.

sie-NOM.SG.F war-SG.F glücklich LF-NOM.SG.F $^{-}$

'Sie war glücklich [ihrer Natur nach].'

Oft kann man lesen, nominativische Langformen würden eine charakterisierende Lesart vertreten. Inwieweit dies in ihnen selbst angelegt ist, lasse ich hier offen.

Die folgenden Beispiele illustrieren dies zunächst lediglich: 

(79) Rabota
u nas
byla trudnaja.
(Mrázek 1990, 49)
Arbeit-NOM.SG.F bei-PRP uns-GEN war-SG.F schwierig LF-NOM.SG.F
'Unsere Arbeit war schwer.'
(80) Charakter Pavla byl bezuprečnyj. (Mrázek 1990, 49)
Charakter-NOM.SG.M Pavel-GEN.SG war-SG.M tadellos-NOM.SG.M
'Der Charakter Pavels war tadellos.'

Der Gebrauch der markierten präsentischen Kopulaform est' (die fast ausschließlich mit dem Nominativ anzutreffen ist) im Falle eines temporären Kontrasts (vgl. Geist 2006, 290) unterstützt diese Ansicht insofern, als in diesen Fällen fast immer Charakterisierungen vorliegen:
(81) A on u tebja i est' poslušnyj. aber er-NOM bei-PRP du-GEN auch ist-3SG gehorsam-NOM.SG.M 'Aber er [deiner] IST auch gehorsam.' $($ AG 1980, 290)

Lang- und Kurzformen, die von ein und demselben Adjektivstamm abgeleitet sind, können mitunter (mehr oder weniger stark) voneinander abweichende Bedeutungen haben bzw. Interpretationen erhalten. Ferner unterscheiden sie sich mitunter auch in der Realisierbarkeit ihrer Argumente (Geist 2006, 139-140): ${ }^{32}$

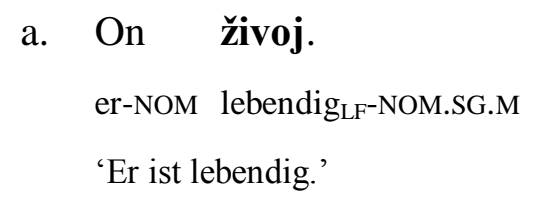

(Geist 2006, 128)

32 Oft ist fraglich, ob den betreffenden Konstituenten überhaupt Argumentstatus zukommt, oder ob es sich nur um Adverbiale handelt. Die zitierten Beispiele sowie ihre Bewertung stammen von Geist (2006). Im Falle von (84a) ist die Kennzeichnung von grippom als ungrammatisch fraglich. So ist dieser Satz nicht zwangsläufig ausgeschlossen, sofern man sich einen Kontext denkt, in dem der Sprecher seinen Mitarbeiter als "dauerhaft an Grippe krankend" betrachtet bzw. die Grippe als quasi charakteristische Eigenschaft darstellt. Vor diesem Hintergrund sind die Aussagen bzgl. der Argumentrealisierung von Lang- und Kurzformen, die Geist (2006) aufzählt, zu relativieren. Nichtsdestotrotz erweist sich die Beobachtung als stabil, dass bei Kurzformen Argumente tendenziell eher als bei Langformen realisiert werden. Meiner Analyse zufolge liegt das im verbalnominalen Charakter der Kurzformen (siehe Abschnitt 4.3). 
b. On živ.

er-NOM lebendig KF-NOM.SG.M

'Er lebt.'

(83) a. Andrej byl očen' vesëlyj.

(Geist 2006, 141)

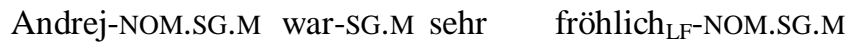

'Andrej war sehr fröhlich [seinem Wesen nach].'

b. Andrej byl očen' vesel.

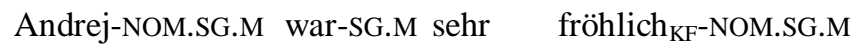

'Andrej war sehr fröhlich [in jenem Moment].'

(84) a. Sotrudnik

byl bol'noj

(*grippom).

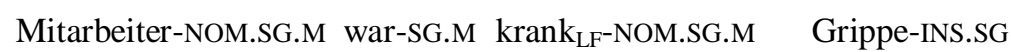

'Der Mitarbeiter war kränklich.'

b. Sotrudnik

byl bolen

(grippom).

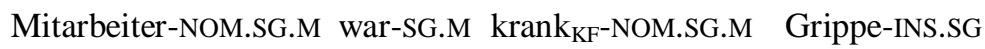

'Der Mitarbeiter war krank (hatte eine Grippe).'

(Geist 2006, 140)

Ferner können von sog. "psychischen" Adjektiven, die innere Zustände des Menschen denotieren, Kurzformen nur im Falle belebter Subjektreferenten verwendet werden (Geist 2006, 140):
a. Vid
u Iry
byl
dovol'nyj. (Geist 2006, 141)

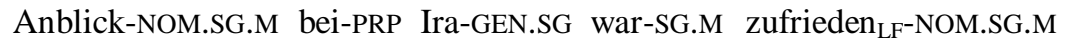

b. *Vid

u Iry

byl dovolen.

Anblick-NOM.SG.M bei-PRP Ira-GEN.SG war-SG.M zufrieden $_{\text {KF-NOM.SG.M }}$

'Ira machte einen zufriedenen Eindruck.' [wörtlich: 'Der Anblick Iras war zufrieden']

(86) a. Prazdnik byl očen' vesëlyj.

(Geist 2006, 141)

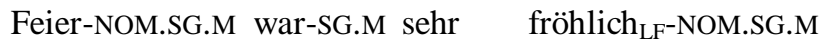

b. *Prazdnik byl očen' vesel.

Feier-NOM.SG.M war-SG.M sehr fröhlich KF-NOM.SG.M

'Die Feier war sehr fröhlich.' 
Bei allen anderen (d. h. nicht-psychischen) Adjektiven sind Kurzformen dagegen auch mit unbelebten Subjektreferenten möglich:

(87) Ego golos

$$
\text { (byl) prijaten. }
$$

(Mrázek 1990, 49)

seine Stimme-NOM.SG.M war-SG.M angenehm KF-NOM.SG.M

'Seine Stimme ist (war) angenehm.'

(88) Obed

$$
\text { tebe uže gotov. }
$$

(Mrázek 1990, 49)

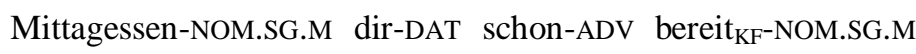

'Das (dein) Mittagessen ist schon fertig (für dich).'
Dom
byl
polon
*(gostej).
Haus-NOM.SG.M war-SG.M voll $_{\mathrm{KF}}$-NOM.SG.M Gast-GEN.PL
'Das Haus war voll von Gästen.'

(Geist 2006, 140)

Das folgende und abschließende Beispiel illustriert einen Fall, der ohne weitere theoretische Erwägungen nicht klar verortet werden kann. Hier stellt sich die Frage, welchen (Satzglied-)Status die beteiligten Konstituenten haben. So könnte der infinitivische Ausdruck Subjektsatz oder Komplementsatz sein. Entsprechend scheint es möglich, die Form veselo 'fröhlich' entweder als ('unpersönliches') Kurzform-Prädikativ, als "Zustandsprädikativum” oder als prädikatives Adverb zu analysieren:

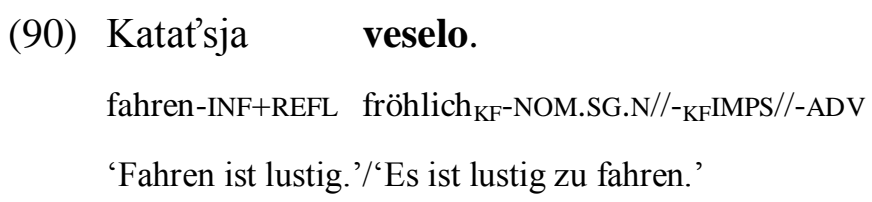

Weitere Erwägungen dazu werden in Abschnitt 5.6.3 erfolgen.

\section{3 "Zustandsprädikativa"}

Der letzte Teil der Bestandsaufnahme widmet sich den sog. "Zustandsprädikativa", für die ich im Weiteren die Bezeichnung "Zustandsprädikative" vorziehe, da ich die Ansicht vertrete, dass keine spezielle lexikalische (Sub-)Kategorie "Prädikativum" angenommen werden muss. Vielmehr soll gelten, dass der Ge- 
brauch als "Zustandsprädikativum" lediglich einen Sonderfall der Verwendung prädikativer Nomina darstellt, der eine syntaktische Null als Subjektausdruck involviert. Mehrheitlich handelt es sich bei diesen Ausdrücken um Qualitätsadjektive (siehe Abschnitt 4.4.3). Die Details meiner Analyse sowie ein Abriss der Auffassungen über "Zustandsprädikativa" in der Fachliteratur werden in Abschnitt 5.6.3 gegeben. Im Folgenden wird zunächst - wiederum nacheinander für das Polnische und Russische - ein deskriptiver Überblick geboten.

\subsubsection{Polnisch}

Die Besonderheit von Zustandsprädikativen besteht darin, dass sie (im Satz oder darüber hinaus) auf keinen konkreten Referenten verweisen, auf den der von ihnen bezeichnete psychische, physische, Natur- oder auch Modalzustand zutrifft, ${ }^{33}$ so dass der Eindruck entsteht, dass das bloße 'Herrschen' eines Zustands, aber kein Träger desselben, denotiert werde:

(91) Jest zimno.

$\mathrm{K} 06,6$ ist-IMPS kalt-IMPS

'Es ist kalt.'

(92) Jeść lody w zimie jest niezdorowo. esse-INF Eis-ACC.PL in-PRP Winter-LOC.SG ist-IMPS ungesund-IMPS

'Eisessen im Winter ist ungesund.'

(Rothstein 1993, 730)

(93) Nie wolno spacerować z świeżym opatrunkiem.

NKJP

NEG frei-IMPS spazieren-INF mit-PRP frischer Verband-INS.SG

'Man soll nicht mit einem frischen Verband spazieren gehen.'

33 Zustandsprädikative können auch modale Zustände bzw. Eigenschaften bezeichnen, vgl. z. B. russ. vozmožno 'möglich', neobchodimo 'unumgänglich' oder poln. potrzebne 'nötig', możliwe 'möglich'. Sie sind dann einfach Adjektive mit modaler Bedeutung und als solche nicht mit den sog. "Modalprädikativa” wie z. B. russ. nado 'nötig', možno 'möglich' oder poln. trzeba 'nötig', można 'nötig' deckungsgleich, da letztere - ähnlich wie Modalverben - grammatikalisierte Modalausdrücke sind, die keine eigenen Propositionen instanziieren, sondern bestehende Propositionen modalisieren (vgl. u. a. Zybatow 1994; Hansen 2009). In diesem Sinne halte ich Fortuins (2000) Einschätzung, dass "Modalprädikativa”, nicht aber Zustandsprädikative, das 'prädikative Zentrum' im Satz bilden könnten, für zweifelhaft bzw. mindestens irreführend. 
Zustandsprädikative zeigen im Polnischen typischerweise den Marker -o, der 'unpersönlich' (IMPS) ist. ${ }^{34}$ Mit ihnen wird in präsentischen Sätzen die Kopulaform jest oft ausgelassen bzw. kann 'unausgesprochen' bleiben: ${ }^{35}$

\section{(94) Jak (jest) chłodno! \\ wie ist kalt-IMPS}

'Wie kalt es ist!'

Zustandsprädikative werden ferner von (optionalen) Dativausdrücken begleitet, die den vom jeweiligen Zustand Betroffenen (EXPERIENCER) oder 'Bevorteilten' (BENEFIZIENT) bezeichnen:

(95) Przykro mu, że była wpadka. peinlich-IMPS ihm-DAT dass war-SG.F Reinfall-NOM.SG.F 'Ihm ist peinlich, dass es ein Reinfall war.'

Auf Grund der Optionalität der Dative ist ihre Bewertung als 'Satzsubjekt' wenig überzeugend (vgl. Bondaruk \& Szymanek 2007). Eine Analyse, der zufolge es sich bei ihnen um interne (und somit optionale, vgl. Bierwisch 1997, 245) Argumente der Zustandsprädikative oder um Adjunkte handelt, ist plausibler.

Zustandsprädikative im Polnischen zeigen nicht ausschließlich den Marker -o. Es gibt auch Fälle, in denen der ansonsten den Adverbien vorbehaltene Marker - $e$ (regressiv palatalisierend) verwendet bzw. bevorzugt wird: ${ }^{36}$

34 Diese Markierung zeigt sich auch bei den no/to-Formen. Wie Ukrainisch, unterscheidet Polnisch das 'unpersönliche/merkmallose' - o vom 'persönlichen/merkmalhaften' - $e$ (Sg. n.). Im Russischen besteht diese formale Unterscheidung nicht, so dass - $o$ im Prinzip ambig ist. Ferner dient es auch als Adverbmarker. Diese 'Vielseitigkeit' hat folgerichtig Analysen hervorgebracht, die Zustandsprädikative, wenn nicht als "Prädikativa", so doch entweder als prädikative Adverbien oder als Kurzformen werten (siehe auch Abschnitt 5.6.3).

35 Diese Form von "Nullkopula" ist ihrer Natur nach anders als die alternativlose Nullform im Russischen. Bei letzterer besteht die Lautform regulär aus 'nichts' $(\varnothing)$, im Polnischen dagegen liegt mit jest durchaus eine Lautform vor, die aber unter bestimmten Umständen, auf die ich in Abschnitt 5.6.3 eingehe, nicht (unbedingt) artikuliert werden muss (jest).

36 Es gibt Lexeme, die beide Markierungen zulassen, was dafür spricht, diese Marker als gleichermaßen merkmallos zu betrachten, vgl. przyjemno : przyjemnie 'angenehm' (sehr selten przyjemno), pożyteczno : pożytecznie 'nützlich' (sehr selten pożyteczno). Andere Zustandsprä- 
(96) Czytać jest przyjemnie.

(Bąk 1999, 412)

lesen-INF ist-IMPS angenehm-ADV[IMPS]

'Lesen ist angenehm.'

(97) Czytać dobre książki jest pożytecznie.

(Bąk 1999, 413)

lesen-INF gute Bücher-ACC.PL ist-IMPS nützlich-ADV[IMPS]

'Gute Bücher zu lesen ist nutzbringend.'

(98) Dobrze

byłoby, gdyby rodzice

K06, 34

gut-ADV[IMPS] sein-LPT-IMPS+KONJ wenn+KONJ Eltern-NOM.PL.MPS

przyjaciółki byli rozsądni[.]

Freundin-GEN.SG.F sein-LPT-PL.MPS vernünftig-NOM.PL.MPS

'Es wäre gut, wenn die Eltern der Freundin vernünftig wären.'

(99) Tam nie jest bezpiecznie, kochanie.

K06, 23

dort-ADV NEG ist-IMPS sicher-ADV[IMPS] Liebe-NOM.SG

'Dort ist es nicht sicher, meine Liebe.'

Beide Marker - $o$ und $-e$ haben gemeinsam, keine Kongruenzmerkmale auszudrücken. Diese Gemeinsamkeit ist m. E. Grundlage für ihre Austauschbarkeit. Relevant für die Wahl ist, ob das betreffende Lexem 'üblicherweise' oder generell die $o$-Markierung als Zustandsprädikativ zulässt (vgl. Komárek 1954, der Vergleichbares im Tschechischen beschreibt). So liefert das NKJP z. B. für poln. bezpieczno 'unsicher' nur einen einzigen Treffer, der zudem die Adverbverwendung repräsentiert. Sowohl als Zustandsprädikativ als auch als Adverb dominiert bei diesem Lexem -e. Auf 'kerngrammatische' Ursachen kann man dies m. E. nicht zurückführen, so dass Idiosynkrasien ausschlaggebend sein dürften.

Bis dato wurden ausschließlich Zustandsprädikative beschrieben, die auf adjektivischen Wurzeln bzw. Stämmen beruhen. Daneben gibt es auch solche, die desubstantivisch und dabei in keiner Weise besonders markiert/markierbar sind:

dikative haben nur die Markierung -e, z. B. dobrze (*dobro) 'wohl' (dobro ist Substantiv '(das) Gute'; als Zustandsprädikativ auch dobra), bezpiecznie (*bezpieczno) 'unsicher' (sehr selten und nur als Adverb bezpieczno). Die meisten Zustandsprädikative lassen nur -o zu, z. B. miło (*mile) 'lieb, angenehm' (mile nur als Adverb). 
$\begin{array}{llll}\text { (100) Wstyd mówić } & \text { o } & \text { tym. } \\ \text { Scham } & \text { sprechen-INF } & \text { über-PRP } & \text { das-LOC.SG }\end{array}$

'Es ist peinlich, darüber zu sprechen.'

(101) Czas było jechać.

(Skibicki 2007, 280)

Zeit war-IMPS fahren-INF

'Es war Zeit zu fahren.'

(102) tego już nie sposób było zrozumieć NKJP das-GEN.SG schon NEG Weise war-IMPS verstehen-INF 'das war (schon) nicht mehr zu verstehen'

Gegen die Ansicht, dass es sich bei diesen desubstantivischen Elementen um rein nominale Einheiten (Substantive) handelt, spricht die Tatsache, dass sie mit der ‘unpersönlichen' Form byto auftreten (siehe auch Bondaruk \& Szymanek 2007, 83). Ferner spricht dagegen auch der Umstand, dass sich mit diesen Elementen nie Attribute verbinden, wie man es von Substantiven erwarten würde. Stattdessen treten Adverbien auf, was sie eher als Verben (vgl. u. a. Bartnicka 1972) bzw. als Verbalnomina (siehe Abschnitt 4.3) ausweist:

\section{(103) *Wielki / Bardzo mi było wstyd. groß-NOM.SG.M sehr-ADV mir-DAT war-IMPS Scham} 'Ich war sehr beschämt.'

(Bondaruk \& Szymanek 2007, 83)

Schließlich gehören in den Bereich der Zustandsprädikative auch Strukturen, in denen infinitivartige Perzeptionsprädikate auftreten, die dabei (fast immer) die Möglichkeit der Wahrnehmung (mit) ausdrücken. Im Polnischen sind dies u. a. widać 'zu sehen', stychać 'zu hören' oder czuć 'zu fühlen':

(104) Stąd widać całą wioskę. (Fehrmann, Junghanns \& Lenertová 2007, 4) von-hier seh-INF ganzes Dorf-ACC 'Von hier ist das ganze Dorf zu sehen.' 
(105) Nawet z Brukseli już jest widać, co się dzieje w Słowacji [...]. sogar aus Brüssel-GEN schon ist-3SG seh-INF was sich tut in Slowakei 'Sogar aus Brüssel ist schon zu sehen, was sich in der Slowakei tut ...'

Aus diesen Beispielen wird deutlich, dass diese "Infinitivischen Perzeptionsprädikate" (IPP; Fehrmann, Junghanns \& Lenertová 2007; Junghanns \& Lenertová 2010) den Akkusativ am internen Argument lizenzieren. Jedoch sind sie, wie besonders widać illustriert, offenbar keine 'echten' Verbformen mehr, denn zu widać liegt heute - im Unterschied zu widzieć 'sehen' (widzę, widzisz ...) - kein Flexionsparadigma (mehr) vor (*widam, *widasz ...). Wie der Korpusbeleg (105) zeigt, können IPP auch mit dem overten präsentischen jest auftreten, auch wenn meist die "Nullkopula" 'erscheint'. Dies macht die IPP den Zustandsprädikativen ähnlicher als den "Modalprädikativa", da letztere niemals mit jest auftreten (siehe Abschnitt 5.6.3). Schließlich sind IPP nur prädikativ verwendbar und stets 'unpersönlich', d. h., ein denkbares wahrnehmendes AGENS-Subjekt ist nicht realisierbar. Das wahrnehmende Individuum kann im Polnischen - im Gegensatz zu anderen Zustandsprädikativen und anders als mit russischen IPP (siehe unten) - auch nicht in Form eines Dativs realisiert werden:

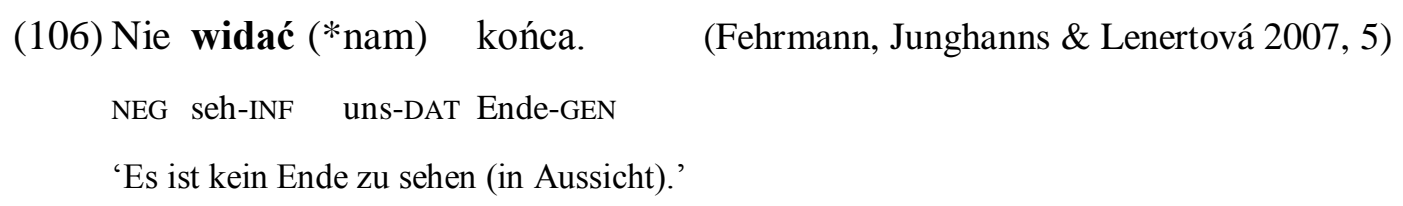

\subsubsection{Russisch}

Die russischen Zustandsprädikative haben im Großen und Ganzen die gleichen Eigenschaften wie ihre polnischen Entsprechungen. Deadjektivische Zustandsprädikative zeigen hier jedoch fast ausnahmslos den 'unpersönlichen' Marker - $o$ :
(107) Bylo
ticho.
(Mrázek 1990, 61)
war-SG.N[IMPS] still-IMPS
'Es war still.' 


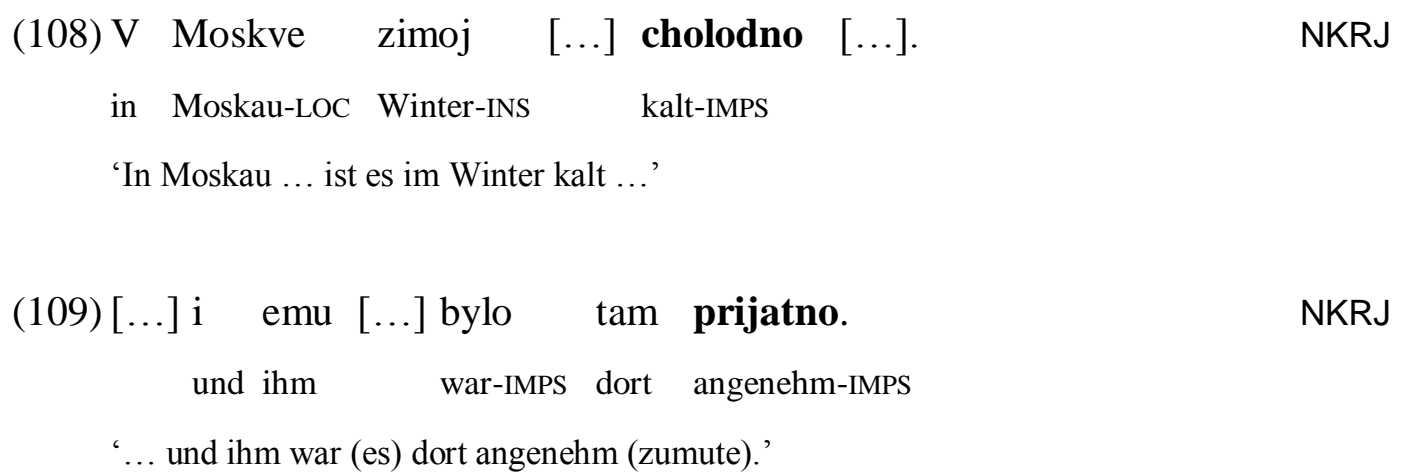

Als Unterklasse der Zustandsprädikative können im Russischen Perzeptionsprädikative wie z. B. vidno 'sichtbar' oder slyšno 'hörbar' gelten, die auf Stämmen mit Wahrnehmungsbedeutung basieren. Ihre Spezifik liegt darin, dass sie fast immer zu einer modalen Lesart führen; außerdem ist zu bemerken, dass diese Zustandsprädikative trotz ihrer adjektivisch-adverbartigen Form zur Selektion 'direkter Objekte' im Akkusativ fähig sind, wodurch sie verbal(nominal) wirken und den weiter oben beschriebenen IPP im Polnischen vergleichbar sind:

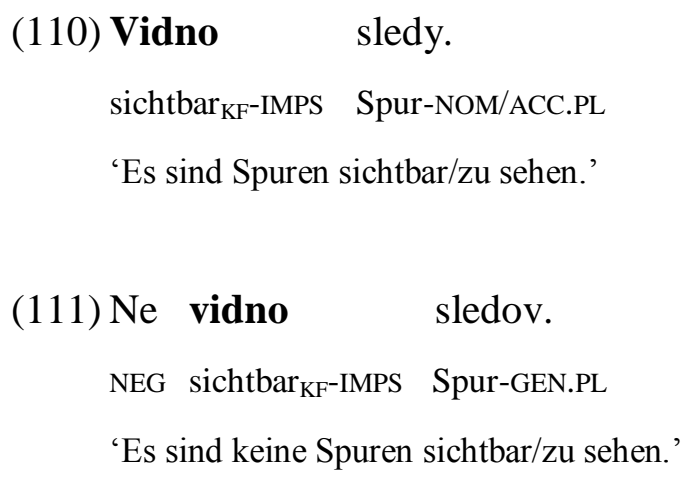

Der negierte Satz deutet darauf hin, dass das direkte Objekt im affirmativen Fall tatsächlich den Akkusativ trägt, auch wenn die normative Grammatik den Nominativ vorsieht. In diesem Zusammenhang ist die Tatsache von Belang, dass meist 'uneindeutige' Fälle von (unbelebten) Objekten vorliegen, deren Nominativ- und Akkusativformen zusammenfallen. Sicherlich ist nicht unplausibel, hier von einer 'Vermeidungsstrategie' der Sprecher auszugehen, durch die eine eindeutige Festlegung auf den Objektskasus umgangen wird, was ermöglicht, der Norm mindestens nicht zu widersprechen.

Das Russische hat aber neben deadjektivischen auch 'richtige' bzw. deverbale IPP, so z. B. vidat' 'zu sehen' oder slychat' 'zu hören', die von der normativen Grammatik zwar als umgangssprachlich ausgewiesen werden, jedoch gut belegt 
sind. Das folgende negierte Beispiel zeigt, dass auch die russischen IPP Akkusativobjekte lizenzieren:
(112) Vo t'me
čeloveka $[\ldots]$ ne bylo vidat'.
NKRJ
in Dunkelheit-LOC Mensch-GEN NEG war-IMPS seh-INF
'In der Dunkelheit war der Mensch ... nicht zu sehen.'

Hinsichtlich der IPP gleichen sich also Russisch und Polnisch. Jedoch zeigen Fehrmann, Junghanns \& Lenertová $(2007,6)$, dass im Russischen - anders als im Polnischen - mit IPP Dative möglich sind, die den Wahrnehmenden bezeichnen. ${ }^{37}$ Wie im Polnischen, so liegen auch im Russischen eine ganze Reihe von Zustandsprädikativen vor, die desubstantivisch sind:
(113) Mne pora echat'. mir Zeit-NOM.SG.F fahr-INF
'Für mich ist es (an der) Zeit zu fahren.'
(114) $[$ B]ylo pora na avtobus. war-IMPS Zeit-NOM.SG.F auf Autobus-ACC '... es war Zeit, den Bus zu nehmen.'

(Š\&L 1990, 442)

Das 'unpersönlich' bylo zeigt, dass nicht das Existenzverb byt' vorliegt, da dann die kongruierende Form byla wie in (115) zu erwarten wäre:
(115) Byla tišina.
war-SG.F Stille-NOM.SG.F
'Es war [herrschte] Stille.'

(Mrázek 1990, 61)

In Fällen wie (115) kann von Existenzsätzen ausgegangen werden, worauf auch die Ersetzbarkeit von 'war' durch 'herrschte' (oder 'bestand') in der Übersetzung

37 Mit Fehrmann, Junghanns \& Lenertová $(2007,14)$ könnte dies daran liegen, dass das Polnische keine Dative mit dem Status von Argument-Adjunkten (vgl. Grimshaw 1990), sondern nur bloße EXPERIENCER-Dative (reine Adjunkte) zulässt. Das Russische dagegen lässt auch ersteren Dativtyp zu, der für IPP nötig ist, bei denen das externe Argument 'ausgeblendet' wird (vgl. Details zur 'Ausblendung' in Abschnitt 4.3). 
hindeutet. Auch negierte Beispiele wie das folgende mit né bylo 'es gab nicht' und dem Genitiv der Negation belegen die existenzielle Natur dieser Aussagen:

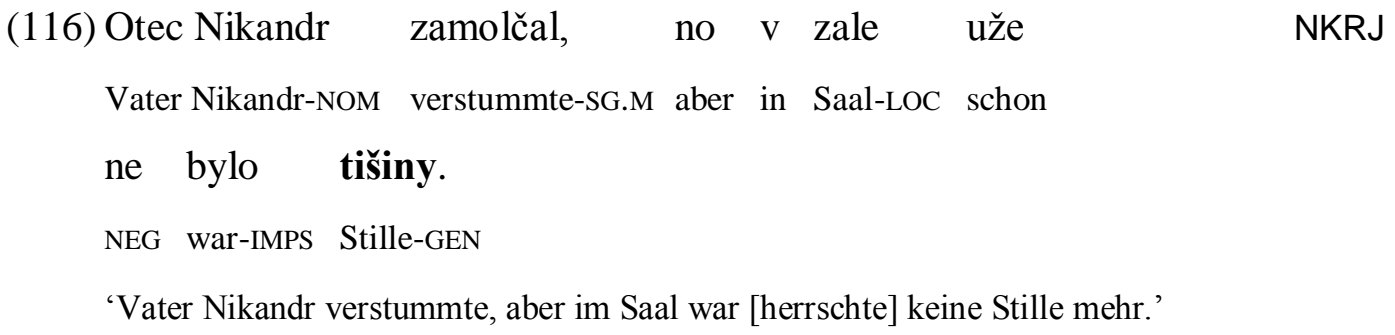

Für die Analyse der Zustandsprädikative verweise ich auf Abschnitt 5.6.3. Im folgenden Abschnitt werde ich zunächst die wichtigsten bestehenden Arbeiten zu den relevanten Variationen besprechen. 


\section{Besprechung relevanter Analysen zu den Variationen}

In diesem Abschnitt werden die wichtigsten bestehenden Beschreibungen bzw. Analysen des Phänomens der Kasus- und Formalternation in russischen und seltener bzw. eingeschränkter - in polnischen Kopulasätzen vorgestellt und besprochen. $\mathrm{Zu}$ zeigen ist, worin deren Vorteile und Schwächen bestehen.

\subsection{Semantisch-deskriptiver Ansatz}

Auf die Mehrzahl dieser Ansätze, darunter Švedova (1952), Potebnja (1958), Isačenko (1962), Mrázek (1964), Jakobson (1971a), Chvany (1975), Borkovskij (1978) oder die AG (1980), werde ich nicht im Detail eingehen, da ihre Aussagen zur Kasusvariation und zur Alternation von Lang- und Kurzformadjektiven (dies stets zum Russischen) überwiegend deskriptiv und somit nicht explikativ sind. Das heißt keinesfalls, dass diese Arbeiten nicht wertvolle Informationen und Hinweise auf Erklärungsmöglichkeiten enthalten. Gerade Darstellungen wie Jakobson (1971a) oder Isačenko (1962) sind wegweisend, wenn es um die generelle Richtung geht, der eine erklärende Beschreibung der interpretativen Effekte in Kopulasätzen folgen kann und sollte.

Die grundlegende Leistung der o. g. und weiterer Arbeiten im "rein semantischen Lager" besteht darin, erkannt zu haben, dass Adjektivform und Kasusmarkierung mit bestimmten Lesarten bzw. Semantiken korrelieren. Darüber hinaus werden für letztere diverse Beschreibungen und teilweise auch Erklärungen wie z. B. "permanent vs. temporär", "neutral vs. markiert", “Ganzes vs. Teil”, "Feststellung vs. Werturteil”, “[ \pm Zeitbezug]", “[ $[ \pm$ episodisch]”, “[ \pm Marker für resultative Aktualisierung]" u. ä. angeboten. All diese Charakterisierungen sind insofern hilfreich, als sie darauf hinweisen, welche 'Semantik' sich v. a. mit der expliziten Markierung des Prädikatstatus verbindet, was also zunächst den Instrumental und die Kurzformen betrifft, im Weiteren aber auch - substraktiv - den Nominativ und die Langformen. Freilich sagen diese Charakterisierungen nichts darüber aus, in welcher sprachlichen Domäne sie angesiedelt sind. Da kaum einmal ein Standpunkt in dieser Frage bezogen wird, kann man die "rein semantischen" Ansätze weder als deterministisch noch als non-deterministisch klassifizieren. 
Im Folgenden werde ich auf drei Arbeiten aus dem "semantischen Lager" näher eingehen, die m. E. in Bezug auf die vorliegende Arbeit besonders gewinnbringend sind, und zwar Nichols (1981), Ueda (1992) und Richardson (2001).

\subsubsection{Nichols (1981)}

Nichols (1981) nennt in ihrer Analyse insgesamt 28 Faktoren in sechs Gruppen, die für die Kasuswahl am russischen Prädikatsnomen ursächlich sein sollen. ${ }^{38}$ Sie kommt zu dem Schluss, dass keiner dieser Faktoren als primär gelten könne. Für die vorliegende Arbeit ist ihre Beschreibung bedeutsam, da sie überzeugend zeigt, dass die Kasusmarkierung nicht nur von einem bestimmten Faktor determiniert wird, sondern dass es sich um eine ganze Reihe von Faktoren handelt, die sowohl die Kasus- und Formenwahl als auch sich gegenseitig (lediglich) beeinflussen. ${ }^{39}$ Man kann also höchstens von tendenziellen, nicht aber von absoluten Kriterien sprechen (vgl. auch Hentschel 1991). Wichtig ist Nichols' Arbeit auch dadurch, dass die Faktoren, die sie zusammenstellt, ganz verschiedenen - sowohl grammatischen als auch konzeptuellen - Ebenen angehören. Das heißt, dass die Wahl von Kasus und Adjektivform weder als rein grammatisches noch als rein pragmatisches Phänomen gelten kann. Vielmehr wird die Wahl offenbar von sehr heterogenen Faktoren beeinflusst, die ihrerseits auf alle denkbaren Absichten des Sprechers zurückgeführt werden können. In jedem Fall spricht die empirisch fundierte Arbeit von Nichols (1981) gegen die deterministische Vorannahme, die Kasusvariation sowie die Alternation in der Adjektivform werde durch eine bestimmte, im grammatischen System angelegte Invariante gesteuert.

38 Neben der lexikosemantischen Klasse der Prädikative nennt sie semantische und pragmatische Faktoren wie den Stil (umgangssprachlich/mündlich $\rightarrow$ NOM vs. formal/schriftlich $\rightarrow$ INS), den Typ eines möglichen Kontrolleurs (Subjekt $\rightarrow$ NOM vs. Nicht-Subjekt $\rightarrow$ INS; Subjekt finiter Prädikate $\rightarrow$ NOM vs. Subjekt infiniter Prädikate $\rightarrow$ INS) u. a. Auch könne die Deklinationsklasse und das Paradigma des Prädikativs relevant sein (Maskulina/Neutra/Pluralia $\rightarrow$ INS vs. Feminina $\rightarrow$ NOM). Letzterer Faktor hat besonders im gesprochenen Bereich Einfluss, was auf eine Interaktion der Faktoren bzw. der zugehörigen Domänen hindeutet.

39 Dies erinnert an eine Analyse im Sinne der Optimalitätstheorie (OT), innerhalb derer die relevanten Faktoren in einer bestimmten Abfolge ge-rank-t werden. Vgl. auch Hentschel (1991), der zeigt, wie verschiedene Typen von Faktoren die Kasuswahl am Prädikativ und sich gegenseitig beeinflussen. Auch seine hierarchische Listen von Kriterien ähnelt einem OT-Ranking. 


\subsubsection{Ueda (1992)}

Die Arbeit von Ueda (1992) beschäftigt sich auf der Basis empirischer Daten mit der Variation russischer Kurz- und Langformadjektive. Allein durch dieses Untersuchungsobjekt, das ansonsten deutlich hinter den substantivischen Prädikativen zurücksteht, ist die Arbeit bedeutsam. Ueda (1992) gibt sehr konkrete Beschreibungen für die Interpretationen der russischen Adjektivformen mit ihren möglichen Kasusmarkierungen. Die Arbeit schließt in vielerlei Hinsicht an Timberlake (1986) an, greift andererseits aber auch der späteren Analyse Geists (2006) voraus, wenn sie zu dem Schluss kommt, dass die Kurzformen lediglich eine Eigenschaft eines Referenten denotieren, während die Langfomen im Nominativ eine "unique", jene im Instrumental hingegen eine "non-unique" Interpretation zeigen. Das bedeutet, dass die Langformen im Instrumental eine Eigenschaft denotieren und gleichzeitig implizieren, dass es sich dabei nicht um die einzige Eigenschaft des Referenten handelt, was mitunter der Interpretation gleichkommen kann, dass der Sprecher eine andere Eigenschaft erwartet (hätte). Eben hierin liegt die Parallele zu Timberlakes (1986) “temporalem" sowie “modalem” Instrumental und zu dem von Geist (2006) angenommenen "Bezug auf eine spezifische Topiksituation", die in (denkbarer) Konkurrenz zu mindestens einer Alternativsituation steht.

Leider versäumt Ueda, diese Aussagen in Bezug auf jene wichtigen Fälle zu relativieren, in denen die treffend beobachteten Interpretationen, und zwar trotz der entsprechenden morphologischen Form, nicht gegeben sind. Dies führt letztlich zu Übergeneralisierungen.

\subsubsection{Richardson (2001)}

Richardson (2001) geht es um russische adjektivische Sekundärprädikate. Bezeichnend ist die Verwandtschaft zu der späteren, jedoch eher syntaxzentrierten Analyse Markmans (2008). Diese besteht v. a. in der Annahme, dass das Vorhandensein der instrumentalischen Markierung eine eventive Interpretation mit sich bringe, während die nominativische Markierung eine stative bzw. non-eventive Interpretation 'erzeuge'. Während Markman (2008) unter "eventiv" die Anwesenheit eines Ereignisarguments versteht, formalisiert Richardson (2001) ihr Verständnis von "Eventivität” nicht, lässt aber durchblicken, dass sie die Denota- 
tion nicht nur eines, sondern zweier Zustände meint, was wiederum einem $\mathrm{Zu}$ standswechsel gleichkommt, der auch in einigen diachronen Arbeiten als Bedeutung des alt(nord)slavischen prädikativen Instrumentals genannt wird (so u. a. bei Lunt 1974, 133; Moser 1994, 10ff.).

Meiner Ansicht nach kann die Eventivitätshypothese, ob von Richardson (2001) oder Markman (2008), kaum standhalten, sofern man beachtet, dass die Kopula auf (Kimsche) Zustände referiert (vgl. u. a. Maienborn 2003a, 2003b), was sich nicht zuletzt auch in ihrer ausschließlichen Imperfektivität äußert (vgl. u. a. Geist 2006, 169-171). Letztlich sind Zustände (states) keine Ereignisse (events). Nichtsdestotrotz weist auch Richardsons (2001) Arbeit darauf hin, dass der Instrumental in der Tat die Lesart eines "Zustandswechsels" bzw. von (scheinbarer) "Eventivität" (Markman 2008), von “non-uniqueness" (Ueda 1992) oder "Kontrastivität" (Kuznetsova \& Rakhilina 2010; Kuznetsova 2013), die einer "spezifischen Topiksituation" (Geist 2006) oder auch 'einfach' eine "temporäre” Lesart (u. a. AG 1980) erhalten kann, nicht aber von sich aus hat. Der Punkt, der bei Richardson (2001) - ebenso wie bei den anderen entsprechenden Arbeiten - nur geringe oder keine Beachtung findet, ist, dass der Instrumental die genannten Lesarten nur im Falle seiner potenziellen Opposition zum Nominativ zeigt/erhält. Ist letzteres nicht der Fall, so lassen sich solche 'interpretativen Effekte' nicht erkennen bzw. ist der Instrumental lediglich ein morphologischer Marker ohne spezielle Zusatzsemantik. Ebendies werde ich mich bemühen, mit meinem Ansatz zu erfassen. Dabei sollen die genannten und meist zutreffend beschriebenen Lesarten durchaus nicht negiert werden; zentral sind jedoch ihr Zustandekommen und v. a. auch ihr mögliches Ausbleiben (siehe dazu Abschnitt 6).

Mit diesen Ausführungen beende ich die Besprechung des "rein semantischen Lagers" von Ansätzen zur Beschreibung/Erklärung der Kasus- und Formvariationen in russischen und polnischen Kopulasatzstrukturen. Der folgende Abschnitt wendet sich einer Klasse von Ansätzen zu, die diese Variation(en) sowohl auf syntaktische als auch auf semantische Aspekte zurückführen (oft, indem semantische Anteile in die Syntax verschoben werden). 


\subsection{Semantosyntaktischer Ansatz}

\subsubsection{Matushansky (2000)}

Eine bedeutende Arbeit der semantosyntaktischen Richtung ist Matushansky (2000), die russische prädikative NPn v. a. in Präteritälsätzen behandelt. Die Autorin stellt die Hypothese auf, dass die Anwesenheit eines extended Small Clause (xSC; Matushansky 2000, 292), den sie später als funktionale Aspektprojektion (AspP) identifiziert (Matushansky 2000, 296-298), für die Lizenzierung des Instrumentals verantwortlich sei. Im Umkehrschluss werde im Falle präsentischer Kopulasätze, die den Instrumental ausschließen, nur ein einfacher SC und somit keine AspP projiziert. Matushansky $(2000,301)$ führt dies auf das 'defiziente' Präsens zurück. Evidenz sieht sie darin, dass im Falle des letzteren weder durch eine (overte) Kopulaform noch durch die Satzsyntax der Verbalaspekt sichtbar werde. Sofern demnach keine AspP vorliege, könne auch kein Instrumental lizenziert werden und "[...] the predicate case is checked via the unspecified mechanism of case agreement." (Matushansky 2000, 300). So verbindet Matushansky (2000) die An- bzw. Abwesenheit einer syntaktischen AspP mit dem Fehlen oder Vorhandensein von semantischem Aspekt, was sie wiederum mit der morphosyntaktischen Lizenzierung des Instrumentals verbindet; vgl. (1): ${ }^{40}$

40 Weitere Evidenz sieht Matushansky (2000, 289-291) darin, dass Nominativ- im Gegensatz zu Instrumental-NPn (i) keine wh-Bewegung und (ii) kein Scrambling in eine Kontrastfokusposition erlauben sowie (iii) nicht aus faktiven Nebensätzen extrahiert und (iv) nicht topikalisiert werden können (siehe auch Bailyn 2012, 195). All diese Punkte involvieren die Bewegung des Prädikativs in eine satzinitiale Position, was m. E. deutlich auf die Informationsstruktur verweist. Andererseits könnte die Bewegung eines nominativischen Prädikatsnomens in eine satzinitiale Position dazu führen, dass diese das (referierende) Satzsubjekt sei; so könnte mindestens der Eindruck eines prädizierend-identifizierenden Satzes (Geist 2006) entstehen. Interessant ist, dass Kurzformen, nicht aber prädikative Langformen, diese Bewegungen absolvieren können. Sowohl bei Kurzform-APn als auch bei Instrumental-NPn handelt es sich m. E. um explizit-prädikative Formen, deren Satzfunktion unmittelbar deutlich ist, was sie wiederum relativ unabhängig von bestimmten Strukturpositionen macht. Anders ausgedrückt: Eine explizit-prädikative Form ist auch in einer derivierten Position bzgl. ihrer Satzfunktion eindeutig identifizierbar, während dies bei einer nicht-expliziten Form (etwa einer Nominativ-NP [primär: Subjektsfunktion] oder einer Langform [primär: Attributsfunktion]) eine uneindeutige syntaktische Struktur verursachen kann, womit übereinstimmt, “[...] daß die primäre Funktion von Kasusmarkierungen eine möglichst transparente Signalisation der syntaktischen Struktur [...] ist" (Hentschel 1993a, 110). Somit wäre die "Unbeweglichkeit" nominativischer NPn nicht eigentlich strukturell verursacht, sondern dem Bestreben nach Transparenz geschuldet. 
(1) a. $\left[\mathrm{xSC}=\mathrm{AspP} \operatorname{Asp}^{0}\left[\mathrm{sC}=\operatorname{PredP} \operatorname{Pred}^{0} \mathrm{NP}_{\mathbf{I N S}}\right]\right]$

b. $\quad\left[\mathrm{SC}=\operatorname{PredP}_{\mathrm{P}} \operatorname{Pred}^{0} \mathrm{NP}_{\mathrm{NOM}}\right]$

Matushansky $(2000,301)$ selbst weist darauf hin, dass ihre Arbeit drei Kernbereiche des Phänomens unberücksichtigt lasse, namentlich (i) die Interaktion zwischen prädikativem Kasus, Modus und Negation, (ii) den Kontrast zwischen Kurz- und Langformadjektiven und (iii) die empirische Tatsache, dass instrumentalische NPn generell akzeptabler bzw. häufiger als nominativische sind.

Abgesehen von diesen 'Lücken' fragt man sich in Anbetracht der o. g. Hypothese, inwiefern präteritale, futurische oder konjunktivische Kopulasätze mit nominativischem Prädikatsnomen "aspektlos" sein können bzw. ob eine solche Annahme aus aspektologischer Sicht überhaupt haltbar ist. ${ }^{41}$ Wie sowohl Maienborn (2003a, 2005a) als auch Geist (2006) zeigen, ist der semantische Aspekt der Kopula stets der imperfektive, da sie auf (Kimsche) Zustände referiert, was natürlich auch auf Präsenssätze zutrifft, auch wenn diese ggf. eine "Nullkopula" enthalten. Eine Analyse, die die Kasusmarkierung in russischen Kopulasätzen an das Vorhandensein von Aspekt koppelt, ist fraglich, zumal in polnischen präsentischen Kopulasätzen ganz regelmäßig overte Verb- und Aspektmorphologie vorhanden ist, was die Abwesenheit von semantischem Aspekt (bzw. einer AspP) in den russischen Sätzen noch zweifelhafter macht. Da ich davon überzeugt, dass (i) jeder Satz auf einer Verbform aufbaut (wenn sie auch "stumm" sein kann) und dass (ii) jede Verbform über semantischen Aspekt verfügt, kann ich Matushansky (2000) nicht zustimmen.

\subsubsection{Harves (2002)}

Ebenfalls "semantosyntaktisch" (und der Arbeit Matushanskys verwandt) ist die Analyse von Harves (2002), die jedoch das Phasenmodell nach Chomsky (2000, 2001, 2008) und darin einen Gegensatz zwischen "schwachen" und "starken"

41 Dem Ansatz Matushanskys (2000) ist Markman (2008) vergleichbar, die jedoch durch die Annahme eines Default-Aspekts absolute “Aspektlosigkeit” vermeidet. Geist (2006, 77-80) weist darauf hin, dass Matushansky (2000) byt' als "aspektlos" betrachtet, sofern ein nominativisches Prädikativ vorliegt. Aspektlose Verben sollte es per definitionem aber nicht geben. Die Behauptung solcher Verben ähnelt der nicht minder problematischen Annahme "kasusloser" Kurzformen (siehe dazu Abschnitt 5.6.1). 
Phasen zugrunde legt. Als "starke" Phase bezeichnet sie einen Small Clause, der von einem Asp-Kopf mit den Merkmalen [+INST,+EPP,+Asp] dominiert wird (vgl. Harves 2002, 112). Eine "schwache" Phase hingegen wird von einem AspKopf selegiert, bei dem diese Merkmale durchweg negativ sind: ${ }^{42}$
a. $\quad\left[\mathrm{AspP} \operatorname{Asp}^{[+\mathrm{INST},+\mathrm{EPP},+\mathrm{Asp}]}\left[\operatorname{PredP} \operatorname{Pred}^{0}\right.\right.$ NP INS ]]
("starke" Phase)

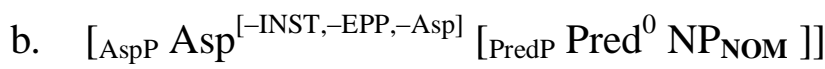
("schwache" Phase)

Während demnach im Falle der "starken" Phase der Instrumental durch 'merkmalhaltiges' Asp ${ }^{0}$ lizenziert wird, kann dies im Falle der "schwachen" Phase nicht erfolgen, so dass das Zuweisen des Nominativs $\mathrm{T}^{0}$ überlassen bleiben muss.

Im Kern unterscheidet sich die Analyse von Harves (2002) nur wenig von Matushanskys (2000) Vorschlag, da die Instrumentalzuweisung gleichermaßen mit der Aspektspezifikation in Verbindung gebracht wird. Wie Bondaruk (2013b, 188) bemerkt, bringt die Verlagerung in das Phasenmodell keine nennenswerten Vorteile, zumal die Annahme "starker" und "schwacher" Phasen stipulativ ist und die Zuweisung des Nominativs durch $\mathrm{T}^{0}$ Multiple Agree notwendig macht, was mindestens in diesem Bereich durchaus fraglich bzw. umstritten ist. So ist in Bezug auf Harves (2002) dasselbe Fazit wie in Bezug auf Matushansky (2000) zu ziehen, nämlich dass ein Ansatz, der letztlich von "aspektlosen” Verben ausgeht, kaum haltbar erscheint.

\subsubsection{Markman (2008)}

Direkt auf Matushansky (2000) baut die Analye von Markman (2008) auf. Jedoch nimmt Markman nicht an, dass der Nominativ mit der völligen Abwesenheit von Aspekt einhergehe. Sie behauptet aber etwas nicht minder 'Radikales', indem sie zwei distinkte Ausformungen von byt' ansetzt, die sich hinsichtlich der Anwesenheit eines Ereignisarguments unterscheiden. Das ist insofern 'radikal', als es bedeutende theoretische Konsequenzen für die Aspektkategorie hat.

Markman postuliert ein "non-eventives" byt' (mit Nominativ) und ein "eventives" byt' (mit Instrumental). ${ }^{43}$ Ihr Konzept von "Eventivität" erinnert an Richardson

42 Das ergibt die einigermaßen paradoxe Situation eines Asp-Kopf mit dem Merkmal [-Asp].

43 Die Formen von byt' sind für Markman bloße Auxiliare, die sie im Strukturbaum als Belegung eines (funktionalen) Kopfes $\mathrm{F}^{0}$ lokalisiert (vgl. Markman 2008, 203), vgl. (i): 
(2001), die jedoch prädikative Adjektive betrachtet und ihre Annahmen nicht in dem Maße formalisiert. Und während Richardson (2001) unter "Eventivität" im Prinzip die Denotation eines Zustandswechsels versteht, bezeichnet dieser Begriff bei Markman (2008) die Anwesenheit eines Ereignisarguments (event argument). Dieses Argument wird ihr zufolge (sie geht mit Bowers (1993) von einer Prädikationsphrase aus) durch einen speziellen Subtyp von Pred", namentlich "eventives Pred $^{0 "}$ "bzw. "PredEv", eingebracht (vgl. Markman 2008, 200).

Auf der anderen Seite ergibt sich nicht, wie man erwarten könnte, dass bei "noneventivem" byt' ein Zustandsargument vorliegt; letzteres soll stattdessen gar kein Sachverhaltsargument involvieren (vgl. Markman 2008, 208). Der "non-eventive" Pred-Kopf ist laut Markman daher lediglich eine identische Abbildung, die die Bedeutung der prädikativen NP unverändert weiterreicht:

(3) a. [PredEvP PredEv ${ }^{0}$ NP $\left.{ }_{\text {INS }}\right]$

(“eventiv")

b. [PredP Pred $\left.^{0} \mathrm{NP}_{\text {NOM }}\right]$

("non-eventiv")

Diese Annahmen haben bedeutende Folgen für den Aspekt: Im Fall von "eventivem" byt' geht Markman vom 'normalen' imperfektiven Aspekt aus, der eine 'Laufzeit' für die Ereigniszeit derart festlegt, dass das Intervall der letzteren mit dem Referenzzeitintervall überlappt (Markman 2008, 200). So üblich und unproblematisch diese Annahme ist, so verwegen ist Markmans These zum Aspekt bei “non-eventivem" byt'. Letzteres habe nämlich nur einen Default-Aspekt, der keine 'Laufzeit' festlege (vgl. Markman 2008, 202-203). Diese Annahme ist für Markman folgerichtig, da sie für das “non-eventive" byt' überhaupt kein Sachverhaltsargument ansetzt, das Voraussetzung für die Festlegung einer 'Laufzeit' ist.

Diese Aspekttheorie verbindet Markman (2008) mit Matushansky (2000), die im Falle des Nominativs gar keine Aspektspezifizierung vorsieht. Da Markman (2008) immerhin einen Default-Aspekt annimmt, ergibt sich bei ihr - wenigstens auf den ersten Blick - kein so prinzipielles Problem wie bei Matushansky (2000),

(i) $\left.\left[{ }_{\mathrm{TP}} \mathrm{T}^{0}\left[\mathrm{AspP} \operatorname{Asp}^{0}{ }_{[\mathrm{FP}} \mathbf{F}^{0}\left[\operatorname{Pred(Ev)P} \operatorname{Pred}(\mathrm{Ev})^{0} \mathrm{NP}\right]\right]\right]\right]$.

Die Annahme, dass es sich bei diesen Formen um bloße Auxiliare und nicht um die eigentlichen Träger der Kopulabedeutung handelt, teile ich. Gleiches gilt für ihre Strukturposition, sofern man berücksichtigt, dass ich keine AspP annehme und anstelle von Pred(Ev)P eine 'einfache' VP für die Kopulabedeutung ansetze; ferner sind die Formen von byt' m. E. lexikalische Verbalauxiliare (daher in $\mathbf{V}^{\mathbf{0}}$ ), vgl. (ii) (siehe auch Abschnitt 5.2):

(ii) [ [IP $\mathrm{I}^{0}$ [vP $\left.\mathbf{V}^{0}\left[\mathrm{vp} \mathrm{V}^{0} \mathrm{NP}\right]\right]$. 
denn der Default-Aspekt kann ja als eine gewisse Aspektspezifikation gelten, so dass "non-eventives" byt' nicht vollends "aspektlos" erscheint. Jedoch ist zu beachten, dass im Falle des Default-Aspekts das Ereigniszeitintervall unmittelbar (also ohne 'Umweg' über die Referenzzeit) in Beziehung zur Auswertungs- bzw. Äußerungszeit gesetzt wird. Folglich sind die Formen von "non-eventivem" byt' lediglich Tempusformen ohne Aspektspezifikation. Die Schlussfolgerung kann nur lauten, dass der Default-Aspekt letztlich doch kein Aspekt ist, was Markman (2008) auf eine Ebene sowohl mit Matushansky (2000) als auch mit Harves (2002) stellt. Aus welchen Gründen diese Behauptung m. E. problematisch ist, habe ich bereits oben dargelegt.

Ferner ist die Annahme, dass Kopulasätze mit nominativischen Prädikatsnomina keinerlei Sachverhaltsargument involvieren, in einer Davidsonschen Semantik einigermaßen widersprüchlich. Worauf referieren Verben bzw. Sätze, wenn kein referenzielles Argument im Spiel ist? Wie kann in einem Satz, der derart ohne Sachverhaltsbezug ist, ein Verbmodus zustande kommen (vgl. hierzu u. a. Zimmermann 2009)? Auf diese Fragen kann es bei Zugrundelegung des Modells von Markman (2008) m. E. keine befriedigenden Antworten geben.

Auch erscheint die Annahme eines event-Arguments in Markmans "eventiven" Kopulasätzen (mit instrumentalischen Prädikatsnomina) problematisch. Während Richardsons (2001) "Eventivität" die Denotation eines Zustandswechsels meint, die man mit einer semantischen Komponente wie BECOME erfassen könnte, behauptet Markman, dass byt' ein (inhomogener) event im Davidsonschen bzw. Vendlerschen Sinne sei. Damit wiederum stellt sich Markman gegen die Ansicht, dass die Kopula bzw. die Kopulabedeutung auf einen Zustand referiert. Noch grundsätzlicher stellt sich die Frage, was man sich eigentlich unter "eventivem" sein vorzustellen hat, falls es sich dabei nicht lediglich um werden handeln soll. ${ }^{44}$ Schließlich bleibt Markman (2008) sehr vage, wenn es um prädikative APn geht (die o. g. Annahmen beziehen sich durchweg auf NPn). In einer Fußnote zieht sie in Erwägung, dass Adjektive schon im mentalen Lexikon mit einem Zustandsargument ausgestattet sein könnten. Während diese These per se nicht zwangs-

44 Wie die Autorin selbst feststellt, ist die Eventivitätshypothese auch in Bezug auf die sekundäre Prädikation (mit Instrumental-APn und -NPn) problematisch, da diese schwerlich als "eventiv" gewertet werden kann. M. E. berührt auch dies eben angesprochene Problematik. Letztlich ist die Behauptung nicht haltbar, dass sämtliche Kopulasätze mit instrumentalischen Prädikatsnomina eine "eventive" Interpretation oder Lesart haben sollen. 
läufig unplausibel ist, wirkt es widersprüchlich, wenn den "non-eventiven" Formen von byt' jedes Sachverhaltsargument (und eine 'wirkliche' Aspektspezifikation) abgesprochen bzw. ihnen nicht wenigstens ein Zustandsargument zuerkannt wird, während das Vorhandensein des letzteren bei Adjektiven möglich sein soll.

\subsubsection{Matushansky (2008)}

Einen sprachübergeifenden und dabei nicht aspektbezogenen Ansatz, der sich teilweise in der Distributed Morphology (DM) bewegt, verfolgt Matushansky (2008). Die technischen Grundannahmen, die Matushansky (2008) macht, sind die folgenden: Zum einen werden Kasus von Köpfen an ihr Komplement zugewiesen. Dabei gilt generell, dass ein $v$-Kopf den Akkusativ und der T-Kopf den Nominativ zuweist. Aber die an der Satzoberfläche erscheinenden Kasusformen sind der Autorin zufolge (meist) Kombinationen aus mehreren privativen Kasusmerkmalen. Im Sinne von DM nimmt sie an, dass die lautliche Realisierung eines bestimmten 'Bündels' solcher Merkmale sprachspezifisch unterschiedlich durch Einsetzungsregeln erfolgt. Die Kernprinzipien dieser Regeln seien wiederum impoverishment und underspecification (vgl. Matushansky 2008, 222).

Kopulasätze mit nominativischen Prädikatsnomina haben nach Matushansky (2008) die folgende Satzstruktur (nota bene ohne $v \mathrm{P}$ und VP):

(4) $\mathrm{TP}>$ PredP $>\mathrm{NP}_{\text {NOM }}$

(vgl. Matushansky 2008, 222)

Die Abwesenheit von $v \mathrm{P}$ sowie VP korrespondiert mit der Abwesenheit einer Verbform in diesen Sätzen, die im Russischen stets präsentisch sind (vgl. Matushansky 2008, 223). ${ }^{45}$ Während $\mathrm{T}^{0}$ das Kasusmerkmal [nominative] zuweist, weist $\operatorname{Pred}^{0}$ das Merkmal [predicative] zu. Laut Matushansky (2008, 223) ergeben sich die Oberflächenkasus aus dem Faktum, dass der Subjektausdruck ausschließlich im Skopus von $\mathrm{T}^{0}$ steht, während der prädikative Ausdruck im Skopus sowohl

45 Es ist nicht klar, wie Matushansky (2008) unter diesen Annahmen russische Kopulasätze erfassen will, die trotz einer (overten) Verbform den Nominativ am Prädikatsnomen zeigen, also z. B. im Präteritum (vgl. auch Bondaruk 2013b, 192). Daraus folgt auch, dass diese Analyse zwar russische, nicht aber ohne Weiteres polnische Präsenssätze erfassen kann. 
von $\mathrm{T}^{0}$ als auch von Pred ${ }^{0}$ ist. Demnach gelten (mindestens für das Russische) die relevanten Einsetzungsregeln in (5): ${ }^{46}$

(5) a. [nominative] $\rightarrow$ NOM

(vgl. Matushansky 2008, 223)

b. [nominative, predicative] $\rightarrow \mathrm{NOM}$

Dagegen sollen Sätze mit instrumentalischen Prädikatsnomina sogar zwei vPn beinhalten, von denen die eine "eventive" Semantik hat und somit ein Ereignisargument sowie das entsprechende 'Kasusmerkmal' [eventive] einbringt, während die andere $\nu \mathrm{P}$ das externe Argument bzw. den Subjektausdruck einführt und den Akkusativ (Merkmal [accusative]) zuweist:

(6) $\quad \mathrm{TP}>v \mathrm{P}_{\mathrm{EVENT}}>v \mathrm{P}>\mathrm{VP}>$ PredP $>\mathrm{NP}_{\text {INS }}$

(vgl. Matushansky 2008, 222)

Die relevanten Einsetzungsregeln lauten wie in (7):

(7) a. [predicative, eventive] $\rightarrow$ INSTR

(Matushansky 2008, 223)

b. [nominative] $\rightarrow \mathrm{NOM}$

c. [accusative] $\rightarrow$ ACC

Das Prädikatsnomen steht nicht nur im Skopus von $v_{\text {EVENT }}^{0}$ und Pred ${ }^{0}$, sondern erhält vier Kasusmerkmale von den Köpfen $\operatorname{Pred}^{0}, v^{0}, v_{\text {EVENT }}^{0}$ und $\mathrm{T}^{0}$. Dass letztlich aber nur die beiden Kasusmerkmale [predicative] und [eventive] für die Einsetzung des Instrumentals relevant sind, führt Matushansky im Sinne von DM auf die Prinzipien von impoverishment und underspecification zurück, die uns ihrer Ansicht nach erlauben, "[...] to account for the fact that not all features assigned to a given terminal affect its surface representation.” (Matushansky 2008, 223)

Ein russisches Beispiel wäre der Satz in (8a), dem nach Matushansky (2008) die syntaktische Struktur in (8b) zukommt:

46 Die Regel in (5b) schlussfolgere ich aus Matushanskys (2008) Aussagen. 
(8)

a. Ja sčitaju eë lingvistkoj.

ich-NOM halte-1.SG sie-ACC.SG Linguistin-INS.SG

(Matushansky 2008, 214)

'Ich halte sie für eine Linguistin.'

b. [TP $\mathrm{T}^{0}\left[{ }_{v \mathrm{P}} v_{\text {EVENT }}^{0}\left[{ }_{v \mathrm{P}} \mathrm{ja}\left[v^{\prime} v^{0}\right.\right.\right.$ [vP sčitaju [PredP eë [Pred Pred $^{0}$ lingvistkoj ]]]]]]]

Dies ist ein Beispiel sekundärer Prädikation, die Matushansky (2008) in ihrer Arbeit primär thematisiert. Ein 'einfacher' Kopulasatz mit Instrumental-NP wäre demnach wohl wie in (9) zu analysieren. Denkt man Matushanskys Konzept konsequent weiter, muss ein solcher Satz "eventiv" sein und $v_{\text {EVENT }}^{0}$ enthalten: ${ }^{47}$

(9) a. Ja byla lingvistkoj.

ich-NOM war-SG.F Linguistin-INS.SG

'Ich war Linguistin.'

b. $\quad\left[\mathrm{TPP}^{0}\left[v^{\prime} \mathrm{ja}_{\mathrm{i}}\left[{ }_{v \mathrm{P}}\right.\right.\right.$ byla $\mathrm{a}_{v \text {-EvenT }}\left[\operatorname{PredP} t_{\mathrm{i}}\left[\right.\right.$ Pred $^{\prime}$ Pred $^{0}$ lingvistkoj $\left.\left.\left.\left.]\right]\right]\right]\right]$

Daraus, dass das Prädikativum im Einflussbereich sowohl von $\operatorname{Pred}^{0}$ als auch von $v_{\text {EVENT }}^{0}$ ist, folgt nach Matushansky $(2008,223)$ seine Ausstattung mit den beiden Kasusmerkmalen [predicative] und [eventive] und somit die Markierung mit dem Oberflächenkasus Instrumental. Als Besetzung von $v_{\text {EVENT }}^{0}$ ist byla ein "eventives" Verb (beachte die Parallele zu Markmann 2008).

Ein präsentischer Kopulasatz mit Nominativ dagegen soll eine viel bescheidenere Struktur haben, "[a]ssuming that in the absence of an overt copula the small clause merges as the complement of T (see Bailyn \& Rubin 1991, among others, for Russian)" (Matushansky 2008, 221): ${ }^{48}$

(10) a. Ja - lingvistka. ich-NOM [bin-1.SG] Linguistin-NOM.SG 'Ich bin (eine) Linguistin.'

b. [Tе $\mathrm{T}^{0}$ [PredP ja [Pred Pred $^{0}$ lingvistka ]]]

47 Einen 'unteren' $v$-Kopf setze ich nicht an. Aus Matushanskys Ausführungen lässt sich dazu nichts ableiten. Da sie das 'prädikative Subjekt' ansonsten in Spec-PredP ansetzt, und da ein Akkusativmerkmal hier keine Rolle spielt, halte ich eine zweite $v$ P für verzichtbar. Die Bewegung des Subjektausdrucks nach Spec- $v$ P folgere ich daraus, dass $v_{\text {EVENT }}^{0}$ die Position des overten und "eventiven" byla ist.

48 Es ist nicht auszuschließen, dass sich der Subjektausdruck in der Folge nach Spec-TP bewegt. 
Wie Bondaruk (2013b) in ihrer Besprechung des Aufsatzes von Matushansky (2008) richtig bemerkt, stößt die Analyse eines Satzes mit Nominativ-Prädikativ und overter Form von byt' auf Probleme, denn “[...] Matushansky's model [...] links nominative case on the predicate either with the lack of the copula verb [...] or with the identity interpretation" (Bondaruk 2013b, 192). ${ }^{49}$ Einziger Ausweg ist (wie bei Markman 2008) die stipulative Annahme zweier Ausprägungen von byt', und zwar einer "eventiven" und einer "non-eventiven", die in einem $v$-Kopf über der PredP anzusetzen wäre, wie (11b) zeigt: ${ }^{50}$
(11) a. Ja byla lingvistka.
ich-NOM war-SG.F Linguistin-NOM.SG
'Ich war (eine) Linguistin.'

b. [TP $\mathrm{T}^{0}\left[{ }_{v \mathrm{P}} \mathrm{ja}\left[v^{\prime}\right.\right.$ byla ${ }_{v}$ [PredP $t_{\mathrm{i}}$ [Pred’ bylapred lingvistka $\left.\left.\left.\left.]\right]\right]\right]\right]$

Sicher ist, dass in den Fällen mit nominativischem Prädikatsnomen der Kopf $v_{\text {EVENT }}^{0}$ nicht anwesend sein darf, da ansonsten der Instrumental zu erwarten wäre. Demnach liegt in Kopulasätzen mit nominativischen Prädikatsnomina stets eine "non-eventive" Variante von byt' vor. Wie ich bereits im Zusammenhang von Markman (2008) geäußert habe, ist es durchaus nicht diese Variante, die konzeptuelle Probleme bereitet, sondern vielmehr die Annahme einer "eventiven" Ausprägung mit Ereignisargument. Allein dieser Punkt lässt an der Analyse von Matushansky (2008) - ebenso wie an denen von Matushansky (2000), Richardson (2001) und Markman (2008) - zweifeln. Bondaruk (2013b, 192-193) nennt noch weitere Kritikpunkte, u. a. das theoretische Problem der Zuweisung mehrerer Kasusmerkmale an ein Element (zumal die 'Kasusmerkmale' mitunter sehr weit vom traditionellen Kasuskonzept entfernt sind), die aus Matushanskys (2008) Kasustheorie folgende Notwendigkeit der Definition von Barrieren, die die Kasusperkolation blocken sowie die Annahme von Einsetzungsregeln à la DM. Am

49 Matushansky $(2008,223)$ schreibt, dass bei Identitätssätzen der Nominativ an der zweiten NP nur folgerichtig sei, denn "identity be has no eventuality argument".

50 Falls der $v$-Kopf das Kasusmerkmal [accusative] zuweist, muss die Merkmalskombination [predicative, accusative] im Russischen qua impoverishment als 'effektlos' betrachtet werden bzw. ('auch nur') zum Oberflächenkasus Nominativ führen. 
bedeutsamsten ist jedoch sicherlich Bondaruks (2013b, 193) treffender Einwand, dass Matushanskys (2008) Analyse nicht auf die polnischen Daten anwendbar ist.

\subsection{Syntaktischer Ansatz}

Die (rein) syntaktischen Analysen gehen davon aus, dass die Kasusvariationen vollends auf syntaktischen Verhältnissen bzw. Mechanismen beruhen.

Syntaktisch in diesem Sinne sind auch die Analysen russischer Kopulasätze von Moro (1997) und den Dikken (2006), die hier dennoch nicht im Detail besprochen werden, da sie nicht auf die Kasusmarkierung eingehen. Stattdessen befassen sie sich mit der Ableitung 'kanonischer' im Unterschied zu 'inversen' Wortfolgen in diesen Sätzen, ${ }^{51}$ die - wie etwa aus den Arbeiten von Geist (2006) und Bondaruk (2013b) deutlich wird - in engem Zusammenhang mit dem Unterschied zwischen "prädizierenden” und "spezifizierenden” Kopulasätzen stehen. Bedeutsam für die weitere Diskussion ist den Dikkens (2006) RelP-Analyse (einschließlich seiner Kritik an Moro 1997), da sie später von Pereltsvaig (2007) aufgegriffen wird. Zu letzterer Arbeit komme ich in Kürze. Zunächst aber geht es um Roy (2006).

\subsubsection{Roy (2006)}

Etwas salopp kann man sagen, dass Roy (2006) Semantik syntaktisch 'verpackt'. Um den Ansatz in gebotener Kürze vorzustellen, komme ich direkt dazu, dass sie zwei unterschiedliche Strukturen für russische Kopulasätze mit nominativischen vs. instrumentalischen Prädikatsnomina ansetzt. Die Syntax im (12) soll Nominativsätzen zugrunde liegen:

51 Moro (1997) geht von einer "symmetrischen" Struktur aus, in der Subjektausdruck und Prädikatsnomen Schwester-DPn in einem Small Clause (SC) sind, vgl. (i):

(i) $\left.\quad\left[\mathrm{AgrP}[e]\left[\mathrm{Agr}^{\prime} \operatorname{Agr}^{0}\left[\mathrm{TP}^{0} \mathrm{~T}_{\mathrm{VP}} b e_{\mathrm{V}^{\circ}}[\mathrm{sC} \mathrm{DP} \mathrm{DP}]\right]\right]\right]\right]$

(Moro 1997, 91)

Den Dikken (2006) kritisiert, dass dieser SC 'kopflos' sei. Seine Alternative besteht in der Annahme einer Rel[ator]P mit dem Prädikatsnomen (YP) im Komplement und dem Subjektausdruck (XP) im Spezifikator; vgl. (ii):

(ii) $\quad\left[{ }_{R P} \mathrm{XP}\left[\mathrm{R}^{\prime} \mathrm{R}^{0} \mathrm{YP}\right]\right]$

(den Dikken 2006, 11)

RelP ist im Prinzip ein kategorisierter SC; siehe (iii-a). Beachte, dass der Hauptunterschied zu Bowers' (1993) PrP in (iii-b) sowie auch zu Citkos (2008) $\pi$ P in (iii-c) nur im Label besteht:
(iii) a. $\left[\mathrm{sc} \ldots \mathrm{F}^{0} \ldots\right]$
b. $\left[\operatorname{PrP} \ldots \operatorname{Pr}^{0} \ldots\right]$
c. $\left[\pi \mathrm{P} \ldots \pi^{0} \ldots\right]$ 
Nominativische Prädikatsnomina sind Roy (2006) zufolge also keine NPn oder DPn, sondern Num[ber]Pn. Die Formen von byt' lokalisiert Roy in $\mathrm{T}^{0}$ (womit sie als bloße Auxiliare gelten können) und nimmt an, dass sie sich später weiter nach $\operatorname{Agr}_{S}{ }^{0}$ bewegen. Der Subjektausdruck (die DP) hat seinen Ursprung in Spec-PredP und bewegt sich über Spec-TP nach Spec-Agr ${ }_{S} P$. Das Prädikatsnomen (die NumP) bewegt sich wiederum obligatorisch in einen weiteren Spezifikator der TP. Entscheidend an letzterer Bewegung ist, dass sich das Prädikativ danach nicht mehr im Skopus von Pred $^{0}$ befindet. Diese Bewegung ist laut Roy obligatorisch. Letzteres folge daraus, dass NumPn events denotieren, und dass diese wiederum von einem sog. "max-Operator” gebunden werden müssen. Dieser Operator sei 'stark', wodurch auch die NumP, die von ihm gebunden wird, ein 'starker' Ausdruck sei. Dies schließlich, so Roy, erzwinge die erwähnte syntaktische Bewegung der NumP aus PredP heraus (vgl. Bondaruk 2013b, 196-197). So ergibt sich für den Beispielsatz in (13a) letztlich die syntaktische Analyse in (13b):
(13) a. Oleg byl direktor fabriki. (vgl. Bondaruk 2013b, 185)
Oleg-NOM war-SG.M Direktor-NOM Fabrik-GEN
'Oleg war ein Fabrikdirektor.'

b. [AgrSP Oleg byl $_{\mathrm{k}}\left[\mathrm{TP} t_{\mathrm{i}}{ }^{\prime}[\mathrm{NumP}\right.$ direktor $\left.\left.]\left[\mathrm{T}^{\prime} t_{\mathrm{k}}\left[\operatorname{PredP} t_{\mathrm{i}}\left[\operatorname{Pred}^{\prime} \operatorname{Pred}^{0} t_{\mathrm{NumP}}\right]\right]\right]\right]\right]$

Schon hier fällt die große Zahl an Bewegungen auf. Ferner ist die Annahme einer $\operatorname{Agr}_{S} \mathrm{P}$ nach heutigen Maßstäben relativ überraschend (vgl. Bondaruk 2013b, 198). Gravierender wirkt für mich jedoch die Annahme des "max-Operators" als (einzige!) Begründung für die - an sich fragwürdige - Bewegung des Prädikativs nach Spec-TP. Die Kategorisierung des letzteren als "NumP" gehört ebenfalls zu den Annahmen von Roy (2006), die man - nach ökonomischen Gesichtspunkten lieber vermeiden würde. Das gilt umso mehr, als der ihnen zugeschriebene Bezug auf events automatisch bedeutet, dass auch Sätze mit nominativischen Prädikatsnomina in jedem Fall auf Ereignisse (nicht auf Zustände) referieren (müssen).

Für Kopulasätze mit instrumentalischen Prädikatsnomina sieht die Struktur nach Roy anders aus. Sie unterscheidet sich in zwei Punkten deutlich von der o. g. Syntax, namentlich durch die Präsenz einer AspP und die Anwesenheit einer $\mathrm{Cl}\left[\right.$ assifier]P im Komplement von Pred $^{0}$ : 
(14)

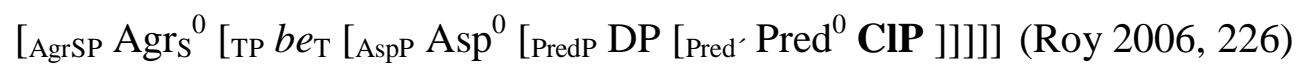

Folgt man Roy, sind instrumentalische Prädikative also CIPn. Diese seien im Gegensatz zu den o. g. NumPn nicht 'stark' und verbleiben daher in situ, wo ihnen $\operatorname{Pred}^{0}$ den Instrumental zuweisen könne. Die Autorin nimmt ferner an, dass CIPn nicht einfach events, sondern count eventualities denotieren. Für letztere wiederum gelte, dass sie durch einen Aspekt-Operator existenziell gebunden werden müssen, was wiederum impliziere, dass bei Präsenz einer ClP auch stets eine AspP projiziert werden müsse, da das Ereignisargument der ClP ansonsten nicht abgebunden werden könnte. Der Beispielsatz mit Instrumental in (15a) sollte somit die syntaktische Struktur in (15b) haben:

\section{(15) a. Oleg byl direktorom fabriki. (vgl. Bondaruk 2013b, 185) \\ Oleg-NOM war-SG.M Direktor-INS Fabrik-GEN \\ 'Oleg war Fabrikdirektor.'

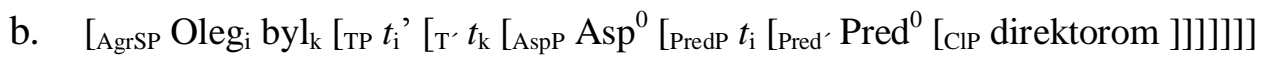

Was präsentische Kopulasätze mit "Nullkopula" betrifft, so haben diese laut Roy die o. g. NumP-Struktur. Die "Nullkopula" deutet ihr zufolge auf die Abwesenheit einer AspP hin, was wiederum bedeute, dass nur eine NumP, nicht aber eine CIP vorhanden sein könne, weil das Ereignisargument der letzteren sonst nicht abgebunden würde.

Im Grunde dient Roys (2006) Analyse insgesamt der technischen Untermauerung ihrer Sichtweise, dass Nominativsätze aus interpretativer Sicht definierend Instrumentalsätze hingegen charakterisierend seien.

Wenn auch Roys (2006) Analyse in sich stimmig ist und den Vorteil hat, nicht wie z. B. Matushansky (2000, 2008) oder Markman (2008) - zwei verschiedene Ausprägungen von byt' ansetzen zu müssen, so wirken die meisten ihrer syntaktischen (und auch semantischen) Annahmen doch sehr stipulativ. Die Argumentation ist sogar zirkulär, als einerseits die Existenz von NumPn und ClPn behauptet wird, um die Ab- oder Anwesenheit von AspP zu erklären, während auf der anderen Seite die Projektion von AspP als Voraussetzung für die Projektion der (instrumentalischen) ClP dargestellt wird. 
Die interpretativen Effekte 'definierend' vs. 'charakterisierend' begründet Roy (2006) nicht, sondern reformuliert sie de facto lediglich in Form des Gegensatzes der von ihr stipulierten Kategorien "Num" und "Cl". Letztlich lösen sich die vermeintlichen Vorteile gegenüber den besprochenen Analysen von Matushansky (2000, 2008), Richardson (2001), Harves (2002) und Markman (2008) auf, da Roys Modell die einheitliche Behandlung der byt'-Formen als T-Auxiliare durch die Annahme von NumP und ClP 'erkauft'. Und wie schon bei Matushansky (2000), Harves (2002) oder Markman (2008), besteht auch bei Roy (2006) das tiefgreifende Problem, eine Aspektspezifikation nur in Instrumentalsätzen vorzusehen. $^{52}$

\subsubsection{Pereltsvaig (2007)}

Pereltsvaig (2007) ist insofern 'originell', als sie zwei absolut differierende syntaktische Strukturen für russische Kopulasätze mit Nominativ und Instrumental ansetzt. In Bezug auf Nominativsätze bedient sie sich dafür bei Moro (1997), während sie sich in Bezug auf Instrumentalsätze an Arbeiten wie Bowers (1993), oder den Dikken (2006) orientiert. Schon an dieser Stelle sei festgestellt, dass ihre Analyse zu der sehr problematischen Folgerung führt, Nominativsätze seien stets Identitätssätze (vgl. ganz ähnlich Matushansky 2008).

Im Kern dem Ansatz von Moro (1997) folgend, nimmt Pereltsvaig (2007) für Nominativsätze die folgende "symmetrische" Grundstruktur an: ${ }^{53}$

Originell an Pereltsvaigs (2007) Vorschlag ist nicht nur, dass diese Struktur "symmetrisch" ist und im Komplement von TP keinerlei SC oder VP auftritt, ${ }^{54}$

52 Bondaruk (2013b, 198) kritisiert, dass Roys Annahmen auch zwei distinkte Ausprägungen von PredP implizieren würden, von denen nur eine den Instrumental am Prädikatsnomen checken könne. M. E. trifft das nicht zwangsläufig zu, da die Instrumentalzuweisung durch $\operatorname{Pred}^{0}$ als optional betrachtet werden könnte.

53 Moros (1997) Struktur besteht aus einem Small Clause (SC) mit zwei Schwester-DPn:

(i) $\ldots\left[\right.$ [vp $b e_{\mathrm{v}}[\mathrm{sc}$ DP DP ]]

(vgl. Moro 1997, 91)

Den Dikken $(2006,62)$ kritisiert, dass Moros SC keinen Kopf habe. Dies trifft auf Pereltsvaigs Struktur nicht zu, da sie überhaupt keinen SC beinhaltet. 
sondern auch, dass keine der beiden DPn nach Pereltsvaigs (2007, 222) Auffassung ein Argument ist, da sie weder in der Komplement- noch in der Spezifikatorposition eines lexikalischen Kopfes stehen. Auf Grund der Gleichrangigkeit beider DPn lässt sich nicht bestimmen, welche DP Subjektausdruck und welche Identitätsausdruck sei. Erst durch die Bewegung einer der DPn nach Spec-TP kommt eine "asymmetrische" Struktur zustande:

(17) $\left[\mathrm{TP} \mathrm{DP}_{\mathrm{i}} b y t_{\mathrm{T}}^{\prime}\left[\mathrm{DP} \mathrm{DP} t_{\mathrm{i}}\right]\right]$

(Pereltsvaig 2007, 236)

Die Gleichrangigkeit beider DPn ist nach Pereltsvaig die Ursache für die Identitätslesart; demnach leitet sich diese Interpretation direkt aus der Syntax ab.

Was Instrumentalsätze angeht, so nimmt Pereltsvaig (2007) eine von Grund auf “asymmetrische" Syntax an. Ihre Analyse lässt sich mit der von Bowers (1993), den Dikken (2006), Geist (2006), Citko (2008) u. a. vergleichen, da auch sie eine spezifische Kategorie zur Etablierung von "Prädikation" ansetzt. Dabei nimmt sie, wie Geist (2006), die Kategorie $v^{0}$ an, die lexikalisch als byt'-Form realisiert werde. Dies ist ein deutlicher Unterschied zu jenen o. g. Arbeiten, die eine funktionale Prädikationskategorie wie etwa $\operatorname{Pr}(\mathrm{ed})^{0}, \operatorname{Rel}^{0}$ oder $\pi^{0}$ ansetzen. Das lexikalische Verb byt' in $v^{0}$, so Pereltsvaig (2007), vergibt den Instrumental an die prädikative Komplement-NP, bei der es sich somit um ein "Argument" des lexikalischen Kopulaverbs handele; vgl. (18):

(18) $\left[{ }_{v \mathrm{P}} \mathrm{DP} b y t_{v}^{\prime} \mathrm{NP}\right.$ INS ]

(vgl. Pereltsvaig 2007, 222)

Zusammenfassend heißt das, dass nur Instrumentalsätze wirklich Kopulasätze, Nominativsätze dagegen stets Identitätssätze seien. Interpretative Effekte wie “temporal vs. permanent" entlang der Kasusvariation, wie sie von deskriptiven Grammatiken des Russischen beschrieben werden, kann es damit im Grunde nicht geben, sofern nicht die Behauptung gemacht werden soll, dass die 'permanente' Lesart stets und ausnahmslos deckungsgleich mit der Identität zweier referenzieller Ausdrücke sei. Anders formuliert: Nominativsätze sind Pereltsvaig zufolge keine Kopulasätze, da sie keinen prädikativen Ausdruck enthalten, sondern 'nur'

54 Diese Annahme ist nicht nur originell, sondern auch problematisch, da ihr zufolge Nominativsätze "Nominalsätze" ohne eine verbale Basis sind. 
zwei referenzielle DPn. Ferner nimmt sie zwei grundsätzlich verschiedene Ausprägungen von byt' an, namentlich (i) eine funktionale in $\mathrm{T}^{0}$ in den nominativischen Identitätssätzen sowie (ii) eine lexikalische in $v^{0}$ in den instrumentalischen Kopulasätzen. ${ }^{55}$

Ein weiterer Kritikpunkt lautet, dass es wenig inituitiv wirkt, für russische byt'Sätze derart differierende syntaktische Strukturen anzunehmen. Zwar unterscheiden sich die Strukturen dieser Sätze auch in den Analysen von Matushansky (2000, 2008), Harves (2002), Markman (2008) oder Bailyn (2013), aber keine dieser Arbeiten geht so weit, eine "symmetrische" Struktur für die Nominativsätze anzusetzen, die keinerlei Verbform als Basis aufweist. Insbesondere letztere Annahme sowie die Ansicht, Nominativsätze seien stets Identitätssätze, sind m. E. hinreichende Gründe, die Analyse von Pereltsvaig (2007) abzulehnen.

\title{
3.3.3 Bailyn (2012)
}

Eine rezente und in den Kontext einer größeren Syntaxtheorie eingebettete Analyse der russischen Kasusvariation formuliert Bailyn (2012). ${ }^{56}$ Ausgangspunkt ist Bailyns (2012, 194-195) Argumentation gegen Ansätze, die für Sätze mit Kasusvariation jeweils die gleiche syntaktische Struktur postulieren. Für ihn, der einen explizit syntaktischen Ansatz verfolgt (vgl. Bailyn 2012, 196-199), sind lediglich Sätze mit dem Nominativ (dem Kongruenzkasus) Fälle primärer Prädikation. Es handele sich dabei um "[...] non-verbal sentences, with a primary predicator (the verb to be) whose present tense form happens to be (morphologically) null” (Bailyn 2012, 194). Dagegen seien “[a]pparent primary predicates marked with Instrumental [...] in fact secondary predicates” (Bailyn 2012, 195). Solche Sätze haben laut Bailyn die gleiche Syntax wie Sätze mit 'offensichtlichen' Sekundärprädikaten, wie z. B. durakom ‘(als) Narr’ in Beispiel (19):

\author{
(19) Saša kažetsja durakom. (Bailyn 2012, 183) \\ Sascha-NOM scheint-3SG-REFL Narr-INS \\ 'Sascha scheint ein Narr (zu sein).'/'Sascha erscheint als Narr.'
}

\footnotetext{
55 Hier zeigt sich eine Parallele zu dem Vorschlag von Geist (1999), die ebenfalls ein funktionales $b y t_{1}^{\prime}$ (+ Nominativ) und ein lexikalisches $b y t_{2}^{\prime}$ (+ Instrumental) annimmt.

56 Die Annahmen in dieser Arbeit entsprechen vielfach jenen in Bailyn (2001).
} 
Mit Bowers (1993, 1997) geht Bailyn von der Kategorie "Pred" aus, die in allen Fällen von Prädikation vorliege. Alle T-Köpfe, so Bailyn weiter, selegieren somit eine PredP (= primäre Prädikation), wozu andererseits aber nur bestimmte V-Köpfe in der Lage seien (= sekundäre Prädikation). PredP könne ferner auch ein Adjunkt sein (ebenfalls = sekundäre Prädikation).

Kernthese Bailyns ist, dass der Instrumental unmittelbar durch Pred ${ }^{0}$ zugewiesen werde. Der Pred-Kopf muss dazu aber "leer", d. h. frei von Informationen sein, die an den Schnittstellen interpretierbar sind. Ist Pred $^{0}$ dagegen "gefüllt", dann 'absorbiere' es das Instrumentalmerkmal, woraus folge, dass weder der Instrumental noch ein anderer Kasus zugewiesen werden kann (Bailyn 2012, 194). Das heißt, dass beim Instrumental "leeres" Pred $^{0}$ involviert sein muss, was Bailyns Annahmen zufolge nur bei sekundärer Prädikation der Fall ist. Bei primärer Prädikation sei Pred ${ }^{0}$ hingegen immer "gefüllt", was den Instrumental ausschließe, so dass nur der Kongruenzkasus (Nominativ) in Frage kommt.

Ich habe erwähnt, dass Bailyn Kopulasätze mit dem Instrumental strukturell mit Sätzen wie (19) vergleicht. Letztere basieren ihm zufolge auf Verben, die lexikalisch ein Small Clause-(SC)-Komplement regieren (Bailyn 2012, 183-184). Dazu gehören u. a. kazat'sja 'scheinen' und sčitat' 'halten (für)'. Für den kažetsja-Satz in (19) nenne ich in (20) die Repräsentation, die Bailyn selbst ansetzt:

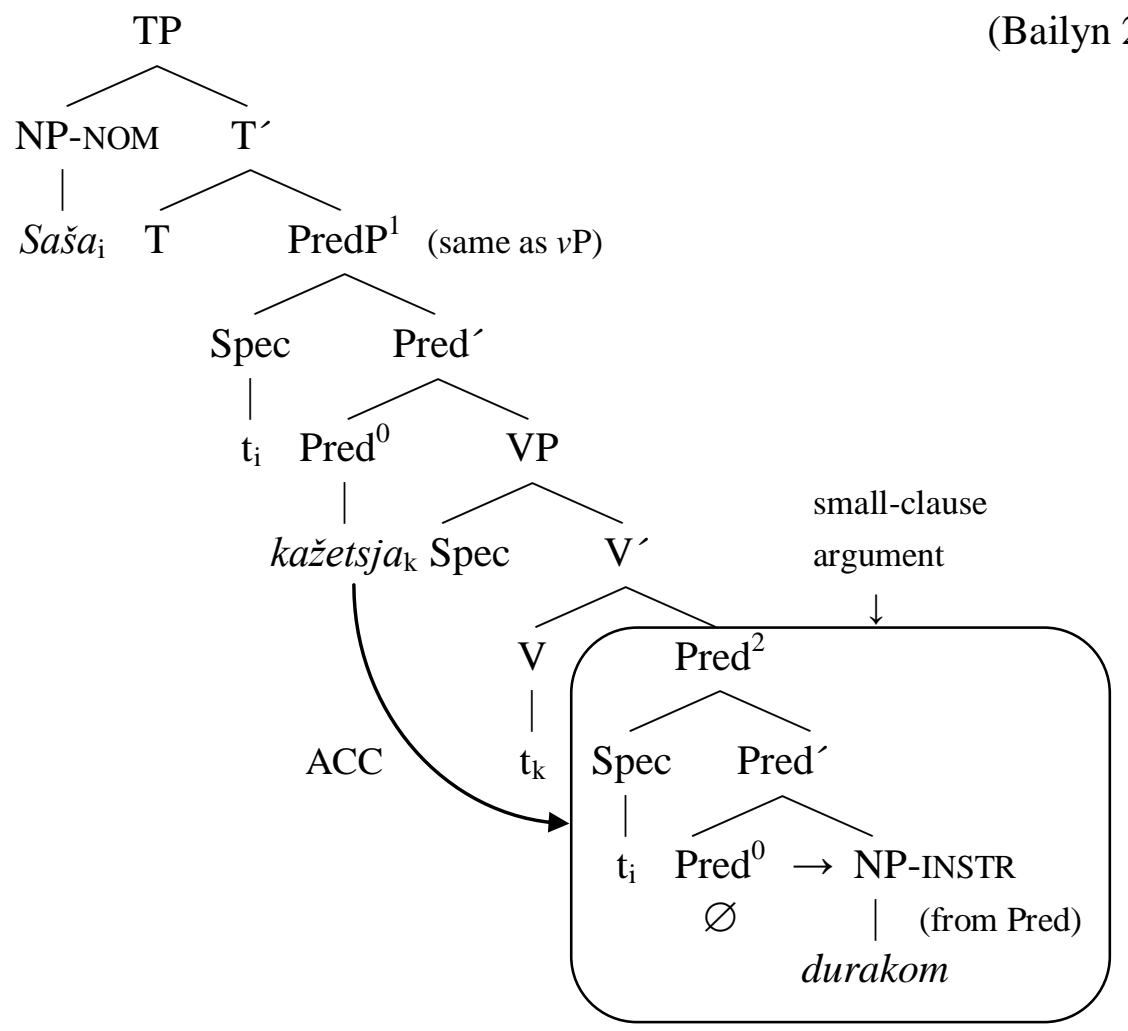


Demnach weist kažetsja seinem SC-Komplement den Akkusativ zu. Ferner hebt es das Subjekt des SC an (Raising). Kažetsja besetzt letztlich die obere PredP $(=v \mathrm{P})$, während das untere $\operatorname{Pred}^{0}$ (Kopf des SC) "leer" ist und so den Instrumental zuweisen kann. Daher erscheint das Prädikatsnomen durakom im Instrumental und könne als sekundäres Prädikat gelten. Die Analyse erfasst, warum in solchen Konfigurationen nur der Instrumental, nicht aber der Kongruenzkasus erscheinen kann. Letzteres wird durch Bailyns Analyse für den sčitat'-Satz in (21) noch deutlicher, die ich daher in (22) ebenfalls zitiere: ${ }^{57}$
(21) Ja sčitaju ego durakom. (vgl. Bailyn 2012, 183) ich-NOM halte für-1SG ihn-ACC Narr-INS 'Ich halte ihn für einen Narren.'

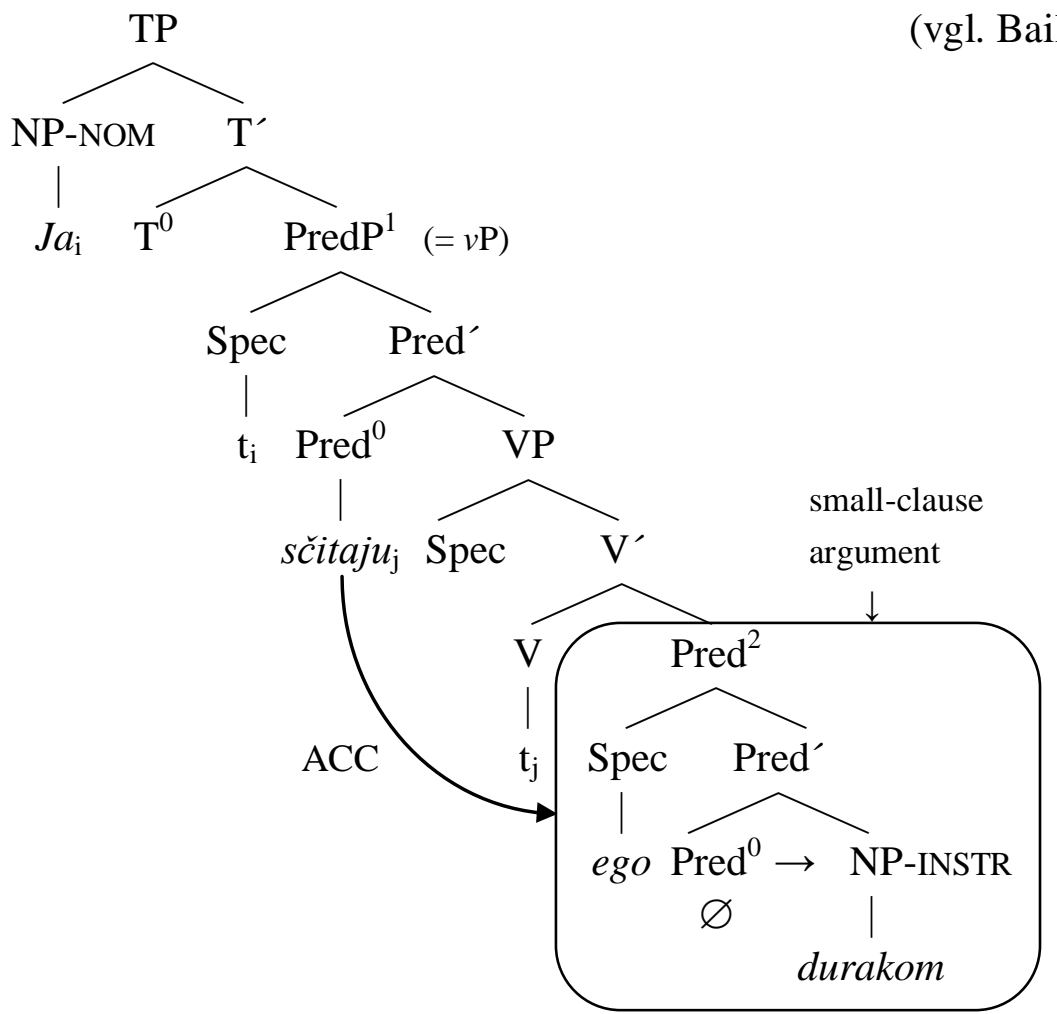

Dem zufolge ist sčitat' - analog zu seiner englischen Entsprechung consider - ein ECM-Verb (vgl. Bailyn 2012, 182, Fn. 14). Anders als kazat'sja, das seinem externen Argument keine Thetarolle zuweist und so das Subjekt des SC anhebt und

57 Bailyns $(2012,184)$ Struktur zeigt durakom (offenbar ein Kopierfehler) als AP, die mit ego aus dem SC koindiziert ist. In (22) ist dies 'korrigiert'. 
zum Satzsubjekt 'befördert', vergibt sčitat' diese Thetarolle, weshalb das SC-Subjekt in situ bleibt. Dort erhält es jedoch von Pred $^{0}$ keinen Kasus. Da sčitaju aber dem SC als Ganzem den Akkusativ zuweist, kann das SC-Subjekt 'ausnahmsweise' von dieser Kasuszuweisung profitieren und so via ECM in der Akkusativform $e$ go erscheinen. ${ }^{58}$

Dass Pred $^{0}$ als Kopf des SC nur den Instrumental vergeben kann, erklärt sich in beiden o. g. Strukturen aus seiner "Leere". Letztere schließt laut Bailyn gleichzeitig den Kongruenzkasus aus, weshalb etwa der Satz in (23) ungrammatisch sei:

$$
\begin{array}{cccc}
\text { (23) *Ja sčitaju ego } & \text { duraka. } \\
\text { ich-NOM halte für-1SG } & \text { ihn-ACC } & \text { Narr-ACC }
\end{array}
$$

Die Argumentation lautet, dass - würde "leeres" Pred ${ }^{0}$ neben dem Instrumental auch den Kongruenzkasus zulassen - dieser Satz grammatisch sein sollte. So gelingt es Bailyn (2012) in der Tat, die Daten zu erfassen und - mindestens für die genannten Fälle - ein konsistentes Bild zu zeichnen.

Es gibt jedoch besonders einen Punkt an jenem Teil seiner Analyse, der sich auf Fälle bezieht, die man üblicherweise als primäre Prädikation betrachtet, die der Autor aber explizit als Fälle sekundärer Prädikation bezeichnet. Es sind dies Kopulasätze mit instrumentalischen Prädikatsnomina. Zur Annäherung an das Problem sei zunächst Bailyns Analyse für solche Sätze genannt, die er als primäre Prädikation betrachtet, namentlich Kopulasätze mit nominativischem (kongruierendem) Prädikatsnomen:

$$
\begin{aligned}
& \text { a. Boris byl muzykant. } \\
& \text { Boris-NOM was musician-NOM } \\
& \text { 'Boris was a musician (in his very nature).' }
\end{aligned}
$$

58 M. E. ist für das Russische kein ECM nötig. Die fraglichen Verben müssen nicht als Selegierer von SC-Komplementen betrachtet werden. Der semantische "Gehalt" letzterer kann stattdessen als Teil der grammatischen Bedeutung dieser Verblexeme gelten. Der Instrumental kann also als Realisierungsform 'prädikativer' Argumente betrachtet werden. Sog. "halbkopulative” Verben wie javljat'sja 'scheinen', stat' 'werden', ostat'sja 'bleiben' usw. ähneln ersteren, da der Instrumental auch an ihren Komplementen obligatorisch ist. Dagegen ist er im Falle der Kopula(bedeutung) lediglich optional (siehe Abschnitt 5.5). 


\section{b. Boris - muzykant. \\ Boris-NOM $\varnothing \quad$ musician-NOM \\ 'Boris is a musician.'}

Bailyn geht hier von einem primären Prädikator ("gefülltes" Pred") aus. Dass so ein "gefülltes" Pred $^{0}$ wie in $(24 b)$ auch phonetisch leer sein kann, erklärt Bailyn $(2012,194)$ damit, dass es doch insofern "overt" sei, als es - in der Rolle des primären Prädikats - mit Tempus assoziiert ist. Sofern Pred ${ }^{0}$ demnach also doch "gefüllt" ist, wird der Instrumental 'absorbiert'. Folglich kommt nur der Nominativ in Frage, der durch Multiple Agree von $\mathrm{T}^{0}$ sowohl an den Subjektausdruck als auch an das Prädikatsnomen vergeben wird. Ich gebe die Struktur, die Bailyn (2012, 194) für solche Sätze präsentiert, mittels indizierter Klammerung wieder: ${ }^{59}$

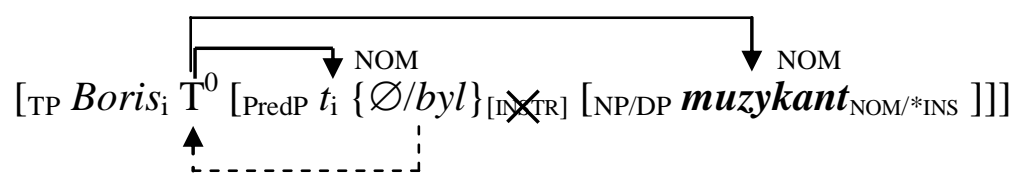

Demnach sei also Pred ${ }^{0}$ sowohl als Nullform (Präsens) als auch in Form von byl (Präteritum) "gefüllt", was den Instrumental ausschließe. Somit muss $\mathrm{T}^{0}$ als Kasusgeber sowohl für den Subjektausdruck als auch für das Prädikativ 'einspringen' (Multiple Agree). Soviel zur primären Prädikation.

Zurück zu Kopulasätzen mit Instrumental, die Bailyn zufolge dieselbe Syntax wie kazat'sja-Sätzen haben und Fälle sekundärer Prädikation sein sollen. Zwar verdeutlicht er seine Analyse für diese Kopulasätze nicht anhand einer Baumstruktur, jedoch macht er sie durch Glossierungen deutlich, indem er einen Kopulasatz mit Instrumental unmittelbar einem kazat'sja-Satz gegenüberstellt:

(26) a. On he $^{-}$-NOM 'He was a soldier.'

$$
\text { byl }
$$

was soldatom.

(vgl. Bailyn 2012, 195)

59 In Bailyns $(2012,194)$ Strukturbaum steht Boris $s_{\mathrm{i}}$ (mit Index!) in Spec-PredP, während in Spec-TP eine nicht weiter markierte DP erscheint. Es wirkt plausibel, dass Boris dieser DP entspricht, weshalb ich die Bewegung von Boris aus Spec-PredP nach Spec-TP darstelle (Bailyn setzt diese Bewegung auch in anderen Strukturbäumen an). 


\section{b. Saša kažetsja durakom. \\ Sasha $\mathrm{i}_{\mathrm{i}}$-NOM seems $\quad\left[\right.$ PREDP $_{\mathrm{i}} \quad \mathrm{t}_{\mathrm{i}}$ fool-INSTR $]$ \\ 'Sasha seems (to be) a fool.'}

Die Formen byl in (26a) und kažetsja in (26b) sind jeweils die Primärprädikatoren im oberen Pred ${ }^{0}\left(=v^{0}\right)$. Beide haben nach Bailyn einen SC als Komplement. Wie kažetsja, so ist auch byl ein Raising-Verb, das das SC-Subjekt anhebt. Das untere "leere" $\operatorname{Pred}^{0}$ (der Sekundärprädikator) schließlich vergibt in beiden Fällen den Instrumental an das Prädikativ. Soweit kann der Analyse (in ihrem Zusammenhang) durchaus gefolgt werden.

Das gravierende und m. E. nicht zu lösende Problem im Kontex der Annahme, Kopulasätze mit Instrumental seien Fälle sekundärer Prädikation, besteht m. E. darin, dass von einem primären Prädikat eine gewisse deskriptive Bedeutung zu erwarten ist. Worin aber soll letztere bei dem angeblichen Primärprädikat byl in (26a) bestehen? Dass kažetsja in (26b) eine solche Bedeutung hat, ist nicht zu bezweifeln. Diese Bedeutung, die als Primärprädikation gelten kann, würde dann durch den SC um eine Sekundärprädikation 'angereichert'. In (26a) ist das gleiche SC-Komplement gegeben.

Es stellt sich also die Frage, was für eine 'Bedeutung' byl als Primärprädikat beisteuern könnte. Wenn man ihm keine deskriptive Bedeutung geben kann, müsste es als bloßes Auxiliar gelten. Diese Annahme ist aber abzulehnen, da ein Auxiliar als Belegung des oberen Pred-Kopfes, d. h. der Position des Primärprädikats, völlig inadäquat ist. Schriebe man byl dagegen die 'normale' Kopulabedeutung zu, würde diese durch den SC um eine zweite Kopulabedeutung 'angereichert'. Dann würden zwei gleichartige Prädikationen nebeneinander vorliegen, was kaum sinnvoll erscheint. Schließlich kann auch die Annahme, byl wäre hier Existenzverb mit der deskriptiven Bedeutung 'existieren', die Analyse kaum retten, da sich zeigt, dass dies (i) eine recht eigenartige Satzbedeutung ergäbe und (ii) im Präsens die explizite Existenzform est' zulassen sollte, was nicht der Fall ist; vgl. (27a). Auch der Ausschluss der negierten Existenzaussage mit net (+ 'Genitivsubjekt') in (27b) bestätigt, dass die Form von byt' oben nicht das Existenzverb vertritt.

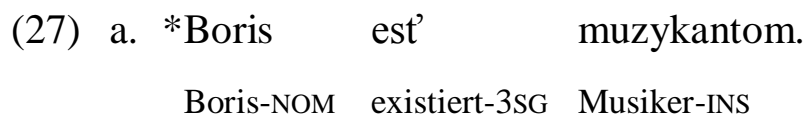




\section{b. *Borisa net muzykantom. \\ Boris-GEN existiert nicht Musiker-INS}

Ich sehe keine Möglichkeit, Bailyns (2012) Hypothese vor dem Hintergrund dieser semantischen Erwägungen zu legitimieren. Ich sehe auch nicht, inwiefern seine syntaktische Unterscheidung zwischen primärer (Nominativ) und sekundärer Prädikation (Instrumental) der Beantwortung der Frage nach dem Zustandekommen der interpretativen Effekte dienlich ist. Dennoch sind diverse Aspekte seines Modells zu begrüßen, darunter die Unterbringung der Kopulabedeutung in einem speziellen syntaktischen Kopf, die Annahme, dass auch "stumme" Köpfe semantisch "gefüllt" sein können (vgl. die "Nullkopula") sowie die Trennung der Kasuszuweisung von den byt'-Formen.

\subsubsection{Bondaruk (2013b)}

Bondaruks (2013b) Arbeit ist einerseits die rezenteste und umfassendste unter den hier vorgestellten, andererseits thematisiert sie die Kasusvariation an prädikativen $\mathrm{DPn}^{60}$ und $\mathrm{APn}$ in polnischen Kopulasätzen, wobei sie auch Analysen zum Russischen in ihre Betrachtung einfließen lässt.

Eine hervorzuhebende Folgerung Bondaruks besteht darin, dass Instrumentalsätze stets prädikativ sind, während Nominativsätze entweder prädikativ oder äquativ sein können (vgl. Bondaruk 2013b, 210). Dem ist m. E. zuzustimmen (vgl. auch Geist 2006). Freilich interessieren an dieser Stelle nur diejenigen Nominativsätze, die als 'echte' Kopulasätze gelten können.

Ebenso ist der Aussage Bondaruks (2013b, 210) zuzustimmen, dass Nominativsätze in ihrer Verwendung und Deutung relativ stark beschränkt seien, während dies bei Instrumentalsätzen nicht der Fall ist (vgl. schon Bogusławski 2001, 127). Die Restriktionen bestehen v. a. in der Wahl der grammatischen Person des Subjektausdrucks (meist liegt die 1. oder 2. Ps. vor) sowie in der lexikalischen Klasse des Prädikativs, bei dem es sich in Nominativsätzen meist um eine Berufs- oder Nationalitätenbezeichnung, einen Titel oder einen emotional gefärbten Ausdruck handele (vgl. Bondaruk 2013b, 210; siehe erneut auch Bogusławski 2001).

60 Bondaruk (2013b) verwendet für prädikative Substantive/Substantivgruppen das Label DP. 
Schließlich ist im Kontext meiner eigenen Analyse von großer Relevanz, dass Bondaruk die Formen von być von der Kasuszuweisung entkoppelt (wie u. a. auch Bailyn 2012) und sie als Raising-Verben (und damit als bloße Auxiliare) in einem Kopf $v^{0}$ über der PredP ansetzt (Bondaruk 2013b, 163-167):

(28) $\left[\mathrm{TP}^{0} \mathrm{~T}^{0}\left[{ }_{\nu \mathrm{P}}\right.\right.$ byćc $_{v}\left[\operatorname{PredP} \ldots\right.$ Pred $\left.\left.\left.^{0} \ldots\right]\right]\right]$ (vgl. Bondaruk 2013b, 183)

Bondaruk (2013b) argumentiert klar syntaktisch, indem sie die Kasusvariation direkt auf unterschiedliche Mechanismen der Kasusmarkierung zurückführt. Mit Bowers (1993) nimmt sie in prädizierenden und spezifizierenden Kopulasätzen generell eine PredP an, deren Komplement eine prädikative DP oder AP sei. Zentral ist, dass der Pred-Kopf (a) non-defektiv oder (b) defektiv sein könne: Ist er non-defektiv, weist er den vollen Umfang an phi-Merkmalen auf und kann den Instrumental valuieren. Ist er dagegen defektiv, so hat er keinerlei phi-Merkmale und kann keinen Kasus valuieren (Bondaruk 2013b, 182-183).

Abgesehen von dieser Hypothese arbeitet Bondaruk mit den folgenden Mechanismen zur Ableitung der Kasusformen: Im Falle des Nominativs rechnet sie mit lokal begrenzter, d. h. auf den jeweiligen “Clause" beschränkter Kasuskongruenz (case agreement), der sie wiederum Feature Sharing nach Pesetsky \& Torrego (2007) zugrunde legt. Ganz explizit argumentiert sie in diesem Kontext gegen das in der Literatur ebenfalls vorgeschlagene Multiple Agree, wie es z. B. auch Bailyn (2012) verwendet (vgl. Bondaruk 2013b, 211). Im Falle des Instrumentals geht sie dagegen davon aus, dass dieser Kasus am Prädikatsnomen durch den non-defektiven Pred-Kopf valuiert werde.

Was prädikative APn betrifft, so folgert Bondaruk (2013b), dass deren konsequente Nominativmarkierung Folge der Kasus- und phi-Merkmalskongruenz zwischen Subjektausdruck und prädikativem Adjektiv sei, welche durch Vermittlung von $\mathrm{T}^{0}$ zustande komme (Bondaruk 2013b, 206). Auch in diesem Fall spricht sie sich gegen Multiple Agree aus.

Hinsichtlich der Interpretation der Kopulasätze folgt sie insofern der bereits besprochenen Arbeit Roys (2006), als auch sie (vgl. Bondaruk 2013b, 210) Instrumentalsätze als 'charakterisierend' und Nominativsätze als 'definierend' bewertet. Insbesondere mit letzterer Schlussfolgerung habe ich Probleme, da nicht klar ist, wie sich der Zusammenhang zwischen den von Bondaruk behaupteten Mechanismen der Kasusmarkierung und den von Roy übernommenen Lesarten gestaltet. In 
keinem Fall legt Bondaruk, wie Roy (2006), eine "NumP” bzw. "ClP" zugrunde. Ob die semantischen bzw. pragmatischen Konzepte von 'Charakterisierung' bzw. 'Definierung' aus den Mechanismen der Kasusvaluierung vs. -kongruenz folgen, kann ich nicht völlig ausschließen, halte es aber doch für unwahrscheinlich.

Ferner ist Bondaruks (2013b) Annahme eines defektiven Pred-Kopfes m. E. zwar zunächst 'unauffällig', ließe sich aber de facto auch als völlige Abwesenheit einer PredP interpretieren, was zu derselben Situation wie bei Markman (2008) führt, die ihre "non-eventive" PredP als identische Abbildung modelliert. Ob "defektiv" oder "semantisch leer", sind solche Pred-Varianten letztlich doch 'effektlos' und somit im Prinzip überflüssig. Ihre Annahme scheint einem allgemeinen Bestreben nach Symmetrie bzw. der Einhaltung der Nullhypothese, dass alle prädizierenden Kopulasätze eine PredP enthalten müssen, geschuldet. Mit diesem Problem steht in engem Zusammenhang, dass Bondaruk (2013b) - ebensowenig wie die meisten Arbeiten zu diesem Thema - nicht klarmacht, was eigentlich unter "Prädikation" zu verstehen sei bzw. worin die (konzeptuelle) Leistung des abstrakten Pred $^{0}$ bestehen soll. Sofern Pred ${ }^{0}$ nur ein "stummer" Kopf zur Etablierung einer rein syntaktisch basierten "Prädikation" ist (siehe so auch Bailyn 2012), ist m. E. an der Legitimität seiner Annahme zu zweifeln, da weder morphologische noch semantische Evidenz für diese 'Spezialkategorie' vorliegt. Sofern Pred $^{0}$ hingegen Träger einer spezifischen "Prädikationsbedeutung" ist, muss er, wie schon Bowers (1993) annimmt, jeder Form von "Prädikation" zugrunde liegen. Dann aber wäre doch recht verwunderlich, dass $\operatorname{Pred}^{0}$ eine "defektive" Variante haben soll, die gänzlich 'unfähig' ist, da sie weder Kasus zuweisen kann noch phi-Merkmale hat. Noch verwunderlicher wirkt dann Bondaruks (2013b) Ansicht, dass sich aus dieser 'Unfähigkeit' die 'definierende' Lesart der prädizierenden Nominativsätze ergebe.

Aus den genannten Erwägungen heraus scheint mir die Bondaruks Analyse, trotz ihrer syntaktischen 'Rafinesse' sowie der o. g. (für meine Analyse mitunter sehr vorteilhaften) Aspekte nicht befriedigend und generell $\mathrm{zu}$ 'technisch', um dem sehr variablen Charakter der Kasusvariation adäquat und flexibel Rechnung zu tragen. Die hier vertretene, weniger 'technische' "Oppositionsanalyse" kann (mindestens) die gleichen Vorhersagen hinsichtlich der Lesarten von Kopulasätzen mit Nominativ und Instrumental machen. Sie ist zudem in der Lage, den jeweils bestehenden Kontext sowie die verfügbaren Alternativrealisierungen des Prädikatsnomens zu berücksichtigen. Letztere haben m. E. einen bedeutenden Einfluss auf die Lesart, die die tatsächlich gewählte Form erhält. Auf 'technischer' Seite ist im 
Prinzip nichts weiter als die Annahme erforderlich, dass die Kopulabedeutung, die ihrer Natur nach schlicht ein Verb ist, den Instrumental am Prädikatsnomen lizenzieren kann, aber nicht muss. Der Sprecher hat folglich, sofern die syntaktische Umgebung nicht beschränkend wirkt, die Wahl zwischen einem explizit markierten oder einem nicht explizit markierten Prädikatsnomen. Insofern er diese Wahl hat, wird er davon Gebrauch machen. Die interpretativen Effekte, die seine Wahl hervorruft, lassen sich m. E. voll und ganz auf dieses Konzept der Ikonizität bzw. der expliziten Markiertheit zurückführen (siehe hierzu Abschnitt 6).

\subsection{Semantopragmatischer Ansatz}

Diese "Klasse" von Ansätzen geht in aller Regel von einer bestimmten "Eigenbedeutung" der möglichen Kasusmarkierungen aus. Die Arbeiten bleiben jedoch im Gegensatz zu den rein semantischen Analysen - nicht bei dieser Annahme stehen, sondern beziehen auch die Domäne der Pragmatik mit ein, indem sie davon ausgehen, dass die angesetzten "Eigenbedeutungen" jeweils auch eine kontextuelle bzw. konzeptuelle Dimension haben bzw. erfordern, um eine endgültige Interpretation zu erhalten. Das bedeutet, mit anderen Worten, dass zwar von einer semantischen Invariable auf grammatischer Ebene ausgegangen wird, dass deren schließliche Interpretation aber erst auf extragrammatischer/pragmatischer Ebene erfolgt. In diesem Sinne stehen alle hier angesiedelten Analysen der ZweiEbenen-Semantik (siehe Abschnitt 4.7) nahe.

\subsubsection{Timberlake (1986)}

Timberlake (1986) untersucht russische adjektivische Prädikatsnomina innerhalb der sekundären Prädikation. Er hat die Vorannahme, dass die Kasusmarkierungen als solche jeweils eine bestimmte 'Bedeutung' haben:

\section{[Nominativ]}

The nominative implies that the state designated by the predicate complement holds on the narrated occasion, but does not contrast with alternative possible views on which the state might be expected not to hold on that occasion. In temporal terms, the state typically has held for some time prior to the narrated time. Even if it has not - if there has been a change of state - the inception of the state is not focused on. (Timberlake 1986, 139) 


\section{[temporaler Instrumental]}

$[\ldots]$ the instrumental in this usage indicates that the inception of the state represents a significant departure from the prior state of affairs and, further, that it is a necessary development from the circumstances that obtain locally around the narrated time. (Timberlake 1986, 142)

\section{[modaler Instrumental]}

[...] does not involve the inception of a state over time. It appears to be motivated instead by a contrast between expected and actual state, in the following way. From prior circumstances (previous experience, general principle), the speaker anticipates that a certain state would not hold at the narrated occasion; the instrumental signals that the state does hold despite expectations to the contrary. (Timberlake 1986, 146)

Aus den o. g. generellen Bedeutungen folgen für Timberlake gewisse Präferenzen für den einen oder anderen Kasus (vgl. Timberlake 1986, 151ff.). In der folgenden (keine Vollständigkeit beanspruchenden) Aufstellung gilt, dass links von ">" die Wahrscheinlichkeit des Instrumentals größer ist:

(29) a. Koordination > Nicht-Koordination

b. emphatische Gradmodifizierer > keine Modifizierer ${ }^{61}$

c. kein Komplement > zusätzliches Komplement

d. Aspekt und "Subaspekt": impf. prozessual > perf. / impf iterative ${ }^{62}$

Diese Wahrscheinlichkeiten bezüglich der Wahl der Kasusmarkierung bringt den Ansatz Timberlakes (1986) in die Nähe der "Tendenzanalyse" von Hentschel (1991), die das Ziel hat, empirisch beobachtbare Regelmäßigkeiten bei der Markierungswahl festzustellen (vgl. zum Polnischen Bogusławski 2001). Allerdings besteht ein m. E. gravierender Unterschied darin, dass Timberlake (1986) seine 'Bedeutungen' als Teil des grammatischen Systems im engeren Sinne versteht, während dies bei Hentschel (1991) oder Bogusławski (2001) offenbar nicht der Fall ist, da beide Ikonizität für das Zustandekommen der Lesarten als ausschlaggebend betrachten. Das heißt, dass sie keine fixe Semantik als Basis der Kasus-

61 Beispiele hierfür sind russ. očen' 'sehr', soveršenno 'vollends' u. ä.

62 Bei letzteren sollen beide Kasus gleichermaßen üblich sein. 
wahl betrachten, sondern die Kasus als "Diakritika" (vgl. Hentschel 1993a) rekonstruieren, die lediglich zur Interpretation beitragen (können), diese aber nicht von Grund auf determinieren (siehe Abschnitt 3.5).

Betreffs der o.g. Charakterisierungen der Bedeutung der sekundärprädikativen Kasusformen bestehen starke Ähnlichkeiten zu Uedas (1992) Ausführungen. Hinsichtlich der 'Bedeutung' des Instrumentals ergeben sich ferner Parallelen zwischen Timberlake (1986) und der Analyse Geists (2006). Nach Geist führt die Wahl des Instrumentals am Prädikatsnomen dazu, dass der betreffende Kopulasatz auf eine "spezifische Topiksituation" referiert, die mit wenigstens einer (denkbaren) Alternativsituation kontrastiert. Wenn Timberlake "[...] a different relationship between the actual reported state and the possible expected states" (Timberlake 1986, 162) als 'Bedeutung' des (modalen) Instrumentals erkennt, wird die Parallele zu Geists Aussage m. E. sehr deutlich. Eben deshalb ordne ich beide Ansätze dem "semantopragmatischen Lager" zu.

An Timberlake (1986) ist hervorzuheben, dass er die 'interpretativen Effekte', die mit den Kasusmarkierungen einhergehen können, zutreffend und detailliert erfasst. $\mathrm{Zu}$ kritisieren ist aber, dass es sich nur um das Aufzeigen von möglichen Lesarten und Tendenzen handelt, nicht jedoch um absolute 'Bedeutungen'. Die Aussagen Timberlakes (1986) lassen aber gerade letzteres erwarten. Mindestens lässt er die konkrete Verortung sowohl der Kasusbedeutungen als auch der Quelle der Kasuswahl im Dunkeln. Ebenhier liegt einer der Vorteile der 20 Jahre jüngeren Arbeit von Geist (2006), die ich folgend vorstelle. ${ }^{63}$

\subsubsection{Geist (2006)}

(i) Variation Nominativ vs. Instrumental

Die nun zu besprechende Monographie basiert auf der Dissertation der Autorin (Geist 2004) und stellt eine umfassende Betrachtung u. a. der folgenden Themen dar, die sie primär anhand spanischer und russischer Daten ausführt: ${ }^{64}$

- Kompositionalität in Kopulasätzen;

- Typologie von Kopula- und Identitätssätzen;

63 Allerdings behandelt Timberlake adjektivische Prädikatsnomina in sekundärer Prädikation, während Geist (2006) nominativische und adjektivische der primären Prädikation untersucht.

64 Siehe Pitsch (2012b) mit einer detaillierten Besprechung der Arbeit Geists (2006). 
- Variation bzgl. der spanischen Kopula;

- Variation bzgl. der russischen Prädikatsnomina;

- Variation der russischen Kurzform- vs. Langformadjektiv;

- Argumente für und gegen die Annahme einer "Nullkopula".

Für meine Arbeit ist v. a. Geists (2006) Vergleich spanischer und russischer Kopulasätze und der dort auftretenden Alternationen bzw. Variationen von Belang. Während im Russischen die Kasusmarkierung am Prädikatsnomen zwischen Nominativ und Instrumental variiert, liegt in spanischen Kopulasätzen eine Variation zwischen den beiden Kopulalexemen ser und estar vor. Die Vermutung liegt nahe, dass beide Variationen ähnliche oder gar identische 'interpretative Effekte' mit sich bringen.

Da mit Maienborn (2003a, 2005a) bereits eine weitreichende semantopragmatische Analyse der genannten spanischen Variation vorliegt, baut Geist (2006) ihre Betrachtung und v. a. die Analyse der russischen Kasusvariation auf letzterer auf, geht aber durchaus auch auf Unterschiede zwischen beiden Variationen ein. Maienborn (2003a, 2005a) sieht für die beiden spanischen Kopulalexeme ser und estar naheliegenderweise zwei eigenständige Lexikoneinträge vor: ${ }^{65}$
a. $\quad$ ser: $\quad \lambda \mathrm{P} \lambda \mathrm{x} \lambda \mathrm{z}[\mathrm{z} \approx[\mathrm{P}(\mathrm{x})]]$
(Maienborn 2003a, 125)
b. $\quad$ estar: $\quad \lambda \mathrm{P} \lambda \mathrm{x} \lambda \mathrm{z}\left[\mathrm{z} \approx[\mathrm{P}(\mathrm{x})] /\left[\mathrm{s}_{\mathrm{I}}{ }^{\text {top }} \mid \mathrm{R}\left(\mathrm{z}, \mathrm{s}_{\mathrm{I}}^{\text {top }}\right)\right]\right]$
(Maienborn 2003a, 163)

Maienborns Eintrag für das Lexem ser entspricht weitgehend der üblichen "Kopulabedeutung”, wie sie sich schon bei Bierwisch (1988) oder Steinitz (1990) findet. Spezifische Annahmen Maienborns sind, dass die Kopula, zusammen mit einigen anderen "Zustandsverben", auf keinen Davidsonschen, sondern auf einen Kimschen Zustand (vgl. Kim 1976) referiere. ${ }^{66}$ Ferner setzt sie mit Asher (1993) als 'Verknüpfer' zwischen (verbaler) Proposition und referenziellem Zustandsargument einen sog. Charakterisierungsjunktor “ $\approx$ " an, der somit eine ähnliche Funktion wie Bierwischs (1988) Instanziierungsfunktor "INST" hat.

65 Maienborn (2003a) arbeitet im Rahmen der Diskursrepräsentationstheorie (DRT). In (30b) ist der Abschnitt links von "|" die sog. Assertion (der grammatisch invariante Teil) während der Abschnitt rechts in Form einer Präsupposition die Bedingungen für deren 'Auswertung' nennt.

66 Ontologisch ist ein Kimscher Zustand eine abstrakt-mentale Entität ohne räumliche Dimension, der nicht lokal modifizierbar ist (siehe auch Abschnitt 5.1). 
Mit Blick auf die russische Kasusvariation relevanter ist der Lexikoneintrag, den Maienborn für estar vorsieht. Er stellt eine Erweiterung des Eintrags von ser um eine semantische Komponente dar, die Maienborn (2003a) als "Spezifizitätspräsupposition" (SPS) bezeichnet. Dies bedeutet letztlich, dass estar - anders als ser - nicht lediglich auf einen Kimschen Zustand in der außersprachlichen Welt referiert, sondern zudem präsupponiert, dass eine "spezifische Topiksituation" gegeben ist, die in Relation zu diesem Zustand steht. ${ }^{67}$ Die Verwendung von estar setzt demnach voraus, dass die Topiksituation, auf die der Kopulasatz referiert, spezifisch ist. ${ }^{68}$ Diese Spezifizität kann laut Maienborn (2003a) allen interpretativen Effekten Rechnung tragen, die für spanische Kopulasätze mit estar beschrieben werden (siehe Geist 2006, 98-101). Der Gebrauch des 'unmarkierten' Kopulalexems ser hingegen schließt zwar keineswegs aus, dass die Topiksituation spezifisch sein kann, bleibt in dieser Hinsicht jedoch 'neutral'.

Geist (2006) wendet die Vorarbeit Maienborns (2003a, 2005a) auf das Russische an, indem sie die Hypothese aufstellt, dass Sätze mit dem 'markierten' Instrumental am Prädikatsnomen Bezug auf eine "spezifische Topiksituation" nähmen, während solche mit dem 'unmarkierten' Nominativ in dieser Hinsicht 'neutral' blieben. Da sich die Variation im Russischen nicht an der Kopula, sondern am Prädikatsnomen zeigt, verlagert Geist (2006) die Maienbornsche SPS in den Lexikoneintrag des Instrumentalsuffixes. Sie betrachtet den 'prädikativen' Instrumental als semantischen Kasus, da er ihrer Analyse gemäß die Bedeutung des Prädikativs anreichert (Geist 2006, 113). Ihren Lexikoneintrag für das Instrumentalsuffix gebe ich in (31) wieder:

$$
\text { INS: } \lambda \mathrm{P} \lambda \mathrm{x}\left[[\mathrm{P}(\mathrm{x})] \&\left[\mathrm{R}_{1}(\mathrm{~s}, \mathrm{x})\right] /\left(\left[\mathrm{R}_{2}\left(\mathrm{~s}, \mathrm{~s}_{\mathrm{I}}^{\mathrm{top}}\right)\right]\right)_{\alpha}\right]
$$

Die SPS ist hier in Form der semantischen Komponente " $\left[\mathrm{R}_{2}\left(\mathrm{~s}, \mathrm{~s}_{\mathrm{I}}{ }^{\text {top }}\right)\right]$ ” integriert. Dabei handelt es sich um einen optionalen Bedeutungsanteil. Der Index “ $\alpha$ " ist

67 "Die Spezifizitätspräsupposition von estar ist als Bedingung formuliert, die fordert, dass der stative Zustand $\mathrm{z}$ relativ $\mathrm{zu}$ einer spezifischen Topiksituation $\mathrm{s}_{\mathrm{I}}^{\text {top }}$ verankert ist. $\mathrm{R}$ dient als freier Parameter für Relationen. Die Variable für die spezifische Topiksituation $\mathrm{s}_{\mathrm{I}}{ }^{\text {top }}$ wird bei der aspektuellen Spezifikation der Kopula gebunden [...]" (Geist 2006, 98). Schon Klein (1994, 83) deutet - quasi nebenbei - in die gleiche Richtung wie Maienborn (2003), wenn er den spanischen estar-Sätzen bescheinigt, einen Topikzeit-Kontrast zu reflektieren, wohingegen ser-Sätze ebendies nicht tun.

68 Der Begriff der “Topiksituation” entspricht im Prinzip Kleins (1994, 3-9, 36-48) “Topikzeit”. 
laut Geist (2006) wie folgt zu interpretieren: “An die Realisierung dieser Bedeutungskomponente ist eine syntaktische Bedingung geknüpft. Ist die Kasusalternation in der jeweiligen syntaktischen Position möglich, hat $\alpha$ den Wert $[+]$ und die Spezifizitätspräsupposition ist aktiviert. Besteht keine Kasusalternation und ist der INS die einzige Option, hat $\alpha$ den Wert [-], und die Spezifizitätspräsupposition ist neutralisiert." (Geist 2006, 110). Die SPS ist demnach immer dann neutralisiert, wenn der Instrumental nicht prädikativ, sondern in einer anderen Funktion verwendet wird. So will die Autorin mit dem obigen Lexikoneintrag sämtliche ("semantischen") Verwendungsweisen des russischen Instrumentals erfassen. ${ }^{69}$

Diese Annahmen Geists tragen dem Umstand Rechnung, dass die Kasusvariation in russischen Kopulasätzen gewissen Restriktionen unterliegt, die zum Ausschluss jeweils eines Kasus führen können: Liegt kein syntaktisch zugänglicher Kongruenzgeber vor, so kommt der Kongruenzkasus Nominativ nicht in Frage. Im Falle präsentischer Sätze ist wiederum der Instrumental offenbar ausgeschlossen. Ganz der hier vertretenen Ansicht entsprechend, formalisiert Geist durch den o. g. Eintrag, dass der 'prädikative' Instrumental nur dann eine 'spezifische' Bedeutung hat bzw. eine 'spezifische' Lesart evozieren kann, wenn er in potenzieller Opposition zum Nominativ steht. Ist dies nicht der Fall, handelt es sich lediglich um eine zwangsläufig erscheinende Kasusform, die keine 'Eigenbedeutung' hat.

Geist (2006) lässt zwar jene Fälle unerwähnt, in denen der Nominativ die einzig verfügbare Option darstellt - also die durchaus nicht marginalen Präsenssätze $-{ }^{70}$, aber ihre Analyse erfasst diese insofern, als der Nominativ als 'unmarkierte' Kasusform die Bedeutung des Prädikatsnomens zwar nicht mit der SPS anreichert, die Möglichkeit einer spezifischen Interpretation der jeweiligen Topiksituation jedoch dadurch keineswegs ausgeschlossen wird. Insofern kann ein Präsenssatz immer potenziell unspezifisch oder spezifisch sein; dies wird sprachlich aber einfach nicht reflektiert.

Bemerkenswert ist Geists Modellierung russischer Kopulasätze. Sie nimmt zwei unterschiedliche syntaktische Strukturen für Nominativ- und Instrumentalsätze an.

69 Der Kern von Geists (2006) Rekonstruktion der Kasusbedeutung des Instrumentals beruht auf Zimmermann (2003a). Demnach etabliert er eine un(ter)spezifizierte Relation zwischen einem Referenten und einem Sachverhalt (siehe genauer Geist 2006, 106-111).

70 Den Ausschluss des Instrumentals in Präsenssätzen führt Geist (2006, 162-164) auf die 'Defizienz' der Nullkopula (und ihres overten 'Substituts' est') zurück. Diese 'Defizienz' gehe Hand in Hand mit dem 'lautlichen Rückbau' dieser Verbform, für deren strukturelle Realität Geist (2006, 162-176) gute Argumente vorbringt (vgl. auch Geist 2008). 
Außergewöhnlich daran ist, dass sie - anders als die oben betrachteten Analysen den Unterschied nicht in der An- oder Abwesenheit einer AspP oder im Typ der Prädikationsphrase (PrP) sieht, sondern in der Präsenz einer PrP an sich. So erkennt sie lediglich Instrumentalsätzen eine PrP zu ("INS-Prädikative”, vgl. (32b)), nicht aber Nominativsätzen. Letztere beruhen ihr zufolge auf bloßen NPn im Komplement des Kopulaverbs (“Kongruenz-Prädikative”, vgl. (32a)):

(32) a. Kongruenz-Prädikative

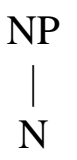

b. INS-Prädikative

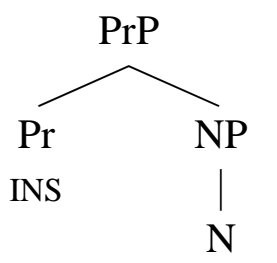

(Geist 2006, 112)

Aus der Struktur der "INS-Prädikative" geht hervor, dass Geist (2006) das Instrumentalsuffix als Belegung des funktionalen Kopfes $\operatorname{Pr}^{0}$ betrachtet. "Um Kasus zu bekommen, adjungiert das Nomen durch Kopf-zu-Kopf Bewegung an Pr und verknüpft sich mit dem INS-Affix." (Geist 2006, 112). Auf diese Modellierung werde ich weiter unten nochmals zu sprechen kommen.

Während Geists o. g. Lexikoneintrag für das Instrumentalsuffix die Vorteile hat, dass er (a) alle ("semantischen") Verwendungsweisen dieses Kasus abdecken kann, und dass er (b) formalisiert, wann die SPS aktiv und wann 'neutralisiert' ist, hat ihre Analyse m. E. doch mindestens einen tiefgreifenden problematischen Aspekt, der kasustheoretischer Natur ist und unmittelbar mit ihrer Einstufung des Instrumentals als "semantischem” Kasus (Geist 2006, 113) zusammenhängt.

Ich bin der Überzeugung, dass die Assoziierung einer bestimmten Kasusmarkierung mit einer 'Gesamtbedeutung' à la Jakobson (1971a) zu Komplikationen führt, wenn man an die Bandbreite der Verwendungen gewisser Kasusformen denkt, darunter sehr prominent der hier interessierende Instrumental. Dass es das Instrumentalsuffix selbst sei, das die jeweilige Semantik bzw. Lesart verantworte, ist deterministisch bzw. birgt die Gefahr von Übergeneralisierungen, insofern die behauptete 'Gesamtbedeutung' entweder zu allgemein oder aber zu spezifisch ist, um sämtliche beobachtbaren Lesarten - und nur diese - abzuleiten.

Ferner bedeutet eine derartige "semantische Aufladung" der morphologischen Kasusmarker, wie sie von Geist (2006) praktiziert wird, dass deren angebliche Bedeutungsanteile in Sprachen ohne Kasusmorphologie gänzlich anders(wo) enko- 
diert sein müssen, bzw. dass solche Sprachen über eine ganze Batterie von "Nullkasusmarkern" verfügen müssten.

Mit Blick auf den Lexikoneintrag für das Instrumentalsuffix sind zwei Punkte besonders zu hinterfragen, die ich hier nur nenne: Zum einen halte ich es für problematisch, einer morphologischen Kasusmarkierung eine funktionale Kategorie zu geben (bei Geist hat das Instrumentalsuffix die Kategorie [+Pr]). Zum anderen scheint mir aus lexikontheoretischer und ökonomischer Perspektive ein Modell attraktiver, das ohne optionale semantische Anteile in Lexikoneinträgen auskommt, die nur unter speziellen Bedingungen 'aktiv' sind oder 'neutralisiert' werden müssen, was fraglos "mentalen Aufwand" bedeutet.

Schließlich erzwingt die diskutierte Sichtweise die Unterscheidung von "strukturellen" und "semantischen" Kasus, wobei erstere lediglich 'bedeutungslose' Marker wären, die von einem Regens auf vorhersagbare Weise gefordert werden, während letztere per se 'bedeutungshaft' und syntaktisch im Prinzip selbständig bzw. 'autolizenzierend' sein müssten (vgl. den “nackten” Instrumental).

Eine theoretisch gleichermaßen elegantere wie wünschenswertere Lösung besteht m. E. darin, alle Kasusformen als 'strukturell' in dem Sinne zu behandeln, dass sie stets 'regiert' sind, und zwar unabhängig davon, ob durch die Selektionsbeziehung zwischen ihnen und ihrem Regens eine 'greifbare' Bedeutung wie in (33a) oder 'nur' eine Satzfunktion wie in (33b) reflektiert wird:

$$
\begin{aligned}
& \text { a. On pisal pis'mo karandašom. } \\
& \text { er-NOM schrieb-SG.M Brief-ACC Bleistift-INS } \\
& \text { 'Er hat einen Brief mit dem Bleistift geschrieben.' }
\end{aligned}
$$

b. On rukovodil institutom $[\ldots]$

$$
\text { er-NOM leitete-SG.M Institut-INS }
$$

'Er hat das Institut geleitet ...'

NKRJ

Im Beispiel (33a) liegt im traditionellen Verständnis ein "semantischer" Instrumental vor, der das 'Instrument' einer Handlung nennt. In (33b) wird üblicherweise von "strukturellem" Instrumental gesprochen, da er lediglich das interne Argument bzw. 'direkte Objekt' des Verbs markiert. Die (syntaktische) Gleichbehandlung beider Fälle würde bedeuten, auch für den "semantischen" Instrumental in (33a) ein strukturelles Regens etwa in Form einer stummen Präposition mit der 
(möglichen) Bedeutung INSTRUMENT anzusetzen. ${ }^{71}$ In (33b) ist dagegen das Verb selbst als unmittelbares Regens zu betrachten. In beiden Fällen wäre der Instrumental eine bloße morphosyntaktische Form, die von einem syntaktischen Kopf, namentlich $\mathrm{P}^{0}$ bzw. $\mathrm{V}^{0}$, 'regiert' wird; vgl. (34):

(34) a. On pisal pis'mo $\left[\varnothing_{\mathbf{P}}[\mathrm{NP}\right.$ karandašom $] .^{72}$

b. On [ rukovodil $v$ [NP institutom ]].

Ob die Kasusform eine Semantik zum Ausdruck bringt oder aber nur eine syntaktische Funktion erfüllt, hängt also vom jeweiligen Regenten ab. Die zugrunde liegende Relation ist in jedem Fall eine syntaktische. Der 'prädikative' Instrumental wäre bei dieser Sichtweise ebensowenig ein "semantischer" Kasus wie alle anderen Belege dieser Kasusmarkierung - auch er ist lediglich das Resultat der Rektion durch einen syntaktischen Kopf, in diesem Falle des Kopulaverbs. Ein Kopulasatz ist somit strukturell parallel zum obigen rukovodil-Beispiel: ${ }^{73}$

(35) a. On [ rukovodil $\mathbf{v}$ [NP institutom ]].

(Rus)

b. On [ bylv [NP studentom ]].

Nunmehr möchte ich auf die o. g. syntaktische Analyse zurückkommen, die Geist für ihre so genannten "INS-Prädikative" ansetzt. Ich wiederhole diese zunächst:

71 Tatsächlich etabliert die leere Präposition wahrscheinlich eine un(ter)spezifizierte Relation, die erst auf konzeptueller Ebene spezifiziert wird. Auf jeden Fall vergibt sie, ebenso wie das Verb rukovodit' 'leiten' in (33b), eine thematische Rolle an ihr internes Argument, an dem sie ferner den ('strukturellen') Instrumental 'regiert'.

72 Evidenz für diese Analyse kommt auch aus dem Sprachvergleich. So wird die im Russischen leere und m. E. unterspezifizierte Präposition im Deutschen overt und explizit als mit realisiert:

(i) On pisal pis'mo [ $\varnothing_{\mathbf{P}}[\mathrm{NP}$ karandašom ]].

(ii) Er hat einen Brief [ mit $_{\mathbf{P}}$ [DP dem Bleistift ]] geschrieben.

(Deu)

Sowohl der russ. Instrumental in (i) als auch der dt. Dativ in (ii) sind lediglich 'Erfüllungen' der Anforderungen des jeweiligen Regens (russ. $\varnothing / \mathrm{dt}$. mit), haben aber selbst keine Bedeutung (diese 'steckt' immer im Regens).

73 Später werde ich die Analyse so modifizieren, dass die Kopulabedeutung in einem leeren V-Kopf sitzt, über dem ein V-Auxiliar (das vermeintliche Kopulaverb) die nötigen Merkmale ausbuchstabiert. Also selegiert nicht die byt'-Form, sondern der leere V-Kopf den Instrumental (siehe Bondaruk 2013b, die Gleiches für die polnischen być-Formen annimmt); vgl. (i):

(i) On byl ${ }_{\mathrm{AUX}}\left[\varnothing_{\mathbf{V}}[\mathrm{NP}\right.$ studentom $\left.]\right]$.

(Rus) 
Es sei daran erinnert, dass Geist das Instrumentalsuffix (INS) als Belegung des Kopfes $\operatorname{Pr}^{0}$ betrachtet. Um Kasus zu erhalten, adjungiert der Kopf der NP an $\operatorname{Pr}^{0}$, wobei er mit der SPS ausgestattet wird. Es sei ferner daran erinnert, dass sie im Instrumental einen "semantischen Kasus" erblickt. Im Vergleich mit der vorab angedeuteten Sicht auf Kasusformen fällt auf, dass auch Geist einen relativ 'abstrakten' Kopf (nämlich $\operatorname{Pr}^{0}$ ) mit einer bestimmten Semantik ansetzt, dessen Komplement eine NP ist. In dieser speziellen Hinsicht liegt also nur ein geringer Unterschied zur Analyse in (37) vor, die den zuvor von mir formulierten Erwägungen folgt, denen zufolge der Kasusmarker ein leeres Regens lediglich 'reflektiert':

\section{(37) $\left[\mathrm{PP} \mathrm{P}^{0} \mathrm{NP}-\mathrm{INS}\right]$}

Freilich besteht ein Unterschied in der Etikettierung, da in Geists Struktur eine PrP, hier aber eine PP vorliegt. Letztere ist der Ansicht geschuldet, alle "semantischen" Kasus seien zugrunde liegend PPn. ${ }^{74}$ Ein zweiter Unterschied besteht, wie gesagt, darin, dass die Autorin das Instrumentalsuffix als Belegung des Kopfes $\operatorname{Pr}^{0}$ behandelt, während es in (37) bereits mit (dem Kopf) der NP verbunden ist. Diese Gegenüberstellung soll keine 'Berichtigung' der von Geists Struktur sein (deren Annahme eines "semantischen" prädikativen Instrumentals ich ohnedies ablehne), sondern lediglich zeigen, dass sich beide Modellierungen quasi ineinander übersetzen lassen. Von einem "semantischen" Kasus kann aber nur im Falle der Analyse Geists (2006) gesprochen werden, da sie die 'Kasusbedeutung' unmittelbar im Kasussuffix unterbringt. Im Fall der PP-Analyse hingegen ist das Kasussuffix ein semantisch leerer, morphologischer Marker, der von einer phonetisch leeren, aber bedeutungstragenden Präposition 'regiert' wird. Klar ist, dass der Kasusmarker auch in (37) die betreffende Semantik sprachlich zum Ausdruck

74 Sofern man die Kategorie der Präposition auf Grund ihrer Geschlossenheit und ihrer rein 'negativen' Merkmale $([-\mathrm{V},-\mathrm{N}])$ als funktionale Kategorie klassifiziert $([+\mathrm{P}])$, könnte sie ohne Weiteres die Stelle der stipulierten funktionalen Kategorie "Prädikation" einnehmen. 
bringt, jedoch ist er dort nicht ihr unmittelbarer Träger, sondern reflektiert sie lediglich. $^{75}$

Was die Kasusvaration betrifft, so ist Geists (2006) Arbeit überaus wertvoll, da sie ein formalisiertes Modell mit sehr konkreten Annahmen zur 'Semantik' der Instrumentalmarkierung präsentiert. Naturgemäß bieten gerade derart konkrete Vorschläge viel Raum für Kritik, die sich aber im Falle von Geist (2006) nur als fruchtbar erweisen kann, da sich ihre Arbeit in besonderer Weise als Ausgangspunkt aller weiteren Erwägungen hinsichtlich der Kasusvariation in russischen Kopulasätzen empfiehlt. Festzuhalten ist, dass sie den Instrumental direkt mit dem (optionalen) Bedeutungsanteil der SPS assoziiert, und dass sie beachtet, dass diese 'Bedeutung' nur dann 'aktiv' ist, wenn der Instrumental in Konkurrenz zum Nominativ steht. Ebendiese Beobachtung muss in jede theoretische Arbeit zur fraglichen Variation einfließen, da sich ansonsten ein verschobenes Bild ergäbe, denn die russische Kasusvariation findet - signifikant anders als die spanische Kopulavariation - nicht konsequent und unabhängig von syntaktischen und weiteren Faktoren statt. Diese Beobachtung ist auch das Fundament für die hier vertretene "formbasierte" Analyse der russischen sowie polnischen Variationen, die ich in Abschnitt 6 detailliert vorstellen werde.

\section{(ii) Variation Langform vs. Kurzform}

Die Arbeit von Geist (2006) ist auch deswegen hervorzuheben, weil sie nicht ausschließlich die Kasusvariation, sondern auch den Gegensatz zwischen Kurz- und Langformadjektiven im Russischen in die Betrachtung einbezieht, der mindestens in der gehobenen russischen Standardsprache bis heute besteht (vgl. "Modell B" nach Guiraud-Weber 1993).

Inkonsequent wirkt jedoch Geists (2006) Zugrundelegung eines anderen theoretischen Rahmens zur Erfassung dieser Adjektivvariation. Während sie im Kontext der Kasusvariation eine leicht modifizierte Form des lexikalistischen Minimalismus im Sinne Bierwischs nutzt, arbeitet sie bei ihrer Ableitung der Adjektivvariation mit der Distributed Morphology (DM), die geradezu antilexikalistisch ist.

75 Mit Stechow (2007b) ließe sich auch von der "Sichtbarmachung" der betreffenden Semantik durch das Kasussuffix sprechen. Mit Zimmermann $(2012,2013)$ könnte man, quasi aus umgekehrter Perspektive, sagen, dass die morphosyntaktische Information, die das Kasussuffix transportiert, "verspätet" im leeren Kopf $\mathrm{P}^{0}$ interpretiert wird. 
Konkret analysiert die Autorin nur die russischen Langformen als 'richtige' Adjektive, während sie die Kurzformen als (im Sinne von DM) syntaktisch derivierte Verbformen betrachtet. Strukturell stellt sie dies wie folgt dar (Geist 2006, 153):

(38) a. $\quad v \mathrm{P}$ mit der $\mathrm{L}[\mathrm{ang}] \mathrm{F}[\mathrm{orm}]$

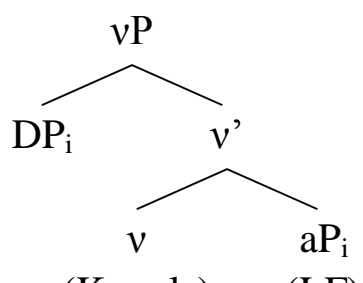

(Kopula) b. $\quad$ P mit der K[urz]F[orm]

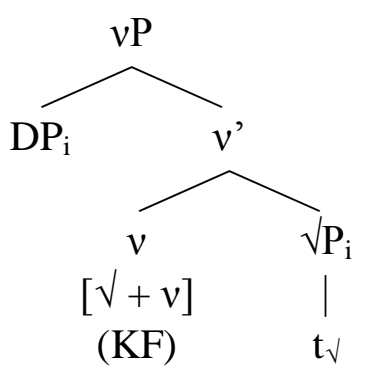

Kurz- sowie Langformen basieren demnach gleichermaßen auf unkategorisierten Wurzeln $(\sqrt{ })$, deren weiteres 'Schicksal' innerhalb der syntaktischen Derivation sich jedoch anders gestaltet:

Wie (38a) zeigt, wird bei der Derivation einer Langform eine Wurzel von einem 'leichten' (funktionalen) Kopf $a^{0}$ selegiert und so in ein Adjektiv überführt. ${ }^{76} \mathrm{Im}$ folgenden Schritt wird die so entstandene $a \mathrm{P}$ von einem funktionalen Kopf $v^{0}$, dessen Belegung das Kopulaverb ist, selegiert. Im Spezifikator der $v \mathrm{P}$ kann nun der Subjektausdruck (DP), der semantisch zur Wurzel gehört (oben durch den Index markiert), syntaktifiziert werden. Hier ist festzuhalten, dass es in diesem Fall das Kopulaverb in $v^{0}$ ist, das Zustandsreferenz hat, während das Adjektiv in $\operatorname{der} a \mathrm{P}$ lediglich ein einstelliges Prädikat mit seiner deskriptiven Bedeutung ist.

76 Der Kopf $a^{0}$ ist bei Geist $(2006,151)$ nicht unmittelbar lexikalisch belegt, sondern abstrakt. In der $a$ P sieht sie auch einen Agr-Kopf mit phi-Merkmalen vor. Mit DM nimmt sie Einsetzungsregeln an, die dafür sorgen, dass in der 'morphologischen Komponente' aus der Informationenskette $[\sqrt{ }+a+$ Agr $]$ eine Langform 'entsteht' (Geist 2006, 150-152).

Bzgl. der Langformen nimmt sie an, dass sie den "Bezug auf eine kontextuell zu spezifizierende Vergleichsklasse" (Geist 2006, 151) herstellen. Dieser Bezug sei die Semantik von $a^{0}$. Den "Vergleichsklassenbezug" formalisiert Geist (2006, 134) auf der Basis von Zimmermanns (1992, 264) "Modifikationstemplate". Ich halte die Assoziierung der Langformen mit diesem "Vergleichsklassenbezug" für zu weitreichend, da sie m. E. nicht in jedem Fall Bezug auf eine solche nehmen. Der fragliche Begriff meint laut Geist übrigens eine implizite Eigenschaft, die dem Referenten nebst der vom Adjektiv explizit bezeichneten Eigenschaft zugeschrieben, aber erst konzeptuell spezifiziert, wird. Der Satz in (i) hat demnach zwangsläufig die Interpretation 'Anna war gut und ...', wobei “...” erst konzeptuell durch eine bestimmte weitere Eigenschaft bzw. 'Klasse' (z. B. 'Frau', 'Freundin', 'Kollegin', 'Mensch' o. ä.) befüllt würde.

(i) Anna byla dobraja / dobroj. (Rus) Anna war gut LF$^{-N O M}$ gut $_{\mathrm{LF}}$-INS 
Im Gegensatz dazu, so Geist (2006, 148-150), ist bei der Derivation einer Kurzform kein 'leichtes' $a^{0}$ involviert. Kurzformen sind demnach keine Adjektive. Die Wurzel werde vielmehr unmittelbar von $v^{0}$ selegiert und damit in eine Verbform überführt. Zu beachten ist, dass in diesem Fall bereits der erwähnte $v$-Kopf über Zustandsreferenz verfügt, und dass daher kein Kopulaverb i. e. S., sondern nur ein Aspekt-/Tempusauxiliar zur Ausbuchstabierung der entsprechenden Merkmale nötig ist (vgl. Geist 2006, 154). Was an der Oberfläche als Kurzform erscheint, ist der Autorin zufolge also eine Verbform, die von sich aus einen "stativen Zustand" bezeichnet (Geist 2006, 150). Es handelt sich um eine $v \mathrm{P}$, in deren Spezifikator der Subjektausdruck realisiert wird. Ein Kopulaverb ist weder zur Etablierung von Zustandsreferenz noch zur Syntaktifizierung des Subjektausdrucks erforderlich. Formen von byt', die mit Kurzformen auftreten, sind demnach laut Geist (2006, 152-157) lediglich Ausbuchstabierungen der funktionalen Köpfe $\mathrm{Asp}^{0}$ und $\mathrm{T}^{0}$.

Das 'Brisante' an Geists Aussagen ist, dass Kurzformen keine Adjektive, sondern Verbformen repräsentieren. Sie referieren also selbst auf einen (Kimschen) $\mathrm{Zu}$ stand, und realisieren auch selbst das Subjekt. Ein Kopulaverb ist deshalb überflüssig. Da dennoch Formen von byt' mit Kurzformen auftreten, können diese nur Auxiliare sein. Was die Autorin mit dieser Verbalitätshypothese besser als andere Ansätze erklären kann, sind die folgenden (mitunter nur postulierten) Eigenschaften russischer Kurzformen (siehe Geist 2006, 138-142):

1. Kurzformen haben keinen Kasus. Da Kasus eine nominale Kategorie sei, könne die Verbalität der Kurzformen dies erklären.

2. Kurzformen kongruieren mit dem 'höflichen' Pronomen $V y$ 'Sie' grammatisch, Langformen dagegen semantisch. Ursache sei wiederum die Verbalität der Kurzformen, denn auch verbale Partizipien kongruieren stets grammatisch.

3. Langformen, nicht aber Kurzformen, lassen die Konversion zu Substantiven zu. Dies weise darauf hin, dass Kurzformen nicht nominal seien.

4. Kurzformen nehmen häufiger als Langformen Komplemente, bei manchen Kurzformen sind diese sogar obligatorisch. Damit seien Kurzformen wiederum eher Verben als Nomina ähnlich.

5. Kurzformen hätten eine andere Bedeutung als Langformen: Erstere sollen auf "stative Zustände", letztere auf "vergleichsklassenbezogene Eigenschaften" referieren. 
Wie ich in Abschnitt 4.3.5 begründen werde, ist Geists Annahme einer (tendenziellen) Verbalität der Kurzformen zuzustimmen. Ich werde allerdings weder von DM Gebrauch machen, noch werde ich behaupten, Kurzformen seien (syntaktisch oder lexikalisch) voll und ganz Verbformen. Meine Ansicht besteht vielmehr darin, dass Kurzformen Verbalnomina sind, die weder ganz Nomina noch ganz Verben sind bzw. Eigenschaften beider Klassen in sich vereinen.

\subsection{Formbasiert-pragmatischer Ansatz}

\subsubsection{Hentschel (1991)}

Hentschel (1991) nimmt eine vergleichsweise außergewöhnliche Position innerhalb der Forschung zur Motivation der variierenden Kasusmarkierung in nordslavischen (russischen) Kopulasätzen ein. Er geht von dem Standpunkt aus, dass es sich beim 'prädikativen' Instrumental um ein redundantes Signal handele, etwa dem Genitiv der Negation oder dem Gegensatz Akkusativ-Genitiv im Falle belebter Referenten vergleichbar. ${ }^{77}$ Wie beim Genitiv der Negation handelt es sich laut Hentschel auch beim Instrumental um "ein Element der Ausdrucksebene natürlicher Sprachen, welches zusammen mit mindestens einem anderen ein und dasselbe inhaltliche bzw. grammatisch-inhaltliche Moment repräsentiert" (Hentschel 1991, 221). Letzteres Zitat ist im Kontext der vorliegenden Arbeit nicht genug hervorzuheben, bringt es doch den Kern der hier vertretenen Sichtweise zum Ausdruck, dass die Kasusvariation in russischen und polnischen Kopulasätzen nämlich keine echt-semantische, sondern eine bloß 'formbasierte' (ikonische) ist, die auf der Opposition mindestens zweier konkurrierender Ausdrucksformen in derselben Position bzw. Funktion beruht. Diese 'Formbasiertheit' in Verbindung mit der Ansicht, dass die jeweilige sprachliche Form erst im bzw. durch den (syntaktischen) Kontext ihre finale Interpretation erhält und erhalten kann, lässt mich bei Hentschel (1991) und vergleichbaren Arbeiten von "formbasiert-pragmatischen" Ansätzen sprechen.

Jakobson (1971a) betrachtet als das 'ideale Subjekt' eines Satzes mit transitivem Prädikat ein Substantiv mit belebtem Referenten. Für Hentschel wird durch den Nominativ v. a. das belebte Subjekt explizit gekennzeichnet. Er stellt sich die

77 In der 'Belebtheitskategorie' sieht Hentschel "nichts anderes als eine explizite Kennzeichnung eines belebten Substantivs und somit idealen Subjekts als Nicht-Subjekt" (Hentschel 1991, 224). Etwa die gleiche Funktion schreibt er auch dem 'prädikativen' Instrumental zu. 
Aufgabe herauszufinden, “ob nicht für das PrNInstr [Prädikatsnomen im Instrumental] als deutlicherem Signal [verglichen mit dem Nominativ] ähnliche Präferenzen [wie z. B. beim Belebtheits-Akkusativ/Genitiv oder Negationsgenitiv] des Auftretens zu ermitteln sind, die als gezielte Tendenzen zur Steigerung der Identifikationsmöglichkeit des PrN und somit des Subjekts zu interpretieren sind" (Hentschel 1991, 224).

Hentschel lehnt die traditionelle Erklärungsdichotomie temporär vs. permanent ab. Durch eine quantitative Untersuchung kommt er zu dem Schluss, dass diese angenommene semantische Opposition keinen Einfluss auf die Kasusmarkierung hat. ${ }^{78}$ Die ermittelten Unterschiede verweisen laut Hentschel "klar in den Bereich der Zufälligkeit" (Hentschel 1991, 226). ${ }^{79}$

Hentschel greift vier von Røed (1966) postulierte semantische Gruppen auf, die Auswirkungen auf die Kasuswahl am Prädikatsnomen haben sollen:

(39) a. Zuweisung von Charaktereigenschaften:

b. Zuweisung von zeitweilig-zufälligen Eigenschaften:

c. Zuordnungen von Berufen oder Tätigkeiten.

d. Abstrakta oder abstrakte Aussagen.

Da Røed es versäumt habe, das Zufallsmoment bei seinen Quantifizierungen zu kontrollieren, sei ihm entgangen, dass die Unterschiede zwischen (39a) und (39b) im 20. Jahrhundert nicht (mehr) signifikant wären. Des Weiteren weist Hentschel (1991, 226) darauf hin, dass die vier Gruppen stets als streng komplementäre Belegmengen betrachtet worden seien, was aber solchen Beispielen wie (40) widerspreche:

78 Das Korpus umfasst 509.000 Wortformen, davon 55\% schöne, 35\% Informationsliteratur und $10 \%$ gesprochene Sprache. Einer der Texte ist ungewöhnlich, da in ihm der Nominativ gegenüber dem Instrumental im Verhältnis 3:2 überwiegt, während bei allen anderen Texten der Instrumental den Nominativ im Verhältnis von 6:1 dominiert. Hentschel zufolge ist er archaisch. Der älteste Text ist aus den 1960er Jahren, die Mehrheit stammt aus den 1970er-1980er Jahren. Von 351 relevanten Belegen sind 101 mit Nominativ und 250 mit Instrumental. Bei den Informationstexten liegt der Nominativanteil bei unter fünf Prozent, während er in der schönen Literatur und gesprochenen Sprache ein Fünftel aller Fälle ausmacht (Hentschel 1991, 225).

79 In einer Kontrollberechnung werden 54 Belege, die explizite lokale oder temporale Einschränkungen enthalten, mit 55 Belegen, die nicht beschränkt werden, verglichen, wobei sich kein signifikanter Unterschied zum Ergebnis der Hauptuntersuchtung zeigt. 
(40) [...] počuvstvovala, čto žit' so svekrov'ju budet nesladko, [...] i staruche, vpročem, kakoj staruche, ona byla togda šumlivoj, suetnoj požiloj ženščinoj, nado bylo pojti navstreču.

'... sie fühlte, dass es nicht angenehm werden würde, mit der Schwiegermutter zu leben, ... und dass man der Alten - im Übrigen, welcher Alten? Sie war damals eine lärmende, hektische betagte Frau - entgegenkommen musste.'

Hier werden einerseits mit den Attributen šumlivyj 'lärmend' und suetnyj 'hektisch' permanente Charaktereigenschaften benannt, andererseits erfolgt aber durch togda 'damals' eine zeitliche Einschränkung. Hier käme es also bei der o.g. strengen Betrachtungsweise zu einem Konflikt zwischen (39a) und (39b).

Die auffälligste von Røeds Gruppen ist die in (39d) mit i. w. S. abstrakten Prädikativen (wozu auch prädikative Sätze gehören). 95\% der prädikativen Substantive dieser Art stehen im 20. Jahrhundert im Instrumental. Auch Hentschel (1991, 227) findet diesen Zustand durch seine Erhebung bestätigt. Abstrakta lassen also eine eindeutige Tendenz zum Instrumental erkennen.

Zurück zu Hentschels (1991) These, dass die Kasusvariation genauso wie die spezifische Markierung belebter Objektausdrücke durch den Akkusativ-Genitiv ein Phänomen sei, das die betroffene Konstituente morphologisch explizit als Nichtsubjekt kennzeichnet und das Subjekt damit implizit hervorhebt. Laut Hentschel wurde diesem Ansatz bis dato in der Forschung kaum Beachtung gezollt, einzig Nichols (1985) bilde hier eine Ausnahme, indem sie die Belebtheit des "Kontrolleurs" des Prädikatsnomens, d. h. des Subjektausdrucks, erwähnt. Nichols (1985, 375) kommt jedoch zu dem Ergebnis, dass die Belebtheit des Referenten des Prädikativs bzw. des Satzsubjekts in Kopulasätzen keine Rolle für die Kasuswahl spiele. Da man “in derartigen Sätzen jedoch von weitgehender Kongruenz zwischen Subjekt und PrN hinsichtlich der Belebtheit ausgehen” (Hentschel 1991, 228) könne, spricht sich der Autor gegen die Schlussfolgerung Nichols' aus und nimmt quasi den entgegen gesetzten Standpunkt ein. Substantive mit unbelebten Referenten im Prädikat tendierten hochsignifikant stärker zum Instrumental als solche mit belebten Referenten. Zudem sei unter den semantischen Faktoren der für die Korrelation mit der Kasusmarkierung berechnete Wert mit Bezug zur Be- 
lebtheit der am deutlichsten ausgeprägte überhaupt. ${ }^{80}$ Grundsätzlich stellt Hentschel also Unbelebtheit als 'Trigger' für den Instrumental fest. Das semantische Merkmal [ \pm belebt] ist demnach das wortsemantische Kriterium mit dem stärksten Einfluss auf die Kasuswahl am Prädikatsnomen (vgl. Hentschel 1991, 228).

Paradox erscheint, dass, während "belebte Substantive als direkte Objekte eine andere flexionsmorphologische Form als das Subjekt erhalten”, beim Prädikatsnomen die Belebtheit gewissermaßen zu flexionsmorphologischer Homonymie ('doppelter Nominativ') mit dem Subjektausdruck führt. Hentschel greift in diesem Zusammenhang erneut auf Jakobson (1971a) zurück, der zumindest für transitive Verben Substantive mit belebten Referenten für 'ideale Subjekte' und solche mit unbelebten Referenten für 'ideale Objekte' hält. Hentschel meint, dass eine solche Aussage auch für Prädikatsnomina zutreffend sei. Seine Untersuchung zeigt, dass in $90 \%$ der Fälle Subjekt und Prädikatsnomen im Merkmal [ \pm belebt] übereinstimmten (Hentschel 1991, 228).

Hentschel greift nunmehr in diesem Kontext Mayerthaler (1980) auf, der mit Bezug auf den Markiertheitsbegriff der Prager Schule ein Hierarchisierungsmodell flexionsmorphologischer "Natürlichkeit" aufstellt, das auf drei Ebenen anzuwenden ist. So unterscheidet Mayerthaler zum einen formale von semantischer Markiertheit (die Mayerthaler "Merkmalhaftigkeit" nennt) und nimmt noch die Möglichkeit der Symbolisierungsmarkiertheit an.

Formal sei z. B. die Endung des Gen. Sg. m. - $a$ im Russischen markierter als die Nullendung $-\varnothing$ des Nom. Sg. m. Sie ist andererseits aber weniger markiert als die Endung des Ins. Sg. m. -om. Über die formale Markiertheit entscheide die Länge der Wortform bzw. der Endung, und zwar gemessen in der Gesamtzahl der phonologischen Segmente. Semantisch sei z. B. der Plural markierter als der Singular, jedoch weniger markierter als der Dual. Die Symbolisierungsmarkiertheit schließlich sei dort am geringsten, wo semantische und formale Markiertheit zusammentreffen. So seien z. B. die Nicht-Nullendungen für den Gen. Pl. unmarkiert gegenüber der entsprechenden Nullendung.

In diesem Verständnis und aufbauend auf Jakobsons (1971a) Einschätzung sind laut Hentschel (1991) Subjekte mit belebten Referenten semantisch unmarkiert und solche mit unbelebten Referenten markiert, während Objekte mit unbelebten

80 Dieser Wert beträgt $r=-0,304$. Das negative Vorzeichen spricht für eine Tendenz zum Nominativ, da dieser dem Wert 0, der Instrumental dagegen dem Wert 1 entspricht. Nichtzufällige Werte liegen unter -0,113 bzw. über 0,113 (Hentschel 1991, 228, Fn. 12). 
Referenten semantisch unmarkiert, solche mit belebten Referenten hingegen markiert seien. Somit ließe sich die Entwicklung der Belebtheitskategorie "als Synchronisierung von semantischer und formaler Markiertheit verstehen, als Entwicklung zur Ikonizität: das markiertere, das belebte direkte Objekt [...] erhält die merkmalhaftere Kennzeichnung der Genitivform" (Hentschel 1991, 229).

Übertragen auf die Kasusmarkierung an Prädikatsnomina heiße dies, dass Prädikatsnomina mit unbelebten Referenten markierter seien, was wiederum indirekt vom Subjektausdruck determiniert werde, da ja der Standardfall in der Kongruenz zwischen Subjekt und Prädikatsnomen hinsichtlich ihrer Belebtheit bestehe. Ganz ähnlich wie im Falle von direkten Objekten mit belebten Referenten, erhalte nun ein Prädikatsnomen mit unbelebtem Referenten als der markiertere Fall eine besondere Kennzeichnung, namentlich den Instrumental. Hentschel fasst seine Schlüsse wie folgt zusammen:

Beide Kategorien, die der Belebtheit und der prädikative Instrumental, sind als Phänomene der unterschiedlichen Behandlung von belebten und nicht belebten Substantiven zu werten, und zwar bei potentiellem flexionsmorphologischem $\mathrm{Zu}$ sammenfall zwischen Subjekt und direktem Objekt einerseits und Subjekt und PrN andererseits. Die merkmalhaftere Kennzeichnung - Genitivform einerseits, Instrumental andererseits - erhält stets bzw. bevorzugt die markiertere Ausprägung des direkten Objekts bzw. des PrN. (Hentschel 1991, 229)

Der Frage, warum die Auswirkungen der Belebtheit beim Akkusativ-Genitiv zur Konstituierung der 'Belebtheitskategorie' geführt haben, beim prädikativen Instrumental aber 'nur' zu einem stochastischen Zusammenhang, geht Hentschel nicht mehr nach - er will dem "an anderer Stelle nachgehen".

Auf Grund seiner Korpusuntersuchungen kommt der Autor zu folgenden weiteren Ergebnissen: Bei der Prüfung des Einflusses des Genus auf die Kasuswahl stellt er einen signifikanten Zusammenhang zwischen dem Maskulinum und dem Nominativ einerseits, sowie zwischen dem Femininum ${ }^{81}$ und Neutrum und dem Instru-

81 Hentschel (1991, 229, Fn. 17) verweist auf Nichols' (1985, 358f.) Feststellung, die femininen Langformen im Sg. hätten eine ausgeprägte Tendenz zur Kongruenz mit dem 'Kontrolleur', d. h. also zum Nominativ. Hentschel (1991) wertet das als Stütze für seine Argumentation, dass in markierten Kontexten das jeweils deutlichere Signal bevorzugt wird. Der Ins. Sg. f. der Langformen auf -oj fällt mit Gen., Dat. und Lok. Sg. zusammen, während die Form des Nom. Sg f. auf - aja von allen anderen Formen distinkt ist (wie es bei den Substantiven die Form des Instrumentals in allen Genera und Numeri ist!). 
mental andererseits fest. Beim Tempus korreliere das Futur signifikant mit dem Instrumental, das Präteritum im gleichen Maße mit dem Nominativ (sic!). Beim Verbmodus tendiere der Konjunktiv stärker zum Instrumental, ohne dass dies jedoch Signifikanz erreiche. Dasselbe gelte für die Negation. Alle diese Korrelationen seien aber längst nicht so ausgeprägt wie die zwischen der Kasuswahl und der Belebtheit bzw. Unbelebtheit des Referenten. "Prinzipiell korreliert der Instrumental mit semantisch markierten Kontexten, der Nominativ mit weniger markierten. [...] Der Instrumental ist [...] das deutlichere Signal für das PrN im Vergleich zum Nominativ" (Hentschel 1991, 230). Diese deutlichere Signal würde laut Ökonomieprinzip besonders dann bevorzugt, wenn eine gewisse Erschwernis der Identifikation vorliege, wie z. B. bei Nebensätzen gegenüber Hauptsätzen, bei Frage- und Befehlssätzen gegenüber Aussagesätzen, bei stärker markierten bzw. 'komplexeren' Tempus- und Modusformen gegenüber weniger markierten sowie auch bei längeren gegenüber kürzeren Sätzen bzw. bei Sätzen mit markierter Wortfolge (vgl. Hentschel 1991, 230).

Letztlich werden folgende 'Trigger' für die Wahl des Instrumentals bzw. des Nominativs am Prädikatsnomen von Hentschel (1991) festgestellt:

\begin{tabular}{|l|l|}
\hline \multicolumn{1}{|c|}{ Tendenz zum Instrumental } & \multicolumn{1}{c|}{ Tendenz zum Nominativ } \\
\hline Struktur ist ein Nebensatz & Struktur ist ein (bloßer) Hauptsatz \\
\hline Subjekt erscheint nicht-initial & Subjekt erscheint initial \\
\hline kein (overter) Subjektausdruck & overter Subjektausdruck \\
\hline $\begin{array}{l}\text { Subjektausdruck ist keine nominativische } \\
\text { Wortform }\end{array}$ & $\begin{array}{l}\text { Subjektausdruck ist eine nominativische } \\
\text { Wortform }\end{array}$ \\
\hline $\begin{array}{l}\text { keine Numeruskongruenz zwischen } \\
\text { Subjektausdruck und Prädikativ }\end{array}$ & $\begin{array}{l}\text { Numeruskongruenz zwischen Subjekt- } \\
\text { ausdruck und Prädikativ }\end{array}$ \\
\hline 'längerer' Satz & 'kürzerer' Satz \\
\hline Kopulaverb im Futur & $\begin{array}{l}\text { Kopulaverb im Präteritum, Präsens; } \\
\text { Nullkopula }\end{array}$ \\
\hline Prädikativ ist femin oder neutral & Prädikativ ist maskulin \\
\hline $\begin{array}{l}\text { Prädikativ mit unbelebtem Referenten } \\
\text { (Konkreta und Abstrakta) }\end{array}$ & Prädikativ mit belebtem Referenten \\
\hline
\end{tabular}

Die Unterschiede bei den Genera lassen sich laut Hentschel (1991, 232) auf die Belebtheit zurückführen, da bei den Feminina und Neutra bis auf wenige Ausnahmen Substantive mit unbelebten Referenten vorlagen. Der Autor gesteht ein, 
dass sich ansonsten aber unmittelbar kein gemeinsamer Nenner als Erklärung für die Wahl der Kasusmarkierung aufdrängt. Bei genauerer Betrachtung sei jedoch nicht zu übersehen, dass ein gemeinsamer Nenner im Moment der Markiertheit liege. Alle genannten Faktoren, die als 'Trigger' für den Instrumental aufgeführt werden, stellten den markierten Teil der jeweiligen Korrelation dar. "Alle diese markierten Kontexte tendieren zur merkmalhaften Kennzeichnung durch den Instrumental. Die Tendenz zum PrN läßt sich also als Entwicklung in Richtung Ikonizität interpretieren” (Hentschel 1991, 232).

In seiner Rolle als explizitem Signal mit der negativen 'Bedeutung' des 'NichtSubjekts" steht der prädikative Instrumental laut Hentschel in einer Reihe mit der Ausbildung der 'Belebtheitskategorie' und dem (seit dem Spätgemeinostslavischen) zunehmenden Gebrauch von Personalpronomina in Subjektstellung. Als die zu Grunde liegende Ursache für alle drei Phänomene postuliert er "eine allgemeine Tendenz zur Herausstellung der Subjektsrolle in den vergangenen Jahrhunderten" (Hentschel 1991, 233). Die drei Erscheinungen seien ferner gleichermaßen redundante Signale, da das, was sie anzeigen, in unmarkierten Kontexten i. d. R. auch durch andere Phänomene signalisiert wird. Nur in gewissen Kontexten würden sie zum "alleinigen Garanten einer fehlerfreien Kommunikation" (Hentschel 1991, 233).

Die Lehrmeinung einer Verbindung zwischen Instrumental und 'dem Vorübergehenden' bzw. zwischen Nominativ und 'Konstanz' erneut angreifend, stellt der Autor die m. E. überaus berechtigte Frage, ob diese Sichtweise nicht "wenigstens zum Teil exogener Natur" und "sozusagen ein Erziehungserfolg der normativen Sprachwissenschaft” (Hentschel 1991, 233) sei. Zwar schließt Hentschel die Möglichkeit, dass diese Nuancierung im bewussten Sprachgebrauch als Stilmittel verwendet wird, durchaus nicht aus, aber man sollte sie seines Erachtens angesichts der wesentlich deutlicheren Zusammenhänge (zur "Markiertheit") nicht als den hauptsächlichen Faktor missverstehen.

Abgesehen von der ohnedies lobenswerten Datenerhebung, die Hentschel (1991) betreibt, ist seine Arbeit meiner Meinung nach die erste über die russische Kasusvariation, die das Prinzip der Ikonizität als mögliche Grundlage der Kasuswahl am Prädikatsnomen in Betracht zieht und die daher die Charakterisierung "formbasiert" verdient. $\mathrm{Zu}$ beachten ist, dass Hentschel ganz primär jene Fälle russischer Kopulasätze beachtet, in denen Nominativ und Instrumental konkurrieren können. In ihnen, so die deutliche Schlussfolgerung seiner Arbeit, über- 
nimmt der Instrumental die Rolle eines expliziten Signals, das in redundanter Weise - wodurch die diagnostizierte Explizitheit ja erst zustande kommt! - die jeweils markierte Ausprägung von vielerlei möglichen Korrelationen bzw. Gegensätzen kenntlich macht. Anders ausgedrückt, markiert der Instrumental am Prädikatsnomen, sofern an seiner Stelle auch der Nominativ in Frage kommt, eine auf bestimmte Weise markierte Lesart.

Was Hentschel (1991) kaum anspricht, sind jene Fälle, in denen der Instrumental oder der Nominativ die jeweils einzig mögliche Markierungsoption am Prädikatsnomen sind. Wie ich bereits zitiert habe, würden sie dann (wenn also kein anderes Phänomen bzw. keine andere Form für an ihrer Stelle verwendet werden kann) zum "alleinigen Garanten einer fehlerfreien Kommunikation" (Hentschel 1991, 233). Sicherlich kann dieser Einschätzung nicht widersprochen werden; jedoch ist mehr dazu zu sagen, welche interpretativen Folgen eine solche Situation in Bezug auf die ‘Signalfunktion' der prädikativen Kasusmarker hat.

\subsubsection{Hentschel (1993a)}

Der Titel des Beitrags "Haben Kasus Bedeutungen oder sind sie eine diakritische Kategorie" deutet schon klar an, was Hentschel (1993a) in ihm vorschlägt, dass nämlich die Variation von Nominativ und Instrumental (und zwar bei prädikativen Substantiven sowie Adjektiven) "zu einem großen Teil [diakritisch motiviert ist], und [dass] die Art, wie sich das diakritische Prinzip in der Kasuswahl niederschlägt, bestätigt, daß die primäre Funktion von Kasusmarkierungen eine möglichst transparente Signalisation der syntaktischen Struktur, die Differenzierung von Prädikat und Argumenten im Satz ist.” (Hentschel 1993a, 110).

Hentschel schließt sich in diesem Sinne der Ansicht von z. B. Potebnja (1958) und Kacnel'son (1972) an, die Nominativ und Instrumental in prädikativer Position als isofunktional betrachten, wodurch aber - was auch für meine in Abschnitt 6 dargestellte Theorie bedeutsam ist - "nicht ausgeschlossen wird, daß der Kasusgegensatz punktuell für stilistische oder perspektivische (wichtig/unwichtig) Nuancierung ausgenutzt werden k a n n." (Hentschel 1993a, 109-110)

Zu Beginn seines Aufsatzes erwähnt Hentschel (1993a, 101), dass Isačenko (1983, 491 ff.) gegen die Sichtweise polemisiert, der 'prädikative' Instrumental habe sich im Altrussischen auf Grund seiner größeren syntaktischen Transparenz gegenüber dem Kongruenzkasus (dem 'doppelten' Nominativ) durchgesetzt. 
Isačenko "verweist zum einen darauf, daß in vielen Fällen auch bei doppelter Kasusverwendung überhaupt keine Gefahr der Ambiguität gegeben ist, und zum anderen darauf, daß viele Sprachen bei einer homonymen Markierung von Subjekt und substantivischem Prädikat bleiben, so z. B. weitgehend das Serbokroatische." (Hentschel 1993a, 101)

Zweifelsohne muss man Isačenko (1983) in seiner Polemik Recht geben, jedoch nur in Hinsicht auf Annahmen, die die Kasuswahl am Prädikatsnomen ausschließlich bzw. einseitig mit dem Bestreben nach syntaktischer Transparenz erklären wollen. Hentschels (1993a) Theorie ist jedoch nicht derart einseitig, wobei auch an seine zuletzt besprochene Arbeit (Hentschel 1991) zu erinnern ist, in der er explizit schreibt, dass der Instrumental lediglich ein redundantes Signal für den (syntaktischen) Prädikatsstatus - und damit eben auch ggf. verzichtbar - sei. Er ist dies, da es sich um "ein Element der Ausdrucksebene natürlicher Sprachen [handelt], welches zusammen mit mindestens einem anderen ein und dasselbe inhaltliche bzw. grammatisch-inhaltliche Moment repräsentiert” (Hentschel 1991, 221). Noch anders formuliert: "Der Instrumental ist [...] das deutlichere Signal für das $\operatorname{Pr}$ [ädikats]N[omen] im Vergleich zum Nominativ" (Hentschel 1991, 230) bzw. "[...] the agreeing Case [der Nominativ] represents the unmarked form with the Instrumental showing up when additional conditions obtain" (Hinterhölzl 2001, 105), wobei die zuletzt genannten Zusatzbedingungen namentlich eine explizite Kennzeichnung des prädikativen Status des Ausdrucks erforderlich machen. Isačenkos (1983) Polemik kann also insofern etwas 'Wind aus den Segeln genommen' werden, als die Etablierung des 'prädikativen' Instrumentals keineswegs eine quasi 'unvermeidliche' Entwicklung darstellte, sondern Folge einer allgemeinen Tendenz zur Ikonizität (vgl. Hentschel 1991, 229) erklärt werden kann, die nicht unbedingt Eineindeutigkeit impliziert, sondern durchaus auch in redundanter Weise bestimmte 'Momente' verdeutlichen bzw. hervorheben kann. Dabei ist durchaus möglich, dass die betreffenden 'Momente' in früheren Sprachzuständen der betrachteten Sprache oder in anderen Sprachen keinerlei besondere Hervorhebung erfahren.

$\mathrm{Zu}$ den für meine eigene Arbeit überaus relevanten Einsichten Hentschels (1993a) gehört u. a. sein Blick auf die russischen Kurz- und Langformen:

Da die Kurzform im modernen Russischen nicht mehr in attributiver Funktion verwendet wird, muß sie als die vom diakritischen Standpunkt vorteilhafte Variante 
angesehen werden. Die Langformen sind hingegen auch mögliche attributive Formen und somit aufgrund ihrer formalen Eigenschaften allein keine eindeutigen Signale für den prädikativen Status eines Adjektivs. Die geringste diakritische Potenz hat die Langform im Nominativ, die als solche auch Attribut des Subjekts sein kann. (Hentschel 1993a, 102)

Hier finden sich einige meiner eigenen Schlussfolgerungen so gut wie exakt wieder, namentlich, dass die Kurzformen explizit prädikative Formen sind (m. E. sind sie dies auch, weil es sich um Verbalnomina handelt; siehe die Abschnitte 4.3 und 4.4.3), dass die Langformen im Instrumental den Kurzformen in Bezug auf 'Prädikativität' annähernd vergleichbar sind (und erstere daher auch seit Langem und immer mehr verdrängen; ${ }^{82}$ siehe auch Corbett 2004, 208) und dass nominativische Langformen - im Russichen immer und im Polnischen mindestens potenziell - "Prädikatsphrasen" und keine echten Sekundärprädikate sind (siehe Abschnitt 5.4).

Bemerkenswert (einfach) ist auch die Ansicht, die Hentschel in Bezug auf den Gegensatz zwischen identifizierenden und prädizierenden (bei ihm: qualifizierenden/klassifizierenden) Kopulasätzen sowie der Kasusmarkierung am Prädikatsnomen in ihnen äußert. Auch hier zeigt sich deutlich seine Ablehnung einer zugrunde liegenden semantischen Distinktion "permanent vs. temporär”:

Es ist eine vielfach gemachte Beobachtung, daß in Identifikationssätzen [...] der prädikative Instrumental im Slavischen wesentlich weniger verbreitet ist als in Qualifikations- bzw. Klassifikationssätzen (vgl. Rothstein 1986). In typischen Identifikationssätzen mit zwei Indexausdrücken (Personalpronomen oder Eigennamen) ist das sicher der Fall (vgl. Weiss 1978). Dieses hat aber absolut nichts mit dem semantischen Gegensatz konstant vs. temporär zu tun, wie Jakobson [1971a] und Wierzbicka [1980] postulieren, sondern damit, daß eine Differenzierung der beiden Nominalphrasen in Identifikationssätzen kommunikativ nicht relevant ist: Während bei ,A ist identisch mit B' auch ,B ist identisch mit A' wahr ist, impliziert ,A ist ein Element der Klasse B' natürlich nicht ,B ist ein Element der Klasse A'. (Hentschel 1993a, 106)

82 Ich verwende die Einschränkung “annähernd”, da die instrumentalische Langform Hentschel zufolge restringierter als die Kurzform ist (was m. E. aber nur die geschriebene Standardsprache volle Gültigkeit hat); vgl.: "Die Langform im Instrumental zeigt [...] nur in solchen Sätzen eine nennenswerte Verbreitung, die folgende Bedingungen erfüllen: Erstens, neben dem Adjektiv als Prädikat müssen auch das Subjekt [...] und die Kopula an der syntaktischen Oberfläche realisiert sein. [...] Zweitens muß das Subjekt vor der Kopula mit folgendem adjektivischen Prädikat stehen [...]. Die Kurzform ist dagegen in allen Kontexten des Kopulasatzes ein eindeutiges Signal für den prädikativen Status des Adjektivs [...]” (Hentschel 1993a, 104). 
Diese Annahme Hentschels ist natürlich nicht nur im Rahmen der vorliegenden Arbeit diskussionswürdig. Gerade auch im Hinblick auf die Klassifikation von 'sein'-Sätzen etwa von Geist (2006) oder Bondaruk (2013b) kann man spekulieren, auf welcher Ebene des Sprachsystems der postulierte Gegensatz von Identitäts-, spezifizierenden, klassifizierenden, ... und/oder prädizierenden (Kopula-) Sätzen seine Basis haben soll oder kann. Im Kontext der vorliegenden Arbeit ist Hentschels (1993a) insofern von Bedeutung, als sie klar die "formbasierte" Richtung vorzeichnet und diese sogar über "Kopulasätze" im engeren Sinne hinaus auf die Identitätssätze anwendet.

Hentschel (1993a) weist noch auf viele weitere Faktoren hin, die die Kasuswahl am Prädikatsnomen beeinflussen (können) und die allesamt insofern "formbasiert" sind, als sie mit dem syntaktischen Umfeld zu tun haben, in den das Prädikatsnomen eingebettet ist. So stellt er $u$. a. folgende Punkte als relevant fest:

- Ist der Subjektausdruck ein Subjektpronomen, tritt laut Hentschels Untersuchung der Nominativ am Prädikatsnomen in ca. 45\% der Fälle auf, fehlt das Subjektpronomen an der Oberfläche, sinkt dessen Anteil auf 13\%. Hier merkt Hentschel an, dass ein bloßer Nominativ (ohne Subjekt) zu einer 'Fehldekodierung' des Satzes als Existenzsatz führen kann (vgl. Hentschel 1993a, 103).

- Letzterer Befund wiederholt sich auch bei den Adjektiven, da im Falle eines "Nullpronomens" in Subjektposition der Anteil der (explizit prädikativen und nominativischen) Kurzform vom 59\% auf 83\% steige (Hentschel 1993a, 104).

- Im Falle zweier voller Nominalphrasen in einem Kopulasatz liegt der Anteil des Instrumentals am Prädikatsnomen bei $80 \%$, was daran liege, dass hier die syntaktischen Funktionen nicht ohne Weiteres bzw. per se dekodierbar seien. Hentschel (1993a, 106) folgert, dass bei kategorialer Identität der beiden Satzglieder die Wahrscheinlichkeit (bzw. kommunikative Notwendigkeit) ihrer Differenzierung durch den Kasus steigt.

- Die Langform im Nominativ wird gemieden, wenn in demselben Satz eine andere adjektivische Wortform mit der gleichen flexionsmorphologischen Markierung als Bestandteil des Subjektausdrucks vorhanden ist (vgl. Hentschel 1993a, 107).

- Schon in Hentschel (1992) hat der Autor gezeigt, dass die Abfolge der Konstituenten im Kopulasatz ebenfalls Einfluss auf die Wahl der Markierung am Prädikatsnomen hat: Sofern eine von der 'üblichen' Abfolge Subjekt - Kopula - Prädikatsnomen ab- 
weichende Sequenz vorliegt (so z. B. Prädikatsnomen - Subjekt - Kopula), ist die Häufigkeit der syntaktisch transparenteren Prädikatsmarker (Instrumental bzw. Kurzform) deutlich höher (vgl. Hentschel 1993a, 107-108).

- Der Instrumental am Prädikatsnomen im Falle präsentischer russischer Kopulasätze (mit der "Nullkopula") ist keine strikt grammatisch, sondern eher eine kommunikativ determinierte Erscheinung, die der Vermeidung von Ambiguität dient, denn "[z]wei unmittelbar aneinander grenzende Nominalphrasen, eine im Nominativ und eine im Instrumental, ohne ein verbales Element in ihrem Kontext, bergen die Gefahr, als elliptischer Satz mißverstanden zu werden, besonders natürlich in der gesprochenen Sprache. [...]." (Hentschel 1993a, 108). Wie in Abschnitt 5.5.1 gezeigt wird, geht es hier nicht ausschließlich um die Abwesenheit eines verbalen Elements. Zentral scheint die Abwesenheit jedweden Materials neben den beiden NPn. Der Folgerung Hentschels, dass es die hohe Wahrscheinlichkeit einer 'Fehldekodierung' als Ellipse eines Vollverb wie z. B. 'arbeiten (als)', 'dienen (als)' usw. ist, die den Instrumental im Russischen ausschließt, schließe ich mich an.

Die Arbeit von Hentschel (1993a) ist für die vorliegende Analyse enorm wertvoll, weist sie ihr doch den grundsätzlichen Weg. Durch eine "formbasierte" Analyse, die keine semantische (invariante) Distinktion annimmt, kann den beobachtbaren Verhältnissen und Lesarten im Russischen ebenso wie im Polnischen Rechnung getragen werden, ohne bestimmte Wortformen bzw. Suffixe (etwa das Instrumentalsuffix) mit einer 'gefährlich' spezifischen Semantik zu 'belasten' und am Ende genötigt zu sein, eine 'Neutralisierung' anzunehmen, wenn diese Semantik von den sprachlichen Daten - trotz der Form, die sie beinhalten soll - nicht bestätigt wird (vgl. so u. a. bei Geist 2006). Es ist eine Tatsache, dass die Formen des Prädikatsnomens im Polnischen und Russischen nicht immer eine bestimmte Lesart evozieren. Wie schon oft angesprochen, ist letzteres gerade dann der Fall, wenn nur eine einzige Form - bedingt durch die syntaktischen Verhältnisse - möglich ist. In diesem Fall kann das Fehlen irgendwelcher 'interpretativer Effekte' einfach dadurch erklärt werden, dass eine Opposition der einzig möglichen Form zu anderen Formen überhaupt nicht in Frage kommt. Man kann, mit anderen Worten, nicht wählen, und wo man nicht wählen kann, muss das zwangsläufig Vorhandene dasselbe leisten bzw. abdecken, was unter anderen Umständen ggf. von mehreren Form-Kandidaten zum Ausdruck gebracht werden kann. Diese Analyse hat den Vorteil, die Flexibilität der nordslavischen Kasusvariation in Kopulasätzen getreu 
abzubilden, indem sie non-deterministisch und somit ebenso flexibel wie ihr Untersuchungsgegenstand selbst ist.

\subsubsection{Guiraud-Weber (1993)}

Bei dem franzöischen Beitrag Guiraud-Webers (1993) handelt es sich um eine "formbasierte" Arbeit, die ihren Fokus auf Gebrauch und Verbreitung der russischen Kurzformen setzt. Diese Analyse hebt sich von den meisten anderen slavistischen Arbeiten ab, indem sie nicht von einer monolithischen Normsprache ausgeht, die alleiniges Untersuchtungsobjekt ist, sondern die ganz natürliche Möglichkeit in Betracht zieht, dass innerhalb ein und desselben (abstrakten) Sprachsystems eine Anzahl sich voneinander unterscheidender "Modelle" bzw. synchroner Sprachzustände existiert. Verschiedene Sprecher (bzw. sogar ein und derselbe Sprecher) können demnach (je nach Äußerungssituation, Stil- und Funktionalebene usw.) von unterschiedlichen Modellen Gebrauch machen. Einfach reformuliert heißt das, dass es nicht nur ein "Russisch" oder "Polnisch" gibt, sondern mithin diverse Formen derselben, die sich in bestimmter Hinsicht voneinander unterscheiden (müssen). Da Guiraud-Weber (1993) mit Blick auf die russischen Kurzformen von zwei nebeneinander bestehenden bzw. konkurrierenden Modellen ausgeht, bezeichnet sie ihre Herangehensweise als "bisynchronisch". Im folgenden Zitat verdeutlicht die Autorin ihre Grundannahmen (meine Übersetzung):

La méthode bisynchronique consiste à chercher à ramener l'ensemble des faits d'un état de langue donné non pas à un seul modèle, comportant un ensemble de règles, mais à deux modèles différents, $\mathrm{A}$ et $\mathrm{B}$, comportant chacun un ensemble de règles, qui peuve être partiellement communes aux deux modèles, mais sont nécessairement en certains points différents. Les faits dont il ne peut être rendu compte par le modèle A, sont rapportés au modèle B. En cas d'hésitation, une de deux variantes possibles est ramenée au modèle A, l'autre au modèle B. (Garde 1988: 64-65).

La méthode bisynchronique [...] permet de prendre en compte toutes les variantes réellement coexistantes dans la langue contemporaine sans être tenté d'en sacrifier une partie pour sauvegarder la logique interne du modèle. (Guiraud-Weber 1993, 83)

[Die bisynchronische Methode besteht darin, die Gesamtheit der Fakten eines gegebenen Sprachzustands zusammenzutragen, [und zwar] nicht für ein einzelnes Modell, das eine Menge von Regeln enthält, sondern für zwei unterschiedliche Modelle, A und B, die jeweils eine eigene Menge von Regeln enthalten; diese 
können teilweise beiden Modellen gemeinsam sein, müssen sich aber notwendigerweise in bestimmten Punkten unterscheiden. Die Tatsachen, denen durch Modell A nicht Rechnung getragen werden kann, werden Modell B zugeordnet. Im Zweifelsfall wird eine der beiden möglichen Varianten Modell A, die andere Modell B zugeordnet.

Die bisynchronische Methode [...] erlaubt, alle real koexistierenden Varianten der gegenwärtigen Sprache zu berücksichtigen, ohne versucht zu sein, einen Teil davon zu opfern, um die interne Logik des Modells zu retten.]

Bezogen auf die russischen Kurzformen läuft dieser bisynchronische Ansatz darauf hinaus - worin er auf deskriptiver Ebene übrigens den Beobachtungen u. a. von Kiparsky (1967) oder Corbett (2004) gleichkommt -, sie nur einem der beiden "Modelle", nämlich "Modell B", des Russischen zuzuordnen, während sie in "Modell A" weitgehend fehlen (vgl. Guiraud-Weber 1993, 89-91). Exakter formuliert, meint die Autorin, dass Sprecher des Russischen, die "Modell A" präferieren (was ihr zufolge primär im modernen Russischen der Fall ist), den Gebrauch von Kurzformen weitestmöglich (sofern die Grammatik diese also nicht erzwingt) vermeiden und auf Langformen im Nominativ oder Instrumental zurückgreifen (vgl. Guiraud-Weber 1993, 89). In "Modell B" dagegen wird die Kurzform 'nach allen Regeln der Kunst' verwendet (man könnte polemisierend behaupten, dass sich die entsprechenden 'Gebrauchsanweisungen' in der Akademiegrammatik finden).

Die relativ einfache Schlussfolgerung lautet, dass "Modell A" weitaus simpler als Modell B ist, da die Kurzformen regelmäßiger Bestandteil von Modell B sind und so die Auswahl möglicher adjektivischer Formen in der Prädikatposition von Kopulasätzen gewissermaßen verdoppeln. ${ }^{83}$

Das bisynchronische Modell nach Guiraud-Weber (1993) kann schematisch wie in (42) dargestellt werden. Während das komplexere "Modell B" alle drei adjektivischen Formen vollumfänglich beinhaltet, schließt "Modell A" die Kurzformen quasi aus. Diese Darstellung ist insofern vereinfacht, als "Modell A" Kurzformen

83 Vgl.: "Le modèle A qui est beaucoup plus simple que le modèle B [...]" [Modell A, das viel einfacher als Modell B ist ...] (Guiraud-Weber 1993, 90). Vgl. ferner: "Le modèle B est beaucoup plus complexe car il double les possibilité syntaxiques par la présence constante du FC [...]" [Modell B ist viel komplexer, da es die syntaktischen Möglichkeiten durch die konstante Präsenz der Kurzform verdoppelt ...] (Guiraud-Weber 1993, 91). 
dann durchaus doch aufweisen kann, wenn deren Gebrauch obligatorisch ist (vgl. Guiraud-Weber 1993, 89): $:^{84}$

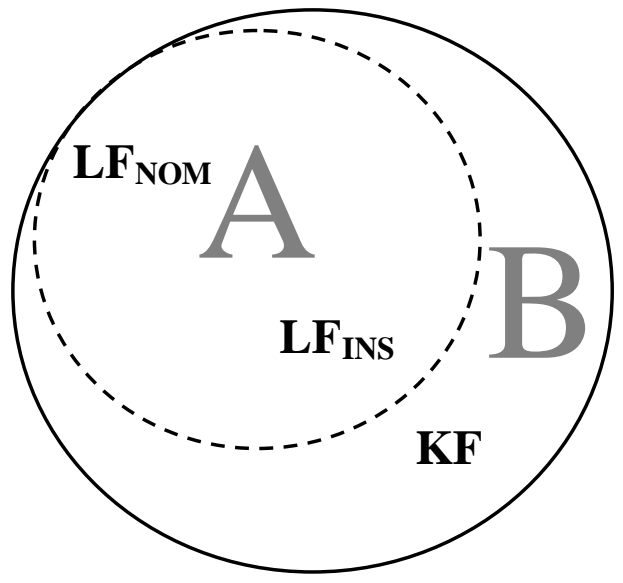

Diese Modellierung ist für die vorliegende Arbeit von immenser Bedeutung, da Guiraud-Weber durch sie zeigt, dass nicht nur ein einziges (monolithisches) "grammatisches Gefüge" vorliegt, sondern von mehreren solcher Gefüge ausgegangen werden kann. In jedem Fall kann in Bezug auf die russischen Kurz- und Langformen davon ausgegangen werden. Ich würde sogar soweit gehen zu behaupten, dass von mehr als nur zwei Modellen auszugehen ist, wenn man weitere Faktoren wie Idiolekt, Soziolekt, Stileben, mögliche Fachsprachen, mögliche Bilingualität oder dialektale Herkunft des Sprechers etc. einbezieht. Aber GuiraudWeber erfasst die primäre Tatsache, indem sie zeigt, dass Sprecher das eine oder das andere "Modell” bevorzugen (oder sogar ausschließlich verwenden) können, und dass dies Auswirkungen auf den Gebrauch, die Frequenz und nicht zuletzt auch auf die Bewertung bzw. Interpretation der jeweils betrachteten Formen hat. Im konkreten Fall bedeutet der bi- bzw. polysynchronische Ansatz, dass nicht jeder Sprecher zwangsläufig alle normativ vorgesehenen Prädikativformen bzw. -markierungen auch wirklich als Option betrachtet und verwendet. Sofern er ein Sprecher der "gepflegte Hochsprache" (Isačenko 1962, 151) ist, die man sicherlich mit Guiraud-Webers (1993) "Modell B" gleichsetzen darf, hat er alle drei Optionen - die Kurzform sowie die beiden Langformen - zur Auswahl und wird sie auf der Basis seines grammatischen Wissens bewusst einsetzen. Wie die Auto-

84 Das ist v. a. dann der Fall, wenn Kurzform und Langform deutlich andere Bedeutungen haben

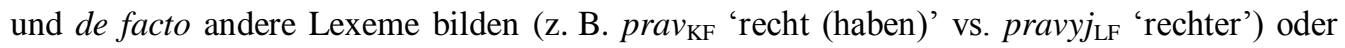
wenn von einem Lexem nur die Kurzformbildung möglich ist (z. B. rad 'froh'). 
rin feststellt, ist es aber gerade im modernen Russischen eher der Fall, dass die Sprecher das "Modell A" präferieren, in dem der Gebrauch der Kurzformen auf ein mögliches Minimum reduziert ist (vgl. Corbett $(2004,208)$ der in den letzten 200 Jahren den Rückgang der Kurzformen beobachtet).

Dass dieser Ansatz "formbasiert" ist, versteht sich von selbst. Er lässt sich ferner auch in einen "oppositionsbasierten” Ansatz 'umrechnen', wie ich ihn in dieser Arbeit verfolge. Es ergibt sich nämlich aus dem Gesagten, dass in einem Modell mit drei potenziell wählbaren Prädikativformen mehr und andersartige Oppositionen zustande kommen als in einem Modell mit nur zwei oder sogar mit nur einer Form. Der Vorteil einer solchen form- sowie oppositionsbasierten Sichtweise ist ein hohes Maß an Flexibilität zur Erfassung der offensichtlich hochgradig sprecherbezogenen und kontextbedingten Erscheinung der Kasus- und Formvariation in russischen sowie auch in polnischen Kopulasätzen. Abschnitt 6 wird dieses form- und oppositionsbasierte Konzept im Detail vorstellen.

\subsubsection{Bogusławski (2001)}

Die Arbeit von Bogusławski (2001) kommt in vielen zentralen Annahmen den Analysen Hentschels (1991, 1993a) entgegen. Sie befasst sich allerdings mit dem Polnischen und dort 'nur' mit prädikativen Substantiven (NPn).

Bogusławski betrachtet den Gegensatz zwischen Nominativ und Instrumental an polnischen substantivischen Prädikatsnomina als "Variation im eigentlichen Sinne", worunter er das (völlige) Fehlen semantischer Unterschiede versteht:

Zwischen den Spracheinheiten "Kopula + Nom.", "Kopula + Instr." besteht tatsächlich das Verhältnis der Variation im eigentlichen Sinne; sie sind keine zwei verschiedenen semantischen Entitäten. Meine Voraussetzung dabei ist, daß wir den Terminus Variation eben auf das Fehlen der semantischen Unterschiede (im strengen Sinne) beziehen. (Bogusławski 2001, 127)

Aufschlussreich ist in diesem Zusammenhang einmal mehr ein Vergleich mit der Analyse Geists (2006). Während Bogusławski den Instrumental als Default betrachtet, der im Vergleich zum Nominativ "negativ bestimmt” sei, kommt Geist zu dem geradezu konträren Schluss, wenn sie den (russischen) Nominativ als "neutral" einstuft bzw. ihm jene semantische Komponente (die Maienbornsche “Spezifizitätspräsupposition”) abspricht, die dem Instrumentalsuffix zueigen sei. 
Schwerer als diese Differenz wiegt, dass Bogusławski (wie Hentschel) in der Kasusvariation ein lediglich 'diakritisches' Phänomen erblickt, wohingegen Geist wenn auch in Bezug auf den Instrumental - eine "semantischen Formel" aufstellt, die Bogusławski explizit ausgeschlossen wissen will:

Man sieht sich außerstande, eine semantische Formel für den [...] Nominativ [...] anzusetzen, die einen ausreichend allgemeinen Charakter hätte und zugleich untilgbar ("uncancellable") wäre; d. h. eine Paraphrase (oder irgendeine andere Repräsentation), die nicht widerspruchslos verneint werden könnte. (Bogusławski 2001, 127)

Die "Untilgbarkeit", von der Bogusławski hier spricht, ist etwas, das jede Analyse des "semantischen Lagers", die eine Invariante als Grundlage der Variationen betrachtet, anstreben muss, da einmal vorhandene semantischen Anteile nicht getilgt werden können (siehe Zimmermann 1999, 134). Eine solche 'Neutralisierung' findet sich aber de facto bei Geist (2006).

Bogusławski stellt sehr detailliert jene Fälle im Polnischen zusammen, in denen der Nominativ prinzipiell un/zulässig ist. Diese Kompilation möchte ich hier wenigstens z. T. nachvollziehen, da es sich bei ihr um die geschlossenste ihrer Art mit Blick auf die Verhältnisse im Polnischen handelt. Der Autor stellt zunächst die folgenden Punkte fest:

- Nicht-Personen als Subjekte von Kopulasätzen lassen prinzipiell keine nominativischen Prädikatsnomina zu (Bogusławski 2001, 109-112).

- Bei Partizipialformen der Kopula ist der Nominativ ausgeschlossen (Bogusławski 2001, 112-113).

- Beim Infinitiv der Kopula ist der Nominativ nur zulässig (aber nie obligatorisch) bei (i) charakterisierenden Ausdrücken, deren primäre (idiomatisierte!) Markierung der Nominativ sei (z. B. pies na kobiety 'scharf auf Frauen'); bei (ii) Eigennamen und Ethnonymen in der metonymischen Bedeutung 'eine Rolle spielen, welche derjenigen ähnlich ist, die von einer bestimmten Person gespielt wurde' (insb. auch mit taki); bei (iii) Nachnamen in der metonymischen Bedeutung ‘Angehörige(r) der Familie/Sippe X' (Bogusławski 2001, 113).

- Bei unpersönlichen Sätzen mit dem Reflexivmarker się (jest/byto/będzie się ...) ist ausschließlich der Instrumental möglich (Bogusławski 2001, 114). 
- Bei Subjektausdrücken, die eine numerische Quantifikation beinhalten, die in Präteritum und Konjunktiv eine neutrale/'impersonale' Form des Verbs (było) erfordern (z. B. dwóch), ist ausschließlich der Instrumental zulässig (Bogusławski 2001, 115).

Als Hauptbereich der Kasusvariation identifiziert Bogusławski (2001, 117) Sätze mit Personenbezeichnungen und finiten Kopulaformen. Für das Erscheinen des “doppelten Nominativs" in solchen Sätzen müssen die Prädikatsnomina ihm zufolge die folgenden notwendigen Bedingungen erfüllen:

- Sie müssen sonst als standardisierte Bestandteile 'kurzer' Subjektphrasen auftreten (z. B. dureń 'Dummkopf'). Bei Eigennamen sei es auch häufig so, dass diese sonst als Bestandteile vollständiger Subjektphrasen auftreten (Bogusławski 2001, 116):

(i) On jest dureń. 'Er ist ein Dummkopf.'

(ii) Mój brat jest Stefan. 'Mein Bruder ist Stefan.'

- Sie dürfen weder ganz allgemeine Konzepte für Personen noch ausdrücklich Einzelmengen denotieren (Bogusławski 2001, 116-117):

(iii) Pilot tego UFO jest człowiekiem/*człowiek. 'Der Pilot dieses UFOs ist ein Mensch.'

(iv) Osoba, o którą chodzi, jest mężczyzną/*mężczyzna. 'Die Person (...) ist ein Mann.'

- Sie dürfen nicht von attributiven oder argumenttragenden Ergänzungen begleitet werden (Bogusławski 2001, 117-119).

- Sie dürfen nicht mit iterierenden Adverbialen auftreten (z. B. dwaltrzy/kilka razy 'zwei/drei/ein paar Mal') (Bogusławski 2001, 119-120).

Schließlich, so Bogusławski (2001, 120-121), ist die nominativische Markierung in einigen ‘lexikonbasierten' Fällen praktisch die einzig mögliche, an der auch die genannten Bedingungen kaum etwas ändern können, so z. B. (43a-d): ${ }^{85}$

85 Vgl. Geist (2006, 69-70), die russische 'idiomatisierte' Ausdrücke nennt, die den Nominativ am Prädikatsnomen ebenso 'fordern':

(i) a. On byl vylityj otec.

'Er war ganz der Vater'.

b. Ona byla koža i kosti.

'Sie war Haut und Knochen.'

c. On byl živoj trup.

'Er war ein lebendiger Leichnam.'

(Der Instrumental würde zum Verlust des idiomatischen Charakters führen.) 
(43) a. Ona była herod-baba.

'Sie war ein Dragoner.'

b. On był zewłok człowieka.

'Er war ein lebendiger Leichnam.'

c. On był skóra i kości.

'Er war Haut und Knochen.'

d. Ona jest wykapana matka.

'Sie ist ganz die Mutter.'

Andererseits liegen laut Bogusławski (2001, 122) aber auch 'lexikonbasierte' Substantive vor, die als Prädikatsnomen auf den Instrumental festgelegt sind, z. B. nicość 'Nichtigkeit', piękność 'Schönheit', znakomitość 'Bekanntheit'.

Ferner beobachtet der Autor eine Reihe anderer, namentlich charakterisierender und bewertender Ausdrücke, die im Prädikat in ihrer Markierung schwanken, wobei der Nominativ aber überwiege, z. B. bankrut 'Bankrott', brudas 'Schmutzfink', leniuch 'Faulpelz', polityczny trup 'politische Leiche', skurwysyn 'Hurensohn', śpioch 'Schlafmütze' (Bogusławski 2001, 122). ${ }^{86}$

Schließlich werden noch fünf weitere Typen von Prädikativen mit ihrem spezifischen Markierungsverhalten beschrieben, die ich hier aus Platzgründen vernachlässige (siehe Bogusławski 2001, 122-125).

Unbedingt erwähnt werden sollten die Feststellungen zu negierten Deklarativ- und Interrogativsätzen (Bogusławski 2001, 125-127). In diesen sei der Instrumental die absolut vorherrschende Form. Diesen Befund wertet Bogusławski als Folge der zuvor beschriebenen Regeln zur Nominativverwendung. So trete der Nominativ - abgesehen von einigen wenigen metonymisch gebrauchten oder 'lexikonbasierten' Fällen - in beiden genannten Satztypen nur dann auf, wenn diese als Korrekturen verwendet würden. Dies wird vom Autoren mit der Annahme in Verbindung gebracht, der "doppelte Nominativ" verfüge über die charakteristische Eigenschaft der "Konsequenzialität", die sich ihrerseits ausschließlich mit nicht negierten Ausdrücken verbinde. Diese Eigenschaft sei ein Reflex des (von Bogu-

86 Es wird vermerkt, dass der Nominativ durch eine Temporalangabe deutlich beschränkt werde. Das deutet m. E. darauf hin, dass Sätze, in denen diese Lexeme als Prädikative auftreten, tendenziell als Idiome gewertet werden, dass ihr Idiomcharakter jedoch nicht so ausgeprägt wie bei den zuvor genannten Wendungen ist, so dass der Instrumental und/oder eine Temporalangabe den idiomatischen Charakter mindern oder ganz aufheben kann. 
sławski demnach als kongruenzgebend betrachteten) Subjektnominativs, da Subjektausdrücke praktisch nie negiert seien. Als solche würden sie auch nur mit geeigneten, also nicht negierten Prädikaten verknüpft. In einem negierten Kopulasatz entspricht die Negation i. d. R. dem bloßen Fehlen der prädizierten Eigenschaft. Solch ein Fehlen habe wiederum keine spezifizierbaren Konsequenzen, die aber der seiner Natur nach "konsequenziale” Nominativ erfordere. Daher seien, so Bogusławski, in negierten Kopulasätzen nominativische Prädikative nahezu ausgeschlossen, der "nicht konsequenziale" Instrumental dagegen werde in ihnen folgerichtig (und signifikant) bevorzugt.

Bogusławskis Betrachtung ist für die vorliegende Arbeit wertvoll, da sie dem “formbasierten" Lager angehört und sich auf die Verhältnisse im Polnischen konzentriert, was ansonsten generell vergleichsweise selten ist. Sowohl die Nullhypothese als auch die Schlussfolgerung Bogusławskis decken sich mit jenen von Hentschel (1991, 1993a) und besagen, dass der Kasusvariation keine semantische Distinktion i. e. S. zugrunde liegt. Anhand von Bogusławskis Detailbeobachtungen offenbart sich aber m. E., dass die Kasusvariation im Polnischen eingeschränkter als im Russischen erfolgt.

Der Instrumental ist an substantivischen Prädikatsnomina im Polnischen in einem weit höheren Maße als im Russischen der Normalfall (vgl. eine mögliche Erklärung in Abschnitt 5.5.2). Dem Autor kann daher zugestimmt werden, wenn er schreibt, dass "die instrumentalische Markierung negativ (by default) bestimmt" (Bogusławski 2001, 127) sei. Das heißt, der Instrumental erscheint am polnischen substantivischen Prädikatsnomen, falls nicht auf Grund irgendwelcher 'speziellen' Umstände der 'markierte' “doppelte Nominativ” zum Einsatz kommt. Letztlich belegt Bogusławskis Arbeit, dass sowohl für das Russische als auch für das Polnische der "formbasierte" Ansatz greift, auch wenn für beide Sprachen die konkreten Bedingungen der Wahl der einen oder anderen Form unterschiedlich aussehen mögen.

\subsection{Rein struktureller Ansatz}

"Rein strukturell" sind Ansätze, die das Vorhandensein einer wie auch immer beschaffenen semantischen Distinktion grundsätzlich ablehnen, die aber auch keine pragmatische oder "formbasierte" Erklärung für die Variationen anerkennen. Vielmehr kommen sie zu dem Schluss, dass in (russischen) Kopulasätzen der 
Instrumental heute der Default an (v. a. substantivischen) Prädikatsnomina sei. In Bezug auf adjektivische Prädikative herrschen z. T. gegensätzliche Ansichten, da zum einen behauptet wird, der Instrumental sei auch hier der Default, zum anderen mitunter aber die Sichtweise zu finden ist, adjektivische Prädikatsnomina erschienen bevorzugt im Kongruenzkasus. Diese Ansichten korrelieren i. d. R. mit den jeweils zugrunde gelegten Annahmen zur Frage, ob prädikative Adjektive, die im Instrumental stehen, (a) 'nur' Attribute eines prädikativen "Nullsubstantivs" oder (b) 'richtige' Prädikatsnomina sind (vgl. u. a. Babby 1975, Bailyn 1994; Babby 1999; Geist 2006, 2010). Im ersteren Falle kann der Instrumental als bloßes Ergebnis der Kongruenz zwischen attributiver AP und Bezugs-NP erklärt werden, im letzteren Falle dagegen muss er von der Kopula(bedeutung) an die prädikative AP zugewiesen werden (oder anders zustande kommen).

Wie auch immer diese Ansichten konkret aussehen, es fällt auf, dass die Situation, die "rein strukturelle" Ansätze für das moderne Russische behaupten, derjenigen im heutigen Polnischen in vielen markanten Punkten entspricht. ${ }^{87}$ Es gehört sicher zu den 'attraktiven' Aspekten dieser Analysen, mindestens für den nordslavischen Raum eine gemeinsame Tendenz im Bereich der Prädikativmarkierung ansetzen zu können, im Rahmen derer eine ursprünglich semantische Distinktion in eine strukturell determinierte Kasuswahl am Prädikatsnomen übergeht. Die heutigen Daten des Polnischen, deutlicher noch aber des Russischen, sprechen jedoch gegen diese Hypothese bzw. dafür, dass der beschriebene Zustand 'zu schön ist, um wahr zu sein' oder auf jeden Fall noch nicht erreicht ist.

Ein bemerkenswert konsequenter Vertreter der "rein strukturellen" Perspektive ist die Studie von Krasovitsky et al. (2008), die für das heutige Russische im Prinzip die oben erwähnten Annahmen macht. Der Nominativ an prädikativen Substantiven wird als bloßes "Rudiment" bewertet, dessen völlige Verdrängung durch den Instrumental nur eine Frage der Zeit ist: "In our view, semantics had only a subsidiary role in this morphosyntactic process, supporting variation at some stages, while ultimately being ousted in favour of a single rule, namely: instrumental case marking on predicate nouns." (Krasovitsky et al. 2008, 113)

Vor dem Hintergrund der empirischen Tatsache, dass dieser Schlussfolgerung der keineswegs 'rudimentäre' Charakter des “doppelten Nominativs" im heutigen

87 Etwa mit Verweis auf die Betrachtungen von Bogusławski (2001) wird aber klar, dass den heutigen polnischen Daten durch eine "rein strukturelle" Sicht nicht umfassend Rechnung getragen werden kann. 
Russischen zuwider läuft, stellt auch Kuznetsova (2013) die Konklusion von Krasovitsky et al. (2008), die auf der statistischen Auswertung eines relativ kleinen Korpus basiert, in Frage. ${ }^{88}$ Sie kommt durch eine eigene Korpusanalyse zu einem völlig anderen Schluss:

Krasovitsky et al. (2008) reject the semantic explanation and, using data from a small corpus, show that the semantic distribution described above applies to the 19th century. In the 20th century, however, the semantic rule of variation in case marking has been replaced by an overall syntactic rule: all secondary predicates that were previously in the domain of variation now tend to use the instrumental case. In this paper I test their hypotheses on a larger amount of data - the R[ussian] $\mathrm{N}$ [ational] C[orpus] - and show that Nom[inative] C[ase] is still used in modern Russian. (Kuznetsova 2013, 52-53) (99 $^{8}$

Der "rein strukturelle" Standpunkt ist offensichtlich zu starr, um dem sehr variablen Charakter der Form- und Kasusvariationen in polnischen und russischen Kopulasätzen adäquat Rechnung tragen zu können.

\subsection{Fazit}

Nach dieser umfangreichen und dennoch nur kursorischen Besprechung einer Reihe vorliegender Ansichten und Analysen zur russischen und polnischen Kasusund Formvariation in Kopulasätzen ist m. E. deutlich geworden, dass weder der rein semantische noch der zuletzt besprochene rein strukturelle Ansatz Erfolg versprechen. Ebensowenig erfolgreich erweisen sich rein syntaktische Ansätze. Auch "Mischformen" (semantisch-syntaktisch, semantisch-pragmatisch) lassen Fragen offen. Allen genannten "Lagern” ist gemeinsam, dass sie übergeneralisieren bzw. deterministisch sind. Sie weisen bestimmten Formen (u. a. AG 1980; Isačenko 1962; Ueda 1992; Geist 2006, 2010), syntaktischen Strukturen (u. a. Matushansky 2000; Markman 2008; Bailyn 2012) oder auch ganzen "Konstruktionen” (u. a. Kuznetsova \& Rakhilina 2010) eine jeweils spezifische Bedeutung zu. Daraus folgt in allen diesen Fällen die Festlegung der entsprechenden Größe auf die postulierte Semantik. Gerade jene Fälle aber, die die Variationen im Polnischen

88 Das Korpus enthält 1.853 Belege, die über vier Perioden zu je 50 Jahren von 1801 bis 2000 verteilt sind (vgl. Krasovitsky et al. 2008, 103).

89 Mit ist nicht klar, warum Kuznetsova nur von "secondary predicates" spricht, da Krasovitsky et al. (2008) ganz generell "predicate nouns" untersuchen. 
und mehr noch im Russischen 'inkonsequent' erscheinen lassen (verglichen z. B. mit der spanischen Kopulavariation), da in ihnen nur eine einzige Form des Prädikatsnomens möglich ist, lassen alle diese Ansätze an ihre Grenzen stoßen bzw. zwingen sie zur Annahme zusätzlicher 'Neutralisierungsmechanismen'.

Als einzig gangbarer Weg hat sich der "formbasierte" erwiesen, den u. a. Hentschel (1991, 1993a), Guiraud-Weber (1993) und Bogusławski (2001) beschreiten. Er ist flexibel genug, auch die zuletzt genannten Fälle zu erfassen, da er einerseits von keiner semantischen (grammatischen) Distinktion ausgeht, andererseits die Realität interpretativer Effekte aber nicht ausschließt, diese jedoch als bloße Lesarten auffasst, die primär durch das (syntaktische) Umfeld zustande kommen. Mein Beitrag in Abschnitt 6 wird darin bestehen, diesen "formbasierten" Ansatz um eine "oppositionalistische” Komponente zu erweitern, was ermöglicht, die jeweils verfügbaren Prädikativformen relativ zueinander zu gewichten und dies (wenigstens grundlegend) mit den Lesarten zu korrelieren. 


\section{Theoretischer Rahmen}

\subsection{Grammatikmodell}

Im Sinne des Minimalistischen Programms (Chomsky 1993, 1995a) wird in dieser Arbeit von einer modular aufgebauten Grammatik für natürliche Sprachen ausgegangen, deren Module über so genannte Schnittstellen (Interfaces) miteinander kommunizieren. Die Schnittstellen sind so beschaffen, dass sie die auf den beiden jeweils relevanten Seiten gegebenen Informationen enkodieren bzw. dekodieren und so einen Informationsaustausch zwischen ihnen gewährleisten können.

Mentales Lexikon und Syntax sind als Module unmittelbar dem Sprachsystem im engeren Sinne zuzurechnen. Während das mentale Lexikon der Ort ist, an dem lexikalische Einheiten mit der für ihre Weiterverarbeitung notwendigen Menge sie definierender phonetischer, grammatischer, semantischer usw. Informationen abgelegt sind (siehe Abschnitte 4.2 und 4.4), wird die Syntax als Mechanismus begriffen, der lexikalische Einheiten zu Einheiten höherer Komplexität verknüpft (siehe Abschnitt 4.5).

Für die Syntax als verknüpfender Instanz dienen die lexikalischen Einheiten aus dem mentalen Lexikon - die ggf. zuvor durch die Morphologie, die als Subsystem des mentalen Lexikons betrachtet wird, zusammengesetzt (flektiert) worden sind - als Eingabe (Input), was wiederum eine Schnittstelle zwischen Lexikon und Syntax voraussetzt. Letztere kann zum einen als "Numeration" (vgl. Chomsky 1995a, 225ff.) vorgestellt werden, d. h. als die durch die Äußerungsabsichten des Sprechers vorbestimmte Menge derjenigen lexikalischen Einheiten, die an der syntaktischen Derivation beteiligt werden sollen (aus denen letztlich also die Satzaussage komponiert werden soll). Eine solche Menge lexikalischer Einheiten allein lässt jedoch offen, im welcher Abfolge ihre Syntaktifizierung zu erfolgen hat und wie ihre hierarchischen Beziehungen unter- und zueinander beschaffen sein müssen. Somit ist es notwendig, die Reihenfolge der Einsetzung lexikalischer Einheiten unter terminale Knoten in der Syntax als in irgendeiner Form (prä-)determiniert zu betrachten. Die Einsetzungsabfolge lässt sich dabei als regelhaft aus den Subkategorisierungs- und Selektionseigenschaften in den Lexikoneinträgen jener lexikalischer Einheiten rekonstruieren, die Teil der erwähnten "Numeration" bzw. "Sprecherauswahl" sind. So ergibt sich letztlich das Bild einer durch lexikalische Informationen determinierten syntaktischen Strukturbildung, wie sie u. a. in den Arbeiten Manfred Bierwischs, Dieter Wunderlichs und Ilse Zimmermanns 
deutlich zum Ausdruck kommt (siehe auch Abschnitt 4.2). Somit wird neben der "Numeration" die Argumentstruktur als zwischen Lexikon und Syntax vermittelnde Schnittstelle angesehen, welche die Anzahl und auch die Hierarchie der zu syntaktifizierenden lexikalischen Einheiten - bzw. derer Argumente - angibt bzw. prädeterminiert.

Im Ergebnis einer jeden (erfolgreichen) syntaktischen Derivation liegen komplexe Laut- und Bedeutungsrepräsentationen vor, die wiederum als Eingabe für die beiden sog. "Performanzsysteme" dienen, die Chomsky (1993, 1995a) als artikulatorisch-perzeptives System (A-P) sowie als begrifflich-intentionales System (C-I) bezeichnet. Beide stehen ihrerseits über die ihnen zugeordneten Schnittstellensysteme der Phonetischen Form (PF) sowie der Logischen Form (LF) mit der Syntax in Verbindung. Schematisch ergibt sich das Grammatikmodell in (1):

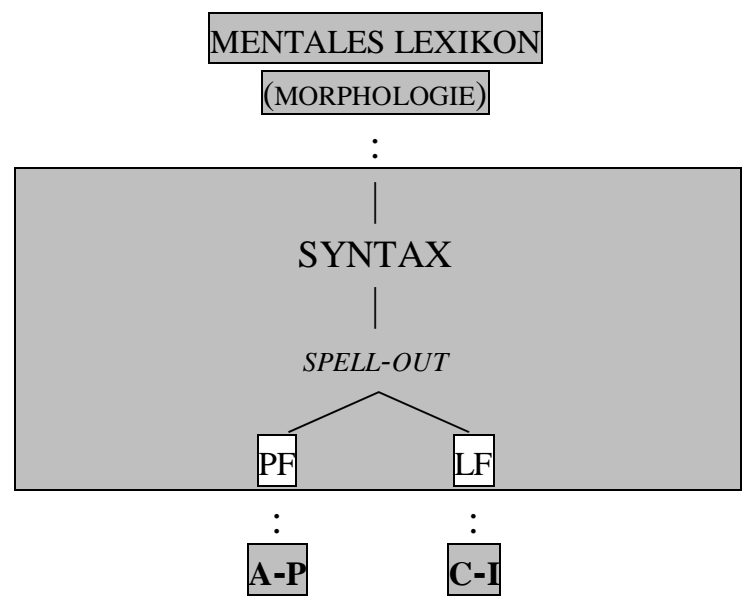

Freilich handelt es sich in vielen Punkten um eine Vereinfachung. Jedoch werden hier v. a. zwei Aspekte durchaus deutlich:

Zum einen handelt es sich um eine syntaktozentrische Architektur (vgl. u. a. Jackendoff 2010, 67), d. h. die Syntax als Verknüpfungsapparat steht im Mittelpunkt der Bildung und Verarbeitung von Satzstrukturen und damit Äußerungen. Sie ist vermittels spezifischer Schnittstellen mit anderen kognitiven Systemen bzw. Modulen verbunden, die zur Produktion und Rezeption sowie zur begrifflich-inhaltlichen Erfassung sprachlicher Zeichen bzw. Signale benötigt werden. Hierher gehört einerseits das Performanzsystem, das die 'physische' Produktion und Rezeption von Lautereignissen ermöglicht (“A-P”), sowie andererseits jenes mentale System, das dem Menschen die begriffliche bzw. konzeptuelle Erfassung und Verarbeitung von Bedeutungen und Interpretationen erlaubt (“C-I"). 
Ferner ergibt sich aus diesem Grammatikmodell, dass die Informationen aus dem mentalen Lexikon als Input die Grundlage einer jeder syntaktischen Derivation darstellen. Die Betrachtung des Baus und Funktionierens des mentalen Lexikons mit seinen Einheiten, den Lexikoneinträgen, ist somit eine grundsätzliche Notwendigkeit im Rahmen eines jeden Versuchs, morphosyntaktische Operationen formal zu erfassen und zu rekonstruieren.

Somit wird davon ausgegangen, dass Lexikon, Morphologie und Syntax jene Module sind, die den "inneren Zirkel” bzw. "Kern" des Sprachsystems bilden. Durch die erwähnten Schnittstellensysteme kommunizieren die aus der syntaktischen Derivation entstehenden Repräsentationen mit kognitiven Systemen, die dem Sprachsystem nur im weiteren Sinne angehören und so gewissermaßen dessen “äußeren Zirkel” bzw. seine "Peripherie” bilden. Das folgende Zitat aus Chomsky (1995a) entspricht diesen Ausführungen:

\begin{abstract}
A language consists of a lexicon and a computational system. The computational system draws from the lexicon to form derivations, presenting items from the lexicon in the format of $\mathrm{X}$-bar theory. Each derivation determines a linguistic expression, an SD [structural description], which contains a pair $(\pi, \lambda)[P F, L F]$ meeting the interface conditions. (Chomsky 1995a, 186)
\end{abstract}

So muss zunächst eine wenigstens grundsätzliche Theorie über die Beschaffenheit des mentalen Lexikons eingeführt werden, bei der es insbesondere um die Struktur und Rolle seiner Einheiten, der Lexikoneinträge, gehen muss. Ein weiteres Ziel muss darin bestehen, der Natur und dem Charakter der Argumentstruktur als Schnittstelle zwischen Lexikon und Syntax näher zu kommen und so das $\mathrm{Zu}$ sammenwirken der zuletzt genannten Module nachzuvollziehen.

Es zeigt sich, dass die Ausführungen zum mentalen Lexikon unmittelbar in solche zur Syntax übergehen bzw. deren Voraussetzung bilden. Hier müssen v. a. die der Verknüpfung lexikalischer Einheiten zugrunde liegenden Prinzipien geklärt werden, so etwa das "Format der X-Bar-Theorie", das im obigen Zitat bereits Erwähnung findet. Weiterhin müssen, soweit vonnöten, Annahmen zu morphologischen Operationen im Lexikon sowie zur Satzstruktur und zur syntaktischen Derivation formuliert werden, nach denen nachfolgend syntaktische Strukturen - wenn auch stets nur so weit wie jeweils notwendig - rekonstruiert werden. 


\subsection{Mentales Lexikon und Lexikoneinträge}

Grundlegend den Annahmen von Bierwisch (1983, 1990, 1996, 1997, 2007, 2009), Wunderlich (1997) und Zimmermann (1992, 2003a, 2003b, 2009) folgend, gehe ich davon aus, dass lexikalische Einheiten in Form von Lexikoneinträgen im mentalen Lexikon abgelegt sind.

Primär kommen Lexikoneinträge durch den Spracherwerb zustande, werden also inhaltlich sozusagen durch "Erfahrungswerte" befüllt, die v. a. der sprachliche Input des Lerners liefert. Diese Primärausstattung wird später (sobald die Ebene der "kreativen Morphologie" erreicht ist; vgl. u. a. Gagarina 2003; Gülzow \& Gagarina 2006) durch morphologische Ableitungen ergänzt bzw. begleitet, durch welche aus bestehendem lexikalischen Material mittels produktiver Analogbildungen neue lexikalische Einheiten deriviert werden (können). In diesem Sinne lässt sich ( $\mathrm{ab}$ dem Beginn der erwähnten Entwicklungsstufe) zwischen primärem und sekundärem Wachstum des mentalen Lexikons unterscheiden.

Die Lexikoneinträge beinhalten alle relevanten Informationen zur Bestimmung der lautlichen Gestalt der jeweiligen lexikalischen Einheit, ihrer kategorialen $\mathrm{Zu}$ ordnung ("Wortart"), Angaben zu ihrer Kombinierbarkeit mit anderen Einheiten (was gleichermaßen relevant für die Flexion, für Wortbildungsprozesse sowie für die syntaktische Projektion sein kann) und schließlich auch zu ihrer grammatisch determinierten (invarianten) Bedeutung. Im Folgenden wird dies anhand eines allgemeinen Musters für Lexikoneinträge exemplifiziert:

$$
\begin{array}{llll}
/ \ldots / & {[\alpha \mathrm{V}, \beta \mathrm{N}]} & \lambda x_{n} \ldots \lambda x_{1} & {\left[x_{1} \ldots x_{n}\right]} \\
-\mathrm{PF}- & -\mathrm{GM}- & -\mathrm{AS}- & -\mathrm{PAS}-
\end{array}
$$

Die Phonetische Form (PF) beinhaltet Informationen zur Lautgestalt des Lexems. Die grammatischen Merkmale (GM) sichern die Einordnung des Lexems in eine bestimmte (lexikalische oder funktionale) Kategorie. Hier erscheinen ferner auch wortartgebundene Finitheits- und/oder Kongruenzmerkmale, sofern eine flektierte Form gegeben ist. Damit entsprechen die GM im Prinzip dem, was Chomsky (1986) als "kategoriale Merkmale" bezeichnet.

Somit verbleiben Argumentstruktur (AS) und Prädikat-Argument-Struktur (PAS) als Teile von Lexikoneinträgen. Die PAS kann auch als "grammatisch determinierte Bedeutung" des Lexems charakterisiert werden, d. h. als diejenige Bedeu- 
tung, die unabhängig von außersprachlichen (konzeptuellen, pragmatischen, situativ-kontextuellen) Faktoren für das Lexem in invarianter Form Gültigkeit besitzt (siehe u. a. Zimmermann 2009, 485). In der hier verwendeten Notation wird die PAS als eine (mehr oder weniger detailliert dekomponierte) Funktor-ArgumentStruktur erfasst, die in aller Regel aus Basisprädikaten (primes = semantische Konstanten mit fixer konzeptueller Interpretation) und Individuenvariablen (Argumentpositionen) zusammengesetzt ist. Für Verben, zu deren Natur der Bezug auf Sachverhalte in der außersprachlichen Welt (Realität) gehört, wird seit Davidson (1967a) neben den üblichen Argumentvariablen zudem eine Situationsvariable angenommen. Dabei kann es sich - je nach theoretischem Modell und Verbtyp - um ein allgemeines Sachverhaltsargument oder um spezielle Subtypen von Sachverhaltsargumenten (z. B. ein Zustands- oder Ereignisargument) handeln. Higginbotham (1985) spricht hier von einem "hidden argument". In einer bestimmten Phase der Satzstrukturbildung wird diese Argumentstelle existenziell (ab)gebunden, was als Entsprechung der grammatischen Kategorie des Verbmodus betrachtet werden kann (vgl. u. a. Zimmermann 2009). Mit Bierwisch (1988) gehe ich davon aus, dass die Sachverhaltsvariable durch den Instanziierungsfunktor INST mit der Proposition des Verbs (der PAS) verbunden bzw. in Relation gesetzt wird. INST weist den Sachverhalt, auf den durch den Gebrauch des Verbs verwiesen wird, demnach als eine "Instanz" der betreffenden verbalen Proposition aus. So wird die Proposition, die ein Verb von sich aus in lediglich allgemeiner Form bezeichnet, auf einen konkreten Sachverhalt in der außersprachlichen Welt bezogen. Ein verbaler Lexikoneintrag hat somit die generelle Form in (3):

$$
/ \ldots / \quad[+\mathrm{V},-\mathrm{N}] \quad \lambda x_{n} \ldots \lambda x_{1} \lambda s \quad\left[s \operatorname{INST}\left[x_{1} \ldots x_{n}\right]\right]
$$

In der AS, die hier im Prinzip als Schnittstelle zwischen mentalem Lexikon und Syntax betrachtet wird (vgl. u. a. Bierwisch 2009, 288, 292), sind Anzahl und hierarchische Position der Argumente einer lexikalischen Einheit (hier eines Verbs) fixiert. Die Individuenvariablen $x_{\mathrm{n}} \ldots x_{1}$ in der PAS stellen die Argumentpositionen des jeweiligen Lexems dar (vgl. u. a. Bierwisch 1990, 177; Steube 1997, 222). Diese Variablen können durch Operatoren gebunden werden, wodurch die spätere syntaktische Projektion der Argumente (prä-)determiniert wird. Im hier verwendeten Modell werden $\lambda$-Abstraktoren verwendet, um die Argumentpositionen in der PAS zu binden und auf diese Weise syntaktifizierbare Argumentstellen 
als Teile der AS zu "erzeugen". Es ist die derart beschaffene AS, auf die die Syntax zugreift bzw. die von der Syntax "gesehen" wird, wenn es zur Projektion der Argumente einer lexikalischen Einheit (etwa einer Verbform) kommt. ${ }^{90}$

Somit kommt die "semantische Selektion" (Chomskys s-selection) eines Lexems auf der Basis der Bindung von Variablen in der SF durch $\lambda$-Abstraktoren zustande. Die thematischen Relationen zwischen Argument(position)en und verbalem Prädikat ergeben sich aus der relativen Position der betreffenden Variablen in der (dekomponierten) PAS, die sich in der Abfolge der Argumentstellen in der AS in umgekehrter Reihung spiegelt. Das bedeutet, dass die Hierarchie der Argumentstellen innerhalb der AS mit der Einbettungstiefe der Argumentpositionen in der PAS in engem Zusammenhang steht. "The more to the left an abstractor is in the so-called lambda prefix [in der AS], the lower is the position of the argument that it binds in the hierarchy [in der PAS]" (Junghanns 2008, 3).

Argumentstellen (in der AS) können mit morphosyntaktischen Merkmalen versehen sein, die als "lexikalische Adressen" oder Annotationen bezeichnet werden. Durch derartige Annotationen werden Anforderungen an die morphosyntaktische Realisierung des betreffenden Arguments fixiert, sofern sie nicht durch generelle Prinzipien ohnehin determiniert werden und somit vorhersagbar bzw. "strukturell" sind. Sie erscheinen demnach nur im Falle idiosynkratisch geforderter Realisierungsformen. So entsprechen sie teilweise der "Subkategorisierung", wie sie von Chomsky (1965) angenommen wird, d. h. der Determinierung der Form von Argumenten, die eine lexikalische Einheit 'regiert' bzw. 'akzeptiert' (siehe auch Bierwisch 2009, 284). Die Subkategorisierung ist in Lexikoneinträgen folglich jeweils durch die AS repräsentiert. Bei Argumentstellen, die nicht in dieser Form annotiert sind, ist die Realisierungsform der betreffenden Argumente strukturell prädikabel bzw. muss generellen (sprachspezifischen oder universellen) Prinzipien folgen.

Unter Annahme derart beschaffener Lexikoneinträge sind alle für die Lautform, Kategorie, das Projektionsverhalten sowie für die grammatisch determinierte Bedeutung relevanten Informationen einer lexikalischen Einheit im mentalen Lexikon verortet. Diese Annahmen bedeuten für die vorliegende Arbeit eine explizit lexikalistische Sichtweise, der Positionen gegenüberstehen, denen zufolge man

90 Sofern eine Argumentposition in der PAS von keinem $\lambda$-Abstraktor gebunden wird, kann das entsprechende Argument nicht syntaktifiziert, sondern lediglich interpretiert werden. Bei solchen freien Argumentstellen wird auch von "semantischen Parametern" gesprochen. 
Teile der Argumentstruktur und Semantik von Lexemen aus dem mentalen Lexikon in die Syntax verlagern (siehe u. a. Borer 2005) oder auch 'nur' die Argumentstruktur aus Lexikoneinträgen ausschließen könne (vgl. u. a. Dölling 2005a; Dölling \& Heyde-Zybatow 2007). Eine solche Sichtweise führt m. E. jedoch zur partiellen Überlappung bzw. "Verwischung" grammatischer Module respektive sprachlicher Domänen. Da ich in einer solchen Überlappung einen theoretischen Nachteil sehe bzw. eine klare Aufgabenteilung der Module annehmen will, soll die Syntax hier als dasjenige Modul gelten, welches auf der Basis einer möglichst minimalen Zahl von Prinzipien die Verknüpfung lexikalischer Einheiten bewerkstelligt. Eine solchermaßen klare Arbeitsteilung wird durch die dargelegten Annahmen gewährleistet.

\subsection{Morphosyntaktische Merkmale}

\subsubsection{Wortarten}

Ich werde von der mittlerweile quasi ‘traditionellen' generativen Wortartenlehre abweichen, da ich die Kategorisierung von 'normalen' (primär attributiv verwendeten) Adjektiven als 'verbal und nominal' $[+\mathrm{V},+\mathrm{N}]-$ mindestens für die betrachteten Sprachen - für unangemessen halte. ${ }^{91}$ Adjektive und Substantive bilden in diesen Sprachen seit jeher eine natürliche Klasse, die sich v. a. in ihrer Flexion und damit in ihrer 'Nominalität' äußert. ${ }^{92}$

91 Während ich hinsichtlich der kategorialen Merkmale diese Trennung von der traditionellen Einteilung konsequent befolge, bleibe ich zum Zwecke der Darstellung und des besseren Verständnisses im Fließtext sowie in den Baumdiagrammen bei den traditionellen Bezeichnungen. So werde ich sowohl bei Kurz- als auch bei Langformen mitunter einfach von 'Adjektiven' sprechen, und ebenso werden Infinitive und Partizipien meist nach wie vor einfach als 'Verbformen' bezeichnet. Diese 'Inkonsequenz' wird aus lexikalistischer Sicht dadurch relativiert, dass die diversen Formen eines Lexems (z. B. dobra $_{\mathrm{KF}}$ und dobraja $_{\mathrm{LF}}$ 'gut' / čitaju $u_{1 . \mathrm{SG}}$ und čitat' ${ }_{\mathrm{INF}}$ 'lesen') auf Grund ihrer gemeinsamen Wurzel bzw. ihres gemeinsames Stammes zusammengehören, und zwar ungeachtet der Kategorie, der sie durch bestimmte Suffixe letzten Endes angehören (also z. B. $\operatorname{dobra}_{[+\mathrm{V},+\mathrm{N}]}$ vs. dobraja $a_{[-\mathrm{V},+\mathrm{N},+\mathrm{a}]}$ 'gut' / čitaju $u_{[+\mathrm{V},-\mathrm{N}]}$ vs. čitat ${ }_{[+\mathrm{V},+\mathrm{N}]}$ ).

92 Im Altkirchenslavischen flektieren Substantive und (Kurzform-)Adjektive noch identisch nach Kasus, Numerus, Genus; vgl. die $o$-stämmigen Singularbeispiele in (i) und (ii):

(i) N $r a b-\imath, \mathrm{G} r a b-a, \mathrm{D} r a b-u$, A $r a b-b$, I $r a b-o m b, \mathrm{~L} r a b-\check{e} \quad$ 'Knecht' (Substantiv)

(ii) N dobr-b, G dobr-a, D dobr-u, A dobr-b, I dobr-omb, L dobr-ě 'gut' (Adjektiv)

Von diesen "nominal" flektierenden Kurzformen werden die Langformen durch Anfügen eines

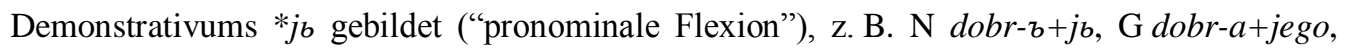
D dobr-u+jemu usw. (siehe Trunte ${ }^{5} 2005$, 38-40). Man nimmt an, dass sie Definitheit aus- 
Ebensowenig adäquat ist $\mathrm{m}$. E. die Kategorisierung infiniter Verbformen als rein 'verbal' $([+\mathrm{V},-\mathrm{N}])$. Hier sollte auf die 'Mischkategorie' $[+\mathrm{V},+\mathrm{N}]$ zurückgegriffen werden, die ich 'normalen' Adjektiven soeben abgesprochen habe. Dies kommt auch der verbreiteten Ansicht entgegen, dass Partizipien sowohl an der verbalen also auch an der nominalen Kategorie teilhaben. ${ }^{93}$ Auch der Infinitiv hat mindestens nominale Wurzeln (vgl. Bielfeldt 1961, 235). Eine Sonderstellung nehmen die (primär im Russischen noch anzutreffenden) Kurzform‘adjektive' ein, die nurmehr prädikativ verwendbar sind. Sie haben m. E. die gleiche Kategorie wie Partizipien $-[+\mathrm{V},+\mathrm{N}]$. Es handelt sich, mit anderen Worten, um keine 'normalen' Adjektive (mehr - diese Rolle haben heute die Langformen), sondern um Verbalnomina (vgl. ähnlich Geist 2006, 2010).

In Aufstellung (4) nenne ich die möglichen Kombinationen der angesetzten lexikalischen Merkmale $[ \pm \mathrm{V}]$ 'verbal' ${ }^{94}$ und $[ \pm \mathrm{N}]$ 'nominal' ([ $\left.\pm \mathrm{a}\right]$ 'adjektivisch' bezeichnet eine nominale Subkategorie) ${ }^{95}$

drückten, die Kurzformen dagegen nicht. In vielen slavischen Sprachen wurde später eine der Bildeweisen präferiert (im Polnischen die Langform). Im Russischen haben sich beide Typen gehalten, jedoch sind Kurzformen heute auf den prädikativen Gebrauch beschränkt. Ich werde sie daher anders kategorisieren als die 'echt-adjektivischen' Langformen.

93 Partizipien haben als $[+\mathrm{V},+\mathrm{N}]-$ Einheiten "Kurzformendungen”, z. B. russ. pisal-a, napisan- $a$ 'geschrieben'. Nach einer möglichen Konversion zu einem 'normalen' Adjektiv können sie "Langformendungen" erhalten, z. B. napisann-aja. Die Partizipien Präs. sowie Prät. Aktiv werden nicht prädikativ, sondern nur attributiv verwendet; vgl. pišušč-aja (*pišušč-a) 'schreibende', napisavš-aja (*napisav̌s-a) 'geschrieben habende'. Zum jeweiligen Verblexem gehören Partizipien (wie Infinitive) trotz ihrer verbalnominalen Merkmalsauszeichnung $[+\mathrm{V},+\mathrm{N}]$ auf Grund dessen, dass sie - wie alle anderen Formen des Lexems - von einem Verbstamm abgeleitet sind.

94 Da ich Aspekt als lexikalisch betrachte, da er also bei Verben in der Syntax bereits vorhanden und spezifiziert ist, setze ich ihn hier voraus. Jede (rein) verbale Form hat immer auch ein Aspektmerkmal ([ \pm Perf $])$.

95 Fett markiert sind die ausschlaggebenden (eigentlich identifizierenden) Merkmale.

Präpositionen, die oft als lexikalisch klassifiziert werden, betrachte ich als funktional bzw. als lexikalisch-funktionalen Hybriden. Es wirkt irrelevant, ob für sie rein negative lexikalische Merkmale $([-\mathrm{V},-\mathrm{N}])$ oder ein funktionales Merkmal (z. B. [+P]) angesetzt werden. Außer Zweifel steht, dass Präpositionen eine geschlossene Klasse bilden und Ähnlichkeiten mit Konjunktionen haben $([+\mathrm{C}])$, die stets als funktional klassifiziert werden.

Womöglich kann das subkategorisierende Merkmal [ \pm a] bei den Nomina auch durch das von Wunderlich (1996) angesetzte Merkmal [ \pm dep] 'referenziell abhängig' ausgetauscht werden. 
(4)

\begin{tabular}{|c|c|c|}
\hline$[+\mathbf{V},-\mathrm{N}]$ & Verben & reine Verba \\
\hline$[+\mathrm{V},+\mathrm{N}]$ & Infinita (Kurzformen) & Verbalnomina \\
\hline$[-\mathrm{V},+\mathbf{N},-\mathrm{a}]$ & Substantive & \multirow{2}{*}{ reine Nomina } \\
\hline$[-\mathrm{V},+\mathrm{N},+\mathbf{a}]$ & Adjektive (Langformen) & \\
\hline
\end{tabular}

\subsubsection{Finita und Infinita}

Sehen wir uns im nächsten Schritt die Verben genauer an. Reine Verba sind im vorliegenden Modell lediglich die 'finiten' Verbformen, unter denen ich solche verstehe, die von sich aus Modus und Tempus ausdrücken. Nur diese Verbformen haben eine Person-/Numerus-Spezifikation, vgl. (5): ${ }^{96}$

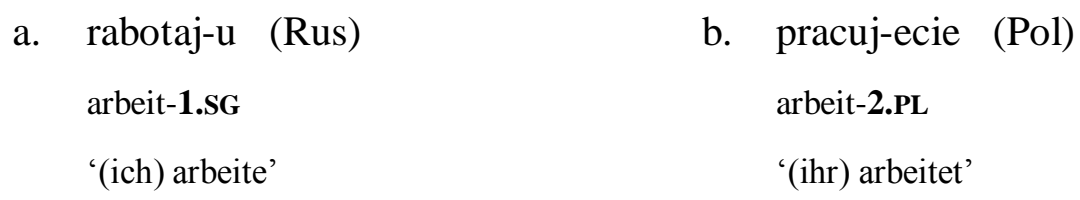

Da die 'finiten' Verbformen im Nordslavischen stets die Merkmale Indikativ und Präsens vertreten, ist es m. E. überflüssig, für letztere überhaupt Merkmale anzusetzen. ${ }^{97}$ Folglich ist eine Verbform finit, wenn sie 'voll flektiert' bzw. 'persönlich' ist. ${ }^{98}$ So kann der Gegensatz zwischen Finita und Infinita als Gegensatz

96 Das eigentliche Finitheitsmerkmal ist die grammatische Person. Isačenko (1962) spricht von einer prädikativen Kategorie. Der Numerus sei “[...] bei den finiten Verbalformen keine selbständige Kategorie. Er ist an den Begriffsinhalt der grammatischen Person gebunden. Persönliche Verbalformen kongruieren im Numerus mit dem Subjekt" (Isačenko 1962, 279-280).

97 Ich nehme also kein [ \pm Past]-Merkmal an. Das heißt nicht, dass dieses Merkmal absolut nonexistent wäre. Vielmehr ist es im Polnischen und Russischen mit dem Verlust der alten synthetischen Tempusformen Aorist und Imperfekt verloren gegangen. M. E. führte dieser Verlust dazu, dass nur noch das Präsens synthetisch gebildet wurde, übernahm doch die ursprüngliche Perfektperiphrase die Funktion eines "Globalpräteritums". Neben den relativ zahlreichen Infinita gibt es mit der "Präsensform" also nur noch eine finite Form (auch die stets finiten I-Auxiliare sind formal "Präsensformen"; siehe Abschnitt 4.6.2).

Ich sehe vom Imperativ ab, dessen Formen als Satzmodusmarker hier keine Rolle spielen. Sie können in mein System als Träger eines Submerkmals [+Imp] integriert werden, womit sie markierter als "Präsensformen" wären (vgl. hierzu u. a. Zimmermann 2009, 489-490).

98 Dass die Infinita keine Person-Spezifikation haben können (und damit auch keine Modus- und Tempusmarkierung), lässt sich im vorliegenden Modell auf ihre teilweise Nominalität bzw. (dadurch gleichsam) verminderte Verbalität zurückführen. 
zwischen $[+\mathrm{V},-\mathrm{N}]-$ und $[+\mathrm{V},+\mathrm{N}]-$ Formen modelliert werden. Nur Finita sind demnach reine Verba, während Infinita Verbalnomina sind: ${ }^{99}$

(6) a. $[+\mathrm{V},-\mathrm{N} \ldots]$

b. $[+\mathrm{V},+\mathrm{N} \ldots]$

Ferner soll mit Zimmermann (2013) gelten, dass Finita Person und Numerus in Bezug auf ihre externe Argumentstelle spezifizieren. Das heißt, dass die Personund Numerusmerkmale nicht unmittelbar zur Verbform gehören, sondern als Annotation zu deren ranghöchster (externer) Argumentstelle. Als solche stellen sie Anforderungen an den einzusetzenden Subjektausdruck (vgl. Zimmermann 2013, 221). Folglich sind bei einer finiten Verbform immer Person und Numerus als Annotation an der ranghöchsten Argumentstelle vorhanden, was mit deren Fähigkeit korreliert, einen Subjektausdruck zu realisieren: ${ }^{100}$

$$
[+\mathrm{V},-\mathrm{N} \ldots+\max ] \quad \ldots \lambda x_{[ \pm 1, \pm 2, \pm \mathrm{PI}]} \lambda s[s \operatorname{INST}[\ldots x \ldots]]
$$

Infinite Verbformen als Verbalnomina $([+\mathrm{V},+\mathrm{N}])$ haben keine solche Annotation und können das Argument daher nicht ohne Weiteres realisieren:

$$
[+\mathrm{V},+\mathrm{N} \ldots+\max ] \quad \ldots \lambda x \lambda s[s \operatorname{INST}[\ldots x \ldots]]
$$

\section{Infinitive und Partizipien}

Der Unterschied zwischen Infinitiven und Partizipien kann über das Wortebenenmerkmal $[ \pm \max ]$ erfasst werden: Während das Infinitivsuffix (russ. $\left\{t^{\prime}\right\}$, poln. $\left\{c^{\prime}\right\}$ ) eine 'maximale' $[+\mathrm{V},+\mathrm{N}]-$ Wortform deriviert, führt das Anfügen eines Partizipial-

99 Ich nehme an, dass verbale Wurzeln bzw. Stämme die Merkmale [+V,-N] haben, also 'echtverbal' sind. Erst durch das Antreten der Infinitiv- oder Partizipmorphologie wird diese Merkmalskombination überschrieben und $\mathrm{zu}[+\mathrm{V},+\mathrm{N}]$. Wie schon weiter oben bemerkt, ergibt sich die Zusammengehörigkeit der finiten $([+\mathrm{V},-\mathrm{N}])$ und infiniten $([+\mathrm{V},+\mathrm{N}])$ Formen $\mathrm{zu}$ einem Lexem aus der ihnen gemeinsamen Basis (Wurzel/Stamm). Aus diesem Grund kann man auch sagen, dass Infinitive und Partizipien deverbale Verbalnomina sind - ihre Basis ist $[+\mathrm{V},-\mathrm{N}]$ (dagegen sind die ebenfalls verbalnominalen Kurzform 'adjektive' (eher) denominal, denn ihre Wurzeln sind $\left[\alpha \mathrm{V},+\mathrm{N},(+\mathrm{a})_{\alpha}\right]$; siehe Abschnitt 4.4.3).

100 " $[ \pm$ max $]$ " zeigt an, ob eine Form voll flektiert ist oder nicht (vgl. u. a. Zimmermann 2003b). 
suffixes nicht direkt zu einer voll flektierten Form (hier ist noch ein Kongruenzmarker ([+Agr]) hinzuzufügen):

(9) i. $[+\mathrm{V},+\mathrm{N},+\max ]$

(Infinitiv)

ii. $[+\mathrm{V},+\mathrm{N},-\max ]$

(Partizip)

Unter den Infinita sind die Infinitive folglich am wenigsten markiert. Die Partizipien haben dagegen Kongruenzmarker für Numerus und Genus. ${ }^{101}$ Auf die Kurzform'adjektive', die den Partizipien in vielerlei Hinsicht ähneln, jedoch als grundlegend eher nominale Formen keinen eigenen Sachverhaltsbezug besitzen, werde ich weiter unten zurückkommen.

\subsubsection{Aktiv und Passiv}

In russischen und polnischen Modus-/Tempusperiphrasen werden sowohl passivische ( $n / t$ - und $m$-Partizip ${ }^{102}$ ) als auch aktivische (Infinitiv, $l$-Partizip, Kurzform) Infinita bzw. Verbalnomina gebraucht. "Aktiv" und "Passiv" sind dabei m. E. keine morphosyntaktischen, sondern argumentstrukturelle 'Größen'. Ihnen entspricht in der Argumentstruktur die 'Unbelassenheit' ("Aktiv") oder 'Ausblendung' ("Passiv") der ranghöchsten syntaktifizierbaren Argumentstelle. Als Passivierungsinput eignen sich Transitiva, da sie über ein internes Argument verfügen, das zum syntaktischen Subjekt 'befördert' werden kann, sobald das externe Argument 'ausgeblendet' ist. ${ }^{103}$ Unter 'Ausblendung' verstehe ich mit Fehrmann, Jung-

101 Sätze mit Adverbialpartizipien (Gerundien) werden m. E. präpositional eingeleitet. Die Adverbialpartizipien sind eine relativ junge Wortform, deren heutige Gestalt auf ursprünglich nominativische Formen regulärer Partizipien zurückgeht (vgl. Eckert, Crome \& Fleckenstein 1983, 178). Letztere modifizierten Nominativausdrücke, was ihre heutzutage präskriptiv geforderte Festlegung auf Koreferenz mit dem Matrixsubjekt befördert haben dürfte (vgl. Růžička 1999, 12). Semantisch etablieren sie adverbiale Relationen zwischen Zeiten/Sachverhalten, was für ihren präpositionalen Charakter spricht (siehe auch Abschnitt 5.3.2.2).

${ }^{102}$ Das $m$-Partizip (Präs. Pass.) liegt nur im Russischen vor, wohin es “[...] als eine mehr oder weniger künstliche Form [...] aus dem Altslavischen übernommen worden [...] [ist]. Die Form auf -мый ist eine ausgesprochene Buchform geblieben; anderen slavischen Sprachen, die dem Einfluß des Kirchenslavischen weniger ausgesetzt waren, ist sie so gut wie völlig fremd.” (Isačenko 1962, 337, § 198).

103 Das schließt nicht aus, dass auch bei Intransitiva eine 'Ausblendung' der ranghöchsten Argumentstelle erfolgen kann, was jedoch keine 'Passivierung” i. e. S. bewirkt, sondern 'nur' die 
hanns \& Lenertová (2010, 219-220) die Ersetzung des ranghöchsten Arguments durch eine ungebundene "Dummyvariable" $z:{ }^{104}$

(10) a. $\quad \lambda y \lambda x \lambda s[s$ INST $[\ldots x \ldots y \ldots]]$

(“Aktiv")

b. $\lambda y \quad \lambda s[s$ INST $[\ldots z \ldots y, \ldots]]$

("Passiv")

Was das $n / t$-Partizip betrifft, so ist zu betonen, dass es in beiden Sprachen als Prädikat von Passivsätzen aller Verbmodi und Tempora verwendet wird. Es ist somit kein "Partizip Präteritum Passiv", sondern vielmehr lediglich ein Partizip mit passivierender Wirkung auf die Argumentstruktur, das keine temporale Festlegung hat (ebenso wie das $m$-Partizip oder das aktivische $l$-Partizip). Die "Passivierung" im o. g. Sinne geht hier auf das Suffix $\{n / t\}$ zurück, das auch die Merkmale $[+\mathrm{V},+\mathrm{N},-\max ]$ einbringt. Die Modus-/Tempus-Spezifikation folgt nicht aus der Partizipmorphologie, sondern aus demjenigen I-Kopf, der das Partizip selegiert und ggf. von ausbuchstabierenden V-Auxiliaren 'sichtbar gemacht' wird (siehe Abschnitt 5.2).

Während das n/t-Partizip im Polnischen regulär von Verbstämmen beider Aspekte gebildet wird, konzentriert sich seine Bildung im Russischen heute auf Perfektiva. ${ }^{105}$ Andererseits besitzt das Russische mit dem $m$-Partizip ein zweites Passiv-

nonkanonische oder Nicht-Realisierung des betreffenden Arguments. Abschnitt 5.3.2 zeigt, dass eine 'Ausblendung' auch bei der Sekundärprädikation erfolgt.

104 "The variable $z$, representing the blocked external argument, is not yet existentially bound, therefore, a by-phrase is possible (e. g., njan'koj 'by the nanny'). At CS, $z$ is either interpreted as coreferential with the referent of the $b y$-phrase, or it is existentially quantified per default if no $b y$-phrase is realized. If a $b y$-phrase is realized, it is necessarily referentially identified with the blocked external argument.” (Fehrmann, Junghanns \& Lenertová 2010, 220).

105 Die Bindung des Verbalaspekts an den Partiziptyp folgt im Russischen normativen Vorgaben, die aber mindestens für die Standardsprache als soweit gefestigt gelten müssen, dass ein Verstoß gegen sie als Verletzung der grammatischen Wohlgeformtheit (nicht lediglich der Norm) aufgefasst wird. In älteren Sprachzuständen konnten im Russischen noch alle Partizipien von jeweils beiden Aspekten gebildet werden. Bis heute hat sich die Möglichkeit der Bildung imperfektiver $n / t$-Partizipien für eine Reihe von Verblexemen erhalten, vgl. z. B. bit/-yj 'geschlagen' (vgl. Isačenko 1962, 343). Was letztere betrifft, so spricht Schoorlemmer von marginalen Erscheinungen, die "do not affect the grammatical system as such" (Schoorlemmer 1995, 226). Borik (2012, 116-117) relativiert diese Sicht, indem sie zeigt, dass die imperfektiven Passivpartizipien relativ oft prädikativ auftreten (wenngleich deutlich häufiger attributiv). Sie folgert, dass der Aspekt offenbar weder eine hinreichende noch notwendige Bedingung für die Bildung der $n / t$-Partizipien ist. Als notwendige (jedoch nicht hinreichende) Voraussetzung für ihre Bildung betrachtet sie Transitivität (Borik 2012, 118). 
partizip, das ausschließlich vom imperfektiven Aspekt gebildet wird (Isačenko 1962, 337). In diesem Sinne könnte man sagen, das Russische besitze sowohl ein perfektives als auch ein imperfektives Passivpartizip. Da das $m$-Partizip jedoch nur sehr selten zum Ausdruck des Passivs verwendet wird, und da das $n / t$-Partizip in bestimmten Fällen auch von imperfektiven Verben gebildet wird (vgl. Borik 2012, 116-117), scheint diese Aussage zu generell. Zwar entspricht die Passivperiphrase mit $m$-Partizip - v. a. auf Grund des stets imperfektiven Aspekts an diesem Partizip - einem deutschen "Vorgangspassiv", vgl. (11):

\footnotetext{
(11) Ivan byl uvažaem.

Ivan-NOM war-SG.M geachtet IMPF-MPT-SG.M

'Ivan wurde geachtet.'
}

Jedoch wird diese Periphrase im heutigen Russischen kaum noch verwendet. Die dominierende Ausdrucksform für ein imperfektives "Vorgangspassiv" ist das "Reflexivpassiv", in dem eine imperfektive Verbform mit dem Reflexivmarker -sja verwendet wird (vgl. zur Wirkungsweise des Reflexivmarkers Fehrmann, Junghanns \& Lenertová 2010, 210-211):
(12) Kniga čitaetsja.
Buch-NOM les IMPF-3.SG-REFL
'Das Buch wird gelesen.' (Rus: "Reflexivpassiv")

In diesem Sinne sind die russischen $m$-Partizipien, die zudem nicht von allen Verblexemen gebildet werden können (vgl. etwa *pišem/-yj von pisat' 'schreiben'), als marginal zu werten (weitaus häufiger treten sie als lexikalisierte Attribute mit modalem Bedeutungsanteil auf, z. B. nerušimyj 'unzerstörbar'). Was man heute de facto vorfindet, ist also einerseits das perfektive partizipiale Passiv mit $n / t$-Partizip und andererseits das imperfektive "Reflexivpassiv". 106

\footnotetext{
106 Der Unterschied zwischen "Vorgangs-" und "Zustandspassiv" kann m. E. im Polnischen und Russischen einerseits auf der Basis des Aspekts am Partizip oder andererseits durch eine "Resultativierung" desselben entstehen (siehe auch Fn. 180 in Abschnitt 5.2.3). Hinsichtlich des ersteren Falls ist zu beachten, dass ein perfektives Passiv interpretativ zwar eher einem "Zustands-" als einem "Vorgangspassiv" entspicht, mit diesem jedoch nicht deckungsgleich ist.
} 


\subsection{4 "l-Formen"}

Auch auf den Status der " $l$-Formen" muss eingegangen werden. Die " $l$-Form" spielt als die Verbform für aktivische Periphrasen in der Grammatik slavischer Sprachen seit jeher eine zentrale Rolle. Ich gehe davon aus, dass der Status der "l-Formen" im Russischen und Polnischen insofern gleich ist, als es sich um infinite (verbalnominale) Partizipien handelt. Das waren sie bereits im (Spät-)Urslavischen (vgl. u. a. Trost 1968). Zusammen mit Formen des Präterital- bzw. Konjunktivauxiliars (I-Auxiliare; siehe Abschnitte 4.6.2, 4.6.3 und 5.2.4) dienen sie dem periphrastischen Ausdruck der entsprechenden Modi und Tempora. Sie sind aber in diesen Periphrasen - und zwar sowohl im Polnischen als auch im Russischen - infinit und ohne spezifische grammatische Eigenbedeutung. Mit anderen Worten, es handelt sich nicht um (finite) "Präteritalformen" oder gar “Konjunktivformen". ${ }^{107}$ Vielmehr markiert das Suffix $\{l\}$ die Verbalnominalität bzw. Partizipialität der Form. Argumentstrukturelle Änderungen führt dieses Suffix nicht durch (es ist aktivisch). Seine Merkmale $[+\mathrm{V},+\mathrm{N} \ldots]$ werden von den erwähnten I-Auxiliaren mit der Präteritum- bzw. Konjunktivsemantik selegiert, so dass die Periphrasen mit den entsprechenden Bedeutungen entstehen. ${ }^{108}$ Ich möchte anmerken, dass sich Infinitive und $l$-Partizipien - insofern beide aktivisch sind und in Periphrasen eingesetzt werden - einigermaßen ähnlich sind. So wirken $l$-Partizipien wie "kongruierende Infinitive", durch deren Einsatz das mögliche Spektrum aktivischer Periphrasen im Slavischen weit über den Infinitiv hinaus erweitert wurde und wird. ${ }^{109}$

107 Andere Vorschläge bringen (i) Sørensen (1975) und (ii) Schümann (2010). Sie nehmen an, die russische " $l$-Form" habe (i) eine Distanz- bzw. (ii) eine Negationssemantik, die gleichermaßen den präteritalen ('Distanz zur Äußerungszeit' bzw. ' $\neg$ Präsens') und konjunktivischen ('Distanz zur aktuellen Welt' bzw. ' $\neg$ Realis') Gebrauch erklären könne.

${ }^{108}$ Zur Gewährleistung einer eindeutigen Selektionsbeziehung zwischen I-Auxiliar und $l$-Partizip schließe ich nicht aus, dass das Suffix $\{l\}$ ein identifizierendes Zusatzmerkmal [+L] einbringt, auf das das I-Auxiliar 'anspringt'. Wenn man auf ein solches Zusatzmerkmal verzichtet, ist davon auszugehen, dass Periphrasen auf usueller Basis gebildet werden. Als (diachrone) Evidenz dafür können (a) die generelle Instabilität bzw. Wandelbarkeit und (b) die relative Zufälligkeit in der Zusammensetzung von Periphrasen gelten.

109 Bezeichnenderweise kann im polnischen periphrastischen Futur ohne Bedeutungsunterschied neben dem Infinitiv auch das $l$-Partizip verwendet werden:

(i) Anna będzie pracować/pracowała.

Anna AUX arbeit-INF/arbeit-LPT.SG.F

'Anna wird arbeiten.' 


\subsection{5 "Kurzformen"}

Wie weiter oben angedeutet wurde, behandele ich die v. a. im geschriebenen Russischen noch gebräuchlichen "Kurzformadjektive" anders als ihre "langförmigen" Entsprechungen. Letztere gelten mir synchron als 'normale' bzw. 'typische' Adjektive mit der Merkmalsauszeichnung $[-\mathrm{V},+\mathrm{N},+\mathrm{a}]$, innerhalb derer das subkategorisierende Merkmal [+a] sie von den Substantiven absetzt (siehe auch folgender Abschnitt). Als 'typisch adjektivisch' gelten mir die Langformen v. a. deshalb, weil sie über das volle Kasusparadigma verfügen, auf dessen Basis sie NPn mit beliebigen Merkmalskombinationen attributiv modifizieren können.

Auf der anderen Seiten haben Kurzformen - ebenso wie (prädikative) Partizipien - lediglich nominativische Flexionsformen. Ich führe dies nicht darauf zurück, dass sie "kasuslos" wären, sondern darauf, dass sie nicht (mehr) nur reine Nomina, sondern Verbalnomina mit den Merkmalen $[+\mathrm{V},+\mathrm{N}]$ sind. Diese Merkmalskombination mit verbalem Anteil macht sie - ebenso wie die "kurzförmigen" Partizipien - zu explizit prädikativen Formen:

(13) a. $[-\mathrm{V},+\mathrm{N},+\mathrm{a} \ldots]$

( "Langform")

b. $[+\mathrm{V},+\mathrm{N} \ldots]$

("Kurzform")

Im Weiteren vereinfache ich die Merkmalsnotation, indem ich nur positive Merkmale angebe. Sie genügen zur Identifikation, wenn man voraussetzt, dass nicht angegebene Merkmale negativ oder ganz abwesend sind: ${ }^{110}$
a. $[+a \ldots]$
b. $[+\mathrm{V},+\mathrm{N} \ldots]$

(“Langform")

("Kurzform")

Auf das unterschiedliche Verhalten von "Qualitätsadjektiven" und "Beziehungsadjektiven" in Bezug auf die Bildbarkeit von Kurzformen komme ich im folgen-

\footnotetext{
Von dieser Kombination aus bud-Auxiliar und l-Partizip, die auf ein ursprüngliches Futurum exactum zurückgeht (Stichwort 'Wandelbarkeit von Periphrasen'), macht das Russische heute keinen Gebrauch.

${ }^{110}$ Die Angabe des Merkmals [+N] in (14a) erübrigt sich, da [+a] eine nominale Subkategorie ist. Es gilt also die Regel “wenn [+a], dann [+N]” (nicht aber umgekehrt!).
} 
den Abschnitt zu sprechen. An dieser Stelle sei lediglich vermerkt, dass die (aus diachroner Sicht) "pronominalen” Kongruenzmarker, die an Langformen auftreten (egal, ob es sich um 'echte' Adjektive oder um Adjektivierungen handelt), Wurzeln oder Stämme mit dem Merkmal [+a] selegieren, während die "nominalen" Marker, die sich auch an nominativischen Formen von Substantiven sowie an prädikativen Partizipien finden, die Merkmale $[+\mathrm{V},+\mathrm{N}]$ bzw. $[-\mathrm{V},+\mathrm{N}]$ fordern. Als Illustration nenne ich die Lexikoneinträge der Kongruenzmarker $\{-a j a\}$ und $\{-a\}$, die beide für Nom. Sg. f. stehen (mögliche Synkretismen blende ich aus): ${ }^{11}$
a. laja/ $[\mathrm{NOM},+\mathrm{Fem},+\max ] \quad \lambda P_{[+\mathrm{a}]}[P]$
(“Langformendung")
b. /a/ $\quad[\mathrm{NOM},+\mathrm{Fem},+\max ] \quad \lambda P_{[ \pm \mathrm{V},+\mathrm{N}]}[P]$
(“Kurzformendung”)

Vorteilhaft an der hier vorgestellten Merkmalsarchitektur ist, dass man für die seit jeher "nominalen" Suffixe auch nominale Selektionsmerkmale ansetzen kann. Im 'traditionellen' Modell müsste das Suffix $\{-a\}$ sowohl an nominale (Substantive, Kurzformadjektive) als auch an verbale Formen (Partizipien) antreten können und somit variable, aber in keiner nachvollziehbaren Verbindung zueinander stehende Selektionsvorgaben machen. Nach dem obigen Modell fordert sowohl das "nominale" $\{-a\}$ als auch das "pronominale" $\{-a j a\}$ im Prinzip jeweils etwas "Nominales', jedoch ist dieses 'Nominale' bei $\{-a j a\}$ rein nominal, während es bei $\{-a\}$ teilweise verbal, d. h. verbalnominal, sein darf. Die Analyse von infiniten Verbformen und Kurzformen als Verbalnomina erfasst unmittelbar, warum sich letztere mit $\{-a\}$ verbinden, während $\{-a j a\}$ ausschließlich an reine Nomina der Subkategorie 'Adjektiv' gefügt werden kann.

Der folgenden Abschnitt erörtert v. a. die Korrelation zwischen Argumentstruktur und Syntaktifizierung bei den drei Hauptwortarten Verb, Substantiv und Adjektiv. Hier wird auch nochmals vertiefend auf die Differenzen zwischen Langformen und Kurzformen eingegangen.

111 Die Notation " $\lambda P[P]$ ” gibt an, dass die Anfügung dieses Suffixes an eine Basis keinerlei semantische Anteile zu deren Bedeutung hinzufügt, sondern lediglich ihre identische Abbildung bewirkt (seine SF wird unverändert 'weitergereicht'). Die Notation ist zum Zwecke der besseren Lesbarkeit technisch vereinfacht, insofern möglicherweise vorhandene weitere Argumentstellen des selegierten Ausdrucks nicht berücksichtigt werden. Diese vereinfachte Notationsweise wird durchgehend angewendet. 


\subsection{Argumentstruktur und Syntaktifizierung}

Dem Verhältnis zwischen Lexikon und Syntax wird hier eine relativ 'simple' Annahme zugrunde gelegt: Lexikalische Einheiten projizieren entsprechend ihrer Kategorie und Argumentstruktur. Ich gehe daher davon aus, dass die grammatischen Merkmale (GM) sowie die Argumentstruktur (AS) die für die Syntax sichtbaren bzw. relevanten Teile von Lexikoneinträgen sind. In diesem Sinne kann v. a. die AS als Schnittstelle zwischen Lexikon und Syntax betrachtet werden, wie es weiter oben bereits getan wurde. Die lexikalischen Kategorien verhalten sich hinsichtlich der syntaktischen Realisierung ihrer möglichen Argumente unterschiedlich. Im Folgenden werden daher Verben, Substantive und Adjektive einzeln besprochen. Präposition(alphras)en wurden in Abschnitt 1.2.6 behandelt.

\subsubsection{Verben}

Es ist bekannt, dass Verben die lexikalische Kategorie sind, die man am unmittelbarsten mit "Prädikativität" assoziiert. Das liegt daran, dass Verben auf Sachverhalte referieren, und dass Sachverhalte (unabhängig davon, ob es sich um Ereignisse, Prozesse, Zustände etc. handelt) modal und temporal verortet werden können und müssen, um Aussagen bzw. Assertionen zu tätigen. Die Kategorien des (Verb-)Modus und des Tempus werden von der AG (1980, 10, § 1714; S. 86, $\S 1894)$ als Grundlage der predikativnost' 'Prädikativität' dargestellt, die ihrerseits - frei paraphrasiert - die Verbindung zwischen Sprache (als Zeichensystem) und der außersprachlichen Wirklichkeit herstellt. Es handelt sich um Kategorien, die ihre sprachliche Realisierung im Rahmen von bzw. an verbalen Formen erfahren. Es ist somit durchaus plausibel, dem Verbum die charakteristische Eigenschaft und Fähigkeit des 'Prädikativseins' zuzuerkennen.

Die Argumentstruktur von Verben hat die Besonderheit, dass die designierte Argumentstelle, die - sofern vorhanden - dem externen Argument ('Subjekt') entspricht, nicht identisch mit der referenziellen ist (anders als bei Substantiven; vgl. Bierwisch 1997, 245). Die verbale Referenz ist also getrennt vom 'Subjekt'.

Meist werden vier Verbklassen unterschieden, denen die Zahl der Partizipanten zugrunde gelegt wird: 
(16) a. nullstellige Verben

b. einstellige (intransitive) Verben

c. zweistellige (monotransitive) Verben

d. dreistellige (ditransitive) Verben (keine Partizipantenargumente)

(ein Partizipantenargument)

(zwei Partizipantenargumente)

(drei Partizipantenargumente)

Bierwisch (1997) gibt für Verben die allgemeine AS in (17) mit strukturell vergebenen Kasus im Deutschen an: ${ }^{12}$

$\underset{[\mathrm{ACC}]}{\lambda x_{3}}\left\{\begin{array}{cccc}\lambda x_{2} & \}\end{array}\right\} \underset{[\mathrm{DAT}]}{\lambda x_{1}} \quad \lambda x_{0}$

(vgl. Bierwisch 1997, 249)

Zunächst fällt auf, dass die ranghöchste und dabei referenzielle Argumentstelle $\lambda x_{0}$ nicht annotiert ist. Ursache dafür ist, dass das referenzielle Argument von Verben bzw. Verba, das auf einen Sachverhalt in der außersprachlichen Realität referiert, keine syntaktische Realisierung erfährt. Es wird im Laufe der Strukturbildung vom funktionalen Kopf $\mathrm{I}^{0}$ (bzw. $\mathrm{T}^{0} / \mathrm{Mod}^{0}$ ) gebunden, was der Spezifikation von Verbmodus entspricht. Die designierte bzw. externe Argumentstelle $\lambda x_{1}$ ist mit dem Nominativ annotiert, d. h. sie muss in ebendiesem Kasus realisiert werden. Sofern zwei interne Argumente vorliegen, muss das rangniedere $\left(\lambda x_{3}\right) \mathrm{im}$ Akkusativ, das ranghöhere $\left(\lambda x_{2}\right)$ im Dativ erscheinen. Liegt nur ein internes Argument vor, erscheint es im Akkusativ. Diese generellen Vorgaben an die Realisierung verbaler Argumente lassen sich unverändert auf das Polnische und Russische übertragen. Da es sich um strukturell determinierte und somit vorhersagbare Selektionsmerkmale handelt, ist ihre Nennung unnötig (oben erfolgt sie zum Zwecke der Darstellung). Anzugeben sind allein solche Merkmale, die idiosynkratisch und damit nicht vorhersagbar sind.

Was die Partizipantenargumente von Verben betrifft, so ist ferner festzuhalten, dass diese i. d. R. obligatorisch syntaktifiziert werden (Bierwisch 1997, 245). ${ }^{113}$ Im Folgenden dient das russische Verblexem OTKRYVAT' ‘öffnen' als Beispiel. Es soll gezeigt werden, wie bei Verben eine semantische Dekomposition aussehen und deren Verhältnis zur AS beschaffen sein kann.

\footnotetext{
112 Die Kasus unterscheidet er mittels der Merkmale $[ \pm$ Obj] 'Objective' und $[ \pm \mathrm{Obl}]$ 'Oblique' (Bierwisch 1997, 248), die ich in 'einfache' Kasusmerkmale 'übersetze'.

${ }^{113}$ Diese Eigenschaft teilen Verben mit Präpositionen sowie mit Kurzformen.
} 
Das erwähnte Verblexem ist monotransitiv. Ferner handelt es sich um ein kausatives Verb, denn seine deskriptive Bedeutung beinhaltet eine Kausierungsrelation. In der Literatur wird hierfür meist das Prädikat CAUSE verwendet (vgl. Davidson 1967b; Dowty 1979; Wunderlich 1997; Bierwisch 2002, 2005). Während durch die relativ häufig anzutreffende Notation $[x$ CAUSE ...] suggeriert wird, eine Entität $x$ verursache, dass ein bestimmter Zustand eintrete, muss präziser gelten, dass es sich bei CAUSE um keine Relation zwischen einem Individuum und einem Zielzustand, sondern vielmehr um eine Relation zwischen zwei Sachverhalten handelt (vgl. u. a. Levin \& Rappaport Hovav 1995; Bierwisch 2002, 2005; Fehrmann, Junghanns \& Lenertová 2011, 2012; Junghanns 2012). CAUSE ist somit ein Prädikat vom Typ $<\mathrm{t},<\mathrm{t}, \mathrm{t}>>$. Dabei ist der 'Verursacher' bzw. das AGENS-Argument in eine nicht näher spezifizierte Handlung involviert, die ihrerseits die eigentliche Ursache für das Eintreten bzw. Bestehen des Folge- oder Zielzustands ist, in dem sich schließlich das THEMA-Argument befindet. ${ }^{114}$

In (18) zitiere ich nach Junghanns $(2012,14)$ die entsprechend gestaltete AS und PAS (ohne Aspektsemantik) für das Lexem OTKRYVAT':

(18) $\lambda y \lambda x \lambda s$

$\left[[s\right.$ INST $[P x]]:\left[\left[s\right.\right.$ CAUSE $\left.s^{\prime}\right]:\left[s^{\prime}\right.$ INST [BECOME [OPEN $\left.\left.\left.\left.\left.y\right]\right]\right]\right]\right]$

Die PAS erfasst die erwähnte Bedeutungsstruktur kausativer Verben. Hier sind mit $s$ und $s^{\prime}$ zwei Sachverhalte ivolviert, die zueinander in der Relation der Kausierung stehen. In der AS erscheint neben den Argumentstellen für die Partizipanten jedoch nur das ranghöchste Sachverhaltsargument $s$, während das in der PAS tiefer eingebettete Argument $s^{\prime}$ nicht erscheint. Da die AS jener Bestandteil des Eintrags ist, den die Syntax als 'Anleitung' zur Argumentrealisierung 'sehen kann', werden in der VP - neben der Verbform selbst - lediglich die beiden verbalen Argumente ('Subjekt' und 'Objekt') projiziert. Das Sachverhaltsargument $s$ bleibt syntaktisch unrealisiert, wird aber in $\mathrm{I}^{0}$ existenziell abgebunden und spezifiziert (vgl. Zimmermann 2009). Da hier ein indikativisches Beispiel vor-

\footnotetext{
114 In Kleins (1994, 85) Terminologie handelt es sich beim monotransitiven OTKRYVAT' um einen “2-state lexical content” (vgl. auch Mueller-Reichau 2013, 194).
} 
liegt, bringt derselbe Kopf $\mathrm{I}^{0}$ auch das semantische Präsens ( $\left.\mid \mathrm{PRES} \|\right)$ als grammatische Bedeutung ein: ${ }^{115}$

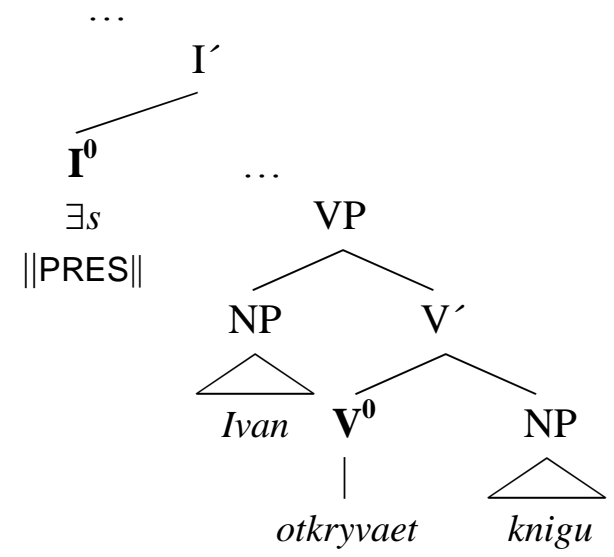

Das zweite Sachverhaltsargument $s^{\prime}$ spielt in der Syntax überhaupt keine Rolle. Die kausative Verbform otkryvaet stellt folglich eine Einheit dar, in der zwar zwei Sachverhalte 'stecken', von welchen jedoch nur der kausierende die Grundlage zur verbmodal-temporalen Einordnung darstellt. ${ }^{116}$

Es sollte ersichtlich geworden sein, dass die AS für die syntaktische Strukturbildung zentral ist. Nur was in der AS als Argumentstelle erscheint, kann und muss ggf. syntaktisch realisiert werden. Was lediglich Teil der PAS ist, wird zwar interpretiert, kann aber nicht (kanonisch) syntaktifiziert werden (die entsprechenden Argumentpositionen sind dann lediglich "semantische Parameter"). Ferner ist festzuhalten, dass Verben bzw. Verba über Sachverhaltsbezug verfügen, der sich im Lexikoneintrag in Form eines referenziellen Sachverhaltsarguments niederschlägt. Dieses Sachverhaltsargument erfährt im Unterschied zu den Partizi-

115 Im folgenden und allen weiteren Baumdiagrammen verwende ich trotz der oben beschriebenen 'neuartigen' Merkmalsarchitektur die traditionellen Kategorienetiketten (Label). Ich werde also sowohl rein verbale $([+\mathrm{V},-\mathrm{N}])$ als auch verbalnominale $([+\mathrm{V},+\mathrm{N}])$ Formen verbaler Lexeme mit "V" bezeichnen. Ebenso werde ich sowohl die verbalnominalen Kurzformen $([+\mathrm{V},+\mathrm{N}])$ als auch die rein nominalen Langformen $([-\mathrm{V},+\mathrm{N},+\mathrm{a}])$ mit " $\mathrm{A}$ ” bezeichnen.

116 Siehe Junghanns (2012), der diese Tatsache als Grund für die zwangsläufige Einheit von Raum und Zeit der beiden von Lexemen wie OTKRYVAT' denotierten und in Relation zueinander gesetzten Sachverhalte erkennt (de facto wird diskutiert, dass lokale Adverbiale über $s$ prädizieren (können), so dass ein Individuum, das in einen Sachverhalt involviert ist, zwangsläufig mit diesem Sachverhalt lokalisiert wird).

In meinen eigenen Verbeinträgen folge ich Klein (1994), so dass in $\mathrm{I}^{0}$ nicht das Ereigniszeitargument $s$, sondern das Topikzeitargument $t$ existenziell abgebunden (und - im Indinikativ mit der Äußerungszeit $\mathrm{t}^{0}$ in Beziehung gesetzt) wird. 
pantenargumenten keine syntaktische Realisierung. Für letztere gilt, dass sie sofern keine idiosynkratischen Selektionsanforderungen vorliegen - in den strukturell determinierten Kasusformen Nominativ, Dativ und Akkusativ realisiert werden. Im Lexikoneintrag anzugeben sind lediglich idiosynkratische Anforderungen an die Argumente, was generell bzw. nicht nur für verbale Lexeme gilt.

Im vorangehenden Abschnitt habe ich die Annahme von Verbalnomina vorgestellt. Hierzu zählen m. E. der Infinitiv sowie die (prädikativ gebrauchten) Partizipien und Kurzformen (die also im Unterschied zu den Langformen keine 'richtigen' Adjektive sind). Diese Formen, von denen mindestens die beiden erstgenannten fraglos grundlegend verbal sind, haben m. E. die lexikalische Merkmalskombination $[+\mathrm{V},+\mathrm{N}]$ und nicht - wie finite Verbformen $-[+\mathrm{V},-\mathrm{N}]$. Gemeinsam ist finiten und infiniten Verbformen somit das Merkmal $[+\mathrm{V}]$. Dieses Merkmal ist auch dafür verantwortlich, dass diese Formen ihre Argumente i. d. R. syntaktifizieren (müssen). ${ }^{117}$ Wie Geist $(2006,139-140)$ zeigt, trifft dies nicht nur auf die generell als Verbformen betrachteten Finita und Infinita zu, sondern auch auf die nur prädikativ verwendbaren Kurzform'adjektive'. Indem man all diesen Formen das Merkmal [+V] (unabhängig vom Merkmalswert $[ \pm \mathrm{N}]$ ) zugesteht, findet die ihnen gemeinsame obligatorische Argumentrealisierung eine unmittelbare Erklärung. Das bei den Infinita und Kurzformen positive Merkmal [+N] wiederum kann deren 'Infinitheit' sowie eine mitunter abweichende, weil nominale (d. h. optionale) Form der Argumentrealisierung erfassen (dies betrifft die Kurzformen).

\subsubsection{Substantive}

Neben Verben verfügen auch Substantive über referenzielle Kraft (vgl. u. a. Bierwisch 1997, 245). Diese Eigenschaft zeigt sich auch bei Substantiven an ihrer ranghöchsten, referenziellen Argumentstelle $\left(\lambda x_{0}\right)$. Freilich verweist letztere nicht

117 Junghanns \& Lenertová (2010) erwägen für "Infinitivische Perzeptionsprädikative" (IPP) wie z. B. poln. widać 'zu sehen (sein)', stychać 'zu hören (sein)'; russ. vidát' 'zu sehen (sein)', slychát' 'zu hören (sein)' usw., die unpersönlich sind, aber Akkusativobjekte lizenzieren, die Kategorisierung $[+\mathrm{V},+\mathrm{N},+$ Praed], die sich ihnen zufolge im Rahmen eines Sprachwandelprozesses aus $[+\mathrm{V},-\mathrm{N}]$ entwickelt habe und für die genannten Eigenschaften verantwortlich sei. Insbesondere wird erwogen, dass der Akkusativ als "Rest" der früheren Verbalität ([+V]) zugewiesen werden könnte. Wenn man für Infinitive generell die Merkmale $[+\mathrm{V},+\mathrm{N}]$ ansetzt, wirkt der Schritt zum Prädikativ nicht so groß und die Nähe zu Kurzformen umso geringer (neben russ. vidát', slychát' liegen die Kurzformen vidno, slyšno mit identischen Eigenschaften vor). 
auf einen Sachverhalt, sondern auf eine Entität in der außersprachlichen Welt. Wiederum ebenso wie bei Verben, erfährt die referenzielle Argumentstelle auch bei Substantiven (bei denen sie identisch mit der externen Argumentstelle ist) keine syntaktische Realisierung. Sie wird vielmehr (je nach theoretischem Modell) durch einen semantischen Operator (ein semantisches Template, einen type shift) oder aber syntaktisch durch einen non-/overten D-Kopf hinsichtlich ihres Referenzbereiches festgelegt, sprich definit oder indefinit spezifiziert. ${ }^{118}$ Im Hinblick auf prädikative Substantive (NPn) ist festzuhalten, dass diese nicht referieren. Sie gleichen eher Adjektiven, insofern sie nonreferenziell sind (vgl. Geist 2013, 492-493). Es scheint also so, dass erst die Selektion durch D ${ }^{0}$ (bzw. die Anwendung eines entsprechenden type shift) aus dem referenziellen Potenzial von Substantiven wirkliche referenzielle Kraft werden lässt. ${ }^{119}$

Für die morphosyntaktische Realisierung der Argumente von (deutschen) Substantiven nennt Bierwisch (1997) das Muster in (20):

$\begin{array}{ccc}\lambda x_{2} & \lambda x_{1} & \lambda x_{0} \\ {[\mathrm{GEN}]} & {[\mathrm{GEN}]}\end{array}$

(vgl. Bierwisch 1997, 249)

Wie bereits erwähnt, erfährt die referenzielle Argumentstelle $\lambda x_{0}$ keine syntaktische Realisierung, weshalb sie keine Annotation aufweist. Mögliche interne Argumente von Substantiven erscheinen strukturell im Genitiv. Für letztere gilt, dass sie optional realisiert werden, was ein bedeutsamer Unterschied zu Verben und Verbalnomina sowie zu Präpositionen ist, deren Argumente konsequent syntaktifiziert werden. Andererseits sind auf diese Weise die rein nominalen Substantive und (Langform-)Adjektive verbunden, da auch letztere ihre Komplemente lediglich optional realisieren (vgl. Bierwisch 1997, 245).

\footnotetext{
${ }^{118}$ Insofern erfüllt der funktionale Kopf $\mathrm{D}^{0}$ (sofern angenommen) innerhalb der nominalen Domäne eine vergleichbare Funktion wie $\mathrm{I}^{0}$ in der verbalen Domäne. Das deutet darauf hin, dass sich die nominale Kategorie der Definitheit mit der verbalen des Modus vergleichen lässt.

${ }^{119}$ Mitunter werden im Komplement der Kopula keine NPn, sondern DPn angesetzt (so z. B. von Bondaruk 2013b). Dafür scheint z. B. zu sprechen, dass etwa im Englischen und Deutschen der indefinite Artikel mit prädikativen Substantiven erscheint. Dagegen spricht aber die Nonreferenzialität prädikativer Substantive, die unter den obigen Annahmen unmittelbar mit der Abwesenheit von $\mathrm{D}^{0}$ korreliert. Eine Erklärung findet sich z. B. bei Geist (2006, 28-29), die mit Verweis auf Kamp \& Reyle (1993), Milsark (1974), Longobardi (1994) und Zamparelli (2000) annimmt, dass es sich beim fraglichen Artikel um ein Expletivum handele.
} 
Der Standardfall für Substantive ist ein einziges Argument, bei dem es sich um ihr referenzielles handelt. Somit sind Substantive im Lexikon i. d. R. als einstellige Prädikate abgelegt. Ein Lexikoneintrag für die polnische Substantivwurzel dom'Haus' dürfte demnach wie in (21) aussehen:

(21) $/ \mathrm{dom} / \quad[-\mathrm{V},+\mathrm{N},-\mathrm{a},-\max ] \quad \lambda x[x$ HOUSE $] \in\langle\mathrm{e}, \mathrm{t}\rangle$

$\lambda x$ ist die designierte Argumentstelle, die auf den Referenten des Substantivs verweist und syntaktisch nicht realisiert wird. Diese Argumentstelle wird im Weiteren durch einen D-Kopf definit oder indefinit spezifiziert. Zimmermann (2013) schlägt für das Russische den folgenden Eintrag für einen "Zeroartikel” vor, der die in/definite Bindung der referenziellen Argumentstelle von Substantiven bewerkstelligt (der Eintrag folgt meiner Notationsweise): ${ }^{120}$

Sofern eine NP Komplement dieses D-Kopfes ist, wird deren referenzielles Argument entsprechend gebunden. Demnach gelangen NPn vom Typ <e,t> aus dem Lexikon in die Syntax, die dort durch $\mathrm{D}^{0}$ in generalisierte Quantoren überführt werden und so als verbale Argumente fungieren können.

Wie schon erwähnt, haben Substantive die Merkmale in (23a), Adjektive (Langformen) dagegen jene in (23b):

(23) a. $[-\mathrm{V},+\mathrm{N},-\mathrm{a}]$

(Substantive)

b. $[-\mathrm{V},+\mathrm{N},+\mathrm{a}]$

(Adjektive LF $_{\text {) }}$

Beide Kategorien sind reine Nomina ohne jede Verbalität. Im Vergleich zu den Langformen (die im Polnischen und Russischen den 'adjektivischen Normalfall' repräsentieren) sind Substantive der unmarkierte Fall dieser Klasse. Im Gegensatz

120 "Stumme" D-Köpfe werden für das Russische u. a. auch von Geist (2011) angenommen. Zimmermanns Eintrag erzeugt einen generalisierten Quantor. Demnach selegieren Subjektausdrücke das Verb bzw. die V'-Ebene, nicht aber umgekehrt.

Alternativ zu D-Köpfen kann (für das Polnische und Russische) angenommen werden, dass im Lexikon entsprechende type shift-Operatoren oder semantische Templates zur Verfügung stehen, die das Gleiche leisten. Demnach würden Substantive, die als verbale Argumente fungieren sollen, bei Bedarf an diesen Gebrauch angepasst. 
$\mathrm{zu}$ den rein nominalen Langformen, sind Kurzformen Verbalnomina mit den Merkmalen $[+\mathbf{V},+\mathrm{N} \ldots]$ und somit infiniten Verbalformen vergleichbar - mehr dazu im folgenden Abschnitt.

\subsubsection{Adjektive}

Adjektive und Substantive werden traditionell in der Klasse der Nomina zusammengefasst. Aus der hier angewendeten lexikontheoretischen Perspektive ist dies insofern gerechtfertigt, als Adjektive - ebenso wie Substantive - überwiegend als Einheiten des Typs <e,t> im Lexikon abgelegt sind und ihre internen Argumente ebenfalls nur optional realisieren. Anders als Substantive haben Adjektive jedoch keine referenzielle Kraft, was sie dazu prädestiniert, als Prädikative oder als Attribute zu fungieren. ${ }^{121}$

Was die Argumente von Adjektiven betrifft, so verfügen sie i. d. R. über ein externes Argument, das dem Referenten entspricht, auf den die denotierte Eigenschaft zutrifft. Sofern interne Argumente vorliegen, werden diese im Normalfall optional realisiert und erscheinen strukturell im Dativ: ${ }^{122}$

$$
\underset{[\mathrm{DAT}]}{\lambda x_{1}} \quad \lambda x_{0}
$$

(vgl. Bierwisch 1997, 249)

Dieses Schema ist auch für das Russische und Polnische gültig (womöglich mit Ausnahme der Dativzuweisung). Die Mehrzahl adjektivischer Lexeme hat - ebenso wie Substantive - nur ein ein externes Argument. Damit gilt auch für adjektivische Wurzeln bzw. Stämme, dass sie im Lexikon meist als einstellige Prädikate vorliegen, was der vereinfachte Eintrag für den russischen Stamm škol’n'schulisch; Schul-' in (25) illustriert:

$$
/ \text { škol’n/ }[-\mathrm{V},+\mathrm{N},+\mathrm{a}] \quad \lambda x \exists y[x \underline{\mathrm{R}}[y \mathrm{sCHOOL}]] \in\langle\mathrm{e}, \mathrm{t}\rangle \quad \text { (Beziehungsadjektiv) }
$$

\footnotetext{
121 Sind sie ersteres, referieren Adjektive überhaupt nicht. Sind sie letzteres, verweisen sie - als "Trittbrettfahrer" - lediglich auf Basis der Unifizierung ihres externen Arguments mit dem referenziellen Argument ihres jeweiligen Bezugssubstantivs auf einen Referenten.

${ }^{122}$ Nach Bierwisch (1997) haben Adjektive und Präpositionen das gleiche Realisierungsschema.
} 
Bei škol’nyj 'schulisch; Schul-' handelt es sich um ein sog. Beziehungsadjektiv ${ }^{123}$, was in diesem Eintrag durch eine unspezifizierte Relation $(\underline{\mathrm{R}})$ angedeutet wird, die zwischen dem Referenten des Adjektivs und dem 'Individuum' "[y SCHOOL]", dessen Referent abgebunden ist, besteht. Es kann gelten, dass die Relation durch das Derivationssuffix $\{n\}$ etabliert wird, wenn es an die Wurzel škol- tritt und so das Adjektiv ableitet. Die Merkmale $[-\mathrm{V},+\mathrm{N},+\mathrm{a}]$ gehen also bei den Beziehungsadjektiven auf Derivationssuffixe wie z. B. russ./poln. $\{n\},\{s k\},\{o v\} /\{o w\}$ etc. zurück. Mit den resultierenden Adjektivstämmen sind ausschließlich LangformKongruenzmarker verbindbar, so z. B. russ. $\{a j a\},\{o e\},\{o g o\},\{y m\}$ oder poln. $\{y\},\{e\},\{e g o\},\{y c h\}$ etc. Letzere müssen folglich in ihren Lexikoneinträgen so beschaffen sein, dass sie nur morphologische Einheiten mit dem Merkmal [+a] 'akzeptieren'; vgl. dazu den exemplarischen Eintrag für russ. $\{a j a\}$. Ich gebe der Klarheit halber alle (also auch negativ spezifizierte) Merkmale an:

$$
\text { /aja/ } \quad[\mathrm{NOM},+\mathrm{Fem},+\max ] \quad \lambda P_{[-\mathrm{V},+\mathrm{N},+\mathrm{a},-\max ]}[P]
$$

("Langformendung")

Folgend vollziehe ich die Derivation der russischen Flexionsform školnaja schrittweise nach. Zugrunde liegt die substantivische Basis škol- mit dem Eintrag in (27a). In (27b) ist der Eintrag des Ableitungssuffixes $\{n\}$ formuliert, das beim Antreten an die vorgenannte Basis eine unspezifizierte Relation zu einem neu eingeführten Referenten $(\lambda x)$ herstellt. ${ }^{124}$ In $(27 \mathrm{c})$ nenne ich das Ergebnis der Anfügung von $\{n\}$ in (27b) an škol- in (27a), also den Stamm des resultierenden Beziehungsadjektivs. In (27d) ist der Eintrag des Langform-Kongruenzmarkers $\{$ aja $\}$ wiederholt, und in (27e) wird der derivierte Lexikoneintrag für školnaja genannt:

$$
\begin{aligned}
& \text { (27) a. } / \text { škol } / \quad[-\mathrm{V},+\mathrm{N},-\mathrm{a},-\mathrm{max}] \quad \lambda x[x \mathrm{SCHOOL}] \\
& +\quad \text { b. } / \mathrm{n} / \quad[-\mathrm{V},+\mathrm{N},+\mathrm{a},-\max ] \quad \lambda P_{[-\mathrm{V},+\mathrm{N},-\mathrm{a},-\max ]} \lambda x \exists y\left[x \underline{\mathrm{R}}\left[\begin{array}{ll}
P & y
\end{array}\right]\right. \\
& =\mathrm{c} . / \text { škol'n } / \quad[-\mathrm{V},+\mathrm{N},+\mathrm{a},-\max ] \quad \lambda x \exists y[x \underline{\mathrm{R}}[y \mathrm{SCHOOL}]] \\
& + \text { d. laja/ } \quad[\mathrm{NOM},+\mathrm{Fem},+\max ] \quad \lambda P_{[-\mathrm{V},+\mathrm{N},+\mathrm{a},-\mathrm{max}]}[P] \\
& =\text { e. /škol'naja/ }[-\mathrm{V},+\mathrm{N},+\mathrm{a}, \mathrm{NOM},+\mathrm{Fem},+\max ] \quad \lambda x \exists y[x \underline{\mathrm{R}}[y \text { SCHOOL }]]
\end{aligned}
$$

\footnotetext{
${ }^{123}$ Siehe zur traditionellen Unterscheidung zwischen Beziehungs- und Qualitätsadjektiven Geist (2006, 125, Fn. 1) sowie schon Abschnitt 2.2.2.

${ }^{124}$ Phonologisch ist $\{n\}$ derart beschaffen, dass es die vorangehende Basis palatalisiert.
} 
Die bisher gemachten Aussagen zu Adjektiven und die mit ihnen assoziierten Merkmale $[-\mathrm{V},+\mathrm{N},+\mathrm{a}]$ gelten für Beziehungsadjektive wie russ. škol’n- 'schulisch; Schul-' oder poln. miejsk- 'städtisch; Stadt-'. Neben dieser lexikosemantischen Klasse von Adjektiven, die ausschließlich in der Langform auftreten, liegen im Polnischen und Russischen auch sog. Qualitätsadjektive vor. Letztere bezeichnen Eigenschaften des Referenten nicht - wie Beziehungsadjektive - als Relation zu einem Individuum oder einer Substanz, sondern unmittelbar durch ihre deskriptive Bedeutung (siehe Geist 2006, 125). Entsprechend handelt es sich fast immer um unabgeleitete bzw. 'ursprüngliche' Adjektive und somit um Adjektivwurzeln, nicht -stämme. ${ }^{125}$ Bezeichnend bei den Qualitätsadjektiven ist, dass diese i. d. R. sowohl eine Lang- als auch eine Kurzform haben können, vgl. z. B. russ. $d o b r-a_{\mathrm{KF}}$ : dobr-aja $a_{\mathrm{LF}}$ 'gut', poln. pewien- $\varnothing_{\mathrm{KF}}$ : pewn- $y_{\mathrm{LF}}$ 'gewiss'. Sie haben somit neben der primär attributiv verwendeten Langform auch eine explizit prädikative Kurzform. ${ }^{126}$ Wie bereits mehrfach erwähnt, betrachte ich Kurzformen nicht als 'normale' (rein nominale) Adjektive (wie die Langformen), sondern als Verbalnomina, also als $[+\mathrm{V},+\mathrm{N}]$-Einheiten, die Gemeinsamkeiten mit infiniten Verbformen haben, darunter z. B., dass sie als Satzprädikat auftreten:

$$
\begin{array}{lll}
\text { a. Anna budet } & \text { rabotat'. } \\
\text { Anna-NOM AUX-3.SG } & \text { arbeit-INF } \\
\text { 'Anna wird arbeiten.' } &
\end{array}
$$

b. Kniga budet pročitana.

Buch-NOM AUX-3.SG PERF-les-NPT-SG.F

'Das Buch wird gelesen werden.'

125 Ausnahmen sind z. B. russ. tëmnyj/poln. ciemny 'dunkel', die, obwohl semantisch Qualitätsadjektive, ein Suffix zeigen, das sonst der Derivation von Beziehungsadjektiven dient. Es handelt sich wohl um Formen, die früh als Beziehungsadjektive von substantivischen Basen abgeleitet wurden, ihrer Bedeutung nach aber Qualitäten bezeichnen. Geist $(2006,125)$ ist daher zuzustimmen, dass "[d]ie Grenze zwischen Beziehungs- und Qualitätsadjektiven [...] fließend" ist. Anzumerken ist ferner, dass Qualitätsadjektive intersektiv sowie nicht-intersektiv sein können, was aber ein semantischer Unterschied ist, der für die Morphosyntax zweitrangig ist.

${ }^{126}$ Da Beziehungsadjektive stets der Einengung ihres Referenten auf einen bestimmten Bereich dienen, kommen sie kaum prädikativ vor und haben (wohl deswegen) keine Kurzformen:
(i) školnaja kniga
schulisches Buch
(ii) ?? kniga
byla
škol'naja / *škol’na
Buch war schulisch $_{\mathrm{LF}} / \mathrm{schulisch}_{\mathrm{KF}}$
'Schulbuch'
?"Das Buch war schulisch.'

(Rus) 
c. Anna budet dobra.

(Kurzform)

Anna-NOM AUX-3.SG gut $_{\mathrm{KF}}$-SG.F

'Anna wird gut sein.'

Dass für die spezifischen Eigenschaften der Kurzformen nicht ihre Kongruenzmarkerierung verantwortlich zu machen ist, liegt m. E. auf der Hand. Die Qualitätsadjektive bzw. deren Wurzeln selbst dürften vielmehr so beschaffen sein, dass sie die Bildung sowohl von Kurzformen als auch von Langformen zulassen und die entsprechenden (v. a. Distributions-) Eigenschaften mit sich bringen.

Will man zunächst erfassen, wie aus der Wurzel dobr- eine Langform dobraja abgeleitet wird, muss man annehmen, dass diese Wurzel selbst jene Merkmale hat, die bei Beziehungsadjektiven vom Ableitungssuffix (z. B. $\{n\}$ ) eingebracht werden, d. h. die rein nominalen Merkmale $[-\mathrm{V},+\mathrm{N},+\mathrm{a}]$. Nur, wenn dem so ist, kann ein Langform-Kongruenzmarker wie $\{a j a\}$ antreten. Gleichzeitig muss die Wurzel eines Qualitätsadjektivs aber auch die Ableitung der Kurzform zulassen. Dies wiederum kann sie nur, wenn die Merkmale $[+\mathrm{V},+\mathrm{N}]$ verfügbar sind.

Folglich muss die Wurzel eines Qualitätsadjektivs gleichermaßen die Merkmalskombinationen $[-\mathrm{V},+\mathrm{N},+\mathrm{a}]$ und $[+\mathrm{V},+\mathrm{N}]$ 'bedienen' können. Das lässt sich durch die Annahme der konflationierten Merkmalsmenge $\left[\alpha \mathrm{V},+\mathrm{N},(-\mathrm{a})_{\alpha}\right]$ erreichen, deren stabiler Kern das Merkmal [+N] ist, während Variabilität hinsichtlich $[ \pm \mathrm{V}]$ und - in Abhängigkeit von dessen Wert - auch hinsichtlich des nominalen Submerkmals [+a] besteht (es kann positiv sein oder ganz fehlen). Diese Konflation baue ich in den folgenden Lexikoneintrag der russ./poln. Wurzel dobr- ein:

(29) $/$ dobr/ $\left[\alpha \mathrm{V},+\mathrm{N},(+\mathrm{a})_{\alpha}\right] \quad \lambda x[x \mathrm{GOOD}] \in\langle\mathrm{e}, \mathrm{t}\rangle$

(Qualitätsadjektiv)

Man kann nun - wie weiter oben bereits für das Beziehungsadjektiv škol’naja geschehen - die Derivation der flektierten Langform russ. dobraja durchspielen:

$$
\begin{array}{lllll}
(30) & \text { a. } & / \text { dobr } / & {\left[\alpha \mathrm{V},+\mathrm{N},(+\mathrm{a})_{\alpha}-\mathrm{max}\right]} & \lambda x[x \mathrm{GOOD}] \\
+ & \text { b. } & \text { /aja/ } & {[\mathrm{NOM},+\mathrm{Fem},+\mathrm{max}]} & \lambda P_{[-\mathrm{V},+\mathrm{N},+\mathrm{a},-\max ]}[P] \\
= & \text { c. } & / \text { dobraja/ } & {[-\mathrm{V},+\mathrm{N},+\mathrm{a}, \mathrm{NOM},+\mathrm{Fem},+\max ]} & \lambda x[x \mathrm{GOOD}]
\end{array}
$$

Durch das Kongruenzsuffix $\{a j a\}$ wird die variable Wurzel dobr- quasi auf die rein nominale Adjektivform dobraja festgelegt. 
Der Kurzform-Kongruenzmarker $\{a\}$ muss anders funktionieren. Namentlich muss er andere Selektionsmerkmale haben, die seine Verbindbarkeit sowohl mit substantivischen, partizipialen als auch qualitätsadjektivischen Wurzeln bzw. Basen gewährleisten. Diese Merkmale müssen $[ \pm \mathrm{V},+\mathrm{N},-\mathrm{a},-\mathrm{max}]$ lauten und sind im Suffixeintrag (31) untergebracht:

$$
\text { /a/ } \quad[\mathrm{NOM},+\mathrm{Fem},+\max ] \quad \lambda P_{[ \pm \mathrm{V},+\mathrm{N},-\max ]}[P]
$$

(“Kurzformendung”)

Die schrittweise Ableitung der russischen Kurzform dobra aus der Wurzel dobrläuft demnach wie folgt ab:

$$
\begin{array}{lllll}
(32) & \text { a. } & / \text { dobr } / & {\left[\alpha \mathrm{V},+\mathrm{N},(+\mathrm{a})_{\alpha}-\max \right]} & \lambda x[x \mathrm{GOOD}] \\
+ & \text { b. } & / \mathrm{a} / & {[\mathrm{NOM},+\mathrm{Fem},+\max ]} & \lambda P_{[ \pm \mathrm{V},+\mathrm{N},-\max ]}[P] \\
= & \text { c. } & / \text { dobra } / & {[+\mathrm{V},+\mathrm{N}, \mathrm{NOM},+\mathrm{Fem},+\max ]} & \lambda x[x \mathrm{GOOD}]
\end{array}
$$

Durch $\{a\}$ ist aus der variablen Wurzel dobr-also die verbalnominale Einheit bzw. Kurzform dobra geworden. Dadurch ist sie - im Gegensatz zur rein nominalen Langform dobraja - nicht attributiv, sondern nur prädikativ verwendbar. Ferner werden auf Grund des positiven Merkmals [+V] ggf. vorhandene interne Argumente von Kurzformen obligatorisch realisiert:

$$
\begin{aligned}
& \text { a. Dom byl polon *(gostej). }
\end{aligned}
$$

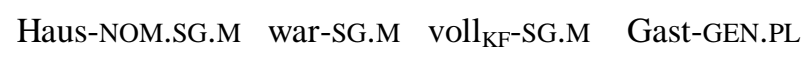

$$
\begin{aligned}
& \text { 'Das Haus war voll von Gästen.' } \\
& \text { b. Éto palto *(Kate) dlinno. } \\
& \text { dieser Mantel-NOM.SG.N Katja-DAT } \text { lang }_{\text {KF-SG.N }} \\
& \text { 'Dieser Mantel ist Katja zu lang.' }
\end{aligned}
$$

Liegen dagegen die Langformen polnyj 'voll' bzw. dlinnyj 'lang' vor (egal, ob attributiv oder prädikativ), sind die betreffenden Argumente optional (vgl. Geist 2006, 140), was der Nominalität der Langformen zuzuschreiben ist. ${ }^{127}$

\footnotetext{
127 Geist $(2006,140)$ nennt noch zwei weitere Beispielsätze. Der erste enthält das Adjektiv bolen : bol'noj 'krank'. Mit ihm ist ein Instrumental zur Bezeichnung der Krankheit (z. B. grippom 'an Grippe') fakultativ möglich, bei attributivem Gebrauch aber ausgeschlossen. Hier ist fraglich, ob es sich um ein Argument oder eine freie Angabe (Adjunkt) handelt. M. E. ist letzeres der
} 
Auch die übrigen Kriterien, die Geist (2006, 138-142) im Rahmen ihrer eigenen Argumentation anbringt, um zu begründen, dass die russischen Kurzformen verbal seien (was m. E. zu weit geht, da sie 'nur' verbalnominal sind), können durch die vorliegende Analyse $([+\mathbf{V},+\mathrm{N}])$ erfasst werden (Beispiele von Geist):

(34) a. grammatische Kongruenz mit $V y$ 'Sie':

$V y_{\mathrm{PL}[\mathrm{f.sg}]}$ byli togda molody KF-PL $/$ molod $_{\mathrm{KF}-\mathrm{f} . \mathrm{sg} .}$.'Sie waren damals jung.'

b. keine Konversion Adjektiv $\rightarrow$ Substantiv: staroe $_{\mathrm{LF}}$ i $_{\text {novoe }} \mathrm{LF} /$ staro $_{\mathrm{KF}}$ i novo $_{\mathrm{KF}}$ 'das Alte und das Neue'

c. nur belebte Referenten bei 'psychischen Adjektiven': Prazdnik byl očen' vesëlyj $j_{\mathrm{LF}} /$ vesel $_{\mathrm{KF}}$. 'Die Feier war sehr fröhlich.'

Die grammatische Kongruenz mit der Höflichkeitsform $V y$ 'Sie' verbindet die Kurzformen mit den infiniten $l$-Partizipien, wie das Beispiel unter (34a) illustriert. Dass keine Konversion zum Substantiv möglich ist, kann ebenfalls auf den verbalen Anteil der Kurzformen zurückgeführt werden, da dieser deren attributive Verwendung ausschließt, die ihrerseits Voraussetzung für eine Substantivierung ist (siehe auch Abschnitt 5.6.2). Die Verbalität der Kurzformen mag auch Ursache dafür sein, dass mit ihren 'psychischen' Ausprägungen nur belebte Referenten akzeptabel sind, wie (34c) zeigt (eventuell ist vesel aber auch bereits ein eigenständiges Lexem mit eigener Bedeutung und belebten Referenten).

Schließlich sei zur Untermauerung des o. g. Ableitungsvorschlags für polnische und russische verbalnominale Kurzformen demonstriert, dass er den Vorteil hat, auch substantivische und partizipiale Formen einheitlich (ohne sich widersprechende Zusatzannahmen) mittels der Kurzform-Kongruenzsuffixe ableiten zu können. Ich wiederhole dazu die Ableitung der Kurzform dobra und stelle Parallelderivationen für die nominativische Substantivform škola 'Schule' und das $l$-Partizip rabotala (ohne Aspekt) daneben:

Fall, und der Ausschluss beim attributiven Gebrauch hat keine syntaktischen, sondern informationsstrukturelle Ursachen (beachte, dass der Instrumental bei isolierter Verwendung akzeptabel ist!). Das zweite Beispiel enthält sklonen 'geneigt'. Hier wiederum ist eine $k$-PP in allen Gebrauchsweisen obligatorisch. Es scheint, dass sklonen ohne diese PP uninformativ ist. Auch bei (33b) lässt sich fragen, ob der Dativ nicht von einer leeren Kategorie eingeführt wird. 


$$
\begin{aligned}
& \text { (35) a. /dobr/ }\left[\alpha \mathrm{V},+\mathrm{N},(+\mathrm{a})_{\alpha}-\max \right] \quad \lambda x[x \text { GOOD }] \\
& + \text { b. /a/ }[\mathrm{NOM},+\mathrm{Fem},+\max ] \quad \lambda P_{[ \pm \mathrm{V},+\mathrm{N},-\max ]}[P] \\
& =\text { c. } / \text { dobra } /[+\mathrm{V},+\mathrm{N}, \mathrm{NOM},+\mathrm{Fem},+\max ] \quad \lambda x[x \mathrm{GOOD}] \\
& \text { (36) a. /škol } / \quad[-\mathrm{V},+\mathrm{N},-\mathrm{a},-\max ] \quad \lambda x[x \text { SCHOOL }] \\
& + \text { b. /a/ }[\mathrm{NOM},+\mathrm{Fem},+\max ] \quad \lambda P_{[ \pm \mathrm{V},+\mathrm{N},-\max ]}[P] \\
& =\quad \text { c. /škola/ }[-\mathrm{V},+\mathrm{N},-\mathrm{a}, \mathrm{NOM},+\mathrm{Fem},+\mathrm{max}] \quad \lambda x[x \text { SCHOOL }] \\
& \text { (37) a. } / \operatorname{rabota}(\mathrm{j}) / \quad[+\mathrm{V},-\mathrm{N},-\max ] \quad \lambda x \lambda s[s \text { INST }[x \text { WORK }] \\
& + \text { b. } / 1 / \quad[+\mathrm{V},+\mathrm{N},-\max ] \quad \lambda P_{[+\mathrm{V},-\mathrm{N},-\max ]}[P] \\
& =\text { c. } / \text { rabotal } / \quad[+\mathrm{V},+\mathrm{N},-\max ] \quad \lambda x \lambda s[s \text { INST }[x \text { WORK }]] \\
& + \text { d. la }\left[\quad[\mathrm{NOM},+\mathrm{Fem},+\max ] \lambda P_{[ \pm \mathrm{V},+\mathrm{N},-\max ]}[P]\right. \\
& =\mathrm{e} . / \text { rabotala } / \quad[+\mathrm{V},+\mathrm{N}, \mathrm{NOM},+\mathrm{Fem},+\max ] \quad \lambda x \lambda s[s \text { INST }[x \text { WORK }]]
\end{aligned}
$$

Während sich Kurzformen und Substantive im Wert des Merkmals [ $\pm \mathrm{V}]$ unterscheiden $([+\mathrm{V}]=$ Verbalnomen vs. $[-\mathrm{V}]=$ reines Nomen $)$, gleichen sich Kurzformen und Partizipien in ihren Merkmalen $[+\mathrm{V},+\mathrm{N}]$. Der Unterschied zwischen ihnen besteht auf referenzieller Seite, da nur Partizipien, und zwar auf Grund ihrer verbalen Abkunft, Sachverhaltsreferenz haben, nicht aber die eher nominal basierten Kurzformen. Dies ist auch Grund dafür, dass die prädikativ verwendeten Kurzformen, nicht aber die Partizipien, eine "Kopula" bzw. die abstrakte Kopulabedeutung brauchen (siehe Abschnitt 5.1).

Schließlich ist darauf hinzuweisen, dass Langformen - im Unterschied zu den Kurzformen - regulär bzw. primär attributiv bzw. als Modifikatoren fungieren. Daneben können die Langformen aber durchaus auch prädikativ verwendet werden (siehe Abschnitt 2.2), was in der vorliegenden Arbeit naturgemäß bedeutsam ist. Hier kann man sich im Prinzip mit den einstelligen und so bereits von sich aus prädikativ verwendbaren Lexikoneinträgen der Langformen zufriedengeben, sofern man z. B. mit Heim \& Kratzer (1998) eine semantische Regel Predicate Modification annimmt, auf deren Basis Prädikate andere Prädikate desselben logischen Typs modifizieren können. Im Russischen und Polnischen besteht letztere Option zum attributiven Gebrauch ausschließlich für die Langformen, was 
ich auf deren reine Nominalität zurückführe. ${ }^{128}$ M. E. erklären sich die unterschiedlichen Eigenschaften von Lang- und Kurzformen konsequent durch die reine Nominalität der ersteren im Gegensatz zur Verbalnominalität der letzteren. In Abschnitt 5.6.1 werde ich detaillierter auf den prädikativen Gebrauch polnischer und russischer Lang- und Kurformen eingehen.

\subsection{Syntax}

Der Verknüpfung lexikalischer Einheiten bzw. "atomarer syntaktischer Einheiten" (Zimmermann 1999, 131) werden folgende Schemata zugrunde gelegt:
(38) a. $\left[\alpha^{\prime} \boldsymbol{\alpha} \beta\right]$
(39) a. $\left[\beta^{\prime} \boldsymbol{\beta} \alpha\right]$
b. $\left[\alpha^{\prime} \beta \boldsymbol{\alpha}\right]$
b. $\left[\beta^{\prime} \alpha \boldsymbol{\beta}\right]$

Diese Schemata (vgl. u. a. Bierwisch 1997, 251) beschreiben in generalisierter Form die Verknüpfungsoperation Merge, durch die zwei Elemente $\alpha$ und $\beta$ zu einem komplexen Element $\alpha^{\prime}$ bzw. $\beta^{\prime}$ verbunden werden, wobei $\alpha^{\prime}$ bzw. $\beta^{\prime}$ gewissermaßen Erweiterungen von $\alpha$ respektive $\beta$ (bzw. der für $\alpha$ bzw. $\beta$ charakteristischen Merkmale) repräsentieren. ${ }^{129}$ Da $\alpha$ oder $\beta$ seine Merkmale projiziert, wird es als "Kopf' bezeichnet. Das, wozu es projiziert, wird "Phrase" genannt. Was die Wahl zwischen den Abfolgen in (38a/b) bzw. in (39a/b) betrifft, so ist anzunehmen, dass sprachspezifische Parameter (head parameter; vgl. Chomsky 1981), kategoriale, phonologische oder gänzlich idiosynkratische Eigenschaften von $\alpha$ respektive $\beta$ dafür verantwortlich sind.

Als syntaktisches Projektionsformat sehe ich das X-Bar-Schema vor, dem zufolge in strikt binärer Form von Köpfen zu Phrasen ("maximalen Projektionen”) proji-

${ }^{128}$ Geist $(2006,2010)$ nimmt an, dass die russischen Langformen (auch in prädikativer Funktion) (semantisch) attributiv seien, was sie mit dem Konzept des "Vergleichsklassenbezugs" erfasst. Damit wird den Langformen aber m. E. zuviel Eigenbedeutung 'aufgebürdet'.

Dass nur Langformen ein volles Kasusparadigma, Kurzformen hingegen nur nominativische Formen besitzen, lässt sich m. E. direkt aus den hier gemachten Annahmen ableiten. Nur reine Nomina (Substantive und Langformen) haben demnach ein volles Kasusparadigma, reine Verba dagegen haben überhaupt keines. Verbalnomina (darunter Partizipien und Kurzformen) wiederum sind auch in dieser Hinsicht 'gemischt', da sie mit den nominativischen Formen nur über einen Teil des Kasusparadigmas verfügen.

129 Anstelle von $\alpha^{\prime}$ bzw. $\beta^{\prime}$ ließe sich für das komplexe Element auch $\gamma$ schreiben, das Merkmale entweder von $\alpha$ oder von $\beta$ haben könnte. 
ziert wird. ${ }^{130}$ Durch Merge werden also nur jeweils zwei Einheiten zu einer Einheit höherer Komplexität (einer Phrase oder einem Phrasenteil) verbunden:

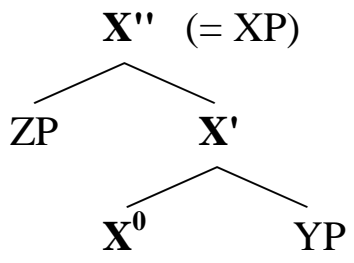

$\mathrm{X}$ steht als Platzhalter für jede mögliche lexikalische oder funktionale Kategorie. Eine lexikalische Einheit wird jeweils in der Stufe $X^{0}$, d. h. als syntaktischer “Kopf”, generiert. Als “Kopf” projiziert sie gemäß (40) über mögliche Zwischenstufen zu einer Phrase. Dabei ist es generell so, dass nie mehr als zwei Projektionsstufen vorliegen bzw. möglich sind. Die Position YP wird als "Komplement" bezeichnet und ist selbst stets phrasal. Letzteres gilt auch für die Position ZP, die als "Spezifikator" bezeichnet wird. ${ }^{131}$

Syntaktische Phrasen haben endozentrischen Charakter, d. h., der Kopf vererbt seine Merkmale an die höheren Projektionsstufen, so dass eine Phrase letztlich immer die Merkmale und Eigenschaften ihres Kopfes trägt. So wird etwa ein lexikalischer Verbkopf $\left(\mathrm{V}^{0}\right)$ zu einer (ebenso lexikalischen) Verbphrase (VP) projizieren, ein funktionaler Komplementiererkopf $\left(\mathrm{C}^{0}\right)$ dagegen $\mathrm{zu}$ einer (ebenso funktionalen) Komplementiererphrase (CP), vgl. (41a) und (41b):

${ }^{130}$ Dem lange und nach wie vor weithin anerkannten X-Bar-Schema steht in neueren Arbeiten mitunter die Bare Phrase Structure (Chomsky 1995b) gegenüber, in der die rigide Ordnung innerhalb von Phrasen aufgehoben ist. In dieser Arbeit wende ich sie nicht an.

${ }^{131}$ In verbalen Syntagmen erscheinen in der Komplementposition in aller Regel Objektausdrücke, in der Spezifikatorposition dagegen Subjektausdrücke. Oftmals sind die Spezifikatorpositionen aber lediglich "Landeplätze" für Phrasen, wenn diese - aus welchem Grund auch immer - syntaktisch bewegt werden (müssen). So ist Spec-IP oft "Landeplatz" für in den informationsstrukturellen Hintergrund verschobene Argumente, während Spec-CP oft für wh-Ausdrücke als Landeplatz dient. Nach Spec-NegP können sich sog. Negative Polarity Items wie z. B. russ. ničego 'nichts' bewegen, wenn Negative Concord (Mehrfachnegation) vorliegt. Welche Spezifikatorposition für welchen Typ von Ausdruck als Landeplatz dient, ist also keineswegs arbiträr, sondern in der Regel semantisch, informationsstrukturell oder phonologisch begründet bzw. begründbar. Bewegungen nur zum Zweck des syntaktischen Merkmalsabgleichs nehme ich (mit Zimmermann 2012) nicht an. 
(41) a.

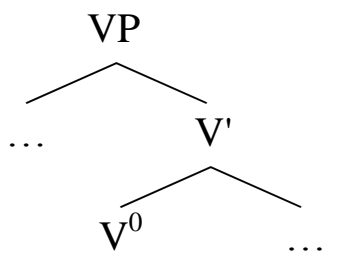

b.

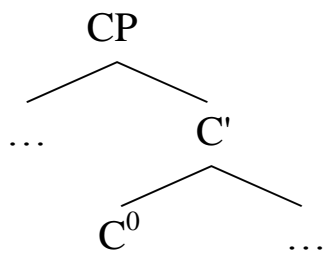

Durch das X-Bar-Schema kann man der Verknüpfung lexikalischer Einheiten auf der Grundlage möglichst minimaler Prinzipien (v. a. das der strikt binären Verzweigung) einheitlich Rechnung tragen. Ferner lässt sich die Hierarchie der verknüpften Einheiten darstellen, die sich auch in der linearen Abfolge der Konstituenten äußert. ${ }^{132}$ Ein weiterer Vorteil dieser syntaktischen Grundstruktur besteht darin, dass durch die Projektion von Köpfen zu Phrasen "Landepositionen" für bewegte Konstituenten ent- und bereitstehen, namentlich in Form von Spezifikatorpositionen bzw. von festen “Andockstellen” für modifizierende Adjunkte. ${ }^{133}$ Für die Modellierung von Satzstrukturen genügt für die Zwecke dieser Arbeit die Feststellung, dass jeder Satz auf einer lexikalischen Verbphrase (VP) beruht und bottom up, also sukzessive von unten nach oben, auf dieser aufgebaut wird. In diesem Sinne ist es berechtigt, den Satz als eine "erweiterte Projektion des Verbs" zu betrachten. Ein flektiertes Verb, also der Kopf einer VP, projiziert seine Argumente entsprechend seiner Argumentstruktur, die in seinem Lexikoneintrag verankert ist. Wenn dies geschehen ist, sind sowohl die deskriptive Bedeutung der Verbform als auch ihre Argumente Teil der syntaktischen Struktur.

Um den grammatischen Kategorien, die mit Verben (und Sätzen) verbunden sind, Rechnung zu tragen, werden über der VP die verschiedensten funktionalen Projektionen angenommen, etwa für Genus verbi (VoiceP), für Agentivität oder für Präsenz eines externen Arguments (meist $v \mathrm{P})$, für den Verbalaspekt (AspP) ${ }^{134}$, für

${ }^{132}$ Dieser Zusammenhang kann durch syntaktische Bewegungen von Phrasen und/oder Köpfen verschleiert werden. Solche Bewegungen sind, Zimmermann (2012) folgend, Folge phonologischer, semantisch-skopaler oder informationsstruktureller Erfordernisse; sie werden explizit nicht zum Zwecke des Merkmalsabgleichs angenommen.

133 An welcher Stelle eine Adjunktion an die Matrix anknüpfen kann, hängt v. a. davon ab, worauf sich das Adjunkt als Modifikator bezieht. Es ist eine Frage der Argumentstruktur bzw. des logischen Typs der beteiligten Ausdrücke. Ein attributives Adjektiv z. B. muss vom Typ $<<e, t>,\langle e, t>>$ sein, um eine NP vom Typ <e,t> modifizieren zu können (was syntaktisch auf eine AP-Adjunktion an NP hinausläuft; siehe dazu im Detail Abschnitt 5.4).

${ }^{134}$ Eine AspP wird bspw. in den minimalistischen Arbeiten von Luka Szucsich angesetzt, Migdalski (2012) schlägt vor, dass in slavischen Sprachen keine TP, sondern ausschließlich mit einer AspP zu rechnen sei. 
die Satznegation (NegP) oder generell für die Satzpolarität (PolP), für die Flexion an sich (IP), für das Tempus (TP - dies entweder als Alternative zur IP oder lediglich als deren Bestandteil im Sinne von Split-INFL; siehe Pollock 1989), ferner auch für den Verbmodus $(\mathrm{Mo}(\mathrm{o}) \mathrm{dP})^{135}$, für die Satzart und/oder für den Satzmodus (CP), für die illokutionäre Kraft (ForceP), für den informationsstrukturellen Status des Topikexponenten (TopP) oder des Fokusexponenten (FocP) u. a. m. Die Kriterien für die Annahme derartiger funktionaler Kategorien und folglich ihrer Anzahl sowie ihrer internen Hierarchie sind Gegenstand anhaltener Diskussion. Der vorliegenden Arbeit liegt in diesem Zusammenhang die Vorstellung zugrunde, dass stets eine Modellierung zu bevorzugen ist, die weniger ('neuer') Kategorien und Annahmen als andere Modelle bedarf. In diesem Sinne werde ich bemüht sein, mit so wenigen funktionalen Kategorien wie möglich auszukommen.

Für Substantive bzw. Nomina wird mithin von einem funktionalen Kategorienbestand ausgegangen, der den Verben analog sei. Hier spielt naturgemäß die Kategorie der 'Determiniertheit' (Definitheit, Spezifizität) eine zentrale Rolle, die seit Abney (1987) in aller Regel als funktionale Determinansphrase (DP) in der Syntax auftritt, und deren Komplement jeweils die lexikalische Substantiv- oder Nominalphrase (NP) ist. In Artikelsprachen sitzen in $\mathrm{D}^{0}$ overte oder stumme (definite) Artikel oder ggf. andere Arten von Determinierern. Sofern man bestrebt ist, DPn als universalen Teil nominaler Syntagmen zu betrachten, müssen in NichtArtikel-Sprachen wie dem Russischen oder Polnischen stumme D-Köpfe angenommen werden, wie es u. a. Geist (2011) tut. Eine Alternative dazu könnte sein, das "Problem" der Definitheit/Spezifizität semantisch-lexikalisch abzuhandeln, indem man anstelle von syntaktischen D-Köpfen entsprechende semantische Templates (siehe u. a. Zimmermann 1992) bzw. type shift-Operationen im Lexikon vorsieht (siehe z. B. Bošković 2005, 2010). Ferner steht der Weg offen, Sprachen wie Russisch oder Polnisch Definitheit als grammatische Kategorie vollends abzusprechen (siehe z. B. Bunčić im Druck). Mitunter wird neben DP in der nominalen Domäne mit noch weiteren funktionalen Projektionen gerechnet, z. B. mit einer Num[eral]P, einer Poss[essor]P oder einer 'leichten' $n \mathrm{P}$ (siehe v. a. Adger 2003). In dieser Arbeit spielt die interne Struktur nominaler Syntagmen

\footnotetext{
135 Vgl. z. B. Zimmermann (2009), die für das Russische eine ModP (Verbmodus) über TP (Tempus) ansetzt. Emonds \& Veselovská (2012) nehmen sogar ausschließlich eine MoodP an, da sie vorschlagen, im Tschechischen und Englischen sei ausschließlich der Verbmodus syntaktisch repräsentiert.
} 
und auch die Frage nach der Repräsentiertheit von Definitheit und/oder Spezifizität keine Rolle, daher lege ich mich nicht darauf fest, ob im Russischen und Polnischen DPn anzunehmen sind oder nicht bzw. ob oberhalb von NP mit einer Reihe weiterer Projektionen zu rechnen ist oder nicht. Dementsprechend werde ich in dieser Arbeit durchweg das Label NP für Substantivgruppen verwenden, ohne damit einen der o. g. theoretischen Standpunkte abzulehnen oder zu verteidigen.

Als syntaktische Grundausstattung polnischer und russischer Sätze betrachte ich die Projektionsabfolge in (42):

$$
\mathrm{CP}>\mathrm{IP}>\ldots(>\mathrm{NegP})>\mathrm{VP}^{*}(>\mathrm{XP}) \quad \mathrm{X} \in\{\mathrm{A}, \mathrm{N}, \mathrm{P}\}
$$

Der Asterisk an VP deutet an, dass mehrere VP-Projektionen möglich sind, von denen die "tiefste" das Satzprädikat enthält. Es geht hier um die Erfassung analytischer Strukturen, in die (lexikalische) Auxiliare bzw. Modalverben involviert sind. Die eingeklammerte XP, deren Kopf von einem Adjektiv, einem Substantiv oder von einer Präposition gestellt werden kann, deutet an, dass als Satzprädikat auch diese drei nicht-verbischen Kategorien in Frage kommen, und zwar in den für diese Arbeit besonders relevanten Kopulasätzen.

Den Verbalaspekt betrachte ich als lexikalische Kategorie, die von Verbformen aus dem mentalen Lexikon bereits mitgebracht wird. Er wird daher syntaktisch nicht gesondert repräsentiert (vgl. so u. a. auch Zimmermann 2013).

Auf die Annahme der funktionalen Kategorie v ('leichtes Verb') werde ich verzichten, da ich überzeugt bin, dass flektierte Verbformen mit sämtlichen Argumenten (darunter auch ihr externes Argument) aus dem Lexikon in die Syntax kommen, und dass solche "Kategorien" wie Agentivität oder auch Genus verbi (oder was sonst mitunter mit $v^{0}$ assoziiert wird) nicht syntaktischer Natur ist, sondern aus der deskriptiven Eigenbedeutung von Verblexemen und/oder ihrer Argumentstruktur folgt (und damit aus ihren Lexikoneinträgen; vgl. u. a. auch Junghanns 2008).

Von einer Neg[ations]P gehe ich nur aus, wenn Satznegation vorliegt. Ich weiche also von Zimmermann $(2009,2013) \mathrm{ab}$, indem ich nicht generell eine Pol[aritäts]$\mathrm{P}$ [hrase] vorsehe. Ich folge ihr jedoch darin, dass im Falle von Negation der funktionale $\mathrm{Kopf}_{\mathrm{Neg}}{ }^{0}$ nicht overt als Negationspartikel russ. ne/poln. nie 'nicht' realisiert wird. Vielmehr sind ne bzw. nie Präfixe und werden als solche schon im 
Lexikon (morphologisch) mit der Verbform verbunden. Letztere hat dadurch schon in $\mathrm{V}^{0}$ ein morphosyntaktisches Merkmal [+Neg], durch das die Präsenz der NegP in der Satzsyntax lizenziert wird bzw. das - umgekehrt - von $\mathrm{Neg}^{0}$ selegiert wird (vgl. Zimmermann 2013). Auch in diesem Fall handelt es sich um die "verzögerte Interpretation morphologischer Informationen", denn während das Präfix ne/nie lediglich ein morphosyntaktisches Merkmal einbringt, aber keine Bedeutung hat, steckt die logische Negation in $\mathrm{Neg}^{0}$, das durch das Präfix jeweils nur “sichtbar gemacht" (vgl. erneut Stechows 2007b "Auxiliarprinzip") wird. ${ }^{136}$

Anstelle der in jüngeren Arbeiten weit verbreiteten T[empus]P wähle ich hier das Label IP (Inflection Phrase bzw. "Flexionsphrase"), da m. E. an dieser Stelle im Strukturbaum nicht allein das (semantische) Tempus, sondern auch der Verbmodus repräsentiert sind (vgl. u. a. Zimmermann 2009, die eine gesonderte ModP für letzteren annimmt). Beide Kategorien korrelieren derart, dass der (unmarkierte) Indikativ die Präsenz von Tempus impliziert (bzw. umgekehrt), während der (markierte) Konjunktiv die Abwesenheit jeglicher Tempusspezifikation impliziert (er ist also "tempusneutral"; vgl. u. a. Vinogradov 1952, 286). Da beide Kategorien interagieren und integral mit der verbalen Flexion zusammenhängen, halte ich das Label "IP" für geeigneter als "ModP" oder "TP", die jeweils nur eine Kategorie thematisieren. ${ }^{137}$

Schließlich wird ein Satz durch CP ("Komplementiererphrase") abgeschlossen; ihr Kopf legt die Satzart und den Satzmodus (vgl. u. a. Zimmermann 2009) fest und hat im Falle eingebetteter Sätze in aller Regel die Form einer Subjunktion.

Die Leistungen der einzelnen Projektionen fasse ich in (43) zusammen (zur Unterscheidung von upper vs. lower auxiliaries siehe Abschnitt 5.2.1):

${ }^{136}$ Ich schließe nicht aus, dass NegP in der Satzsyntax keine eigenständige Projektion, sondern vielmehr lediglich eine Adjunktion an VP sein kann.

${ }^{137}$ In der Tat ist es so, dass dem Tempus bis dato weit mehr Aufmerksamkeit in der linguistischen Forschung zugekommen ist als dem Verbmodus. "Verbmodus" wird oft mit "Satzmodus" vermischt oder beide werden einfach unter "Modus" zusammengefasst (so ist es nach wie vor umstritten, ob der Imperativ ein Satz- oder Verbmodus ist). Eine klare Trennung nimmt Zimmermann (2009) vor (die den Imperativ übrigens in die Satzmodi einreiht). Die komplexe Semantik des Verbmodus erschwert seine Erfassung gravierend (siehe u. a. Zimmermann 2013 mit einem Vorschlag für Indikativ, Konjunktiv und Konditional). Mezhevich (2008) hat mit Bezug auf Russisch und Hebräisch einen interessanten Vorschlag zur Erfassung des Verbmodus (verstanden als Indikativ vs. Konjunktiv) gemacht, der auf der Relationierung von bestimmten Zeit(intervall)en beruht und so analog zu Aspekt und Tempus funktioniert. 
(43) $\mathrm{CP}$

IP

( NegP

$\left(\mathrm{VP}^{l+n}\right.$

$\mathrm{VP}^{l}(\mathrm{XP})$
Satztyp/Satzmodus

Verbmodus(/Tempus)(-Auxiliare)

Satznegation ausbuchstabierende (Modal-)Auxiliare )

[lower auxiliaries]

[upper auxiliaries]

)
Satzprädikat (Sachverhaltsreferenz, deskriptive Bedeutung, Argumente)

Über die genannten Punkte hinausgehende Annahmen zur Satzstruktur sind für die Zwecke dieser Arbeit nicht notwendig. Relevanter ist die Interaktion zwischen der funktionalen Ebene (v. a. I-Köpfe) und der lexikalischen Ebene (V-Köpfe), bzw. zwischen grammatischer Bedeutung und ihrer morphologischen Realisierung bzw. "Sichtbarmachung". Diesem Wechselverhältnis widmet sich der folgende Abschnitt.

\subsection{Semantik und Morphosyntax}

\subsubsection{Flexionsmorphologie als Reflex semantischer Operatoren}

Indem ich einer Annahme Stechows folge, ${ }^{138}$ lege ich den weiteren Ausführungen die Ansicht zugrunde, dass morphologische Flexive der bloßen 'Sichtbarmachung' semantischer Operatoren ('wirklich' bedeutungstragender Einheiten) dienen können. Letztere rekonstruiere ich als funktionale Köpfe in der Satzstruktur, die Wortformen mit einer passenden morphologischen Markierung c-kommandieren (siehe Pitsch 2013). In diese Richtung geht auch Zimmermann (2009, 2012, 2013), wenn sie "[f]ür Kasus-, Numerus-, Tempus- und Modusflexive [...]” annimmt, dass “[...] ihre semantische Interpretation verzögert - mit Hilfe von leeren Köpfen - erfolgt” (Zimmermann 2013, 217). In der vorliegenden Arbeit

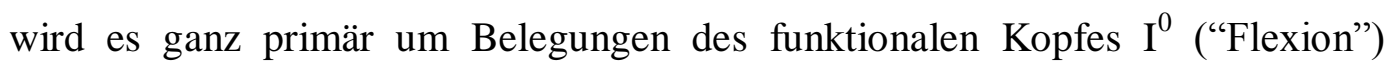

${ }^{138}$ Vgl. v. a. sein Auxiliarprinzip: "Jedes Auxiliar (Hilfsverb) ist semantisch leer, hat aber ein Merkmal, das einen koverten Operator [...], der in seinem Spezifikator steht, sichtbar macht." (Stechow 2007b, 41). Ich folge hier der Idee der 'Sichtbarmachung', nicht aber der Annahme, dass der semantische Operator im Spezifikator der sichtbar machenden Form steht. Mit Zimmermann (2013) soll vielmehr gelten, dass die Operatoren (leere) funktionale Köpfe in der syntaktischen Struktur sind, die VPn mit einer bestimmten Merkmalsausstattung selegieren. 
gehen, werden hier doch die verbalen Kategorien Verbmodus und Tempus 'verwaltet'. ${ }^{139}$

Flexionsmorphologie 'reflektiert' also lediglich bestimmte grammatische Bedeutungen, ist jedoch nicht ihr Träger. Auf diese Weise kann die Flexionsmarkierung von Wortformen in vielen Fällen von ihrer eigentlichen grammatischen Bedeutung dissoziiert werden. So ergibt sich ein Modell von der Semantik-Morphosyntax-Schnittstelle, in dem morphologische Flexive oft keine Eigenbedeutung haben, sondern als sprachliche Zeichen grammatische Bedeutungen lediglich 'sichtbar machen'. Wie bereits beschrieben, sind diese Bedeutungen im Strukturbaum in (ggf. leeren) funktionalen Köpfen angesiedelt. Sie befinden sich somit in einer hierarchisch hohen Position, so dass sie die entsprechenden Flexionsformen c-kommandieren und auf diese Weise mit ihrer Bedeutung versehen.

Eine wünschenswerte Konsequenz dieser Modellierung ist, dass die Syntax uneingeschränkt als bloßer Kombinationsmechanismus betrachtet werden kann, der lexikalische Einheiten auf der Basis ihrer morphosyntaktischen Merkmale verknüpft (Merge; siehe Abschnitt 4.5). Da semantische Operatoren als funktionale Köpfe (mitunter als upper auxiliaries) rekonstruiert werden, determinieren dieselben morphosyntaktischen Merkmale (qua Selektion) auch deren Kombinierbarkeit mit bestimmten verbalen Flexionsformen. Letztere werden vorab im Lexikon (Antritt der Flexionsmarker) mit den relevanten Merkmalen (nicht aber mit der jeweiligen Semantik) ausgestattet.

Im Rahmen der Sprachproduktion zeichnen grammatische Flexive demnach Wortformen als Selektionsobjekte bestimmter funktionaler Köpfe aus, die bestimmte grammatische Bedeutungen tragen. In der Gegenrichtung ermöglichen sie die Rekonstruktion der oft 'unsichtbaren' semantischen Operatoren und damit jener invarianten Bedeutungsanteile, die zum Verständnis sprachlicher Äußerungen unverzichtbar sind. Letztlich wird so durch Flexive durchaus Bedeutung ausgedrückt, jedoch nur indirekt, da sie deren 'Reflex', nicht aber deren Träger sind.

139 Sofern die Belegung von $\mathrm{I}^{0}$ "stumm" ist, kann in der Tat von einem "Operator" gesprochen werden, ist sie dagegen overt und flektiert, passt besser die Charakterisierung als "Auxiliar" (konkret als upper auxiliary). Schließlich ist möglich, dass $\mathrm{I}^{0}$ zwar overt ist, aber nicht flektiert - in diesem Fall kann man entweder von einem "overten Operator" oder aber von einem "unterspezifizierten Auxiliar" sprechen (vgl. z. B. russ. by). Demnach kann man die zuerst erwähnten "stummen" I-Köpfe auch als "unterspezifizierte Nullauxiliare" betrachten, allerdings nur, wenn die von ihnen selegierte Verbform in $\mathrm{V}^{0}$ infinit ist (so im russischen Präteritum). Sofern sie finit ist, liegt doch ein “Operator” vor (so z. B. im Präsens). 
Ebendiese Indirektheit meint auch Zimmermann (2013), wenn sie von "verzögerter Interpretation morphosyntaktischer Informationen" spricht.

Das Beispiel in (44) soll diesen Mechanismus schematisch verdeutlichen:

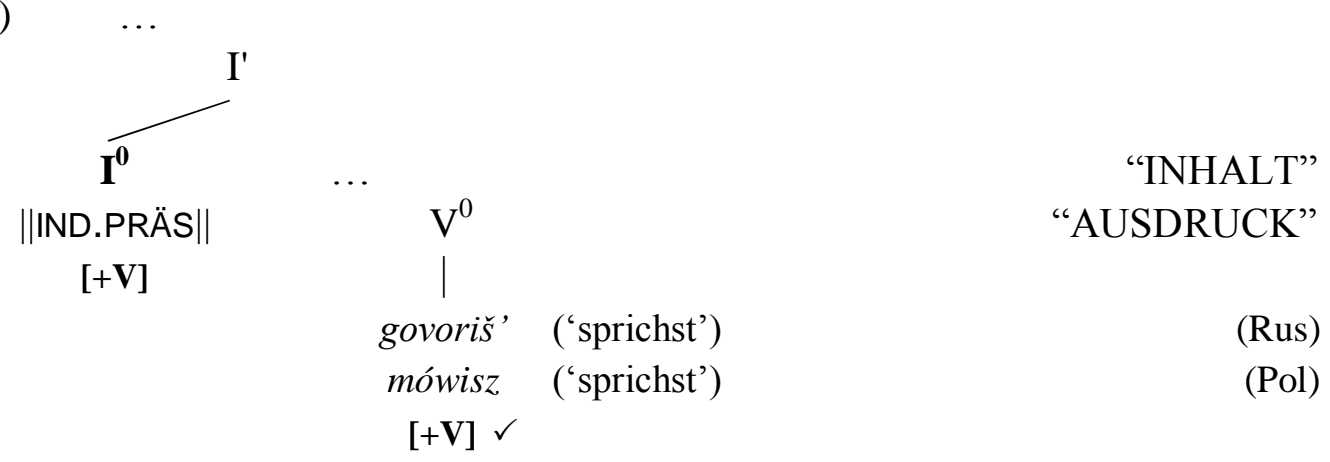

Das hier vorliegende $\mathbf{I}^{\mathbf{0}}$ trägt die Verbmodus- und Tempusbedeutungen "Indikativ" und "Präsens". Über das Merkmal $[+\mathrm{V}]$ selegiert $\mathrm{I}^{0}$ finite Verbformen (siehe Abschnitte 4.3 und 4.6.3). ${ }^{140}$ Dabei c-kommandiert $\mathrm{I}^{0}$ die Verbform in $\mathrm{V}^{0}$. Feature Checking stellt sicher, dass beide Einheiten 'zusammenpassen'. Bedeutsam ist, dass die Flexionsmarker an der Verbform lediglich morphosyntaktische Informationen repräsentieren, nicht aber die entsprechenden semantischen Anteile beinhalten. Demnach hat die Verbform lediglich ihre deskriptive Bedeutung, obwohl sie ein Suffix aufweist, das auf 'Finitheit' hinausläuft. Ort der Tempus- und Verbmodusbedeutung ist jedoch der semantische Operator in $\mathrm{I}^{0}$. Die Verbindung und scheinbare Einheit zwischen Semantik und Morphologie bzw. zwischen "Inhalt" und "Ausdruck" kommt also syntaktisch zustande, namentlich in Form der Selektion passend markierter Wortformen durch funktionale Köpfe.

Im folgenden Abschnitt wird dieses Modell der Interaktion zwischen Morphosyntax und Semantik anhand der Wechselbeziehung zwischen verschiedenen Belegungen von $\mathrm{I}^{0}$ sowie den von ihnen selegierten Verbformen im Detail dargestellt.

\subsubsection{Semantische Operatoren und Auxiliare in $I^{0}$}

Ich betrachte Aspekt als lexikalische Kategorie. Verbale Aspektformen behandele ich somit im Prinzip als gegebene lexikalische Einheiten im mentalen Lexikon. Ihre arbiträre Gestalt sowohl im Polnischen als auch im Russischen (Präfigierung

${ }^{140}[+\mathrm{V}]$ genügt als Selektionsmerkmal, da alle weiteren Verbformen zusätzliche positiv spezifizierte Merkmale aufweisen. Siehe dazu Abschnitt 4.6.3, Fn. 153. 
mittels einer Vielzahl verschiedener Präfixen, Suffigierung, Suppletion; mitunter fehlen Aspektpartner völlig, vgl. u. a. Bunčić 2013) deutet darauf hin, dass die Aspektformen im Rahmen des Spracherwerbs erworben (rote-learned) werden. Aspekt wird also nicht als syntaktische Kategorie betrachtet. Entsprechend wird auch keine Aspektphrase (AspP) angenommen. Jede russische und polnische verbale Form, die flektiert als $\mathrm{V}^{0}$ in die Syntax kommt, ist im vorliegenden Modell bereits mit morphologischem und semantischem Verbalaspekt ausgestattet. ${ }^{141}$

Nach Paslawska \& Stechow (2003a, 307) ist nicht nur von zwei, sondern von drei semantischen Aspekten (im Russischen) auszugehen, namentlich IMPERFEKTIV, PERFEKTIV und PERFEKT. Auf morphologischer Seite stehen dem nur zwei Markierungsoptionen gegenüber, namentlich "Imperfektiv" und "Perfektiv", wobei die semantischen Aspekte PERFEKTIV und PERFEKT gleichermaßen von der "Perfektiv"-Markierung 'reflektiert' werden.

Aus der Kombination dieser semantischen Aspekte mit den drei semantischen Tempora PRÄSENS, PRÄTERITUM, FUTUR ${ }^{142}$ ergeben sich die möglichen Interpretationen (einschließlich der Lesarten eines Perfekt Präsens, Perfekt Präteritum und Perfekt Futur, die jeweils spezifische Kontexte voraussetzen).

Wie ebenfalls bereits erörtert, sind alle Verblexeme mit einem referenziellen Sachverhaltsargument ausgestattet, das vom Verbalaspekt gebunden und in Relation zum Topikzeitintervall gesetzt wird. Letzteres wiederum wird im Rahmen der syntaktischen Strukturbildung schließlich durch den Verbmodus (gemeinsam mit Tempus in $I^{0}$ repräsentiert) aufgegriffen und entweder indikativisch oder konjunktivisch (respektive konditional) spezifiziert.

${ }^{141}$ Dies impliziert nicht, dass morphologischer und semantischer Aspekt generell deckungsgleich seien. Es kann vielmehr gelten, dass allen Verbstämmen im Lexikon auf der Basis von Präfigiertheit und/oder Suffigiertheit bzw. bloßer Idiosynkrasie je einer der semantischen Aspekte zugeordnet wird. So ist ein Verbstamm im Lexikon zu Beginn zwar morphologisch, noch nicht jedoch semantisch im/perfektiv (vgl. Paslawska \& Stechow 2003b, 10). Er könnte erst folgend durch eine semantische Operation, die noch im Lexikon erfolgt, auch semantischen Aspekt erhalten. Auf diese Weise kann auch der "sekundären Imperfektivierung" Rechnung getragen werden, ohne den Verbalaspekt syntaktisch repräsentieren zu müssen. Letzten Endes ist Aspekt demnach keine syntaktisch-funktionale, aber auch keine vollends lexikalische Kategorie. Seine Grammatikalisierung ist fortgeschritten, aber noch nicht abgeschlossen.

142 Die Annahme eines semantischen FUTUR weicht von der Ansicht ab, dass es der perf. Aspekt in Kombination mit dem PRÄSENS sei, der im Russischen und Polnischen für eine lediglich 'futurische' Interpretation sorge (vgl. z. B. Kempgen ${ }^{2}$ 2008, 172-173; Zimmermann 2013, 222). Paslawska \& Stechow (2003b, 15-16) argumentieren dafür, dass auch das Russische über ein semantisches FUTUR verfügen muss, was folglich analog auch für das Polnische gilt. 
Im Falle des Indikativs lokalisiert das Tempus zudem das Topikzeitintervall relativ zur Äußerungszeit $\mathrm{t}^{0}{ }^{143}$ Im Falle des Konjunktivs, der im Russischen und Polnischen 'analytisch' ausgedrückt wird, erfolgt keine temporale Verortung. Was die Konjunktiv- und Konditionalsemantik betrifft, so folge ich Zimmermanns rezentem Vorschlag, dem zufolge der Konjunktiv der Indikativbedeutung ohne Tempusspezifikation entspricht und ihr eine Präsupposition hinzufügt, "die besagt, dass dem epistemischen Modell des modalen Subjekts [...] die Proposition [...] nicht angehört." (Zimmermann 2013, 225). Die konditionale Lesart wiederum wird bei Bedarf durch ein modifizierendes Template ermöglicht (Zimmermann 2013, 225) und stellt so eine kontextuell verursachte Modifikation bzw. Erweiterung der konjunktivischen Lesart dar, die man 'nicht sehen kann'. Indem ich mit Stechow (2007b) und Zimmermann (2012, 2013) darin übereinstimme, dass Flexionsmorphologie oft nur der 'Sichtbarmachung' semantischer Operatoren dient, soll gelten, dass die drei semantischen Tempora PRÄSENS, PRÄTRITUM und FUTUR durch abstrakte semantische Operatoren etabliert werden, die in der Satzsyntax als funktionale I-Köpfe auftreten. Welcher I-Kopf konkret vorliegt, wird durch entsprechende Flexive an der lokal nächsten Verbform und ggf. durch eine mögliche overte Realisierung des I-Kopfes selbst (als upper auxiliary) 'reflektiert'. Da I ${ }^{0}$ nicht nur für die Spezifikation von semantischem Tempus, sondern auch des Verbmodus verantwortlich ist, binden die I-Köpfe gleichzeitig die Topikzeitvariable indikativisch (= bloße Existenzquantifikation; Zimmermann 2009, 486-487) oder konjunktivisch/konditional (siehe oben; Zimmermann 2013, 224-225).

In (45) und (46) nenne ich Lexikoneinträge für die indikativischen I-Köpfe, die für die semantischen Tempora PRÄSENS und FUTUR verantwortlich sind. Bei ihnen handelt es sich um 'richtige' semantische Operatoren in dem Sinne, dass sie bereits finite Verbformen mit Modus-/Tempussemantik versorgen. Die finiten Verben in $\mathrm{V}^{0}$ haben nur ihre deskriptive Bedeutung und eine Aspektsemantik. ${ }^{144}$

\footnotetext{
${ }^{143}$ Man kann diskutieren, ob der Verbmodus das Tempus impliziert oder umgekehrt. Vgl. letztere Sichtweise bei Lehmann (2007): "Die Subkategorie Indikativ der Kategorie Modus wird vom Tempus impliziert - gibt es ein Tempus, ist der Modus Indikativ.” (Lehmann 2007, 22).

${ }^{144}$ Finite Verben realisieren ihr externes Argument bereits in Spec-VP, weshalb in (45) und (46) kein $\lambda x$ auftritt. Im Unterschied zu den upper auxiliaries bzw. I-Auxiliaren in Präteritum und Konjunktiv selegieren die I-Köpfe in (45) und (46) also VPn des Typs <e,t>, erstere aber VPn des Typs $\langle\mathrm{e},\langle\mathrm{e}, \mathrm{t}\rangle>$. Letztlich sind die I-Auxiliare also Raising-Verben vergleichbar, die die
} 
(45) a. $1 \varnothing /$

(PRÄSENS)

b. $[+\mathrm{I}]$

(Pol/Rus)

c. $\lambda P \exists t\left[\left[t \mathrm{AT} \mathrm{t}^{0}\right] \wedge[P t]\right]$

$\lambda P \in[+\mathrm{V}]$

(46) a. $1 \varnothing /$

(FUTUR)

b. $[+\mathrm{I}]$

(Pol/Rus)

c. $\lambda P \exists t\left[\left[\mathrm{t}^{0}\right.\right.$ BEFORE $\left.\left.t\right] \wedge[P t]\right]$

$\lambda P \in[+\mathrm{V},+$ Perf $]$

Das Präteritum wird im Gegensatz zu Präsens und Futur im Russischen und Polnischen nicht 'synthetisch', sondern 'analytisch' ausgedrückt. Die betreffende Struktur geht auf eine frühere (urslavische) Perfektperiphrase zurück, bestehend aus Perfektauxiliar und l-Partizip. Im modernen Polnischen ist diese Struktur relativ unbeschadet erhalten geblieben, wenngleich sich ihre Bedeutung in Folge des Verlusts von Aorist und Imperfekt sowie des "Aufstiegs" der Aspektdistinktion zu einem “Globalpräteritum” (vgl. Lehmann 2009) gewandelt hat. ${ }^{145}$ Letzteres gilt auch für das russische Präteritum, dessen Oberflächengestalt sich jedoch auf Grund des Wegfalls der Lautform der ursprünglichen Perfektauxiliare heute auf die $l$-Partizipien beschränkt und so einen 'synthetischen' Eindruck macht. ${ }^{146}$ Als Vertreter der polnischen Präteritalauxiliare nenne ich in (47) den Eintrag des Enklitikons - $(e) m$ für die 1. Ps. Sg. sowie in (48) den des phonetisch leeren Auxiliars der 3. Ps. Sg. und Pl. Die Präteritalauxiliare sind allesamt upper auxiliaries

ranghöchste Argumentstelle ihres Komplements 'anheben' und syntaktisch als 'ihr' Subjekt realisieren, ihm aber keine Thetarolle zuweisen.

145 Anders als in den nordslavischen Sprachen hat das Perfekt im Bulgarischen und Makedonischen und z. T. auch im BKS seine kompositionale Perfektbedeutung erhalten. Ursächlich ist der Umstand, dass letztere Sprachen noch über Aorist und Imperfekt verfügen, die der Norden größtenteils aufgegeben und deren Funktionen er auf die frühere Perfektperiphrase übertragen hat, die so die erwähnte Globalbedeutung erhielt (vgl. u. a. Sadnik 1966; Eckert, Crome \& Fleckenstein 1983, 171-173).

${ }^{146}$ Evidenz gegen die Behauptung, das russische Präteritum sei ein 'synthetischer' Ausdruck mit finiten $l$-Formen, ist die Tatsache, dass letztere auch im Konjunktiv auftreten, dort aber mit der "Partikel" by. Wären die $l$-Formen von sich aus mit PRÄTERITUM assoziiert, müsste diese Bedeutung im Falle ihres konjunktivischen Gebrauchs 'neutralisiert' werden, was mindestens im hier zugrunde gelegten Semantikmodell ausgeschlossen ist (siehe Zimmermann 1999, 134). 
und als solche k/overte Belegungen von $\mathrm{I}^{0}$. Es handelt sich um finite Auxiliare, da sie dem ranghöchsten Argument des selegierten $l$-Partizips die syntaktische Realisierung als Subjektausdruck ermöglichen. Der Subjektausdruck wird hier - anders als im 'synthetischen' Präsens und Futur - erst in Spec-IP generiert: ${ }^{147}$

(47) a. $/(\mathrm{e}) \mathrm{m} /$

(PRÄTERITUM, 1. Ps. Sg.)

b. $[+\mathrm{I}]$

c. $\lambda P \lambda x_{[+1,-2,-\mathrm{Pl}]} \exists t\left[\left[t\right.\right.$ BEFORE $\left.\left.\mathrm{t}^{0}\right] \wedge[P x t]\right]$

$\lambda P \in[+\mathrm{V},+\mathrm{N}, \pm$ Perf,+ Agr $]$

(48) a. $1 \varnothing /$

(PRÄTERITUM, 3. Ps. Sg./Pl.)

b. $[+\mathrm{I}]$

c. $\lambda P \lambda x_{[-1,-2, \pm \mathrm{PI}]} \exists t\left[\left[t\right.\right.$ BEFORE $\left.\left.\mathrm{t}^{0}\right] \wedge[P x t]\right]$

$\lambda P \in[+\mathrm{V},+\mathrm{N}, \pm$ Perf,+ Agr $]$

Das Russische hat in seiner Entwicklung alle overten Formen des Präteritalauxiliars aufgegeben (siehe u. a. Isačenko 1983, 371-372). Geblieben ist ein "stummes" und dabei hinsichtlich seiner Person-/Numeruskombination "variables" Auxiliar, dessen Eintrag ich in (49) nenne. Der "Gewinn" in Folge dieses Wandels liegt auf der Hand: Anstelle von (mindestens) fünf paradigmatischen Formen für das Präteritalauxiliar, wie sie heute im Polnischen im Lexikon vorliegen müssen, genügt dem Russischen nur eine einzige "flexible" Nullform. Der Vergleich mit dem Nullauxiliar für die 3. Person im Polnischen (und auch mit anderen v. a. südslavischen Sprachen) legt die Vermutung nahe, dass der Prozess des Abbaus der Lautform des Präteritalauxiliars ein sprachübergreifendes Phänomen ist, das zuerst die (im Person-/Numerusparadigma unmarkierten) Formen der 3. Person und folgend alle Personen und Numeri erfassen kann:

${ }^{147}$ Dass es sich beim Subjektausdruck wirklich um das externe Argument des lexikalischen Verbs (Satzprädikats) handelt, ist gesichert, da das I-Auxiliar lediglich eine Argumentstelle für dieses Argument bereitstellt, diese aber nicht mit keiner Thetarolle ausstattet (mangels lexikalischdeskriptiver Bedeutung). Das 'Subjekt' wird also nur 'angehoben'. 
(49) a. $1 \varnothing /$

(PRÄTERITUM, 'variabel')

b. $[+\mathrm{I}]$

c. $\quad \lambda P \lambda x_{[ \pm 1, \pm 2, \pm \mathrm{Pl}]} \exists t\left[\left[t\right.\right.$ BEFORE $\left.\left.\mathrm{t}^{0}\right] \wedge[P x t]\right]$

$$
\lambda P \in[+\mathrm{V},+\mathrm{N}, \pm \text { Perf },+ \text { Agr }]
$$

Nach Zimmermann (2013, 225) besteht der semantische Konjunktiv darin, dass die Indikativsemantik durch eine Präsupposition angereichert wird, die besagt, dass die denotierte Proposition nicht Teil des epistemischen Modells $\left(\mathrm{M}_{\mathrm{EP}}\right)$ des modalen Subjekts (u) ist. In (50) übernehme ich diesen Vorschlag, indem ich die entsprechende Semantik in den Lexikoneintrag des polnischen Konjunktivauxiliars der 1. Ps. Sg. bym integriere. Hinter “|” erscheint tiefergestellt die von Zimmermann angesetzte Präsupposition, die die Geltung der Proposition 'irrealisiert'. Die Variable " $u$ " für das modale Subjekt ist ein Parameter, der erst kontextuell identifiziert wird. Die "Atemporalität” des Konjunktivs rührt daher, dass keine Verortung des Referenzzeitintervalls relativ zur Äußerungszeit erfolgt: ${ }^{148}$

(50) a. /bym/

(KONJUNKTIV, 1. Ps. Sg.)

b. $[+\mathrm{I},+$ Konj $]$

c. $\quad \lambda P \lambda x_{[+1,-2,-\mathrm{Pl}]} \exists t\left[\begin{array}{lll}P & x t] \mid \neg[\wedge \exists t[P & \left.x t] \cap \mathrm{M}_{\mathrm{EP}} \mathrm{u}\right]\end{array}\right.$ $\lambda P \in[+\mathrm{V},+\mathrm{N}, \pm$ Perf,+ Agr $]$

Im Russischen sind die Formen des Konjunktivsauxiliars zu einer scheinbaren "Partikel" by erstarrt. M. E. handelt es sich aber - wie schon beim stummen Präteritalauxiliar - um eine "variable" Auxiliarform. Die Variabilität hinsichtlich der Merkmale Person und Numerus findet ihre morphologische Entsprechung im Fehlen jeglicher Flexionsmarkierung:

$$
\begin{aligned}
& \text { a. } / \mathrm{b}(\mathrm{y}) / \\
& \text { b. }[+\mathrm{I},+ \text { Konj }]
\end{aligned}
$$



$$
\begin{aligned}
& \lambda P \in[+\mathrm{V},+\mathrm{N}, \pm \text { Perf },+ \text { Agr }]
\end{aligned}
$$

\footnotetext{
148 Über das Verbmodusmerkmal [+Konj], das diese I-Auxiliare einbringen, selegieren bestimmte Matrixverben konjunktivische (non-faktive) Nebensätze (russ. (čto)by-/poln. (̇e)by-Sätze).
} 
Mit den by-Auxiliaren kann sowohl im Polnischen als auch im Russischen neben dem l-Partizip auch der Infinitiv auftreten, und zwar in 'unpersönlichen' Sätzen. ${ }^{149}$ Meist treten solche infinitivischen Konjunktive in (non-faktiven) Komplement- oder Adjunktsätzen auf, die von der Konjunktion żelčto 'dass' eingeleitet werden, an die das Konjunktivauxiliar obligatorisch enklitisiert; vgl. (52a/b):

$$
\begin{aligned}
& \text { a. ..., żeby pracować. } \\
& \text { dass+KONJ.AUX arbeit-INF } \\
& \text { b. ..., čtoby rabotat'. } \\
& \text { dass+KONJ.AUX arbeit-INF } \\
& \text { '..., um zu arbeiten.' }
\end{aligned}
$$

In solchen Sätzen liegt nach Standardannahmen ein PRO-Subjekt vor, dass von einem Antezedens im Matrixsatz (oder arbiträr) kontrolliert wird. Man kann annehmen, dass die externe Argumentstelle des infinitivischen Satzprädikats bis zum Schluss nicht syntaktifiziert wird, und dass dem das phonetisch und semantisch leere Pronomen PRO entspricht. ${ }^{150}$ Sicherlich ist die Nichtsyntaktifizierbarkeit des externen Arguments nicht allein dem Infinitiv geschuldet, sondern auch den Eigenschaften der Auxiliarform by, die die syntaktische Realisierung des betreffenden Arguments offensichtlich ebenfalls ausschließt. Ich nehme daher eine spezielle ‘unpersönliche' Form des Konjunktivauxiliars an, die im Polnischen und Russischen gleichermaßen by lautet. Diese Form hat - im Unterschied zu den homonymen polnischen Formen der 3. Person bzw. zur russischen "Einheitsform" - keine Nullendung, sondern ist 'endungslos'. Folglich annotiert das 'unpersön-

149 Švedova \& Lopatin (21990, 277-278) nennen neben den "kanonischen" Verbindungen von by mit $l$-Formen und Infinitiven noch die relativ seltene Kombination mit anderen (attributiven) Partizipien, z. B. obretšij by ('potenziell gefundener'). Diesem Befund wird durch die o. g. Selektionsmerkmale " $[+\mathrm{V},+\mathrm{N}, \pm$ Perf, + Agr $]$ " entsprochen, die im Prinzip alle Partizipien (nicht nur $l$-Partizipien) zulassen.

150 "An argument position is occupied by a semantically empty pronoun. This is an expression without meaning and without logical type, often called PRO. This expression is moved at the left periphery and creates a $\lambda$-abstract. We will call this kind of movement PRO-movement. [...] At LF, PRO is deleted. PRO originates in a case-less position." (Stechow 2012. 2184) Vgl. auch Stechow (2009, 145). Seinen Annahmen liegt die PRO-Theorie nach Heim \& Kratzer (1998) zugrunde. Ich denke, dass man Kontrolle anstelle durch das syntaktische PRO auch rein semantisch erklären kann, indem die ungebundene Argumentstelle schließlich per default existenzquantifiziert wird und als kontrollierter Parameter in die Interpretation eingeht. 
liche' by die externe Argumentstelle nicht mit Person- und Numerusmerkmalen. Die fraglichen Sätze haben also zwar Verbmodus, aber kein (kanonisches) Satzsubjekt, da die Struktur dafür keine Kongruenzmerkmale bereitstellt. ${ }^{151}$

'Unpersönliches' by versieht die ranghöchste Argumentstelle des infinitivischen Prädikats also nicht mit einer Annotation. Das referenziell ungebundene $\lambda x$ muss daher per default gebunden und dann als Parameter kontrolliert werden, was durch ein geeignetes Antezedens in der Matrix oder aber 'arbiträr' erfolgt: ${ }^{152}$

$$
\begin{aligned}
& \text { a. /by/ } \\
& \text { b. }[+\mathrm{I},+ \text { Konj }] \\
& \text { c. } \quad \lambda P \lambda x \exists t\left[\begin{array}{lll}
P & x & t
\end{array}\right] \mid \neg\left[\wedge \exists t\left[\begin{array}{lll}
P & x & t
\end{array}\right] \cap \mathrm{M}_{\mathrm{EP}} \mathrm{u}\right] \\
& \lambda P \in[+\mathrm{V},+\mathrm{N}, \pm \text { Perf }]
\end{aligned}
$$

(KONJUNKTIV, ‘unpersönlich')

(Pol/Rus)

Der Vergleich der folgenden Satzstrukturen illustriert Ähnlichkeiten und Differenzen zwischen Infinitiv und $l$-Partizip im analytischen Konjunktiv:

$$
\begin{array}{llll}
\text { a. } & \ldots, \text { żeby } & \text { [PRO }] & \text { pracować. } \\
& \text { dass+KONJ.AUX.IMPS } & \text { arbeit-INF } \\
& \text { '..., um zu arbeiten.' } & & \\
\text { b. } & \ldots, \text { żebyś } & \text { pro } & \text { pracowała. } \\
& \text { dass+KONJ.AUX-2SG } & {[2 . \mathrm{SG}]} & \text { arbeit-LPT-SG.F } \\
& \text { ‘..., damit du arbeitest.' } &
\end{array}
$$

Das infinitivische Satzprädikat in (54a) ist ideal zum Ausdruck des 'Unpersönlichen' geeignet, während das $l$-Partizip in (54b) zusammen mit einem nominati-

${ }^{151}$ Im Rahmen ihrer Analyse slavischer Impersonale mit Akkusativen nehmen Fehrmann, Junghanns \& Lenertová (2013) einen T-Kopf an, der zwar ein Tempusmerkmal, aber keine Kongruenzmerkmale aufweist. Diesen Kopf kann man als [+T,-Agr] erfassen. De facto ist auch das hier vorgeschlagene 'unpersönliche' by ein solches "non-agreeing T" $\left(/ \mathrm{I}^{0}\right)$. Vgl. auch Franks (1995) und Madariaga (2011), die für russische Infinitivsätze wie Mne pospat' by! 'Könnte ich nur ein wenig schlafen!' annehmen, diese seien ge-Tense-t ([+T]). Dass auch in solchen Sätzen keine Kongruenzmerkmale gegeben sind, liegt auf der Hand. Auch in letzterem Beispiel dürfte also ‘unpersönliches' by vorliegen.

${ }^{152}$ Die Abwesenheit einer Person-/Numerus-Annotation an $\lambda x$ korreliert mit der Abwesenheit des Selektionsmerkmals [+Agr] an $\lambda P$, was nur die Selektion eines Infinitivs zulässt. Umgekehrt korrelieren oben bei den 'persönlichen' I-Auxiliaren die vorhandenen Annotationen mit der Präsenz von [+Agr], was die Selektion von $l$-Partizipien zur Folge hat. 
vischen Subjektausdruck (pro) auftreten kann, mit dem es in Numerus und Genus kongruiert (als Partizip muss es im Unterschied zum Infinitiv kongruieren). Diesen Vergleich kann man analog auch für das Russische durchführen, wenn auch dort mangels Kongruenzmorphologie am Auxiliar nie explizit wird, ob 'unpersönliches' oder 'persönliches' by vorliegt. Folglich ist der Unterschied nur an der Art des infiniten Satzprädikats auszumachen, was jedoch durchaus hinreichend ist:

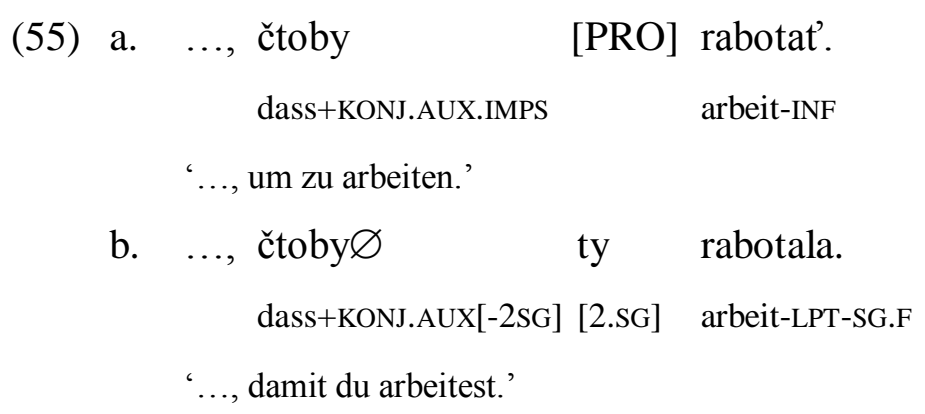

\subsubsection{Die Interaktion zwischen $I^{0}$ und $V^{0}$}

Bisher wurde skizziert, dass der Großteil flexionsmorphologischer Markierungen an Verbformen der "Sichtbarmachung" semantischer Anteile dient, die sich in der Struktur in einer hierarchisch höheren Position befinden. Nunmehr soll die Interaktion zwischen den relevanten Ebenen, $\mathrm{I}^{0}$ (IP) und $\mathrm{V}^{0}$ (VP), dargestellt werden. Zunächst ist darauf hinzuweisen, dass die funktionalen Einheiten in $\mathrm{I}^{0}$ nur dann "semantische Operatoren" im wörtlichen Sinne sind, wenn sie eine finite Verbform 'lediglich' mit ihrer Semantik ausstatten, ansonsten aber morphosyntaktisch nicht in Erscheinung treten. Dies ist der Fall bei finiten "Präsensformen" (siehe Abschnitt 4.3), die man traditionell als 'synthetische' Verbformen bezeichnet, da alle Informationen morphologisch direkt an ihnen selbst realisiert sind.

Sofern der bereits im mentalen Lexikon spezifizierte Aspekt dieser "Präsensformen" der imperfektive ist, werden sie vom indikativisch-präsentischen I-Kopf (siehe (45) oben) selegiert und reflektieren bzw. erhalten auf diese Weise dessen Modus- und Tempusbedeutung. Mit Zimmermann $(2012,2013)$ ist von "verzögerter Interpretation" zu sprechen, weil die Verbform erst durch $\mathrm{I}^{0}$ mit grammatischen Bedeutungen ausgestattet wird, in $\mathrm{V}^{0}$ jedoch noch 'bedeutungslos' ist. Die 
folgende Struktur veranschaulicht diesen Mechanismus der Versorgung einer finiten imperfektiven Verbform in $\mathrm{V}^{0}$ durch den Indikativ/Präsens-Operator in $\mathrm{I}^{0}:^{0.153}$



Ist die "Präsensform" in $\mathrm{V}^{0}$ dagegen perfektiv, wird sie vom indikativisch-futurischen I-Kopf (siehe (46) oben) selegiert, der ihr wiederum seine grammatische Bedeutung verleiht:

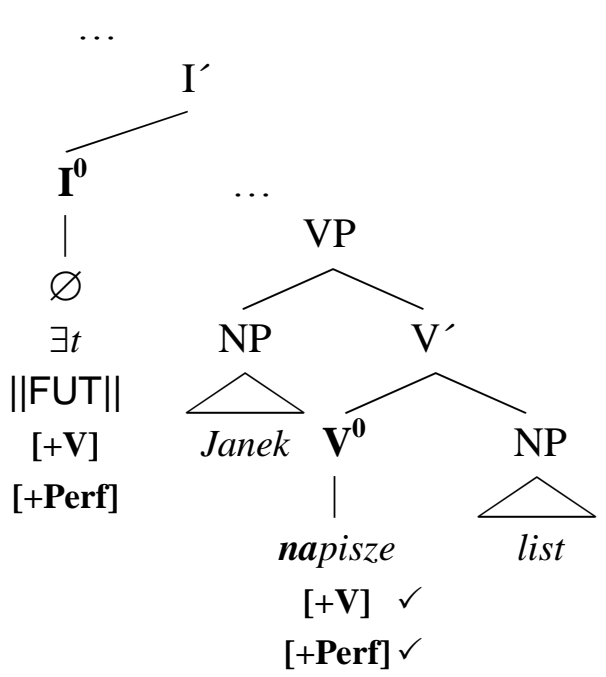

Beim PRÄSENS und beim FUTUR wird die morphologische Aspektmarkierung der Verbform also indirekt genutzt, um semantisches Tempus zu reflektieren. Derselbe indirekte Mechanismus zur Versorgung mit FUTUR kommt auch beim 'analytischen' Futur zur Anwendung. Bekanntlich kann sowohl im Russischen als auch im Polnischen die Futurbedeutung (i) durch perfektive "Präsensformen" (so-

${ }^{153}[+\mathrm{V}]$ genügt hier als Selektionsmerkmal, da alle übrigen verbalen Flexionsformen weitere positive Merkmale besitzen. Die "Präsensformen" sind demnach am wenigsten markiert. 
eben beschrieben) oder (ii) durch imperfektive Infinitive (im Polnischen auch l-Partizipien) im Zusammenspiel mit den "Futurauxiliaren" (będ-/bud-Formen) ausgedrückt werden. Der Ausdruck von FUTUR ist also aspektuell: Eine perfektive "Präsensform" repräsentiert die Kombination FUTUR + PERFEKTIV, die Periphrase mit dem "Futurauxiliar" hingegen die Kombination FUTUR + IMPERFEKTIV.

Jedoch ist bei letzterer Kombination derselbe Mechanismus zwischen $\mathrm{I}^{0}$ und $\mathrm{V}^{0}$ wirksam wie beim bereits beschriebenen perfektiven Futur, was zunächst überraschen mag, da das 'analytische' Futur nun einmal imperfektiv ist.

Der Unterschied zwischen beiden Futurformen besteht darin, dass beim 'analytischen' Futur eine Form des będ-/bud-Auxiliars zwischen dem futurischen Operator in $\mathrm{I}^{0}$ und dem infiniten Satzprädikat interveniert (es handelt sich um ein V-Auxiliar; Abschnitt 5.2.1). Diese Form 'imitiert' gewissermaßen eine 'normale' perfektive "Präsensform". Da es sich jedoch um ein Auxiliar ohne deskriptive Bedeutung und ohne semantischen Aspekt handelt, ${ }^{154}$ führt seine 'Mimikry' lediglich zur Versorgung mit dem semantischen FUTUR, und zwar durch denselben Indikativ-Futur-Operator in $\mathrm{I}^{0}$ wie beim "perfektiven Futur" oben in (57). Da die "Futurauxiliare" imperfektive Infinita (Verbalnomina) selegieren, ${ }^{155}$ und da in der Struktur allein letztere über semantischen Aspekt verfügen, wird folglich letztlich deren Aspekt interpretiert:

${ }^{154}$ Ich analysiere die "Futurauxiliare" folglich als morphosyntaktisch ('formal') perfektiv, jedoch als semantisch ('tatsächlich') aspektlos (siehe auch Pitsch 2013).

${ }^{155}$ Die Formen des będ-/bud-Auxiliars erscheinen nur im Rahmen des aktivischen 'analytischen' Futurs mit imperfektiven Infinita. Wenn sie im partizipialen Passiv die FUTUR-Versorgung bewerkstelligen, 'akzeptieren' sie durchaus auch perfektive Partizipien, vgl. (i):

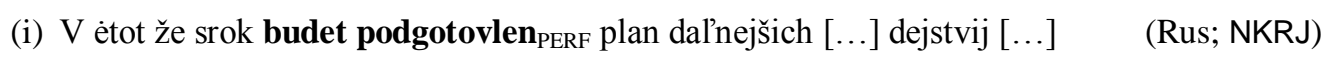

'In diesem Zeitraum wird ein Plan der nächsten [...] Aktionen vorbereitet werden ...'

Es stimmt also nicht, dass das będ-lbud-Auxiliar ausschließlich Imperfektiva selegiert. M. E. stellt es keine Anforderungen an den Aspekt seines Komplements. Dass im "Aktiv" stets nur der imperfektive Aspekt gewählt wird, dient m. E. der Vermeidung von Redundanz. Insofern bereits das 'synthetische' Futur perfektiv ist, würde ein 'analytisches' perfektives Futur eine Doppelung im System bedeuten. Dafür spricht auch, dass etwa im Bulgarischen, das sowohl bei imperfektiven als auch perfektiven Verben eine Art 'analytisches' Futur (Partikel šte) verwendet, ein 'synthetisches' perfektives Futur (ohne šte) in Hauptsätzen unmöglich ist, was ebenfalls auf Redundanzvermeidung zurückgeführt werden kann. Letztlich unterscheiden sich die Sprachen lediglich in den konkret verwendeten Ausdrucksformen, denen aber der gleiche 'Fundus' denkbarer Ausdrucksformen zugrunde liegt (Parametrisierung). 
(58)

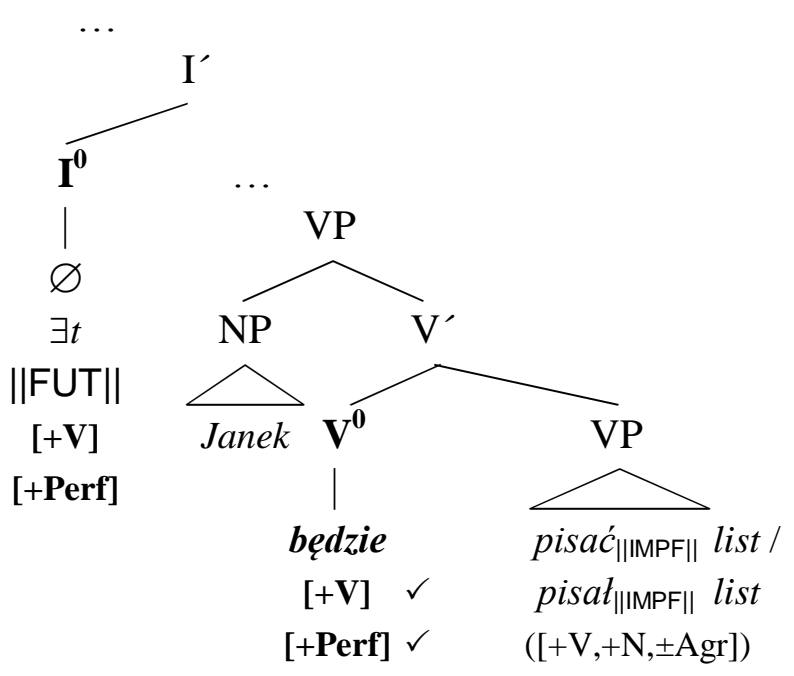

(59)

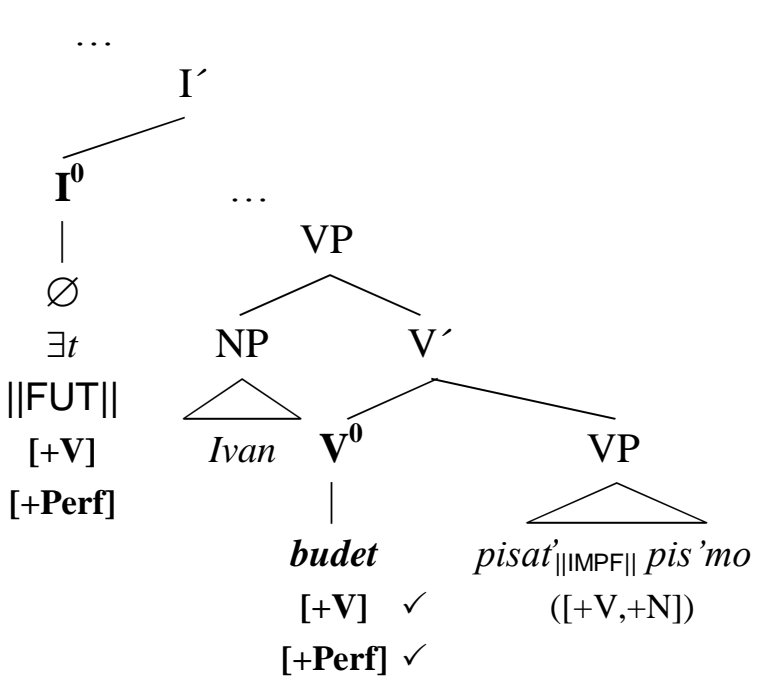

(Rus)

Das Auxiliar dient somit dem Zweck, die Projektion des futurischen I-Kopfes zu ermöglichen, wodurch die Gesamtstruktur mit FUTUR versorgt wird. Die semantische Aspektlosigkeit des Auxiliars ermöglicht, dass die infinite Verbform als eigentliches Satzprädikat ihren semantischen Aspekt zur Geltung bringen kann. In diesem Sinne sind das 'analytische' (imperfektive) und das 'synthetische' (perfektive) Futur strukturell annähernd parallel.

Anders stellt sich die Interaktion bei Präteritum und Konjunktiv dar. Wie im letzten Abschnitt beschrieben, involvieren diese Periphrasen upper auxiliaries bzw. Auxiliarformen, die unmittelbar overte I-Köpfe sind (siehe Abschnitt 5.2). Im Gegensatz zu solchen V-Auxiliaren bzw. lower auxiliaries wie z. B. dem będ-/budAuxiliar, die man auf Grund ihres "Verbseins" auch als "lexikalische Auxiliare" charakterisieren mag (vgl. Borsley \& Rivero 1994), sind upper auxiliaries in $\mathrm{I}^{0}$ (oder I-Auxiliare) grammatikalisierte (funktionale) Einheiten, die nurmehr ihrer 
Gestalt nach an Formen des Lexems byćlbyt' erinnern. Für letztere Sichtweise spricht nicht nur ihre grammatische Funktion, ${ }^{156}$ sondern auch ihre sehr spezifische morphonologische Form. ${ }^{157}$ Nichtsdestotrotz sind bei ihnen verbale Eigenschaften erhalten geblieben, darunter ihre finite Gestalt. Das heißt, sie buchstabieren ebenso wie 'normale' Finita Person- und Numerusmerkmale aus, so dass das externe Argument des infiniten Satzprädikats in Spec-IP als nominativischer Subjektausdruck syntaktifiziert werden kann.

Das Präteritum und der Konjunktiv haben jeweils spezifische I-Auxiliare. Wie in (47)-(49) weiter oben gezeigt wird, hat das I-Auxiliar des Präteritums indikativisch-präteritale Semantik, die es dem selegierten l-Partizip 'verleiht':

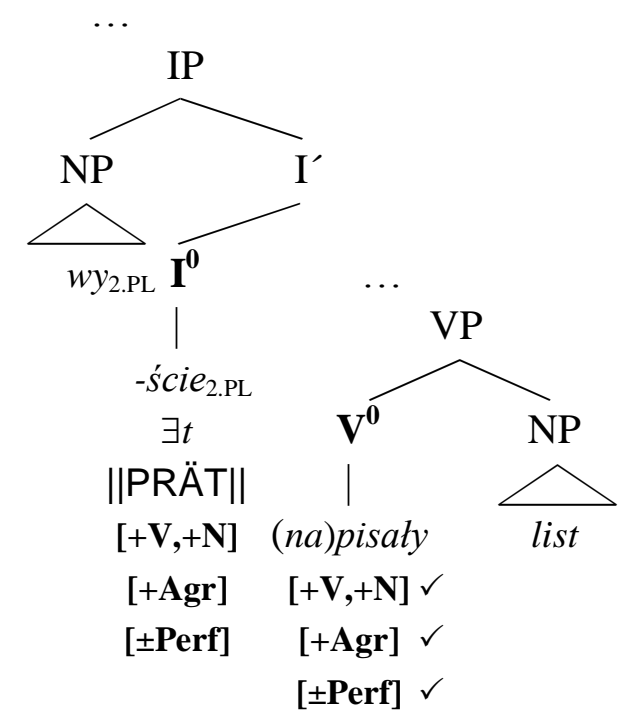

Diese Struktur kann zwei Oberflächenabfolgen zeitigen, die in (61a) und (61b) gezeigt werden. Sie ergeben sich aus der Enklitizität des Präteritalauxiliars, in Folge derer entweder das Auxiliar selbst an das Subjektpronomen klitisiert oder

${ }^{156}$ Man denke etwa an die Konjunktivauxiliare (bym, byś, by etc.), die kaum mehr als 'Verwandte' des heutigen Lexems byćlbyt' wahrgenommen werden.

${ }^{157}$ Es handelt sich stets um Klitika. Zu erwähnen ist ferner die extreme Reduziertheit der polnischen Präteritalauxiliare (- $m,-\dot{s},-\varnothing$ etc.) bzw. der komplette Verlust ihrer Lautform im Russischen. Schließlich fällt auf, dass upper auxiliaries ausschließlich finite Formen haben. Im Gegensatz dazu können lower auxiliaries auch infinit sein; vgl. z. B. das polnische Bsp. in (i):

(i) Każdy chce być kochany. jeder will-3SG AUX-INF geliebt-PART-NOM.SG.M 'Jeder will geliebt werden.' 
das $l$-Partizip in eine Position links vom Auxiliar bewegt wird. Die Wahl ist m. E. primär stilistisch motiviert, wobei (61b) neutraler ist: ${ }^{158}$
a. Wyście
napisały
list.
ihr+PRÄT.AUX-2.PL PERF-schreib-LPT-PL
Brief-ACC
b. Wy napisałyście
list.
ihr PERF-schreib-LPT-PL+PRÄT.AUX-2.PL Brief-ACC
'Ihr habt einen Brief geschrieben.'

Da das Präteritalauxiliar im heutigen Russischen keinerlei overte Formen mehr hat, kann vor dem Hintergrund des Ökonomieprinzips angenommen werden, dass im mentalen Lexikon nurmehr eine einzige Nullform mit unterspezifizierter Person-/Numerusspezifikation gegeben ist. An der Satzoberfläche ist im russischen Präteritum also stets nur das $l$-Partizip sichtbar, was sicherlich als Ursache dafür zu sehen ist, dass die meisten Sprecher und Grammatiken die "l-Form" als "Präteritalform" betrachten. Die hier vorgelegte Analyse spricht dagegen und für die volle Parallelität des russischen zum polnischen Präteritum.

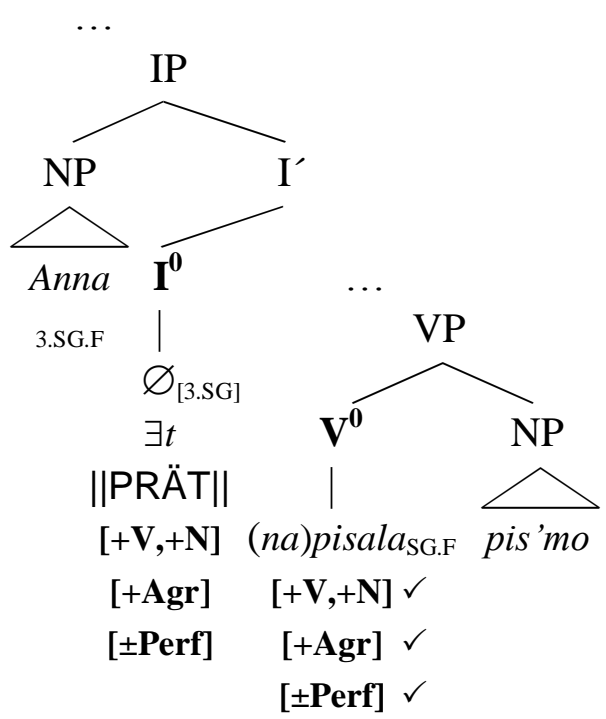

(Rus)

In der gleichen Weise 'analytisch' wie das Präteritum ist in beiden Sprachen auch der Konjunktiv. Im vorangehenden Abschnitt wurde beschrieben, dass auch in die

\footnotetext{
${ }^{158}$ Bei diesem Beispiel ist zu bedenken, dass Polnisch eine Nullsubjektsprache ist, dass also 'normalerweise' kein overtes Subjektpronomen gebraucht wird. In diesem Fall bestünde die Möglichkeit zu einer Abfolge wie in (61a) gar nicht, so dass nur (61b) verbliebe.
} 
Konjunktivperiphrase ein I-Auxiliar involviert ist (erkennbar am Segment by-). Ferner wurde erwähnt, dass der polnische und russische Konjunktiv 'atemporal' ist, also keine Verortung des Topikzeitintervalls relativ zur Äußerungszeit vornimmt. Ebenso wurde neben den 'persönlichen' Formen des by-Auxiliars eine 'unpersönliche' Form vorgeschlagen, die nicht mit $l$-Partizipien, sondern mit ebenso 'unpersönlichen' Infinitiven auftritt. ${ }^{159}$ Die Struktur in (63) zeigt eine polnische Konjunktivform mit dem ‘persönlichen’ I-Auxiliar byśmy (1. Ps. Pl.):

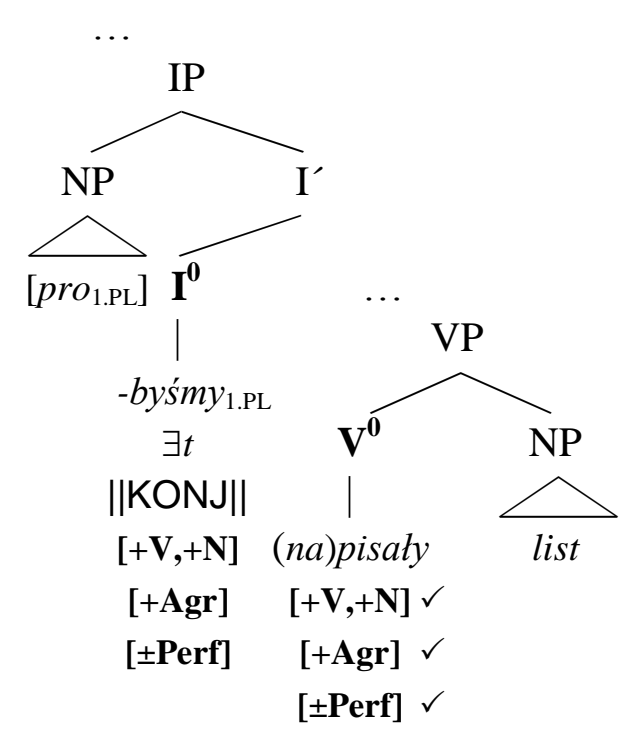

Diese Struktur kann dem Satz in (64) zugrunde liegen:

pro Napisałybyśmy list.

[1.PL] PERF-schreib-LPT-PL+KONJ.AUX-1.PL Brief-ACC

'Wir würden den/einen Brief schreiben.'/'Wir hätten den/einen Brief geschrieben.'

Im Falle des Vorhandenseins der satzeinleitenden Konkjunktion $\dot{z} e$ 'dass' erscheint das enklitische Konjunktivauxiliar adjazent an dieser: ${ }^{160}$

${ }^{159}$ Obwohl es bereits aus der Analyse des Präteritums hervorgegangen sein dürfte, möchte ich hervorheben, dass das $l$-Partizip auch im Rahmen des Konjunktivs nichts weiter als eine spezifische infinite Form ist und keine grammatische Eigenbedeutung hat. Ebendies ermöglicht seine Selektion sowohl durch das Präterital- als auch durch das Konjunktivauxiliar.

${ }^{160}$ Es handelt sich wahrscheinlich um keine syntaktische, sondern um eine Bewegung auf PF. 
(65)

$$
\begin{aligned}
& \text {..., żebyśmy pro napisały list. } \\
& \text { dass+KONJ.AUX-1.PL [1.PL] PERF-schreib-LPT-PL Brief-ACC } \\
& \text { '..., dass (/damit) wir den/einen Brief schreiben (würden).' }
\end{aligned}
$$

Das 'unpersönliche' by hingegen steht mit einem Infinitiv und entsprechend mit einem PRO-Subjekt: ${ }^{161}$

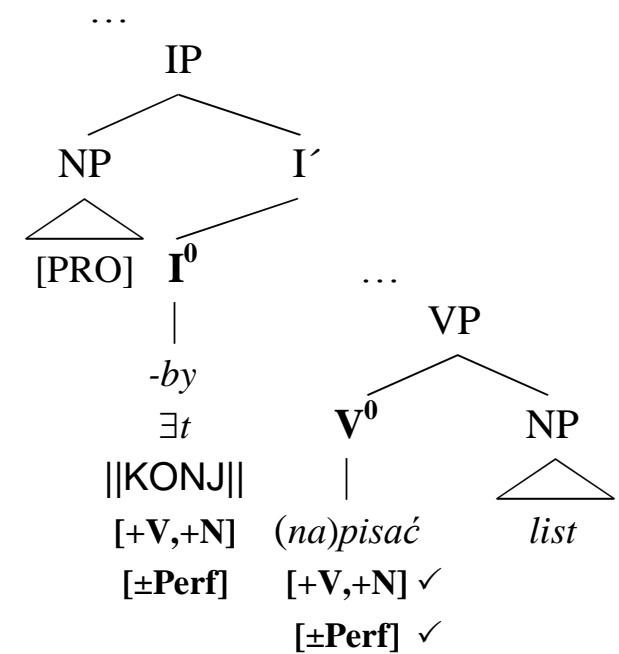

Diese 'unpersönliche' Variante des Konjunktiv tritt fast ausschließlich in Nebensätzen auf, die von $\dot{z} e$ eingeleitet werden, an das by obligatorisch antritt:

$$
\begin{aligned}
& \text { (67) ..., żeby } \\
& \text { PRO napisać list. } \\
& \text { dass+KONJ.AUX.IMPS PERF-schreib-INF Brief-ACC } \\
& \text { '..., um den/einen Brief zu schreiben.' }
\end{aligned}
$$

Der russische Konjunktiv funktioniert parallel zu seinem polnischen Äquivalent, jedoch mit der 'typisch' russischen Eigenheit, dass das I-Auxiliar keine Flexionsmarker zeigt, also stets nur by lautet, und zwar unabhängig davon, ob es 'persönlich' oder 'unpersönlich' ist. Zunächst in (68) zur 'persönlichen' Variante:

${ }^{161}$ Das Tschechische kennt kein 'unpersönliches' by mit Infinitiv und scheint folglich in dieser Hinsicht anders parametrisiert zu sein als das Polnische und das Russische. 
(68)

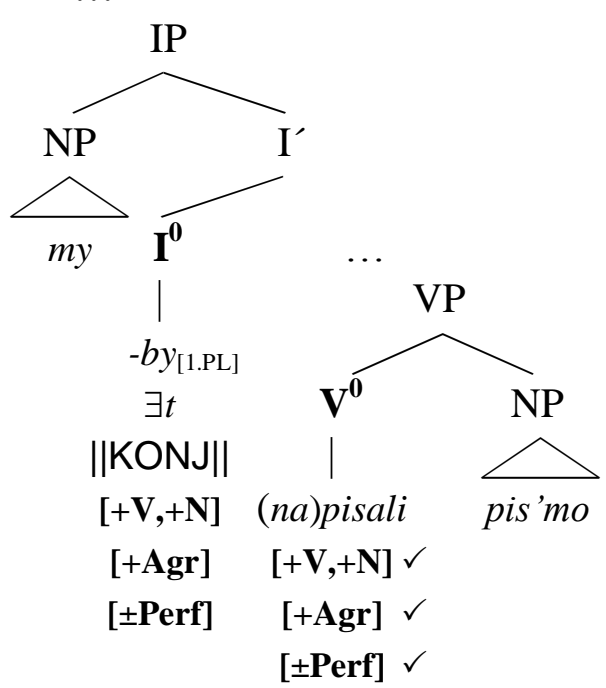

(Rus)

Aus dieser Struktur können sich die folgenden Abfolgen ergeben, wobei die zweite die üblichere ist. Beachte, dass by auch im Russischen enklitisch ist, was aber orthographisch durch Getrenntschreibung verschleiert wird:
a. My by
napisali
pis'mo.
wir KONJ.AUX-[1.PL]
PERF-schreib-LPT-PL
Brief-ACC
b. My napisali
by pis'mo.
wir PERF-schreib-LPT-PL KONJ.AUX-[1.PL] Brief-ACC
'Wir haben einen Brief geschrieben.'

Orthographisch nicht verschleiert wird die Enklise von by im Falle von Nebensätzen mit der Konjunktion čto 'dass'. Hier wird stets čtoby geschrieben, weshalb diese "Form" nicht selten als spezifische Konjunktion (als Einheit) betrachtet wird (vgl. u. a. Vinogradov 1952, 291-292; die Gegensicht vertritt Junghanns 1994):
(70) .., čtoby
my napisali
pis'mo.
dass+KONJ.AUX-[1.PL] wir PERF-schreib-LPT-PL Brief-ACC
'..., dass wir haben einen Brief schreiben (/schreiben würden/geschrieben hätten).'

(Rus)

Die 'unpersönliche' Variante unterscheidet sich an der Satzoberfläche nicht in der Form von by, sondern darin, dass hier anstelle eines $l$-Partizips ein 'unpersönlicher' (akongruenter) Infinitiv das Satzprädikat stellt: 


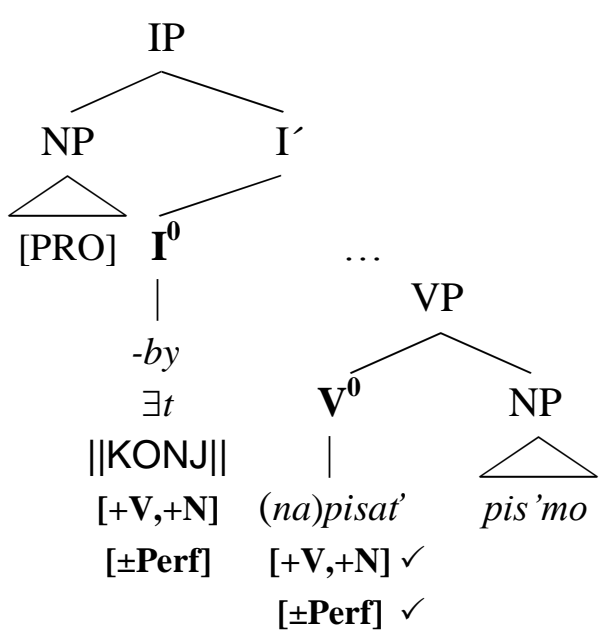

(Rus)

Auch im Russischen treten diese infinitivischen Konjunktive primär in Nebensätzen auf, die von čto eingeleitet werden, so dass hier meist čtoby vorliegt:

$$
\begin{aligned}
& \text {..., čtoby PRO napisat' pis'mo. } \\
& \text { dass+KONJ.AUX.IMPS PERF-schreib-INF Brief-ACC } \\
& \text { '..., um den/einen Brief zu schreiben.' }
\end{aligned}
$$

Damit ist die Interaktion zwischen IP- und VP-Ebene im Polnischen und Russischen grundlegend beschrieben. Im Folgenden werde ich in gebotener Kürze die zentralen Charakteristika der "Zwei-Ebenen-Theorie der Bedeutung" vorstellen, die in der vorliegenden Arbeit eine bedeutende Rolle spielt, wenn es um die Bewertung und Interpretation der fraglichen Variationen geht.

\subsection{Zwei-Ebenen-Semantik}

Für diese Arbeit erweist sich die "Zwei-Ebenen-Theorie der Bedeutung" (vgl. Bierwisch 1983, 1986, 1987, 2007; Dölling 1997; Lang 1987; Lang \& Maienborn 2011; Maienborn 1997) als überaus fruchtbar. Ihr Kern besteht in der Unterscheidung zwischen einer grammatisch determinierten ('innersprachlichen') und einer extragrammatisch determinierten ('außersprachlichen') Bedeutungsebene lexikalischer Einheiten (sowie qua Kompositionalität auch ganzer Phrasen und Sätze). Die grammatisch determinierte Bedeutung wird auch als "Semantische Form" (SF) bezeichnet, während das Zustandekommen von Lesarten (Interpretationen) 
durch extragrammatische Faktoren im Begriff des "Konzeptuellen Systems" (Conceptual System = CS) zusammengefasst wird.

Der Vorteil dieser Dichotomie besteht darin, lexikalischen Einheiten eine grundständige (invariante) Semantik zuordnen, ihre konkrete Interpretation in Satz und Text aber auf konzeptuelle (nicht i. e. S. grammatische) Faktoren zurückführen zu können.

Auf diese Weise ist es unnötig, lexikalischen Einheiten im mentalen Lexikon eine Bedeutungsrepräsentation (bzw. mehrere) zuzuordnen, die sämtliche empirisch beobachtbaren Lesarten abzudecken imstande ist (sind). Sofern sich durch nachvollziehbare Implikaturen und Inferenzen, die aus Faktoren wie dem (Prä-)Kontext und dem Weltwissen von Sprecher und Hörer folgen, die jeweilige Interpretation (bzw. ein interpretativer Effekt) erklären lässt, kann die SF lexikalischer Einheiten möglichst minimal gehalten werden, was minimalistischen Zielen entspricht. Man kann und muss, mit anderen Worten, auf der SF-Ebene nur die absolut notwendigen invarianten Bedeutungsanteile repräsentieren, auf deren Grundlage später auf der CS-Ebene - unter Einbeziehung des Weltwissens und/oder des Kontexts - die eine oder andere Lesart (Interpretation) zustande kommt. ${ }^{162}$ Was prädikative Ausdrücke angeht, so ist das Zwei-Ebenen-Modell insofern sehr attraktiv, als es die Lesarten bzw. interpretativen Effekte zu erfassen erlaubt, die mit den Formvariationen in polnischen und russischen Kopulasätzen einhergehen. Überaus vorteilhaft ist, dass man den Formen dabei keine spezielle (invariante) Eigenbedeutung auf SF zuschreiben muss, sondern die Lesarten/Effekte der CS-Ebene zuordnen kann, und zwar auf der Basis der potenziell verfügbaren Opposition (Konkurrenz) mindestens zweier Formen in ein und derselben syntaktischen Position bzw. Funktion. Siehe Details hierzu in Abschnitt 6.

\footnotetext{
${ }^{162}$ Oft liegen auf SF un(ter)spezifizierte (“offene” oder "freie”) Prädikat- bzw. Relationsvariablen vor, die erst auf CS spezifiziert werden. Hinsichtlich solcher "freier" Variablen nehmen Maienborn \& Geldermann (2013) an, dass deren "[...] ökonomischste/beste Spezifizierung [...] diejenige [ist], die mit den wenigsten kontextuell nicht lizenzierten Zusatzannahmen auskommt" (Maienborn \& Geldermann 2013, 130). Sie verweisen hier auf Langs $(1985,106)$ Unspecified Argument Rule.
} 


\section{Kopulasätze}

\section{1 "Prädikation" und "Prädikativität"}

Dieser Abschnitt widmet sich aus verschiedenen Perspektiven den Begriffen "Prädikation" und "Prädikativität". ${ }^{163}$ Beide referieren auf Grundeigenschaften von Sätzen bzw. Satzaussagen: zum einen auf deren Fähigkeit, sprachlich enkodierte Propositionen mit Sachverhalten in der außersprachlichen Wirklichkeit zu verbinden; zum anderen auf den Umstand, dass dies mittels der grammatischen Kategorien Modus (Satz- sowie Verbmodus) und Tempus geschieht. ${ }^{164}$

Üblicherweise werden Prädikation und Prädikativität mit Verbformen in Verbindung gebracht, denen die erwähnten grammatischen Kategorien eigen sind bzw. die - mindestens in den meisten indoeuropäischen Sprachen - kraft ihrer lexikalischen Kategorie und den damit verbundenen morphologischen Möglichkeiten Modus und Tempus realisieren können. Es ist offensichtlich, dass Kopulasätze, deren Prädikat von einem Nicht-Verb (dem Prädikativ) gestellt wird, insofern einen besonderen Satztyp darstellen. Nichtsdestoweniger liegt auch in Kopulasätzen eine Verbform vor, namentlich eine Form des ‘sein'-Lexems.

Insofern ist es wenig überraschend, dass die Realisierung der grammatischen "Prädikativität" (d. h. von Modus und Tempus) der "Kopula" obliegt, während die semantische "Prädikation" (d. h. der Ausdruck der deskriptiven Bedeutung des Satzprädikats) dem Prädikativ zukommt. Der vorliegende Abschnitt geht auf diese Arbeitsteilung ein, wobei verschiedene Zitate aus einschlägigen Arbeiten herangezogen werden. Ferner ist zu klären, worin genau "Prädikation" semantisch und (morpho-)syntaktisch besteht und wie sie formal erfasst werden kann. ${ }^{165}$

${ }^{163}$ Eine sprachübergreifende Darstellung der grundlegenden Vorstellungen von “(primärer sowie sekundärer) Prädikation" seit Aristoteles bietet Hackmack (2000).

${ }^{164}$ Ferner sind auch die Kategorien Aktionsart, Verbalaspekt, Genus verbi, Person, Numerus, und ggf. auch Genus involviert. Sie treten jedoch in ihrer Bedeutung insofern hinter den Kategorien Modus und Tempus zurück, als es sich (i) beim Genus verbi (Diathese) im Slavischen um keine unmittelbar morphologisch realisierte Kategorie handelt (sie wird periphrastisch oder 'reflexiv' umgesetzt), (ii) bei Aktionsart und Aspekt um lexikalische Kategorien, die also jedem Verblexem inhärent eigen sind, und (ii) bei Person, Numerus und Genus 'nur' um Kategorien zum Ausdruck von Kongruenzmerkmalen (Verb-Subjekt-Kongruenz) handelt. Was die Kategorie der Person betrifft, so kann sie auch als prädikative Kategorie verstanden werden, wie es z. B. bei Isačenko (1962, 272-279) der Fall ist (siehe auch Abschnitt 4.3).

${ }^{165}$ Was "Prädikativität" im o. g. Sinne betrifft, so wird weiterhin vorausgesetzt, das es sich lediglich um die sprachspezifisch ausgeprägte Realisierung der verbalen Kategorien von Modus und 
Das erste Zitat in diesem Zusammenhang stammt von Busch (1960) und befasst sich sehr grundsätzlich mit Kopula- bzw. - genereller - mit "Seinssätzen". Wegen ihrer Grundsätzlichkeit und Relevanz für die vorliegende Arbeit zitiere ich diese Passage in extenso:

In den meisten indogermanischen Sprachen bilden die Seinssätze eine einheitliche Satzform, die durch das Prädikatsverbum 'sein' konstituiert und ausdrücklich als Seinssatzform gekennzeichnet ist. Das Verbum 'sein' tritt in diesen Sätzen als selbständiges oder als unselbständiges Prädikat auf und drückt dabei wie ein normales Verbum die grammatischen Kategorien der Person, des Numerus, des Tempus, des Modus und des verbalen Genus aus ${ }^{[\mathrm{Fn} .]}$. Selbständiges Prädikat ist das Verbum 'sein', indem es mit Hilfe der genannten Kategorien das "verbale Sein”, d. h. das (zeitlich-wirkliche) Befindlichsein des vom Subjektsnomen bezeichneten Seienden

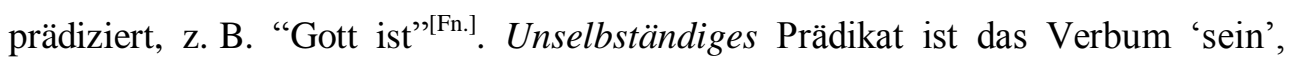
indem es mit Hilfe derselben Kategorien das (zeitlich-wirkliche) Befindlichsein des vom Subjektsnomen bezeichneten Seienden in der vom Prädikatsnomen bezeichneten Befindlichkeit ausdrückt. Dabei kann das Prädikatsnomen entweder ein Wasoder Wie-sein oder eine Faktizität (Situation) als Befindlichkeit des subjektischen Seienden bezeichnen: Wenn das Prädikatsnomen, indem es im Nominativ steht, ein Was- oder Wie-sein des subjektischen Seienden bezeichnet, so nennt man das Prädikatsverbum 'sein' gewöhnlich "Copula", z. B. in Sätzen wie "Der Himmel ist blau", "Du warst mein Freund", "Wir werden froh sein"166. Wenn das Prädikatsnomen dagegen, indem es mit oder ohne Präposition in einem obliquen Kasus steht oder indem es ein Adverb (ein impersonales Prädikatsnomen) ist, eine Faktizität (Situation) des subjektischen Seienden bezeichnet, so nennt man das Prädikatsverbum 'sein' gewöhnlich "Existenzverbum”, z. B. in Sätzen wie "Wir Menschen sind auf der Erde", "Er war in guter Stimmung", "Hier wäre jetzt Wüste".

Da also in den Seinssätzen das Verbum 'sein' als solches nur die jedem Verbum als Verbum zugrundeliegende und darum "abstrakte" Bedeutung des "verbalen Seins", des "Befindlichseins" hat, ist nicht seine Stellung als selbständiges[,] sondern seine Stellung als unselbständiges Prädikat als die normale anzusehen: Normalerweise bildet es - als unselbständiges Prädikat - zusammen mit dem jeweiligen Prädikatsnomen, das eben das "konkrete" Was- oder Wie-sein bzw. die "konkrete" Faktizität (Situation) des subjektischen Seienden bezeichnet, das "ganze" (komplexe) Prädikat, wobei das Prädikatsnomen, gleichgültig welcher Wortart, als

Tempus handelt. In diesem Verständnis ist "Prädikativität" die Eigenschaft bzw. Fähigkeit von Verbformen, die genannten Kategorien morphologisch zu realisieren.

${ }^{166}$ Fn. im Original: "Nach J. Wackernagel, Vorlesungen über Syntax I (1920) S. 23, war es Abaelard, der den 'Ausdruck 'Copula' zur Bezeichnung von 'esse' in syntaktischer Funktion' aufgebracht hat. Vgl. K. von Prantl, Geschichte der Logik im Abendland (1855) II S. 196.” 
Prädikatsnomen nicht über verschiedenartige korrelative Prädikatsformen, z. B. Kasus, verfügt, sondern dem Ausdruck der jeweiligen (grammatisch-kategorialen) Form der Prädikation, d. h. den Ausdruck des jeweiligen (zeitlich-wirklichen) Befindlichseins des subjektischen Seienden, ganz dem Prädikatsverbum 'sein' überläßt und selbst nur den "konkreten" prädikativen Gehalt, d. h. die "konkrete" Befindlichkeit des subjektischen Seienden, ausdrückt. (Busch 1960, 9-11)

Zwar weichen mehrere Punkte in dieser Aussage von den hier vertretenen Ansichten $a b,{ }^{167}$ die grundsätzlichen Eigenschaften von Kopulasätzen - auch im Gegensatz zu “Existenzsätzen”, die auf dem Vollverb ‘(da) sein' (= 'existieren') beruhen - kommen jedoch klar zum Ausdruck: In Kopulasätzen besteht eine Arbeitsteilung zwischen dem Prädikatsnomen und der kopulativen Verbform (die so heißt, da sie das Satzsubjekt syntaktisch mit dem Prädikatsnomen 'verkuppelt'). Diese Teilung besteht darin, dass die deskriptive Bedeutung bzw. das Prädikat (verstanden als sprachliches Zeichen für eine Eigenschaft, die einem Referenten zugeschrieben wird) durch das Prädikatsnomen eingebracht bzw. ausgedrückt wird, während die "Kopula" als Verbform die grammatischen und der "Prädikativität" im o. g. Sinne zugrunde liegenden Kategorien Modus und Tempus realisiert. Die Kopula stellt somit einen "verbalen Rahmen" bereit, in den das Prädikatsnomen seine deskriptive Bedeutung füllt. Das Kopulaverb ist insofern in der Tat "semantisch leer", als es keine eigene deskriptive Bedeutung besitzt. Andererseits ist das Prädikatsnomen auf Grund seiner Nominalität unfähig, die genannten prädikativen grammatischen Kategorien zu realisieren.

Man kann also auch formulieren, dass Kopula und Prädikatsnomen auf jeder Ebene (Semantik, Morphologie, Syntax) komplementär zueinander sind bzw. eine Einheit bilden, innerhalb der keiner auf den anderen verzichten kann.

Aus vergleichender Perspektive lässt sich die Besonderheit von Kopula-Prädikativ-Strukturen auch wie folgt erfassen: Was bei 'normalen' Verbformen 'synthetisch', d. h. mittels meist nur einer verbalen Wortform, ausgedrückt wird, ist im Falle von Kopula-Prädikativ-Strukturen auf stets (mindestens) zwei Wortformen

167 So habe ich eine durchaus andere Sicht auf den Status von PPn, die mit 'sein' auftreten (vgl. Abschnitt 1.2.6). Auch, was prädikativ gebrauchte Adverbien betrifft, bin ich nicht der Ansicht, dass diese automatisch zu "Existenzsätzen" gehören. Ferner können Prädikatsnomina im (Nord-)Slavischen durchaus die Kasuskategorie realisieren. Es ist fraglich, ob Busch (1960) den slavischen prädikativen Instrumental als obliquen (semantischen) oder strukturellen Kasus betrachten würde. Für mich ist er letzteres. 
verteilt, von denen nur eine - die "Kopula" - verbal ist. Neben ihr steht das Prädikatsnomen als Träger der deskriptiven Bedeutung. Insofern kann man die "Kopula" auch als "verbalen Rahmen" für das Prädikatsnomen betrachten. Anhand des Eintrags in (1) für die Stämme des polnischen Verbs pracować 'arbeiten' soll dieser Vergleich und Gegensatz illustriert werden: ${ }^{168}$

\section{(1) $/ \operatorname{prac}\{$ owa/uj $\} / \quad[+\mathrm{V},-\mathrm{N}] \quad \lambda x \lambda s[s$ INST $[x$ WORK $]]$}

Es wurde schon erläutert, dass Verben über ein "hidden argument" (Higginbotham 1985) verfügen, das auf einen Sachverhalt referiert, der durch die verbale Proposition sprachlich beschrieben wird. Der Instanziierungsfunktor INST vermittelt zwischen der Proposition und der Sachverhaltsvariable $s$, indem er den Sachverhalt als eine konkrete (reale/außersprachliche) "Instanz" der an sich abstrakten Proposition ausweist. Im obigen Lexikoneintrag ist demnach die charakteristisch verbale Sachverhaltsreferenz durch " $[s$ INST ... $]$ ” und die Proposition durch " $[x$ WORK $]$ " repräsentiert, wobei sich zeigt, dass die Proposition ein Argument des INST-Funktors ist. ${ }^{169}$ Beide Komponenten sind in einem 'normalen' Verblexem wie pracować quasi synthetisiert, indem sie mit ein und derselben phonetischen Repräsentation assoziiert bzw. durch ein und dieselbe Wortform realisiert werden.

Bei einer Kopula-Prädikativ-Struktur hingegen ist nach gängiger Sichtweise (vgl. u. a. Bierwisch 1988, 46; Steinitz 1990, 121-122; Zimmermann 1999, 128; Maienborn 2003a, 125) der charakteristisch verbale Bezug auf einen Sachverhalt von der deskriptiven Bedeutung bzw. Proposition getrennt. Die beiden Komponenten, die bei einer 'normalen' Verbform eine untrennbare Einheit bilden, erhalten in Form von Kopula und Prädikativ eine jeweils selbständige sprachliche Realisierung. Als Beispiel mag poln. być studentem '(ein) Student sein’ dienen. Das

${ }^{168}$ Die Segmente innerhalb geschweifter Klammern sind komplementär, so dass die PF parallel die beiden Stämme/pracowa/ und /pracuj/ repräsentiert. Welcher Stamm zur Anwendung kommt, hängt v. a. von den phonologischen Eigenschaften der antretenden Suffixe ab (weitere Details in Pitsch 2013).

${ }^{169}$ Den INST-Funktor führt Bierwisch $(1988,23-24)$ mit Verweis auf die fact-function von Reichenbach (1947) ein. An anderer Stelle schreibt er: "Notice that for [...] verbs [...], the Argument Structure provides the event-position $\lambda \mathrm{e}$ based on the argument e of the operator INST [...]. This accounts for the reference of verbal constructions to states or events assumed by Higginbotham (1985) and others." (Bierwisch 1997, 242). 
Prädikatssubstantiv studentem kann durch (2), die Kopulabedeutung durch (3) repräsentiert werden: ${ }^{170}$

(2) /student-/ $[+\mathrm{N} \ldots] \quad \lambda x[x$ STUDENT $]$

(3) $\quad / \ldots / \quad[+\mathrm{V} \ldots] \quad \lambda P \lambda x \lambda s[s$ INST $[P x]]$

Die deskriptive Bedeutung des Prädikatssubstantivs (“[ $x$ STUDENT]”) wird für " $\left[\begin{array}{l}P \\ x\end{array}\right]$ " in die Kopulabedeutung eingesetzt und ist somit deren Komplement. Dies zeigt erneut, dass die Kopulabedeutung in der Tat einen "verbalen Rahmen" für das Prädikativ darstellt. Ihre Aufgabe besteht jedoch nicht allein hierin. Mindestens ebenso zentral ist die Tatsache, dass die Kopulabedeutung als 'Eigenleistung' den Bezug auf einen Sachverhalt herstellt (“[ $s$ INST [...]]”). ${ }^{171}$ Ferner ist sie als verbale Einheit dasjenige Element im Satz, an dem sich die Kategorien Modus und Tempus sowie Person und Numerus morphologisch manifestieren können (in Form entsprechender Auxiliare; siehe Abschnitt 5.2).

Die Tabelle in (4) fasst die Unterschiede zwischen 'normalen' Verbformen und den beiden Teilen von Kopula-Prädikativ-Strukturen zusammen:

\begin{tabular}{|c|c|c|c|}
\cline { 2 - 4 } & $\begin{array}{c}\text { 'normale' } \\
\text { Verbform }\end{array}$ & \multicolumn{2}{|c|}{ Kopula-Prädikativ-Strukturen } \\
\cline { 3 - 4 } & & Kopulabedeutung & Prädikativ \\
\hline deskriptive Bedeutung & & & \\
\hline Sachverhaltsreferenz & & & \\
\hline Modus/Tempus & & & \\
\cline { 3 - 4 } & & & \\
\hline
\end{tabular}

${ }^{170}$ Kasus- und Kongruenzmerkmale von studentem werden hier ignoriert. Der Eintrag in (3) repräsentiert ausschließlich die Kopulabedeutung, d. h. keine spezifische Flexionsform von być.

${ }^{171}$ Meist wird, sofern 'Zustände' und 'Ereignisse' überhaupt als unterschiedliche Sachverhaltstypen angenommen werden, davon ausgegangen, dass die Kopula (resp. Kopulasätze) auf Zustände referiert. Maienborn (2003a, 2005b, 2007a) nimmt an, dass bestimmte Zustandsverben, darunter auch die Kopula, auf 'Kimsche (K-)Zustände' (Kim 1976) verweisen. Dabei soll es sich um abstrakte (nicht unmittelbar wahrnehmbare) und rein temporale Entitäten handeln, die keine lokale Modifikation zulassen. Im Gegensatz zu K-Zuständen seien Davidsonsche Zustände und Ereignisse (events) wahrnehmbare spazio-temporale Entitäten. Argumente gegen die Annahme von K-Zuständen bringt Dölling (2005b). 
Die grauen Bereiche zeigen, welche morphosyntaktischen und semantischen Leistungen die Formen erbringen. Letztlich steht dem synthetischen Charakter 'normaler' Verbformen der analytische Charakter von Kopula-Prädikativ-Strukturen gegenüber.

Das zweite relevante Zitat stammt aus Barnetovás (1979) Russischer Grammatik und erläutert im Detail den Begriff der "Prädikativität", in dem sich auch das bereits Gesagte wiederfindet:

[...] В русском языке, равно как и в чешском или в других языках индоевропейского строя, предложение как коммуникативная единица языка опирается на предикативность, под которой подразумевается соотнесенность выражаемого в предложении содержания с действительностью. Предикативность заключается в обязательном выражении того, как соотнесено с точки зрения говорящего с действительностью содержание предложения в плане реальности/потенциальности его реализации [...] и, как правило, также во временном плане [...]. (Barnetová 1979, 669, § 893) [... Im Russischen, ebenso wie im Tschechischen oder in anderen Sprachen indoeuropäischer Struktur, stützt sich der Satz als kommunikative Einheit auf die Prädikativität, unter der die wechselseitige Bezogenheit des Satzinhalts mit der Wirklichkeit verstanden wird. Prädikativität besteht im obligatorischem Ausdruck dessen, wie aus Sprechersicht der Satzinhalt mit der Wirklichkeit in Bezug auf Realität/Potenzialität seiner Realisierung und in aller Regel auch in Bezug auf Temporalität in wechselseitiger Beziehung steht ... meine Übers., H. P.]

In den Formulierungen der "wechselseitigen Bezogenheit des Satzinhalts mit der Wirklichkeit" findet sich der bereits angesprochene Sachverhaltsbezug wieder, über den allein verbale Lexeme verfügen und der im Falle der Kopulabedeutung höchstwahrscheinlich Referenz auf einen (Kimschen) Zustand bedeutet. Ferner kommt in den Begriffen der sprecherbezogenen "Realität/Potenzialität" die Kategorie des Verbmodus und durch die Nennung der "zeitlichen Ebene" die Kategorie des Tempus zur Sprache, die ebenfalls bereits als Grundlagen jedweder Satzaussage identifiziert wurden. Dass in den slavischen Sprachen allein Verben fähig sind, diese Kategorien morphologisch zu realisieren, bedeutet, dass jeder Satz auf einer flektierten Verbform basieren muss. Dies muss folglich auch für Kopulasätze gelten (siehe auch Abschnitt 4.5).

In diesem Sinne wird sich hier auch definitiv gegen die Annahme sog. "Nominalsätze" ausgesprochen, die in der russistischen Sprachwissenschaft (vgl. u. a. Schlegel et al. 1992, 265, §573), aber auch in der Indogermanistik (vgl. u. a. 
Benveniste 1966) eine gewisse Tradition hat. Meines Erachtens (siehe auch Belošapkova ${ }^{3} 1997,695$, § 25/II) kann es keine Sätze geben, die nur auf Nomina und damit lediglich auf einer AP oder NP (ohne VP) basieren. Sofern an der sprachlichen Oberfläche keine flektierte Verbform vorliegt, ist vielmehr von einer koverten Verbform auszugehen. Ihre Annahme ist theoretisch unumgänglich, sofern man nicht zu der Stipulation bereit ist, es gäbe Sätze ohne Modus-/Tempusspezifikation. Solche Sätze könnten auf keinen Sachverhalt verweisen. Sie könnten folglich nicht als Aussagen über bzw. als Bezugnahmen auf Vorgänge in der außersprachlichen Wirklichkeit dienen. Solche Sätze könnten demnach keine kommunikative Funktion ausüben und wären im wahrsten Sinne des Wortes 'sinnlos'. Demnach soll hier gelten, dass in allen Sätzen stets eine (prädikative) Verbform vorhanden ist, sei sie overt oder kovert realisiert.

Bis dato wurden die beiden Hauptbestandteile von Kopulasätzen und deren semantische und morphosyntaktische Eigenschaften betrachtet. Schließlich ist $\mathrm{zu}$ bedenken, ob und in welcher Form sich "Prädikation" syntaktisch manifestiert. Bailyn (2012, 198) sagt sehr explizit, "supporting a claim made by Rothstein (1992), [...] that predication is at its core a syntactic relation". Was veranlasst ihn zu dieser syntaktozentrischen Stellungnahme? In seinem Modell (basierend auf Bowers 1993; siehe auch schon Bailyn 2001) wird "Prädikation" durch einen syntaktischen (funktionalen) Kopf einer speziellen Prädikationskategorie $\left(\operatorname{Pr}^{0}\right)$ etabliert. Jedoch versäumt Bailyn zu klären, welche semantischen Konsequenzen die Anwesenheit von $\operatorname{Pr}^{0}$ hat (er will durch diesen Kopf v. a. die möglichen Kasusformen russischer Prädikatsnomina theoretisch ableiten).

Aus den bisherigen Erwägungen folgt, dass "Prädikation" auf semantischer bzw. referenzieller Ebene mit dem charakteristisch verbalen Sachverhaltsbezug gleichgesetzt werden kann. In verbalen Lexikoneinträgen kann man somit "Prädikation" mit der Komponente " $[s$ INST [...]]" identifizieren, wie sie sich auch im Lexikoneintrag der Kopulabedeutung in (3) wiederfindet. Die Präsenz eines Sachverhaltsarguments ist Voraussetzung für die aspektuelle Spezifikation, durch die ein Referenz- bzw. Topikzeitargument eingeführt wird, das wiederum Voraussetzung zur temporalen sowie verbmodalen Spezifikation ist. Die Kopulabedeutung in (3) weiter oben muss in diesem Zusammenhang wie folgt komplettiert werden, wobei der semantische Aspekt der Kopulabedeutung stets und einzig der imperfektive sein kann (es handelt sich um ein Imperfektivum tantum; Geist 2006, 169-171): 
In diesem Lexikoneintrag verbinde ich die Annahme des imperfektiven Aspekts der Kopulabedeutung gleichsam mit Bailyns (2001, 2013) Ansicht, "Prädikation" sei eine syntaktische Relation. Jedoch gilt letzteres m. E. nur für Kopula-Prädikativ-Strukturen, wohingegen "Prädikation" - im Sinne des verbalen Sachverhaltsbezugs - ansonsten eine inhärente und damit lexikalische Eigenschaft verbaler Lexeme ist. Im Falle von Kopulasätzen tritt die derart verstandene "Prädikation" dagegen als separate syntaktische Einheit auf. Dem wird durch den obigen Lexikoneintrag Rechnung getragen, indem es sich hier nicht um den Eintrag des Verblexems byćlbyt' 'sein' handelt, sondern um den Eintrag einer Null $(\varnothing)$, die die Bedeutung und Argumentstruktur repräsentiert, die man traditionell mit dem Kopulalexem 'sein' in Verbindung bringt. Im Prinzip deckt sich also der vorliegende Vorschlag mit Bowers (1993), Bailyn (2001, 2013) und anderen Arbeiten, die die Kopulabedeutung in Form eines speziellen syntaktischen Kopfes repräsentieren, sei es $\operatorname{Pr}(\text { ed })^{0}$ (Bowers 1993; Bailyn 2001, 2012; Markman 2008; Bondaruk 2013b), $\operatorname{Rel}^{0}$ (den Dikken 2006), $\pi^{0}$ (Citko 2008), $v^{0}$ (Geist 2006) o. ä. Im Unterschied zu diesen Arbeiten nehme ich jedoch keine "Spezialkategorie" für die Kopulabedeutung bzw. für "Prädikation" an, sondern gehe davon aus, dass es sich hier um das gewissermaßen 'grundlegendste' Verb überhaupt handelt, was einerseits die Nullform sowie andererseits das Fehlen einer deskriptiven Bedeutung nachvollziehbar werden lässt. Ferner bewährt sich auch hier das Bild des "verbalen Rahmens", in den das Prädikativ seine deskriptive Bedeutung 'gießt' ${ }^{172}$

Die Basis-VP für das bereits genannte Beispiel być studentem '(ein) Student sein' muss demnach wie in (6) syntaktisch repräsentiert werden:

\section{(6) $\left[\mathrm{vP} \varnothing_{\mathrm{V}}[\mathrm{NP}\right.$ studentem $\left.\mathrm{N}]\right]$}

Freilich fehlt hier noch jegliche Form des Verblexems być 'sein'. Wenn es sich, wie hier vorgeschlagen, bei dessen Formen offenbar nicht um Formen der Kopu-

\footnotetext{
${ }^{172}$ Die grammatischen Merkmale weisen das leere $\mathrm{V}^{0}$ mit der Kopulabedeutung in (5) als Infinitiv aus. Die VP mit Prädikatsnomen wiederum ist ambivalent zwischen einem Infinitiv und einem Partizip, da der leere V-Kopf allein einem Infinitiv $([+\mathrm{V},+\mathrm{N}, \ldots-\mathbf{A g r},+\max ])$ entspricht, durch das Prädikatsnomen jedoch Kongruenzmerkmale vorliegen, so dass die gesamte VP letztlich etwas Partizipialem $([+\mathrm{V},+\mathrm{N}, \ldots+\mathbf{A g r},+\max ])$ gleichkommt.
} 
la(bedeutung) selbst handelt, dann liegt der Schluss nahe, dass diese Formen lediglich Auxiliare sind, die der bloßen Ausbuchstabierung bzw. "Sichtbarmachung" morphosyntaktischer Merkmale bzw. verbaler Kategorien dienen. Ich nehme an, dass diese Auxiliarformen in einem weiteren (auxiliaren) V-Kopf generiert werden, der die Basis-VP unmittelbar selegiert: ${ }^{173}$

\section{(7) $\left[\mathrm{vP}\right.$ być $_{\mathrm{V}}\left[\mathrm{vP} \varnothing_{\mathrm{V}}[\mathrm{NP}\right.$ studentem $\left.\left.\mathrm{N}]\right]\right]$}

Im nächsten Abschnitt werde ich die nordslavischen 'sein'-Formen im Detail betrachten. Es ist zu zeigen, welche Formen im Strukturbaum (a) "unten" in einem auxiliaren $\mathrm{V}^{0}$ sitzen und welche Formen (b) “oben” als $\mathrm{I}^{0}$ realisiert werden. Dieses syntaktische Kriterium trennt die sog. lower auxiliaries von den upper auxiliaries. Es wird sich zeigen, dass man nur erstere heute noch als Formen des Verblexems byćlbyt' 'sein' betrachten kann, während letztere synchron soweit grammatikalisiert sind, dass sie als nurmehr funktionale Elemente gelten müssen, die nur noch äußerliche Ähnlichkeit mit dem heutigen Lexem byćlbyt’ aufweisen.

\subsection{Die Formen von 'sein'}

\subsubsection{I-Auxiliare und V-Auxiliare}

In diesem Abschnitt wird erörtert, welche Auxiliartypen es im Polnischen und Russischen an sich gibt, was sie charakterisiert, und welche Aufgaben sie erfüllen. Ich werde an geeigneter Stelle das Tschechische einbeziehen, da sich in dieser Sprache bzw. in ihrer Orthographie die Auxiliare leichter isolieren lassen.

In Grammatiken und Forschungsarbeiten werden u. a. folgende Typen von "Hilfsverben” erwähnt: Perfekt-/Präteritumauxiliar, Konjunktivauxiliar, Passivauxiliar, Kopulaverb, Relator etc. Ob es sich bei all diesen Typen um Auxiliare im eigentlichen Sinne handelt, bleibt meist offen, da kaum je klare Standpunkte hinsichtlich

173 In früheren Theoriestadien wurde mitunter die spezifisch auxiliare Projektion AuxP verwendet. Ein Auxiliar ist demnach eine [+V]-Einheit mit dem subkategorisierenden Merkmal [+Aux]. Es handelt sich hier m. E. aber lediglich um notationelle Varianten; vgl. (i):

(i) $\left[\right.$ AuxP być cux $_{\text {Aux }}\left[\varnothing_{\mathrm{V}}[\mathrm{NP}\right.$ studentem $\left.\left.\mathrm{N}]\right]\right]$

Wie auch später in Abschnitt 5.3.2, so scheint mir hier die Option des syntaktischen Matchings (Haider 1988) der leeren mit der overt realisierten V-Projektion denkbar, so dass (i) ggf. auf (ii) zu reduzieren ist, worin "być ${ }_{\mathrm{V}}$ " Auxiliar und Kopulabedeutung in sich vereint:

(ii) $\left[\mathrm{Aux}_{\mathrm{VP}}\right.$ być $_{\mathrm{Aux} / \mathrm{V}}[\mathrm{NP}$ studentem $\left.\mathrm{N}]\right]$ 
der Eigenbedeutung der fraglichen Formen, ihrer möglichen Sachverhaltsreferenz bzw. ihrer ggf. reinen Ausbuchstabierungsfunktion bezogen werden.

Ich vertrete die Ansicht, dass im Rahmen polnischer, russischer und tschechischer periphrastischer Strukturen grundsätzlich die beiden in (8) genannten Typen von Auxiliaren auftreten. Periphrastisch sind u. a. bestimmte Modus-/Tempusformen, Sätze mit Modalverben sowie Kopulasatzstrukturen.

\section{(8) a. V-Auxiliare (lower auxiliaries) \\ b. I-Auxiliare (upper auxiliaries)}

Die Unterscheidung zweier grundlegender Typen von Auxiliaren findet sich mit Bezug auf das Polnische z. B. bei Borsley \& Rivero (1994) und Dornisch (1997) und mit Bezug auf das Tschechische u. a. bei Toman (1980), Veselovská (1995) und Kosta (2001) (siehe auch Skoumalová 2003). ${ }^{174}$

Es wirkt zunächst so, als würden sowohl V- als auch I-Auxiliare in den drei untersuchten Sprachen gleichermaßen von Flexionsformen des 'sein'-Lexems gestellt, d. h. tschech. být, poln. być und russ. byt'. Es wird sich jedoch zeigen, dass nur die V-Auxiliare synchron als Flexionsformen dieses Lexems gelten können.

Wie die Bezeichnung "I-Auxiliar" und auch die Alternative upper auxiliary verraten, wird dieser Auxiliartyp relativ weit "oben” im Strukturbaum - namentlich in $\mathrm{I}^{0}$ - generiert. Ein V-Auxiliar oder lower auxiliary dagegen wird bei Bedarf als zusätzlicher V-Kopf oberhalb des infiniten Satzprädikats $\left(\mathrm{V}^{\mathrm{infin}}\right)$ generiert:

\footnotetext{
${ }^{174}$ In Bezug auf das Russische finden sich kaum systematische Unterscheidungen. Die Schulgrammatik ist in Bezug auf 'Hilfsverben' generell einigermaßen 'nachlässig', insofern sie die Begriffe svjazka 'Kopula' und vspomogatelnyj glagol 'Hilfsverb' oft synonym verwendet.
} 
(9)

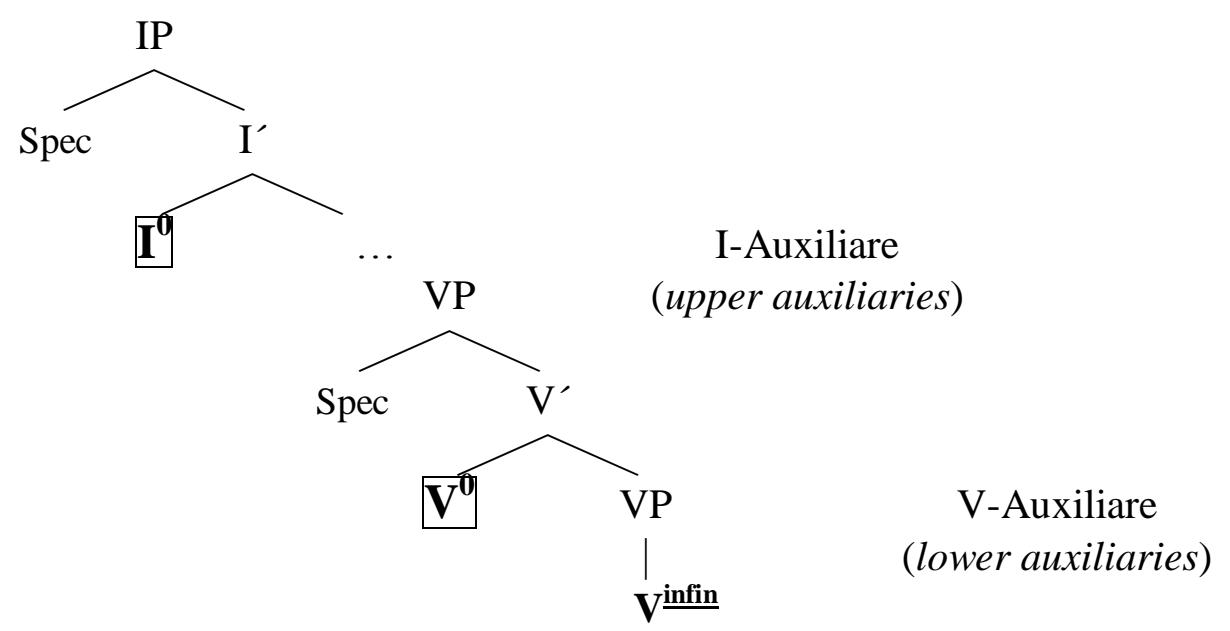

In Anbetracht dieser Verteilung ist Borsley \& Rivero (1994) zuzustimmen, wenn sie zwischen "lexikalischen Auxiliaren" (V-Auxiliare) und "funktionalen Auxiliaren" (I-Auxiliare) unterscheiden. Da den I-Auxiliaren eine hierarchisch höhere Position als den V-Auxiliaren zukommt, können letztere von ersteren selegiert und 'mit Bedeutung ausgestattet' werden (nicht jedoch umgekehrt). Die 'Lexikalität” der V-Auxiliare mag auch Begründung dafür sein, dass sie in einem Satz mehrfach auftreten können, wogegen von den "funktionalen" I-Auxiliaren jeweils nur ein Exemplar möglich ist. Schließlich sind I-Auxiliare stets (en)klitisch, V-Auxiliare dagegen non-klitisch. ${ }^{175}$ Das tschechische Beispiel in (10) illustriert diese Eigenschaften zum Teil:

\section{(10) Já bych byl trápen bolestí. \\ I-AUX V-AUX INFINITUM}

'ICH würde vom Schmerz gequält werden (/worden sein).'

Das konjunktivische I-Auxiliar bych ist enklitisch (es lehnt sich an das overte Subjektpronomen já 'ich' an). Dieses I-Auxiliar selegiert $l$-Partizipien. In diesem Fall selegiert es direkt ein V-Auxiliar, namentlich byl. Zusammen mit letzterem konstituiert das I-Auxiliar so die Konjunktivperiphrase. Das V-Auxiliar ist non-

175 Toman (1980, 305-308) nennt für die tschechischen präteritalen I-Auxiliare folgende weitere Besonderheiten: Sie können nicht mit dem Negationspräfix ne- versehen werden; sie haben die Allegroform $-s$ in der 2. Ps. Sg. (vgl. die Vollform jsi); sie können bei Nennung des Subjekts in der 1. Ps. ausgelassen werden; ihre 2. Ps. Sg. si hat im Unterschied zum V-Auxiliar in der gesprochenen Sprache nicht die Alternativform seš. 
klitisch. Im Unterschied zum I-Auxiliar, das selbst die Spezifikation des Konjunktivs vornimmt, hat es keine Eigenbedeutung, sondern buchstabiert mit Hilfe seiner Morphologie den Verbmodus für das eigentliche Satzprädikat trápen (mit) aus, das dazu auf Grund seiner Infinitheit nicht in der Lage ist. Analog sind die Strukturen im Polnischen und im Russischen:

(11) Filar byl by <był budowany.

V-AUX I-AUX INFINITUM

'Der Pfeiler wäre gebaut worden (/würde gebaut werden).'

NKJP

(12)

$\begin{array}{lcccc}\text { Inače sledujuščej seriej min ja } & \text { byl } & \text { by } & \langle\text { byl> } & \text { ubit. } \\ & \text { V-Aux } & \text { I-Aux } & & \text { INFINITUM }\end{array}$

'Ansonsten wäre ich durch die nächste Serie von Minen getötet worden.'

NKRJ

Für Borsley \& Rivero (1994) gehören das Konjunktiv- und das Präteritalauxiliar zu den "funktionalen" Auxiliaren, während z. B. das Futurauxiliar (będ-/bud-) für sie ein "lexikalisches" Auxiliar darstellt (vgl. auch bereits die Abschnitte 4.6.2 und 4.6.3). Generell lässt sich sagen, dass alle Auxiliare, die der Ausbuchstabierung von Tempus- und Kongruenzmerkmalen dienen, V-Auxiliare darstellen. Hierzu gehören auch Formen von Modalverben ("Modalauxiliare”) wie poln. móc 'können', musieć ‘müssen' oder russ. moč’ ‘können'; freilich haben letztere im Unterschied zu 'normalen' Auxiliaren durchaus eine modale 'Eigenbedeutung':

$\begin{array}{rccc}\text { (13) Kto } & \text { by } & \text { mógl } & \text { przypuszczać ? } \\ & \text { I-AUX } & \text { V-AUX } & \text { INFINITUM }\end{array}$

'Wer könnte das vermuten?'/‘Wer hätte das vermuten können?'

NKJP

Es gibt weitere Unterschiede zwischen den beiden Auxiliartypen: V-Auxiliare, die der bloßen Ausbuchstabierung von Tempus- und Kongruenzmerkmalen dienen, lassen die Bildung sämtlicher paradigmatischer Formen des Lexems byćlbyt' zu; sie haben entsprechend in der 3. Person (Präsens Indikativ) overte Lautform, namentlich tschech. je 'ist'/jsou 'sind' und poln. jest 'ist'/sq 'sind' (im Russischen 
sind diese Formen "stumm"). ${ }^{176}$ I-Auxiliare hingegen zeigen einen begrenzten Formbestand, darunter in der 3. Person ihres (formalen) Präsensparadigmas Nullformen. ${ }^{177}$ Darüber hinaus haben I-Auxiliare nicht lediglich ausbuchstabierende Funktion, sondern bringen - worin ihre grundlegende Rolle besteht - als 'Eigenbedeutung' eine bestimmte Modus-/Tempussemantik ein. Das heißt, dass I-Auxiliare - anders als V-Auxiliare - grammatische Kategorien und Bedeutungen nicht nur "sichtbar machen", sondern selbst einbringen und repräsentieren. Sie sind in diesem Sinne tatsächlich "grammatische" bzw. "funktionale" Elemente. In Tabelle (14) fasse ich die Eigenschaften von I- und V-Auxiliaren zusammen:

${ }^{176}$ Sofern die Formen von byćlbyt' lediglich V-Auxiliare ohne Eigenbedeutung sind, stellt sich die Frage nach dem Zustandekommen von Derivaten wie z. B. russ. probyt' : probyvat' 'verweilen', zabyt' : zabyvat' 'vergessen', otbyt' : otbyvat' 'abreisen; ableisten', pobyt' : pobyvat' 'sich eine Zeitlang aufhalten' (ähnlich im Polnischen; vgl. dazu u. a. auch Matushansky 2000). Es ist zu erwarten, dass solche Präfixe wie pro-, po- etc. im Rahmen der Derivation an semantisch gehaltvolle Verbstämme antreten, was der Ansicht zuwider liefe, das Lexem byćlbyt' sei semantisch leer. Diesem Einwand wäre zu begegnen, indem man die genannten Lexeme einfach als 'gegeben' (also als nicht mehr produktiv deriviert) betrachtet, was gerade in Fällen wie zabyt' 'vergessen' nahezuliegt. Wenn man von Derivaten ausgeht, könnte es sich ferner womöglich auch um Ableitungen vom Existenzverb być/byt' handeln. Eine andere Erklärung wäre, dass die semantisch leeren Verbstämme by-/bud- bereits im Lexikon mit der leeren Kopulabedeutung 'fusionieren' können. Auf diese Weise könnten sie die Kopulabedeutung 'annehmen', wenn dies notwendig wird, etwa im Falle des Antretens von Derivationspräfixen wie pro-, po- usw.

177 Nullformen für die 3. Person liegen im Westslavischen (Polnischen, Tschechisch, Slovakisch etc.) und naturgemäß im Russischen (wo alle Formen "stumm" sind) vor. Im Südslavischen ist das I-Auxiliar in der 3. Person dagegen noch overt, wenn sich auch bereits partielle Schwunderscheinungen zeigen, die potenziell für den vermeintlichen Modus "Renarrativ" eine Rolle spielen (vgl. u. a. Levin-Steinmann 2004; Sonnenhauser 2012; Sonnenhauser \& Meermann 2013). Letzteres stellt sich für mich als bloße Ausnutzung der Formvaration "overtes vs. kovertes I-Auxiliar" dar, die mit keiner realen semantischen Distinktion einhergeht (vgl. Abschnitt 6, in dem dieses Konzept ausbuchstabiert und auf die Variationen an polnischen und russischen Prädikatsnomina angewendet wird). 


\begin{tabular}{|l|l|l|}
\multicolumn{1}{|c|}{ I-Auxiliare } & \multicolumn{1}{c|}{ V-Auxiliare } \\
\hline Kategorie & {$[+\mathrm{I}]$ (stets finit) } & {$[+\mathrm{V}]$ (finit oder infinit) } \\
\hline Lokation & in $\mathrm{I}^{0}$ & in (additivem) $\mathrm{V}^{0}$ \\
\hline Charakter & funktional/grammatisch & lexikalisch/ausbuchstabierend \\
\hline Klitizität & (en)klitisch & non-klitisch \\
\hline Anzahl & höchstens eine Form & mehrere Formen möglich \\
\hline Paradigma & eingeschränkt (nur 'Finita') & komplett \\
\hline Funktion/en & - Modus/ Tempus-Spezifikation & \\
& $\begin{array}{l}\text { - ggf. Ausbuchstabierung von Finit- } \\
\text { heits- und Kongruenzmerkmalen }\end{array}$ & $\begin{array}{l}\text { - Ausbuchstabierung von Finit- } \\
\text { heits- und Kongruenzmerkmalen }\end{array}$ \\
\hline
\end{tabular}

Die Verteilung der Formen von tschech. být, poln. być und russ. byt' 'sein' auf die beiden Auxiliartypen ist wie in (15):

\begin{tabular}{|c|c|c|}
\hline (15) & I-Auxiliare & V-Auxiliare \\
\hline Cze & $\begin{array}{l}\text { Konjunktivauxiliar: } \text { bych }_{<\mathrm{CL}>} \text { etc. } \\
\text { Präteritalauxiliar: } j s e m_{<\mathrm{CL}>} \text { etc. }\end{array}$ & $\begin{array}{l}\text { Ausbuchstabierer: nonklit. Formen von být } \\
\text { Modalverben: moct, muset etc. }\end{array}$ \\
\hline Pol & $\begin{array}{l}\text { Konjunktivauxiliar: } \text { by }_{<\mathrm{CL}>} \text { etc. } \\
\text { Präteritalauxiliar: }(e) m_{<\mathrm{CL}>} \text { etc. }\end{array}$ & $\begin{array}{l}\text { Ausbuchstabierer: nonklit. Formen von być } \\
\text { Modalverben: móc, musieć etc. }\end{array}$ \\
\hline Rus & $\begin{array}{l}\text { (unterspez.) Konjunktivauxiliar: } b y_{<\mathrm{CL}>} \\
\text { (unterspez.) Präteritalauxiliar: } \varnothing\end{array}$ & $\begin{array}{l}\text { Ausbuchstabierer: Formen von } b y t^{\prime} \\
\text { Modalverb: } m o \check{c}\end{array}$ \\
\hline
\end{tabular}

Im folgenden Abschnitt wird diese Verteilung im Detail betrachtet. Es geht darum zu zeigen, dass man in der Tat mit zwei ‘Sätzen' von Auxiliaren zurechtkommt.

\subsubsection{Bestand und Distribution der V-Auxiliare}

Im Folgenden werden für die betrachteten Sprachen die Gebrauchsweisen der V-Auxiliare illustriert. Diese finden im Tschechischen, Polnischen und Russischen gleichermaßen sowohl im partizipialen Passiv, im periphrastischen (imperfektiven) Futur als auch in Kopulasatzstrukturen Verwendung. Natürlich ist letzterer Gebrauch für die vorliegende Arbeit von besonderem Interesse. Zunächst nenne ich deskriptiv die grundlegenden Paradigmen der V-Auxiliare (ohne Partizipien, Gerundien und Imperativformen): 


\begin{tabular}{|c|c|c|c|}
\hline (16) Tschechisch & Ps. & Sg. & PI. \\
\hline \multicolumn{2}{|l|}{ Infinitiv } & \multicolumn{2}{|c|}{ být } \\
\hline \multirow{3}{*}{ Präsens } & 1 & jsem & jsme \\
\hline & 2 & jsi $(\operatorname{seš})$ & jste \\
\hline & 3 & je & jsou \\
\hline \multirow{3}{*}{ Präteritum } & masc & $b y l$ & byli \\
\hline & fem & byla & byly \\
\hline & neutr & bylo & byla \\
\hline \multirow{3}{*}{ Futur } & 1 & $b u d u$ & budeme \\
\hline & 2 & budeš & budete \\
\hline & 3 & bude & budou \\
\hline
\end{tabular}

\begin{tabular}{|c|c|c|c|}
\hline (17) Polnisch & Ps. & Sg. & PI. \\
\hline \multicolumn{2}{|l|}{ Infinitiv } & \multicolumn{2}{|c|}{ być } \\
\hline \multirow{3}{*}{ Präsens } & 1 & jestem & jesteśmy \\
\hline & 2 & jesteś & jesteście \\
\hline & 3 & jest & $s q$ \\
\hline \multirow{3}{*}{ Präteritum } & masc & byt & byli/byty \\
\hline & fem & byta & \multirow{2}{*}{ byty } \\
\hline & neutr & byto & \\
\hline \multirow{3}{*}{ Futur } & 1 & będę & będziemy \\
\hline & 2 & będziesz & będziecie \\
\hline & 3 & będzie & $b e ̨ d a$ \\
\hline
\end{tabular}




\begin{tabular}{|c|c|c|c|}
\hline (18) Russisch & Ps. & Sg. & PI. \\
\hline \multicolumn{2}{|l|}{ Infinitiv } & \multicolumn{2}{|c|}{ byt $t^{\prime}$} \\
\hline \multirow{3}{*}{ Präsens } & 1 & \multirow{3}{*}{\multicolumn{2}{|c|}{$\varnothing$}} \\
\hline & 2 & & \\
\hline & 3 & & \\
\hline \multirow{3}{*}{ Präteritum } & masc & byl & \multirow{3}{*}{ byli } \\
\hline & fem & byla & \\
\hline & neutr & bylo & \\
\hline \multirow{3}{*}{ Futur } & 1 & budu & budem \\
\hline & 2 & budeš & budete \\
\hline & 3 & budet & budut \\
\hline
\end{tabular}

Dass in der Tat dieselben V-Auxiliare sowohl in das partizipiale Passiv, das periphrastische Futur als auch in Kopulasatzstrukturen involviert sind, zeigen die folgenden Vergleiche, zunächst anhand des Tschechischen:
a. pro Budeš trápen bolestí?
[2SG] wirst-2SG gequält-SG.M Schmerz-INS
'Wirst du vom Schmerz gequält werden?'
b. pro Budeš učitel?
[2SG] wirst-2SG Lehrer-NOM
'Wirst du (ein) Lehrer sein/werden?'
(Kopulasatzstruktur)
c. pro Budeš pracovat?
[2SG] wirst-2SG arbeiten ${ }_{\mathrm{IMPF}}-\mathrm{INF}$
'Wirst du arbeiten?'
(periphrastisches Futur)

Es gibt keinen plausiblen Grund, die Form budeš in (19a) als distinkt von budeš in (19b) oder (19c) zu betrachten: In allen drei Beispielen ist sie overt und nonklitisch, und stets reflektiert sie das semantische FUTUR. Traditionell wird die Verwendung von budeš (und der übrigen Flexionsformen von být) als "Hilfsverb" von der als "Kopula" unterschieden: "Hilfsverb" wäre budeš demnach in (19a) und (19c), "Kopula" hingegen in (19b), und zwar, weil es dort scheinbar den Status eines Vollverbs bzw. des (verbalen Teils des) Satzprädikats aufweist. Die kaum wünschenswerte Unterscheidung zweier homonymer Formen budešs (Auxi- 
liar) und budeš2 (Kopula) lässt sich umgehen, indem man - wie bereits in Abschnitt 5.1 vorgeschlagen - in der Kopulasatzstruktur in (19b) die Kopulabedeutung nicht unmittelbar in budeš, sondern in einem abstrakten V-Kopf verortet. Der Vergleich der drei obigen Sätze zeigt dann volle Parallelität, da budeš in allen drei Fällen eine infinite Verbform mit FUTUR 'versorgt' (zur Erinnerung: der bedeutungstragende FUTUR-Operator sitzt in $\mathrm{I}^{0}$ ). Es handelt sich also lediglich um ein ausbuchstabierendes (und insofern "lexikalisches") Auxiliar zur Sichtbarmachung von Merkmalen, die weder die overten Infinita in (20a) und (20b) noch der koverte (und somit ebenfalls infinite) V-Kopf in (20b) realisieren können: ${ }^{178}$

$\begin{array}{rcccccr}\text { (20) a. } & \text { Budeš } & \text { trápen }_{\mathrm{V}} & \text { (bolestí) } & ? & \text { (partizipiales Passiv) } \\ \text { b. } & \text { Budeš }_{\mathrm{V}} & \varnothing_{\mathrm{V}} & \text { učitel }_{\mathrm{N}} & ? & \text { (Kopulasatzstruktur) } \\ \text { c. } & \text { Budeš }_{\mathrm{V}} & \text { pracovat }_{\mathrm{V}} & & ? & \text { (periphrastisches Futur) }\end{array}$

Analog ist die Situation im Polnischen, wo die overten und non-klitischen Formen der 3. Person des V-Auxiliars jest 'ist'/sa 'sind' lauten. Wie im Tschechischen, so können auch im Polnischen mit den V-Auxiliaren Passivpartizipien beider Aspekte verwendet werden, jedoch kennt das Polnische keine 'Kurzformen' dieser Partizipien, sondern nur 'Langformen'. Ferner ist anzumerken, dass mit perfektiven Passivpartizipien neben Formen von być auch solche des Auxiliars zostać (vgl. dt. 'werden') auftreten, die ebenfalls non-klitisch und in allen paradigmatischen Formen overt sind: ${ }^{179}$

${ }^{178}$ Eine Alternative zur vorliegenden Analyse kann Geists $(2006,160)$ Vorschlag eines variablen Lexikoneintrags für die Formen von russ. byt' darstellen, dem zufolge sie entweder Kopulaverben (Sachverhaltsbezug vorhanden), Aspektauxiliare oder bloße Tempusauxiliare (letztere beiden ohne Sachverhaltsbezug) sein können. M. E. ist die hiesige Analyse vorteilhafter, da sie auf derart variable Einträge verzichten kann, und da so die Formen von byt' im Lexikon vollends einheitlich repräsentiert werden können. Indem ein abstrakter V-Kopf Träger der Kopulabedeutung ist, ist die Arbeitsteilung zwischen den lexikalischen Einheiten klar geregelt (die produktiven Formen von byt' sind immer V-Auxiliare; Modus-/Tempusauxiliare sind nurmehr die unproduktiven bzw. grammatikalisierten Formen von $b y t$ ).

${ }^{179}$ Die Formen im polnischen partizipialen (Vorgangs-)Passiv sind wie folgt verteilt:
(i) $b y c ́$ + imperfektives $n / t$-Partizip
(impf. Vorgangspassiv),
(ii) zostać + perfektives $n / t$-Partizip
(perf. Vorgangspassiv).

Die Kombination in (iii) ist kein partizipiales (Vorgangs-)Passiv, sondern eine Kopulasatzstruktur bzw. ein "Zustandspassiv" (adjectival passive bei Borik 2012):

(iii) być + perfektives $n / t$-Partizip (resultativ; siehe Fn. 180) ("Zustandspassiv"). 
(21) a. Ja jestem budzony.

(partizipiales Passiv)

ich-NOM bin-1SG geweckt ${ }_{\mathrm{IMPF}}$-SG.M

'ICH werde geweckt.'

(Cienkowski 1970, 210)

b. Dziecko zostało urodzone przez 13-letnią

Kind-NOM wurde-SG.N geboren-SG.N durch 13-jähriges-ACC.F

dziewczynkę.

Mädchen-ACC.F

'Das Kind wurde von einem 13-jährigen Mädchen geboren.' $\quad$ (Sadziński 1989, 139)

c. Ja jestem nauczycielem.

(Kopulasatzstruktur)

ich bin-1SG Lehrer-INS

'ICH bin Lehrer.'

d. pro Będę pracować.

(periphrastisches Futur)

[1SG] werde-1SG arbeiten $_{\text {IMPF-INF }}$

'Ich werde arbeiten.'

An dieser Stelle kann gut gezeigt werden, dass die V-Auxiliare in der Tat in einem zusätzlichen V-Kopf über dem infiniten V-Kopf des Satzprädikats sitzen. Der übliche Nachweis hierfür ist die Position des Auxiliars relativ zum Negationsmarker nie 'nicht', der linear links von den V-Auxiliaren erscheint; vgl. (22):
a. Ja nie jestem budzony.
(partizipiales Passiv)
ich-NOM NEG bin-1SG geweckt $_{\mathrm{IMPF}}$-SG.M
'ICH werde nicht geweckt.'
b. Ja nie jestem nauczycielem.
ich NEG bin-1SG Lehrer-INS
'ICH bin nicht Lehrer.'
c. Ja nie będę pracować.
ich NEG werde-1SG arbeiten IMPF-INF
'ICH werde nicht arbeiten.'
(Kopulasatzstruktur)

Ich werde unten im Zusammenhang mit dem Russischen noch auf den Gegensatz zwischen partizipialem Vorgangspassiv und adjektivischem “Zustandspassiv" näher eingehen. 
Die Plazierung des Negationsmarkers nie unmittelbar links vom Satzprädikat würde, sofern sie überhaupt möglich ist, keine Satz-, sondern Konstituentennegation herbeiführen; vgl. dies am Beispiel des periphrastischen Futurs:

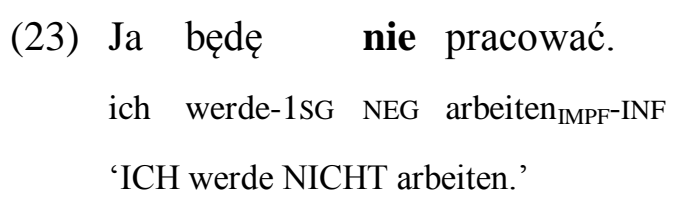

Der spätere Vergleich mit den I-Auxiliaren wird zeigen, dass der Negationsmarker dort nicht am Auxiliar, sondern am infiniten Satzprädikat erscheint. Ruft man sich die allgemeine Satzstruktur im Polnischen und Russischen in Erinnerung (CP > IP > NegP > VP* ...), kann das nur heißen, dass V-Auxiliare unterhalb der NegP, I-Auxiliare aber oberhalb davon sitzen.

Auch im Polnischen kann man alle Formen des V-Auxiliars gleichartig behandeln, wenn man für Kopulasatzstrukturen einen abstrakten V-Kopf annimmt, der die eigentliche Kopulabedeutung trägt, und dessen Merkmale stellvertretend von der jeweiligen Form des V-Auxiliars ausbuchstabiert werden; vgl. (24):
(24) a. Jestem budzony $_{\mathrm{V}}$. (partizipiales Passiv) b. Jestem $_{\mathrm{V}} \varnothing_{\mathrm{V}} \quad$ nauczycielem $_{\mathrm{N}}$. (Kopulasatzstruktur) c. Będę pracować $_{\mathrm{V}}$. (periphrastisches Futur)

Ebenso wie im Tschechischen und Polnischen, werden auch im Russischen zusammen mit Passivpartizipien Formen von byt' verwendet, die non-klitisch sind, ein volles Paradigma sowie durchgehend overte Lautform haben. Eine bedeutsame Ausnahme stellen jedoch ihre "Präsensformen" dar, die durchweg "stumm" sind; vgl. den Passivsatz in (25a) sowie den Kopulasatz in (25e):

\footnotetext{
(25) a. Političeskimi silami $\varnothing$ podgotovlen zakonoproekt. (part.

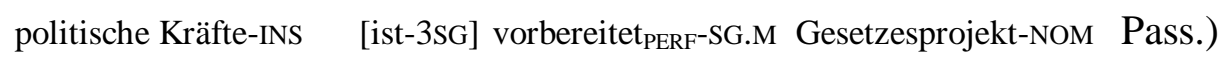
'Von den politischen Kräften wird ein Gesetzesprojekt vorbereitet.'

b. Magazin budet otkryt (prodavcom) v 9 časov.

Geschäft-NOM wird sein-3SG geöffnet ${ }_{\mathrm{KF}}$-SG.M Verkäufer-INS um 9 Uhr

'Das Geschäft wird (vom Verkäufer) um 9 Uhr geöffnet werden.'
} 
c. Magazin budet otkryt s 9 do 20 č[asov]. (KopulaGeschäft-NOM wird sein-3SG geöffnet $\mathrm{KF}^{-S G . M}$ von 9 bis $20 \mathrm{Uhr}$ sätze) 'Das Geschäft wird von 9 bis 20 Uhr geöffnet sein.'

d. Ty byla učitel'nicej.

$\mathrm{du}$ war-SG.F Lehrerin-INS

'Du warst Lehrerin.'

e. Ty $\varnothing \quad$ učitel'nica.

du [bist-2sG] Lehrerin-NOM

'Du bist (eine) Lehrerin.'

f. My budem rabotat'. (periphrastisches Futur)

wir werden-1PL arbeiten IMPF-INF

'Wir werden arbeiten.'

Auch für das Russische gilt, dass mit der Annahme eines abstrakten V-Kopfes mit Kopulabedeutung die parallele Analyse aller relevanten Formen von byt' als V-Auxiliare möglich ist (siehe zu otkryt $t_{\mathrm{A}}$ in (26b) Fn. 180 weiter unten):

(26) a. Magazin budet $_{\mathrm{V}}$ otkryt $_{\mathrm{V}}$ (prodavcom). (partizipiales Passiv)

b. Magazin budet $_{\mathrm{V}} \quad \varnothing_{\mathrm{V}} \quad$ otkryt $_{\mathrm{A}}$ (Kopulasatzstruktur)

c. On budet $_{\mathrm{V}}$ rabotat' $_{\mathrm{v}}$ (periphrastisches Futur)

$\mathrm{Zu}$ den passivischen Sätzen und Übersetzungen in (25b) und (25c) ist zu bemerken, dass sie aus Schlegel et al. $(1992,101)$ stammen, wo sie als Beispiele für die "morphologische" Nichtunterscheidung zwischen Vorgangspassiv und "Zustandspassiv" im Russischen dienen sollen. Dieser Ansicht schließe ich mich an, jedoch ist mehr dazu zu sagen. Da es sich um ein Thema handelt, das nicht unmittelbar mit dem Gegensatz von V- und I-Auxiliaren zu tun hat, tue ich letzteres in Form eines Exkurses.

\subsubsection{Exkurs: Vorgangspassiv und "Zustandspassiv" im Russischen}

Der Unterschied zwischen (25b) und (25c) wird lediglich durch temporale Modifikationen, namentlich einerseits die punktuelle Zeitangabe $v 9$ časov 'um 9 Uhr' und andererseits das Zeitintervall s 9 do 20 č. 'von 9 bis 20 Uhr', deutlich. Ferner spielt die instrumentalische by-Phrase prodavcom 'vom Verkäufer' eine wichtige 
Rolle, die nur beim partizipialen Vorgangspassiv (dem 'echten' Passiv) akzeptabel ist, nicht aber beim "Zustandspassiv" (das kein 'echtes' Passiv ist, sondern eine Kopula-Prädikativ-Struktur).

Eine Vorgangslesart kann nur ein 'echtes' partizipiales Passiv haben, während die Zustandslesart aus der "Resultativierung" des Partizips (mit der im Deutschen auch dessen Adjektivierung einhergeht) folgt; vgl. dazu auch (26a) mit (26b) oben. ${ }^{180}$ Nur solche Sätze, in denen ein verbales (nicht "resultativiertes") Partizip vorliegt, stellen somit ein Vorgangspassiv dar. Was das "Zustandspassiv" angeht, so hat sich in der (primär germanistischen) Forschung heute weitgehend die Ansicht durchgesetzt, dass es sich dabei um eine Kopulasatzstruktur handelt (siehe u. a. Rapp 1996; Maienborn 2007b; Maienborn \& Geldermann 2013, 128). Die Zustandslesart solcher Strukturen lässt sich darauf zurückführen, dass einerseits die Kopulabedeutung im Satz vorhanden und andererseits die partizipiale Form keine eigentliche Verbform, sondern vielmehr eine Adjektivierung bzw. Resultativbildung ist (Fn. 180). Durch letzteren Prozess wird das Topikzeitargument existenziell abgebunden und repräsentiert im Weiteren nur noch ein Ereignis, das einem neu eingeführten Folgezustand vorausgegangen ist. Diese Abbindung des ursächlichen Ereignisses mag auch erklären, warum eine by-Phrase unnatürlich bzw. inakzeptabel ist: Da sich der Sprecher durch die Verwendung des "Zustands-

${ }^{180}$ Das Label "A" an otkryt in (26b) folgt der traditionellen Kategorienarchitektur und ist an den Analysen Zimmermanns (2003b) sowie Maienborn \& Geldermanns (2013) orientiert, die für deutsche Partizipialformen im "Zustandspassiv" (Kopulasätze!) deren Adjektivierung ansetzen. $\mathrm{Da}$ ich Kurzformen generell als Verbalnomina $([+\mathrm{V},+\mathrm{N}])$ betrachte, kommt letzteres für die russischen und polnischen Partizipialformen nicht in Frage. Den genannten Analysen ist aber m. E. insoweit zu folgen, als es sich bei Formen wie otkryt in Fällen wie (26b) um Resultativbildungen handelt. Deren "Resultativierung" erfolgt entweder bereits im Lexikon oder erst in der Syntax durch ein semantisches Template (affixlose Anreicherung der SF; vgl. Zimmermann 1992). Dabei wird ihr Topikzeitargument existenziell abgebunden und mittels der Relation RESULT als Quelle eines neu eingeführten Folgezustands $s^{\prime}$ ausgewiesen. So bleibt bei solchen Formen nurmehr das interne Argument ungebunden (es kommt also zu einem Wechsel des logischen Typs). So wird im Kopulasatzbeispiel (26b) über magazin 'Geschäft' prädiziert, es werde in der Zukunft die Eigenschaft des Geöffnet(worden)seins aufweisen. In meinem Modell lässt die beschriebene "Resultativierung" die Kategorie $[+\mathrm{V},+\mathrm{N}]$ der Partizipialform unberührt. Vgl. den Eintrag in (i) [vor] mit dem in (ii) [nach der "Resultativierung"]:

(i) /otkryt/ $\quad[+\mathrm{V},+\mathrm{N}, \ldots] \quad \lambda y \lambda t \exists s[[t \supseteq \tau(s)]: s$ INST [z OPEN $y]]<\mathrm{e},\langle\mathrm{e}, \mathrm{t}\rangle>$

(ii) /otkryt/ $\quad[+\mathrm{V},+\mathrm{N}, \ldots] \quad \lambda y \exists s^{\prime} \exists t \exists s\left[\left[s^{\prime}\right.\right.$ RESULT $\left.t\right]:[[t \supseteq \tau(s)]: s$ INST [z OPEN $\left.\left.y]\right]\right]<e, t>$ Beachte, dass durch den Wechsel des Typs zu <e,t> ein einstelliges Prädikat entsteht, was der entstandenen Resultativform etwas Adjektivisches verleiht. Hierin könnte man eine Entsprechung zu der für das deutsche "Zustandspassiv" angenommenen Adjektivierung sehen. 
passivs" auf den Folgezustand (als Eigenschaft des Subjektreferenten) konzentriert, spielt der Agens des verursachenden Ereignisses eine nur untergeordnete bzw. gar keine Rolle. Es muss daher 'seltsam' wirken, ihn in Form einer by-Phrase zu nennen.

Wenn gesagt wird, das russische partizipiale Passiv sei ambig zwischen Vorgangs- und Zustandslesart (vgl. u. a. Lehmann 2009; Borik 2012), so kann dem zugestimmt werden, allerdings nur mit gewissen Einschränkungen. ${ }^{181}$ Das partizipiale Passiv hat stets und nur die Vorgangslesart, wogegen Kopulasätze mit "resultativierten" Partizipialformen stets und nur die Zustandslesart haben können. Der Eindruck, das partizipiale Passiv könne auch die Zustandslesart haben, ist m. E. ein Irrtum, der zum einen aus der häufigen Homonymie dieser Passivsätze mit Kopulasätzen (d. h. "Zustandspassiven”) sowie andererseits aus dem überwiegend perfektiven Aspekt der $n / t$-Partizipien im Russischen folgt: Letzterer sorgt für eine 'abgeschlossene' Sichtweise des Sprechers auf die Handlung. Diese 'Abgeschlossenheit' kommt der Zustandslesart sehr nahe, entspricht ihr aber nicht vollends, da beim partizipialen Passiv das Ereignis, das einen Zustand zur Folge hat, eben nicht - wie beim resultativen "Zustandspassiv" - abgebunden und so gewissermaßen 'kaltgestellt' wird. Das bedeutet, dass bei einem partizipialen Passiv mit perfektivem $n / t$-Partizip ein im Verlauf befindliches Ereignis geschildert wird, dessen Abschluss (bzw. Folgezustand) gleichzeitig ebenfalls perspektiviert bzw. thematisiert wird. Bei einem "Zustandspassiv" dagegen steht einzig der Folgezustand (als Eigenschaft des Subjektreferenten) im Zentrum der Äußerung. Man kann zweierlei Evidenz anbringen, um zu zeigen, dass das russische partizipiale Passiv in der Tat die Vorgangslesart hat. Zum einen liegen zwar nicht viele, aber im einzelnen sehr frequente imperfektive $n / t$-Partizipien wie z. B. bit 'geschlagen' vor (siehe u. a. Borik 2012). Die Passivsätze mit ihnen zeigen deutlich die Vorgangslesart (eine Übersetzung mit “Zustandspassiv" ist ausgeschlossen):

${ }^{181}$ Paslawska \& Stechow (2003a) vertreten sogar die extreme Ansicht, das russische partizipiale Passiv sei stets - und unzweideutig - stativ (Gegenargumente bringt u. a. Borik 2012). 


\section{(27) Bill rasskazyval, kak uže byl bit v avtobuse \\ Bill erzählte wie schon war-SG.M geschlagen ${ }_{\mathrm{KF}}$-SG.M in Bus-LOC \\ odnoj beremennoj baboj za popytku ustupit' ej mesto. \\ eine schwangere Frau-INS für Versuch-ACC abtreten-INF ihr-DAT Platz-ACC}

'Bill erzählte, wie er schon einmal im Bus von einer schwangeren Frau für den Versuch geschlagen $\{$ wurde/worden war/*war\}, ihr seinen Platz zu überlassen.'

NKRJ

Freilich zeigen solche Passivsätze lediglich, dass das russische partizipiale Passiv nicht zwangsläufig perfektiv ist. ${ }^{182}$ Im gegebenen Kontext ist Beispiel (28) aussagekräftiger:

${ }^{182}$ De facto ist das russische partizipiale Passiv überwiegend perfektiv, da die $n / t$-Partizipien bei der absolut überwiegenden Zahl der Verblexeme nur von den Perfektiva gebildet werden; imperfektive $n / t$-Partizipien sind schlichtweg unüblich, vgl. ${ }^{\vee}$ sdelan : *delan 'gemacht', ' pročitan : *čitan 'gelesen', 'otkryt : *otkryvan 'geöffnet' usw. (dagegen aber im Polnischen: 'zrobiony : ' robiony 'gemacht', ‘ przeczytan : ' czytan 'gelesen', ' otworzonylotwarty : ‘ otwierany 'geöffnet'). In Folge dieser heute mehrheitlichen Beschränkung des n/t-Partizips auf perfektive Formen ist das partizipale Passiv folglich auch mehrheitlich auf den Ausdruck eines perfektiven Passivs spezialisiert. Zum Ausdruck eines imperfektiven Passivs wird heute meist das sog. "Reflexivpassiv" verwendet. Bei den wenigen Verblexeme, die imperfektive $n / t$-Partizipien zulassen, ist der reflexive Gebrauch meist anders belegt:

(i) Mal'čik b'ëtsja. (echt-reflexiv/Antipassiv)

Junge schlägt-REFL

'Der Junge schlägt sich (selbst).'/‘Der Junge schlägt (/prügelt).'

(ii) Mal'čik $\varnothing$ bit.

(passivisch)

Junge [AUX] geschlagen

'Der Junge wird geschlagen.'

Der reflexive Gebrauch ist hier primär der echt-reflexiven sowie der Antipassiv-Lesart vorbehalten; somit zeigt das imperfektive partizipiale Passiv ohne Verwechslungsgefahr die passivische Lesart an. Bei anderen Verblexemen wie z. B. myt' 'waschen' ist auf Grund ihrer deskriptiven Bedeutung die Antipassiv-Lesart sehr unwahrscheinlich, weshalb die Reflexivform 'nur' die echt-reflexive sowie die passivische Lesart ausdrücken muss, was offenbar den Verzicht auf die partizipiale Ausdrucksform, die seit jeher relativ buchsprachlich ist, legitimiert. Satz (iv) ist m. E. höchstens in älteren Texten anzutreffen (das $n / t$-Partizip war im Russischen früher regelmäßig von beiden Aspekten bildbar):

(iii) Mal'čik moetsja. (echt-reflexiv/passivisch/?'Antipassiv) Junge wäscht-REFL

'Der Junge wäscht sich.'/'Der Junge wird gewaschen.'

(iv) "Mal'čik $\varnothing$ myt.

Junge [AUX] gewaschen 


\section{(28) Sejčas političeskimi silami $[\ldots]$ podgotovlen $\quad$ zakonoproekt $[\ldots]^{183}$

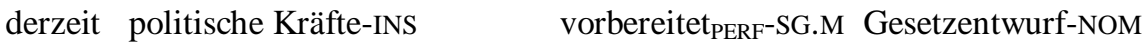 'Derzeit wird von den politischen Kräften [...] ein Gesetzentwurf vorbereitet [...].'}

Die by-Phrase zeigt, dass es sich um ein 'echtes' Passiv handelt (vgl. Borik 2012, 119), und zwar trotz des perfektiven Aspekts des n/t-Partizips. Das Adverb sejčas 'derzeit' verweist ebenfalls auf einen zum Sprechzeitpunkt im Verlauf befindlichen Vorgang - und nicht auf einen Zustand des Vorbereitet-Seins. ${ }^{184}$ Da dieser Satz ein 'echtes' Vorgangspassiv darstellt, ist hier - wie in allen partizipialen Passivsätzen - von einem V-Auxiliar auszugehen, das in diesem Fall "stumm" ist und so INDIKATIV und PRÄSENS reflektiert. Bei podgotovlen muss es sich folglich um ein verbales Partizip (nicht um eine Resultativbildung) handeln. Dieser 'echte' Passivsatz hat deutlich und trotz des perfektiven Aspekts die Vorgangslesart. Der Präsident der Ukraine, der diesen Satz äußerte, dürfte das partizipiale Passiv mit perfektivem Partizip mehr oder weniger bewusst gewählt haben (und nicht etwa ein "Reflexivpassiv" mit imperfektiver Verbform), da er den absehbaren (von ihm geplanten und prospektiv als erfolgreich betrachteten) Abschluss der Vorbereitung des Gesetzentwurfs thematisieren wollte, obwohl die Vorbereitungen zum Zeitpunkt seiner Äußerung noch liefen. Die Kombination aus beidem wird eben durch das präsentische partizipiale Passiv mit perfektivem Partizip erreicht. ${ }^{185}$ Letztlich bestätigt sich die Aussage Schoorlemmers (1995) und anderer Arbeiten, dass das russische partizipiale Passiv (mit Kurzform-Partizipien) ambig zwischen

${ }^{183}$ Ich danke Uwe Junghanns für den Hinweis auf dieses Beispiel (Quelle: interfax.ru).

${ }^{184}$ Das heißt auch, dass Sätze mit $n / t$-Partizipien ohne overtes Auxiliar nicht zwangsläufig präterital zu interpretieren sind (vgl. Lehmann 2009; Paslawska \& Stechow 2003a). Die präteritale Interpretation haben sie m. E. nur, wenn es sich um Kopulasätze handelt (was freilich fast immer der Fall ist, da das perfektive partizipiale Passiv im Präsens nur sehr selten vorkommt), und zwar deswegen, weil der denotierte Folgezustand ein vorangehendes Ereignis impliziert, das relativ zu ihm in der Vergangenheit liegt. In einem 'echten' Passivsatz dagegen ist das verursachende Ereignis (noch) im Verlauf begriffen. Beachte, dass beide Sätze formal präsentisch sind, sich aber in ihrer temporalen Interpretation unterscheiden:

(i) $\mathrm{I}^{\mathrm{PRÄS}}$ Zakonoproekt $\varnothing_{\mathrm{AUX}}$ podgotovlen $\mathrm{V}_{\mathrm{V}} . \quad$ (part. Passiv präsentische Interpration) 'Der Gesetzentwurf wird vorbereitet.'

(ii) $\mathrm{I}^{\mathrm{PRÄS}}$ Zakonoproekt $\varnothing_{\mathrm{AUX}} \varnothing_{\mathrm{V}}$ podgotovlen ${ }_{\mathrm{A}} . \quad$ (Kopulasatz $\sim$ präteritale Interpration) 'Der Gesetztentwurf ist vorbereit.' [ 'Der Gesetzentwurf wurde vorbereitet.']

${ }^{185}$ Der Interpretation des Satzes ist daher auch ein futurischer 'Beigeschmack' eigen, der aber nur den (prospektiven) Folgezustand, nicht aber das (ablaufende) Ereignis betrifft, das diesen $\mathrm{Zu}-$ stand herbeiführt. 
der Zustands- und der Vorgangslesart sei (siehe auch Borik 2012, 122). Jedoch besteht diese Ambiguität nur an der Oberfläche, da 'tiefenstrukturell' ein gravierender Unterschied zwischen einem 'echten' Passivsatz und einer Kopula-Prädikativ-Struktur bzw. dem "Zustandspassiv" besteht. Diesen Gegensatz wiederhole ich hier nochmals an den bereits oben genannten Beispielen:

(29) a. Magazin budet $_{\mathrm{V}}$ otkryt $\mathrm{v}$. 'Das Geschäft wird geöffnet werden.'

b. Magazin budet $_{\mathrm{V}} \varnothing_{\mathrm{V}} \quad$ otkryt $_{\mathrm{A}}$. (Kopulasatz: Zustandslesart) 'Das Geschäft wird geöffnet sein.'

Sätze mit diesem Bestand an Formen sind in der Tat oberflächlich ambig zwischen Vorgangspassiv und “Zustandspassiv” bzw. zwischen 'echtem' partizipialem Passiv und einer Kopulasatzstruktur mit departizipialem Prädikatsadjektiv. Eine instrumentalische by-Phrase (z. B. prodavcom 'vom Verkäufer') ist daher nur bei (29a) akzeptabel und kann als Hilfsmittel zur Desambiguierung verwendet werden.

Eindeutig(er) sind Sätze mit Langform-Partizipien, die immer als Kopulasätze mit adjektivierten Partizipien zu analysieren sind und hinsichtlich derer Borik (2012) von einem "adjectival passive" spricht. Sie lassen daher niemals eine by-Phrase $\mathrm{zu}$, und bei ihnen ist anstelle von departizipialen Adjektiven auch eine deutsche Übersetzung mit ursprünglichen (Primär-)Adjektiven möglich, sofern solche lexikalisch vorhanden sind (vgl. Maienborn \& Geldermann 2013, 131):

(30) Magazin budet $_{\mathrm{V}} \varnothing_{\mathrm{V}}$ otkrytyj. (Kopulasatz: reine Eigenschaft) 'Das Geschäft wird \{geöffnet/offen\} sein.'

Damit beende ich den Exkurs zu den Passivtypen im Russischen.

\subsubsection{Bestand und Distribution der I-Auxiliare}

Zunächst stelle ich auch hier den Bestand der I-Auxiliare deskriptiv dar. Beachte, dass alle Formen (en)klitisch sind: 


\begin{tabular}{|l|c|c|c|}
\cline { 2 - 4 } \multicolumn{1}{c|}{ (31) Tschechisch } & Ps. & Sg. & PI. \\
\hline \multirow{4}{*}{ präteritales I-Auxiliar } & 1 & jsem & jsme \\
\cline { 2 - 5 } & 2 & $j s i$ & jste \\
\hline \multirow{3}{*}{ konjunktivisches I-Auxiliar } & 3 & & $\varnothing$ \\
& 1 & bych & bychom/bysme \\
\cline { 2 - 5 } & 2 & bys & byste \\
\cline { 2 - 5 } & 3 & \multicolumn{2}{|c|}{ by } \\
\hline
\end{tabular}

\begin{tabular}{|l|c|c|c|}
\cline { 2 - 4 } \multicolumn{1}{c|}{$(32) \underline{\text { Polnisch }}$} & Ps. & Sg. & PI. \\
\hline \multirow{4}{*}{ präteritales I-Auxiliar } & 1 & $(e) m$ & śmy \\
\cline { 2 - 5 } & 2 & $(e) s ́$ & ście \\
\hline \multirow{3}{*}{ konjunktivisches I-Auxiliar } & 3 & \multicolumn{2}{|c|}{$\varnothing$} \\
\cline { 2 - 5 } & 1 & bym & byśmy \\
\hline & 2 & byście \\
\cline { 2 - 5 } & 3 & \multicolumn{2}{|c|}{ by $\varnothing$} \\
\hline
\end{tabular}

\begin{tabular}{|l|c|c|c|}
\cline { 2 - 4 } \multicolumn{1}{l|}{ (33) Russisch } & Ps. & Sg. & Pl. \\
\hline präteritales I-Auxiliar & $1 / 2 / 3$ & \multicolumn{2}{c|}{$\varnothing$} \\
\hline konjunktivisches I-Auxiliar & $1 / 2 / 3$ & \multicolumn{2}{c|}{ by $\varnothing$} \\
\hline
\end{tabular}

Es fällt sofort auf, dass im Russischen die Tendenz zum Abbau der Auxiliarformen am weitesten fortgeschritten ist. Anders könnte man auch formulieren, dass sich die Formen der 3. Person, wie sie in den heutigen westslavischen Sprachen vorliegen, im Russischen jeweils als generelle Formen durchgesetzt haben.

Ferner soll hier demonstriert werden, dass bestimmte Formen im Polnischen und Russischen (aber auch im Tschechischen) in der Tat als I-Auxiliare klassifiziert werden können, indem gezeigt wird, dass sie sich in ein und derselben Strukturposition im Satz befinden und eine vergleichbare Bedeutung und Funktion haben. Ich beginne mit dem Tschechischen, wo die I-Auxiliare v. a. orthographisch durch die Schreibung als separate Wortform gut isolierbar sind. Zu den I-Auxiliaren gehören zwei Reihen auxiliarer Formen, namentlich (a) jene, die zusammen mit l-Partizipien die Präteritumperiphrase bilden, sowie (b) jene, die gemeinsam mit l-Partizipien oder dem Infinitiv den analytischen Konjunktiv konstituieren. Ich konzentriere mich bei diesem Vergleich auf Beispiele mit $l$-Partizipien: 
(34)

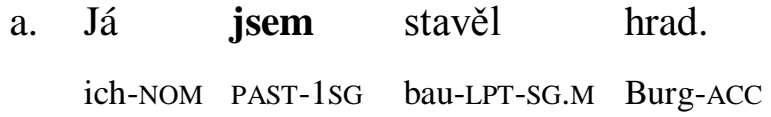
'ICH habe eine Burg gebaut.'/'ICH baute eine Burg.'
b. Já bych stavěl hrad.
ich-NOM KONJ-1SG bau-LPT-SG.M Burg-ACC
(Konjunktiv)
(Präteritum)
'ICH würde eine Burg bauen.'/'ICH hätte eine Burg gebaut.'

Diese beiden Beispiele der 1. Ps. Sg. zeigen, dass die Präteritum- und die Konjunktivperiphrase lediglich durch das Auxiliar unterschieden sind. Das deutet darauf hin, dass es das Auxiliar und nicht das Infinitum ist, das die relevante Bedeutung repräsentiert bzw. etabliert. Dass diese Auxiliare in $\mathrm{I}^{0}$ (und nicht, wie die V-Auxiliare, in einem zusätzlichen V-Kopf) sitzen, zeigt wiederum die Abfolge in negierten Sätzen (siehe auch bereits Abschnitt 5.2.2): ${ }^{186}$

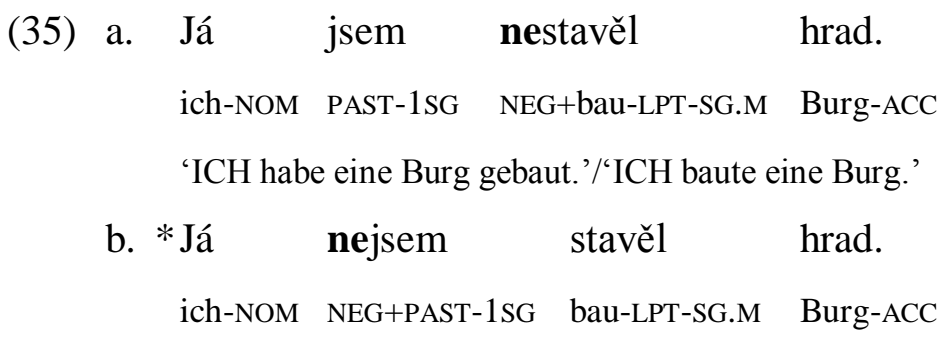

${ }^{186}$ In der tschechischen Standardsprache liegen auch ein Antepräteritum (Plusquamperfekt) und ein Konditional (Konjunktiv) Präteritum vor. In beiden kann die Negation entweder am Vollverb oder am V-Auxiliar auftreten (Beispiele aus MČ 1986, 427; vgl. auch Junghanns 1999):
(i) a. byl jsem nechválil (Cze, Antepräteritum)
V-AUX-LPT I-AUX NEG-lob-LPT
b. nebyl jsem chválil
NEG-V-AUX-LPT I-AUX lob-LPT
'(ich) hatte nicht gelobt'
(ii) a. byl bych neprosil (Cze, Kond. Präteritum)
V-AUX-LPT I-AUX NEG-bitt-LPT
b. nebyl bych prosil
NEG-V-AUX-LPT I-AUX bitt-LPT
'(ich) hätte nicht gebeten'
Beachte, dass die semantische Negation in $\mathrm{Neg}^{0}$ so oder so das Satzprädikat im Skopus hat, egal, ob das sie ausbuchstabierende Präfix ne- am Vollverb oder am V-Auxiliar erscheint. Dies ist hier durch die generelle Position von NegP über VP* gesichert (siehe Abschnitt 4.5) 
(36)
a. Já bych nestavěl hrad.
ich-NOM KONJ-1SG NEG+bau-LPT-SG.M Burg-ACC
'ICH würde keine Burg bauen.'/‘ICH hätte keine Burg gebaut.'
b. *Já nebych stavěl hrad.
ich-NOM NEG+KONJ-1SG bau-LPT-SG.M Burg-ACC
(Konjunktiv)

Im Präteritum und Konjunktiv erscheint der Negationsmarker sowohl im Tschechischen, Polnischen als auch Russischen stets am infiniten Satzprädikat, nicht aber am Auxiliar. Da die NegP unterhalb von IP, aber oberhalb von VP projiziert wird, lässt dies den Schluss zu, dass die relevanten Auxiliare in $\mathrm{I}^{0}$ sitzen. ${ }^{187}$

In der 3. Person ist das präteritale I-Auxiliar im Tschechischen phonetisch leer, während im Konjunktiv die quasi "endungslose" Form by vorliegt:

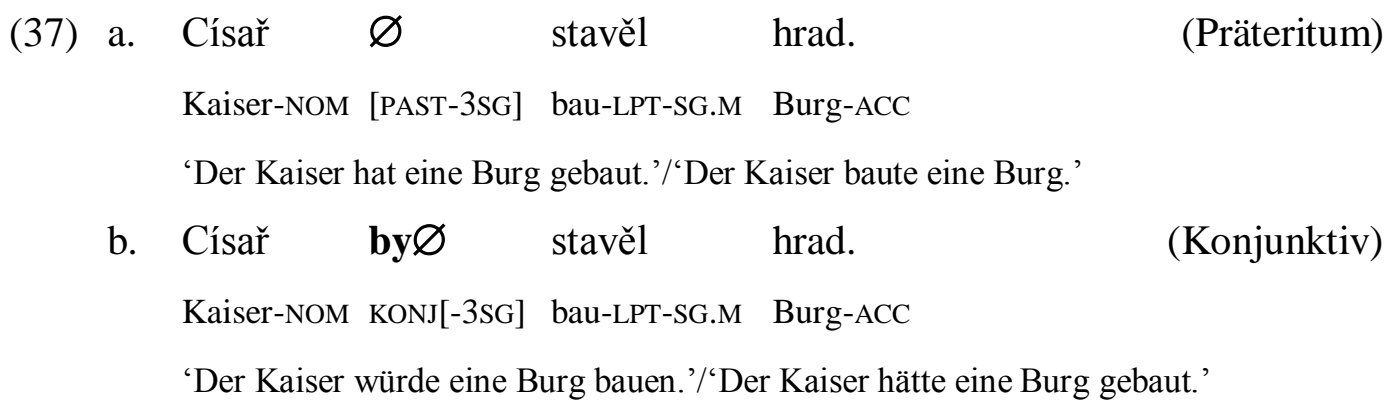

Exakt dieselbe Situation zeigt sich im Polnischen. Ein Unterschied besteht jedoch darin, dass die enklitischen I-Auxiliare heute primär verbadjazent sowie in der Orthographie nicht separat erscheinen. Ihre Basisposition ist dennoch $\mathrm{I}^{0}$ (siehe auch Abschnitte 5.2.1, 5.2.2):
a. Ja
zbudowałam
szkołę.
ich-NOM bau-LPT-SG.F+PAST-1SG Schule-ACC
'ICH habe die/eine Schule gebaut.'/'ICH baute die/eine Schule.'

(Präteritum)

${ }^{187}$ Selbst wenn man, wie Zimmermann (2012, 2013), den Negationsmarker als Verbpräfix (oder als Kopf-Adjunkt an $\mathrm{V}^{0}$ ) analysiert, widerspricht dies der Argumentation für die Lokalisierung der I-Auxiliare nicht, denn offensichtlich kann der Negationsmarker nur mit lexikalischen, nicht jedoch mit funktionalen verbalen Formen verbunden werden. Auch dies spräche für die Verortung der hier relevanten Auxiliare in $\mathrm{I}^{0}$, da dies bedeutet, dass es sich bei ihnen um funktionale Einheiten handelt, mit denen sich der Negationsmarker nicht verbinden kann. Im Russischen ist der "Negationstest" auf Grund der generellen "Unsichtbarkeit" des präteritalen I-Auxiliars nur im Falle des Konjunktivs aussagekräftig. 
$\begin{array}{llll}\text { b. } & \text { Ja } & \text { zbudowałabym } & \text { szkołę. }\end{array}$

In der 3. Person liegen wiederum "Nullformen" vor: Eine volle Nullform im Fall des Präteritums, und eine Nullendung im Fall des ('persönlichen') Konjunktivs: ${ }^{188}$
a. Państwo zbudowało $\varnothing \quad$ szkołę. $\quad$ (Präteritum)
Staat-NOM bau-LPT-SG.N+[PAST-3SG] Schule-ACC
'Der Staat hat die/eine Schule gebaut.'/'Der Staat baute die/eine Schule.'
b. Państwo zbudowałoby $\varnothing \quad$ szkołę.
Staat-NOM bau-LPT-SG.N+[KONJ-3SG] Schule-ACC
'Der Staat würde die/eine Schule bauen.'/'Der Staat hätte die/eine Schule gebaut.'

Das Russische besticht dadurch, dass das Paradigma des präteritalen I-Auxiliars gänzlich "stumm" ist. Wie schon in Abschnitt 5.2.1 gezeigt wurde, genügt daher die Annahme, dass das russische Präteritalauxiliar heute als semantischer Operator bzw. als unterspezifiertes Nullauxiliar im mentalen Lexikon abgelegt ist. Die Lage im Konjunktiv ist analog, da by quasi stets eine "Nullendung" hat bzw. ebenfalls als semantischer Operator bzw. als unterspezifiziertes Konjunktivauxiliar gelten kann:

\footnotetext{
${ }^{188}$ Besonders im Polnischen wirken die präteritalen I-Auxiliare wie bloße "Endungen" für Person und Numerus, die aber freilich auch eine Modus-/Tempusbedeutung tragen. Mit Junghanns (1995), der sich damit auf die russischen bud-Formen bezieht, könnte man auch von "Quasiaffixen" reden, da durch sie Modus, Tempus, Person und Numerus "exzeptionell" realisiert werden. Die 'Endungshaftigkeit' der polnischen Präteritalauxiliare zeigt sich auch daran, dass sie sich am Konjunktivauxiliar offenbar als reine Person-/Numerusendungen wiederfinden, weswegen die by-Formen mitunter auch in die Komponenten $b y$ - und jene "Endungen" aufgespalten werden (ich lehne diese Sichtweise ab, da die vermeintlichen Komponenten der by-Formen im polnischen Satz niemals separat erscheinen können; ebenso zeigen ihre tschech. Entsprechungen ein klar idiosynkratisches Paradigma, vgl. Toman 1980 - die polnischen by-Formen beruhen m. E. auf analogischem Ausgleich). Vertreter der "Endungshypothese" sind zum Teil Franks \& Bański (1999), wenn sie annehmen, dass sich die polnischen präteritalen I-Auxiliare 'schizophren' verhalten, da sie angeblich einerseits als Auxiliarklitika, andererseits als Flexionsmarker auftreten können (m. E. sind sie stets Auxiliare).
} 
$(40)$
a. Ja $\varnothing \quad$ rabotal.
ich-NOM [PAST-1SG] arbeit-LPT-SG.M
'Ich habe gearbeitet.'/'Ich arbeitete.'
b. Ja by $\varnothing \quad$ rabotal.
ich-NOM KONJ[-1SG] arbeit-LPT-SG.M
'Ich würde arbeiten.'/'Ich hätte gearbeitet.'

(Präteritum)

(Konjunktiv)

Die 3. Person unterscheidet sich im Russischen nicht von den übrigen Personen:
(41)
a. Marija $\varnothing \quad$ rabotala.
Marija-NOM [PAST-3SG] arbeit-LPT-SG.F
'Maria hat gearbeitet.'/'Maria arbeitete.'
(Präteritum)
b. Marija by $\varnothing$ rabotala.
Marija-NOM KONJ[-3SG] arbeit-LPT-SG.F
(Konjunktiv)
'Maria würde arbeiten.'/‘Maria hätte gearbeitet.'

\subsubsection{Fazit}

Dieser Abschnitt hat gezeigt, dass in den betrachteten Sprachen eine generelle Zweiteilung von Auxiliaren anzunehmen ist, die nach ihrer syntaktischen Position als V- und als I-Auxiliare bezeichnet wurden. Während die V-Auxiliare rein ausbuchstabierende Funktion und somit keine Eigensemantik haben, liegt letztere bei den I-Auxiliaren in Form einer Modus-/Tempus-Spezifikation vor. Zu den eigentlichen I-Auxiliaren in den betrachteten Sprachen gehören die Formen des Präteritum- sowie des Konjunktivauxiliars. Im weiteren Sinne sind zu den I-Auxiliaren aber auch die stets phonetisch leeren "semantischen Operatoren" für PRÄSENS und FUTUR zu zählen, die oben in Abschnitt 4.6.2 beschrieben wurden.

Die Haupteigenschaften von V- und I-Auxiliaren lassen sich wie in (42) zusammenfassen:

\begin{tabular}{|c|c|c|}
\hline (42) & V-Auxiliare & I-Auxiliare \\
\hline (en)klitisch & - & + \\
\hline non-overte/endungslose 3. Person & - & + \\
\hline modal-temporale Eigensemantik & - & + \\
\hline
\end{tabular}


Diese phonetischen und semantischen Eigenschaften, ihre Basispositionen in der Satzstruktur und schließlich auch ihre Funktion lassen die I-Auxiliare deutlich als funktionale bzw. 'grammatische' Elemente hervortreten. Ihren Ursprung in den paradigmatischen (präsentischen, aoristischen sowie konjunktivischen) Formen des Verblexems (späturslav./aksl.) byti 'sein' können sie freilich nicht verhehlen, jedoch sollte klar geworden sein, dass es sich heute um vollends grammatikalisierte bzw. 'fossilierte' Formen handelt, die nicht mehr in direktem Zusammenhang mit den aktuellen Flexionsformen von byt/byćlbyt' stehen, die das Formenreservoir der "lexikalischen" V-Auxiliare bilden.

\subsection{Primäre und sekundäre Prädikation}

\subsubsection{Primäre Prädikation}

In Abschnitt 5.1 bin ich für "Kopulasätze" zur VP-Grundstruktur in (43) gelangt:

(43) $\left.\left[\mathrm{vP} V\left[\mathrm{vp}_{\mathrm{V}} \varnothing_{\mathrm{V} P} \mathrm{X}\right]\right]\right] \quad \operatorname{mit} \mathrm{X} \in\{\mathrm{A}, \mathrm{N}, \mathrm{P}\}$

Die Position "V" wird im Falle primärer Prädikation von einer Form des V-Auxiliars, d. h. von einer Flexionsform des Lexems byćlbyt' eingenommen, das der "Sichtbarmachung" eines bestimmten I-Operators dient bzw. durch ein overtes oder kovertes I-Auxiliar selegiert wird (siehe Abschnitte 4.6.2, 4.6.3).

Unter "primärer Prädikation" verstehe ich alle Fälle, in denen der abstrakte V-Kopf mit der Kopulabedeutung und dem prädikativen Ausdruck in seiner Komplementposition das in/finite Satzprädikat stellt und eine eigene verbmodale und ggf. temporale Spezifikation aufweist. Dies kann sowohl in Haupt- als auch in Nebensätzen der Fall sein. Es handelt sich also stets um Satz- bzw. satzwertige Strukturen inklusive einer IP-Projektion. ${ }^{189}$

${ }^{189}$ Infinite Satzteile, z. B. in Kontrollverbstrukturen, betrachte ich als overt oder non-overt eingeleitete CPn ohne IP, was dem Fehlen von Modus und Tempus entspricht; vgl. (i-a/b):

(i) a. Ivan chočet $\left[{ }_{C P} \varnothing_{C}\left[{ }_{v P}\right.\right.$ rabotat' $\left.\left._{V}\right]\right]$. 'Ivan will arbeiten.'

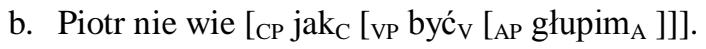

'Peter weiß nicht, wie er [so] dumm sein soll.'

(Witkoś 2011, 55)

Die Merkmale $[+\mathrm{V},+\mathrm{N}]$ des Infinitivs (vgl. Abschnitt 4.3) werden unmittelbar vom C-Kopf bzw. mittelbar vom Matrixverb selegiert, was Koreferenz von Matrix- und Infinitivsubjekt zur 
Im Folgenden werden einige Beispiele (jeweils in (a)) mit ihrer syntaktischen Repräsentation (jeweils in (b)) gezeigt. Das soll auch zeigen, dass die hier vertretene Analyse generell anwendbar ist. Den abstrakten V-Kopf, der die Kopulabedeutung trägt, hebe ich der Deutlichkeit halber fett hervor $\left(\varnothing_{\mathbf{V}}\right)$. Ggf. erfolgende Bewegungen werden nicht dargestellt. ${ }^{190}$ Zunächst einige Hauptsatzstrukturen:

\section{Indikativ Präsens}

(44) a. Jan jest moim przyjacielem.

'Jan ist mein Freund.'

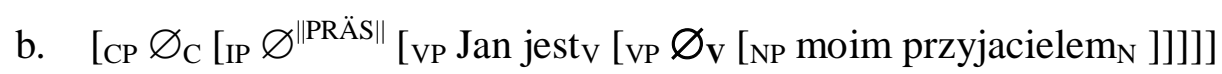

(45) a. Ivan - moj drug.

'Ivan ist mein Freund.'

b. [C $\left.\varnothing_{\mathrm{CP}}\left[{ }_{\text {IP }} \varnothing^{\|\mathrm{PRÄS}\|}\left[\mathrm{vP} \operatorname{Ivan} \varnothing_{\mathrm{V}}\left[\mathrm{vP} \varnothing_{\mathrm{V}}\left[\mathrm{NP} \operatorname{moj} \operatorname{drug}_{\mathrm{N}}\right]\right]\right]\right]\right]$

\section{Indikativ Präteritum}

(46) a. Byłam szczęśliwa.

'Ich war glücklich.'

b. [CP $\varnothing_{\mathrm{C}}\left[\right.$ IP pro $-\mathrm{m}^{\|\mathrm{PRÄT}\|}\left[{ }_{\mathrm{VP}}\right.$ była $\left.\left.\left.{ }_{\mathrm{V}}\left[\mathrm{vP} \varnothing_{\mathrm{v}}\left[\mathrm{AP}_{\text {szczęśliwa }}\right]\right]\right]\right]\right]$

(47) a. Ja byla sčastliva.

'Ich war glücklich.'

b. [C $\varnothing_{\mathrm{C}}\left[\mathrm{IP}\right.$ ja $\varnothing^{\|\mathrm{PRÄT}\|}\left[\mathrm{vP}\right.$ byla ${ }_{\mathrm{V}}\left[\mathrm{vP} \varnothing_{\mathrm{V}}\left[\mathrm{AP}\right.\right.$ sčastliva $\left.\left.\left.\left.{ }_{\mathrm{A}}\right]\right]\right]\right]$

Folge hat ("semantische Kontrolle"). Von solchen infiniten CPn, die sekundärer Prädikation nahe stehen (siehe unten), sind Nebensätze zu unterscheiden, die verbmodal und ggf. temporal spezifiziert sind und somit eine IP enthalten; vgl. (ii-a/b):

(ii) a. Aleksandr utverždaet, [CP čto ${ }_{\mathrm{C}}\left[\mathrm{IP}\right.$ oni $\varnothing_{\mathrm{I}}\left[\mathrm{VP}\right.$ byli [VP $\varnothing_{\mathrm{V}}\left[{ }_{\mathrm{AP}}\right.$ nekrasivy $\left.\left.\left.\left._{\mathrm{A}}\right]\right]\right]\right] . \quad$ (Rus)

'Alexander behauptet, dass sie unschön waren ...'

b. Dvuch let učëby [...] bylo v vojnu dostatočno, [CP čto ${ }_{\mathrm{C}}$ [IP by [vP rabotat' technikom]]].

'Zwei Jahre Lehre waren im Krieg ausreichend, um als Techniker zu arbeiten.'

Hier liegen eindeutig IPn vor. In Bezug auf Beispiele wie (ii-b) gehen auch Franks (1995) und Madariaga (2011) davon aus, dass ein Merkmal [+T] in $\mathrm{T}^{0}$ anzusetzen sei (nach meinen Annahmen entspricht dem [+Konj] in $\mathrm{I}^{0}$ ).

${ }^{190}$ Das würde in diesen Beispielen die Klitika in $\mathrm{I}^{0}$ bzw. die Verbformen in $\mathrm{V}^{0}$ betreffen, da erstere an der Oberfläche in aller Regel adjazent zu letzteren erscheinen. Ich schließe nicht aus, dass diese Oberflächenabfolgen auch durch Bewegung auf PF zustande kommen können und damit nicht syntaktisch, sondern postsyntaktisch bzw. phonologisch basiert sind. 


\section{Konjunktiv}

(48) a. Bylibyśmy wolni.

'Wir wären frei.'

b. $\quad\left[\mathrm{CP} \varnothing_{\mathrm{C}}\left[\mathrm{IP}\right.\right.$ pro -byśmy ${ }^{\|\mathrm{KONJ}\|}\left[\mathrm{vP}\right.$ byli $\left.\left.\left.{ }_{\mathrm{V}}\left[\mathrm{VP} \varnothing_{\mathrm{V}}\left[\mathrm{AP}_{\mathrm{WO}} \mathrm{wolni}_{\mathrm{A}}\right]\right]\right]\right]\right]$

(49) a. My byli by rady.

'Wir wären sehr froh.'

b. [CP $\varnothing_{\mathrm{C}}\left[\mathrm{IP} \mathrm{my}-\mathrm{by}{ }^{\|\mathrm{KONJ}\|}\left[\mathrm{vP}\right.\right.$ byliv $\left.\left.\left.\left[\mathrm{vP} \varnothing_{\mathrm{V}}\left[\mathrm{AP}_{\mathrm{Aady}}\right]\right]\right]\right]\right]$

An diesen Beispielen wird deutlich, dass die abstrakte Kopulabedeutung einheitlich im leeren V-Kopf oberhalb des nominalen Prädikatausdrucks lokalisiert ist. In der VP-Projektion darüber buchstabiert eine Flexionsform des V-Auxiliars die jeweiligen Finitheits- und Kongruenzmerkmale aus, die dem noch 'weiter oben' in der Struktur projizierten I-Kopf (der selbst die Form eines Auxiliars haben kann) entsprechen, und die der nominale (nicht-verbische) Prädikatausdruck nicht realisieren kann. Je nachdem, an welcher Strukturposition eine finite ("präsentisch" flektierte) Verbform sitzt, wird die Syntaktifizierung des nominativischen Subjektausdrucks ermöglicht. Bei einem finiten V-Auxiliar wird es demnach in Spec-VP, bei einem finiten I-Auxiliar hingegen in Spec-IP generiert (hier liegt der primäre Unterschied zwischen ‘synthetischen' und ‘analytischen' Verbformen).

Dieselben Mechanismen wirken auch in Komplement- (d.h. Subjekt- und Objekt-) sowie in Adjunkt- bzw. adverbialen Nebensätzen, auf die ich hier jedoch nicht weiter eingehen werde. Im folgenden Abschnitt konzentriere ich mich vielmehr auf das Phänomen der "sekundären Prädikation", das in einer Betrachtung von Kopulasatzstrukturen nicht zu ignorieren ist. ${ }^{191}$ Im Mittelpunkt meiner Behandlung dieses Phänomens steht sein Verhältnis zur "primären Prädikation” bzw. die Frage, was diese beiden Prädikationstypen verbindet und unterscheidet.

191 Vgl. z. B. Bailyn (2012), in dessen Argumentation die sekundäre Prädikation - weit über ihr traditionelles Verständnis hinaus - eine überaus bedeutsame Rolle spielt (Bailyn behauptet, sie sei in jeden Fall der Instrumentalzuweisung involviert); vgl. auch Abschnitt 3.3.3. 


\subsubsection{Sekundäre Prädikation}

\subsubsection{Generelle Annahmen}

Unter "sekundärer Prädikation" wird hier eine zusätzlich zur Haupt- oder Matrixsituation $\left(\mathbf{s}_{1}\right)$ versprachlichte Nebensituation $\left(s_{2}\right)$ verstanden, deren impliziter Partizipant (u) mit einem der Partizipanten der Matrixsituation (x/y/z) koreferent ist bzw. koreferent interpretiert wird: ${ }^{192}$
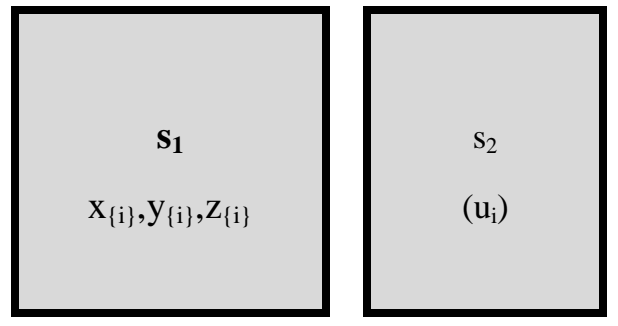

Durch ihren Partizipantenbezug unterscheiden sich Sekundärprädikate grundlegend von Adverbialen mit Situationsbezug (Beispiele von Renz 2009,1):

$\begin{array}{llll}\text { Piotr } & \text { pił } & \text { kawę } & \text { nagi. } \\ \text { Peter-NOM } & \text { trank } & \text { Kaffee-ACC } & \text { nackt-NOM }\end{array}$

'Peter trank den Kaffee nackt.'

$\begin{array}{llll}\text { Piotr } \quad \text { pił } \quad \text { kawę } & \text { szybko. } \\ \text { Peter-NOM } & \text { trank } & \text { Kaffee-ACC } & \text { schnell-ADV } \\ \text { 'Peter trank den Kaffee schnell.' }\end{array}$

(Adverbial) Situationsbezug

Die Kombination aus eigener Situationsreferenz und Partizipantenbezug unterscheidet "sekundäre Prädikate" wiederum von adjektivischen "Prädikatsphrasen" (Wilder 1994) respektive “appositiven Adjunkten” (Bailyn 2012), die ihrer Natur nach attributiv sind und lediglich Partizipantenbezug aufweisen (Abschnitt 5.4) Sekundäre Prädikation basiert m. E. stets auf einer Kopulasatzstruktur. Die von ihr denotierte Situation ist immer ein Zustand, in dem ein Referent eine bestimmte

${ }^{192}$ Vgl. Strigin $(2008,391)$, der folgende Interpretationshypothese aufstellt: "Any referent of the primary situation may serve as a referent of the secondary predicate if this can plausibly be inferred." Die Bedingung der Möglichkeit einer plausiblen Inferierung verweist auf die Rolle der konzeptuellen Ebene (Zwei-Ebenen-Semantik, siehe Abschnitt 4.7) bei der Herstellung von (semantischen) Kontrollrelationen (siehe auch weiter unten). 
Eigenschaft hat, die vom Prädikatsnomen versprachlicht wird. Der fragliche Referent erhält keine sprachliche Realisierung (ist also implizit) und wird koreferent mit einem der Partizipanten der Primärsituation interpretiert.

Ferner wird die Topik- bzw. Referenzzeit des Sekundärprädikats durch jene der primären Situation 'beschränkt' bzw. relativ zu ihr lokalisiert. ${ }^{193}$ Hinterhölzl (2001, 100-101) spricht mit Bezug auf die fraglichen russischen Prädikate (satzfinal und im Instrumental) von einer temporal restringierten Lesart. Sofern man dem folgt, ist die sekundäre der primären Prädikation zweifach untergeordnet, und zwar (i) hinsichtlich ihres Partizipantenbezugs und (ii) in Bezug auf die Verortung ihrer Referenzzeit.

Wie Hinterhölzl $(2001,108)$ aber zu Recht bemerkt, ist es weder der Fall noch theoretisch wünschenswert, dass im Rahmen der sekundären Prädikation die beiden involvierten Situationsargumente semantisch identifiziert werden. Er schreibt, dass "[i]f anything is to be identified it is the external argument of the verb and the individual argument of the depictive [...]" (Hinterhölzl 2001, 108). Würden die beiden Situationsargumente identiziert, so würde de facto nur über eine einzige Situation gesprochen.

Wie ich weiter unten erörtere, halte ich die von Hinterhölzl (2001) beschriebene temporale Restriktion der Sekundär- durch die Primärsituation für die Folge ihrer 'Relationierung' bzw. der Inbeziehungsetzung ihrer jeweiligen Referenzzeiten. Letztlich werden beide Situationen in eine unterspezifizierte Relation zueinander gesetzt, was bei sekundären Prädikaten, die auf einer Kopulasatzstruktur aufbauen, v. a. durch den imperfektiven Aspekt, andererseits aber auch durch den jeweiligen Kontext, üblicherweise als "Gleichzeitigkeit" interpretiert wird.

Was die Syntax betrifft, so wird die sekundäre Prädikation in der Fachliteratur oft als ein Small Clause-(SC)-Adjunkt analysiert: ${ }^{194}$

$$
\left[\mathrm{sC} \ldots\left[\mathrm{sC} \cdot \mathrm{F}^{0} \ldots\right]\right]^{195}
$$

${ }^{193}$ Vgl. Timberlakes $(1986,142)$ "temporalen Instrumental”, für den Gleiches gilt (er stellt eine der Ausdrucksformen für Sekundärprädikationen im Russischen dar).

${ }^{194}$ Diese Analyse geht auf Stowell (u. a. 1981, 1983) zurück, der sie für englische there-Sätze nutzt (Gegenargumente bringt u. a. Williams 1984). Bowers $(1993,591)$ diskutiert die Probleme der SC-Analyse im Kontext von Prädikationsstrukturen. Wilder (1994) unterscheidet zwei Typen von SC-Adjunkten: VP- und NP-Adjunkte. Erstere nennt er "sekundäre Prädikate", letztere "Prädikatsphrasen" (vgl. auch Dolińska 2012, 115); siehe Abschnitt 5.4. 
In solchen SCs wird mitunter die syntaktische Entsprechung einer semantischen Prädikationsbeziehung erblickt (vgl. Maienborn 2003a, 22). Wie ich oben in Abschnitt 5.1 dargestellt habe, besteht das semantische Wesen von "Prädikation" m. E. aus der (typisch verbalen) Sachverhaltsreferenz, was bedeutet, dass zu ihrer syntaktischen Repräsentation die Annahme eines V-Kopfes genügt, insofern diese in ihm angelegt ist. Das bedeutet, dass ich mich der Analyse von Bowers (1993) in Bezug auf die Annahme eines "stummen" prädikativen Kopfes zwar anschließe, dass ich jedoch nicht seine Ansicht teile, dass es eine spezifische funktionale Kategorie "Prädikation" $\left(\operatorname{Pr}^{0}\right)$ gebe. Eine solche ist meiner Ansicht nach überflüssig. Ich begnüge mich in Bezug auf die Basis sowohl der primären als auch der sekundären Prädikation mit der Annahme des mehrfach erwähnten leeren V-Kopfes bzw. einer 'einfachen' VP-Struktur: ${ }^{196}$

(54) $\left[\mathrm{vp} \ldots\left[\mathrm{v}, \mathrm{V}^{0} \ldots\right]\right]$

Zwischen der sekundären und der Matrixsituation besteht, wie bereits erwähnt, eine Relation, die in aller Regel auf "Gleichzeitigkeit" hinausläuft:

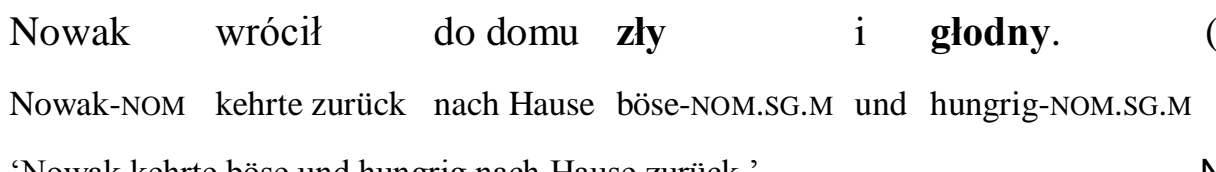

'Nowak kehrte böse und hungrig nach Hause zurück.'

NKJP

['Nowak kehrte nach Hause zurück, und währenddessen/dabei war er böse und hungrig.']

(56) Wraca do domu radosna.

(vgl. Chachulska 2008, 46)

kehrt zurück nach Hause froh-NOM.SG.F

'Froh kehrt sie nach Hause zurück.'

['Sie kehrt nach Hause zurück, und währenddessen/dabei ist sie froh.']

${ }^{195} \mathrm{~F}^{0}$ steht für einen beliebigen funktionalen Kopf, denn "All SMALL CLAUSES are projections of a functional head" (den Dikken 2006, 62). Dieses Zitat stammt aus den Dikkens Kritik an Moros (1997) 'symmetrischer' Analyse russischer Kopulasätze, in der 'kopflose' SCs auftreten.

${ }^{196}$ Im Prinzip sind Phrasen wie "Pr(ed)P" (Bowers 1993; Matushansky 2000; Markman 2008; Bailyn 2012; Bondaruk 2013b u. a.), "RelP" (den Dikken 2006), “ $\pi \mathrm{P} "$ (Citko 2008) und auch " $v$ P" (bei Geist 2006) nur Variationen der Annahme eines SC, dessen Kopf eine bestimmte funktionale Kategorie hat. Die Zugrundelegung einer 'simplen' VP erlaubt den generellen Verzicht auf solcherlei 'Spezialkategorien'. 
Diese Relation scheint nicht die einzige zu sein, die vorliegen kann. Dies gilt insbesondere, wenn man auch Fälle wie die folgenden als "sekundäre Prädikation" einstuft (siehe u. a. Hinterhölzl 2001; von ihm stammen auch die Übersetzungen):

(57) a. Golodnyj, on vernulsja domoj.

'When he was hungry, he returned home.'

b. On ženilsja na nej p'janyj.

'He married her (at time $\mathrm{t}$ ) and he was drunk (at $\mathrm{t})$.'
(Hinterhölzl 2001, 100)

"circumstantial reading"

"pure depictive reading"

Hinterhölzl (2001) spricht bei (57a) von zirkumstanzieller Lesart und bei (57b) von reiner depiktiver Lesart. Bemerkenswert ist, dass diese Lesarten im Russischen stets mit einer Kongruenzmarkierung am Adjektiv einhergehen, während der Instrumental eine andere, namentlich die temporal restringierte Lesart mit sich zu bringen scheint: ${ }^{197}$

(58) On ženilsja na nej molodym.

'When he married her, he was young.'
(Hinterhölzl 2001, 100) "temporally restricted reading"

Ich betrachte nur letzteren Fall (mit Instrumentalmarkierung) als Sekundärprädikation im engeren Sinne, also als Relationierung zweier Situationen bzw. derer Referenzzeiten. Aus letzterem folgt m. E. die 'temporale Restriktion', die Hinterhölzl (2001) ganz richtig als "Lesart" bezeichnet. Der Instrumental an den russischen Formen ist m. E. insofern hierauf zurückführbar, als der sekundären Prädikation jener "stumme" V-Kopf zugrunde liegt, der bereits weiter oben im Zusammenhang mit der primären Prädikation als Träger der Kopulabedeutung identifiziert wurde: Er ist gleichermaßen für die (eigenständige) Situations-

197 Sekundärprädikative russische Adjektive im Instrumental sind nicht auf die satzfinale Position beschränkt:

(i) Molodym on, verno, byl romantikom [...]

(Rus; NKRJ)

'Jung war er wohl ein Romantiker ...'

Hier liegt die zirkumstanzielle Lesart vor, vgl. die Paraphrase 'Als er jung war, war er wohl ein Romantiker'. Diese Lesart wird m. E. informationsstrukturell durch die Voranstellung des Sekundärprädikats erreicht. Es ist also wohl nicht der Fall, dass nur kasuskongruente Adjektive diese (und die rein-depiktive) Lesart haben können. Aber im Unterschied zu den Instrumentalformen referieren letztere bzw. deren Syntagmen auf keinen eigen(ständig)en Sachverhalt. 
referenz des Sekundärprädikats als auch für die Lizenzierung des Instrumentals am Prädikatsnomen verantwortlich. ${ }^{198}$

\subsubsection{Gerundiale Strukturen}

Růžička (1990) kommt in seiner Betrachtung russischer gerundialer (adverbialpartizipialer) Nebensatzstrukturen $\mathrm{zu}$ dem Schluss, dass diese von einer "stummen" Präposition eingeleitet werden, die eine semantische Relation der Gleich- bzw. der Vorzeitigkeit zwischen primärer und sekundärer Situation herstellt.

Gleichsinnig nehmen u. a. auch Steube (1987), Junghanns (1994) und Zimmermann $(1999,2003 b, 2009)$ an, dass verschiedene Typen von Adverbialsätzen von PPn eingeleitet werden, die jeweils eine - ggf. unterspezifizierte - Relation zur Matrix etablieren. Die PP-Analyse sollte demnach auf gerundiale Nebensatzstrukturen wie die in (59) und (60) anwendbar sein:

Będąc sześćdziesięcioletnim emerytem jeszcze pracował (Pol) seiend-GER sechzigjähriger Rentner-INS noch arbeitete-LPT.SG.M

'Als sechzigjähriger Rentner arbeitete er noch.' (http://www.pons.eu) [oder: 'Obwohl er ein sechzigjähriger Rentner ist, ...'] [wörtlich: 'Ein sechzigjähriger Rentner seiend, ...']

(60) Buduči ne tol'ko izvestnym istorikom, Morison obraščaet (Rus) seiend-GER NEG nur berühmter Historiker-INS Morisson-NOM wendet-3SG vnimanie na finansy. NKRJ [gekürzt] Aufmerksamkeit-ACC auf Finanzen-ACC 'Indem/da/weil er nicht nur ein berühmter Historiker ist, richtet Morisson seine Aufmerksamkeit auf die Finanzen.' [wörtlich: 'Nicht nur ein berühmter Historiker seiend, ...']

Für eine un(ter)spezifizierte Relation sprechen die diversen deutschen Übersetzungsoptionen. Folgt man dagegen Růžička (1990, 370), so basieren diese Les-

${ }^{198}$ Für die Fälle in (57a/b), wo die Adjektive Kongruenzkasus zeigen, nehme ich mit Wilder (1994) bzw. Bailyn (2012) an, dass "Prädikatsphrasen" bzw. "appositive Adjunkte" vorliegen. Demnach sind diese Adjektive attributive NP-Adjunkte (ohne eigenen Situationsbezug); siehe Abschnitt 5.4. 
arten auf den zugrunde liegenden, spezifizierten temporalen Relationen der Gleich- bzw. Vorzeitigkeit. Dieser Ansicht widerspreche ich und folge vielmehr Zimmermann (1993, 2003b), die von einer un(ter)spezifizierten (adverbialen) Relation ausgeht, die erst im Kontext bzw. auf konzeptueller Ebene eine bestimmte Interpretation erhält.

Ehe ich darauf eingehe, wende ich mich jedoch der syntaktischen Analyse des o. g. polnischen Gerundialsatzes zu:

(61) $\left[{ }_{\mathrm{PP}} \varnothing_{\mathrm{P}}\left[\mathrm{CP} \varnothing_{\mathrm{C}}\left[\mathrm{vP}\right.\right.\right.$ będąc $_{\mathrm{V}}\left[\mathrm{VP} \varnothing_{\mathrm{V}}\left[\mathrm{NP} \ldots\right.\right.$ emerytem en $\left.\left.\left.\left._{\mathrm{N}}\right]\right]\right]\right]$

Die leere Präposition 'beinhaltet' die erwähnte un(ter)spezifizierte Relation. Der leere C-Kopf stellt eine bloße identische Abbildung dar. Für Růžička (1990) hat das Gerundium als Satzprädikat seinen Ursprung im 'untersten' $\mathrm{V}^{0}$ und bewegt sich letztlich in die leere C-Position, um "unmittelbar unter die Rektion der Präposition" (Růžička 1990, 365) zu kommen. Da der C-Kopf offenbar weder Lautform noch Bedeutung hat, halte ich seine Annahme jedoch für verzichtbar. Da ich będac - wie alle Flexionsformen von być - als bloßes V-Auxiliar betrachte, befindet es sich auch ohne Annahme des C-Kopfes (und ohne syntaktische Bewegung dorthin) in einer satzinitialen Oberflächenposition, zumal in dieser infiniten Struktur - anders als etwa bei Růžička (1990, 366) - m. E. keine IP vorliegt. Im Sinne dieser Erwägungen 'reduziere' ich die Struktur auf (62):

(62) $\left[\mathrm{PP} \varnothing_{\mathrm{P}}\left[\mathrm{vP}\right.\right.$ będąc $_{\mathrm{V}}\left[\mathrm{vP} \varnothing_{\mathrm{V}}\left[\mathrm{NP} \ldots\right.\right.$ emerytem $\left.\left.\left.\left._{\mathrm{N}}\right]\right]\right]\right]$

Wie bereits bekannt, setze ich die Kopulabedeutung in $\varnothing_{\mathrm{V}}$ an. Dieser Kopf nimmt das prädikative Substantiv als Komplement und lizenziert den Instrumental. Die gleiche Struktur lässt sich auch auf das russische Beispiel übertragen:

(63) $\left[\mathrm{PP} \varnothing_{\mathrm{P}}\left[\mathrm{vP}\right.\right.$ budučiv $_{\mathrm{V}} \varnothing_{\mathrm{V}}\left[\mathrm{NP} \ldots\right.$ istorikom $\left.\left.\left.{ }_{\mathrm{N}}\right]\right]\right]$

Die Nullpräposition in den letzten beiden Beispielen entspricht der erwähnten un(ter)spezifizierten semantischen Relation $(\underline{\mathbf{R}}){ }^{199}$

199 Vgl. Hinterhölzl $(2001,101)$, der von einem "unselective temporal operator" spricht. Vgl. ferner Zimmermann (2003b, 637), die innerhalb eines semantischen Templates zur Anpassung 
(64) a. [ [PP $\varnothing_{\mathrm{P}}\left[\mathrm{vP}\right.$ będąc $\mathrm{c}_{\mathrm{V}}\left[\mathrm{vp} \varnothing_{\mathrm{V}}[\mathrm{NP} \ldots\right.$ emerytem $\left.\left.\left.\mathrm{N}]\right]\right]\right]$

b. [PP $\varnothing_{\mathrm{P}}\left[\mathrm{vP}\right.$ budučiv [vp $\varnothing_{\mathrm{V}}\left[\mathrm{NP} \ldots\right.$ istorikom $\left.\left.\left.\left.{ }_{\mathrm{N}}\right]\right]\right]\right]$

$\underline{\mathbf{R}}$

Beachte, dass nicht nur die Gerundialformen będąc/buduči, sondern ebenso auch bywszy/byvši auftreten können. Bei letzteren handelt es sich formal um Formen des "Adverbialpartizips der Vorzeitigkeit". Was sie betrifft, so können exakt dieselben Annahmen wie für będąclbuduči, also die formalen "Adverbialpartizipien der Gleichzeitigkeit”, gemacht werden:

(65) a. [Pp $\varnothing_{\mathrm{P}}\left[\mathrm{vp}\right.$ bywszyv $\left[\mathrm{vP} \varnothing_{\mathrm{V}}[\mathrm{NP} \ldots\right.$ emerytem $\left.\left.\left.\mathrm{N}]\right]\right]\right]$

b. $\quad\left[\mathrm{pP} \varnothing_{\mathrm{P}}\left[\mathrm{vP}\right.\right.$ byvšii $\left[\mathrm{vP} \varnothing_{\mathrm{V}}\left[\mathrm{NP} \ldots\right.\right.$ istorikom $\left.\left.\left.\left._{\mathrm{N}}\right]\right]\right]\right]$

\section{$\underline{\mathbf{R}}$}

Das liegt daran, dass der Unterschied zwischen den "Adverbialpartizipien der Gleichzeitigkeit" auf - $a c /-a$ und den "Adverbialpartizipien der Vorzeitigkeit" auf $-w s z y /-v(\check{s} i)$ heute ein rein morphologischer Unterschied ist (vgl. Isačenko 1962, 325). ${ }^{200}$ Semantisch-interpretativ entscheidet (heute) allein ihr (semantischer) Aspekt. Es kann die einfache Regel als gültig betrachtet werden, dass imperfektive Gerundialformen stets die Relation der Gleichzeitigkeit, perfektive Gerundialformen hingegen stets die Relation der Vorzeitigkeit ausdrücken. Hierzu Beispiele mit den Gerundialformen des russischen Verblexems čitat'/pročitat' 'lesen', wobei ich die relevanten Bedeutungen/Lesarten auf den Ebenen SF und CS angebe:

(66) a. [pP $\varnothing_{\mathrm{P}}[\mathrm{vp}\{$ čitaj-a/čita-vši $\}$ knigu $\left.]\right]$

(imperfektiv)

$\underline{\mathbf{R}} \quad\|\mathrm{IMPF}\|$

"Gleichzeitigkeit"

dt. Partizipien II an den Gebrauch als adverbiale Modifikatoren eine unterspezifizierte Adverbialrelation $\left(\underline{\mathrm{R}}_{\mathrm{adv}}\right)$ ansetzt, die ebenfalls erst konzeptuell (auf CS) spezifiziert wird.

${ }^{200}$ Gemeint sind semantisch-interpretative Unterschiede. Differenzen zwischen den beiden morphologischen Formen bestehen durchaus hinsichtlich Bildungsmöglichkeiten und usuellem Gebrauch (siehe Isačenko 1962, 324-333). So sind nicht von allen Lexemen jeweils beide Gerundialformen bildbar. Ferner besteht eine 'ordnende' Tendenz, die Formen auf - $a c /-a$ primär von imperfektiven, die auf -wszy/-vši dagegen primär von perfektiven Verben zu bilden. 
b. $\quad\left[\mathrm{PP} \varnothing_{\mathrm{P}}[\mathrm{vP}\{\right.$ pročtj-a/pročita-vši $\}$ knigu $\left.]\right]$

(perfektiv)

R $\quad \|$ PERF $\|$

"Vorzeitigkeit"

Dass diese beiden Lesarten bei byćlbyt' 'sein' (bzw. bei der von dessen Formen reflektierten Kopulabedeutung) nicht vorliegen können, ist nicht verwunderlich, sofern man beachtet, dass es sich um ein Imperfectivum tantum (Geist 2006, 169171) handelt. Der o. g. Regel folgend, kommt es für die Vorzeitigkeitslesart daher nicht in Frage. Möglich ist nur die Lesart der "Gleichzeitigkeit": ${ }^{201}$

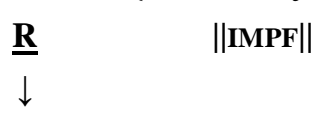

"Gleichzeitigkeit"

(nur imperfektiv)

Als intermediäre Zusammenfassung kann man also festhalten, dass gerundialen Nebensatzstrukturen auf grammatischer Ebene (SF) eine un(ter)spezifizierte Relation in Form einer "stummen" Präposition zugrunde liegt, die erst auf konzeptueller Ebene (CS) spezifiziert wird. Entscheidend für ihre Lesart ist dabei der semantische Aspekt der Gerundialform, deren morphologische Markierung als “Adverbialpartizip der Gleichzeitigkeit” bzw. als "Adverbialpartizip der Vorzeitigkeit" heute sekundär ist. Liegt imperfektiver Aspekt vor, so ergibt sich die Lesart der "Gleichzeitigkeit", liegt dagegen perfektiver Aspekt vor, erhält man "Vorzeitigkeit". Dies gilt für alle Verblexeme, die über zwei semantische Aspekte verfügen. ${ }^{202}$ Im Falle der Kopulabedeutung, die durch die morphologischen Ge-

${ }^{201}$ Es ist offensichtlich, dass die russ. Gerundialform buduči (eine ursprünglich echt-ostslavische Bildung!) auf keiner produktiven Ableitung (mehr) beruht, sondern als 'unregelmäßige' Form im Lexikon abgelegt ist. Die polnische Entsprechung będac dagegen kann als regelhafte Ableitung vom Stamm będ-gelten, wenngleich auch diese Bildung insofern idiosynkratische Züge trägt, als das "Adverbialpartizip der Gleichzeitigkeit" gewöhnlich vom Präsensstamm abgeleitet wird, der bei być (je)s- lautet; vgl. (ja) jestem, (ty) jesteś, ... (oni) sq. Zu erwarten wäre theoretisch die Form *sac (vgl. tschech. jsouc).

202 Die Annahme von Paslawska \& Stechow (2013a, 307), man müsse von drei anstelle von nur zwei semantischen Aspekten im Russischen ausgehen, lässt sich mit dieser Aussage vereinbaren, da die beiden semantischen Aspekte PERFEKTIV und PERFEKT, die den Autor/inn/en zu- 
rundialformen będąclbywszy bzw. budučilbyvši ausbuchstabiert werden kann, kommt dagegen nur die Lesart der "Geichzeitigkeit" in Frage, da es sich um ein Imperfectivum tantum handelt.

\subsubsection{Strukturen ohne Gerundien}

Im Vergleich zu den Gerundialstrukturen erscheinen jene Strukturen, die hier als Grundlage der eigentlichen Sekundärprädikation betrachtet werden, wie 'reduzierte Varianten'. Da die Gerundialformen von byćlbyt' in ihnen nicht auftreten, sind sie zum einen zwar 'weniger explizit' als erstere, dafür aber auch schlichtweg 'kürzer'. In Analogie zu den Gerundialstrukturen gehe ich auch bei ihnen von einer "stummen" Präposition aus, die eine un(ter)spezifizierte semantische Relation zur Matrix herstellt. Abgesehen von der Abwesenheit der Adverbialpartizipien (V-Auxiliare) entsprechen sich folglich die syntaktischen Strukturen von Gerundialsätzen und Sekundärprädikationen:

(68) $\left[\mathrm{PP} \varnothing_{\mathrm{P}}\left[\mathrm{VP} \varnothing_{\mathrm{V}}\left[\{\mathrm{A} / \mathrm{N}\} \mathrm{P}\{\mathrm{A} / \mathrm{N}\}^{0}\right]\right]\right]$

(Pol/Rus)

\section{$\underline{\mathbf{R}}$}

Anders als bei Gerundialsätzen selegiert in diesem Fall die Nullpräposition kein auxiliares Adverbialpartizip, sondern unmittelbar den ebenfalls infiniten leeren V-Kopf, der die Kopulabedeutung trägt. Für diese 'reduzierte' Variante nenne ich zunächst russische Beispiele, in denen das Prädikatsnomen regelmäßig im Instrumental erscheint (die kasuskongruenten Adjektive im Russischen werden in Abschnitt 5.4 behandelt):

(69) On vernulsja domoj p'janym.

er-NOM kehrte zurück nach Hause betrunken-INS.SG.M

'Er kehrte betrunken nach Hause zurück.'

folge gleichermaßen hinter dem morphologischen perfektiven Aspekt stecken können, auf CS ebenso gleichermaßen die Lesart der "Vorzeitigkeit" herbeiführen würden. 
(70) On vernulsja domoj oficerom.

er-NOM kehrte zurück nach Hause Offizier-INS.SG

'Er kehrte als Offizier nach Hause zurück.'

Die Syntax dieser Beispiele ist nach den obigen Annahmen die in (71):

(71) a. [ $\left.{ }_{\mathrm{PP}} \varnothing_{\mathrm{P}}\left[\mathrm{VP} \varnothing_{\mathrm{V}}\left[\mathrm{AP}_{\mathrm{AP}} \mathrm{p}^{\prime} \mathrm{janym}_{\mathrm{A}}\right]\right]\right]$

b. $\quad\left[{ }_{\mathrm{PP}} \varnothing_{\mathrm{P}}\left[\mathrm{VP} \varnothing_{\mathrm{V}}[\mathrm{NP}\right.\right.$ oficerom $\left.\left.\mathrm{N}]\right]\right]$

Auf die semantische Komposition dieser Strukturen gehe ich weiter unten ein.

Im Polnischen ist die Lage etwas anders als im Russischen, da sekundärprädikativ verwendete Adjektive heute kasuskongruent auftreten (vgl. Chachulska 2008, 46). Ihre instrumentalische Markierung ist fast völlig ausgeschlossen. ${ }^{203}$

(72) Wraca do domu radosna (*radosną).

kehrt zurück nach Hause froh-NOM.SG.F froh-INS.SG.F

'Froh kehrt sie nach Hause zurück.'

(Chachulska 2008, 46)

(73) Nowak wrócił do domu zly i głodny.

Nowak-NOM kehrte zurück nach Hause böse-NOM.SG.M und hungrig-NOM.SG.M

'Nowak kehrte böse und hungrig nach Hause zurück.'

NKJP

Sekundärprädikativ verwendete NPn können, wenn dies auch veraltet wirkt (Chachulska 2008, 45-48), ebenfalls in dieser Struktur auftreten. Wie im Russischen, zeigen sie dann stets den Instrumental:

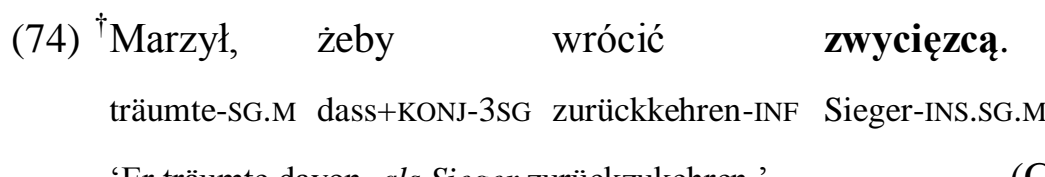
'Er träumte davon, als Sieger zurückzukehren.'

Die relevanten Beispiele haben die bereits bekannte Syntax in (75):

${ }^{203}$ Im Instrumental können sie nurmehr mit den gerundialen und partizipialen Formen von być auftreten, vgl. u. a. Bogusławski (2001, 112-113). 
(75) a. [ $\left[{ }_{\mathrm{PP}} \varnothing_{\mathrm{P}}\left[\mathrm{VP} \varnothing_{\mathrm{V}}\left[{ }_{\mathrm{AP}} \operatorname{radosna}_{\mathrm{A}}\right]\right]\right]$

b. $\left[\mathrm{PP} \varnothing_{\mathrm{P}}\left[\mathrm{VP} \varnothing_{\mathrm{V}}[\mathrm{NP}\right.\right.$ Zwycięzcą $\left.\mathrm{N}]\right]$

Der Instrumental an polnischen NPn in der sekundären Prädikation ist heute, wie gesagt, 'veraltet'. Stattdessen treten NPn üblicherweise als Komplemente von jako 'als; wie' auf:

(76) [Ale pojechał do Moskwy na parę tygodni] i wrócił aber fuhr nach Moskau auf ein paar Wochen und kehrte zurück

\section{jako fanatyczny trockista.}

als fanatischer Trotzkist-NOM.SG.M

NKJP

'[...] und kehrte als fanatischer Trotzkist zurück.'

Diese 'analytische' Ausdrucksform wird mitunter auch im Russischen verwendet:

$$
\text { [...] a vernulsja kak lakej. }
$$

aber kehrte zurück als Lakai-NOM.SG

'... aber [er] kehrte als Lakai zurück.'

Auch für diese Form der sekundären Prädikation nehme ich die bereits dargestellte syntaktische Struktur an, gehe aber von einer "syntaktischen Kompression" der involvierten Köpfe $\mathrm{P}^{0}$ und $\mathrm{V}^{0}$ zu einer Art "Präpositionalverb" jako/kak aus (folglich ein Kopf mit kombinierter Semantik aus un(ter)spezifizierter Relation und Kopulabedeutung). Dieser "Kompression" lege ich Haiders (1988) Konzept der Matching Projections zugrunde: ${ }^{204}$

(78) a. $\left[{ }_{\mathrm{PP}} \mathrm{jako}_{\mathrm{P}}\left[\mathrm{VP} \varnothing_{\mathrm{V}}\left[\mathrm{NP}_{\mathrm{NP}}\right.\right.\right.$ trockista $\left.\left.\left._{\mathrm{N}}\right]\right]\right] \rightarrow\left[\mathrm{P} / \mathrm{VP}\right.$ jakop/v $\left[\mathrm{NP}\right.$ trockista $\left.\left._{\mathrm{N}}\right]\right]$

b. $\quad\left[\mathrm{PP} \mathrm{kak}_{\mathrm{P}}\left[\mathrm{VP} \varnothing_{\mathrm{V}}\left[\mathrm{NP}_{\mathrm{NP}}\right.\right.\right.$ lakej $\left.\left.\left._{\mathrm{N}}\right]\right]\right] \rightarrow\left[\mathrm{P} / \mathrm{VP} \operatorname{kak}_{\mathrm{P} / \mathrm{V}}[\mathrm{NP}\right.$ lakejN $\left.]\right]$

\footnotetext{
${ }^{204}$ Haiders (1988) Matching-Konzept sieht vor, kategorial ähnliche Projektionen zusammenzuführen, sofern leere Köpfe dazwischen vorliegen. Man kann daher hier die Kritik anbringen, dass $\mathrm{P}$ nicht mit V kompatibel sei. Sofern man aber eine grundlegende Parallelelität zwischen $\mathrm{C}$ und $\mathrm{P}$ ansetzt (vgl. Emonds 1985), kann diese Kritik relativiert werden, insofern eine 'Einheitskategorie' P/C (im Kern relatorisch) ebenso mit V wie mit $\mathrm{N}$ kompatibel wäre.
} 
Am Ende dieses Abschnitts werde ich auf jako/kak zurückkommen. Zunächst aber gilt es, die Komponenten der 'unge-match-ten' sekundärprädikativen Struktur semantisch zu erfassen. Ich berücksichtige dabei die 'reduzierten' Strukturen ohne Gerundialformen und setze die o. g. Annahmen betreffs der Interaktion von semantischem Aspekt und un(ter)sezifizierter Relation in $\varnothing_{\mathrm{P}}$ voraus. Aus diesen Annahmen geht hinsichtlich der 'reduzierten' Varianten klar hervor, dass diese die Lesart der "Gleichzeitigkeit" hervorrufen, da die Kopulabedeutung in $\varnothing_{\mathrm{V}}$ den semantischen imperfektiven Aspekt hat:

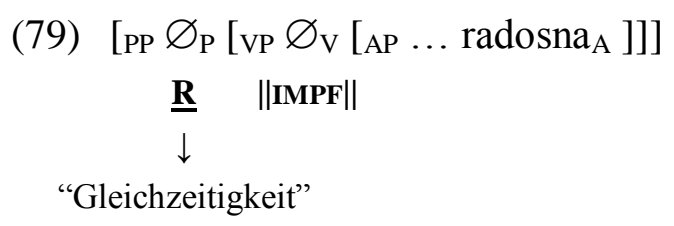

Bei der Darstellung der semantischen Komposition gehe ich bottom-up vor, beginne also mit dem Prädikatsnomen. Als Beispiel dient das obige polnische Sekundärprädikat. Die AS und PAS des Prädikatsnomens radosna - und damit auch die der prädikativen AP - sehen wie folgt aus (vgl. Abschnitt 4.4.3):

(80) $\lambda x[x$ HAPPY $]$ [AP $\left.\operatorname{radosna}_{\mathrm{A}}\right]$

Die AP ist das Komplement des leeren V-Kopfes, der die Kopulabedeutung trägt. Dessen Lexikoneintrag ist bereits aus Abschnitt 5.1 bekannt:

$$
\mid \varnothing / \quad[+\mathrm{V},+\mathrm{N},- \text { Perf, }+ \text { max }] \quad \lambda P \lambda x \lambda t \exists s[[t \subseteq \tau(s)]: s \text { INST }[P x]]
$$

Die Einsetzung der AP als Komplement dieses Kopfes führt zur Projektion einer VP mit der Bedeutungscharakterisierung in (82):

$$
\lambda x \lambda t \exists s[[t \subseteq \tau(s)]: s \text { INST }[x \operatorname{HAPPY}]] \quad \quad\left[\mathrm{VP} \varnothing_{\mathrm{V}}\left[\mathrm{AP}_{\operatorname{radosna}}\right]\right]
$$

Damit ist Sachverhaltsreferenz und eine (infinite) Verbform im Satz gegeben. Diese VP könnte auch Grundlage primärer Prädikation sein. Im Falle der interessierenden sekundären Prädikation aber ist sie Komplement der schon erwähnten Nullpräposition, die eine un(ter)spezifizierte Relation zwischen der Referenz- 
zeit des Sekundärprädikats $(t)$ und der Referenzzeit des Primärprädikats $\left(t^{\prime}\right)$ etabliert. Im Rahmen dessen wird die Referenzzeit des Sekundärprädikats existenziell gebunden $(\exists t)$ sowie eine Argumentstelle für die Referenzzeit des zu modifizierenden Primärprädikats eingeführt $\left(\lambda t^{\prime}\right)$. Ferner wird das externe Argument der o. g. VP 'ausgeblendet', also durch die "Dummyvariable" $z$ ersetzt (siehe Abschnitt 4.3). Diese Annahmen gehen in Lexikoneintrag (83) für $\varnothing_{P}$ auf: 205

$$
\mid \varnothing / \quad[+\mathrm{P}] \quad \lambda P_{[+\mathrm{V},+\mathrm{N}]} \lambda t^{\prime} \exists t\left[\left[t^{\prime} \underline{\mathrm{R}} t\right] \wedge[P z t]\right]
$$

Setzt man die obige VP als Komplement dieser Nullpräposition ein, erhält man als Bedeutung der gesamten PP (84): ${ }^{206}$

$$
\lambda t^{\prime} \exists t \exists \mathrm{s}\left[\left[t^{\prime} \underline{\mathrm{R}} t\right] \wedge[[t \subseteq \tau(s)]: s \text { INST }[z \mathrm{HAPPY}]]\right] \quad\left[{ }_{\mathrm{PP}} \varnothing_{\mathrm{P}}\left[\mathrm{VP} \varnothing_{\mathrm{V}}\left[{ }_{\mathrm{AP}} \operatorname{radosna}_{\mathrm{A}}\right]\right]\right]
$$

Um diese PP als syntaktisches Adjunkt zur Modifikation einer Matrix-VP verwenden zu können, ist zuvor ihre Anpassung an den attributiven Gebrauch erforderlich. Hierzu greife ich auf die semantische Regel Predicate Modification von Heim \& Kratzer $(1998,65)$ zurück, auf deren Basis Prädikate eines bestimmten logischen Typs als Modifikatoren anderer Prädikate desselben Typs eingesetzt werden können. In unserem Fall ist die PP ein einstelliges Prädikat vom Typ $\langle e, t>$. Ebendiesen Typ hat die zu modifizierende VP (ihr einziges $\lambda$-Argument ist ihre Referenzzeit). Folglich ist Predicate Modification anwendbar. Das Ergebnis sieht wie in (85) aus und ist vom 'Modifikator-Typ' $<<\mathrm{e}, \mathrm{t}>,<\mathrm{e}, \mathrm{t}>>$ : $^{207}$

${ }^{205}$ Vgl. hierzu Zimmermanns (2003b, 637) semantisches Template zur Anpassung deutscher Partizipien II an den Gebrauch als adverbiale Modifikatoren.

206 Den Erwägungen zu den Präpositionen jako/kak vorausgreifend, sei bemerkt, dass auch im Falle der gerade behandelten PP-Strukturen ein Matching im Sinne Haiders (1988) denkbar ist:

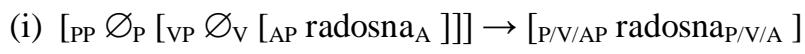

Was bei Haider (1988) durch Matching der syntaktischen Projektionen passiert, wird bei Zimmermann (1993b) durch sukzessiv applizierende semantische Templates bewerkstelligt, die die Anreicherung der SF um die Kopulabedeutung, die Etablierung einer 'offenen' Relation sowie den Kategorienwechsel zur Präposition bewirken. Ich sehe in der Annahme dieser verschiedenen Mechanismen, die effektiv dasselbe bewirken, sich aber hinsichtlich der Domäne unterscheiden, in der sie statthaben, Evidenz für die Realität solcher "Kompressionen".

207 Schon Higginbotham $(1985,568)$ kommt zu Schlussfolgerungen, die auf Predicate Modification hinauslaufen (vgl. Růžička 1990, 367). Es sei bemerkt, dass zur Anpassung an den Gebrauch als Modifikator statt dieser Regel auch die Annahme einer spezifischen funktionalen Modifikationskategorie ("Mod"; vgl. Rubin 1994, Bailyn 1994) oder eines semantischen 


$$
\lambda Q \lambda t^{\prime} \exists t \exists \mathrm{s}\left[\left[Q t^{\prime}\right] \wedge\left[\left[t^{\prime} \underline{\mathrm{R}} t\right] \wedge[[t \subseteq \tau(s)]: s \text { INST }[z \mathrm{HAPPY}]]\right]\right]
$$

Die 'entstandene' Argumentstelle $\lambda Q$ bildet den "Andockpunkt" für die MatrixVP bzw. für deren Bedeutung. Entsprechend wird ihre Referenzzeit durch die adjungierte PP modifiziert. Sehen wir uns das relevante Beispiel nochmals an:

(86) Wraca do domu radosna.

'Als sie nach Hause zurückkehrt, ist sie froh.'

(vgl. Hinterhölzl 2001, 101)

Die parametrische Relation zwischen Sekundär- und Matrixprädikation wird als "Gleichzeitigkeit" interpretiert: Der Referent ist froh zu der Zeit, als er nach Hause kommt. Beachte, dass die Voranstellung des sekundären Prädikats die Lesart in Richtung zirkumstanziell (vgl. Hinterhölzls 2001, 101) verschiebt:

(87) Radosna wraca do domu $[t]$.

'Als sie froh ist, kehrt sie nach Hause zurück.'

(vgl. Hinterhölzl 2001, 101)

In diesem Sinne kann die Stellung des Sekundärprädikats relativ zum Rest des Satzes als einer der Faktoren für das Zustandekommen der Lesart der an sich (auf grammatischer Ebene) un(ter)spezifizierten Relation betrachtet werden. ${ }^{208}$

Die koreferente Lesart zwischen dem Subjektreferenten der VP wraca do doти und dem externen Argument des Sekundärprädikats radosna folgt daraus, dass der erstere die "Dummyvariable" $z$, die in Folge der 'Ausblendung' durch $\varnothing_{\mathrm{P}} \mathrm{zu}$ stande gekommen ist und die den Referenten des Sekundärprädikats vertritt, "semantisch kontrolliert". ${ }^{209}$ Diese "semantische Kontrolle" könnte auch von einem

MOD-Templates (Zimmermann 1992, 264) in Frage kommen. Die Wahl unter diesen Mitteln betrifft primär die Domäne, in der die Anpassung erfolgt.

208 Růžička (1990, 370) äußert sich in vergleichbarer Weise zu den variierenden Positionen russischer Gerundialstrukturen. Auch lässt sich auf diese Weise die Korrelierung von Lesart und syntaktischer Domäne bei Hinterhölzl $(2001,101)$ verstehen. Ferner zeigt sich, dass letztere zu einem nicht unbedeutenden Teil informationsstrukturell basiert ist.

209 "Ich nehme mit Haspelmath (1995) an, daß Kontrolle von verschiedenen Faktoren abhängt, die nicht durch syntaktische oder semantische Unifizierungsmechanismen für die Interrelierung von Kontrolleur und Kontrolliertem erfaßbar sind [Fn.]. Mein Vorschlag benötigt auch keine syntaktische Konstituente für ein PRO.” (Zimmermann 1999, 139). 
beliebigen anderen Partizipanten der Matrixsituation ausgeübt werden (vgl. Strigin 2008, 391), was sich im Polnischen formal an der Kongruenz der Adjektivform zeigt. Dadurch sind die referenziellen Verhältnisse bei der polnischen adjektivischen Sekundärprädikation in aller Regel eindeutig ablesbar:

(88) Nigdy nie widziałem go smutnego. NKJP niemals NEG seh-LPT-SG.M+PRÄT-1.SG ihn-ACC traurig-ACC.SG.M ' $\mathrm{Ich}_{\mathrm{i}} \mathrm{sah} \mathrm{ihn}_{\mathrm{j}}$ niemals traurig $_{* \mathrm{i} / \mathrm{j}}$.'

In (88) kontrolliert das direkte Objekt der Matrix das 'ausgeblendete' externe Argument des Sekundärprädikats semantisch, was an der Satzoberfläche seinen Ausdruck in der Kongruenzmarkierung am Adjektiv findet. Im Russischen dagegen erscheint das Adjektiv - sofern nicht eine "Prädikatsphrase" vorliegt (siehe folgender Abschnitt) - im Instrumental, was ohne Kontext zu Ambiguität führen muss, da das 'ausgeblendete' Argument des sekundären Prädikats als koreferent (a) mit dem Matrixsubjekt oder (b) mit dem Matrixobjekt 'gelesen' werden kann:

$$
\begin{aligned}
& {[\ldots] \text { ja nikogda ne videl } \quad \text { ego grustnym }[\ldots]} \\
& \text { ich niemals NEG seh-LPT-SG.M ihn-ACC traurig-ACC.SG.M } \\
& \text { '... } \text { ich }_{\mathrm{i}} \mathrm{sah} \mathrm{ihn}_{\mathrm{j}} \text { niemals } \text { traurig }_{\mathrm{i} / \mathrm{j}} \ldots
\end{aligned}
$$

Die Bedeutungscharakterisierung für die sekundärprädikative PP mit dem Adjektivkopf grustnym lautet nach den obigen Annahmen wie in (90):

$$
\lambda Q \lambda t^{\prime} \exists t \exists \mathrm{s}\left[\left[Q t^{\prime}\right] \wedge\left[\left[t^{\prime} \underline{\mathrm{R}} t\right] \wedge[[t \subseteq \tau(s)]: s \text { INST }[z \mathrm{SAD}]]\right]\right]
$$

Der semantische Parameter $z$ (Träger der Eigenschaft 'traurig') kann entweder vom AGENS- oder vom THEMA-Partizipanten des Matrixsachverhalts kontrolliert werden. Die Annahme von PRO in der Syntax ist unnötig, da die Kontrolle semantisch erfolgt:

(91) $\ldots\left[\right.$ [vp [vp ja videl $l_{\mathrm{V}}$ ego $]\left[{ }_{\mathrm{PP}} \varnothing_{\mathrm{P}}\left[\mathrm{vP} \varnothing_{\mathrm{V}}\left[\mathrm{AP}\right.\right.\right.$ grustnym $\left.\left.\left.\left._{\mathrm{A}}\right]\right]\right]\right]$

Schließlich möchte ich, wie angekündigt, genauer auf die Präpositionalfügungen aus jako/kak und prädikativen Substantiven eingehen, die sowohl im Polnischen 
als auch im Russischen zum Ausdruck sekundärer Prädikation Verwendung finden, wobei sie für Substantive im Polnischen heute den Regelfall darstellen (vgl. Chachulska 2008):

(92) [...] i wrócił jako fanatyczny trockista. und kehrte zurück als fanatischer Trotzkist-NOM.SG.M

'... und [er] kehrte als fanatischer Trotzkist zurück.'

(93) $[\ldots]$ a vernulsja kak lakej.

aber kehrte zurück als Lakai-NOM.SG

'... aber [er] kehrte als Lakai zurück.'

\subsubsection{Strukturen mit jako und kak}

Wie bereits weiter oben beschrieben, scheint mir auch für diese - oberflächlich durchaus andersartig erscheinende - Struktur der sekundären Prädikation grundlegend dasselbe zu gelten, was bis hierher erörtert worden ist. Meiner Ansicht nach leisten jako bzw. kak dasselbe wie die Komponenten der PP-Strukturen weiter oben, indem sie einerseits eine un(ter)spezifizierte Relation zur Matrix etablieren und andererseits auch die Kopulabedeutung mit ihrem Sachverhaltsbezug enthalten. Wie ich ebenfalls bereits angedeutet habe, lege ich dem eine Form von "syntaktischer Kompression" zugrunde, die man mit dem Konzept der Matching Projections nach Haider (1988) erfassen kann:

(94) a. $\left[{ }_{\mathrm{PP}} \mathrm{jako}_{\mathrm{P}}\left[\mathrm{vP} \varnothing_{\mathrm{V}}\left[\mathrm{NP}_{\mathrm{NP}}\right.\right.\right.$ trockista $\left.\left.\left._{\mathrm{N}}\right]\right]\right] \rightarrow\left[\mathrm{PP}\right.$ jakop $\left.\left[\mathrm{NP}_{\text {trockista }}\right]\right]$

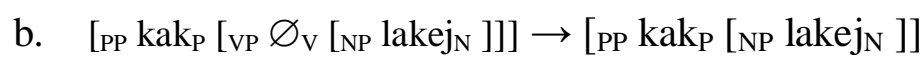

Die involvierten syntaktischen Projektionen werden also auf das Mindeste reduziert. Man kann auch sagen, dass der leere V-Kopf mit der Kopulabedeutung in den P-Kopf "hineinrutscht". Als Metapher mag eine Teleskopstange dienen, die je nach Bedarf aus- oder eingefahren werden kann. ${ }^{210}$ Das bedeutet, dass die overte Präposition jako/kak an sich denselben Lexikoneintrag wie die oben angesetzte Nullpräposition hat, dass aber in Folge des syntaktischen Matching der Projek-

${ }^{210}$ Ganz ähnlich ist das Syntaxmodell mit aufgespaltenem I(nfl)-Kopf nach Pollock (1989). 
tionen die Kopulabedeutung in sie eingeht respektive in ihr aufgeht. Der o. g. Lexikoneintrag für die Nullpräposition sollte demnach in einen Eintrag für eine präpositionale Einheit umgewandelt werden, die entweder "stumm" oder overt sein kann. In (95) nenne ich den angepassten Eintrag für poln. jako:

$$
/\{\varnothing / \text { jako }\} / \quad[+\mathrm{P}] \quad \lambda P_{[+\mathrm{V},+\mathrm{N}]} \lambda t^{\prime} \exists t\left[\left[t^{\prime} \underline{\mathrm{R}} t\right] \wedge[P z t]\right]
$$

Die overte Variante kommt jedoch nur mit prädikativen Substantiven in Frage, was ganz den üblichen Selektionseigenschaften von Präpositionen entspricht.

Als Evidenz für diese Hypothese können m. E. Daten wie der folgende Satz dienen, die zeigen, dass jako in Kombination mit der Kopulabedeutung auftreten kann:

(96) Naucz się języka niemieckiego i poznaj niemiecką kulturę jako będąc gościem jednej z sympatycznych rodzin mieszkających we Frankfurcie.

'Lerne die deutsche Sprache und lerne die deutsche Kultur kennen, indem du Gast einer der sympathischen Frankfurter Familien bist.’ (http://www.frankfurt-invites-friends.de/polska)

Dass jako hier gemeinsam mit dem gerundialen V-Auxiliar będąc auftritt, weist ganz deutlich darauf hin, dass in diesem Falle kein Matching erfolgt ist. Man kann hier Struktur (97) ansetzen, die voll analog zu den o. g. Strukturen sekundärer Prädikate mit Adverbialpartizipien ist:

(97) [pp jakop [vp będąc $c_{\mathrm{V}}\left[\mathrm{vP} \varnothing_{\mathrm{V}}\left[\mathrm{NP}\right.\right.$ gościem $\left.\left.\left._{\mathrm{N}}\right]\right]\right]$

Der obligatorische Instrumental am Prädikatsnomen resultiert hier aus der Zuweisung durch den leeren V-Kopf, dessen Präsenz an der Satzoberfläche durch das V-Auxiliar będac reflektiert wird. Aus alldem kann man schließen, dass bei einem substantivischen Prädikatsnomen und bei Abwesenheit einer Gerundialform tatsächlich Matching stattfindet, indem der leere V-Kopf mit der Kopulabedeutung in die overte Präposition jako quasi "hineinrutscht". Durch dieses Matching verschwindet der leere V-Kopf syntaktisch (nicht jedoch semantisch!), was u. a. auch dazu führt, dass keine Instrumentallizenzierung mehr erfolgt. Da die overte Präposition jako (ebenso wie russ. kak) den Kasus des Bezugsausdrucks lediglich 'weiterreicht', erscheint die prädikative NP kasuskongruent: 
(98) $\left(\mathrm{NP}_{\mathrm{NOM}}\right) \ldots\left[\mathrm{PP}\right.$ jakop $\left[\mathrm{NP}\right.$ gość $\left.\left.\mathrm{NOM}_{\mathrm{N}}\right]\right]$

(Pol)

\section{4 "Prädikatsphrasen"}

Wie im vorangehenden Abschnitt erörtert, erscheinen im Russischen sowohl adjektivische als auch substantivische Sekundärprädikate regelmäßig im Instrumental. Die ‘äußerlich’ sehr ähnlichen Satzstrukturen mit Adjektivformen im Kongruenzkasus (wie z. B. im folgenden Beispielsatz) betrachte ich (für das Russische) hingegen nicht als Sekundärprädikate, sondern als attributive adjektivische NP-Adjunkte bzw. "Prädikatsphrasen" (Wilder 1994, 224) mit Bezug auf eines der Argumente des 'Hauptverbs':

(99)
P'janyj,
on vernulsja domoj.
betrunken-NOM.SG.M er-NOM kehrte zurück nach Hause
'Betrunken kehrte er nach Hause zurück.'
(100) On vernulsja domoj p'janyj. er-NOM kehrte zurück nach Hause betrunken-NOM.SG.M 'Er kehrte betrunken nach Hause zurück.'

Beide Sätze haben m. E. die Basisstruktur in (101):

(101) [vP [NP [AP $\boldsymbol{p}$ 'janyj ] [NP on ]] [v'vernulsja $\mathrm{v}_{\mathrm{v}}$ domoj ]]

Die hervorgehobene adjungierte AP mit dem Kopf p'janyj ist Attribut an der Subjekt-NP mit dem pronominalen Kopf on. Analog zu 'normalen' attributiven APn nehme ich an, dass die AP links an die NP adjungiert. Die Kongruenz in Kasus, Numerus und Genus zwischen der Subjekt-NP und der AP ergibt sich 'automatisch'. Die Oberflächenabfolgen lassen sich auf informationsstrukturell verursachte Bewegungen zurückführen, wie sie u. a. von Junghanns \& Zybatow (1997) und Junghanns (2002) beschrieben werden. Hierauf gehe ich nicht ein. 
Dass Adjektivformen überhaupt in dieser Form als "Prädikatsphrasen" verwendet werden können, ist ihrem Potenzial zum attributiven Gebrauch zu verdanken. ${ }^{211}$ Es ist also kein Zufall, dass diese Verwendung den rein nominalen Langformen vorbehalten ist. Ich nehme an, dass die Adjektive mittels der semantischen Regel Predicate Modification (Heim \& Kratzer 1998, 65) argumentstrukturell und semantisch so angepasst werden, dass sich ihr logischer Typ von <e,t> (einstelliges Prädikat) $\mathrm{zu}\langle<\mathrm{e}, \mathrm{t}\rangle,\langle\mathrm{e}, \mathrm{t}\rangle>$ (intersektiver Modifikator) wandelt.

Indem er sie als "appositive Adjunkte" charakterisiert, behandelt Bailyn (2012) die kongruierenden Adjektivformen (im Russischen) vergleichbar wie Wilder (1994). Jedoch kann ein appositives Adjunkt weitaus 'loser' in die syntaktische Matrixstruktur integriert sein als ein echt-attributives NP-Adjunkt. Insofern folgt durchaus ein syntaktischer Unterschied aus den Analysen Wilders (1994) und Bailyns (2012). Freilich ist es möglich, dass beide Analysen ihre Richtigkeit haben, und dass ein kongruierendes Langformadjektiv somit entweder ein attributives oder ein appositives Adjunkt sein kann. Ich lege mich in dieser Hinsicht nicht fest, zumal es sich bei diesen Fällen um keine Fälle 'echter' Prädikation handelt. Wie schon Wilders (1994) Begriff der "Prädikatsphrase" und schon lange Bailyns (2012) “appositives Adjunkt” andeuten, üben diese Formen zwar im Prinzip die Funktion eines Sekundärprädikats aus (da es sich nun einmal um Prädikate handelt), aber sie unterscheiden sich von 'echter' Sekundärprädikation durch das Fehlen eigener Sachverhaltsreferenz, da keine Kopulabedeutung involviert ist.

Der attributive Gebrauch gehört nicht zum 'Standardverhalten' von Substantiven. Dies und die Tatsache, dass Substantiven die (für Adjektive selbstverständliche) Möglichkeit fehlt, Kongruenzmerkmale zu realisieren, betrachte ich als Ursachen dafür, dass sie als Sekundärprädikate, nicht aber als "Prädikatsphrasen" verwendet werden können. ${ }^{212}$

Wenn für russische sekundäre adjektivische Prädikate mit Kongruenzmarkierung angenommen wird, sie seien "Prädikatsphrasen" im Sinne Wilders (1994) oder “appositive Adjunkte” im Sinne Bailyns (2012), dann muss für die entsprechen-

${ }^{211}$ Hentschel (1993a, 102) schreibt, dass “die Langform im Nominativ [...] als solche auch Attribut des Subjekts sein kann.”

${ }^{212}$ Möglich ist der attributive Gebrauch von NPn, wenn sie appositiv verwendet werden, da hier eine nur sehr 'lose' Einbindung an das syntaktische Umfeld besteht; vgl. Jan, fanatyczny trockista, wrócit do domu 'Jan, fanatischer Trotzkist, kehrte nach Hause zurück'. Hier zeigt sich, dass sowohl die Annahme attributiver "Prädikatsphrasen" (Wilder 1994) als auch appositiver Adjunkte (Bailyn 2012) gerechtfertigt ist. 
den polnischen Adjektivformen, die heute fast nur noch im Kongruenzkasus auftreten, gelten, dass sie stets potenziell ambig zwischen "sekundären Prädikaten" (VP-Adjunkten) und "Prädikatsphrasen" (NP-Adjunkten) bzw. "appositiven Adjunkten" sind. Es ist also möglich, dass der bereits genannte und hier wiederholte polnische Satz mit Kongruenzadjektiv die VP-Basisstruktur in (103) hat:

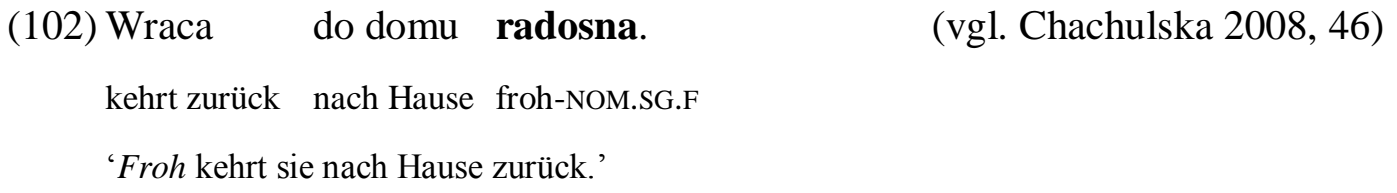

Ebenso ist es aber auch möglich, dass eine andere syntaktische Struktur vorliegt, namentlich jene, die ich den "sekundären Prädikaten" zugrunde lege.

Dass bei Adjektiven, die Bezug auf Partizipanten der Haupthandlung nehmen, verschiedene Typen zu unterscheiden sind, folgert auch Hinterhölzl (2001, 101), der in seinem Beitrag nach semantischen Beschränkungen sucht, die der Kasusmarkierung an adjektivischen Sekundärprädikaten im Russischen zugrunde liegen. Er behandelt alle Adjektivformen, sowohl im Kongruenzkasus als auch im Instrumental, als Sekundärprädikate, was seine Analyse deutlich von Wilders (1994), Bailyns (2012) und auch meinem eigenen Vorschlag absetzt. Korrelierend mit der Kasusmarkierung nennt er verschiedene mögliche Positionen, an denen die Prädikate in der Matrixstruktur adjungieren können, was wiederum unterschiedliche Lesarten zur Folge habe (Hinterhölzl 2001, 100-101):

\begin{tabular}{|c|c|c|}
\hline Domäne & Lesart & Kasusmarkierung \\
\hline CP & circumstantial & \multirow{2}{*}{ Nominativ } \\
\hline IP & pure depictive & \\
\hline VP & temporally restricted & Instrumental \\
\hline
\end{tabular}

Wie aus der vorangehenden Diskussion klar geworden ist, sind für mich die kasuskongruenten Adjektive im Russischen niemals (und im Polnischen nur potenziell) Sekundärprädikate. Mit Wilder (1994) und Bailyn (2012) betrachte ich sie vielmehr als Adjunkte. Die 'rein depiktive' sowie 'zirkumstanzielle' Lesart, 
die Hinterhölzl (2001) nennt, stelle ich durchaus nicht in Abrede. Ich nehme aber an, dass beide Lesarten nicht auf 'echten' Sekundärprädikate mit eigener Sachverhaltsreferenz, sondern lediglich auf "Prädikatsphrasen" bzw. "appositiven Adjunkten” basieren. Auf 'echter' Sekundärprädikation basiert m. E. ausschließlich Hinterhölzls (2001) 'temporal restringierte' Lesart, die er selbst explizit als VP-Adjunkt bezeichnet. Genau diese syntaktische Position habe auch ich im vorangehenden Abschnitt für die Sekundärprädikate angenommen.

Der folgende Abschnitt widmet sich zunächst dem Zustandekommen der u. U. alternativen Kasusmarkierungen an polnischen und russischen Prädikatsnomina, woraufhin in Abschnitt 5.6 prädikative Adjektive näher betrachtet werden.

\subsection{Kasuskongruenz und Kasuszuweisung}

Sprachliche Daten, aus denen hervorgeht, welche Kasusmarkierungen polnische und russische Prädikatsnomina typischerweise zeigen (können), wurden weiter oben in Abschnitt 2 genannt. An dieser Stelle soll es darum gehen, eine Erklärung für diese Markierungen (bzw. für den Ausschluss bestimmter Markierungen unter bestimmten Umständen) zu geben, die möglichst wenig Zusatzannahmen erfordert, aber auch nicht zu Übergeneralisierungen führt.

Meiner Analyse liegt die Überzeugung zugrunde, dass die an der Satzoberfläche sichtbaren Formen von byćlbyt' 'sein' von der Kasusmarkierung am Prädikatsnomen zu trennen sind. ${ }^{213}$ Wie in den Abschnitten 5.1 und 5.2 dargestellt, gehe ich von einer abstrakten Kopulabedeutung aus, die in einem leeren V-Kopf lokalisiert ist. Dieser infinitivartige Kopf $\mathrm{V}^{0}$ ist es, der das Prädikatsnomen in seiner Komplementposition selegiert. Die sichtbaren byćlbyt'-Formen, die sonst auch oft als Formen einer Kopula betrachtet werden, sind m. E. lediglich (V-)Auxiliare zur Ausbuchstabierung von Finitheits- und Kongruenzmerkmalen.

Aus dieser Modellierung folgt, dass die Kasusmarkierung am Prädikatsnomen mit dessen Selektion durch den leeren Kopf $\mathrm{V}^{0}$ mit der Kopulabedeutung zu tun haben muss. Ich wiederhole zunächst seinen Eintrag aus Abschnitt 5.1:

$(105) / \varnothing / \quad[+\mathrm{V},+\mathrm{N},-$ Perf, + max $] \quad \lambda P \lambda x \lambda t \exists s[[t \subseteq \tau(s)]: s$ INST $[P x]] \quad$ (vorläufig)

${ }^{213}$ Im Prinzip ebenso argumentieren z. B. Markman (2008) und Bondaruk (2013b). Die konträre Sicht vertritt u. a. Geist (2006). Quasi eine Mittelposition nimmt Bailyn (2012) ein, da das von ihm angesetzte Pred $^{0}$ entweder mit einer Form von russ. byt' 'gefüllt' oder leer sein kann. 
Dieser Kopf ist de facto ein Infinitiv. Für die Argumentstelle $\lambda P$ wird die Bedeutung des Prädikatsnomens eingesetzt, während $\lambda x$ den (semantisch zu letzterem gehörigen) Referenten vertritt, der später als Subjektausdruck realisiert wird.

\subsubsection{Russisch}

Meine Hypothese lautet, dass der leere V-Kopf im Russischen seinem Komplement, bei dem es sich um ein einstelliges Prädikat handeln muss (andere Beschränkungen sind unnötig), optional den Instrumental zuweist. Dies mache ich in der folgenden Revision des o. g. Lexikoneintrags durch eine Annotation an $\lambda P$ kenntlich. Als "Identifikator" für den Instrumental nutze ich Jakobsons (1971a) Kasusmerkmal ' $\left[ \pm \mathrm{P}\right.$ (eripher)]’: ${ }^{214}$

$(106) / \varnothing / \quad[+\mathrm{V},+\mathrm{N},-$ Perf, + max $] \quad \lambda P_{[ \pm \mathbf{P}]} \lambda x \lambda t \exists s[[t \subseteq \tau(s)]: s$ INST $[P x]]$

Die Optionalität des Instrumentalmerkmals notiere ich mit Hilfe des variablen Vorzeichens “ $\pm ”$ ". Sofern ein nominales Prädikatsnomen eingesetzt wird, kann der Wert "+" vorliegen, was den Instrumental hervorruft:

(107) Anna byla $\varnothing_{\mathrm{V}}$ učitel'nicej.

Anna-NOM AUX-SG.F COP Lehrerin-INS

(Geist 2006, 65)

'Anna war Lehrerin.'

Ebenso kann aber auch der Wert "_" vorliegen, was auf den völlig unmarkierten Nominativ bzw. auf keinerlei Kasuszuweisung hinausläuft: ${ }^{215}$

${ }^{214}$ In Jakobsons Kasussystem ist der Nominativ hinsichtlich aller Merkmale ('Peripher', 'Direktional', 'Quantifizierend') unmarkiert; der Instrumental ist nur hinsichtlich 'P' markiert. Somit entscheidet der Wert von 'P' (siehe auch Zimmermann 2003a):

(i) a. $[-\mathbf{P},-\mathrm{D},-\mathrm{Q}] \rightarrow$ Nominativ (vgl. Jakobson 1971a)

b. $[+\mathbf{P},-\mathrm{D},-\mathrm{Q}] \rightarrow$ Instrumental

215 Ich betrachte den Nominativ als 'Nullkasus', da er sich im Vergleich zu den anderen Kasus rein negativ definiert: Er 'besteht' quasi im Fehlen jedweden Merkmals. Daher kann er m. E. auch nicht zugewiesen, sondern nur durch Kongruenz syntaktisch lizenziert werden. 


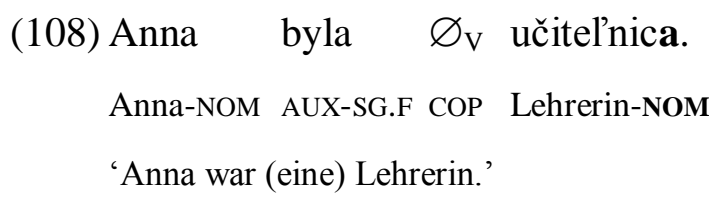

(Geist 2006, 65)

Der Nominativ bedarf der syntaktischen Lizenzierung via Kongruenz mit einem Ausdruck, der mit dieser Kasusmarkierung strukturell versorgt ist, d. h. mit dem Subjektausdruck. Ebendiese syntaktische Herleitung bzw. Begründung der Nominativmarkierung übernehme ich vollends von Geist (2006, 104-106). ${ }^{216}$

Die leere Kopulabedeutung in $\varnothing_{\mathrm{V}}$ kann also (a) den Instrumental zuweisen oder (b) gar keinen Kasus zuweisen, wobei letzteres zwangsläufig den Nominativ herbeiführt, der syntaktisch eines zugänglichen Kongruenzgebers bedarf. Somit basieren die beiden möglichen Kasusmarkierungen an russischen Prädikatsnomina auf zwei grundlegend verschiedenen Mechanismen; vgl. (109):

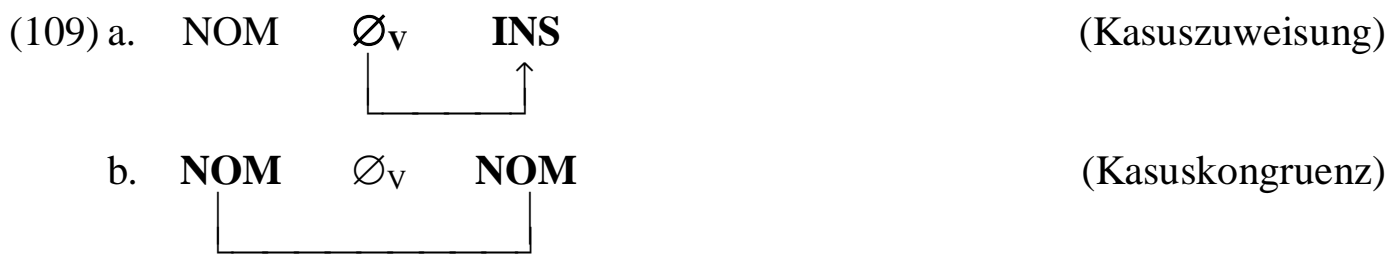

Das bedeutet, dass der Instrumental, sobald er von $\varnothing_{\mathrm{V}}$ zugewiesen wurde, vom syntaktischen Umfeld unabhängig ist. Der Nominativ dagegen bedarf eines Kongruenzgebers in diesem Umfeld. Im Russischen muss letzterer in demselben Clause wie das Prädikatsnomen sein (vgl. "lokal begrenzte Kongruenz" auch bei Bondaruk 2013b). Ist dem nicht so, fällt der Nominativ aus und nur der zugewiesene Instrumental kommt in Frage:

${ }^{216}$ Es gibt Ansätze, die als Kongruenzgeber nicht den Subjektausdruck, sondern das Prädikatsnomen betrachten. So gehen Heidolph, Flämig \& Motsch (1981) sowie Eisenberg (1998) davon aus, dass die Kopula das Prädikatsnomen selegiere und mit dem Nominativ ausstatte, und dass wiederum das Prädikatsnomen den Subjektausdruck syntaktisch und semantisch selegiere und an ihm dabei via Kongruenz auch den Nominativ lizenziere. Indem ich die Einheit aus Kopula und Prädikatsnomen als zusammengesetztes Äquivalent einer 'normaler' Verbform betrachte, ist mir diese Sichtweise fremd, da letztere ihrem Subjekt strukturell den Nominativ zuweisen, ohne eines "internen Kongruenzgebers" zu bedürfen, den die genannten Arbeiten im Prädikatsnomen erblicken. 


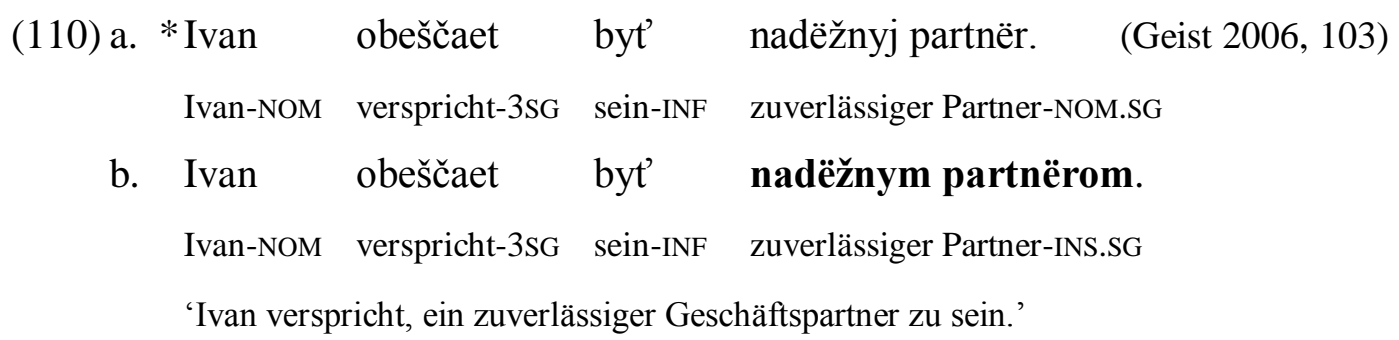

Gleiches gilt im Russischen für prädikative Adjektive (auf die davon abweichende Situation im Polnischen gehe ich unten ein); vgl. (111):

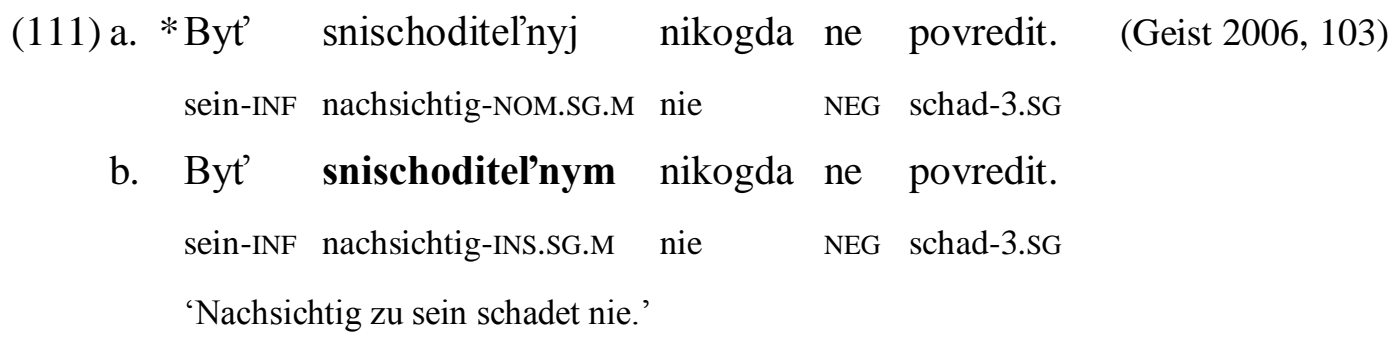

Auf Grund seiner Unabhängigkeit vom syntaktischen Umfeld sollte der Instrumental also immer möglich sein, während der Nominativ nur im Falle der $\mathrm{Zu}$ gänglichkeit eines Kongruenzgebers lizenziert wird. Jedoch ist der Instrumental bekanntlich in präsentischen Kopulasätzen 'blockiert':

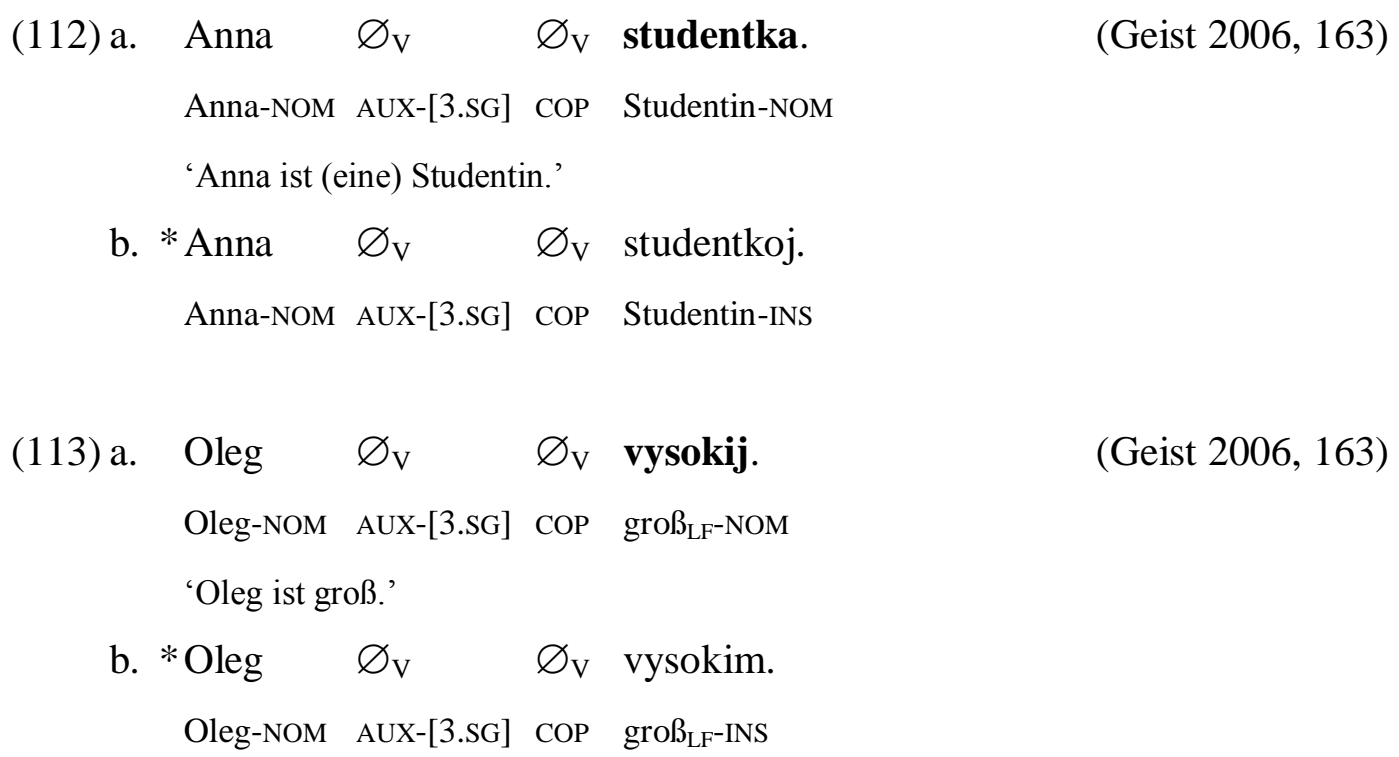

Das widerspricht den obigen Aussagen, denen zufolge der Instrumental auch hier möglich sein sollte. Mitunter wird angenommen, dass sein Ausschluss in präsentischen Sätzen auf 'Defizienz' der "Nullkopula” zurückzuführen sei (vgl. u. a. 
Geist 2006, 163-167). Diese Sichtweise kommt für mich schon deshalb nicht in Frage, weil die sichtbaren Formen von byt' m. E. bloße Auxiliare sind, die mit der Kasusmarkierung am Prädikatsnomen nichts zu tun haben, was folglich auch für die "Nullkopula" (eigentlich nur ein "Nullauxiliar"!) gilt. Gegen die Ansicht, die "Nullkopula" schließe den Instrumental aus, spricht aber noch mehr:

Zunächst ist unzweifelhaft, dass im Rahmen der sekundären Prädikation, bei der am Prädikatsnomen stets der Instrumental erscheint (siehe Abschnitt 5.3.2), letzterer durchaus von einem 'Nullkopf' zugewiesen wird; vgl. (114):

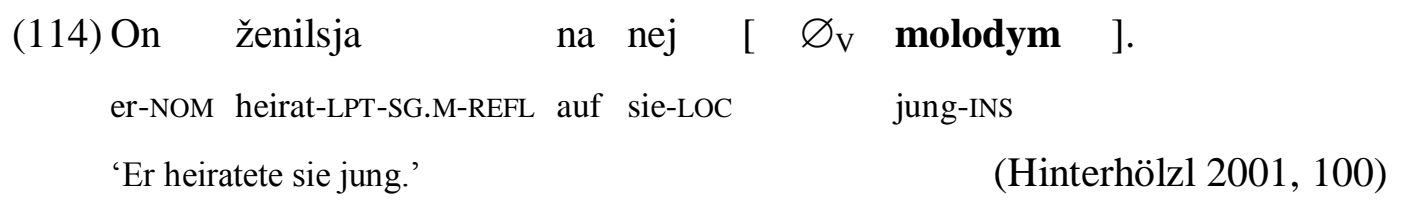

Auf das bloße "Fehlen" der Lautform kann die Nichtzuweisung des Instrumentals also nicht zurückgeführt werden.

Weiterhin kann auch est', die markiert-overte Variante der "Nullkopula", mit dem Instrumental auftreten, was demnach auch von ihr selbst zu erwarten ist: ${ }^{217}$
(115) Ty byl, est' i budeš moim otcom (Geist 2006, 165) du-NOM PAST-SG.M PRES-[2.SG] und FUT-2.SG mein Vater-INS
'Du warst, bist und wirst mein Vater sein.'

Schließlich zeigt ein Blick ins Polnische, dass in Präsenssätzen der Instrumental an prädikativen NPn annähernd obligatorisch ist:

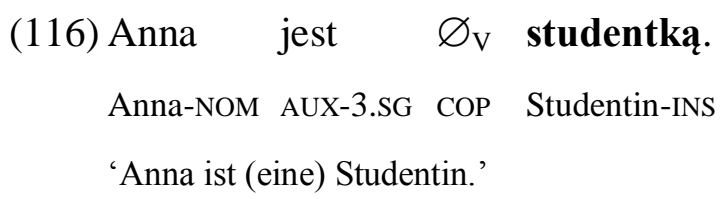

217 Lässt man est' aus, geht der temporale Kontrast, den Geist (2006, 165) feststellt, nicht verloren. Der Präsensbezug wird über die 'rahmenden' Zeiten Präteritum und Futur quasi mitverstanden. So könnte man auch in (i) durchaus eine "Nullkopula" $\left(\varnothing_{\mathrm{V}}\right)$ ansetzen:

(i) Ty byl $\left[\varnothing_{\mathrm{V}}\right]$ i budeš moim otcom. (Rus) Du-NOM PAST [PRES] und FUT mein Vater-INS 'Du warst [bist] und wirst mein Vater sein.', 
Offenbar ist die Abwesenheit einer 'wahrnehmbaren' Verbform in den russischen Präsenssätzen von Bedeutung für die 'Blockierung' des Instrumentals. Jedoch scheint diese Abwesenheit allein dafür nicht hinreichend, da es präsentische Sätze ohne Verbform gibt, die trotzdem den Instrumental zeigen:

(117) Sergej *(u nas) načal'nikom. Sergej-NOM bei uns Chef-INS 'Sergej ist bei uns (der) Chef.'

(118)
Anna *(v bol'nice $)$
medsestroj.
Anna-NOM in Krankenhaus-LOC Krankenschwester-INS
'Anna ist im Krankenhaus Krankenschwester.'

(vgl. Geist 2006, 173)

(vgl. Geist 2006, 173)

Jedoch treten hier lokale PPn auf, deren Abwesenheit den Instrumental wiederum 'blockiert'. Anstelle von PPn kann auch anderes Material vorliegen. Zentral ist offenbar, dass - abgesehen von den beiden NPn - überhaupt 'hörbares' Material vorhanden ist. Das folgenden Beispiel entstammt der Akademiegrammatik (1980, 283), wo es als Beleg für die Möglichkeit des prädikativen Instrumentals in 'kopulalosen' Sätzen genannt wird. Es enthält weder eine Verbform noch eine lokale PP, sondern ein Zeitadverbial. Ich zitiere es nach Hentschel (1993a, 108): ${ }^{218}$

$$
\begin{aligned}
& \text { (119) On pjat' let } \quad \text { brigadirom. } \\
& \text { er-NOM fünf Jahre-GEN Brigadir-INS } \\
& \text { 'Er [ist] fünf Jahre Brigadir [(gewesen)].' }
\end{aligned}
$$

${ }^{218}$ Hentschel zitiert auch (i) ohne weiteres Material. M. E. handelt es dabei (a) um die Ellipse eines Vollverbs wie z. B. služit' 'dienen', (b) um die Ellipse einer Lokalangabe wie z. B. u vas 'bei euch', oder (c) um beides zugleich:

(i) Muž moj voinskim načal'nikom.

(Hentschel 1993a, 108)

Ehemann mein-NOM Militärchef-INS

'Mein Ehemann [dient als]/[ist] Militärchef.'

Es ist nicht zu entscheiden, ob hier Ellipse in einem Kopula- oder in einem anderen Satzes vorliegt. Solche Belege sind daher m. E. kaum brauchbare Evidenz.

Auch zitiert Hentschel den (hier gekürzten) Satz (ii), bei dem es sich eindeutig (beachte die PP $k$ vam) um die Ellipse von prijti 'kommen' und damit um sekundäre Prädikation handelt:

(i) $\mathrm{Ja}[\ldots] \mathrm{k}$ vam posrednikom ot generala. (Hentschel 1993a, 108) ich-NOM zu euch Vermittler-INS von General-GEN 'Ich ... [komme/kam] zu euch als Unterhändler vom General.' 
Geist (2006 174) stellt fest, dass solche 'nackten' Instrumentale in Präsenssätzen nur möglich seien, wenn sie Funktionen oder Tätigkeiten, nicht aber, wenn sie Charakterisierungen oder Status bezeichneten:

(120) Anna V detskom sadu * poslušnym rebënkom / ${ }^{\text {OK }}$ vospitatelem. Anna-NOM im Kindergarten braves Kind-INS.SG.M / Erzieher-INS.SG.M 'Anna ist im Kindergarten Erzieherin.'

(Geist 2006, 174)

$\begin{array}{ccccc}\text { (121) On } & \text { u nas } & \text { *pensionerom } & \text { / } & \text { ok } \\ & \text { načal'nikom. } \\ \text { er-NOM } & \text { bei uns } & \text { Rentner-INS.SG.M } & / & \text { Chef-INS.SG.M }\end{array}$

'Er ist bei uns der Chef.'

(Geist 2006, 174)

Diese Feststellung zeigt für die relevanten Fälle, dass hier keine präsentischen Kopula-Prädikativ-Strukturen, sondern Sätze vorliegen, in denen ein Vollverb, das ein Tätigsein oder Funktionieren bezeichnet, elliptisch ausgelassen wird, was an der Oberfläche lediglich einen adverbialen bzw. sekundärprädikativen Instrumental zur Angabe der konkreten Tätigkeit oder Funktion übrig lässt. Dies ist freilich v. a. in der gesprochenen Sprache bzw. deren Verschriftlichung anzutreffen:

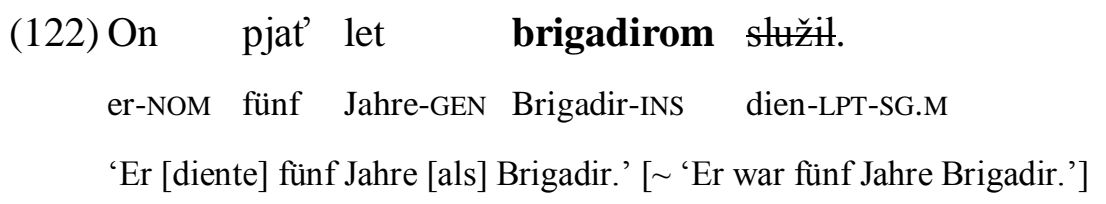

(123) Anna v detskom sadu vospitatelem rabotaet. Anna-NOM im Kindergarten Erzieher-INS.SG.M arbeit-3.SG 'Anna [arbeitet] im Kindergarten [als] Erzieher.' [ 'Anna ist im Kindergarten Erzieher.']

$\begin{array}{rlll}\text { (124) On } & \text { u nas } & \text { načal'nikom } & \text { rabøtaet. } \\ \text { er-NOM } & \text { bei uns } & \text { Chef-INS.SG.M } & \text { arbeit-3.SG }\end{array}$ 'Er [arbeitet] bei uns [als] Chef.' [ 'Er ist bei uns (der) Chef.']

Wie man an den möglichen Übersetzungsparaphrasen erkennt, lassen sich solche Aussagen fast stets auch mit einem Kopulasatz erfassen. Diesen Zusammenhang beobachtet meines Wissens als erster Hentschel (1993a): 
Zwei unmittelbar aneinander grenzende Nominalphrasen, eine im Nominativ und eine im Instrumental, ohne ein verbales Element in ihrem Kontext, bergen die Gefahr, als elliptischer Satz mißverstanden zu werden, besonders natürlich in der gesprochenen Sprache. Es ist in dieser Hinsicht bezeichnend, daß in vielen der Sätze, die die Akademiegrammatik (1982 [1980], 283) als Belege für die Möglichkeit des prädikativen Instrumentals in kopulalosen Sätzen anführt, die Einsetzung eines Vollverbs möglich ist, ohne daß ein wesentlicher Unterschied im propositionalen Gehalt resultiert [...]. (Hentschel 1993a, 108)

Aus all dem geht hervor, dass es sich mindestens beim Großteil präsentischer Sätze, die neben einem Nominativsubjekt einen Instrumental, aber keine overte Verbform enthalten, um keine Kopula-Prädikativ-Strukturen handelt, sondern vielmehr um Sätze mit elliptisch ausgelassenen Prädikaten wie rabotat' 'arbeiten' oder služit' 'dienen', mit denen typischerweise Instrumentale als Angabe einer konkreten Tätigkeit oder Funktion auftreten. Die Ellipse dieser Prädikate wird offenbar gerade durch die Präsenz jener ‘typischen' Instrumentale lizenziert.

Daher sieht Hentschel (1993a) die generelle Gefahr, dass präsentische Kopulasätze mit instrumentalischem Prädikatsnomen mit den fraglichen Ellipsen 'verwechselt' bzw. als solche aufgefasst werden (können). Der Instrumental in präsentischen Kopulasätzen - ob mit 'Nullkopula" oder est' - ist demnach also nicht strukturell-syntaktisch blockiert. Ebensowenig ist sein Ausschluss auf eine 'Defizienz' der 'Nullkopula” zurückzuführen.

Die Schlussfolgerung lautet, dass vom 'prädikativen' Instrumental in einem präsentischen Kopulasatz 'nur' deswegen kein Gebrauch gemacht wird, weil dies eine nicht erwünschte Deutung der resultierenden Struktur als Ellipse eines Vollverbs mit Tätigkeitsbedeutung hervorrufen könnte. Insofern würde der Gebrauch des Instrumentals in diesen Fällen eine Gefahr für die Erreichung der kommunikativen Absichten des Sprechers bedeuten. Um seine Äußerung eindeutig als Kopulasatz auszuweisen, wird der Sprecher den 'doppelten' Nominativ wählen, da letzterer die benannte Gefahr ausschließt. Insofern ist Hentschel zuzustimmen, "[...] daß die primäre Funktion von Kasusmarkierungen eine möglichst transparente Signalisation der syntaktischen Struktur [...] ist" (Hentschel 1993a, 110). Kurzum, dem Nichtgebrauch des Instrumentals in präsentischen Kopulasätzen liegt die Vermeidung ambiger Satzstrukturen zugrunde. 
Ein ebenso bedeutsames wie wünschenswertes Ergebnis dieser Erwägungen besteht darin, dass der "Kopulabedeutung" $\left(\varnothing_{\mathrm{V}}\right)$ bzw. der traditionellen "Nullkopula" (sowie est') die Fähigkeit zur Zuweisung des Instrumentals nicht per se abgesprochen werden muss. Eine weitere wünschenswerte Folge ist, dass man im Rahmen sowohl der (präsentischen) Primär- als auch der Sekundärprädikation ein und denselben "stummen" prädikativen V-Kopf $\left(\varnothing_{\mathrm{V}}\right)$ ansetzen kann, der den Instrumental in beiden Fällen gleichermaßen optional zuweist. Davon wird aber nur im Falle sekundärer Prädikation Gebrauch gemacht (bzw. muss davon mangels eines nominativischen Kongruenzgebers Gebrauch gemacht werden, vgl. Abschnitt 5.3.2), während der Instrumental bei primärer Prädikation aus den genannten Gründen vermieden wird, was folglich zum Nominativ führt, der nota bene wegen des in diesen Strukturen stets vorhandenen Nominativsubjekts auch immer verfügbar ist.

\subsubsection{Polnisch}

Die Situation im Polnischen ist insofern 'übersichtlicher’ als im Russischen, als substantivische Prädikatsnomina heute konequent im Instrumental, adjektivische Prädikative dagegen im Nominativ erscheinen:

(125) Ten wysoki blondyn jest pilotem. dieser große Blonde-NOM ist-3SG Pilot-INS 'Dieser große Blonde ist (ein) Pilot.'

[sie] war-SG.F gelehrig-NOM.SG.F 'Sie war gelehrig.'

Im Polnischen besteht eine stabile Korrelation zwischen der Kategorie des Prädikatsnomens (Substantiv vs. Adjektiv) und seiner Kasusmarkierung (Instrumental vs. Nominativ). Die 'tiefere Ursache' für diese Korrelation sehe ich darin, dass Adjektive per se Eigenschaften bezeichnen und so ohne Weiteres als prädikative Ausdrücke verwendet werden und identifizierbar sind, während Substantive an sich auf 'Dinge' i. w. S. referieren und so im Falle ihrer prädikativen Verwendung eine spezielle 'prädikative' Markierung 'gut brauchen' können. Sofern diese Er- 
klärung zutrifft, braucht man für den polnischen V-Kopf mit der Kopulabedeutung keinen anderen Lexikoneintrag als im Russischen anzusetzen:

(127) $/ \varnothing / \quad[+\mathrm{V},+\mathrm{N},-$ Perf, $+\max ]$

$$
\lambda P_{[ \pm \mathbf{P}]} \lambda x \lambda t \exists s[[t \subseteq \tau(s)]: s \text { INST }[P x]]
$$

Auch hier wird der Instrumental optional zugewiesen, aber im Unterschied zum Russischen ist die nominale Subkategorie des Prädikatsnomens ([ $\pm \mathrm{a}]$; vgl. die Abschnitte 4.3, 4.4.2, 4.4.3) im Polnischen für die Entscheidung des Sprechers das offenbar grundlegende Kriterium. Mit anderen Worten: Während im Russischen die Kasusmarkierung primär pragmatischen und stilistischen Zwecken dient (was v. a. darauf beruht, ob die betreffende Konstituente explizit als prädikativer Ausdruck ausgewiesen wird oder nicht; siehe Abschnitt 6), orientiert sich die Kasuswahl im Polnischen an der Wortart des nominalen Prädikativs und ist damit eher systematisch-kategorialer Natur.

Als Ausnahmen von der o. g. Korrelation haben Fälle zu gelten, in denen Substantive im Kongruenzkasus erscheinen (vgl. v. a. Bogusławski 2001):
(128) Zosia jest wojewodzianka.
Zosia-NOM ist-3SG Voivodentochter-NOM

'Zosia ist Tochter eines Voivoden.'
(Bogusławski 2001, 107)

Offensichtlich wird die Korrelation hier durchbrochen. Im vorliegenden Fall wird sie m. E. ganz bewusst missachtet, was zur Folge hat, dass das Substantiv nicht explizit durch den Instrumental als Prädikativ markiert wird. Nicht anders als in Bezug auf vergleichbare russische Fälle nehme ich an, dass der Sprecher durch das bewusste Nichtverwenden des Instrumentals (bzw. die Verwendung des Nominativs) auf ikonische Weise eine Gleichsetzung von Subjektausdruck und Prädikatsnomen enkodiert. Er setzt, mit anderen Worten, den Subjektreferenten mit der vom prädikativen Substantiv bezeichneten Eigenschaft bzw. 'Klasse' annähernd gleich (bzw. fällt ersterer unter letzteres). Dieses Ausdrucksmittel ist ein rein formbasiertes, das die grammatische Ebene (SF) nicht tangiert. Auf der konzeptuellen Ebene (CS) führt diese 'Auszeichnung' des Prädikatsnomens hingegen zu einer Lesart, die man durchaus mit den (traditionellen) Begriffen 'permanent', 'charakterisierend' oder 'definierend' erfassen könnte (Details in Abschnitt 6). 
Die oben behauptete Korrelation kann auch durch adjektivische Prädikatsnomina im Instrumental durchbrochen werden; vgl. (129):

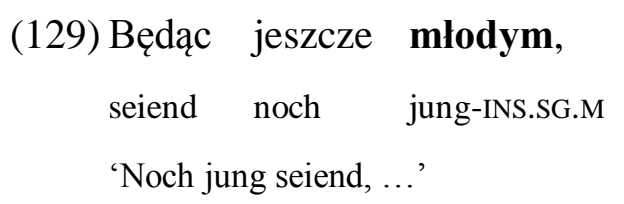

Hier liegt aber m. E. keine ikonisch-formbasierte Motivation wie beim substantivischen Beispiel vor, sondern vielmehr eine strukturell-syntaktische, die aber wiederum auch mit der Wortart des Prädikativs zu tun hat. Da der Nominativ im Polnischen ebenso auf einen Kongruenzgeber wie im Russischen angewiesen ist, scheint er im vorliegenden Beispiel ausgeschlossen zu sein, denn das Gerundium (Adverbialpartizip) będąc projiziert als Infinitum entweder keinen nominativischen (d. h. PRO) oder aber überhaupt keinen Subjektausdruck (wenn Kontrolle semantisch erfolgt). Dem prädikativen Adjektiv im obigen Beispiel fehlt somit ein Kongruenzgeber, so dass der vom "stummen" V-Kopf zugewiesene Instrumental die einzige Möglichkeit zu sein scheint, das Adjektiv mit Kasus zu versorgen:

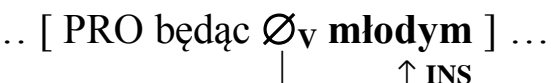
$\uparrow$ INS

Jedoch ist die Lage im Falle von Gerundien im Polnischen nicht so eindeutig, wie das obige Beispiel suggeriert. Der Nominativ am Prädikatsadjektiv ist hier nämlich durchaus nicht ausgeschlossen (wie es im Russischen in entsprechenden Strukturen der Fall ist), vgl. (131):
(131) Prokurator
chyba postanowił być
bezpośredni.
NKJP
Staatsanwalt-NOM wohl beschloss-SG.M sein-INF direkt-NOM.SG.M
'Der Staatsanwalt beschloss wohl, direkt zu sein.'

Wie lässt sich diese Situation, die deutlich von der im Russischen abweicht, erklären? Der Unterschied zwischen beiden Sprachen besteht - rein deskriptiv darin, dass der Nominativ im Russischen restringierter als im Polnischen ist. Namentlich kann er im Russischen, wie bereits bemerkt, nur dann am Prädikats- 
nomen (Adjektiv oder Substantiv) erscheinen, wenn sich ein nominativischer Kongruenzgeber in demselben Clause befindet. Im Polnischen kann er an adjektivischen Prädikatsnomina dagegen auch dann erscheinen, wenn der Kongruenzgeber in einem anderen Clause lokalisiert ist; vgl. (132):

(132) prokurator $_{\mathrm{i}} \ldots$ [ [ $\mathrm{PRO}_{\mathrm{i}}$ być $\varnothing_{\mathrm{V}}$ bezpośredni ]

In dieser Struktur kongruiert das prädikative bezpośredni mit dem Matrixsubjekt prokurator, obwohl sich letzteres in einem anderen Clause befindet. Dies ist im Russischen ausgeschlossen, da die Nominativkongruenz offenbar auf einen Clause beschränkt ist ('nahe Kongruenz').

Die 'ferne Kongruenz' im Polnischen ist aber nur für adjektivische Prädikative eine Option, während sie für Substantive ausgeschlossen ist. Letztere stehen in den fraglichen Fällen stets im Instrumental (vgl. Bogusławski 2001, 112-113). Es ist jedoch zu bemerken, dass Adjektive, wie schon das obige będąc-Beispiel zeigt, in den relevanten Strukturen ebenfalls den Instrumental tragen können. Vgl. dazu auch das folgende Datum von Rothstein (1993), das strukturell mit dem zuletzt genannten Beispielsatz identisch ist, in dem aber nichtsdestotrotz das prädikative Adjektiv nicht im Kongruenzkasus, sondern im Instrumental erscheint:

Postanowił być oszczędnym.
beschloss-SG.M sein-INF sparsam-INS.SG.M
'Er beschloss, sparsam zu sein.'

Dies ist bemerkenswert, da im Matrixsatz ein - wenn auch kovertes - Subjektspronomen im Nominativ vorhanden ist, mit dem das eingebettete Prädikatsadjektiv kongruieren könnte. Dennoch wird die Option der Kasuszuweisung gewählt:

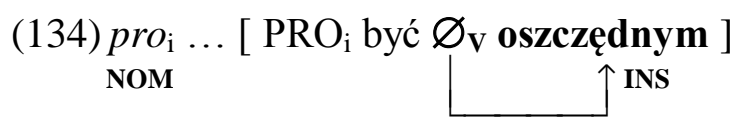

In Strukturen mit infiniten Formen des V-Auxiliars (być, będac, bywszy etc.) hat der polnische (anders als der russische) Sprecher also offenbar die Wahl zwischen dem Nominativ und dem Instrumental am prädikativen Adjektiv. In Anbetracht 
der bereits genannten Tatsache, dass er bei prädikativen Substantiven hingegen auf den Instrumental festgelegt ist, schlussfolgere ich, dass die Wahlfreiheit bei Adjektiven direkt auf deren Wortart bzw. auf ihr attributives Potenzial zurückgeführt werden kann. Im Unterschied zu Substantiven ist eine (wenn nicht die) primäre Funktion von (Langform-)Adjektiven der attributive Gebrauch. Dies zeigt sich auch in ihrer Fähigkeit, Kongruenz mit einem Bezugsausdruck in Kasus, Numerus und Genus morphologisch zu realisieren. Ebendiese Fähigkeit aber fehlt den (primär referenziell verwendeten) Substantiven. Die Option der 'fernen' Kongruenz scheint auf der genannten Fähigkeit der Adjektive zu beruhen, da durch sie die Rekonstruktion des syntaktischen Zusammenhangs von Matrixsubjekt und eingebettetem Prädikatsnomen auch über eine relativ weite Distanz hinweg möglich ist. Bei eingebetteten prädikativen Substantiven würde der Nominativ zu einer intransparenten syntaktischen Struktur führen, da man - vor dem Hintergrund der relativ freien Wortfolge im Polnischen - nicht sicher sein könnte, ob der Staatsanwalt nun Lehrer, oder nicht doch der Lehrer Staatsanwalt zu sein beschloss, vgl. (135): ${ }^{219}$

\section{(135) * Prokurator chyba postanowił być nauczyciel. Staatsanwalt-NOM wohl beschloss-SG.M sein-INF Lehrer-NOM}

Eine solche 'Verwirrung' ist bei einem Adjektiv in prädikativer Verwendung ausgeschlossen, da es selbst klar macht, dass es nicht das Satzsubjekt ist.

Ganz und gar 'blockiert' ist der Nominativ an polnischen Prädikatsadjektiven, wenn es z. B. unter einem "Modalprädikativum" erscheint; vgl. (136):

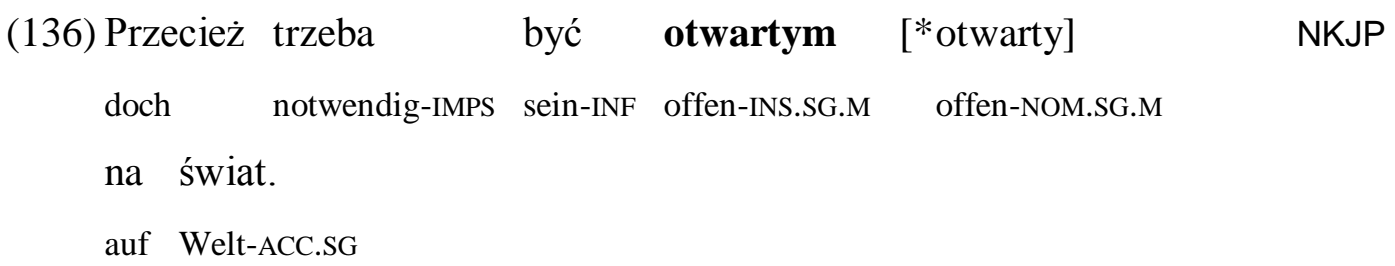

219 Dafür, dass Satz (135) ungrammatisch (und nicht nur nicht akzeptabel) ist, ist sehr wahrscheinlich auch die deskriptive Bedeutung des Vollverbs postanowić 'beschließen' verantwortlich, die eine 'permanente' Lesart des Prädikatsnomens ausschließt. 
Dieser Befund passt ins Bild, da "Modalprädikativa" offenbar keinen nominativischen Subjektausdruck lizenzieren (es handelt sich um 'unpersönliche' Strukturen). Im obigen Beispiel ist also nirgendwo ein geeigneter Kongruenzgeber vorhanden, so dass der zugewiesene Instrumental in der Tat die einzige Markierungsoption für das Prädikatsnomen darstellt (“last resort”).

Im Prinzip ähnlich ist der folgende Fall gelagert; vgl. (137):

\author{
(137) Dawniej było się szczęśliwym / * szczęśliwy. \\ früher war-LPT.IMPS REFL glücklich-INS glücklich-NOM \\ 'Früher war man glücklich.’ ～～(Bondaruk 2013b, 160, Fn. 9)
}

Hierbei handelt es sich ebenso wie beim obigen Satz mit dem "Modalprädikativum" trzeba um einen unperönlichen Satz. Folgt man Fehrmann, Junghanns \& Lenertová (2010), so führt die Verwendung des Reflexivmarkers się hier dazu, dass die externe Argumentstelle des (in diesem Falle zusammengesetzten) Satzprädikats być szczęśliwym 'glücklich sein' durch einen Operator gebunden wird, der arbiträr-human interpretiert wird $\left(\mathrm{OP}_{[\text {arb-hum] }}\right.$; vgl. das vergleichbare generische Pronomen man im Deutschen). Diese Bindung erfolgt semantisch, was heißt, dass in der Syntax kein nominativischer Subjektausdruck projiziert wird. Einem prädikativen Adjektiv im Nominativ würde demnach der Kongruenzgeber fehlen. Als 'Ausweg' kommt dann nur der zugewiesene Instrumental in Frage (vgl. auch Witkoś 2010, 212; Bondaruk 2013b, 175 und 210). ${ }^{220}$

Eine weitere Möglichkeit stellen polnische Beispiele dar, in denen der Instrumental am Adjektiv die einzige Option ist, obwohl im Matrixsatz ein nominativischer Kongruenzgeber vorliegt; vgl. (138):

$\begin{array}{llllll}\text { (138) Piotr } \quad \text { nie } & \text { wie } & \text { jak } & \text { być } & \text { głupim. } & \text { (Witkoś 2011, 55) } \\ \text { Peter-NOM NEG } & \text { wiss-3SG } & \text { wie } & \text { sein-INF } & \text { dumm-INS.SG.M } & \\ \text { 'Peter weiß nicht, wie er dumm sein soll.' } & & \text { (nach Bondaruk 2013b, 210) }\end{array}$

Man kann Bondaruk (2013b, 210) zustimmen, dass der Nominativ hier ausgeschlossen ist, da zwischen Matrix und Einbettung in Form des overten Komple-

\footnotetext{
${ }^{220}$ Was die Kongruenzmerkmale angeht, so kann man die Kombination Sg. m. als Default betrachten, der gewählt wird, wenn völliger Mangel an Kongruenzmerkmalen herrscht.
} 
mentierers in $C^{0}$ eine Art von 'Grenze' (folgend markiert mit “ $\|$ ”) vorliegt, die die Etablierung von Kongruenz verhindert; vgl. (139): ${ }^{221}$

(139) Piotr nie wie [ jak $_{\mathrm{C}}$ być głupim ].

NOM

$\| \quad * \mathrm{NOM} \rightarrow \mathbf{I N S}$

Anders als bei den zuvor betrachteten Fällen mit "Modalprädikativa", in denen gar kein nominativischer Subjektausdruck vorhanden war, liegt hier also zwar ein solcher vor, aber durch die 'Grenze' zwischen Matrix und Einbettung kommt es dennoch zu derselben Situation, dass nämlich die Zuweisung des Instrumentals die einzige Markierungsoption für das prädikative Adjektiv ist.

\subsubsection{Fazit}

Im Ergebnis der Betrachtungen und Erwägungen in diesem Abschnitt bin ich zu dem ergänzten Lexikoneintrag in (140) für den abstrakt-leeren V-Kopf gelangt, mit dem ich die "Kopulabedeutung" assoziiere:

$$
[+\mathrm{V},+\mathrm{N},- \text { Perf },+\max ]
$$

$$
\lambda P_{[ \pm \mathbf{P}]} \lambda x \lambda t \exists s[[t \subseteq \tau(s)]: s \text { INST }[P x]]
$$

Dieser V-Kopf, der einer infinitivischen Verbform entspricht, selegiert prädikative Ausdrücke des logischen Typs <e,t> (einstellige Prädikate), die - sofern dies für sie überhaupt Relevanz hat, da sie rein nominal sind - hinsichtlich des Jakobsonschen Kasusmerkmals $[ \pm \mathrm{P}]$, das den Unterschied zwischen Nominativ und Instrumental repräsentiert, entweder positiv oder negativ spezifiziert sein können. Das Ganze bedeutet eine nur optionale Zuweisung des Instrumentals. ${ }^{222}$

Ein Prädikatsnomen kann somit entweder mit dem Instrumental markiert werden, oder ihm wird vom o. g. V-Kopf überhaupt kein (positiv spezifiziertes) Kasus-

${ }^{221}$ Es ist m. E. nicht eindeutig, dass jak 'wie' die Belegung von $\mathrm{C}^{0}$ ist. Denkbar ist auch, dass es sich um eine lexikalisierte PP in Spec-CP handelt.

${ }^{222}$ Bei sog. "halkopulativen" Verben wie javljat'sja '(etw.) darstellen', ostat'sja 'bleiben', stat' 'werden' usw. ist der Instrumental am Komplementausdruck obligatorisch, so dass das Kasusmerkmal in den Lexikoneinträgen dieser Verben folglich eindeutig [+P] lautet. Das passt gut zu der Tatsache, dass ihr Komplement stets eine Eigenschaft denotiert, die explizit prädikativ und damit als etwas Erworbenes, nicht aber etwas quasi Angeborenes, gewertet wird, vgl. z. B. einen Menschen darstellen, gesund bleiben/werden. Siehe auch Abschnitt 6. 
merkmal bzw. kein Kasus gegeben, so dass es nurmehr über die Kongruenz mit einem zugänglichen Ausdruck den völlig unmarkierten Nominativ erhalten kann, wobei es sich um einen syntaktischen Mechanismus handelt.

Dieser Mechanismus wiederum funktioniert mit Bezug auf prädikative Substantive im Polnischen und Russischen in gleicher Weise. Dagegen müssen prädikative (Langform-)Adjektive im Russischen einen Kongruenzgeber in ihrem eigenen Clause finden ('nahe Kongruenz'), während ihre polnischen Entsprechungen auch mit einem syntaktisch weiter entfernten Kongruenzgeber zurechtkommen ('ferne Kongruenz'). Dieser Unterschied zwischen den beiden betrachteten Sprachen liegt m. E. darin begründet, dass im Polnischen die lexikalische Kategorie des Prädikatsnomens (bzw. sein 'Adjektivsein') für die Kasusmarkierung entscheidender ist als pragmatische Faktoren, die andererseits im Russischen (neben bzw. parallel zu stilistischen Faktoren) den Ausschlag geben. Salopp lässt sich formulieren, dass auch prädikative Adjektive im Polnischen, wenn es irgend geht, kongruieren, während sie im Russischen weitgehend analog zu prädikativen Substantiven behandelt werden. Beidem liegt fraglos die Tendenz zur Vereinheitlichung zugrunde, wenn auch in unterschiedlicher Ausprägung.

\subsection{Die Gebrauchsweisen von (prädikativen) Adjektiven}

Hier wird es um die diversen Gebrauchsweisen bzw. Erscheinungsformen von Adjektiven im Russischen und Polnischen gehen, wobei auch nicht prädikativ verwendete Adjektive einbezogen werden müssen, da die Oberfläche von Kopulasätzen nicht ohne Weiteres erkennen lässt, ob ein Langform-Adjektiv prädikativ oder 'nur' attributiv verwendet wird - was jedoch bedeutende Auswirkungen auf die Interpretation des Adjektivs hat. Im Prinzip soll gezeigt werden, wie und wieso es zu Ambiguitäten kommen kann. Hierum werden sich die Abschnitte 5.6.1 und 5.6.2 drehen.

Ebenfalls werde ich auf die sog. "Zustandsprädikative" eingehen, bei denen es sich m. E. um eine besondere Verwendung ansonsten aber 'ganz normaler' prädikativer Kurzformen handelt. Die Analyse wird in Abschnitt 5.6.3 skizziert.

Zum Einstieg wiederhole ich den Lexikoneintrag der gleichermaßen russischen wie polnischen Wurzel dobr- (ein Qualitätsadjektiv) aus Abschnitt 4.4.3:

$(141) / \mathrm{dobr} / \quad\left[\alpha \mathrm{V},+\mathrm{N},(+\mathrm{a})_{\alpha}-\max \right] \quad \lambda x[x \mathrm{GOOD}] \in\langle\mathrm{e}, \mathrm{t}\rangle$ 
Wird diese Wurzel mit einem Suffix versehen (bspw. dobr-a oder dobr-aja), entsteht eine Form, die wiederum ein einstelliges Prädikat ist (Typ <e,t>). Eine solche Form lässt sich direkt als Prädikatsnomen verwenden. Es ist demnach für die Ebene der grammatischen Bedeutung (SF) gleichgültig, ob ein prädikatives Adjektiv in der Kurz- oder in der Langform auftritt. Auch die Kasusmarkierung bei letzterer ist für die Bedeutungsebene irrelevant. Nichtsdestotrotz sind, wie ich weiter oben gezeigt habe, Kurzformen Verbalnomina, Langformen hingegen reine Nomina. Jedoch ist dieser Unterschied weniger für die Interpretation bedeutsam, als vielmehr für die Distribution der Formen sowie auch für ihre Argumentrealisierung (siehe auch Abschnitt 4.4.3). Das soll nicht ausschließen, dass die Form des Adjektivs nicht ggf. Einfluss auf die Lesart im Satz haben kann. Letzteres ist der Fall, sofern für dieselbe Position mehrere Adjektivformen zur Wahl bzw. in denkbarer Opposition zueinander stehen. Ich werde ein Modell vorstellen, dem zufolge - analog zu prädikativen Substantiven - die Lesarten erst auf konzeptueller Ebene zustande kommen, und zwar auf ikonischer bzw. formbasierter Basis. Obwohl in dieser Arbeit der prädikative Gebrauch von Adjektiven im Mittelpunkt steht, kann der attributive Gebrauch doch nicht gänzlich ausgeblendet werden. Das liegt daran, dass Kopulasätze, an deren Oberfläche lediglich Adjektive sichtbar werden, 'verdeckt' prädikative Substantive enthalten können, seien letztere nun syntaktische "Nullen" oder elliptisch ausgelassen (siehe unten). Das hat freilich Konsequenzen für die Interpretation. Eine umfassende Beschreibung von Kopulasätzen muss demnach auch diese möglichen Fälle berücksichtigen.

Dies wird folgend primär anhand russischer Daten geschehen, da die Distinktion zwischen Kurz- und Langformadjektiven im (geschriebenen) Standardrussischen deutlich ausgeprägter als im Polnischen ist, wo Kurzformen nur noch rudimentär gebildet werden. Dennoch wird das Gesagte grundsätzlich auch auf das Polnische anzuwenden sein. Auf wichtige Unterschiede gehe ich an geeigneter Stelle ein.

\subsubsection{Lang- und Kurzformen}

Der folgende Satz enthält mit dobra 'gut' eine russische Kurzform (KF): 


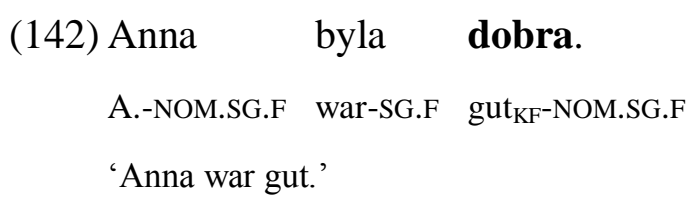

Was ihre Interpretationen betrifft, so bezeichnen russische Kurzformen der Literatur zufolge Eigenschaften von Referenten, ohne dabei irgendwelche zusätzlichen interpretativen Effekte zu evozieren (vgl. u. a. Ueda 1992; Ruferová 1996; Geist 2006). Anders als z. B. Hentschel (1993a, 101), Geist (2006, 138), Kempgen $\left({ }^{2} 2008,164,167\right)$ oder Kamynina $\left({ }^{2} 2010,91\right)$, halte ich es für theoretisch unverzichtbar, auch Kurzformadjektiven die Kategorie Kasus zuzusprechen. ${ }^{223}$ Ebenso wie alle anderen verbalnominalen sowie nominalen Formen in prädikativer Position sind auch die Kurzformen auf den Mechanismus der Kasuskongruenz angewiesen (vgl. Abschnitt 5.5). Sie sind es ganz besonders, da ihr Paradigma im heutigen Russischen und Polnischen auf nominativische Formen beschränkt ist, was m. E. unmittelbar mit ihrer Verbalnominalität zu tun hat, die sie auf den prädikativen Gebrauch beschränkt. ${ }^{224}$ Nur die rein nominalen Langformen sind heute attributiv verwendbar.

Im Polnischen treten Kurzformen weitaus seltener auf als im (v. a. geschriebenen) Russischen. Sie sind meist an bestimmte Lexeme gekoppelt, die auch oder ausschließlich Kurzformen bilden. Meist sind nur maskuline Nominativformen gebräuchlich, da feminine Kurz- und Langformen stets homonym sind: ${ }^{225}$

${ }^{223}$ M. E. ist nicht die Annahme der "Kasushaftigkeit" von Kurzformen, sondern die ihrer "Kasuslosigkeit" die nachzuweisende Hypothese, was jedoch bis dato aussteht. So gilt weiterhin die 'natürliche' Annahme, dass Kurzformen über Kasus verfügen. Beachte auch, dass, sofern den Kurzformen Kasus abgesprochen wird, deren Flexionsendungen eigene "kasuslose" Lexikoneinträge erfordern würden, obwohl sie homonym mit den substantivischen Flexionsmarkern sind; vgl. etwa das Substantiv žen-a (Nom. Sg. f.) 'Ehefrau' mit der angeblich "kasuslosen" Kurzform dobr-a (nur Sg. f.) 'gut'. Siehe auch Abschnitt 4.4.3.

${ }^{224}$ Ausnahmen sind Wendungen wie russ. sred' bela dnja 'bei hellichtem Tage' mit genitivischattributivem bela. Auch polnische Kurzformen sind mehrheitlich nur nominativisch und rein prädikativ. Wenige Lexeme sind Ausnahmen, vgl. w pewien dzień 'an einem gewissen Tage' mit akkusativisch-attributivem pewien. Jedoch sind andere Kasus als der Akkusativ ausgeschlossen (dann treten stets Langformen auf). Ferner ist der Akkusativ nur möglich, wenn er mit dem Nominativ formal zusammenfällt. So kann w pewien dzień ggf. auch als feste Wendung gelten. So oder so sind oblique und attributive Kurzformen im Polnischen eine ebenso marginale Erscheinung wie im Russischen.

${ }^{225} \mathrm{Im}$ Polnischen ist es historisch zu Kontraktionen gekommen, in deren Folge die ursprünglich pronominalen Endungen (vgl. aksl. dobr-a-ja) zunächst zu langvokalischen Endungen (altpoln. 

(143) Piotr
był zdrów
i wesól.
P.-NOM.SG.M war-SG.M gesund KF-NOM.SG.M und fröhlich $_{\mathrm{KF}^{-N O M . S G . M ~}}$
'Peter war gesund und fröhlich.'

(Pol)

Gewissermaßen 'regelmäßig' treten nur neutrale Kurzformen im Polnischen auf, und zwar stets als Zustandsprädikative (siehe genauer Abschnitt 5.6.3):
(144) Ale i
tak było
wesolo.
NKJP
aber auch so war-SG.IMPS gesund $_{\text {KF-NOM.SG.IMPS }}$
'Aber auch so war es lustig.'
(145) Bardzo mi milo.
sehr mir lieb KF-NOM.SG.IMPS
'Es ist mir sehr angenehm.'

Gewisse Lexeme lassen die neutrale Kurzformbildung aber auch beim Gebrauch als Zustandsprädikativ nicht oder kaum noch zu. Statt der 'unpersönlichen' Markierung - $o$ tritt bei ihnen der 'Adverbmarker' $-e$ auf. Ich nehme daher an, dass sowohl $-o$ als auch $-e$ (kongruenz)merkmallos sind:
(146) Tam nie jest bezpiecznie, kochanie. dort NEG ist-IMPS sicher-ADV/IMPS Liebe-NOM.SG 'Dort ist es nicht sicher, [meine] Liebe.'

K06, 23

Diese lexemspezifische Variation zwischen -o und - $e$ betrachte ich morphologisch als "historischen Zufall". Für die Syntax macht es keinen Unterschied, ob die eine oder andere Variante des 'unpersönlichen' Markers vorliegt. Im Russischen gibt es keinen 'Adverbmarker' - $e$ (mehr), weshalb dort ausschließlich - $o$ auftritt.

An dieser Stelle kehre ich zum Russischen zurück. Ich wiederhole zunächst Satz (147) mit einer Kurzform vom Anfang des Abschnitts:

\footnotetext{
dobr- $\bar{a}$ ) und später durch den Verlust der Vokallänge zu kurzvokalischen Endungen (neupoln. dobr-a) mutierten. So ist die heutige 'Kurzform' pewna nicht von der 'Langform' pewna zu unterscheiden. Wahrscheinlich hat dieser Zusammenfall im Polnischen die Schwächung der Kurzform/Langform-Distinktion zum Vorteil der (ohnedies flexibleren) Langformen befördert.
} 


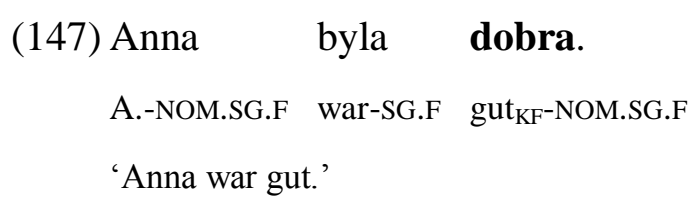

Vgl. in (148) den 'gleichen' Satz, jedoch mit Langformen in prädikativer Position. Im Russischen können letztere im Nominativ oder Instrumental stehen, im Polnischen dagegen fast nur im Nominativ (vgl. Abschnitt 5.5.2): ${ }^{226}$

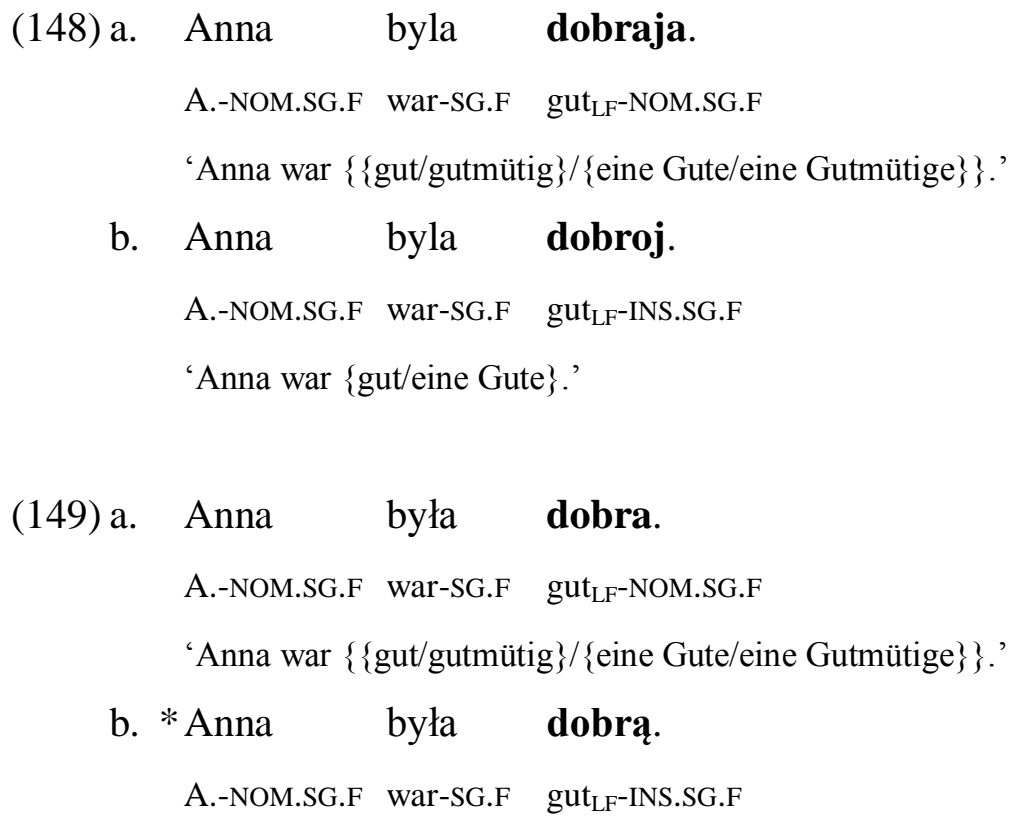

Anders als bei Kurzformen kann ein russischer Sprecher bei Langformen somit i. d. R. zwischen Nominativ und Instrumental wählen. Welche interpretativen Effekte damit assoziiert werden, habe ich bereits in Abschnitt 1.4 erwähnt. In Abschnitt 6 werde ich darauf eingehen, wie es m. E. zu diesen Lesarten kommt. Die klassische "permant vs. temporär"-Deutung lässt sich auch in den deutschen Übersetzungen zu (148) nachvollziehen: Die nominativische Langform in (148a) kann nicht nur als 'gut', sondern auch als 'gutmütig' (ein permanenter Wesenszug) übersetzt werden. Im Fall der instrumentalischen Langform in (148b) hin-

${ }^{226}$ Der Kopulasatz in (149b) ist nur bei Ellipse eines prädikativen Substantivs akzeptabel:

(i) Anna była dobrą keleżanką.

Anna war gute-INS Kollegin-INS

'Anna war eine gute [Kollegin].' 
gegen ist - nota bene ebenso wie bei der Kurzform in (147) - nur die Übersetzung mit 'gut' adäquat (vgl. u. a. Ueda 1992).

Im Polnischen ist es so, dass prädikative Adjektive absolut mehrheitlich in der nominativischen "Langform" auftreten. Abweichungen liegen nur sehr beschränkt bei wenigen Lexemen mit der Option zur Kurzformbildung (z. B. pewny vs. pewien 'gewiss') vor; sehr selten können ausschließlich Kurzformen gebildet werden (z. B. von rad 'gern'). ${ }^{227} \mathrm{Zu}$ den überwiegenden "Langformen" im Nominativ ist zu sagen, dass sie zwar durchaus gleiche Lesarten wie im Russischen haben können, dass letztere jedoch sprachlich nicht explizit werden. Die polnischen Langformen sind, mit anderen Worten, ambig zwischen den verschiedenen Lesarten, die im Russischen durch die Opposition zwischen nominativischen und instrumentalischen Langformen ihre explizite Basis haben (sofern diese Opposition besteht; siehe Abschnitt 5.5.1).

\subsubsection{Mögliche syntaktische Strukturen}

Abgesehen von den o. g. Übersetzungsmöglichkeiten lassen die russischen Langformen - unabhängig von ihrer Kasusmarkierung - stets auch eine deutsche Übersetzung zu, die den indefiniten Artikel und eine Adjektivform zeigt:

(150) a. Anna war eine Gute.

(Deu)

b. Anna war eine gute.

Deutschen Sätzen wie diesen kann, wie (151a) illustriert, ein substantiviertes Adjektiv zugrunde liegen. Ebenso kann aber auch ein phonetisch leeres prädikatives Substantiv $\left(\varnothing_{N}\right)$ mit un(ter)spezifizierter Bedeutung angesetzt werden. Letzteres entspräche einer kontextuell zu bestimmenden Klasse, der der Subjektreferent, von dem durch das Adjektiv noch eine weitere Eigenschaft prädiziert wird, angehört; vgl. (151b): $:^{228}$

${ }^{227}$ In Abschnitt 4.4.3 wird eine Erklärung für die Bildemöglichkeiten von Kurz- und Langformen auf der Basis ihrer kategorialen Merkmale formuliert. Eine Wurzel wie rad-ist als $[+\mathrm{V},+\mathrm{N}]-$ Einheit abgelegt und lässt daher ausschließlich die Bildung von Kurzformen zu.

${ }^{228}$ Vgl. in (i) das englische Äquivalent von (151b), in dem one als overte Entsprechung des angesetzten Nullsubstantivs gewertet werden kann:

(i) Anne was a good one.

(Eng)

'Anna war eine Gute.' 
(151) a. Anna war [NP eine $\mathbf{G u t e}_{\mathbf{N}}$ ]. (prädikatives substantiviertes Adjektiv)

b. Anna war [NP eine [AP gute $\left.\left._{\mathbf{A}}\right] \varnothing_{\mathrm{N}}\right] . \quad$ (prädikatives Nullsubstantiv)

Der Eintrag für das Nullsubstantiv in (151b) sollte wie folgt aussehen, wobei das unspezifizierte Prädikat $P$ als Parameter erst kontextuell bestimmt wird:

$$
[-\mathrm{V},+\mathrm{N},-\mathrm{a},+\max ] \quad \lambda x[P x]
$$

Diese Null wird in (151b) vom Artikel eine begleitet und ist Bezugssubstantiv für das attributive Adjektiv gute. Die V'-Ebene (noch ohne Subjektausdruck) hat demnach die Bedeutung in (153):

(153) $\lambda x \lambda s[s$ INST $[[P x] \wedge[x \mathrm{GOOD}]]]$

Diese Bedeutung hat m. E. auch die $\mathrm{V}^{\prime}$-Ebene in dem Satz mit dem substantivierten Adjektiv eine Gute in (151a), hier jedoch nicht in Folge der Präsenz eines Nullsubstantivs, sondern als Ergebnis der Substantivierung des Adjektivs. ${ }^{229}$ Aus

Auch Heim \& Kratzer (1998) erwägen die Möglichkeit einer solchen Null: "Yet another solution is to posit a certain amount of non-overt structure in VPs of the surface form 'be' + AP or 'be' + PP. Perhaps these VPs contain an invisible predicate that the AP or PP modifies, something like a zero equivalent of a bland noun like 'thing' or 'individual'." (H\&K 1998, 68).

Roy (2013) teilt russische Kopulasätze in drei Klassen: (i) situationsbeschreibend, (ii) charakterisierend und (iii) definierend. In (i) fallen Sätze mit Kurzformen, in (ii) Sätze mit Substantiven im Instrumental oder mit Langformen $+\varnothing_{\text {N-INS }}$ und in (iii) Sätze mit Substantiven im Nominativ oder mit Langformen mit $\varnothing_{\mathrm{N}-\mathrm{NOM}}$. Auch sie nimmt also Nullsubstantive an.

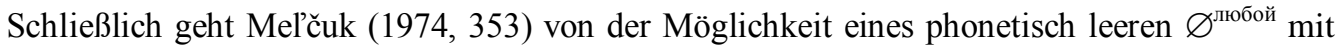
der Bedeutung 'ein beliebiger (Mensch)' aus. Diese Null trete in Sätzen des Typs Solcherlei

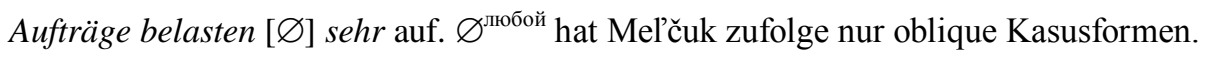

229 Die Substantivierung von Adjektiven setzt deren Anpassung an den attributiven Gebrauch voraus, d. h. aus dem einstelligen Prädikat gut in (i) muss zunächst das Attribut gut in (ii) werden (z. B. durch Predicate Modification nach Heim \& Kratzer 1998, 65):

(i) /gute/ $[-\mathrm{V},+\mathrm{N},+\mathrm{a} \ldots+\max ] \lambda x[x \mathrm{GOOD}] \quad \in\langle\mathrm{e}, \mathrm{t}\rangle$

(ii) /gute/ $[-\mathrm{V},+\mathrm{N},+\mathrm{a} \ldots+\max ] \lambda P \lambda x[[P x] \wedge[x \mathrm{GOOD}]] \quad \in\langle\langle\mathrm{e}, \mathrm{t}\rangle,\langle\mathrm{e}, \mathrm{t}\rangle\rangle$

Erst auf der Basis von (ii) kann es m. E. zur Substantivierung kommen, bei der $\lambda P$ (die Stelle für den Modifikanden) 'blockiert' und $P$ somit zu einem bloßen semantischen Parameter wird:

(iii) $/$ Gute/[-V,+N,-a...+max $] \lambda x[[P x] \wedge[x$ GOOD $]] \in\langle e, t\rangle$

[vgl. (153)!]

Zimmermanns $(1992,264)$ "Modifikationstemplate" hat dieselben Konsequenzen wie die Substantivierung in (ii) bzw. (iii). Zwar nennt Zimmermann (1992) Evidenz für ihr Template, aber 
diesen Erwägungen heraus ist es wahrscheinlich, dass Substantivierungen wie in (151a) dieselbe Interpretation wie Strukturen mit syntaktischer Null wie in (151b) hervorrufen. So lässt sich nicht abschließend klären, welche Struktur jeweils vorliegt. Freilich ist es möglich, dass beide koexistieren bzw. dass eine von beiden präferiert wird.

Schließlich könnte den fraglichen deutschen Sätzen auch die Ellipse des Bezugssubstantivs zugrunde liegen, was voraussetzt, dass das entsprechende Prädikat im Kontext salient ist und daher nicht nochmals explizit genannt werden muss:

Diesen Analysen zufolge liegt bei (155a) ein(e) Substantiv(ierung) als Kopf einer prädikativen NP vor, in (155b) und (155c) dagegen sind attributive Adjektive gegeben, die ein "stummes" oder ein "nicht ausgesprochenes" Bezugssubstantiv modifizieren. Natürlich kann auch eine 'einfache' AP-Struktur wie in (155d) vorliegen, die sich im Deutschen aber deutlich von den bisherigen Strukturen unterscheidet, da prädikative Adjektive keine Flexionsmarkierung erhalten: ${ }^{230}$

(155) a. Anna war [NP eine Gute $_{\mathbf{N}}$ ].

b. Anna war [NP eine $\left[\mathrm{AP}\right.$ gute $\left.\left._{\mathrm{A}}\right] \varnothing_{\mathrm{N}}\right]$.

c. Anna war [NP eine [AP gute $\left._{\mathbf{A}}\right]$ Schülerin $\left._{\mathrm{N}}\right]$.

d. Anna war $\left[\mathrm{AP} \mathbf{g u t}_{\mathbf{A}}\right]$. (prädikative Substantivierung)

(prädikatives Nullsubstantiv)

(Ellipse)

(prädikatives Adjektiv)

Aus syntaktischer Sicht ist festzuhalten, dass (155a-c) Kopulasätze mit prädikativen NPn sind, während nur (155d) auf einer prädikativen AP beruht.

Auch für das Russische kann nicht ohne Weiteres entschieden werden, was für eine Struktur einem Satz wie (148) konkret zugrunde liegt, jedoch sind Substanti-

in Bezug auf Adjektive scheint es mir doch ein zu starkes Instrument zu sein, dessen Verortung im System zudem unklar ist (mentales Lexikon, Syntax, Schnittstelle zwischen beiden?).

Geist (2006, 139) erwähnt, dass russische Kurzformen nicht (mehr) zu Substantiven konvertiert werden können. Dies findet eine plausible Erklärung in deren Verbalnominalität. Nur rein nominale Adjektive (also Langformen) können attributiv gebraucht werden, was soeben als Voraussetzung für deren Substantivierung beschrieben wurde.

${ }^{230}$ Womöglich gehören prädikative Adjektive im Deutschen der verbalnominalen $([+\mathrm{V},+\mathrm{N}])$, attributive Adjektive dagegen der rein nominalen Kategorie $([-\mathrm{V},+\mathrm{N},+\mathrm{a}])$ an 
vierungen von Adjektiven in prädikativer Position relativ unwahrscheinlich. ${ }^{231}$ So erscheint es mit Bezug auf das Russische plausibler anzunehmen, dass (a) eine bloße Adjektivform, (b) ein phonetisch leeres Bezugssubstantiv oder (c) ein elliptisch ausgelassenes Bezugssubstantiv vorliegt. Anders als im Deutschen sind aber im Russischen alle denkbaren syntaktischen Strukturen an der Satzoberfläche homonym. Allein die Verwendung einer Kurzform wie in (156e) lässt sofort erkennen, dass es sich um einen Satz mit prädikativem Adjektiv handelt, da Kurzformen vom attributiven Gebrauch ausgeschlossen sind:

(156) a. 'Anna byla [NP dobraja $\mathbf{a}_{\mathrm{N}}$ ].

(prädikative Substantivierung)

b. Anna byla [NP [AP dobraja d $\left.\left._{A}\right] \varnothing_{N}\right]$. (prädikatives Nullsubstantiv)

c. Anna byla [NP [AP dobraja d $_{A}$ ]ěenicat $]$.

(Ellipse)

d. Anna byla [AP dobraja d $_{\text {] }}$.

(prädikative Langform)

e. Anna byla [AP dobra $_{A}$ ].

(Kurzform)

Anders als Kurz- können die Langformen - mindestens im Falle von Futur, Präteritum, Imperativ, Konjunktiv und infiniten V-Auxiliaren - auch instrumentalisch auftreten:

(157) a. ' Anna byla [NP dobroje $_{\mathbf{N}}$ ].

(prädikative Substantivierung)

b. Anna byla [NP [AP dobroja $\left.\left._{A}\right] \varnothing_{N}\right]$. (prädikatives Nullsubstantiv)

c. Anna byla [NP [AP dobroja $]$ učenicej d $\left._{\mathrm{N}}\right]$. (Ellipse)

d. Anna byla [AP dobroj$\left._{\mathbf{A}}\right]$. (prädikative Langform)

Zentral ist, dass russischen Kopulasätzen mit Langformen theoretisch alle genannten Strukturen zugrunde liegen können, und dass man folglich nicht ohne Weiteres sagen kann, welche Struktur konkret vorliegt. Freilich kann der Kontext hier weiterhelfen (besonders bei Ellipsen), aber ob etwa ein substantiviertes Adjektiv oder ein Nullsubstantiv anzunehmen ist, kann nur auf der Basis theoretischer Erwägungen entschieden werden.

\footnotetext{
${ }^{231}$ Substantivierungen von (Langform-)Adjektiven sind nicht auszuschließen, dürften sich aber auf lexikalisierte Fälle beschränken, vgl. slepoj 'Blinder', gluchoj 'Tauber' etc. Kaum dagegen wird etwa dobryj im Sinne einer deutschen Substantivierung wie 'Guter' verwendet (hier wird eher auf Derivate wie dobrjak 'gutmütiger Mensch’ zurückgegriffen).
} 
Insofern die Langformen demnach per se auf keine der dargelegten Strukturen festzulegen sind, sind sie ebensowenig auf eine bestimmte der aus letzteren folgenden Lesarten festgelegt. Dies gilt gleichermaßen für das Polnische und das Russische, da beide Sprachen über (potenziell attributive) Langformen verfügen. Fasst man das Gesagte zusammen, ergeben sich für russische Adjektive in prädikativer Position unterhalb eines präteritialen, futurischen, imperativischen oder konjunktivischen V-Auxiliars die folgenden Realisierungsoptionen. Die eindeutig prädikative Kurzform setze ich durch graue Schattierung von den Langformen ab:
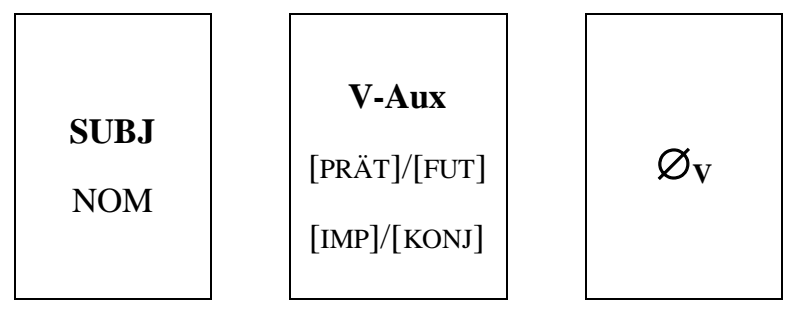

\begin{tabular}{|c|c|}
\hline$\left[{ }_{\mathrm{AP}} \mathrm{KF}\right]$ & $\mathrm{NOM}$ \\
\hline$\left[{ }_{\mathrm{AP}} \mathrm{LF}\right]$ & $\mathrm{NOM}$ \\
\cline { 1 - 1 }$\left[{ }_{\mathrm{NP}} \mathrm{LF} \varnothing_{\mathrm{N}}\right]$ & oder \\
\cline { 1 - 1 }$\left[{ }_{\mathrm{NP}} \mathrm{LF} \mathrm{NP}\right]$ & $\mathrm{INS}$ \\
\hline
\end{tabular}

Es ist zu bemerken, dass sich Kurzformen mit instrumentalischen Langformen im Prinzip decken, da beide gleichermaßen explizit-prädikativ sind. ${ }^{232}$ Jedoch ist eine Kurzform trotzdem immer 'eindeutiger', da sie keinesfalls ein 'verdecktes Attribut' sein kann.

Liegt ein russischer präsentischer Kopulasatz vor, sieht die Lage anders aus:
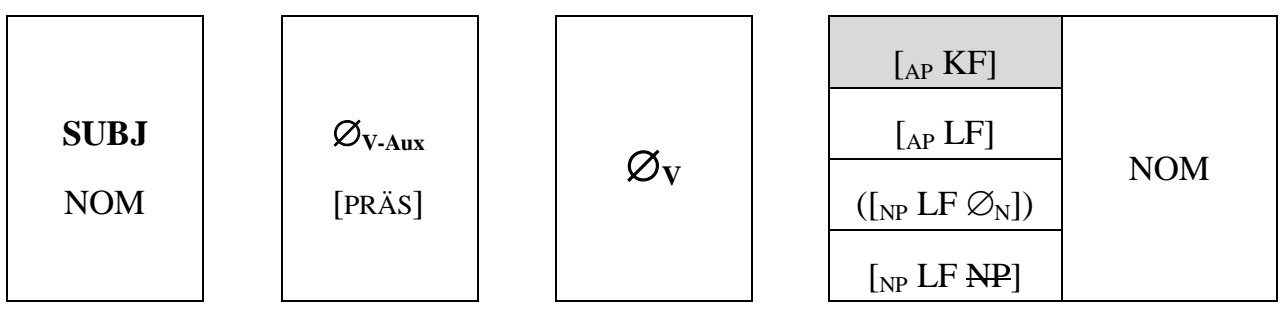

Ist schließlich ein infinites $\mathrm{V}$-Auxiliar gegeben, stehen die folgenden Realisierungsoptionen zur Verfügung: ${ }^{233}$

${ }^{232}$ Das gilt für eine Kurzform immer, für eine instrumentalische Langform jedoch nur dann, wenn sie in potenzieller Opposition zu einer nominativischen Langform steht. Interpretativ bedeutet dies, dass dem Subjektreferenten die Eigenschaft ohne weitere 'Effekte' zugeschrieben wird.

${ }^{233}$ Bei Raising-Strukturen ist auch der Nominativ am Prädikatsnomen zulässig, da sich dann eine syntaktische Spur des 'angehobenen' Subjektausdrucks in Spec-VP befindet, die offenbar die Rekonstruktion des Nominativmerkmals erlaubt (Geist 2006, 105). 
(160)

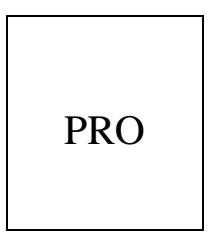

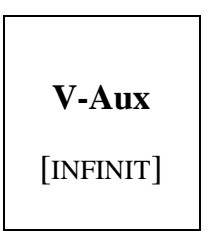

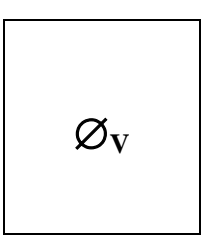

Im Polnischen ist der Instrumental bei prädikativen Adjektiven nur dann zulässig, wenn ein nominativischer Kongruenzgeber in der Struktur absolut unzugänglich ist (siehe oben). Insofern ist die Nominativkongruenz des prädikativen Adjektivs im Polnischen weniger restringiert als im Russischen, wo sie offenbar lokal im jeweiligen Clause hergestellt werden muss. Evidenz dafür wurde bereits im vorangehenden Abschnitt gebracht.

Für polnische Adjektive in Prädikatposition ergibt sich folgendes Gesamtbild:
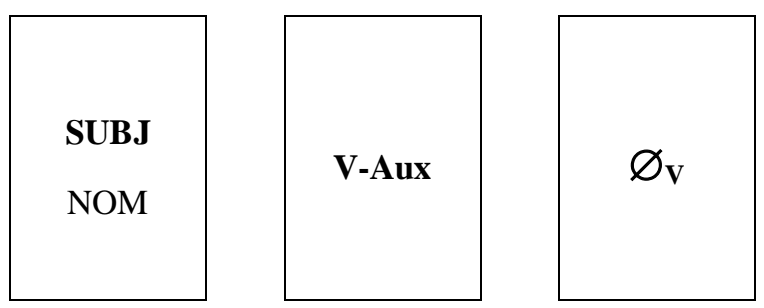

\begin{tabular}{|c|c|}
\hline$\left[{ }_{\mathrm{AP}} \mathrm{KF}\right]$ & \multirow{2}{*}{$\mathrm{NOM}$} \\
\cline { 1 - 1 }$\left[{ }_{\mathrm{AP}} \mathrm{LF}\right]$ & \\
\cline { 1 - 1 }$\left[{ }_{\mathrm{NP}} \mathrm{LF} \varnothing_{\mathrm{N}}\right]$ & \multirow{2}{*}{$\mathrm{INS}$} \\
\cline { 1 - 1 }$\left[\mathrm{NP}_{\mathrm{NP}} \mathrm{LF} \mathrm{NP}\right]$ & \\
\hline
\end{tabular}

Hier ist zu beachten, dass es sich nur bei solchen Kurz- oder Langformen um 'echte' prädikative Adjektive handelt, die ein prädikatives $\mathrm{A}^{0}$ sind. Adjektive, die ein prädikatives Substantiv, das phonetisch leer oder elliptisch ausgelassen ist, lediglich modifizieren, sind keine prädikativen Ausdrücke. Dass diese Attribute im Polnischen im Instrumental erscheinen, ist klar, da prädikative Substantive im Polnischen fast immer den Instrumental erhalten (siehe oben).

Was die Fälle von Matrixstrukturen betrifft, die keinerlei nominativischen Subjektausdruck beinhalten, so erfordern diese eine zweite Tabelle. Hier ist stets nur der Instrumental zulässig; vgl. (162):
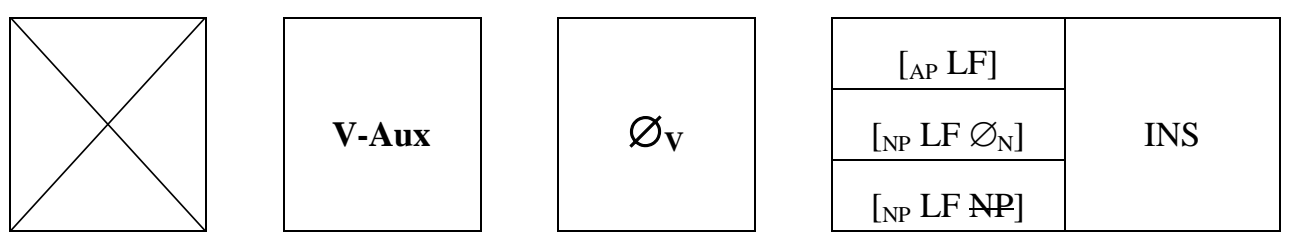

Dieser Abschnitt hat gezeigt, dass sowohl russischen als auch polnischen Kopulasätzen mit Adjektiven in der Komplementposition des abstrakten V-Kopfes (der 
Kopulabedeutung) diverse syntaktische Strukturen zugrunde gelegt werden können, die ich in (163) nochmals zusammenfassend darstelle:

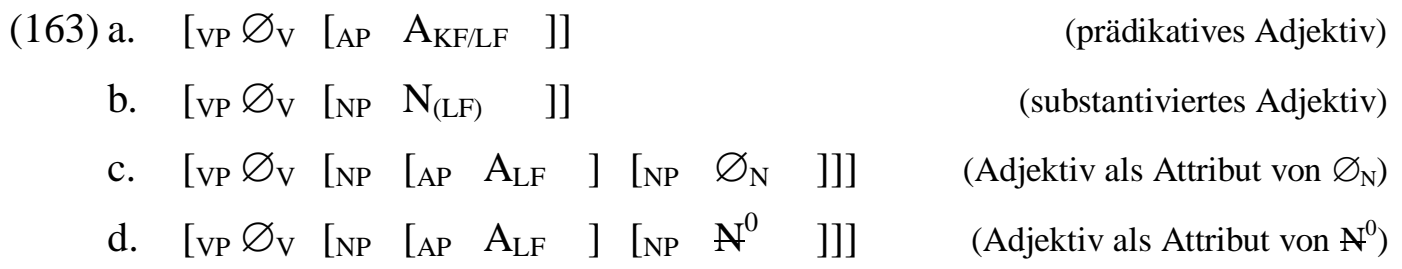

Diese Strukturen fallen an der Satzoberfläche theoretisch zusammen, sofern nicht eine Kurzform verwendet wird, die eindeutig als Prädikativ identifiziert werden kann. Die Langformen sind dagegen potenziell mehrfach ambig. Die vorgeschlagenen Analysen genügen, um alle in der Fachliteratur genannten Lesarten bzw. 'interpretativen Effekte' der (ggf. nur vermeintlich) prädikativen Langformen zu erfassen. Dass Langformen eine 'permanente' und eine 'temporäre' Lesart erhalten können, hat damit zu tun, dass sie kraft ihres Paradigmas sowohl im Nominativ als auch im Instrumental auftreten können. Ferner ergeben sich Übertragungen wie dt. eine guteleine Gute etc. aus der möglichen Präsenz eines Nullsubstantivs, aus der Ellipse eines Bezugssubstantivs oder aus einer Substantivierung.

Von sich aus sollte man weder den Lang- noch den Kurzformen eine spezifische Eigenbedeutung zuschreiben, da man in Erklärungsnöte kommt, wenn eine Adjektivform nicht die erwartete Lesart zeitigt. ${ }^{234}$ Dann müssten, wie bei prädikativen

${ }^{234}$ Gegen die Festlegung der russischen Adjektivformen auf irgendeine spezifische Eigenbedeutung spricht sich bereits Kiparsky (1967) aus, wenn er schreibt: “[...] in sämtlichen Fällen, wo die sogenannte 'kurze' oder Nominalform gebraucht werden kann oder noch gewöhnlich gebraucht wird, kann [...] bereits ohne Verstoß gegen die Sprachrichtigkeit auch die 'lange' oder Pronominalform gebraucht werden. Obgleich es also z. B. noch möglich, ja sogar üblich ist, zu sagen он бо́лен 'er ist krank', она́ больна́ 'sie ist krank', она́ здоро́ва 'sie ist gesund', соба́ки злы 'die Hunde sind böse', ist es kein direkter Fehler, wenn man он больно́й, она́ здоро́вая, соба́ки зль́е sagt. Die Unterscheidung der beiden Formen als etwa 'er ist zeitweilig bzw. ständig krank' ist klare Schulfuchserei, die nicht mehr ernst genommen wird. [...]” (Kiparsky 1967, 107-108; Sperrsatz im Original). Er fügt etwas später hinzu: "Es gibt jedoch, wie Isačenko 1962, 149-151 mit Recht betont, 'in der gepflegten Hochsprache' immer noch Fälle, wo die Nominalform im Nom. 'entweder die einzig mögliche oder doch die üblichere' ist. Diese Fälle gehören aber m. E. nicht mehr in die Morphologie, sondern höchstens in die Syntax, wenn nicht gar nur in die Stilistik." (Kiparsky 1967, 108). Ersteres Zitat spricht für die auch hier vertretene Ansicht, dass Lang- und Kurzformen mithin identische Lesarten haben (können). Auch letzterem Zitat schließe ich mich an, wobei es sich m. E. um eine Frage der 
Substantiven, Zusatzmechanismen ersonnen werden, die dafür sorgen, dass die veranschlagte Eigensemantik 'neutralisiert' wird.

\subsubsection{Zustandsprädikative}

Wie bereits erwähnt, möchte ich die häufig als "Zustandsprädikativa" bezeichneten Formen nicht als eigenständige lexikalische (Sub-)Kategorie bzw. Wortart, sondern als besonderen Verwendungstyp prädikativer Verbalnomina, darunter überwiegend Qualitätsadjektive bzw. Kurzformen, betrachten. In diesem Zusammenhang erscheint mir zunächst ein forschungshistorischer Überblick betreffs der Kategorie der "Prädikativa" angebracht.

\section{Forschungsüberblick}

Ščerba (1928) führt als erster den Begriff "Zustandskategorie" (kategorija sostojanija) in die russistische Sprachbetrachtung ein. Er meint damit prädikative Formen, die psychische, physische, modale oder Naturzustände denotieren. Ščerba zählt hierzu nicht ausschließlich unveränderliche bzw. unflektierbare Wortformen, sondern auch adverbiale Ausdrücke verschiedenen Typs (vgl. Rozental' \& Telenkova 1972, 131). Er ist jedoch unsicher, ob es sich um eine "klare und überzeugende Kategorie" (Ščerba 1928, 17) handele. Vinogradov \& Istrina (1960) grenzen die "Zustandskategorie" auf nicht flektierbare/adverbartige Wortformen ein, denen sie auch die Kurzformadjektive zuordnen. In der Folge werden die vermeintlichen Mitglieder der "Zustandskategorie" mitunter auch einfach als "prädikative Adverbien" beschrieben (z. B. AG 1980). Sofern eine gesonderte "Zustandskategorie" angenommen wird, herrschen divergierende Ansichten über deren Umfang (vgl. z. B. Vinogradov \& Istrina 1960 mit Galkina-Fedoruk 1964).

Innerhalb der "Zustandskategorie" wird meist weiter zwischen "Zustandsprädikativa" und "Modalprädikativa" unterschieden. Dabei bezeichnen erstere i. d. R. eigenständig Zustände, z. B. russ. cholodno oder poln. (jest) zimno 'es ist kalt', während letztere grammatikalisierte Modalausdrücke sind, die eine bestehende

"Stilistik" bzw. Pragmatik handelt, während für die Syntax völlig irrelevant ist, ob eine Langoder Kurzform als Komplement der Kopulabedeutung verwendet wird. Ich danke Uwe Junghanns für den Hinweis auf Kiparskys (1967) Aussagen. 
Proposition modalisieren, z. B. russ. nado oder poln. trzeba 'nötig' (vgl. u. a. Krüger 1996). Isačenko (1954, 359; 1962, 194ff.) sieht hier Vertreter einer relativ jungen Wortart, die er selbst "Prädikativa" nennt. Vor- und Nachteile einer Übertragung auf das Tschechische erwägt z. B. Komárek (1954). In Bezug auf das Polnische verwendet Wolińska $(1978,36)$ den Begriff "Prädikator", wobei sie den kategorialen Status der betreffenden Elemente beiseite lässt (vgl. Bondaruk \& Szymanek 2007, 83). Kritik an der Etablierung dieser neuen Wortart übt Sperber (1972), wie schon Travníček und Šapiro vor ihm (siehe Rozental' \& Telenkova 1972, 131).

Generell herrschen sehr unterschiedliche Ansichten bzgl. des Umfangs und der Kategorie der fraglichen Formen. Während Vostokov (z. B. ${ }^{4} 1839$ ) und nach ihm auch Šachmatov (1927) die Formen der "Zustandskategorie" der verbalen Domäne zuordnen, sieht u. a. Aksakov (1880) in ihnen Kurzformadjektive, die lediglich die Bedeutung von Verben annehmen bzw. diese in ihrem syntaktischen Kontext (erst) erhalten. Anders positionieren sich u. a. Potebnja (1958) und Bogorodickij $\left({ }^{5} 1935\right)$, wenn sie die fraglichen Formen jeweils unterschiedlichen Wortarten zuordnen. Peškovskij sieht sich gar dazu genötigt, sie gar keiner Wortart zuzuordnen (Rozental' \& Telenkova 1972, 131).

Auch in neueren Arbeiten herrscht keine Einigkeit, insbesondere auch bei der Unterscheidung in "Zustands-" und "Modalprädikativa". Laut Zybatow (1994) sind "Zustandsprädikativa" prädikative Adjektive (vgl. meine eigene Analyse), "Modalprädikativa" dagegen (Modalverben vergleichbare) Modalausdrücke. Für Schoorlemmer (1994, 1995) sind "Modalprädikativa" modale Prädikate, in denen Hansen (2009) wiederum “dekategorisierte Modale” erkennt. Nach Fortuin (2000) bilden Modalprädikativa das 'prädikative Zentrum' des Satzes, wozu 'ZZustandsprädikativa" nicht in der Lage seien (diese Rolle spiele dann stattdessen die Kopula als Vollverb). Hentschel (2001) untersucht primär die Möglichkeiten der morphosyntaktischen Realisierung von Experiencer-Ausdrücken mit "Prädikativa”, deren Hauptmerkmal er in der Fähigkeit sieht, quasi autonom als Satzprädikat zu funktionieren. Geist (2006, 2010) analysiert Kurzformen mit den Mitteln der Distributed Morphology als syntaktisch derivierte Verbformen; wenn sie auch nichts zu den "Zustandsprädikativa" sagt, nimmt sie damit im Prinzip die gleiche Position wie schon Vostokov $\left({ }^{4} 1839\right)$ und Šachmatov (1927) (siehe oben).

Für Bartnicka (1972), Wolińska (1978) und Saloni (1974) ist der kategoriale Status der polnischen "Prädikativa" unsicher, sie betonen jedoch deren 'verbale 
Natur'. Bondaruk \& Szymanek (2007, 84) sprechen bei polnischen Wortformen wie wesoło 'fröhlich', smutno 'traurig' etc. von "prädikativen Adverbien", während sie desubstantivische Formen wie wstyd 'Scham', żal 'schade', szkoda 'schade' als Verben (!) kategorisieren. Junghanns \& Lenertová (2010) sehen in "Prädikativa" eine mögliche Unterkategorie lexikalischer Hauptkategorien und setzen dafür ein subkategorisierendes Merkmal [+Praed] an (vgl. Pitsch 2012a).

M. E. zeigt dieser nur kursorische Forschungsstand, wie heterogen die Ansichten in diesem Bereich sind. Aus allgemein-linguistischer Perspektive wirkt die Idee von "Prädikativa" als einer eigenständigen lexikalischen Kategorie mitunter attraktiv. Vor dem Hintergrund minimalistischer Prinzipien und entsprechender theoretischer Ansprüche sollte die Annahme einer 'neuen Wortart' jedoch eingehend geprüft werden, da der Verzicht auf eine derartige 'Bereicherung' des Systems, sofern möglich, zu bevorzugen ist. Ebendarum werde ich mich bemühen.

\section{Bemerkung zu "Modalprädikativa"}

Wie im Forschungsüberblick deutlich wurde, werden als "Prädikativa" nicht lediglich prädikative Formen bezeichnet, die psychische, physische oder Naturzustände ausdrücken, sondern mitunter auch solche, die eine bestimmte Modalität einspeisen. Diese Formen sind hinsichtlich ihrer semantischen Leistung demnach den Modalverben in vielen (auch slavischen) Sprachen vergleichbar, unterscheiden sich jedoch insofern von letzteren, als sie 'unpersönlich' sind, was sich im Ausschluss eines nominativischen Subjektausdrucks zeigt. Mit den "Modalprädikativa" können stattdessen nur optionale Dative erscheinen. Fehlt ein solcher Dativausdruck, wird der Satz bzw. die Modalität generisch interpretiert (vgl. dt. man muss/kann). Der Form bzw. ihrem historischen Ursprung nach lassen die "Modalprädikativa" keine klare Kategorisierung zu, was teilweise zu ihrer Bezeichnung als "Prädikativa", teilweise als Adverbien geführt hat. Folgende Beispiele illustrieren "Modalprädikativa" im Polnischen:

$\begin{array}{cllllll}\text { (164) [T]rzeba } & \text { będzie } & \text { prowadzić } & \text { ją } & \text { do } & \text { lekarza[.] } & \text { NKJP } \\ \text { NECESS } & \text { wird sein } & \text { bring-INF } & \text { sie-ACC } & \text { zu } & \text { Arzt-GEN } & \end{array}$
'Es wird notwendig sein, sie zum Arzt zu bringen.' 
$\begin{array}{ll}\text { (165) Te owoce można jeść. } & \text { ess-INF } \\ \text { dieses Obst-ACC.PL POSS } & \\ \text { 'Dieses Obst kann man essen.' }\end{array}$

Anders als im Russischen, wo im Präsens generell keine overte Form vom byt' als "Kopula" erscheint, ist dies im Polnischen in Form von jest die Regel. Bemerkenswert bei den "Modalprädikativa" ist hierbei, dass sie - auch im Polnischen niemals mit jest auftreten:
(Skibicki 2007, 309)

\author{
niemals mit jest auftreten:
}

(166) Trzeba (*jest) prowadzić ją do lekarza. NECESS ist bring-INF sie-ACC zu Arzt-GEN

'Es ist notwendig, sie zum Arzt zu bringen.'
(167) Te owoce (*jest) można jeść.
dieses Obst-ACC.PL ist POSS ess-INF
'Dieses Obst kann man essen.'

Das ist eine deutliche Differenz zu den Zustandsprädiativen, für die oben festgestellt wurde, dass sie durchaus mit jest auftreten (können). M. E. deutet der Ausschluss des V-Auxiliars bzw. der "Kopula" bei den "Modalprädikativa" auf deren Status als 'grammatische' Einheiten hin. So lässt sich auch eine klare Parallele zu den Modalverben wie poln. musieć 'müssen', móc 'können', russ. moč' 'können' ziehen, da auch letztere formal Verbformen sind, im Satz jedoch lediglich der semantischen Modalisierung dienen. Auch die "Modalprädikativa" treten mit Infinitiven auf, die sie in gleicher Weise, wenn auch 'unpersönlich', modalisieren. Zustandsprädikative dagegen referieren selbständig auf in der Welt bestehende Zustände - sie modifizieren also nicht nur. Daher lassen sie sich auch mit jest verbinden, das in Präsenssätzen quasi den Bezug auf einen Zustand ausdrückt. Dass auf jest $\mathrm{u}$. U. auch verzichtet werden kann, werde ich auf den verbalnominalen Status der Zustandsprädikative zurückführen.

\title{
Analyse
}

Im Folgenden werde ich mich bemühen zu zeigen, dass und wie man auf eine spezielle lexikalische (Sub-)Kategorie "Prädikativum" sowohl im Russischen als 
auch Polnischen verzichten kann, ohne im Hinblick auf die Beschreibung und Analyse von Kopulasatzstrukturen mit Formen wie poln. ciepło 'warm', warto 'wert', wstyd 'Scham' oder russ. prijatno 'angenehm', veselo 'fröhlich', pora 'Zeit' an Explikativität und Aussagekraft etwas zu verlieren.

Ziel meiner Analyse ist es, die 'zustandsprädikative' Verwendung bestimmter Formen, seien diese qualitätsadjektivisch, substantivisch oder infinitivisch, sowie deren Konsequenzen für Argumentrealisierung und Kongruenz zu erklären, ohne eine neue (Sub-)Kategorie ansetzen zu müssen.

Worauf ich mich bei dieser Analyse stützen werde, sind einerseits Komponenten bestehender slavistischer Analysen; andererseits werde ich auch den Vergleich mit nicht-slavischen Sprachen wie dem Deutschen heranziehen, da er m. E. nicht zu vernachlässigende Einblicke v. a. in die Syntax der fraglichen Kopula-Prädikativ-Strukturen gewährt.

Vergleicht man russische bzw. polnische Sätze, die Zustandsprädikative enthalten, mit ihren deutschen Entsprechungen, so fällt auf, dass im Deutschen stets das overte Subjektpronomen es erscheint, für das in beiden slavischen Sprachen keine (overte) Parallele vorliegt:

(168) a. Jest zimno.

b. Es ist kalt.

(169) a. V Moskve zimoj cholodno.

b. In Moskau ist es im Winter kalt.

(170) a. Tam nie jest bezpiecznie, kochanie.

b. Dort ist es nicht sicher, meine Liebe.

(171) a. Czas było jechać.

b. Es war (an der) Zeit zu fahren.

Allerdings ist es im Deutschen nicht immer obligatorisch. Mitunter kann es lediglich ein Platzhalter in der syntaktischen Subjektposition sein, sofern der eigentliche Subjektausdruck, bei dem es sich auch um einen Subjektsatz handeln kann, nicht satzinitial erscheint: 
(172) a. Czytać dobre książki jest pożytecznie.

b-i. Es ist nutzbringend, [gute Bücher zu lesen].

(Deu)

b-ii. [Gute Bücher zu lesen], ist nutzbringend.

(173) a. Katat'sja veselo.

b-i. Es ist lustig, [herumzufahren].

(Deu)

b-ii. [Herumzufahren] ist lustig.

Solcherlei Beispiele lassen es zu, das Zustandsprädikativ jeweils als 'normales' Prädikatsnomen zu analysieren, das mit dem infinitivischen Subjektsatz quasi kongruiert, indem es die Kongruenzmarkierung annimmt, welche der Abwesenheit irgendwelcher Merkmale entspricht. Dies ist im Russischen stets $-o$, während es im Polnischen entweder - o oder - $e$ sein kann. Sofern man also czytać dobre ksiązki bzw. katat'sja jeweils als Subjektsatz ansieht und die folgenden Wortfolgevarianten der Informationsstruktur zuschreibt, erhalten diese Sätze eine wirksame Analyse:

(174) a. Jest pożytecznie czytać dobre książki.

'Es ist nutzbringend, gute Bücher zu lesen.'

b. Veselo katat'sja.

'Es ist lustig, herumzufahren.'

Von zentraler Bedeutung sind hier jene oben exemplifizierten polnischen und russischen Sätze, deren deutsche Übersetzungen zwangsläufig das Subjektpronomen es beinhalten. Diese Zwangsläufigkeit deutet darauf hin, dass es in diesen Fällen Argumentstatus hat und nicht lediglich syntaktischer Stellvertreter eines anderen, wirklichen Subjektausdrucks ist. Dies wiederum lässt die Annahme plausibel sein, dass es selbst referiert, wenn auch auf etwas äußerst Allgemeines bzw. Unkonkretes:

$(175) *($ Es $)$ ist kalt.

Entgegen der Annahme, es handele sich bei kalt in Sätzen wie (175) um rein propositionale bzw. nullstellige Prädikate (siehe u. a. Bierwisch 1988), gehe ich also davon aus, dass kalt auch in einem Satz wie (175) ein einstelliges Prädikat ist, mit 
dem eine Eigenschaft über einen Referenten prädiziert wird. Dasselbe gilt m. E. für die polnischen und russischen Äquivalente solcher deutschen Sätze:

(176) a. (Jest) zimno.

b. Cholodno. beide: 'Es ist kalt.'

(177) a. Było zimno.

b. Bylo cholodno.

beide: 'Es war kalt.'

Die Argumentstruktur und grammatische Bedeutung von kalt bzw. zimno und cholodno ist m. E. daher die in (178):

(178) $\lambda x[x$ COLD $] \in\langle e, t>$

Das ist dasselbe, was ich generell für Adjektive annehme (siehe Abschnitt 4.4.3). Insofern lautet also meine erste Annahme zu den Zustandsprädikativen, dass sie sich nicht von 'normalen' Adjektiven in prädikativer Position unterscheiden. Ebenso wie letztere, weisen auch sie ihrem Referenten (dem externen Argument) die von ihnen bezeichnete Eigenschaft zu.

Der Unterschied zu 'normalen' Kopulasatzstrukturen muss demnach woanders liegen. Hier komme ich auf den obigen Vergleich zu den deutschen Sätzen zurück, die obligatorisch das Subjektpronomen es aufweisen. Ich schrieb, dass der Subjektausdruck es (der kein bloßer syntaktischer Stellvertreterausdruck und daher auch keinesfalls auslassbar ist) referiert, jedoch auf etwas sehr Allgemeines bzw. Unkonkretes. Das relevante deutsche es kann m. E. als 'unverbindliche' Variante des 'verbindlichen' etwas betrachtet werden. Der Sprecher macht durch den Gebrauch von es klar, dass er sich auf keinen konkreten Referenten festlegen will oder kann (verwendet er hingegen etwas, ist klar, dass er das könnte, wenn danach gefragt würde - in diesem Sinne schließt es eine solche Nachfrage im Vornherein aus). ${ }^{235}$

${ }^{235}$ Ich danke Uwe Junghanns für viele wertvolle Gespräche, in deren Verlauf sich diese und weitere Ideen herauskristallisieren konnten. 
Mit coś und čto-nibud'/čto-to haben Polnisch und Russisch durchaus äquivalente Entsprechungen zum deutschen Indefinitpronomen etwas. Eine overte Entsprechung zum beschriebenen deutschen es hingegen fehlt in diesen beiden slavischen Sprachen. Da aber die polnischen und russischen Sätze mit Zustandsprädikativen dieselbe Bedeutung und indefinit-unkonkrete Referenz haben wie ihre deutschen Entsprechungen mit dem fraglichen overten es, liegt es m. E. nahe, in ersteren eine koverte bzw. "stumme" Entsprechung des letzteren anzusetzen. Mit anderen Worten: Ich werde annehmen, dass polnische und russische Kopulasätze mit Zustandsprädikativen ein phonetisch leeres Subjektpronomen enthalten: ${ }^{236}$

(179) a. $\varnothing_{\text {es }}$ było zimno.

b. $\varnothing_{e s}$ bylo cholodno.

Das Nullpronomen, das ich hier mit $\varnothing_{e s}$ bezeichne, sollte im Prinzip die gleiche grammatische Bedeutung wie dt. es/etwas haben. Es bezeichnet folglich etwas Indefinites und nicht zu willentlichen Handlungen Fähiges. In diesem Sinne eignet es sich zum 'Verweis' auf 'Größen', deren Ursprung und Verortung dem Menschen seit jeher unklar sind (und die allenfalls auf etwas - dem Menschen nicht minder unzugängliches - Allmächtiges/Göttliches zurückgeführt werden). In ihrer Analyse slavischer Impersonale mit Akkusativ(objekten), z. B. den sog. Adversitätsimpersonalen, die durch das folgende Beispiel illustriert werden, setzen Fehrmann, Junghanns \& Lenertová (2013) ein leeres Subjektpronomen an, dass sie als indefinit und non-volitional charakterisieren: " $\varnothing_{[+ \text {indef,-vol]". Man }}$ könnte sagen, dass die Verwendung dieser syntaktischen Null die insofern grundlegendste Form des Impersonalen darstellt, als sie sich zu einer Naturkraft-Interpretation eignet, in der man sicherlich die ursprünglichste bzw. älteste Form impersonaler Strukturen vermuten darf: ${ }^{237}$

236 Die Annahme von leeren Subjektpronomina ist auch für slavische Sprachen nicht neu. So geht Mel’čuk (1974) von mehreren Arten von Nullpronomina im Russischen aus, während z. B. Franks (1995, 292-293) in slavischen Sprachen Nullexpletiva in Sätzen mit non-thematischen Subjekten für möglich hält. Auf den Ansatz von Fehrmann, Junghanns \& Lenertová (2013), der von einer syntaktischen Null in den sog. Adversitätsimpersonalen in slavischen Sprachen ausgeht, komme ich im Folgenden detailliert zu sprechen.

${ }^{237}$ Es ist zu betonen, dass $\varnothing_{\text {[+indef,-vol] }}$ nicht selbst eine Naturkraft bezeichnet wie z. B. Mel'čuks (1974) $\varnothing^{\text {стихия }}$, sondern in der Tat lediglich auf eine indefinite und non-volitionale Entität ver- 
Da Aussagen über Naturzustände à la Es ist kalt etc. kaum weniger ursprünglich sein dürften, liegt es nahe, auch in ihnen dasselbe Nullpronomen mit indefiniter und non-volitionaler Bedeutung anzunehmen, d. h. " $\varnothing_{[+ \text {indef,-vol] }}$ " nach Fehrmann, Junghanns \& Lenertová (2013) bzw. " $\varnothing_{e s}$ ", wie ich es weiter oben nannte. Dieses Nullsubjekt wird bei solchen regulär einstelligen Prädikaten wie poln. zimn'kalt', pożyteczn- 'nutzbringend', russ. cholodn- 'kalt', vesel- 'fröhlich' usw. als externes Argument eingesetzt und fungiert demnach als Satzsubjekt. Interpretativ entspricht es dem dt. Pronomen es, wie es ich weiter oben beschrieben habe. Was seine morphosyntaktischen Merkmale angeht, so ist $\varnothing_{e s}$ 'unpersönlich' bzw. 'merkmallos', was sich an der Kongruenzmarkierung der Zustandsprädikative zeigt, die - sofern es sich um adjektivische Formen handelt - ebenfalls stets 'unpersönlich' ist. Als Subjektausdruck hat $\varnothing_{e s}$ jedoch den Nominativ. ${ }^{238}$

Die soeben genannten Beispiele von Zuständsprädikaten basieren allesamt auf Wurzeln oder Stämmen von Qualitätsadjektiven. In der Tat unterscheidet sich die Mehrheit der Zustandsprädikative also lediglich im Subjektausdruck von 'normalen’ Kopulasätzen mit Qualitätsadjektiven in prädikativer Position:

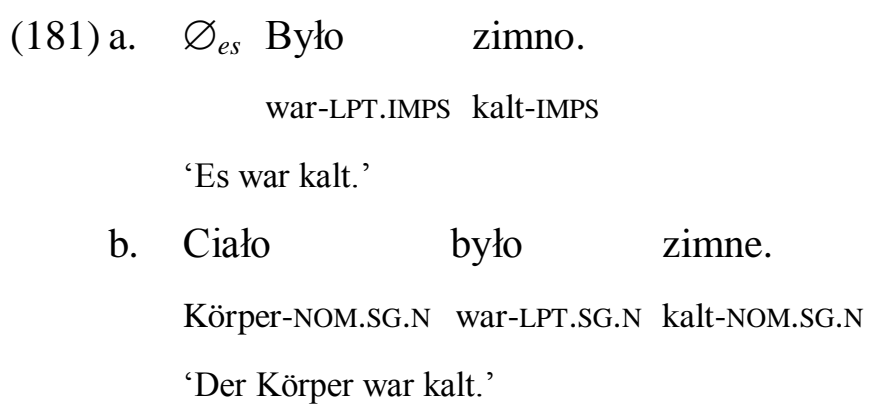

weist, deren 'Offenheit' quasi die Identifikation mit einer Naturkraft zulässt, die laut Fehrmann, Junghanns \& Lenertová (2013) als Instrumental-Adjunkt realisiert werden kann.

${ }^{238}$ Siehe dazu Fehrmann, Junghanns \& Lenertová (2013), die den Nominativ für " $\varnothing_{\text {[+indef,-vol] }}$ explizit annehmen, um den Akkusativ in impersonalen Strukturen zu erklären (vgl. 'Burzios Generalisierung'). 

(182) a. $\varnothing_{e s}$ bylo cholodno.
war-LPT.IMPS kalt-IMPS
'Es war kalt.'
b. Nina byla cholodna.
Nina-NOM.SG.F war-LPT.SG.F kalt $_{\mathrm{KF}}$-NOM.SG.F
'Nina war kalt (/kühl).'
c. Ladon' byla cholodnaja.
Handfläche-NOM.SG.F war-LPT.SG.F kalt $_{\mathrm{LF}}$-NOM.SG.F
'Die Hand(fläche) war kalt.'

(Rus)

Die Markierung ist bei den Zustandsprädikativen stets 'unpersönlich', da das hier angesetzte Nullsubjekt ebenfalls 'unpersönlich' ist. Die Zustandsprädikative, sofern adjektivisch, sind ferner stets Kurzformen, die nach meinen Annahmen aus Abschnitt 4.4.3 Verbalnomina sind $([+\mathrm{V},+\mathrm{N}])$. Dass dem so ist, überrascht insofern wenig, als Zustandsprädikative eben die Qualität von Zuständen bezeichnen, weswegen hierzu naheliegend meist Qualitätsadjektive herangezogen werden. Wie schon für 'persönlich' flektierte Kurzformen in Abschnitt 4.4.3 geschehen, demonstriere ich im Folgenden die Ableitung einer 'unpersönlichen' Kurzform bzw. Zustandsprädikativs, und zwar am russischen Beispiel cholodno 'kalt':

$$
\begin{aligned}
& \text { (183) a. /cholodn/ } \quad\left[\alpha \mathrm{V},+\mathrm{N},(+\mathrm{a})_{\alpha}-\max \right] \lambda x[x \mathrm{COLD}] \\
& + \text { b. } / \mathrm{o} / \quad[\mathrm{NOM}] \quad \lambda P_{[ \pm \mathrm{V},+\mathrm{N},-\max ]}[P] \\
& =\text { c. } / \text { cholodno } /[+\mathrm{V},+\mathrm{N}, \mathrm{NOM}] \quad \lambda x[x \text { COLD }]
\end{aligned}
$$

Das Ergebis in (183c) ist eine Kurzform ohne Kongruenzmerkmale. Als Einsetzungsinstanz für die externe Argumentstelle eignet sich folglich nur ein ebenso 'unpersönlicher' Ausdruck, namentlich das oben beschriebene $\varnothing_{e s}$. Da sowohl die Kurzform als auch $\varnothing_{e s}$ den Nominativ aufweisen, kann das Zustandsprädikativ ordnungsgemäß kongruieren. Es nimmt nicht wunder, dass daher Zustandsprädikative genauso wie alle anderen Kurzformen nicht auftreten können, wenn kein nominativischer Kongruenzgeber in Reichweite ist, wie es z. B. in infiniten Einbettungen der Fall ist, vgl. (184):

(184) a. *... żeby PRO być wesoło.

b. *... čtoby PRO byt' veselo. 
In finiten Nebensätzen sind Zustandsprädikative dagegen problemlos einsetzbar, da $\varnothing_{e s}$ seine eigene, wenn auch indefinit-unkonkrete, Referenz hat, vgl. (185):

(185) a.

... żeby $\varnothing_{e s}$ było wesoło.

b. ... čtoby $\varnothing_{e s}$ bylo veselo.

beide: '..., damit es fröhlich sei/ist.'

Da ich Zustandsprädikative als 'unpersönliche' Kurzformen betrachte, und da Kurzformen in meiner Analyse nicht reine Nomina, sondern Verbalnomina sind, lässt sich m. E. auch relativ einfach die Möglichkeit der Auslassung des finiten V-Auxiliars jest in polnischen Präsenssätzen erklären, vgl. erneut (186):

(186) (Jest) zimno.

'Es ist kalt.'

Das 'Nichtaussprechen' von jest ist hier einerseits damit begründbar, dass diese Form des V-Auxiliars hier nichts weiter als das 'unmarkierte' Tempus Präsens ausbuchstabiert, da dieser Satz, wie gesagt, 'unpersönlich' ist und somit keine Kongruenzmerkmale beinhaltet. Als zweite Basis für die Auslassung kann die verbale Komponente der zustandsprädikativen Kurzformen geltend gemacht werden, durch die sie per se - anders als die rein nominalen Langformen - als Satzprädikate identifiziert werden können (schließlich treten sie nie attributiv auf). Wenn man Kurzformen im Allgemeinen und Zustandsprädikative im Besonderen als Verbalnomina betrachtet, lässt sich leicht ein Bogen zu Intuitionen wie schon der von Aksakov (1880) schlagen, der in ihnen Adjektive erkannte, die die 'Bedeutung von Verben' annehmen bzw. diese in ihrem syntaktischen Kontext erhalten würden. Eine Kategorisierung als $[+\mathrm{V},+\mathrm{N}]$-Einheiten kann dieses 'fluktuierende' Verhalten ohne Weiteres erfassen.

Was schließlich (de)substantivische Zustandsprädikative wie im folgenden Beispiel betrifft, so nehme ich einen Kategorienwechsel im Lexikon, der bei geeigneten Prädikaten mehr oder weniger 'zufällig' erfolgt ist bzw. erfolgen kann: ${ }^{239}$

${ }^{239}$ Wie schon weiter oben ausgeführt, ist ein Indiz dafür, dass es sich bei czas in der Tat um ein Zustandsprädikativ im hier verwendeten Sinne handelt, dass das Pronomen es in der deutschen 
(187) Następnie było czas jechać do Wrocławia.

'Als nächstes war es (an der) Zeit, nach Wrocław zu fahren.'

Das heißt, neben dem 'normalen' Substantiveintrag von czas 'Zeit', der als Flexionsform im Nominativ die Kongruenzmerkmale Sg. und Mask. aufweist, vgl. (188a), ist ein zweiter Lexikoneintrag anzunehmen, der verbalnominal ist und lediglich das Nominativmerkmal hat, vgl. (188b): ${ }^{240}$
(188) a. $\quad / \operatorname{czas} \varnothing / \quad[-\mathrm{V},+\mathrm{N},-\mathrm{a}, \mathrm{NOM},-\mathrm{Pl},+\mathrm{Mask},-\mathrm{Fem}] \quad \lambda p \lambda x[x$ TIME $p]$
b. $/ \operatorname{czas} \varnothing / \quad[+\mathrm{V},+\mathrm{N}, \mathrm{NOM}] \quad \lambda p \lambda x[x$ TIME $p]$

Es kann sein, dass das Nominativsuffix (hier $\{-\varnothing\}$, bei den Feminina $\{-a\}$ ) stets beide Verwendungen zulässt, dass die zustandsprädikative Verwendung aber nur zum Einsatz kommen kann, wenn die Semantik des zugrunde liegenden Substantivs sich zur Charakterisierung eines Zustands eignet, wie es bei 'Zeit' der Fall ist. Andererseits kann es sich auch jeweils um eine idiosynkratische Nebenform handeln, die man quasi als "czas," ins Lexikon schreiben müsste. Aus Platzgründen muss ich diese Frage zukünftigen Forschungsarbeiten überlassen.

Übersetzung obligatorisch ist. Den Infinitiv(satz) betrachte ich daher als inhaltliche Ergänzung (also als Komplement zu czas), keinesfalls aber als möglichen Subjektausdruck.

${ }^{240}$ Dass das Komplement von czas in dessen Gebrauch als Zustandsprädikativ obligatorisch ist, kann auf seinen dann verbalnominalen Charakter zurückgeführt werden (siehe Abschnitt 4.4.3 bzgl. des gleichen Verhaltens von Kurzformen). 


\section{Der "formbasierte" Vorschlag}

\subsection{Ikonische Oppositionen}

Mein Ansatz zur Erklärung des Zustandekommens der beobachtbaren interpretativen Effekte bzw. Lesarten, die sich mit den Variationen in der Kasus- sowie Adjektivform polnischer und russischer Prädikatsnomina verbinden, nimmt ihren Ausgangspunkt in der Ablehnung einer m. E. axiomatischen Annahme der Mehrheit der in den Abschnitten 1.4 und 3 genannten und besprochenen Analysen.

Wie schon weiter oben mehrfach erwähnt, geht diese Mehrheit davon aus, dass die interpretativen Effekte grammatisch verankert seien, d. h. eine invariante Quelle im Sprachsystem hätten. Am wenigsten überrascht diese Blickweise bei Arbeiten des deskriptiv-semantischen Lagers, die der eher traditionellen Sicht verpflichtet sind, bestimmte "Konstruktionen" trügen bestimmte Bedeutungen (recht deutlich wird dies z. B. bei Kuznetsova \& Rakhilina 2010). Sie gehen jedoch i. d. R. nicht darauf ein, ob die Lesarten überhaupt 'grammatisch' verankert sind und wo im Sprachsystem dann ihr Ursprung liegt, oder ob sie nicht vielmehr 'nur' kontextuell basiert sind. So bleibt offen, ob die Lesarten in den (Markierungen der) Prädikatsnomina selbst oder in den resultierenden "Konstruktionen" angelegt sind, oder ob sie stattdessen aus dem Kontext inferiert werden. Freilich kann es kaum als Vorteil gelten, diese Fragen nicht zu stellen oder auszublenden.

Weniger deskriptive bzw. 'technischere' Analysen mit Explikationsanspruch gehen hingegen explizit oder implizit davon aus, dass die interpretativen Effekte in der einen oder anderen Form im Sprachsystem angelegt seien. So suchen sie nach einer syntaktisch-strukturellen und/oder lexikalisch-semantischen Quelle, die sie ggf. als unterspezifiziert beschreiben und mit pragmatischen Mechanismen derart 'unterfüttern', dass die Lesarten auf konzeptueller Ebene (CS) zustande kommen (können). In aller Regel folgern solche Analysen, dass die Effekte auf (bestimmten Teilen) der syntaktischen Struktur (vgl. Matushansky 2000; Pereltsvaig 2007; Markman 2008; Bailyn 2012 u. a.) oder auf speziellen Bedeutungsanteilen in Lexikoneinträgen (etwa im Instrumentaleintrag) basieren (vgl. Timberlake 1986; Geist 2006).

Die vorliegende Analyse weicht von diesen Vorschlägen schon in ihrer gegensätzlichen Ausgangsannahme ab, die besagt, dass die Lesarten nicht in invarianter Weise vom grammatischen System 'vorgegeben' sind. Das heißt, sie haben weder im mentalen Lexikon noch in der Satzsyntax noch gar in "Konstruktionen" eine 
wie auch immer geartete stabile Basis (etwa in Form eines Lexikoneintrags oder einer syntaktischen Struktur).

Dass hingegen Kontext ${ }^{241}$ und Weltwissen eine bedeutsame Rolle für die Entstehung der Lesarten spielen, wird hier keineswegs in Abrede gestellt, sondern begrüßt. In diesem Sinne kann von einem grundsätzlich pragmatischen Ansatz gesprochen werden. Er ist ferner non-deterministisch, da nicht versucht wird, die Lesarten von einer lexikalischen oder syntaktischen Quelle, sondern vielmehr davon abzuleiten, dass die fraglichen Formen - sofern sie variieren - stets in einer ikonischen Opposition zueinander stehen.

Eine Opposition liegt dann vor, wenn "ein Element der Ausdrucksebene natürlicher Sprachen [...] zusammen mit mindestens einem anderen ein und dasselbe inhaltliche bzw. grammatisch-inhaltliche Moment repräsentiert" (Hentschel 1991, 221) bzw. wenn mindestens zwei morphosyntaktische Formen ein und dieselbe syntaktische Position (konkret die Komplementposition des stummen V-Kopfes mit der Kopulabedeutung) besetzen können und sich der Sprecher zwischen ihnen entscheiden kann und muss; vgl. als Beispiele (1) und (2):
a. Zosia jest wojewodzianka.
Äquivalenzmarkierung
Zosia-NOM ist-3SG Voivodentochter-NOM
b. Zosia jest wojewodzianką.
Explizitmarkierung
Zosia-NOM ist-3SG Voivodentochter-INS
beide: 'Zosia ist eine Voivodentochter.'

(2)
a. Puškin byl
velikij poèt.
Äquivalenzmarkierung
Puškin-NOM war-SG.M großer Poet-NOM
b. Puškin byl velikim poètom.
Explizitmarkierung
Puškin-NOM war-SG.M großer Poet-INS
beide: 'Puschkin war eine großer Dichter.'
(Rus)

Ungeachtet irgendwelcher interpretativen Effekte variieren hier zunächst einfach zwei morphologische Formen in derselben syntaktischen Position bzw. Funktion.

\footnotetext{
${ }^{241}$ Der Begriff "Kontext" ist hier weiter als üblich zu fassen. Neben dem Äußerungskontext können auch i. w. S. kontextuelle Faktoren wie das syntaktische Umfeld der Wortform(en), ihr Flexionsparadigma, ihre relative Wortlänge, ihre in der gegebenen Varietät bestehende Zuordnung zu einer bestimmten Stilebene usw. in ihre Bewertung und Lesart mit einfließen.
} 
Unter Ikonizität/Ikonismus/ikonischer Relation verstehe ich eine formbasierte und dabei "sinnbildliche" Beziehung zwischen dem Bezeichnenden (signifiant) und dem Bezeichneten (signifié); vgl. dazu das folgende Zitat von Matthews ( $\left.{ }^{2} 1991\right)$ :

But when we do take care, we will often be able to establish that forms and meanings stand in an iconic or diagrammatic relation. An icon (in the ordinary sense) is a picture, in which there is a schematic correspondence between the painted features and the real features of a man or woman. In a diagram of a circuit there is a similar correspondence between the representations of a switch or a condenser and the positions of a real switch or a real condenser in the circuit itself. Similarly for grammar [...]. (Matthews ${ }^{2} 1991,224-225$ )

Der Begriff diagrammatisch, den Matthews hier als Synonym von ikonisch verwendet, stellt eine direkte Verbindung zu Hentschels (1993a) Konzept von diakritischer Markierung her. Die oben erwähnten Oppositionen sind im uns interessierenden Fall also ikonisch, weil "[d]er [morphologisch markiertere] Instrumental [...] das deutlichere Signal für das Pr[ädikats]N[omen] im Vergleich zum Nominativ [ist]" (Hentschel 1991, 230), ${ }^{242}$ bzw. weil die ausschließlich prädikativ verwendbare Kurzform "als die vom diakritischen Standpunkt vorteilhafte Variante angesehen werden [muss]", der gegenüber “[d]ie [zudem auch attributiv verwendbaren] Langformen [...] aufgrund ihrer formalen Eigenschaften allein keine eindeutigen Signale für den prädikativen Status eines Adjektivs" (Hentschel 1993a, 102) sein können.

Mit anderen Worten, bestimmte morphologische Formen und Marker sind paradigmatisch oder per se 'deutlicher' als andere. In polnischen und russischen Kopulasätzen sind dies i. d. R. der Instrumental und die adjektivische Kurzform. Wenn letztere in Opposition zu ihren jeweils 'weniger deutlichen' Varianten (dem Nominativ bzw. einer adjektivischen Langform) stehen, dann erhalten sie eine ikonische Dimension, innerhalb derer sie explizit den Status der betreffenden Wortform als Prädikat(ausdruck) anzeigen. Diese Anzeige geht damit einher, dass die deskriptive Bedeutung des jeweiligen Lexems eine explizit prädikative Lesart erhält bzw. in dieser Richtung ausgedeutet wird. In aller Regel läuft dies in der Tat auf eine 'temporäre' bzw. 'spezifische' Lesart hinaus. Diese in der Forschung

242 Der Instrumental ist das deutlichere Signal, da er (a) eindeutig die prädikative Funktion des betreffenden Ausdrucks anzeigt (während der Nominativ auch den Subjektausdruck markiert) und da seine Markierung (b) im Kasusparadigma markierter bzw. morpho(no)logisch 'schwerer' als die Nominativmarkierung ist (siehe dazu u. a. die semitische Arbeit von Shapiro 1969). 
seit Langem anzutreffenden Charakterisierungen ergeben sich durch die hier vorgelegte Analyse quasi 'frei Haus'. Auf die Annahme 'grammatischer' Quellen kann so verzichtet werden.

Dafür, dass das Prinzip des Ikonismus zum Zwecke des Ausdrucks bestimmter Konzepte auf allen Ebenen des Sprachsystems wirksam sein kann, spricht sich schon Mayerthaler (1980) im Rahmen seiner Morphologischen Natürlichkeit aus:

Das Prinzip des k.[onstruktionellen] Ik.[onismus] ist nicht nur auf die Morphologie beschränkt, sondern durchzieht alle konstruktionsseitigen Aspekte sprachlicher Organisation [...], also auch die Syntax. (Mayerthaler 1980, 25)

Im Fall polnischer und russischer Kopulasätze operiert der Ikonismus auf den Ebenen der Morphologie und der Satzsyntax: Während ihm morphologische Mittel (nominale Flexionsmarker) zugrunde liegen, wird er erst in der Syntax wirksam, wo seine "primäre Funktion [...] eine möglichst transparente Signalisation der syntaktischen Struktur [...] ist." (Hentschel 1993a, 110). Man kann demnach mit einigem Recht von einem Phänomen sprechen, dass an der Schnittstelle zwischen Morphologie und Syntax operiert bzw. erst dort wirksam wird. Auch dies spricht gegen deterministische Modelle, die den Ursprung sowohl der Variationen als auch der damit verbundenen Lesarten 'tief' im Sprachsystem, d. h. in (nur) einem bestimmten Modul (Lexikon, Morphologie, Syntax, ...) verorten.

Vielmehr "besteht tatsächlich das Verhältnis der Variation im eigentlichen Sinne", so dass die variierenden Formen "keine zwei verschiedenen semantischen Entitäten [sind]" (Bogusławski 2001, 127). Jedoch ist es so, dass die Varation "punktuell für stilistische oder perspektivische [...] Nuancierung ausgenutzt werden kann” (Hentschel 1993a, 109-110), womit die 'klassischen' Dichotomien à la "temporär vs. permanent" gemeint sind. Grundsätzlich gilt, dass die expliziteren Varianten der "Verdeutlichung" der syntaktischen Struktur dienen, indem sie das Prädikatsnomen, soweit dies (dem Sprecher) aus semantischen und/oder morphosyntaktischen Gründen erforderlich erscheint, formal vom Subjektausdruck absetzen. Ganz im Sinne der vorliegenden Analyse erkennt Hentschel $(1991,229)$ hierin die Folge einer allgemeinen Tendenz zur Ikonizität. Wann das jeweils explizitere Signal zum Einsatz kommt, kann dabei m. E. nicht durch eine $(\mathrm{ab})$ geschlossene Liste von Faktoren erfasst und vorhergesagt werden. Jeder Kopulasatz muss in seiner lexikalischen sowie morphosyntaktischen $\mathrm{Zu}$ - 
sammensetzung und in seinem Kontext individuell betrachtet werden, um festzustellen, welche Kriterien den Sprecher bewogen haben (könnten), die jeweilige Form zu wählen. Auch Faktoren wie die Varietät und Stilebene (vgl. die "Modelle" nach Guiraud-Weber 1993) sowie sicherlich auch der Idiolekt des Sprechers sind zu beachten.

Obwohl Bailyn (2012) einen syntaktischen Ansatz verfolgt, geht seine Intuition doch letzten Endes in die gleiche Richtung, wenn er als Ausblick formuliert, er überlasse "a characterization of the semantic distinction to research regarding choice of available structures and not the syntax of predicate case itself." (Bailyn 2012, 198). Offensichtlich betrachtet Bailyn (2012) die interpretativen Effekte nicht als unmittelbare Konsequenz syntaktischer oder semantischer Verhältnisse, sondern verweist im Hinblick darauf, wie sie zustande kommen und zu charakterisieren seien, auf eine Forschung, die die Mechanismen untersucht, die zur Wahl der einen oder anderen verfügbaren Struktur (Form/Markierung) führen - was freilich nur dann Sinn ergibt, wenn mehrere Strukturen (Formen/Markierungen) im Sinne von Oppositionen zur Wahl stehen. Dass und inwiefern die gesuchten Mechanismen ihrer Natur nach formbasiert und ikonisch sind, sollte mittlerweile klar geworden sein.

Es soll also gelten, dass die differenziellen Formen und Marker polnischer und russischer Präsdikatsnomina nicht mehr und nicht weniger als morphosyntaktische Formen sind. Sie haben per se keine Eigenbedeutung und determinieren auch keine spezifischen syntaktischen Teilstrukturen (z. B. AspP, PredEvP, ...). Im Kern wird m. E. durch die Morphosyntax ikonisch ein loserer oder engerer Zusammenhang zwischen Prädikat und Subjekt bzw. - genauer - zwischen der deskriptiven Bedeutung des Prädikatsnomens und dem Referenten des Subjektausdrucks dargestellt. Mit Hentschel (1991, 1993a) basieren somit die interpretativen Effekte auf der mehr oder weniger ausgeprägten morphosyntaktischen Markiertheit des Prädikatsstatus, wovon jedoch nur dann die Rede sein kann, wenn mindestens zwei prädikative Formen konkurriere, wenn also eine Opposition vorliegt.

Grundlegend lassen sich in polnischen und russischen Kopulasätzen mit prädikativen Nomina die beiden Markierungsmuster in (3) ausmachen:
(3) a. NOM COP NOM
b. (NOM) COP PRED

Äquivalenzmarkierung Explizitmarkierung 
Die Äquivalenzmarkierung bringt auf ikonische Weise einen engeren Zusammenhang zwischen Subjektausdruck (links) und Prädikatsnomen (rechts) zum Ausdruck. Da letzteres mit dem Subjektausdruck kasuskongruiert, lässt sich auch von einer symmetrischen Markierung sprechen. Diese Markierungsform setzt einen nominativischen Subjektausdruck voraus, der als Kongruenzgeber für das Prädikatsnomen fungiert. Ferner ist anzumerken, dass der engere Zusammenhang zwischen Subjektausdruck und Prädikatsnomen automatisch mit einer loseren formalen Beziehung zwischen Prädikatsnomen und Kopula (COP) einhergeht. ${ }^{243}$

Die Explizitmarkierung, bei der das Prädikatsnomen eine eindeutig prädikative Form (PRED) hat, reflektiert dagegen ikonisch den umgekehrten Zustand, nämlich einen engeren Zusammenhang zwischen Kopula(bedeutung) und Prädikatsnomen, der sich im Falle der Instrumentalmarkierung auch mit dem Begriff der Rektion beschreiben lässt, was wiederum die Redeweise von einer asymmetrischen Markierung rechtfertigt (vgl. Abschnitt 5.5). Es folgt von selbst, dass die Explizitmarkierung einen loseren bzw. überhaupt keinen formalen Zusammenhang zwischen Prädikativ und Subjektausdruck darstellt, was sich auch darin zeigt, dass ein overt realisierter Subjektausdruck ganz fehlen kann, wie es infinite Strukturen bezeugen. ${ }^{244}$

Es ist also so, dass bei der Äquivalenzmarkierung die morphosyntaktische Relation der Kongruenz als ikonischer Ausdruck der Gleichsetzung des Referenten des Subjektausdrucks mit der Eigenschaft, die das Prädikatsnomen bezeichnet, eingesetzt bzw. ausgenutzt wird (der Subjektreferent 'fällt unter das Prädikät').

Das lässt sich von der Explizitmarkierung nicht behaupten, bei der beide relevanten Ausdrücke im Kasus abweichen. ${ }^{245}$ Eine syntaktische Gleichsetzung der beiden Konstituenten ist somit nicht gegeben. Die syntaktische Absetzung der beiden Ausdrücke voneinander bewirkt auch deren 'Trennung' auf interpretativer

\footnotetext{
${ }^{243}$ Exakter ist von einem engeren Zusammenhang zwischen Prädikativ und abstrakter Kopulabedeutung (im "stummen" V-Kopf) zu sprechen, wobei letztere an der Satzoberfläche durch ein V-Auxiliar, also durch eine Flexionsform von byćlbyt' 'sein', ausbuchstabiert wird.

244 Ausnahmen sind hier Kurzformen, die zwar auf Grund ihrer ausschließlich prädikativen Verwendbarkeit der Explizitmarkierung zuzuordnen sind, die aber dennoch als nominativische Formen auf Kongruenz mit einem Nominativsubjekt angewiesen sind. Im Fall von Kurzformen muss ein nominativischer Subjektausdruck daher zwingend vorhanden sein. Entsprechend kann bei Kurzformen auch nicht von Rektion durch die Kopula(bedeutung) gesprochen werden.

${ }^{245}$ Wiederum bilden die Kurzformen hier eine Ausnahme (siehe vorangehende Fußnote).
} 
Ebene, was tendenziell zur Deutung des Prädikatsnomens als (von außen zugewiesene) Eigenschaft - und nicht als (sozusagen intern schon bestehende) Identifikation, Definition oder Charakterisierung - des Subjektreferenten führt. Die explizite morphosyntaktische Markierung als Prädikatsnomen, so die These, entspricht somit interpretativ einer ebenfalls explizit prädikativen 'Ausdeutung' der deskriptiven Bedeutung des Ausdrucks. Bei vielen prädikativ verwendeten Substantiven und Adjektiven läuft dies in der Tat auf eine Lesart hinaus, in der die bezeichnete Eigenschaft als zeitlich begrenzt aufgefasst wird, so dass z. B. učitelem 'Lehrer' oder professorom 'Professor' als Berufsbezeichnungen gedeutet werden oder bolen bzw. bol'nym 'krank' als temporäre Befindlichkeit (weshalb sie dann auch nicht mit 'kränklich' zu übersetzen sind).

Das ganze Gegenteil ist bei der Äquivalenzmarkierung der Fall, wo das Prädikatsnomen die gleiche Kasusmarkierung wie der Subjektausdruck trägt und somit keine spezielle Markierung aufweist. Die gleichförmige Morphologie zieht die syntaktische Gleichsetzung der beiden relevanten Ausdrücke nach sich, was auf interpretativer Ebene zur Deutung des Prädikatsnomens als mit dem Subjektreferenten identisch bzw. als (quasi) angeborenelunveräußerliche Eigenschaft führt. Dem Referenten wird im Rahmen der Äquivalenzmarkierung also i. d. R. eine Eigenschaft nicht wirklich zugeschrieben. Vielmehr wird etwas, das der Referent (nach Ansicht des Sprechers) bereits besitzt, aufgegriffen und zu seiner Definierung bzw. Charakterisierung verwendet. Damit ist vollends vereinbar, dass Isačenko (1962) von nominativischen Langformen behauptet, sie würden eine (objektive) Feststellung (und keine subjektive Zuschreibung) durch den Sprecher ausdrücken.

Immer und auch hier ist daran zu erinnern, dass diese Erwägungen nur im Falle des Bestehens einer Opposition gelten. Ist die eine oder andere Markierung 'alternativlos', kann auf ikonischer Ebene kein wie auch immer gearteter Kontrast ausgedrückt werden respektive zustande kommen. Das Prädikatsnomen kann dann ausschließlich die syntaktisch lizenzierte Form erhalten - der Sprecher hat also keine Wahl, und eine einzige Form ohne jegliche Alternative ist in ihrer Deutung zwangsläufig 'neutral'. Mit anderen Worten: Sie kann entweder keine oder aber alle möglichen Lesarten erhalten bzw. annehmen, die im Falle des Bestehens einer oder mehrerer Oppositionen auf verschiedene Formen verteilt sind. Dies ist z. B. der Fall in präsentischen Kopulasätzen, in denen nur nominativische Formen von prädikativen Substantiven auftreten. Ganz fraglos kann das Prädikatsnomen učitel’ 
'Lehrer' in (4) sowohl die Lesart einer Berufsbezeichnung als auch eines Wesenszug aufweisen, worüber aber nur der weitere Kontext entscheiden kann. Von einer Äquivalenzmarkierung kann trotz des Nominativs am Prädikatsnomen keine Rede sein, da keine alternative Markierung (der Instrumental) möglich ist:
(4) Ivan byl učitel'.
Ivan-NOM war-SG.M Lehrer-NOM
'Ivan war (ein) Lehrer.'

Die beschriebene Unterscheidung zwischen Äquivalenz- und Explizitmarkierung kann auch wie in (5) schematisiert werden:

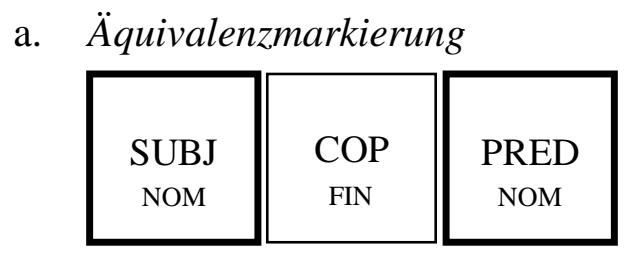

b. Explizitmarkierung

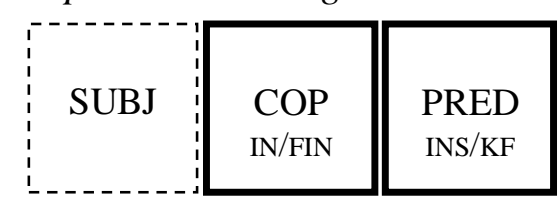

Die jeweils ikonisch ausgedrückte engere Beziehung wird hier durch fett umrandete Kästchen angedeutet. Der Möglichkeit der overten Abwesenheit eines Nominativsubjekts bei der Explizitmarkierung wird durch eine unterbrochene Umrandung entsprochen. Diese Option ist im Übrigen auch der Grund dafür, dass die Explizitmarkierung die einzige Option ist, wenn aus syntaktischen Gründen kein Subjektausdruck projiziert werden kann, wie es im z. B. Russischen stets bei infiniten Formen von byt' der Fall ist; vgl. (6):

(6) $[\ldots]$
Kak daj

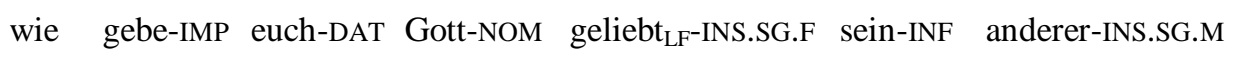
'... gebe Ihnen Gott, daß Sie von einem anderen ebenso geliebt werden.'

Nominativische Formen sind hier ausgeschlossen, da mit byt' ein Infinitiv vorliegt, der kein Nominativsubjekt lizenziert, das als Kongruenzgeber für solche Formen

\footnotetext{
${ }^{246}$ Gedichtzeile und Übersetzung nach: Alexander Puschkin: Gedichte. Russisch/Deutsch. Stuttgart: Reclam 1998, S. 78-79.
} 
fungieren könnte. Das Beispiel zeigt auch, dass es durchaus berechtigt ist, den Kurzformen den Nominativ zuzuerkennen. Wären sie "kasuslos", sollten sie in Strukturen wie (6) verwendbar sein. Es ist also so, dass in (6) die Beschränkung auf die Instrumentalform rein syntaktisch begründet ist. Die Tatsache dieser Beschränkung bewirkt, dass eine ikonische Ausdeutung mangels jedweder Opposition überhaupt nicht in Frage kommt.

Im Polnischen ist die Explizitmarkierung bei prädikativen Substantiven an dieselben syntaktischen Bedingungen wie im Russischen gebunden, wenn hier auch generell eine stärkere Neigung zum Instrumental besteht. Bei prädikativen Adjektiven ist sie auf Grund deren Fähigkeit zur 'fernen Kongruenz' hingegen weniger restringiert. Ganz unumgänglich ist sie nur bei 'unpersönlichen' oder tatsächlich 'subjektlosen' Strukturen (siehe hierzu schon Abschnitt 5.5.2):

(7) Było się szczęśliwym / *szczęśliwy. (vgl. Bondaruk 2013b, 160) war-LPT.IMPS REFL glücklich-INS glücklich-NOM 'Man war glücklich.'

(8) Trzeba być otwartym / *otwarty na świat. NKJP notwendig-IMPS sein-INF offen-INS offen-NOM auf Welt-ACC 'Es ist notwendig, offen für die Welt zu sein.'

Hier ist der Instrumental die einzig verfügbare Form am Prädikatsnomen. Somit besteht keine Opposition zu einer anderen Form. Dies wiederum schließt das Zustandekommen interpretativer Effekte aus. Die Instrumentalform erfährt in diesem Fall keine ikonische Deutung. Von Explizitmarkierung sollte man hier nicht sprechen, da die Äquivalenzmarkierung als Alternative nicht in Frage kommt.

Im folgenden Abschnitt werde ich das Wirken dieser Deutungsmechanismen anhand einiger exemplarischer russischer Belege illustrieren. Hierbei werden sich diverse verschiedene Systeme bzw. "Modelle" herauskristallisieren, die sich primär in der Anzahl der jeweils verfügbaren prädikativen Formen unterscheiden. Entgegen normativer Vorgaben ist folglich von keinem monolithischen und in sich stabilen System auszugehen, sondern von mehreren Subsystemen, die erst in ihrer Gemeinsamkeit ein Gesamtmodell erkennen lassen. So ergibt sich ein flexibles Modell, das in der Lage ist, den existierenden Varianten bzw. Varietäten Rechnung zu tragen. 


\subsection{Verschiedene "Modelle"}

\subsubsection{Prädikative Adjektive im Russischen}

Ich werde diesen Abschnitt ausschließlich auf russischen Daten aufbauen, da wie etwa auch Bogusławski (2001) deutlich macht - das Spektrum von Variationen, Oppositionen bzw. Deutungsgegensätzen in polnischen Kopulasätzen weitaus beschränkter als im Russischen ist. Im eigentlichen Sinne variieren dort lediglich prädikative Substantive (es ist also kein Zufall, dass Bogusławski nur sie betrachtet), aber auch dies eingeschränkter als ihre russischen Entsprechungen, da der Nominativ stets an Sonderbedingungen gebunden ist, um den substantivischen Default des Instrumentals zu 'umgehen'. Jedoch ist der Nominativ auch in diesen Fällen oftmals nicht obligatorisch. Prädikative Adjektive dagegen erhalten, wenn irgend möglich, im Polnischen den Kongruenzkasus, wogegen auf den Instrumental nur 'im Notfall' ausgewichen wird. Auf die(se) Situation im Polnischen werde ich am Ende dieses Abschnitts zurückkommen.

Im Unterschied zum eher 'strukturellen' Polnischen, das den Kasus am Prädikatsnomen v. a. von dessen lexikalischer Kategorie abhängig macht, wird im Russischen die Möglichkeit der Kasusvariation relativ produktiv und systematisch (aus)genutzt, um beim Hörer/Leser bestimmte Lesarten/Deutungen hervorzurufen, was gleichermaßen für prädikative Substantive und Adjektive gilt. Am systematischsten erfolgt dies in präteritalen Kopulasätzen. Dem dürfte so sein, da bereits stattgefundene Sachverhalte bzw. Zustände (offenbar auf Grund dessen, dass man sie rückblickend bewerten kann) am besten in der einen oder anderen Weise dargestellt bzw. perspektiviert werden können, z. B. als ganzheitlich oder partiell, als permanent oder temporär, als spezifisch oder unspezifisch etc. ${ }^{247}$ Auf diese Variationen und Lesarten werde ich mich im Weiteren konzentrieren.

${ }^{247}$ Zukünftige (Futur), hypothetische (Konjunktiv/Konditional) oder 'befohlene' (Imperativ) Zustände werden normalerweise anders perspektiviert. Eine permanente/ganzheitliche Sicht auf sie ist selten, da der Ausgangspunkt ihrer Betrachtung ein jeweils bestehender Zustand ist, von dem sie üblicherweise abweichen. Das führt dazu, dass solche i. w. S. hypothetischen Zustände fast immer als 'temporär' (da noch nicht eingetreten) oder als 'spezifisch' (da vom aktuellen Zustand abweichend) dargestellt werden. Die o. g. non-präteritalen Tempora/Modi werden von Hentschel $(1993,288)$ übrigens als 'irreal' und relativ 'undurchsichtig' bewertet, was die Tendenz zum Instrumental verstärke. Sicherlich spielen die genannten Faktoren zusammen und verursachen gemeinsam, dass die Explizitmarkierung hier bevorzugt wird. 
Da Adjektive von sich aus 'Eigenschaftsbezeichner' sind, ist die Explizitmarkierung (Kurzform/instrumentalische Langform) bei ihnen nur dann auch 'sinnvoll', wenn es sich um "Qualitätsadjektive" handelt, deren deskriptive Bedeutung naturgemäß der Charakterisierung eines (Subjekt-)Referenten dient. ${ }^{248}$ Im Gegensatz dazu sind "Beziehungsadjektive" zuallererst Modifikatoren, die die Spezifizierung ihres Referenten bzw. die Eingrenzung seines Denotats vornehmen. ${ }^{249}$ Aus diesem Grund treten sie fast nur attributiv bzw. kaum prädikativ auf, was eine ikonische Ausdeutung in oben beschriebener Form weitgehend ausschließt. Nicht zufällig bilden "Beziehungsadjektive" kaum Kurzformen, die ja per se prädikativ und der Explizitmarkierung zuzuordnen sind.

Im Folgenden werde ich daher zur Exemplifikation meiner Annahmen zunächst Beispiele mit Qualitätsadjektiven verwenden, bevor ich weiter unten prädikative Substantive behandele. Die folgenden Präteritalsätze in (9) und (10) haben den gleichen deskriptiven Gehalt, illustrieren aber alle drei möglichen Erscheinungsformen von prädikativen Adjektiven im Russischen:

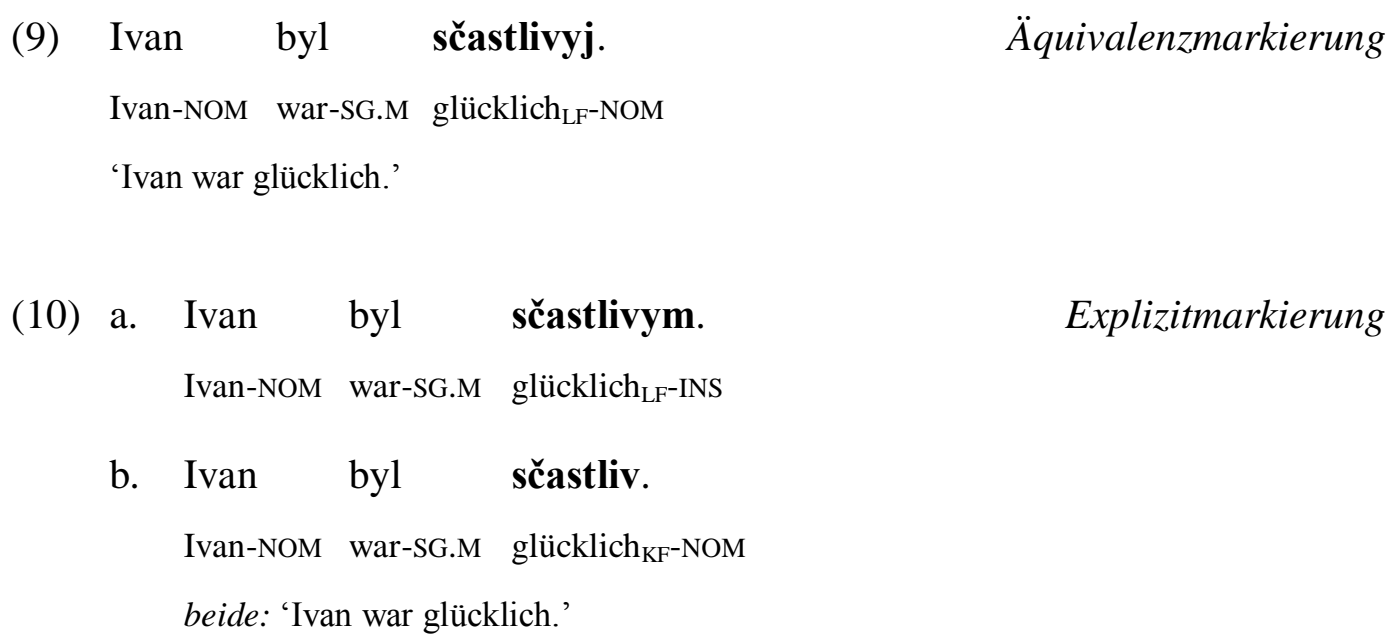

Während (9) der Äquivalenzmarkierung angehört, da das Prädikatsnomen - wie der Subjektausdruck - im Nominativ steht, vertreten beide Varianten in (10) die Explizitmarkierung. Im Falle von (10a) begründet sich dies durch den Instrumental, der die prädikative Funktion des Adjektivs ikonisch verdeutlicht. ${ }^{250}$ Mit

\footnotetext{
248 Daher beantworten "Qualitätsadjektive" i. d. R. die Frage Wie ist der Referent?.

249 Als solche beantworten "Beziehungsadjektive" meist die Frage Was für ein Referent?.

${ }^{250}$ Wie in Abschnitt 5.6.2 ausgeführt, kann hier durchaus eine syntaktische Struktur mit leerem prädikativem Substantiv vorliegen, auf das sich das Adjektiv als Attribut bezieht. Ebenso wäre
} 
Hentschel (1991) kann von Redundanz gesprochen werden, da die Instrumentalmarkierung weder zum Verständnis des Satzes noch für seine Grammatikalität unabdingbar ist, sondern 'lediglich' dessen (innere) Struktur(ierung) verdeutlicht. In (10b) hingegen begründet sich die Zuordnung zur Explizitmarkierung durch die Beschränkung der verbalnominalen Kurzform auf den prädikativen Gebrauch bzw. durch den Ausschluss ihres attributiven Gebrauchs (vgl. Abschnitt 4.3.5). Insofern also klar ist, dass eine Kurzform stets nur prädikativ verwendbar ist, wird die syntaktische Struktur allein schon durch ihre Präsenz verdeutlicht. ${ }^{251}$

Alle drei Optionen (9), (10a), (10b) stehen - mindestens in der gehobenen Standardsprache, die Guiraud-Weber (1993) als "Modell B" erfasst - in Opposition zueinander, da sie im Prinzip austauschbar sind. Daher ist davon auszugehen, dass die verschiedenen Formen auf der Basis ihres 'virtuellen' Nebeneinanders (ihrer potenziellen Konkurrenz) ikonische Lesarten annehmen (werden).

Ein kompetenter Sprecher/Schreiber des Standardrussischen wird also, da er sich seiner Wahl zwischen diesen drei Formen bewusst ist, davon Gebrauch machen und dem Hörer/Leser, der sich ihrer ebenso bewusst sein muss, ${ }^{252}$ damit etwas vermitteln, das über die bloße deskriptive Bedeutung des Adjektivlexems hinausgeht. Dieses "Mehr" folgt, wie oben beschrieben, aus der mehr oder weniger ausgeprägten Markiertheit des prädikativen Status des Adjektivs durch die jeweilige Markierung. So besteht z. B. keine formale Differenz zwischen Subjekt- und Prädikatausdruck im Fall der kongruierenden Langform in (9') [= (9)]:

(9') Ivan byl sčastlivyj.

Äquivalenzmarkierung NOM NOM

Die formale Gleichartigkeit legt als morphosyntaktisches Ikon nahe, eine Gleichsetzung beider Terme auch auf interpretativer Ebene vorzunehmen, so dass die denotierte Eigenschaft 'absolut' auf den Referenten bezogen wird. 'Absolut' kann

eine Ellipse des Bezugssubstantivs denkbar. Beides schließe ich hier aus. Es soll also gelten, dass in (9) und (10) echt-prädikative Adjektivformen bzw. prädikative APn vorliegen.

${ }^{251}$ Vgl. Hentschel (1993a, 102), der schreibt, dass, “[d]a die Kurzform im modernen Russischen nicht mehr in attributiver Funktion verwendet wird, [...] sie als die vom diakritischen Standpunkt vorteilhafte Variante angesehen werden [muß]".

252 Dass dieses Bewusstsein eine Bedingung zum Verständnis der ikonisch enkodierten Lesarten von Prädikatsnomina ist, mag trivial erscheinen, dürfte aber den Schwierigkeiten zugrunde liegen, die Russischlerner häufig mit dieser Erscheinung haben. 
auch mit vertrauteren Begriffen wie 'permanent', 'ganzheitlich', 'unspezifisch', 'non-eventiv' etc. beschrieben werden, die aber allesamt auf denselben Kern der formal-ikonischen Gleichsetzung zurückgeführt werden können.

Erfolgen kann eine solche ikonisch basierte 'Ausdeutung' jedoch nur, wenn - wie im aktuellen Beispiel - eine Opposition zu alternativen Formen besteht. Diese bewirkt, dass sich der interpretative Effekt der Gleichsetzung quasi auch substraktiv ergibt, da die Wahl einer bestimmten Form stets mit der Nicht-Wahl ihrer potenziellen Alternative/n einhergeht. Wie bereits erwähnt, sind die Alternativen im aktuellen Beispiel gleichermaßen der Explizitmarkierung zuzuordnen:

(10') a. Ivan byl sčastlivym.

Explizitmarkierung

b. Ivan byl sčastliv.

(NOM) PRED

Da beide Adjektivformen in (10') [= (10)] im Gegensatz zu jener in (9') explizit prädikativ sind, kommen für die resultierenden Sätze in (10') die vertrauten Lesarten 'temporär', 'teilweise', 'spezifisch', 'eventiv' etc. in Frage, die gemeinsam haben, dass sie keine (völlige) Gleichsetzung von Subjektreferent und Prädikat durch den Sprecher voraussetzen bzw. suggerieren.

Die divergierenden Lesarten der prädikativen Formen sind somit nicht die Folge einer im Sprachsystem (Morphologie/Lexikon/Syntax) von Grund auf angelegten und vorbestimmten Distinktion. Sie sind vielmehr die interpretative Entsprechung des ikonischen bzw. formbasierten Gegensatzes zwischen der gewählten Prädikativform und ihren potenziellen Alternativen. Man könnte auch sagen: Da sich der Sprecher der Alternativen bewusst ist, erhält seine jeweilige Wahl eine zusätzliche Bedeutungsdimension, indem mit der von ihm gewählten Form eine Lesart assoziiert wird, die auf ikonische Weise morphosyntaktisch enkodiert ist bzw. sich an den morphosyntaktischen Verhältnissen orientiert. All das setzt natürlich voraus, dass der Sprecher überhaupt die Wahl zwischen mindestens zwei Formen hat.

Für die (gehobene) russische Standardsprache ergibt sich aus dem Gesagten für prädikative Adjektive in etwa das in (11) skizzierte Gesamtbild. Das Schema zeigt eine asymmetrische Verteilung dreier Formen in einem Raum zwischen jeweils zwei Polen, namentlich gleichsetzend vs. nicht gleichsetzend und attributiv vs. nicht attributiv, denen die beiden Dimensionen Gleichsetzung und Attributivität entsprechen, innerhalb derer die möglichen Formen existieren: 


\begin{tabular}{|c|c|c|c|}
\hline 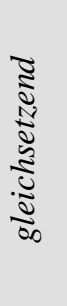 & $\mathbf{L F}_{\text {NOM }}$ & $\mathbf{K F}$ & 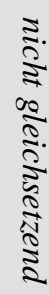 \\
\hline
\end{tabular}

Die Pole gleichsetzend vs. nicht gleichsetzend entsprechen ferner der Äquivalenzbzw. der Explizitmarkierung respektive den Lesarten, die damit einhergehen. Die Dimension der Attributivität hingegen markiert den Unterschied im Gebrauch zwischen der instrumentalischen Langform und der Kurzform, die ja beide der Explizitmarkierung angehören: Während eine Kurzform durch ihre lexikalische Prädikativität Attributivität völlig ausschließt, ist eine Assoziation damit bei einer Langform stets möglich und in unmittelbarer Opposition zu einer Kurzform sogar wahrscheinlich, ${ }^{253}$ denn "[d]ie Langformen sind [...] auch mögliche attributive Formen und somit aufgrund ihrer formalen Eigenschaften allein keine eindeutigen Signale für den prädikativen Status eines Adjektivs." (Hentschel 1993a, 102) Unmittelbar im Anschluss schreibt Hentschel: "Die geringste diakritische Potenz hat die Langform im Nominativ, die als solche auch Attribut des Subjekts sein kann" (Hentschel 1993a, 102), was die sozusagen 'unentschiedene' Position dieser Form zwischen den Polen attributiv und nicht attributiv begründet: Während eine instrumentalische Langform, wenn sie in Opposition zu einer (ebenso explizit prädikativen, aber niemals attributiven) Kurzform steht, die attributive Lesart stets nahelegt, ist letztere bei einer nominativischen Langform zwar nicht ausge-

253 Bei Geist (2006) findet dieser (potenziell) attributive Charakter seine Entsprechung in dem von ihr für prädikative Langformen angesetzten "Bezug auf eine kontextuell zu spezifizierende Vergleichsklasse" (Geist 2006, 151). Demnach sind prädikativ verwendete Langformen immer attributiv, wenn auch der Modifikand unrealisiert und so ein semantischer Parameter bleiben kann. Diese Festlegung ist m. E. zu weitreichend. Der attributive Charakter der Langformen folgt, wie Hentschel (1993a) richtig bemerkt, aus ihren formalen Eigenschaften, zu denen im Russischen und Polnischen ein komplettes Flexionsparadigma und die prinzipielle Möglichkeit ihrer attributiven Verwendung gehört. Diese Möglichkeit besteht auch, wenn sie prädikativ auftreten, so dass die Präsenz eines "stummen" oder elliptisch ausgelassenen Bezugssubstantivs durchaus vermutet werden kann, wenn sie auch nicht zwangsläufig ist. Prädikative Langformen in jedem Fall zu attributiven Formen zu erklären würde bedeuten, ihren durchaus anzutreffenden nicht attributivischen Gebrauch auszublenden, der v. a. in der gesprochenen Sprache anzutreffen ist (vgl. hierzu u. a. die Beobachtungen von Nichols 1981 oder Hentschel 1993a). 
schlossen, jedoch auch nicht derart wahrscheinlich. Die Asymmetrie folgt daraus, dass der nominativischen Langform ein Oppositionspartner innerhalb der Dimension der Attributivität fehlt. ${ }^{254}$

Die oben geschilderte Situation bezieht sich auf die "gepflegte Hochsprache" bzw. die normierte Standardsprache, die auch Vorlage für Guiraud-Webers (1993) "Modell B" ist. Hier stehen alle drei adjektivischen Prädikativformen zur Wahl. Somit ist dieses Modell "[...] viel komplexer [als andere Modelle], da es die syntaktischen Möglichkeiten durch die konstante Präsenz der Kurzform verdoppelt" (Guiraud-Weber 1993, 91).

Es ist logisch, dass sich die Verhältnisse merklich verändern, wenn die Zahl der prinzipiell verfügbaren Formen kleiner ist. Dies ist z. B. der Fall in der razgovornaja reč' ('Umgangssprache') oder etwa auch im journalistischen Register (vgl. Corbett 2004, 208). Hier ist, wenn man Guiraud-Weber (1993) folgt, der Gebrauch der Kurzform auf das Nötigste reduziert, was sie als "Modell A" erfasst. Corbetts (2004) diachrone Daten stützen diese Ansicht, denn sie zeigen, dass die Kurzformen von 1800 bis 2000 merklich zurückgegangen sind, und zwar bemerkenswerterweise begleitet von einer Zunahme der Langformen im Instrumental. Corbett schreibt, dass etwa in der Sprache des Journalismus letztere die Rolle übernommen hätten, die ehedem den Kurzformen zukam, so dass hier nurmehr zwei adjektivische Prädikativformen auftreten: die Langform im Nominativ und die Langform im Instrumental.

Verglichen mit "Modell B", das oben besprochen und in (11) schematisiert wurde, fehlt in "Modell A" auf Grund der Abwesenheit der Kurzform (mit wenigen idiomatisierten Ausnahmen) also der nicht attributive Pol vollends. Somit fehlt auch die Dimension der Attributivität, denn Langformen können zwar im Prinzip attributiv sein, müssen es aber nicht. Sie sind, mit anderen Worten, im prädikativen Gebrauch prinzipiell ambig zwischen Attribut und Nicht-Attribut. Durch ihre Kasusmarkierung können sie aber, wie schon oben in (11), ikonisch die Dimension der Gleichsetzung sichtbar machen, sofern eine potenzielle Opposition besteht. So ergibt sich für das simplere "Modell A" bzw. für die Umgangssprache oder auch die Sprache des Journalismus das Schema in (12):

\footnotetext{
${ }^{254}$ Wären Kurzformen attributiv verwendbar, nähmen ihre Nominativformen diesen Platz ein. Es müsste dann jedoch auch instrumentalische Kurzformen für die Position oben rechts geben.
} 


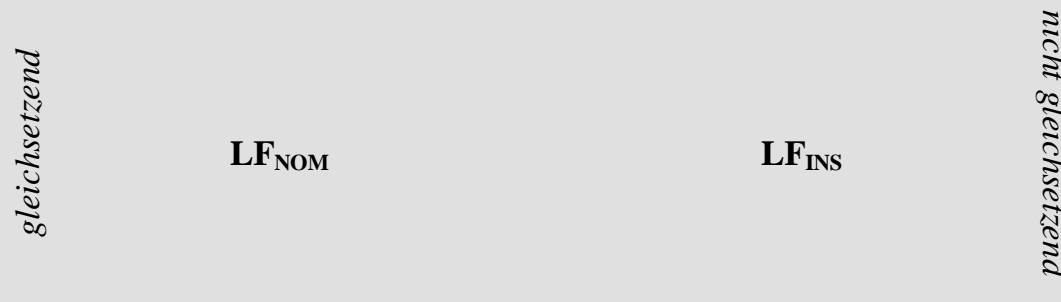

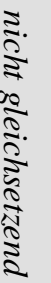

Dieses System von Oppositionen ist also um eine Dimension ärmer, dafür aber voll symmetrisch, da sich hier nur die gleichsetzende nominativische und die nicht gleichsetzende instrumentalische Langform gegenüberstehen. Das "Modell" ist somit 'klarer' und 'einfacher'.

Neben dem maximalen "Modell B" und dem (im Vergleich dazu) reduzierten "Modell A" scheint es noch weitere "Modelle" bzw. Systeme zu geben. So scheint etwa im prostorečie (einer Art 'einfacher' Umgangssprache) die Auswahl an prädikativen Adjektivformen meist noch reduzierter als in den bereits besprochenen Systemen. Ebenso wie in "Modell A" fehlen auch hier die Kurzformen weitestgehend (Ausnahme sind wiederum feste Wendungen), womit auch hier die Dimension der Attributivität fehlt. Somit besteht die Wahl an sich nur zwischen den Langformen mit ihren differenziellen Kasusmarkierungen.

Wie Nichols $(1981,1985)$ beobachtet, entscheidet sich letztere Wahl in der Umgangssprache aber nicht entlang der ikonisch ausgedrückten Dimension der Gleichsetzung, sondern durch andere Faktoren wie z. B. das grammatische Genus des Subjektreferenten und, in direkter Abhängigkeit davon, die Flexionsklasse des Prädikatsadjektivs. Nichols beobachtet, dass feminine Adjektive (im Singular) eher im Nominativ, maskuline, neutrale und Pluraladjektive hingegen eher im Instrumental erscheinen. Eine plausible Erklärung, die lediglich Bezug auf die Flexionsparadigmen nimmt, besteht darin, dass die Nominativendung der langförmigen Feminina - aja im Singularparadigma nur ein einziges Mal auftaucht und damit den höchsten Grad an Eindeutigkeit hat (die Endung -oj für den Instrumental dagegen erscheint synkretisch weitere drei Male im Paradigma). Dasselbe gilt bei den Maskulina und Neutra für die Endung des Instrumentals -ym, der gegenüber die nominativischen Endungen - $y j$ und -oe synkretisch auch im Akkusativ erscheinen. Auch die Bevorzugung der Instrumentalendung -ymi im Plural lässt sich paradigmatisch erklären, da sie im Pluralparadigma nur ein einziges Mal vor- 
kommt, während die Nominativendung -ye wiederum potenziell mit der homonymen Akkusativendung zusammenfällt; vgl. (13):

\begin{tabular}{|c|c|c|c|}
\cline { 2 - 4 } \multicolumn{1}{c|}{} & Sg. f. & Sg. $\mathbf{m} . \mathbf{n}$. & PI. \\
\hline Nom. & $-a j a$ & $-y j /-o e$ & $-y e$ \\
\hline Gen. & $-o j$ & $-e g o$ & $-y c h$ \\
\hline Dat. & $-o j$ & $-e m u$ & $-y m$ \\
\hline Akk. & $-u j u$ & $-y j /-e g o / /-o e$ & $-y e /-y c h$ \\
\hline Ins. & $-o j$ & $-y m$ & $-y m i$ \\
\hline Lok. & $-o j$ & $-o m$ & $-y c h$ \\
\hline
\end{tabular}

Nichols $(1981,1985)$ nennt noch weitere Kriterien, die offenbar die Wahl des Kasus an der Langform in der Umgangssprache beeinflussen. Sie alle sind aber nicht dichotomischer Natur, wie es die Dimension der Gleichsetzung ist, die für die Systeme weiter oben ausschlaggebend ist. ${ }^{255}$ Insofern lässt sich sagen, dass in diesem "Minimalmodell" überhaupt keine Pole existieren, an denen die Kasuswahl der Langformen orientiert ist. Vielmehr ist es so, dass auf Grund 'privativer' Faktoren entweder die nominativische oder die instrumentalische Langform gewählt wird. In diesem Sinne kommen niemals Oppositionen zustande, da ein Faktor oder ein Faktorenbündel unzweideutig zur Wahl der einen oder der anderen Kasusform führt. Unter identischen Bedingungen besteht also keine Wahl. Unter diesen Umständen kann es nicht dazu kommen, dass auf ikonischer Ebene irgendeine zusätzliche Lesart enkodiert wird. Das "Minimalmodell" ist somit frei von Dichotomien, wie das Schema in (14) zeigt:

$$
\mathbf{L F}_{\{\mathrm{NOM}, \mathrm{INS}\}}
$$

Zusammenfassend wurden bisher mit Blick auf prädikative Adjektive im Russischen drei Systeme beschrieben, die sich in der Anzahl der verfügbaren Formen prädikativer Adjektive und, damit zusammenhängend, in den existierenden Dimensionen unterscheiden:

\footnotetext{
${ }^{255}$ Relevant ist laut Nichols u. a. auch die lexikosemantische Klasse des Adjektivs, der Typ eines möglichen Kontrolleurs (Subjekt oder Nicht-Subjekt) sowie die In-/Finitheit der Verbform.
} 


$\begin{array}{llccc} & \text { System } & \text { Formen } & \text { Dimensionen } & \text { [Guiraud-Weber 1993] } \\ \text { a. } & \text { maximal } & 3 & 2 & \text { "Modell B" } \\ \text { b. } & \text { intermediär } & 2 & 1 & \text { "Modell A" } \\ \text { c. } & \text { minimal } & 1 & 0 & -\end{array}$

Ich schließe keineswegs aus, ja halte es für wahrscheinlich, dass noch weitere Systeme bzw. "Modelle" mit den entsprechenden Komponenten existieren. Ich kann es nur zukünftiger (empirischer) Forschung überlassen zu klären, wie diese Systeme im Detail beschaffen sind. Für die aktuelle Darstellung mag die Feststellung genügen, dass nicht nur mit einem einzigen, monolithischen Gefüge von Formen und ikonischen Oppositionen zu rechnen ist, wie es von der normativen Grammatikschreibung meist suggeriert wird. Wahrscheinlicher ist, dass mehrere Subsysteme bestehen, die einen jeweils individuellen Bestand an Formen und Regeln aufweisen. Das Gesamtgefüge setzt sich folglich aus mehren Teilsystemen zusammen, und seine Beschreibung setzt die seiner Teile voraus. ${ }^{256}$

\subsubsection{Prädikative Substantive im Russischen}

Das Spektrum verschiedener "Modelle" ist bei prädikativen Substantiven erwartbar kleiner, da sie lediglich zwei (und nicht, wie den Adjektiven, drei) Erscheinungsformen kennen, namentlich den Nominativ und den Instrumental, die man den o. g. Markierungsmustern wie in (16) zuordnen kann:
a. Ivan
byl
aktër.
Äquivalenzmarkierung
Ivan-NOM war-SG.M Schauspieler-NOM
b. Ivan
byl
aktërom.
beide: 'Ivan war (ein) Schauspieler.'
Explizitmarkierung
Ivan-NOM war-SG.M Schauspieler-INS

\footnotetext{
${ }^{256}$ Möglicherweise lässt sich diese Schlussfolgerung auch im Sinne der Probabilistic Linguistics. So heißt es im Klappentext zur Monographie von Bod, Hay \& Jannedy (2003): "Probabilistic linguistics conceptualizes categories as distributions and views knowledge of language not as a minimal set of categorical constraints but as a set of gradient rules that may be characterized by a statistical distribution.” Ich danke Gerhild Zybatow für diesen Hinweis.
} 
Da Substantive keine Unterscheidung zwischen Kurz- und Langformen kennen, und da es sich bei ihnen um keine primär attributiv verwendete Wortart handelt, erübrigt sich in ihrem Fall die Annahme der Dimension Attributivität. Im Prinzip stellt sich die Situation bei prädikativen Substantiven in der Standardsprache weitgehend so dar wie bei den Adjektiven im simpleren "Modell A" (siehe Schema (12) bzw. (15b) oben), nämlich derart, dass sich Nominativ- und Instrumentalform in direkter Opposition zueinander befinden und auf ikonische Weise (nur) die (eine) Dimension der Gleichsetzung signalisieren, sofern die syntaktischen Verhältnisse beide Formen im Prinzip zulassen; vgl. (17):

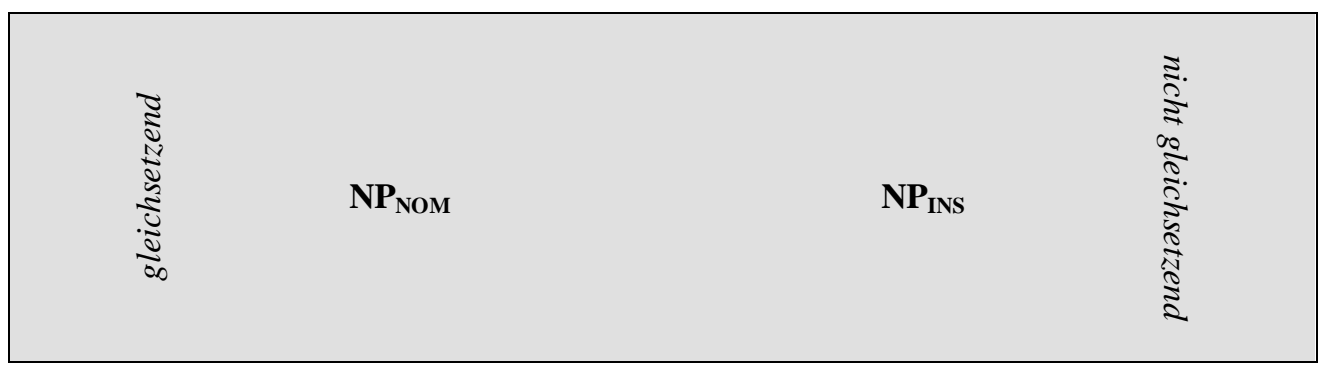

Wenn man die Daten statistischer Erhebungen wie z. B. die von Krasovitsky et al. (2008) nicht völlig ablehnt, kann für das Russische ggf. mit einer Entwicklungstendenz gerechnet werden, innerhalb derer die Nominativform prädikativer Substantive zunehmend vor der Instrumentalform zurücktritt. In einem solchen Szenario würde der Instrumental den Status einer Default-Form annehmen. Falls eine solche Tendenz besteht, würde sie in Bezug auf prädikative Substantive zu einem neuen "Modell" führen, das dem für prädikative Adjektive postulierten "Minimalsystem" in (14) bzw. (15c) weiter oben entspräche. Das Schema für dieses - vorerst rein hypothetische - System sähe wie in (18) aus und wiese keinerlei Oppositionen bzw. Dimensionen auf:

$$
\mathbf{N P}_{\text {INS }}
$$

Jedoch ist vor dem Hintergrund der statistischen Daten und Auswertungen v. a. von Kuznetsova \& Rakhilina (2010) sowie Kuznetsova (2013), die die "rein strukturelle" These von Krasovitsky et al. (2008) merklich in Frage stellen, davon 
auszugehen, dass der Nominativ in Folge des o. g. Entwicklungsszenarios nicht vollends aus dem System verdrängt wird, sondern Teil von ihm bleibt, jedoch im Vergleich zum "Standardmodell” in (17) in seiner Rolle insofern an Eigenständigkeit und Relevanz verliert, als er nurmehr auf 'markierte' Kontexte beschränkt wird und als morphosyntaktisches Ikon nicht mehr für den Pol gleichsetzend steht. Die Instrumentalform ist in diesem neuen "Modell" demnach durchaus auch der prädikative Default, jedoch besteht neben ihr - im Unterschied zum "Radikalmodell" in (18) - in Form des Nominativs doch eine, wenn auch sehr marginale Alternative für spezifische Kontexte. Dieses - ebenfalls hypothetische - "moderate" Modell skizziere ich in (19):

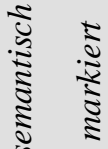
$\mathbf{N P}_{\text {NOM }}$
NP
离

Was die zuletzt genannten hypothetischen Szenarien und "Modelle" angeht, so lässt sich denken, dass sich das in (19) skizzierte moderate "Modell" in der Zukunft zum veranschlagten "Radikalmodell” in (18) entwickelt. Mit Krasovitsky et al. (2008) ist sogar denkbar, dass das "Radikalmodell" aktuell bereits besteht und von (manchen) Sprechern verwendet wird. Als wirklich gesichert gelten kann im Grunde nur das "Standardmodell" in (17) weiter oben. Als Zusammenfassung ergeben sich für russische substantivische Prädikative die gesicherten sowie denkbaren (eingeklammerten) Systeme in (20):

\section{System}

a. $\quad$ Standard

b. moderat

c. radikal

\section{Formen}

2

2

1

\section{Dimensionen}

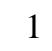

(1) ]

0 ] 


\subsubsection{Prädikative Adjektive im Polnischen}

Im Polnischen ist, wie schon in den Abschnitten 2.2.1 und 5.5.2 beschrieben und ausgeführt wurde, die Form des Prädikatsnomens ganz primär an dessen lexikalische Kategorie (Wortart) gebunden.

Wenn man die wenigen im Polnischen (noch) vorhandenen adjektivischen Kurzformen ausblendet, ergibt sich für diese Sprache (im Vergleich zum Russischen) ein relativ durchsichtiges bzw. 'strukturelles' Gesamtbild. Mit Bezug auf prädikative Adjektive gilt im Polnischen, dass - wenn dies irgend möglich ist - der Kongruenzkasus, also der Nominativ, an ihnen erscheint. Der Instrumental stellt hier lediglich eine 'Notlösung' dar, die nur dann zum Einsatz kommt, wenn der Kongruenzkasus aus syntaktischen Gründen nicht zustande kommen kann. Den Default bei prädikativen polnischen Adjektiven bildet also ganz zweifellos der Nominativ, während der Instrumental in diesem "Modell" insofern ein 'Schattendasein' fristet, als er nur dann gewählt wird, wenn dies syntaktisch erzwungen ist. Dieses "Modell", das für das Polnische den absoluten Standard darstellt, schematisiere ich in (21):

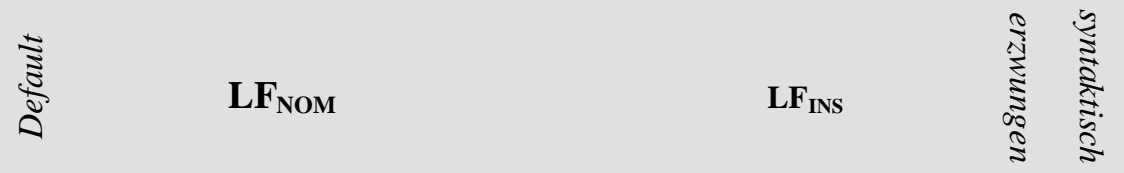

In diesem Modell gibt es keine echten Oppositionen oder Dimensionen. Die Kasusmarkierung am prädikativen Adjektiv kann demnach nicht als ikonisches Signal für irgendeine zusätzliche Lesart eingesetzt oder gewertet werden. Dies entspricht in der Literatur und in Grammatiken vorzufindenen Beschreibungen und Analysen. Das Polnische zeigt einen stark 'strukturellen' Wesenszug bzw. eine Tendenz zur Einheitlichkeit ("eine Satzfunktion - eine Markierung”), die freilich auf Kosten solcher Lesarten geht, wie sie im Russischen, das in dieser Hinsicht deutlich weniger 'strukturell' verfasst ist, durch die morphologische Form bzw. Markierung am Prädikatsadjektiv ausgedrückt werden können. 


\subsubsection{Prädikative Substantive im Polnischen}

Ein etwas anderes Bild zeigt sich bei den prädikativen Substantiven. Zwar stellt den Adjektiven quasi entgegengesetzt - der Instrumental hier den Default dar, jedoch ist der Nominativ nicht lediglich auf syntaktisch erzwungene Kontexte beschränkt. Wie Bogusławski (2001) detailreich beschreibt, tritt der Nominativ an polnischen prädikativen Substantiven vielmehr (aber dies meist fakultativ) in Kontexten auf, die den Default-Instrumental auf bestimmte Art 'neutralisieren', indem sie eine emotionale, identifizierende, metonymische o. ä. Nuance beinhalten bzw. transportieren.

Das bedeutet, dass die Nominativform in diesem "Modell" auf "semantisch markierte' Kontexte beschränkt ist und insofern einen höheren Grad an 'Souveränität' besitzt als der lediglich syntaktisch lizenzierbare Instrumental prädikativer Adjektive. Der Instrumental repräsentiert demgegnüber den dominierenden und annähernd immer einsetzbaren Default. Dies wird in Schema (22) zusammengefasst:

Auch in diesem "Modell" kann von echten Oppositionen bzw. Dimensionen keine Rede sein. Ebenso wie schon bei den prädikativen Adjektiven, stellt sich also das polnische Sprachsystem in Bezug auf prädikative Substantive als vergleichsweise 'strukturell' verfasst dar, indem es die Kasusmarkierung am Prädikatausdruck auch hier in allererster Linie an dessen lexikalische Kategorie koppelt, während sich etwaige semantische Faktoren auf 'markierte' Kontexte beschränken, in denen die Nominativform zum Einsatz kommen kann oder - selten und nur, wenn explizite Trigger wie z. B. der "Überraschungsausdruck" ale 'aber' (siehe Abschnitt 2.1.1) gegeben sind - zum Einsatz kommen muss.

Es fällt auf und ist bemerkenswert, dass das "Standardmodell" in (22) für prädikative Substantive im Polnischen dem vorerst nur prognostizierten "moderaten Modell” im Russischen entspricht, das oben in (19) skizziert wurde. Insofern 
liegt es im Bereich des Möglichen, dass sich das Russische - wenigstens in dieser Beziehung und ggf. auf bestimmte Sprechergemeinschaften beschränkt - dem Zustand annähert, in dem sich das moderne Polnische bereits befindet. Mit dieser 'Aussicht', die ein bloßes Gedankenexperiment darstellt, möchte ich meine Arbeit de facto abschließen.

Der letzte Abschnitt gilt der finalen Zusammenfassung und einem Ausblick. 


\section{Zusammenfassung und Ausblick}

Ich habe mich in dieser Arbeit detailliert mit der "Gammatik prädikativer Ausdrücke" im Russischen und Polnischen beschäftigt. Es ging dabei nicht nur um Kopulasätze und die darin auftretenden Variationen, sondern grundlegender auch um das Verhältnis zwischen Semantik (mentalem Lexikon) und Morphosyntax bzw. zwischen morphologischer Markierung und daraus (potenziell) resultierenden Lesarten oder Interpretationen.

Die fraglichen Variationen fügen sich in dieses Themengebiet ein, da sie für Lesarten verantwortlich sind bzw. sein können, die nicht in ihnen selbst 'stecken' bzw. die im Sprachsystem in keiner invarianten Form angelegt sind. Diese Grundthese der vorliegenden Arbeit hat sich im Laufe der Betrachtungen und der Argumentation bewährt. Nicht zuletzt hat sie den Vorteil, als Explikation nicht weniger flexibel zu sein als das sprachliche Phänomen, das sie zu erfassen sucht. Flexibel ist die vorgelegte Analyse zum einen dadurch, dass sie einerseits der "Form" nicht zuviel Bedeutung aufbürdet, indem sie Ikonizität bzw. "Formbasiertheit" als Erklärungsmuster in Betracht zieht. Andererseits ist sie flexibel, indem sie diverse Sprach-"Modelle" ansetzt, innerhalb derer jeweils unterschiedlich viele prädikative Formen zur Verfügung bzw. Auswahl stehen, woraus sich wiederum jeweils spezifische Verhältnisse zwischen diesen Formen ergeben, die in der Folge die Wahl und schließliche Ausdeutung der vom Sprecher gewählten Form entscheidend steuern. Da es diese "Modelle" (bzw. die in ihnen bestehenden Oppositionen) und eben nicht irgendwelche fixen Invarianten in einem der Teilbereiche des Sprachsystems sind, die zu den beobachtbaren Lesarten der prädikativen Formen im Russischen und Polnischen führen, gelangt man zu einem systematischen Verständnis der Variationen, ohne den problematischen deterministischen Weg beschreiten zu müssen.

Macht man sich die Flexibilität des fraglichen Phänomens bewusst, kann es kaum überraschen, dass in der Fachliteratur eine derartige Vielzahl unterschiedlichster Vorschläge vorzufinden ist. Die hiesige Analyse vermeidet die fundamentalsten Probleme der Mehrzahl dieser Arbeiten, namentlich (a) bloße Deskriptivität sowie (b) eine (deterministische) Erklärung, die Übergeneralisierungen hervorruft. 
In der vorliegenden Arbeit habe ich mich bemüht, detailliert die folgenden Fragen zu behandeln und Antworten darauf zu geben:

- Welche Variationen treten unter welchen Bedingungen in polnischen und russischen Kopulasätzen auf? Welche interpretativen Effekte werden im $\mathrm{Zu}$ sammenhang mit ihnen beobachtet?

- Was für Vorschläge wurden zur Analyse der Variationen bis dato vorgelegt, worin bestehen sie und ihre theoretischen Vor- und Nachteile?

- Die Annahme welcher morphosyntaktischen Merkmale und Kategorien ist mit Blick auf das Russische und Polnische adäquat?

- Wie sehen die Spezifika der prädikativ verwendbaren Wortarten in diesen Sprachen aus? Lassen sich die Daten durch diese Spezifika erklären?

- Wie gestaltet sich die Interaktion zwischen Semantik und Morphosyntax? Welche Folgen ergeben sich für die Syntax russischer und polnischer Sätze?

- Was ist unter "Prädikation" zu verstehen, was unter "Prädikativität"?

- Welche Rolle spielen die Flexionsformen von poln. być/russ. byt' 'sein'? Welche Merkmale und Funktion haben sie konkret?

- Wo liegen Gemeinsamkeiten und Unterschiede zwischen der primären und der sekundären Prädikation? Wann sind "Prädikatsphrasen" (Wilder 1994) anzunehmen und was zeichnet sie aus?

- Wie kommen die verschiedenen Kasusmarkierungen an russischen und polnischen Prädikatsnomina zustande? Nimmt die russische "Nullkopula" in diesem Kontext eine Sonderrolle ein (bzw. ist sie wirklich 'defizient')?

- Ist die Annahme des "Zustandsprädikativums" als eigenständiger Wortart im Nordslavischen theoretisch erforderlich? Worin besteht die Alternative?

- Wie sieht eine "formbasierte" Analyse der fraglichen Variationen aus und was folgt daraus im Hinblick auf die beobachtbaren interpretativen Effekte?

- Welche Rolle spielen ikonische Oppositionen, und wie sind "Modelle" (vgl. Guiraud-Weber 1993) für diesen Ansatz nutzbar zu machen? 
Primär aus Platzgründen konnten in dieser Arbeit u. a. die folgenden Fragen und Vorhaben nicht angesprochen respektive umgesetzt werden:

- Aus welchen Gründen hat sich in den slavischen Sprachen gerade die Instrumentalmarkierung als explizites Signal für die prädikative Funktion von Nominalphrasen etabliert?

- Ist das ikonische/diakritische Prinzip häufig oder selten anzutreffen? Gibt es andere Bereiche oder Phänomene, innerhalb derer es wirksam ist?

- Wie stellt sich die Lage in den südslavischen Sprachen dar? Was hat, abgesehen vom Abbau der Kasusmorphologie, zum Rückgang der (Ausnutzung der) Variationen in Kopulasätzen geführt? Liegen gravierende Unterschiede in Bezug auf Satzstruktur und Interaktion zwischen Semantik und Morphosyntax im Vergleich zu den nordslavischen Sprachen vor?

- Wann werden Unterspezifikation und Ambiguität in sprachlichen Strukturen und Äußerungen bevorzugt, wann dagegen vermieden? Lassen sich hier Tendenzen und Regelmäßigkeiten erkennen? Korrelieren letztere mit der jeweils vorliegenden Varietät (Standard- vs. Umgangs- vs. Fachsprache) bzw. Modalität (mündlich vs. schriftlich)?

- Bestehen, wie es die bestehende Terminologie suggeriert, Parallelen zwischen den sog. "Zustandsprädikativa" (die ich in dieser Arbeit als prädikative Kurzformen analysiere) und den sog. "Modalprädikativa" oder handelt es sich (doch) um völlig unterschiedliche Einheiten? Ist die hier gemachte Andeutung korrekt, der zufolge "Modalprädikativa" grammatische 'Modalisierer' sind, die 'subjektlose' Strukturen erzeugen?

- Welche Möglichkeiten bestehen, um die in der vorliegenden Arbeit aufgestellten Thesen empirisch und experimentell zu überprüfen?

Die zuletzt genannte Frage führt zum Ausblick auf mögliche zukünftige Forschungsvorhaben im Zusammenhang mit dem Thema der aktuellen Arbeit. Hier sehe ich v. a. die Möglichkeit experimenteller (psycholinguistisch fundierter) Studien, anhand derer die vorgelegte Theorie überprüft werden kann. Solcherlei Experimente sind nach meinem Kenntnisstand in der slavistisch-lingustischen Forschung noch relativ selten und würden eine wertvolle Ergänzung zu den mehrheitlich diachron und rein statistisch ausgerichteten Korpusuntersuchungen darstellen, die in Bezug v. a. auf russische Kopula-Prädikativ-Strukturen vorliegen. 


\section{Abkürzungsverzeichnis}

\begin{tabular}{|c|c|}
\hline Fließtext/GLOSSIERUNG & Erklärung \\
\hline Adv./ADV & Adverb(marker) \\
\hline Akk./ACC & Akkusativ \\
\hline AUX & Auxiliar \\
\hline $\mathrm{COP}$ & Kopula \\
\hline Dat./DAT & Dativ \\
\hline $\mathrm{f} . / \mathrm{F}$ & Femininum \\
\hline Fut./FuT & Futur \\
\hline Gen./GEN & Genitiv \\
\hline GER & Gerundium=Adverbialpartizip \\
\hline Imp./IMP & Imperativ \\
\hline impf./IMPF & imperfektiver Aspekt \\
\hline IMPS & impersonal=unpersönlich=akongruent \\
\hline Ind. & Indikativ \\
\hline Inf./INF & Infinitiv \\
\hline INS & Instrumental \\
\hline KF & Kurzform \\
\hline Konj./KONJ & Konjunktiv \\
\hline LF & Langform \\
\hline LOC & Lokativ=Präpositiv \\
\hline LPT & $l$-Partizip \\
\hline m./M & Maskulinum \\
\hline n./N & Neutrum \\
\hline NEG & Negation(smarker) \\
\hline Nom./NOM & Nominativ \\
\hline perf./PERF & perfektiver Aspekt \\
\hline Pl./PL & Plural \\
\hline Präs./PRES & Präsens \\
\hline Prät./PAST & Präteritum \\
\hline PRP & Präposition \\
\hline$\underline{\mathrm{R}}$ & un(ter)spezifizierte semantische Relation \\
\hline REFL & Reflexixmarker \\
\hline$s$ & Sachverhaltsvariable=Situationszeit=Ereigniszeit \\
\hline Sg./SG & Singular \\
\hline$t$ & Topikzeit(variable) \\
\hline$t^{0}$ & Äußerungszeit \\
\hline$\tau$ & temporal trace function (erzeugt 'Laufzeiten') \\
\hline 1. Ps./1 & erste Person \\
\hline 2. Ps./2 & zweite Person \\
\hline 3. Ps./3 & dritte Person \\
\hline$\varnothing$ & Nullform (ohne phonetische Matrix) \\
\hline
\end{tabular}




\section{Literaturverzeichnis}

Abney, S. P. (1987): The English Noun Phrase in Its Sentential Aspect. Cambridge, Mass.: MIT Press.

Adger, D. (2003): Core Syntax. A Minimalist Approach. Oxford [u. a.]: OUP.

Admoni, V. G. (1973): Sintaksis sovremennogo nemeckogo jazyka: sistema otnošenij i sistema postroenija. Leningrad: Nauka.

AG [Akademiegrammatik] (1980) $\rightarrow$ Russkaja grammatika (1980).

Aksakov, K. S. (1880): Polnoe sobranie sočinenij Konstantina Sergěeviča Aksakova. 3: Sočinenija filologičeskija 2 Opyt russkoj gramatiki. Moskva: Bachmetev. Reprint als Aksakov (2011).

Aksakov, K. S. (2011): Opyt russkoj gramatiki: sravnitelnyj vzgljad: Indoevropejskie i slavjanskie jazyki. Moskva: Librokom.

Asher, N. (1993): Reference to Abstract Objects in Discourse. Dordrecht: Springer.

Babby, L. H. (1975): A Transformational Grammar of Russian Adjectives. The Hague, Paris: Mouton.

Babby, L. H. (1999): Adjectives in Russian: Primary vs. Secondary Predication. In: Coats, H., K. Dziwirek \& C. M. Vakareliyska (eds.): Formal Approaches to Slavic Linguistics. The Seattle Meeting. Ann Arbor, Mich.: Michigan Slavic Publ., 1-16.

Bailyn, J. F. (1994): The Syntax and Semantics of Russian Long and Short Form Adjectives: An X'-Theoretic Account. In: Toman, J. (ed.): Formal Approaches to Slavic Linguistics. The Ann Arbor Meeting: Functional Categories in Slavic Syntax. Ann Arbor, Mich.: Michigan Slavic Publ., 1-30.

Bailyn, J. F. (2001): The Syntax of Slavic Predicate Case. ZAS Papers in Linguistics 22: 1-23.

Bailyn, J. F. (2012): The Syntax of Russian. Cambridge [u. a.]: CUP.

Bailyn, J. F. \& B. Citko (1999): Case and Agreement in Slavic Predicates. In: Coats, H., K. Dziwirek \& C. M. Vakareliyska (eds.): Annual Workshop on Formal Approaches to Slavic Linguistics: The Seattle Meeting. Ann Arbour, Mich.: Michigan Slavic Publ., 17-37.

Bailyn, J. F. \& E. J. Rubin (1991): The Unification of Instrumental Case Assignment in Russian. Cornell Working Papers in Linguistics 9: 99-126.

Bąk, P. ( $\left.{ }^{11} 1999\right)$ : Gramatyka języka polskiego: Zarys popularny. Warszawa: WP. 
Barnetová, V. et al. (red.) (1979): Russkaja grammatika. Praha: Academia.

Bartnicka, B. (1972): Konstrukcje typu ciężko nieść i żal porzucić w języku polskim. Prace Filologiczne 23: 103-120.

Bartula, C. (1969): Składnia zdania pojedyńczego w języku staro-cerkiewnosłowiańskim. Kraków: UJ.

Belošapkova, V. A. ( $\left.{ }^{3} 1997\right):$ Sovremennyj russkij jazyk. Moskva: Azbukovnik.

Bendixen, B. \& W. Voigt (1999): Russische Grammatik: Ein Leitfaden. Wiesbaden: Harrassowitz.

Benveniste, É. (1966): La phrase nominale. Problèmes de linguistique générale 1: $151-167$.

Berneker, E. \& M. Vasmer ( $\left.{ }^{7} 1971\right)$ : Russische Grammatik. Berlin, New York: de Gruyter.

Bickes, G. (1984): Das Adjektiv im Deutschen: Untersuchungen zur Syntax und Semantik einer Wortart. Frankfurt am Main [u. a.]: Lang.

Bielfeldt, H. H. (1961): Altslawische Grammatik: Einführung in die slawischen Sprachen. Halle (Saale): Niemeyer.

Bierwisch, M. (1983): Semantische und konzeptuelle Repräsentationen lexikalischer Einheiten. In: Růžička, R. \& W. Motsch (Hgg.): Untersuchungen zur Semantik. Berlin: Akademie, 61-99.

Bierwisch, M. (1986): On the Nature of Semantic Form in Natural Language. In: Klix, F. \& H. Hagendorf (eds.): Human Memory and Cognitive Capabilities - Mechanisms and Performances. Amsterdam: Elsevier, 765-784.

Bierwisch, M. (1987): Semantik der Graduierung. In: Bierwisch, M. \& E. Lang (Hgg.): Grammatische und konzeptuelle Aspekte von Dimensionsadjektiven. Berlin: Akademie, 91-286.

Bierwisch, M. (1988): On the Grammar of Local Prepositions. In: Bierwisch, M., W. Motsch \& I. Zimmermann (Hgg.): Syntax, Semantik und Lexikon. Berlin: Akademie, 1-65.

Bierwisch, M. (1996): Lexikon und Universalgrammatik. In: Weber, N. (Hg.): Semantik, Lexikographie und Computeranwendungen. Tübingen: Niemeyer, 129-165.

Bierwisch, M. (1997): Lexical Information from a Minimalist Point of View. In: Wilder, C., H.-M. Gärtner \& M. Bierwisch (eds.): The Role of Economy Principles in Linguistic Theory. Berlin: Akademie, 227-266. 
Bierwisch, M. (2002): A Case for CAUSE. In: Kaufmann, I. (ed.): More Than Words: A Festschrift for Dieter Wunderlich. Berlin: Akademie, 327-353.

Bierwisch, M. (2005): The Event Structure of CAUSE and BECOME. In: Maienborn, C. \& A. Wöllstein (eds.): Event Arguments: Foundations and Applications. Tübingen: Niemeyer, 11-44.

Bierwisch, M. (2007): Semantic Form as Interface. In: Späth, A. (ed.): Interfaces and Interface Conditions. Berlin, New York: de Gruyter, 1-32.

Bierwisch, M. (2009): Nominalization - Semantic and Syntactic Aspects. In: Giannakidou, A. \& M. Rathert (eds.): Quantification, Definiteness, and Nominalization. Oxford [u. a.]: OUP, 281-320.

Błaszczak, J. \& L. Geist (2001): Zur Rolle des Pronomens tolèto in spezifizierenden Kopulakonstruktionen im Polnischen und Russischen. In: Zybatow, G., U. Junghanns, G. Mehlhorn \& L. Szucsich (eds.): Current Issues in Formal Slavic Linguistics. Frankfurt am Main [u. a.]: Lang, 247-257.

Blom, A. \& S. Daalder (1977): Syntaktische theorie en taalbeschrijving. Muiderberg: Coutinho.

Bod, R., J. Hay \& S. Jannedy (2003) (eds.): Probabilistic Linguistics. Cambridge, Mass.: MIT Press.

Bogorodickij, V. A. ( $\left.{ }^{5} 1935\right)$ : Obščij kurs russkoj grammatiki (iz universitetskich čtenij). Moskva: Gosudarstvennoe social'no-èkonomičeskoe izdat.

Bogusławski, A. (2001): Über Nominativ-Instrumental-Variationen im Polnischen. In: Boeder, W. \& G. Hentschel (Hgg.): Variierende Markierung von Nominalgruppen in Sprachen unterschiedlichen Typs. Oldenburg: BIS, 101-133.

Bondaruk, A. (2010): To as a Verb or as a Pronoun: The Case of Polish Copular Constructions. In: Cyran, E. \& B. Szymanek (eds.): Verb Structures: Between Phonology and Morphosyntax. Lublin: KUL, 53-73.

Bondaruk, A. (2013a): Interplay of Feature Inheritance and Information Structure in Polish Inverse Copular Sentences. In: Junghanns, U., D. Fehrmann, D. Lenertová \& H. Pitsch (eds.): Formal Description of Slavic Languages: The Ninth Conference. Proceedings of FDSL 9, Göttingen 2011. Frankfurt am Main [u. a.]: Lang, 37-64.

Bondaruk, A. (2013b): Copular Sentences in English and Polish: Structure, Derivation and Interpretation. Lublin: KUL. 
Bondaruk, A. \& B. Szymanek (2007): Polish Nominativeless Constructions with Dative Experiencers: Form, Meaning and Structure. Studies in Polish Linguistics 4: 61-97.

Borer, H. (2005): Structuring Sense. Oxford [u. a.]: OUP.

Borik, O. (2012): Past Participle Formation and the Eventive/Adjectival Passive in Russian. In: Chemla, E., V. Homer \& G. Winterstein (eds.): Proceeding of Sinn und Bedeutung 17. Paris: ENS, 115-132.

http://semanticsarchive.net/sub2012/

Borsley, R. \& M. L. Rivero (1994): Clitic Auxiliaries and Incorporation in Polish. Natural Language and Linguistic Theory 12: 373-422.

Boryś, W. (2005): Słownik etymologiczny języka polskiego. Kraków: Wyd. Literackie.

Bošković, Ž. (2005): On the Locality of Left Branch Extraction and the Structure of NP. Studia Linguistica 59: 1-45.

Bošković, Ž. (2010): On NPs and Clauses. Ms. University of Connecticut, Storrs.

Bowers, J. S. (1993): The Syntax of Predication. Linguistic Inquiry 24: 591-656.

Bowers, J. S. (2000): Predication. In: Baltin, M. \& C. Collins (eds.): The Handbook of Contemporary Syntactic Theory. Oxford [u. a.]: OUP, 299-333.

Bunčić, D. (2011): Biscriptality in Slavic and Non-Slavic Languages: A Sociolinguistic Typology. Habilitationsschrift, Univ. Tübingen.

Bunčić, D. (2013): Biaspektuelle Verben als Polyseme: Über Homonymie, Aspektneutralität und die konative Lesart. Die Welt der Slaven 58.1: 36-53.

Bunčić, D. (im Druck): Definitheit als 'verborgene Kategorie' im Russischen? In: Pitsch, H. (Hg.): Linguistische Beiträge zur Slavistik: XXI. JungslavistInnen-Treffen in Göttingen, 13.-15. September 2012. München: Sagner.

Busch, U. (1960): Die Seinssätze in der russischen Sprache. Meisenheim am Glan: Hain.

Chachulska, B. (2008): Prädikativer Instrumental, Kasuskongruenz oder analytische Markierung bei sekundären Prädikaten im Polnischen. In: Schroeder, C., G. Hentschel \& W. Boeder (eds.): Secondary Predicates in Eastern European Languages and Beyond. Oldenburg: BIS, 41-68.

Chomsky, N. (1965): Aspects of the Theory of Syntax. Cambridge, Mass.: MIT Press.

Chomsky, N. (1981): Lectures on Government and Binding. Dordrecht: Foris. 
Chomsky, N. (1986): Knowledge of Language: Its Nature, Origin, and Use. New York: Praeger.

Chomsky, N. (1993): A Minimalist Program for Linguistic Theory. In: Hale, K. \& S. J. Keyser (eds.): The View from Building 20: Essays in Linguistics in Honor of Sylvain Bromberger. Cambridge, Mass.: MIT Press, 1-52.

Chomsky, N. (1995a): The Minimalist Program. Cambridge, Mass.: MIT Press.

Chomsky, N. (1995b): Bare Phrase Structure. In: Webelhuth, G. (ed.): Government and Binding Theory and the Minimalist Program. Oxford: Blackwell, $383-439$.

Chomsky, N. (2000): Minimalist Inquiries: The Framework. In: Martin, R., D. Michaels \& J. Uriagereka (eds.): Step by Step. Cambridge, Mass.: MIT Press, 89-155.

Chomsky, N. (2001): Derivation by Phase. In: Kenstowicz, M. (ed.): Ken Hale: A Life in Language. Cambridge, Mass.: MIT Press, 1-52.

Chomsky, N. (2008): On Phases. In: Freidin, R., C. Otero \& M.-L. Zubizaretta (eds.): Foundational Issues in Linguistic Theory. Cambridge, Mass.: MIT Press, 133-166.

Chvany, C. (1975): On the Syntax of BE-Sentences in Russian. Cambridge, Mass.: MIT Press.

Cienkowski, W. (1970): Gramatyka języka polskiego dla cudzoziemców. II: Fleksja (z ćwiczeniami). Warszawa: UW.

Citko, B. (2008): Small Clauses Reconsidered: Not So Small and Not All Alike. Lingua 118: 261-295.

Comrie, B. S. (1997): The Typology of Predicate Case Marking. In: Bybee, J., J. Haiman \& S. A. Thompson (eds.): Essays on Language Function and Language Type: Dedicated to T. Givón. Amsterdam, Philadelphia: Benjamins, 39-50.

Comrie, B. \& G. Stone \& M. Polinsky ( $\left.{ }^{2} 1996\right)$ : The Russian Language in the Twentieth Century. Oxford: Clarendon.

Corbett, G. G. (2004): The Russian Adjective: A Pervasive Yet Elusive Category. In: Dixon, R. M. W. \& A. Y. Aikhenvald (eds.): Adjective Classes: A Cross Linguistic Typology. Oxford [u. a.]: OUP, 199-222.

Cubberley, P. V. (2002): Russian: A Linguistic Introduction. Cambridge [u. a.]: CUP. 
Dahlitz, G. et al. ( $\left.{ }^{14} 1982\right)$ : Leiffaden der russischen Grammatik. Leipzig: Enzyklopädie.

Davidson, D. (1967a): The Logical Form of Action Sentences. In: Rescher, N. (ed.): The Logic of Decision and Action. Pittsburgh: University of Pittsburgh Press, 81-95. [Reprinted in Davidson (1980), 105-122].

Davidson, D. (1967b): Causal Relations. Journal of Philosophy 64: 691-703. [Reprinted in Davidson (1980), 149-162].

Davidson, D. (1980): Essays on Actions and Events. Oxford: Clarendon.

den Dikken, M. (2006): Relators and Linkers. The Syntax of Predication, Predicate Inversion, and Copulas. Cambridge, Mass.: MIT Press.

Dolińska, J. (2012): Zur Klassifizierung der Prädikative. Diss., Univ. Jena.

Dölling, J. (1997): Semantic Form and Abductive Fixation of Parameters. In: van der Sandt, R., R. Blutner \& M. Bierwisch (eds.): From Underspecification to Interpretation: Working Papers of the Institute of Logic and Linguistics. Heidelberg: ILLH, 113-139.

Dölling, J. (2005a): Semantische Form und pragmatische Anreicherung: Situationsausdrücke in der Äußerungsinterpretation. Zeitschrift für Sprachwissenschaft 24: 159-225.

Dölling, J. (2005b): Copula Sentences and Entailment Relations. Theoretical Linguistics 31: 317-329.

Dölling, J. \& T. Heyde-Zybatow (2007): Verb Meaning: How Much Semantics is in the Lexicon? In: Späth, A. (ed.): Interfaces and Interface Conditions. Berlin, New York: de Gruyter, 33-75.

Dornisch, E. (1997): Auxiliaries and Functional Projections in Polish. In: Browne, W., E. Dornisch, N. Kondrashova \& D. Zec (eds.): Annual Workshop on Formal Approaches to Slavic Linguistics 4. The Cornell Meeting 1995. Ann Arbor, Mich.: Michigan Slavic Publ., 183-209.

Dowty, D. (1979): Word Meaning and Montague Grammar: The Semantics of Verbs and Times in Generative Semantics and in Montague's PTQ. Dordrecht: Reidel.

Eckert, R. \& E. Crome \& C. Fleckenstein (1983): Geschichte der russischen Sprache. Leipzig: Enzyklopädie.

Eisenberg, P. (1998): Grundriß der deutschen Grammatik. 2: Der Satz. Stuttgart, Weimar: Metzler.

Emonds, J. (1985): A Unified Theory of Syntactic Categories. Dordrecht: Foris. 
Emonds, J. \& L. Veselovská (2012): The Cross-Linguistic Homes of Mood and Tense. ZAS Berlin, 24. Febr. 2012 [Handout].

Erben, J. (1980): Deutsche Grammatik: Ein Abriss. München: Hueber.

Fehrmann, D., U. Junghanns \& D. Lenertová (2007): Perzeptionsprädikate in ostund westslavischen Sprachen. Slavistisches Kolloquium, Univ. Leipzig, 14. Nov. 2007.

Fehrmann, D., U. Junghanns \& D. Lenertová (2010): Two Reflexive Markers in Slavic. Russian Linguistics 34: 203-238.

Fehrmann, D., U. Junghanns \& D. Lenertová (2011): Decausatives in a Minimal Theory of Reflexive Marking. Paper presented at FDSL 9, Univ. Göttingen, December 7-9, 2011.

Fehrmann, D., U. Junghanns \& D. Lenertová (2012): Decomposing Decausatives. Paper presented at the Workshop on Decomposition and Natural Classes in Argument Coding, Univ. Leipzig, September 6-7, 2012.

Fehrmann, D., U. Junghanns \& D. Lenertová (2013): Zur Analyse slavischer Impersonale mit Akkusativ. Kolloquium zur slavistischen Linguistik, Univ. Göttingen, 29. Mai 2013.

Filip, H. (2001): The Semantics of Case in Russian Secondary Predicates. Semantics and Linguistic Theory 11: 192-211.

Fortuin, L. J. (2000): Polysemy or Monosemy: Interpretation of the Imperative and the Dative-Infinitive Construction in Russian. Amsterdam, Philadelphia: Benjamins.

Franks, S. (1995): Parameters of Slavic Morphosyntax. Oxford [u. a.]: OUP.

Franks, S. \& P. Bański (1999): Approaches to 'Schizophrenic' Polish Person Agreement. In: K. Dziwirek, H. Coats \& C. Vakareliyska (eds.): Annual Workshop on Formal Approaches to Slavic Linguistics: The Seattle Meeting 1998. Ann Arbor: Michigan Slavic Publ., 123-143.

Franks, S. \& G. Greenberg (1994): The Functional Structure of Slavic Clauses. In: Toman, J. (ed.): Formal Approaches to Slavic Linguistics. The Ann Arbor Meeting: Functional Categories in Slavic Syntax. Ann Arbor, Mich.: Michigan Slavic Publ., 77-108.

Gabka, K. et al. (Hgg.) (1989): Russische Sprache der Gegenwart 3: Syntax. Leipzig: Enzyklopädie [1. Aufl. der Neuf.]. 
Gagarina, N. (2003): The Early Verb Development and Demarcation of Stages in Three Russian-Speaking Cildren. In: Bittner, D., W. U. Dressler \& M. Kilani-Schoch (eds.): Development of Verb Inflection in First Language Acquisition. A Cross-linguistic Perspective. Berlin, New York: de Gruyter, 131-170.

Galkina-Fedoruk, E. M. et al. (red koll.) (1964): Sovremennyj russkij jazyk. 2: Morfologija. Sintaksis. Moskva: MU.

Geist, L. (1999): Russisch byt' ('sein') als funktionale und/oder lexikalische Kategorie. ZAS Papers in Linguistics 14: 1-40.

Geist, L. (2004): Kopulasätze an der Syntax-Semantik-Schnittstelle. Diss., Humboldt-Universität zu Berlin.

Geist, L. (2006): Die Kopula und ihre Komplemente: zur Kompositionalität in Kopulasätzen. Tübingen: Niemeyer.

Geist, L. (2008): Wieviel Verb braucht ein russischer Satz? Überlegungen zur Nullkopula. In: Junghanns, U. (Hg.): Linguistische Beiträge zur Slavistik: XIII. JungslavistInnen-Treffen in Leipzig, 23.-26. September 2004. München: Sagner, 123-141.

Geist, L. (2010): The Argument Structure of Predicate Adjectives in Russian. Russian Linguistics 34: 239-260.

Geist, L. (2011): Indefiniteness and Specificity: Lexical Marking and Information-Structural Conditions. Habilitationsschrift, Univ. Stuttgart.

Geist, L. (2013): Prädikativsätze. In: Meibauer, J., M. Steinbach, H. Altmann (Hgg.): Satztypen des Deutschen. Berlin, New York: de Gruyter, 482-500.

Grice, H. P. (1979): Logik und Konversation. In: Meggle, G. (Hg.): Handlung, Kommunikation, Bedeutung. Frankfurt am Main: Suhrkamp, 243-265.

Grimshaw, J. (1990): Argument Structure. Cambridge, Mass.: MIT Press.

Guiraud-Weber, M. (1993): La méthode bisynchronique dans la description de l'adjectif attribut en russe moderne. Revue des études slaves 65.1: 81-95.

Gülzow, I. \& N. Gagarina (2006): Analytical and Synthetic Verb Constructions in Russian and English Child Language. In: Gagarina, N. \& I. Gülzow (eds.): The Acquisition of Verbs and Their Grammar. The Effect of Particular Languages. Dordrecht: Springer, 229-259.

Hackmack, S. (2000): Prädikation und sekundäre Prädikation. Bremer LinguistikWorkshop, 09.02.2000 [Ms.].

http://www.fb10.uni-bremen.de/iaas/workshop/praedi/hackmack.pdf 
Haider, H. (1988): Matching Projections. In: Cardinaletti, A., G. Cinque \& G. Giusti (eds.): Constituent Structure. Dordrecht: Foris, 101-121.

Hansen, B. (2009): Modals/Modalauxiliare. In: Kempgen, S. et al. (Hgg.): Die slavischen Sprachen: Ein internationales Handbuch zu ihrer Struktur, ihrer Geschichte und ihrer Erforschung. Bd. 1. Berlin, New York: de Gruyter, $468-483$.

Harves, S. (2002): Where Have All the Phases Gone? (Non-)Defective Categories and Case Alternations in Russian. In: Toman, J. (ed.): Annual Workshop on Formal Approaches to Slavic Linguistics: The Second Ann Arbor Meeting. Ann Arbor, Mich.: Michigan Slavic Publ., 97-118.

Haspelmath, M. (1995): The Converb as a Crosslinguistically Valid Category. In: Haspelmath, M. \& E. König (eds.): Converbs in Crosslinguistic Perspective: Structure and Meaning of Adverbial Verbforms - Adverbial Participles, Gerunds. Berlin, New York: de Gruyter, 1-55.

Hauser, M. D., N. Chomsky \& W. T. Fitch (2002): The Faculty of Language: What Is It, Who Has It, and How Did It Evolve? Science 298: 1569-1579.

Heidolph, K. E., W. Flämig \& W. Motsch (1981): Grundzüge einer deutschen Grammatik. Berlin: Akademie.

Heim, I. \& A. Kratzer (1998): Semantics in Generative Grammar. Malden, Mass. [etc.]: Blackwell.

Helbig, G. \& J. Buscha (2001): Deutsche Grammatik: ein Handbuch für den Ausländerunterricht. Berlin: Langenscheidt.

Hentschel, G. (1991): Der prädikative Instrumental beim russischen Substantiv als redundantes Signal in Kopulasätzen. In: Grochowski, M. \& D. Weiss (eds.): Words Are Physicians to an Ailing Mind. München: Sagner, 221-235.

Hentschel, G. (1992): Zum Einfluß der Konstituentenfolge auf die Kasuswahl im Russischen. Lingua 87: 231-255.

Hentschel, G. (1993a): Haben Kasus Bedeutungen oder sind sie eine diakritische Kategorie? Evidenz aus der Kasusvariation im Russischen. Zeitschrift für Slavische Philologie 53.1: 97-112.

Hentschel, G. (1993b): Zur Kasusvariation des prädikativen Substantivs in Kopulasätzen: Syntaktischer Wandel im Polnischen des 16. und 17. Jh. In: Hentschel, G. \& R. Laskowski (eds.): Studies in Polish Morphology and Syntax: Synchronic and Diachronic Problems. München: Sagner, 253-292. 
Hentschel, G. (1995): Passiv in Kopulasätzen? Zu substantivischen Prädikaten mit Subjektfunktion im Russischen und Polnischen. In: Weiss, D. (Hg.): Slavistische Linguistik 1994. Referate des XX. Konstanzer Slavistischen Arbeitstreffens, Zürich, 20.-22.9.1994. München: Sagner, 125-173.

Hentschel, G. (2001): Dative or Prepositional Marking of Noun Phrases in the Context of Russian Adjectival Experiencer Predicates. In: Boeder, W. \& G. Hentschel (Hgg.): Variierende Markierung von Nominalgruppen in Sprachen unterschiedlichen Typs. Oldenburg: BIS, 171-200.

Higginbotham, J. (1985): On Semantics. Linguistic Inquiry 16: 547-593.

Hinterhölzl, R. (2001): Semantic Constraints on Case Assignment in Secondary Adjectival Predicates in Russian. ZAS Papers in Linguistics 22: 99-112.

Hock, W. ( $\left.{ }^{3} 1998\right)$ : Das Urslavische. In: Rehder, P. (Hg.): Einführung in die slavischen Sprachen (mit einer Einführung in die Balkanphilologie). Darmstadt: WBG.

Isačenko, A. V. (1954): Grammatičeskij stroj russkogo jazyka v sopostavlenii s slovackim: Morfologija I-II. Bratislava: SAN.

Isačenko, A. V. (1962): Die russische Sprache der Gegenwart. 1: Formenlehre. Halle (Saale): Niemeyer.

Isačenko, A. V. (1983): Geschichte der russischen Sprache. Bd. 2. Heidelberg: Winter.

Jackendoff, R. (1996): The Architecture of the Language Faculty. Cambridge, Mass.: MIT Press.

Jackendoff, R. (2010): Your Theory of Language Evolution Depends on Your Theory of Language. In: Larson, R. K., V. Déprez \& H. Yamakido (eds.): The Evolution of Human Language. Biolinguistic Perspectives. Cambridge [u. a.]; CUP, 63-72.

Jakobson, R. (1971a): Beitrag zur allgemeinen Kasuslehre. Gesamtbedeutungen der russischen Kasus. In: Selected Writings II: Word and Language. The Hague, Paris: Mouton, 23-71.

Jakobson, R. (1971b): Signe zéro/Das Nullzeichen. In: Selected Writings II: Word and Language. The Hague, Paris: Mouton, 211-222.

Jakobson, R. (1971c): Shifters, Verbal Categories, and the Russian Verb. In: Selected Writings II: Word and Language. The Hague, Paris: Mouton, 130147. 
Jedlička, A. (ved. red.) (1977): Slovník slovanské lingvistické terminologie = Slovar' slavjanskoj lingvističeskoj terminologii = Dictionary of Slavonic Linguistic Terminology. Bd. 1. Praha: Academia.

Junghanns, U. (1994): Syntaktische und semantische Eigenschaften russischer finaler Infinitiveinbettungen. München: Sagner.

Junghanns, U. (1997): On byt' (and byti). In: Junghanns, U. \& G. Zybatow (eds.): Formale Slavistik. Frankfurt am Main [u. a.]: Lang, 251-265.

Junghanns, U. (1999): Generative Beschreibung periphrastischer Konstruktionen des Tschechischen. In: Anstatt, T., R. Meyer \& E. Seitz (Hgg.): Linguistische Beiträge zur Slavistik aus Deutschland und Österreich. VII. JungslavistInnen-Treffen, Tübingen/Blaubeuren 1998. München: Sagner, 133-165.

Junghanns, U. (2002): Untersuchungen zur Syntax und Informationsstruktur slavischer Deklarativsätze. Leipzig: Inst. für Linguistik, Univ. Leipzig.

Junghanns, U. (2008): Argument Structure and Syntax. In: Kempgen, S. et al. (Hgg.): Deutsche Beiträge zum 14. Internationalen Slavistenkongress Ohrid 2008. München: Sagner, 171-181.

Junghanns, U. (2012): Kausative Verben. Präsentation im Kolloquium zur slavistischen Linguistik, Univ. Göttingen, 12. Dez. 2012.

Junghanns, U. \& D. Lenertová (2010): A Case of Linguistic Change: Infinitives as Predicatives. In: Karlík, P. (ed.): Development of Language through the Lens of Formal Linguistics. München: Lincom Europa, 87-103.

Junghanns, U. \& G. Zybatow (1997): Syntax and Information Structure of Russian Clauses. In: Browne, E. W. et al. (eds.): Annual Workshop on Formal Approaches to Slavic Linguistics: The Cornell Meeting 1995. Ann Arbor, Mich.: Michigan Slavic Publ., 289-319.

Kacnel'son, S. D. (1972): Tipologija jazyka i rečevoe myšlenie. Leningrad: Nauka. Kamp, H. \& U. Reyle (1993): From Discourse to Logic. Introduction to Modeltheoretic Semantics of Natural Language, Formal Logic and Discourse Representation Theory. Dordrecht: Kluwer.

Kamynina, A. A. ( $\left.{ }^{2} 2010\right)$ : Sovremennyj russkij jazyk: Morfologija. Učebnoe posobie dlja studentov filologičeskich fakultetov gosudarstvennych universitetov. Moskva: MU.

Kempgen, S. ( 2008$)$ : “Wortarten” als klassifikatorisches Problem der deskriptiven Grammatik: Historische und systematische Untersuchungen am Beispiel des Russischen. Bamberg: Univ. Bamberg. 
Kim, J. (1976): Events as Property Exemplifications. In: Brand, M. \& D. Walton (eds.): Action Theory. Proceedings of the Winnipeg Conference on Human Action. Dordrecht: Reidel, 159-177.

Kiparsky, V. (1967): Russische historische Grammatik. II: Die Entwicklung des Formensystems. Heidelberg: Winter.

Klein, W. (1994): Time in Language. London, New York: Routledge.

Klemensiewicz, Z. (1926): Orzecznik przy formach osobowych słowa BYĆ. Prace Filologiczne 11: 123-181.

Komárek, M. (1954): K otázce predikativa (kategorie stavu) v češtine. Sborník Vysoké školy pedagogické v Olomouci: jazyk a literatura 1954: 7-25.

Kosta, P. (2001): Negace a větná struktura v češtině. In: Hladká, Z. \& P. Karlík (eds.): Čeština - univerzália a specifika 3. Brno: MU, 117-138.

Krasovitsky, A., A. Long, M. Baerman, D. Brown \& G. G. Corbett (2008): Predicate Nouns in Russian. Russian Linguistics 32: 99-113.

Krüger, K. (1996): Modaladjektive im Russischen. In: Jelitte, H. \& E். A. Balalykina (Hgg.): Das Adjektiv im Russischen: Geschichte, Strukturen, Funktionen. Frankfurt am Main [u. a.]: Lang, 31-45.

Kuznetsova, J. (2013): Diachronic Distribution of Predicate Nouns in Russian. Russian Linguistics 37: 51-60.

Kuznetsova, J. \& E. Rakhilina (2010): Russian Depictives and Agreeings. In: Zybatow, G., P. Dudchuk, S. Minor \& E. Pshehotskaya (eds.): Formal Studies in Slavic Linguistics: Proceedings of Formal Description of Slavic Languages 7.5. Frankfurt am Main [u. a.]: Lang, 303-320.

Lang, E. (1985): Symmetrische Prädikate: Lexikoneintrag und Interpretationsspielraum. Eine Fallstudie zur Semantik der Personenstandslexik. Linguistische Studien 127: 75-112.

Lang, E. (1987): Semantik der Dimensionsauszeichnung räumlicher Objekte. In: Bierwisch, M. \& E. Lang (Hgg.): Grammatische und konzeptuelle Aspekte von Dimensionsadjektiven. Berlin: Akademie, 287-458.

Lang, E. \& C. Maienborn (2011): Two-Level Semantics: Semantic Form and Conceptual Structure. In: Maienborn, C., K. von Heusinger \& P. Portner (eds.): Semantics. Berlin, New York: de Gruyter, 709-740.

Larson, R. K. (1988): On the Double Object Construction. Linguistic Inquiry 19: 335-391. 
Lehmann, V. (2007): Linguistik des Russischen. Einführung in die formal-funktionale Beschreibung. 12: Grammatische Kategorien der Wortform. Ms., Univ. Hamburg.

Lehmann, V. (2009): Aspekt und Tempus im Slavischen. In: Kempgen, S. et al. (eds.): Die slavischen Sprachen: Ein internationales Handbuch zu ihrer Struktur, ihrer Geschichte und ihrer Erforschung. Bd. 1. Berlin, New York: de Gruyter, 526-556.

Levin, B. \& M. Rappaport Hovav (1995): Unaccusativity: At the Syntax-Lexical Semantics Interface. Cambridge, Mass.: MIT Press.

Levin-Steinmann, A. (2004): Die Legende vom bulgarischen Renarrativ. Bedeutung und Funktion der kopulalosen 1-Periphrase. München: Sagner.

Longobardi, G. (1994): Reference and Proper Names: A Theory of N-Movement in Syntax and Logical Form. Linguistic Inquiry 25: 609-665.

Lunt, H. G. (1974): Old Church Slavonic Grammar. The Hague, Paris: Mouton.

Madariaga, N. (2011): Infinitive Clauses and Dative Subjects in Russian. Russian Linguistics 35: 301-329.

Maienborn, C. (1997): On the Meaning of Sentence Modifiers: Semantic Indeterminacy and Its Grammatically Induced Specification. In: van der Sandt, R., R. Blutner \& M. Bierwisch (eds.): From Underspecification to Interpretation: Working Papers of the Institute of Logic and Linguistics. Heidelberg: ILLH, 183-202.

Maienborn, C. (2003a): Die logische Form von Kopula-Sätzen. Berlin: Akademie. Maienborn, C. (2003b): Against a Davidsonian Analysis in Copular Sentences. In: Kadowaki, M. \& S. Kawahara (eds.): NELS 44 Proceedings. Amherst: GLSA, 167-186.

Maienborn, C. (2005a): A Discourse-Based Account of Spanisch serlestar. Linguistics 43: 155-180.

Maienborn, C. (2005b): On the Limits of the Davidsonian Approach: The Case of Copula Sentences. Theoretical Linguistics 31.3: 275-316.

Maienborn, C. (2007a): On Davidsonian and Kimian States. In: Comorovski, I. \& K. von Heusinger (eds.): Existence: Semantics and Syntax. Dordrecht: Springer, $107-130$.

Maienborn, C. (2007b): Das Zustandspassiv: Grammatische Einordnung Bildungsbeschränkungen - Interpretationsspielraum. Zeitschrift für germanistische Linguistik 35: 83-15. 
Maienborn, C. (2009): Building Event-based ad hoc Properties: On the Interpretation of Adjectival Passives. In: Riester, A. \& T. Solstad (eds.): Proceedings of Sinn und Bedeutung 13. Stuttgart: OPUS, 35-49.

Maienborn, C. \& S. Geldermann (2013): Expertengeprüft und vom Experten geprüft: Zur Semantik von Nomen-Partizip II-Komposita und ihren präpositionalen Partnern. In: Härtl, H. (ed.): Interfaces of Morphology. Berlin: Akademie, $127-160$.

Markman, V. G. (2008): The Case of Predicates (Revisited): Predicate Instrumental in Russian and Its Restrictions. Journal of Slavic Linguistics 16: $187-246$.

Matthews, P. H. ( $\left.{ }^{2} 1991\right)$ : Morphology. Cambridge [u. a.]: CUP.

Matushansky, O. (2000): The Instrument of Inversion: Instrumental Case and Verb Raising in the Russian Copula. In: Billerey, R. \& B. D. Lillehaugen (eds.): Proceedings of WCCFL 19. Somerville: Cascadilla, 288-301.

Matushansky, O. (2008): A Case Study of Predication. In: Marušič, F. \& R. Žaucer (eds.): Studies in Formal Linguistics: Contributions from Formal Description of Slavic Languages. Frankfurt am Main [u. a.]: Lang, 213-239.

Mayerthaler, W. (1980): Morphologische Natürlichkeit. Wiesbaden: Athenaion.

MČ [Mluvnice češtiny] (1986) $\rightarrow$ Petr et al. (1986).

Mel’čuk, I. A. (1974): O sintaksičeskom nule. In: A. A. Cholodovič (red.): Tipologija passivnych konstrukcij: diatezy i zalogi. Leningrad: Nauka, 343-361.

Mezhevich, I. (2008): A Time-Relational Approach to Tense and Mood. In: Abner, N. \& J. Bishop (eds.): Proceedings of the 27th West Coast Conference on Formal Linguistics. Somerville, Mass.: Cascadilla, 326-334.

Migdalski, K. (2012): Diachronic Interaction between the Syntax of Clitics and the Availability of TP in Slavic. International Workshop 'Clitics and Beyond', Univ. Göttingen, 3. bis 5. Mai 2012 [Handout].

Milsark, G. L. (1974): Existential Sentences in English. Ph. diss., MIT, Cambridge, Mass.

Moro, A. (1997): The Raising of Predicates: Predicative Noun Phrases and the Theory of Clause Structure. Cambridge [u. a.]: CUP.

Moser, M. (1994): Der prädikative Instrumental: Aus der historischen Syntax des Nordostslavischen. Von den Anfängen bis zur petrinischen Epoche. Frankfurt am Main [u. a.]: Lang. 
Mrázek, R. (1964): Sintaksis russkogo tvoritel'nogo: Strukturno-sravnitel'noe issledovanie. Praha: SPN.

Mrázek, R. (1990): Sravnitel’nyj sintaksis slavjanskich literaturnych jazykov. 1: Ischodnye struktury prostogo predloženija. Brno: Univ. J. E. Purkyňe.

Mueller-Reichau, O. (2013): Why kratnost? On Russian Factual Imperfectives. In: Junghanns, U., D. Fehrmann, D. Lenertová \& H. Pitsch (eds.): Formal Description of Slavic Languages: The Ninth Conference. Proceedings of FDSL 9, Göttingen 2011. Frankfurt am Main [u. a.]: Lang, 191-209.

Nichols, J. (1981): Predicate Nominals: A Partial Surface Grammar of Russian. Berkeley: UoCP.

Nichols, J. (1985): Padežnye varianty predikativnych imën i ich otraženie v russkoj grammatike. Novoe v zarubežnoj lingvistike 15: 342-386.

Partee, B. H. (1987): Noun Phrase Interpretation and Type-Shifting Principles. In Groenendijk, J., D. de Jongh, \& M. Stokhof (eds.): Studies in Discourse Representation Theory and the Theory of Generalized Quantifiers. Dordrecht: Foris, 115-143.

Paslawska A. \& A. von Stechow (2003a): Perfect Readings in Russian. In: Alexiadou A., M. Rathert \& A. von Stechow (eds.): Perfect Explorations. Berlin, New York: de Gruyter, 307-362.

Paslawska A. \& A. von Stechow (2003b): Perfekt- und Futurkonstruktionen im Ukrainischen (und Russischen). Ms. [03.04.2003]. www.sfs.uni-tuebingen.de/ astechow/Aufsaetze/Ukrainisch99.pdf

Paslawska, A. \& A. von Stechow (2003c): Tempus-Aspekt-Aktionsarten-Architektur aus typologischer Sicht. Ms. [Juli 2003].

http://www.sfs.uni-tuebingen.de/ astechow/Aufsaetze/Paslawska.visnyk.pdf

Pereltsvaig, A. (2007): Copular Sentences in Russian: A Theory of Intra-Clausal Relations. Dordrecht: Springer.

Pesetsky, D. \& E. Torrego (2007): The Syntax of Valuation and Interpretability of Features. In: Karimi, S., V. Samiian \& W. Wilkins (eds.): Phrasal and Clausal Architecture: Syntactic Derivation and Interpretation. Amsterdam: Benjamins, 262-294.

Petr, J. et al. (1986) (red.): Mluvnice češtiny. Tom 2: Tvarosloví. Praha: Academia. 
Pitsch, H. (2008): Die Nominativ/Instrumental-Alternation in russischen Kopulasätzen: eine vergleichende Analyse zu deren Genese, Motivation und Verortung im grammatischen System mit dem Vorschlag für eine Synthese ausgewählter Ansätze. Magisterarbeit, Inst. für Slavistik, Univ. Leipzig.

Pitsch, H. (2012a): Zur Syntax und Semantik von Modalprädikativa. In: Szucsich, L., N. Gagarina, E. Gorishneva \& J. Leszkowicz (Hgg.): Linguistische Beiträge zur Slavistik: XIX. JungslavistInnen-Treffen in Berlin; 16.-18. Dezember 2010. München: Sagner, 181-194.

Pitsch, H. (2012b): Ljudmila Geist. Die Kopula und ihre Komplemente: zur Kompositionalität in Kopulasätzen. Journal of Slavic Linguistics 20.2: 287-298.

Pitsch, H. (2013): Russian Verb Stems and BYT'. In: Junghanns, U., D. Fehrmann, D. Lenertová \& H. Pitsch (eds.): Formal Description of Slavic Languages: The Ninth Conference. Proceedings of FDSL 9, Göttingen 2011. Frankfurt am Main [u. a.]: Lang, 211-235.

Pitsch, H. (2014): byt' und Prädikatsnomina im Russischen: ein geschärfter Blick auf den "semantischen Ansatz". Erscheint in: Pitsch, H. (Hg.): Linguistische Beiträge zur Slavistik. XXI. JungslavistInnen-Treffen in Göttingen 2012. München: Sagner.

Pollock, J.-Y. (1989): Verb Movement, Universal Grammar, and the Structure of IP. Linguistic Inquiry 20.3: 365-424.

Potebnja, A. A. (1958): Iz zapisok po russkoj grammatike. Moskva: Prosveščenie. Przepiórkowski, A. (2001): Case and Agreement in Polish Predicates. In: Franks, S., T. Halloway King \& M. Yadroff (eds.): Annual Workshop on Formal Approaches to Slavic Linguistics: The Bloomington Meeting. Ann Arbour, Mich.: Michigan Slavic Publ., 257-273.

Rapp, I. (1996): Zustand? Passiv? - Überlegungen zum sogenannten "Zustandspassiv". Zeitschrift für Sprachwissenschaft 15: 231-265.

Reichenbach, H. (1947): Elements of Symbolic Logic. New York: Macmillan.

Renz, M. (2009): Partizipantenbezogene Adverbien im Polnischen und Russischen. Poster, DoktorandInnen-Tag 2009, Fak. III, Univ. Oldenburg. http://www.slavistik.uni-oldenburg.de/download/Renz_Dissertation_Poster.pdf

Reyle, U., A. Rossdeutscher \& H. Kamp (2007): Ups and Downs in the Theory of Temporal Reference. Linguistics and Philosophy 30: 565-635.

Richardson, K. (2001): What Secondary Predicates in Russian Tell Us about the Link between Tense, Aspect and Case. ZAS Papers in Linguistics 26: 1-25. 
Richardson, K. (2007): Case and Aspect in Slavic. Oxford [u. a.]: OUP.

Røed, R. (1966): Zwei Studien über den prädikativen Instrumental im Russischen. Oslo: Universitetsforl.

Rothstein, R. A. (1986): Equation vs. Ascription: The Nominative/Instrumental Opposition in West Slavic. In: Brecht, R. D. \& S. Levine (eds.): Case in Slavic. Columbus, Ohio: Slavica, 312-322.

Rothstein, R. A. (1993): Polish. In: Comrie, B. \& G. G. Corbett (eds.): The Slavonic Languages. London, New York: Routledge, 686-758.

Rothstein, S. (1992): Predication and the Structure of Clauses. Belgian Journal of Linguistics 7: 153-169.

Roy, I. A. (2004): Predicate Nominals in Eventive Predication. University of Southern California Working Papers in Linguistics 2: 30-56.

Roy, I. A. (2006): Non-Verbal Predications: A Syntactic Analysis of Predicational Copular Sentences. Ph. D. diss., Los Angeles, University of Southern California.

Roy, I. A. (2013): Non-Verbal Predication: Copular Sentences at the SyntaxSemantics Interface. Oxford [u. a.]: OUP.

Rozental', D. Ė \& M. A. Telenkova (1972): Spravočnik lingvističeskich terminov: Posobie dlja učitelej. Moskva: Prosveščenie.

Rubin, E. J. (1994): Modification: A Syntactic Analysis and Its Consequences. $\mathrm{Ph}$. D. diss., Cornell University.

Ruferová, J. (1996): K stilističeskomu ispol’zovaniju kratkich i polnych predikativnych prilagatel'nych v sovremennom russkom jazyke. In: Jelitte, H. \& E். A. Balalykina (Hgg.): Das Adjektiv im Russischen: Geschichte, Strukturen, Funktionen. Frankfurt am Main [u. a.]: Lang, 287-295.

Russkaja grammatika (1980). Akademija Nauk SSSR, Institut russkogo jazyka. Tom 2: Sintaksis. Moskva [= AG (1980)].

Růžička, R. (1990): Über Syntax und Semantik russischer Gerundien (Adverbialpartizipien, Deepričastija). Wiener Slawistischer Almanach 25/26, 361-378.

Růžička, R. (1999): Control in Grammar and Pragmatics. A Cross-Linguistic Study. Amsterdam, Philadelphia: Benjamins.

Šachmatov, A. A. (1927): Sintaksis russkogo jazyka 2: Učenie o častjach reči: dopolnenija. Leningrad: AN SSSR.

Sadnik, L. (1966): Der Ersatz von Aorist und Imperfekt durch die l-Periphrase, namentlich im Russischen. Anzeiger für slavische Philologie 1: 16-30. 
Sadziński, R. (1989): Statische und dynamische Valenz: Probleme einer kontrastiven Valenzgrammatik Deutsch-Polnisch. Hamburg: Buske.

Saloni, Z. (1974): Klasyfikacja gramatyczna leksemów polskich. Język Polski 54: 93-101.

Ščerba, L. V. (1928): O častjach reči v russkom jazyke. Russkaja reč’. Novaja serija 2: 5-27.

Schaller, H. (1975): Das Prädikatsnomen im Russischen: Eine beschreibendhistorische Untersuchung. Köln, Weimar: Böhlau.

Schoorlemmer, M. (1994): Dative Subjects in Russian. In: Toman. J. (ed.): Formal Approaches to Slavic Linguistics: The Ann Arbor Meeting. Ann Arbor, Mich.: Michigan Slavic Publ., 129-172.

Schoorlemmer, M. (1995): Participial Passive and Aspect in Russian. Utrecht: OTS.

Schümann, M. (2010): Der russische Partikel-Konjunktiv und der deutsche würde-Konjunktiv im Vergleich. Linguistik Online 44: 4/10.

Shapiro, M. (1969): Aspects of Russian Morphology: a Semiotic Investigation. Cambridge, Mass.: Slavica Publ.

Skibicki, M. (2007): Polnische Grammatik. Hamburg: Buske.

Skoumalová, H. (2003): Multiverb Expressions in Czech. In: Beermann, D. \& L. Hellan (eds.): Proceedings of the Workshop of Multi-Verb Constructions, Trondheim Summer School 2003. Trondheim: NUST.

http://edvarda.hf.ntnu.no/ling/tross/skoumalova.pdf

Sonnenhauser, B. (2012): Auxiliar-Variation und Textstruktur im Bulgarischen. Die Welt der Slaven 57.2: 351-379.

Sonnenhauser, B. \& A. Meermann (2013): Perfektvariation im Serbischen zwischen slavischer und balkanischer Entwicklung. 22. JungslavistInnenTreffen, Univ. München, 13. Sept. 2013 [Handout].

Sørensen, H. C. (1975): Der russische Konjunktiv. Scando-Slavica 23: 145-159.

Sperber, W. (1972): Ist die "Zustandskategorie" eine für die Beschreibung der Grammatik slawischer Sprachen notwendige Wortart? Zeitschrift für Slawistik 17: 401-409. 
Stechow, A. von (2002): Temporal Prepositional Phrases with Quantifiers: Some Additions to Pratt and Francez (2001). Ms.

http://www.sfs.uni-tuebingen.de/ astechow/Aufsaetze/TPPs.final.pdf

Stechow, A. von (2007a): Schritte zur Satzsemantik 1. Ms. http://www.sfs.uni-tuebingen.de/ astechow/Aufsaetze/SchritteI.pdf

Stechow, A. von (2007b): Schritte zur Satzsemantik 2. Ms. http://www.sfs.uni-tuebingen.de/ astechow/Aufsaetze/SchritteII.pdf

Stechow, A. von (2009): Tenses in Compositional Semantics. In: Klein, W. \& P. Li (eds.): The Expression of Time. Berlin, New York: de Gruyter, 129-166.

Stechow, A. von (2012): Syntax and Semantics: An Overview. In: Maienborn, C., K. von Heusinger \& P. Portner (eds.): Semantics: An International Handbook of Natural Language Meaning. Berlin, New York: de Gruyter, 21732223.

Steinitz, R. (1990): Prädikation, Modifikation und Adverbiale. Linguistische Studien. Reihe A: Arbeitsberichte 206: 117-143.

Steinitz, R. (1992): Durative und inchoative Prädikate und die Adverbialkomplemente von Verben. In: Hoffmann, L. (Hg.): Deutsche Syntax: Ansichten und Aussichten. Berlin, New York: de Gruyter, 186-205.

Steinitz, R. (1997): Valenznotwendige Präpositionalphrasen: Weder Argumentnoch Adjunktposition. In: Dürscheid, C., K. H. Rahmers \& M. Schwarz (Hgg.): Festschrift für Heinz Vater zum 65. Geburtstag. Tübingen: Niemeyer, 229-350.

Steube, A. (1987): Grammatical Relations between Prepositions, Conjunctions and the Complementizer "daß" in a REST-Grammer of German. Linguistische Arbeitsberichte 61: 54-74.

Steube, A. (1997): Der russische Aspekt und die Ereignisrolle des Verbs. In: Junghanns, U. \& G. Zybatow (Hgg.): Formale Slavistik. Frankfurt am Main: Vervuert, 213-227.

Stowell, T. (1981): Origins of Phrase Structure. Doctoral diss., Cambridge, Mass.

Stowell, T. (1983): Subjects across Categories. Linguistic Review 2: 285-312.

Strigin, A. (2008): Secondary Predication and the Instrumental Case in Russian. In: Schroeder, C., G. Hentschel \& W. Boeder (eds.): Secondary Predicates in Eastern European Languages and Beyond. Oldenburg: BIS, 381-400.

Švedova, N. Ju. (1952): Polnye i kratkie formy imën prilagatel'nych v sostave skazuemogo v sovremennom russkom jazyke. Russkij jazyk 1952: 73-132. 
Švedova, N. Ju. \& V. V. Lopatin (red.) [= Š\&L] ( $\left.{ }^{2} 1990\right)$ : Russkaja grammatika. Moskva: Russkij jazyk.

Szucsich, L. (2009): Obviation and Feature Sharing in Subjunctive Clauses. In: Zybatow, G., U. Junghanns, D. Lenertová \& P. Biskup (eds.): Studies in Formal Slavic Phonology, Morphology, Syntax, Semantics and Information Structure. Proceedings of FDSL 7, Leipzig 2007. Frankfurt am Main [u. a.]: Lang, 209-220.

Š\&L $\left({ }^{2} 1990\right) \rightarrow$ Švedova \& Lopatin $\left({ }^{2} 1990\right)$.

Timberlake, A. (1985): The Temporal Schemata of Predicates. In: Brecht, R. D. \& M. S. Flier (eds.): Issues in Russian Morphosyntax. Columbus, Ohio: Slavica, 35-57.

Timberlake, A. (1986): The Semantics of Case in Russian Predicate Complements. Russian Linguistics 10: 137-165.

Timberlake, A. (1993): Russian. In: Comrie, B. \& G. G. Corbett (eds.): The Slavonic Languages. London, New York: Routledge, 827-886.

Toman, J. (1980): Weak and Strong: Notes on be in Czech. In: Brettschneider, G. \& C. Lehmann (Hgg.): Wege zur Universalienforschung: Sprachwissenschaftliche Beiträge zum 60. Geburtstag von Hansjakob Seiler. Tübingen: Narr, 305-310.

Trost, K. (1968): Die Perfektperiphrase im Altkirchenslavischen und Altarmenischen: ein Beitrag zur vergleichenden Syntax. Indogermanische Forschungen 73: 87-109.

Trunte, N. H. ( $\left.{ }^{5} 2005\right)$ : Slověnbskъjb językb. Ein praktisches Lehrbuch des Kirchenslavischen in 30 Lektionen. Zugleich eine Einführung in die slavische Philologie. Bd. 1: Altkirchenslavisch. München: Sagner.

Ueda, M. (1992): The Interaction between Clause-Level Parameters and Context in Russian Morphosyntax: Genitive of Negation and Predicate Adjectives. München: Sagner.

Veselovská, L. (1995): Phrasal Movement and $\mathrm{X}^{0}$-Morphology. Word Order Parallels in Czech and English Nominal and Verbal Projections. Ph. D. diss., Univerzita Palackého v Olomouci.

Vinogradov, V. V. (red.) (1952): Sovremennyj russkij jazyk. Morfologija (kurs lekcij). Moskva: MU.

Vinogradov, V. V. \& E. S. Istrina (red. koll.) (1960): Grammatika russkogo jazyka. 2.1: Sintaksis. Moskva: AN SSSR. 
Vostokov, A. Ch. ( $\left.{ }^{4} 1839\right):$ Russkaja grammatika Aleksandra Vostokova, po načertaniju ego že sokraščennoj Grammatiki polněe izložennaja. S. Peterburg": Tipografija Akademii.

Weiss, D. (1978): Identitätsaussagen im Russischen: Ein Versuch ihrer Abgrenzung gegenüber anderen Satztypen. In: Girke, W. (Hg.): Slavistische Linguistik 1977. Referate des III. Konstanzer Slavistischen Arbeitstreffens, Bochum 27.9.77-29.9.77. München: Sagner, 224-259.

Wierzbicka, A. (1980): The Case for Surface Case. Ann Arbor, Mich.: Karoma.

Wilder, C. (1994): Small Clauses im Englischen und in der GB-Theorie. In: Steube, A. \& G. Zybatow (Hgg.): Zur Satzwertigkeit von Infinitiven und Small Clauses. Tübingen: Niemeyer, 219-241.

Williams, E. (1984): There-Insertion. Linguistic Inquiry 15: 131-153.

Wiśniewski, M. (1990): Formalnogramatyczny opis leksemów TO. 2: Słowo to w funkcji spójnika, partykuły lub czasownika niewłaściwego. Acta Universitatis Nicolai Copernici 31: 91-119.

Witkoś, J. (2010): On the Lack of Case on the Subject of Infinitives in Polish. Folia Linguistica 44: 179-238.

Wolińska, O. (1978): Konstrukcje bezmianownikowe we współczesnej polszczyźnie. Katowice: UŚ.

Wunderlich, D. (1991): How do Prepositional Phrases Fit into Compositional Syntax and Semantics? Linguistics 29: 591-621.

Wunderlich, D. (1996): Lexical Categories. Theoretical Linguistics 22: 1-48.

Wunderlich, D. (1997): Cause and the Structure of Verbs. Linguistic Inquiry 28: $27-68$.

Zamparelli, R. (2000): Layers in the Determiner Phrase. New York: Garland.

Zifonun, G., L. Hoffmann \& B. Strecker (1997): Grammatik der deutschen Sprache 1-3. Berlin, New York: de Gruyter.

Zimmermann, I. (1992): Der Skopus von Modifikatoren. In: Zimmermann, I. \& A. Strigin (Hgg.): Fügungspotenzen. Berlin: Akademie, 251-279.

Zimmermann, I. (1999): Partizip II-Konstruktionen des Deutschen als Modifikatoren. ZAS Papers in Linguistics 14: 123-146.

Zimmermann, I. (2003a): On the Semantics of Cases. In: Junghanns, U. \& L. Szucsich (eds.): Syntactic Structures and Morphological Information. Berlin, New York: de Gruyter, 341-380. 
Zimmermann, I. (2003b): German Participle II Constructions as Adjuncts. In: Lang, E., C. Maienborn \& C. Fabricius-Hansen (eds.): Modifying Adjuncts. Berlin, New York: de Gruyter, 627-649.

Zimmermann, I. (2009): Satzmodus. In: Kempgen, S. et al. (Hgg.): Die slavischen Sprachen: Ein internationales Handbuch zu ihrer Struktur, ihrer Geschichte und ihrer Erforschung. Bd. 1. Berlin, New York: de Gruyter, 484-505.

Zimmermann, I. (2012): Leere Köpfe und verzögerte Interpretation. Kolloquium zur slavistischen Linguistik, Univ. Göttingen, 27. Juni 2012 [Handout].

Zimmermann, I. (2013): Selektion und Interpretation morpho-syntaktischer Einheiten. In: Härtl, H. (Hg.): Interfaces of Morphology. Berlin: Akademie, $217-228$.

Zybatow, G. (1994): Infinitive, kleine und große Pros und (in)kohärente Konstruktionen im Russischen. In: Steube, A. \& G. Zybatow (Hgg.): Zur Satzwertigkeit von Infinitiven und Small Clauses. Tübingen: Niemeyer, 141154.

Zybatow, G. \& O. Mueller-Reichau (2011): Typ oder Token? Zum Auftreten von est' in russischen Possessivsätzen. Zeitschrift für Slawistik 56: 305-318.

\section{Quellenverzeichnis}

K06 Krall, H. (2006): Król kier znów na wylocie. Warszawa: Świat Książki.

NKJP Narodowy Korpus Języka Polskiego.

http://nkjp.pl/poliqarp

NKRJ Nacionalnyj Korpus Russkogo Jazyka.

http://www.ruscorpora.ru/index.html 


\section{Appendix}

Dieser Anhang soll an russischen Beispielen zeigen, dass die Erwägungen zur grammatischen Architektur von Verbalaspekt, Tempus und Verbmodus im Nordslavischen, die sich auf Grund der argumentativen Gliederung der Arbeit auf die verschiedenen Abschnitte verteilen, in Bezug auf die Analyse originalsprachlicher Sätze anwendbar und konsistent sind.

Hierfür werde ich zunächst das allgemeine Format beschreiben und folgend mittels vierer Beispiele für russische Kopulasätze (A, B, C, D) sowie zweier Beispiele für russische Nicht-Kopulasätze (E, F) die semantische Amalgamierung der beteiligten lexikalischen und grammatischen Komponenten darstellen. ${ }^{257}$ Wo nötig, werden nähere Informationen und Verweise auf die relevanten Abschnitte der Arbeit gegeben. Die Beispiele der Nicht-Kopulasätze zeigen, dass die vorgeschlagenen theoretischen Erwägungen nicht nur auf Kopula-Prädikativ-Strukturen anwendbar sind. Die semantische Amalgamierung wird jeweils parallel zur syntaktischen Strukturbildung in einem Baumdiagramm nachvollzogen.

\section{Allgemeines Format (“"\#” = "A"/“B"B/“"C"/“"D"/“"E"/“F"}

(\#1) Originalbeispiel

(\#2) a. Lexikoneintrag des Prädikatsnomens (A-D)/des Vollverbs (E und F)

b. Lexikoneintrag der Kopulabedeutung in $\varnothing_{\mathrm{V}}$ (fehlt bei E und F)

c. Bedeutungscharakterisierung der unteren VP

d. Lexikoneintrag des involvierten V-Auxiliars (sofern gegeben)

e. Bedeutungscharakterisierung der oberen VP (sofern gegeben)

f. Lexikoneintrag des involvierten I-Kopfes (Auxiliar/Operator)

g. Bedeutungscharakterisierung der IP

h. Lexikoneintrag des C-Kopfes

i. Bedeutungscharakterisierung der CP (Satzsemantik)

(\#3) Darstellung der syntaktischen Strukturbildung mit paralleler Angabe der semantischen Amalgamierung

${ }^{257}$ Die vier Kopulasatzbeispiele beinhalten ein prädikatives Substantiv (A), Langformadjektiv (B), Kurzformadjektiv (C) sowie ein Zustandsprädikativ (D). Die Nicht-Kopulasätze zeigen ein imperfektives (E) sowie ein perfektives (F) Satzprädikat. 


\title{
A-: Beispiel eines russischen Kopulasatzes mit prädikativem Substantiv
}

\author{
(A1) Anna byla učitel'nicej. \\ Anna war-SG.F Lehrerin-INS.SG.F \\ 'Anna war Lehrerin.'
}

In (A2) werden die Komponenten des Satzes (A1) einzeln dargestellt und kommentiert.

(A2) a. i. /učitel'nicej/

ii. $\quad[+\mathrm{N},-\mathrm{V},-\mathrm{a},+$ Anim, $-\mathrm{D},-\mathrm{Q},+\mathrm{P},-\mathrm{Pl},-\mathrm{Masc},+\mathrm{Fem},+\mathrm{max}]$

iii. $\lambda x[x$ TEACHER $]$

Es handelt sich um ein Substantiv mit belebtem Referenten im Instrumental, das im Lexikon als Einheit vom Typ <e, $\mathrm{t}>$ abgelegt ist (\$4.4.2). Die Einheit projiziert zu NP.

b. i. $\mid \varnothing /$

ii. $[+\mathrm{V},+\mathrm{N},-$ Perf,$+\max ]$

iii. $\lambda P_{[ \pm \mathrm{P}]} \lambda x \lambda t \exists s[[t \subseteq \tau(s)]: s$ INST $[P x]]$

Dies ist der Lexikoneintrag der abstrakten Kopulabedeutung, die als stummer (phonetisch leerer) V-Kopf syntaktifiziert wird. Die prädikative NP in (A2a) kann als Komplement eingesetzt werden ( $\S 5.1$ und 5.5).

c. $\quad \lambda x \lambda t \exists s[[t \subseteq \tau(s)]: s$ INST $[x$ TEACHER $]]$

(untere VP)

Dies ist die Bedeutungscharakterisierung der unteren VP. Auf dieser Ebene ist die prädikative NP in (A2a) bereits eingesetzt. Ein Subjektausdruck kann nicht syntaktifiziert werden, da der leere V-Kopf mit der Kopulabedeutung in (A2b) infinitivisch ist und daher die externe Argumentstelle $\lambda x$ nicht mit Kongruenzmerkmalen versorgen kann.

d. i. /byla/

ii. $[+\mathrm{V},+\mathrm{N},-\mathrm{Perf},-\mathrm{Pl},-\mathrm{Masc},+\mathrm{Fem},+\max ]$

iii. $\lambda P[P]$

Hier liegt ein V-Auxiliar vor. Es hat keine Eigenbedeutung, sondern dient ausschließlich der Ausbuchstabierung von Finitheits- und Kongruenzmerkmalen (§ 5.2). Es ist als 1-Partizip ausgewiesen (§ 4.3.4) und kann somit vom präteritalen oder konjunktivischen I-Auxiliar selegiert werden (\$ 4.6.3). Siehe zur vereinfachten Notation in (A2d) Fn. 111 in Abschnitt 4.3.5. 
Dies ist die Bedeutungscharakterisierung der oberen VP. Da das V-Auxiliar in (A2d) keine semantischen Anteile gebracht hat, handelt es sich um eine identische Abbildung von (A2c).

f. i. $|\varnothing|$

ii. $[+\mathrm{I}]$

iii. $\lambda P_{[+\mathrm{V},+\mathrm{N}, \pm \mathrm{Perf},+\mathrm{Agr}]} \lambda x_{[ \pm 1, \pm 2, \pm \mathrm{Pl}]} \exists t\left[\left[t\right.\right.$ BEFORE $\left.\left.\mathrm{t}^{0}\right] \wedge[P x t]\right]$

Im nächsten Schritt wird dieser I-Kopf projiziert, bei dem es sich um das präteritale I-Auxiliar handelt (§ 4.6.2, Eintrag (49)). Es selegiert 1-Partizipien; somit kommt das V-Auxiliar in (A2d) als Selektionsobjekt in Frage. Das I-Auxiliar bringt die Modus- und Tempusbedeutung INDIKATIV PRÄTERITUM ein. Zudem versorgt es die externe Argumentstelle $\lambda x$ mit variablen Kongruenzmerkmalen, so dass in Spec-IP ein Subjektausdruck mit einer beliebigen Kombination von Person- und Numerusmerkmalen generiert werden kann.

$$
\text { g. } \left.\exists t \exists s\left[\left[t \text { BEFORE } \mathrm{t}^{0}\right] \wedge[[t \subseteq \tau(s)]: s \text { INST [ANNA TEACHER }]\right]\right]
$$

Dies ist die Bedeutungscharakterisierung der IP. Man sieht, dass der Subjektausdruck Anna nunmehr eingesetzt ist. Außerdem sind Verbmodus und Tempus spezifiziert. Entsprechend ist das Situationsargument s existenziell abgebunden (\$ 4.6.2). Der gesamte Term ist vom Propositionstyp $<\mathrm{t}>$.

h. i. $/ \varnothing /$

ii. $[+\mathrm{C}]$

iii. $\lambda p[\mathrm{DECL} p]$

Dieser Eintrag für den 'unmarkierten' C-Kopf folgt Zimmermann (2009, 486). Der phonetisch leere C-Kopf selegiert eine Proposition und macht sie mittels des Satzmodus-Operators DECL deklarativ (Ausstattung mit 'illokutionärer Kraft').

\section{i. $\quad$ DECL $\left[\exists t \exists s\left[\left[t\right.\right.\right.$ BEFORE $\left.\mathrm{t}^{0}\right] \wedge[[t \subseteq \tau(s)]: s$ INST [ANNA TEACHER $\left.\left.\left.]\right]\right]\right](\mathrm{CP})$}

Dies ist die Satzsemantik (grammatische Bedeutung/Semantische Form) von (A1).

Auf der folgenden Seite wird in (A3) die syntaktische Strukturbildung für (A1) dargestellt. Indem die relevanten Knoten mit Bedeutungscharakterisierungen versehen werden, wird auch die semantische Amalgamierung nachvollzogen. Aus Platzgründen wird die Semantische Form der Köpfe lediglich durch Verweise auf (A2) angegeben. Siehe bzgl. der traditionellen Kategorienetikettierung Fn. 115 in Abschnitt 4.4.1! 
(A3)

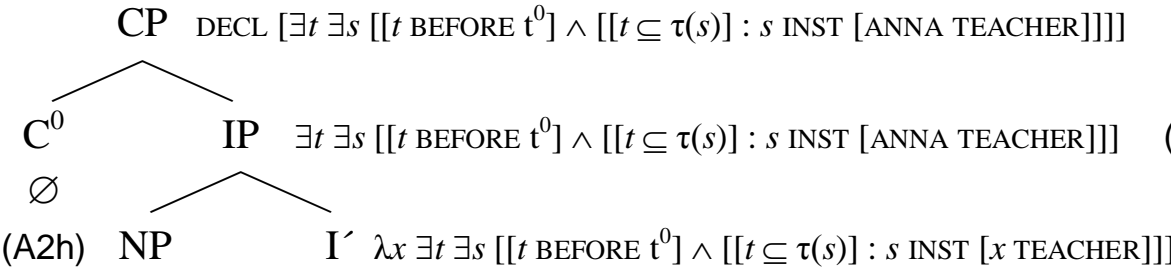

(A2i)

(A2h) NP $\quad \mathrm{I}^{\prime} \lambda x \exists t \exists s\left[\left[t\right.\right.$ BEFORE $\left.\mathrm{t}^{0}\right] \wedge[[t \subseteq \tau(s)]: s$ INST $[x$ TEACHER $\left.]]\right]$

Anna ANNA

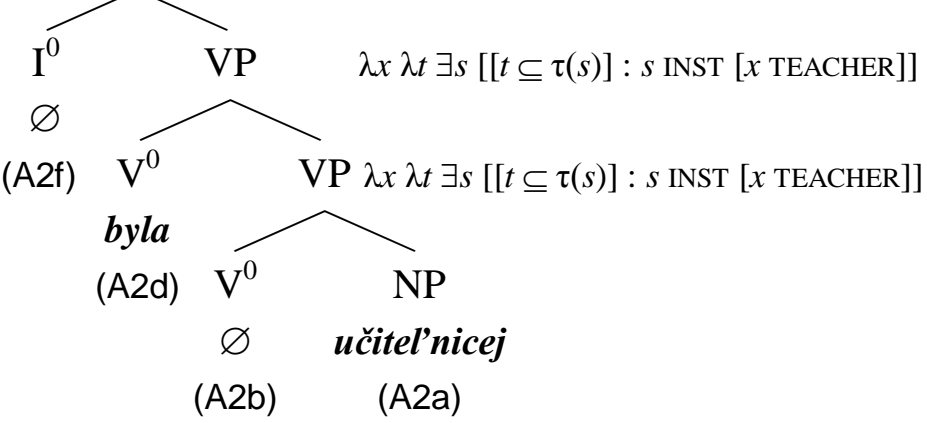

(A2e)

(A2c)

\section{B: Beispiel eines russischen Kopulasatzes mit prädikativer Langform}

(B1) Anna - dobraja.

Anna [ist-3.SG] gut $_{\mathrm{LF}}$-NOM.SG.F

'Anna ist gut.'

In (B2) werden die Komponenten des Satzes (B1) dargestellt und kommentiert.

(B2) a. i. /dobraja/

ii. $\quad[-\mathrm{V},+\mathrm{N},+\mathrm{a},-\mathrm{D},-\mathrm{Q},-\mathrm{P},-\mathrm{Pl},-\mathrm{Masc},+\mathrm{Fem},+\max ]$

iii. $\lambda x[x \mathrm{GOOD}]$

Es handelt sich um ein Langformadjektiv mit Nominativmarkierung, das im Lexikon als Einheit vom Typ <e, $\mathrm{t}>$ abgelegt ist $(\S 4.4 .3)$.

b. i. $\mid \varnothing /$

ii. $[+\mathrm{V},+\mathrm{N},-$ Perf,$+\max ]$

iii. $\lambda P_{[ \pm \mathrm{P}]} \lambda x \lambda t \exists s[[t \subseteq \tau(s)]: s$ INST $[P x]]$

Die prädikative AP in (B2a) wird hierin als Komplement eingesetzt (\$§ 5.1 und 5.5).

c. $\quad \lambda x \lambda t \exists s[[t \subseteq \tau(s)]: s$ INST $[x \mathrm{GOOD}]]$

(untere VP)

Dies ist die Bedeutungscharakterisierung der unteren VP-auf dieser Ebene ist die prädikative AP in (B2a) eingesetzt. Ein Subjektausdruck kann auf Grund der Infinitheit der abstrakten Kopulabedeutung in (B2b) nicht syntaktifiziert werden. 
d. i. $\mid \varnothing /$

ii. $[+\mathrm{V},-\mathrm{N},-$ Perf, + max $]$

iii. $\lambda P \lambda x_{[ \pm 1, \pm 2, \pm \mathrm{P}]}\left[\begin{array}{ll}P & x]\end{array}\right.$

Hier liegt das phonetisch leere, präsentische V-Auxiliar vor. Es hat keine Eigenbedeutung und dient lediglich der Ausbuchstabierung der relevanten Merkmale (§ 5.2). Es ist finit und kann so das externe Argument als Nominativsubjekt syntaktifizieren. Seine eigenen morphosyntaktischen Merkmale erlauben seine Selektion durch den präsentischen I-Operator (§ 4.6.3).

e. $\lambda t \exists s[[t \subseteq \tau(s)]: s$ INST [ANNA GOOD $]]$

(obere VP)

Dies ist die Bedeutungscharakterisierung der oberen VP. Da das V-Auxiliar in (B2d) finit ist, ist hier der Subjektausdruck Anna bereits eingesetzt, und zwar in Spec-VP.

f. i. $|\varnothing|$

ii. $[+\mathrm{I}]$

iii. $\lambda P_{[+\mathrm{V},-\mathrm{N},-\mathrm{Perf}]} \exists t\left[\left[t \mathrm{AT} \mathrm{t}^{0}\right] \wedge[P t]\right]$

Im nächsten Schritt wird dieser präsentische I-Kopf projiziert, bei dem es sich um einen koverten Operator handelt (\$ 4.6.2, Eintrag (45)). Er selegiert imperfektive Finita und akzeptiert somit das V-Auxiliar in (B2d). Ferner bringt er die Modus- und Tempusbedeutung INDIKATIV PRÄSENS ein.

g. $\exists t \exists s\left[\left[t \mathrm{AT} \mathrm{t}^{0}\right] \wedge[[t \subseteq \tau(s)]: s\right.$ INST [ANNA GOOD $\left.\left.]\right]\right]$

Dies ist die Bedeutungscharakterisierung der IP.

h. i. $\mid \varnothing /$

ii. $[+\mathrm{C}]$

iii. $\lambda p[\operatorname{DECL} p]$

Siehe hierzu bereits (A2h).

i. $\quad \operatorname{DECL}\left[\exists t \exists s\left[\left[t \mathrm{AT} \mathrm{t}^{0}\right] \wedge[[t \subseteq \tau(s)]: s\right.\right.$ INST [ANNA GOOD] $\left.\left.]\right]\right]$

Dies ist die Satzsemantik von (B1).

Auf der folgenden Seite wird in (B3) die syntaktische Strukturbildung für (B1) dargestellt. 
(B3)

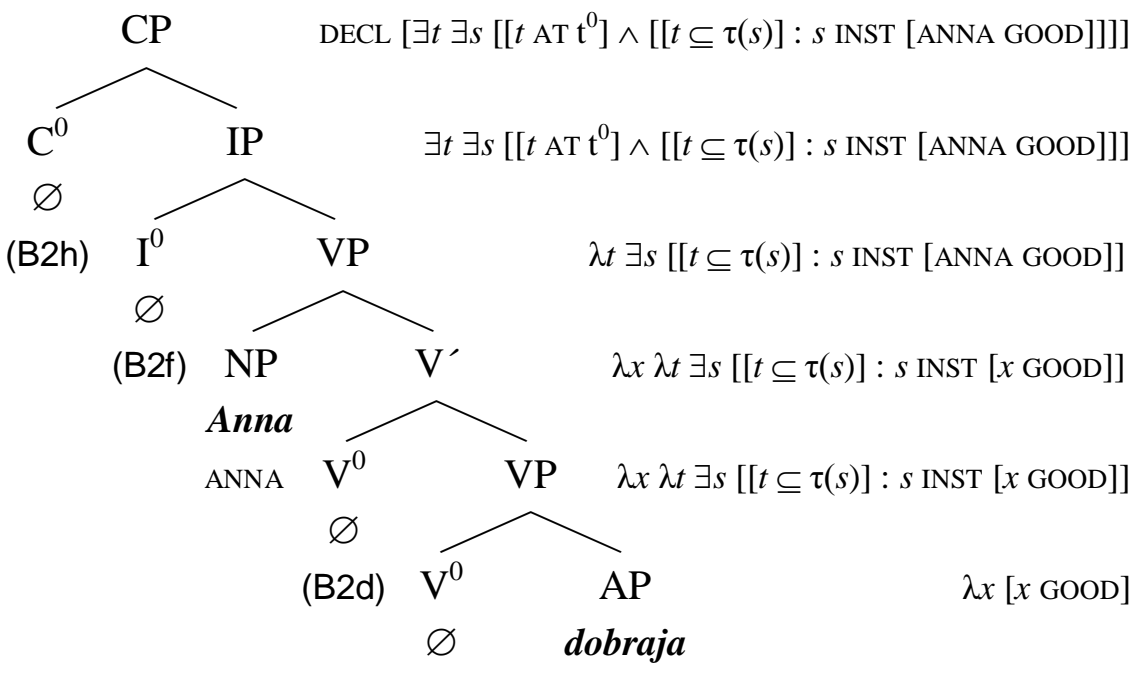

(B2i)

(B2b)

\section{C: Beispiel eines russischen Kopulasatzes mit prädikativer Kurzform}

(C1) Anna byla dobra.

Anna war-LPT-SG.F gut $_{\mathrm{KF}}$-NOM.SG.F

'Anna war gut.'

In (C2) werden die Komponenten des Satzes (C1) dargestellt und kommentiert.

(C2) a. i. /dobra/

ii. $\quad[+\mathrm{V},+\mathrm{N},-\mathrm{D},-\mathrm{Q},-\mathrm{P},-\mathrm{Pl},-\mathrm{Masc},+\mathrm{Fem},+\max ]$

iii. $\lambda x[x \mathrm{GOOD}]$

Es handelt sich um ein Kurzformadjektiv mit Nominativmarkierung, das im Lexikon als Einheit vom Typ <e,t> abgelegt und eine verbalnominale Form ist (§§ 4.4.3 und 5.6.1). Die Kurzform ist somit infiniten Verbformen vergleichbar, weist aber auf Grund ihrer nominalen Herkunft keine Sachverhaltsreferenz auf. Entsprechend ist die Kopulabedeutung in (C2b) notwendig.

b. i. $\mid \varnothing /$

ii. $[+\mathrm{V},+\mathrm{N},-$ Perf, + max $]$

iii. $\lambda P_{[ \pm \mathrm{P}]} \lambda x \lambda t \exists s[[t \subseteq \tau(s)]: s$ INST $[P x]]$

Die prädikative AP in (C2a) wird als Komplement eingesetzt ( $\S 5.1$ und 5.5). Da Kurzformen nur nominativische Formen haben, kann bei ihnen von $[+\mathrm{P}]$ generell kein Gebrauch gemacht werden. Jedoch ist zu beachten, dass es sich bei Kurzformen auch so bereits um explizit prädikative Formen handelt, die so auf die gesonderte Instrumentalmarkierung verzichten können. 
Die Bedeutungscharakterisierung der unteren VP. Die prädikative AP aus (C2a) ist eingesetzt. Wegen der Infinitheit kann (noch) kein Subjektausdruck syntaktifiziert werden.

d. i. /byla/

ii. $[+\mathrm{V},+\mathrm{N},-\mathrm{Perf},-\mathrm{Pl},-\mathrm{Masc},+\mathrm{Fem},+\max ]$

iii. $\lambda P[P]$

Dieses V-Auxiliar ist identisch mit (A2d) weiter oben. Als 1-Partizip kann es vom präteritalen oder vom konjunktivischen I-Auxiliar selegiert werden (§ 4.6.3).

e. $\lambda x \lambda t \exists s[[t \subseteq \tau(s)]: s$ INST $[x \mathrm{GOOD}]]$

(obere VP)

Die obere VP ist bedeutungsidentisch mit der unteren VP, da das V-Auxiliar keinerlei Eigenanteile eingebracht hat und zudem infinit ist. Der Subjektausdruck kann nach wie vor nicht syntaktifiziert werden.

f. i. $/ \varnothing /$

ii. $[+\mathrm{I}]$

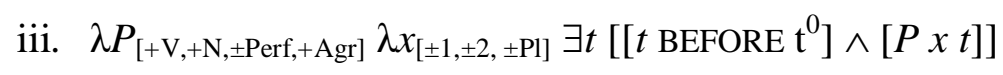

Durch diesen präteritalen I-Kopf wird das V-Auxiliar in (C2d) selegiert und 'erhält' so die Modus-/Tempusbedeutung INDIKATIV/PRÄTERITUM. Qua Annotation an der externen Argumentstelle kann in Spec-IP nun der Subjektausdruck Anna syntaktifiziert werden.

g. $\exists t \exists s\left[\left[t\right.\right.$ BEFORE $\left.\mathrm{t}^{0}\right] \wedge[[t \subseteq \tau(s)]: s$ INST [ANNA GOOD $\left.\left.]\right]\right]$

Das ist die Bedeutungscharakterisierung der IP mit eingesetztem Subjektausdruck sowie mit Modus- und Tempusspezifikation.

h. i. $/ \varnothing /$

ii. $[+\mathrm{C}]$

iii. $\lambda p[$ DECL $p]$

Siehe hierzu bereits (A2h).

i. $\quad$ DECL $\left[\exists t \exists s\left[\left[t\right.\right.\right.$ BEFORE $\left.\mathrm{t}^{0}\right] \wedge[[t \subseteq \tau(s)]: s$ INST [ANNA GOOD $\left.\left.\left.]\right]\right]\right]$ Mit (C2i) liegt die Satzsemantik von (C1) vor.

Auf der folgenden Seite wird in (C3) die syntaktische Strukturbildung für (C1) dargestellt. 
(C3)

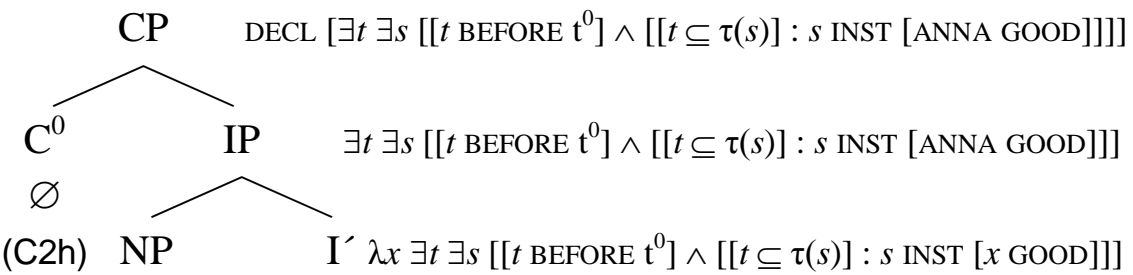

(C2i)

(C2h)

Anna

ANNA

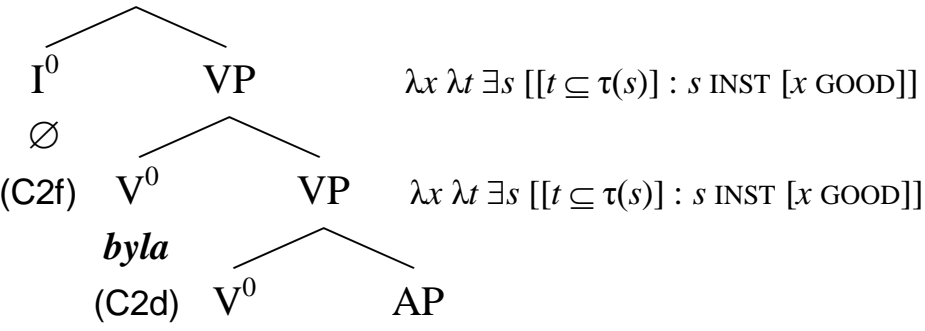

\section{D: Beispiel eines russischen Kopulasatzes mit Zustandsprädikativ}

(D1) Bylo

cholodno.

(Rus)

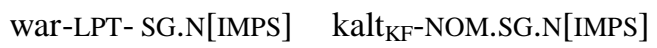

'Es war kalt.'

In (D2) werden die Komponenten des Satzes (D1) dargestellt und kommentiert.

(D2) a. i. /cholodno/

ii. $\quad[+\mathrm{V},+\mathrm{N},-\mathrm{D},-\mathrm{Q},-\mathrm{P},+\max ]$

iii. $\lambda x[x \mathrm{COLD}]$

Es handelt sich um ein Kurzformadjektiv mit Nominativmarkierung, das im Lexikon als Einheit vom Typ <e, $\mathrm{t}>$ abgelegt und eine verbalnominale Form ist (\$§ 4.4.3 und 5.6.1). Zwar zeigt es den Marker-o, der üblicherweise mit den Merkmalen Sg. und Neutr. assoziiert wird, jedoch ist dieser m. E. ambig zwischen (a) den genannten und (b) überhaupt keinen Merkmalen (was den Default darstellt, wenn kein Kongruenzgeber vorhanden ist oder ein vorhandener Kongruenzgeber keine Kongruenzmerkmale hat. Letztere Variante von -o liegt bei Zustandsprädikativen vor, da sie später mit einem Subjektausdruck 'ohne Merkmale' quasi kongruieren müssen. Entsprechend hat die Form cholodno keine Kongruenzmerkmale, sondern lediglich Kasus (vgl. \$ 5.6.3, Eintrag (183b)). 
b. i. $\mid \varnothing /$

ii. $[+\mathrm{V},+\mathrm{N},-$ Perf,$+\max ]$

iii. $\lambda P_{[ \pm \mathrm{P}]} \lambda x \lambda t \exists s[[t \subseteq \tau(s)]: s$ INST $[P x]]$

Da die prädikative AP in (D2a) vom Typ <e,t> ist, kann sie hier als Komplement eingesetzt werden. Siehe ansonsten bereits $(C 2 b)$ oben.

c. $\quad \lambda x \lambda t \exists s[[t \subseteq \tau(s)]: s$ INST $[x \operatorname{COLD}]]$

(untere VP)

Dies ist die Bedeutungscharakterisierung der unteren VP mit der eingesetzten prädikativen AP aus (D2a).

d. i. /bylo/

ii. $[+\mathrm{V},+\mathrm{N},-$ Perf,$+\max ]$

iii. $\lambda P[P]$

Dieses präteritale V-Auxiliar hat die Besonderheit, 'unpersönlich' flektiert zu sein. Wie schon das Zustandsprädikativ cholodno in (D2a), hat auch dieses Auxiliar keine Kongruenzmerkmale (Kasus hat es nicht bzw. braucht es nicht zu haben, da es ein deverbales Verbalnomen ist; Kurzformadjektive hingegen sind denominale Verbalnomina). Da es sich um ein 1-Partizip handelt, kann es vom präteritalen oder konjunktivischen I-Auxiliar selegiert werden (§ 4.6.3).

e. $\lambda x \lambda t \exists s[[t \subseteq \tau(s)]: s$ INST $[x \operatorname{COLD}]]$ (obere VP)

Obere und untere VP sind hier erneut bedeutungsidentisch, weil das V-Auxiliar bylo keine Semantik mitgebracht hat.

f. i. $|\varnothing|$

ii. $[+\mathrm{I}]$

iii. $\lambda P_{[+\mathrm{V},+\mathrm{N}, \pm \mathrm{Perf},+\mathrm{Agr}]} \lambda x_{[ \pm 1, \pm 2, \pm \mathrm{Pl}]} \exists t\left[\left[t \mathrm{BEFORE}^{0}\right] \wedge[P x t]\right]$

Siehe schon (C2f).

$$
\text { g. } \exists x_{[- \text {def,-vol }]} \exists t \exists s\left[\left[t \text { BEFORE } t^{0}\right] \wedge[[t \subseteq \tau(s)]: s \text { INST }[x \text { COLD }]]\right]
$$

Das ist die Bedeutungscharakterisierung der IP mit Subjektausdruck und Modus-/Tempusspezifikation. Hier muss angemerkt und beachtet werden, dass als Subjektausdruck das in $§ 5.6 .3$ vorgeschlagene Nullpronomen $\left(\varnothing_{\text {es }}\right)$ eingesetzt wurde, das selbst keine Kongruenzmerkmale aufweist und daher mit den ebenfalls 'unpersönlichen' Formen bylo aus (D2d) und cholodno aus (D2a) kongruiert. Das Nullpronomen bindet die externe Argumentstelle, so dass dafür nichts Konkretes mehr eingesetzt werden kann. Ferner schränkt es dessen Interpretation auf etwas Indefinites und Non-Volitionales ein (Annotation [-def,-vol]; siehe § 5.6.3, unter (180)). 
h. i. $\mid \varnothing /$

ii. $\quad[+\mathrm{C}]$

iii. $\lambda p[\operatorname{DECL} p]$

Siehe hierzu wiederum schon (A2h).

i. $\quad$ DECL $\left[\exists x_{[- \text {def,-vol }]} \exists t \exists s\left[\left[t\right.\right.\right.$ BEFORE $\left.\mathrm{t}^{0}\right] \wedge[[t \subseteq \tau(s)]: s$ INST $[x$ COLD $\left.\left.]]\right]\right]$

Diese Bedeutungscharakterisierung dürfte der Satzsemantik von (D1) entsprechen.

In (D3) wird die syntaktische Strukturbildung für (D1) dargestellt.

(D3)

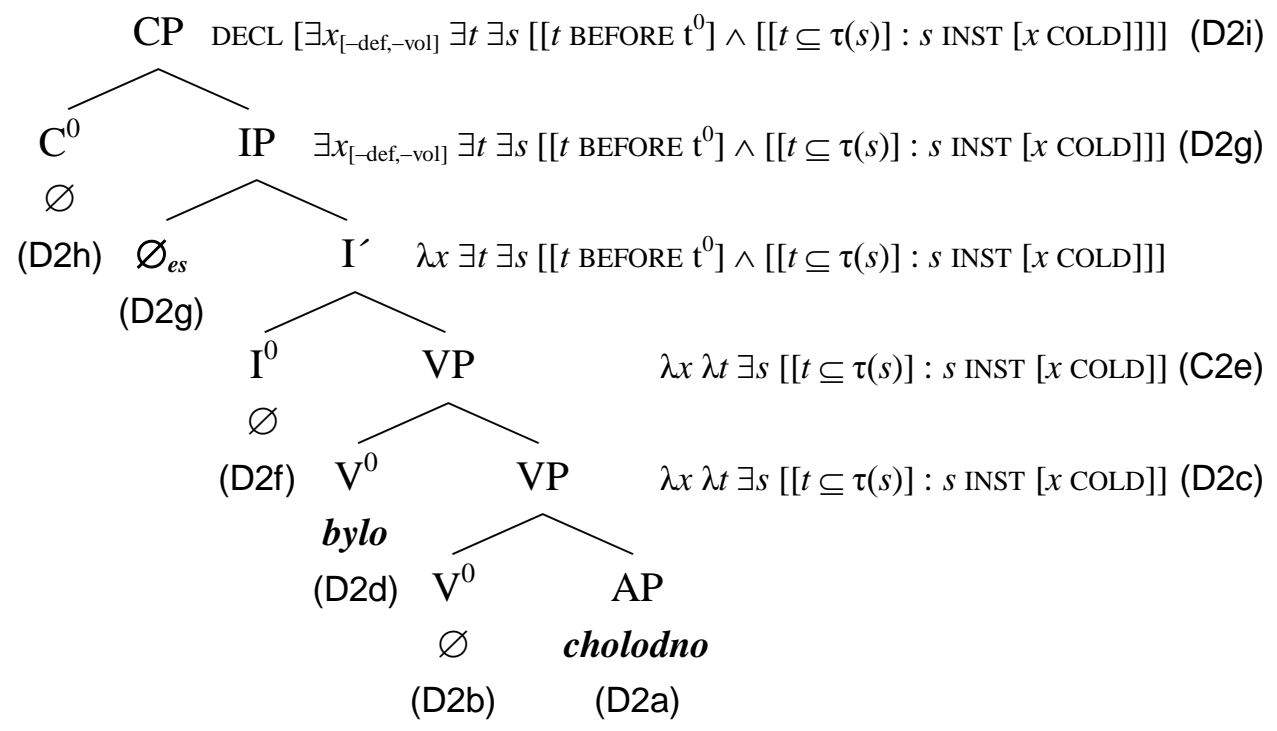




\title{
E: Beispiel eines russischen Nicht-Kopulasatzes (imperfektiv)
}

\author{
(E1) Anna budet rabotat'. \\ Anna wird-3.SG arbeit-INF \\ 'Anna wird arbeiten.'
}

Das Prädikat dieses Satzes ist unergativisch. Laut gängiger Ansicht hat es nur ein Argument mit der Thetarolle AGENS. Der Satz zeigt das periphrastische (imperfektive) Futur.

(E2) a. i. /rabotat'/

$$
\begin{array}{ll}
\text { ii. } & {[+\mathrm{V},+\mathrm{N},- \text { Perf, }+ \text { max }]} \\
\text { iii. } & \lambda x \lambda t \exists s[[t \subseteq \tau(s)]: s \text { INST }[x \text { WORK }]]
\end{array}
$$

Das Verb liegt hier als Infinitiv vor (§ 4.3.2). Der Infinitiv kann das externe Argument nicht syntaktifizieren (keine Annotation an $\lambda x$ ). Das Lexem bezeichnet eine Vendlersche activity und ist imperfektiv.

b.

Diese Komponente ist in Nicht-Kopulasätzen nicht vorhanden, da 'normale' Verbform von sich aus über Situationsbezug und Aspekt verfügen (eine leere Kopulabedeutung ist überflüssig).

$$
\text { c. } \quad \lambda x \lambda t \exists s[[t \subseteq \tau(s)]: s \text { INST }[x \text { WORK }]]
$$

Die Bedeutungscharakterisierung der unteren VP-Ebene ist mit der Bedeutung des infinitivischen V-Kopfes in (E2a) identisch, da der Infinitiv direkt zu VP projiziert.

d. i. /budet/

$$
\begin{aligned}
& \text { ii. }[+\mathrm{V},-\mathrm{N},+ \text { Perf },+\max ] \\
& \text { iii. } \lambda P_{[+\mathrm{V},+\mathrm{N}, \pm \operatorname{Perf}, \pm \mathrm{Agr}]} \lambda x_{[-\mathrm{Pl},-1,-2]}[P x]
\end{aligned}
$$

Mit budet liegt ein V-Auxiliar vor, das keine Eigenbedeutung trägt, sondern lediglich Finitheits- und Kongruenzmerkmale ausbuchstabiert (§ 5.2). Zu letzteren gehört das Aspektmerkmal [+Perf], das bewirkt, dass als Belegung von $I^{0}$ nur der indikativisch-futurische Operator in Frage kommt (§ 4.6.2, Eintrag (46)). Da diesem morphosyntaktischen Aspektmerkmal keine semantische Aspektspezifikation entspricht, ist der semantische Aspekt der infinitivischen Verbform rabotat' für die Interpretation ausschlaggebend. Da dieses V-Auxiliar finit ist (Annotation an $\lambda x$ ), kann in Spec-VP der Subjektausdruck Anna generiert werden. 
Dies ist die Bedeutungscharakterisierung der oberen VP. Da das V-Auxiliar in (E2d) finit ist, ist das externe Argument hier bereits eingesetzt.

f. i. $/ \varnothing /$

ii. $[+\mathrm{I}]$

iii. $\lambda P_{[+\mathrm{V},-\mathrm{N},+ \text { Perf }]} \exists t\left[\left[\mathrm{t}^{0}\right.\right.$ BEFORE $\left.\left.t\right] \wedge[P t]\right]$

Hier kann nur der indikativisch-futurische I-Kopf projiziert werden (§ 4.6.2, Eintrag (46)), da nur er mit einer (formal) perfektiven Verbform kombinierbar ist. Er bringt den Verbmodus Indikativ $(\exists t)$ und das semantische Tempus Futur ein. Es handelt sich hier um kein I-Auxiliar, sondern um einen semantischen Operator (keine Kongruenzmerkmale; siehe § 4.6.2).

$$
\text { g. } \left.\exists t \exists s\left[\left[\mathrm{t}^{0} \text { BEFORE } t\right] \wedge[[t \subseteq \tau(s)]: s \text { INST [ANNA WORK }]\right]\right]
$$

Dies ist die Bedeutungscharakterisierung der IP. Im Vergleich zur oberen VP-Ebene in (E2e) ist die Topikzeit nun existenziell abgebunden. So ergibt sich ein Term vom Propositionstyp <t>.

h. i. $/ \varnothing /$

ii. $[+\mathrm{C}]$

iii. $\lambda p[\mathrm{DECL} p]$

Dies ist derselbe Eintrag für das 'unmarkierte' C-Element wie schon in (A2h).

i. $\quad \operatorname{DECL}\left[\exists t \exists s\left[\left[\mathrm{t}^{0}\right.\right.\right.$ BEFORE $\left.t\right] \wedge[[t \subseteq \tau(s)]: s$ INST [ANNA WORK $\left.\left.\left.]\right]\right]\right]$

Dies ist die Satzsemantik von (E1).

(E3) zeigt die Strukturbildung des Satzes in (E1).

(E3)

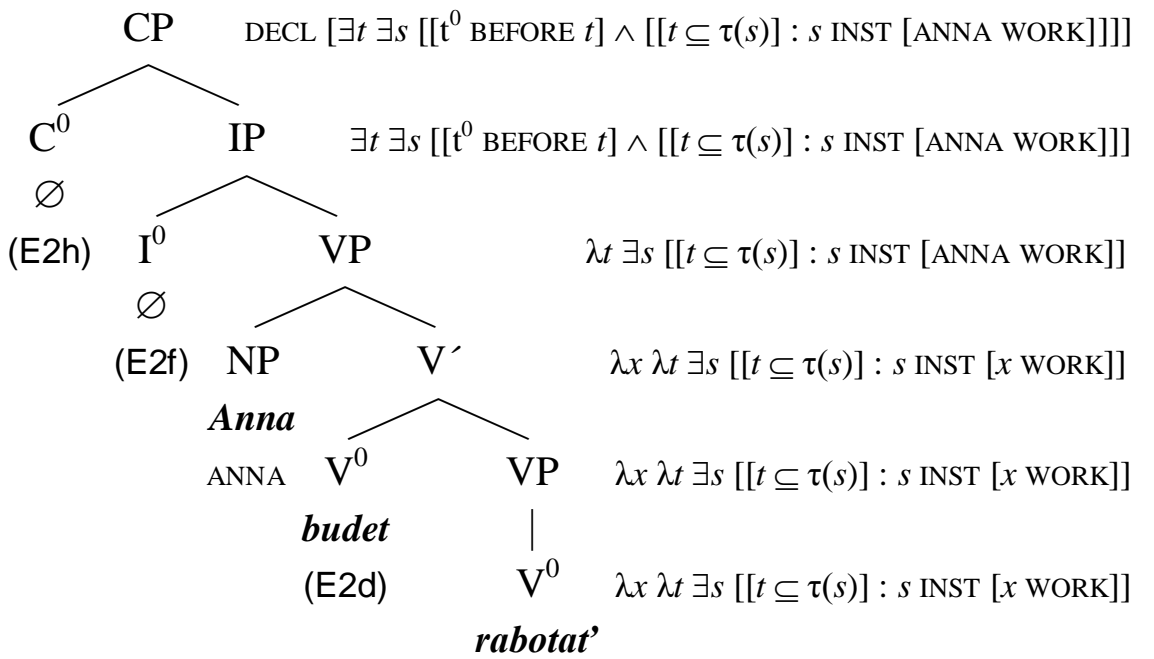




\section{F: Beispiel eines russischen Nicht-Kopulasatzes (perfektiv)}

(F1) My pročitaem knigu.

(Rus)

Anna PERF-les-1.PL Buch-ACC.SG.F

'Wir werden das Buch (durch)lesen.'

Das Prädikat ist monotransitiv und zeigt den perfektiven Aspekt. Die Interpretation des Satzes ist (daher) futurisch. (F2) bildet die V'-Ebene aus Verb und direktem Objekt ab.

(F2) a. i. /pročitaem knigu/

ii. $[+\mathrm{V},-\mathrm{N},+$ Perf, + max $]$

iii. $\lambda x_{[+1,-2,+\mathrm{PI}]} \lambda t \exists s[[\tau(s) \subseteq t]: s$ INST $[x$ READ A BOOK $]]$

Die Verbform ist finit, weshalb das externe Argument syntaktifiziert werden kann. Das direkte Objekt knigu '(ein) Buch' ist bereits als Komplement des Verbs eingesetzt. Die Repräsentation A ВОоК ist-besonders hinsichtlich der Kategorie der In-/Definitheit - vereinfacht.

b.

Die abstrakte Kopulabedeutung fehlt in diesem Nicht-Kopulasatz.

c. $\lambda t \exists s[[\tau(s) \subseteq t]: s$ INST [WE READ A BOOK]]

(untere VP)

Die Bedeutungscharakterisierung der unteren VP-Ebene beinhaltet auch den Subjektausdruck.

d.

In diesem Satz, liegt kein V-Auxiliar vor. Grundlage für die Selektion durch den geeigneten (futurischen) I-Operator sind bereits die morphosyntaktischen Merkmale der Verbform pročitaem, also des finiten Satzprädikats.

e.

Ohne V-Auxiliar liegt auch keine obere VP vor.

f. i. $|\varnothing|$

ii. $\quad[+\mathrm{I}]$

iii. $\lambda P_{[+\mathrm{V},-\mathrm{N},+\mathrm{Perf}]} \exists t\left[\left[\mathrm{t}^{0}\right.\right.$ BEFORE $\left.\left.t\right] \wedge[P t]\right]$

Auch in diesem Satz wird der indikativisch-futurische I-Kopf projiziert (§ 4.6.2, Eintrag (46)), da er sich mit (formal) perfektiven Verbformen kombinieren lässt. Siehe ansonsten (F2f). 
g. $\exists t \exists s\left[\left[\mathrm{t}^{0}\right.\right.$ BEFORE $\left.t\right] \wedge[[\tau(s) \subseteq t]: s$ INST [WE READ A BOOK]]]

Dies ist die Bedeutungscharakterisierung der IP.

h. i. $\mid \varnothing /$

ii. $[+\mathrm{C}]$

iii. $\lambda p[\operatorname{DECL} p]$

Siehe schon (A2h).

i. DECL $\left[\exists t \exists s\left[\left[\mathrm{t}^{0}\right.\right.\right.$ BEFORE $\left.t\right] \wedge[[\tau(s) \subseteq t]: s$ INST [WE READ A BOOK]]]] Dies ist die Satzsemantik von (F1).

In (F3) wird die Strukturbildung für (F1) gezeigt.

(F3) $\mathrm{CP} \operatorname{DECL}\left[\exists t \exists s\left[\left[\mathrm{t}^{0} \operatorname{BEFORE} t\right] \wedge[[\tau(s) \subseteq t]: s\right.\right.$ INST [WE READ A BOOK] $\left.\left.]\right]\right] \quad(\mathrm{F} 2 \mathrm{i})$

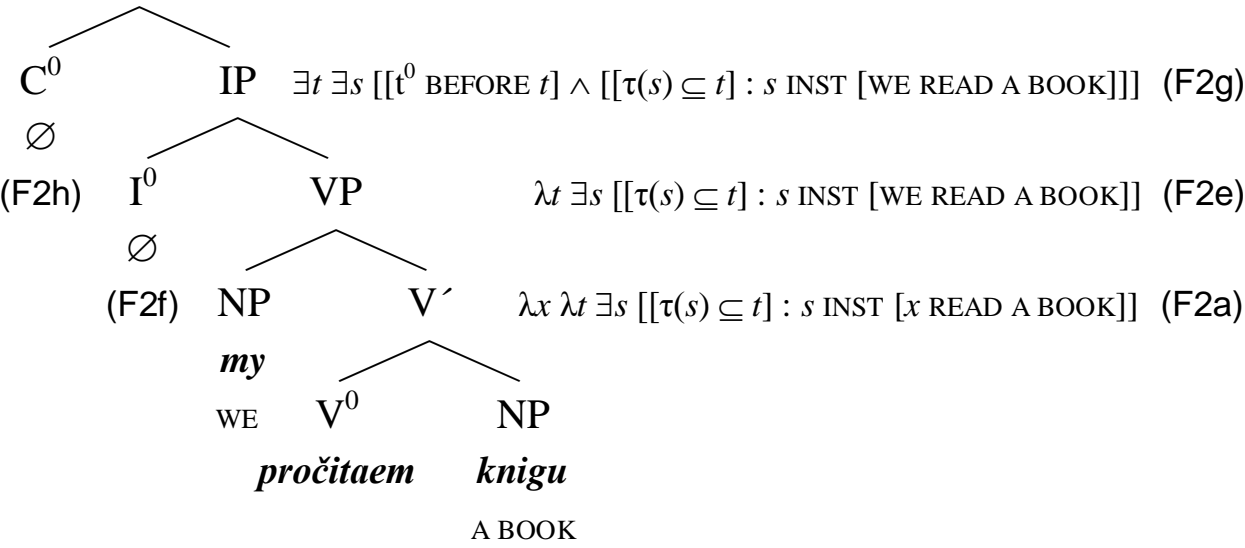

